\title{
LA PITTURA PARIETALE ROMANA COME FONTE DI CONOSCENZA PER LA SCULTURA ANTICA .
}




\section{La pittura parletale romana come fonte di conoscenza per la scultura antica}


Promotor : Prof. Dr. W. J. Th. Peters 


\section{La pittura parietale romana come fonte dl conoscenza per la scultura antica}

\section{PROEFSCHRIFT}

ter verkrijging van de graad van doctor in de letteren

aan de Katholieke Universiteit te Nijmegen

op gezag van de Rector Magnificus

Prof. Dr. J.H.G.I. Giesbers

volgens besluit van het College van Decanen

in het openbaar te verdedigen

op vrijdag 26 september 1986

des namiddags te 1.30 uur precies.

door

Erlc Maria Moormann

geboren te Boxmeer

NIJmegen 1986 



\section{Voor mijn moeder}



Prefazione .. . . . . . . . . . . . . . . . . . . . . 1

Parte I: Testo . . . . . . . . . . . . . . . . . . . 2

Introduzione . . . . . . . . . . . . . . . . . . . 3

Sviluppo della pittura parietale romana . . . . . . . . . . . . . . . . 4

La scultura greca o romana . . . . . . . . . . . . . . . . . . . . . . 9

P'jttura e scullura . . . . . . . . . . . . . . . . . . 12

Punti di partenza e obiettivi della presente ricerca. . . . . . . . . . . 13

I: Elementi scultorei nelle architetture dipinte . . . . . . . . . . . . . 16

Il stile . . . . . . . . . . . . . . . . . . . . . . . . . . 19 Zoccolo . . . . . . . . . . . . . . . . . . . . . . . . . . . . . . 19

Zona mediana. . . . . . . . . . . . . . . . . . . . . . . . . . . . 19

Zona supcriore . . . . . . . . . . . . . . . . . . . . . . . . . . . 25

III stile . . . . . . . . . . . . . . . . . . . . . . . . . 27

Zoccolo . . . . . . . . . . . . . . . . . . . . . . . . . 27

Zona mediana . . . . . . . . . . . . . . . . . . . . . . . . . . . . 29

Zona superiore . . . . . . . . . . . . . . . . . . . . . . . . . . . 29

IV stile . . . . . . . . . . . . . . . . . . . . . . . . . . . . 33

Zoccolo . . . . . . . . . . . . . . . . . . . . . . . . . . 35

Zona micdiana. . . . . . . . . . . . . . . . . . . . . . . . . . . . 37

Zona superiore . . . . . . . . . . . . . . . . . . . . . . . . . . . 46

II : Imitazioni di rilievi . . . . . . . . . . . . . . . . . . . . . . . . . . 51

I stile . . . . . . . . . . . . . . . . . . . . . . 52

II stile . . . . . . . . . . . . . . . . . . 53

III e IV stile . . . . . . . . . . . . . . . . . . . . . . . . . .

Conclusioni . . . . . . . . . . . . . . . . . . . . . . 56

III : Statue in rappresentazioni di giardini . . . . . . . . . . . . . . . . 57

III stile . . . . . . . . . . . . . . . . . . . . . . . . . . . . . . 61

IV stile . . . . . . . . . . . . . . . . . . . . . . 63

Conclusioni . . . . . . . . . . . . . . . . . . . . . . . . 67

IV : Modelli scultorei adoperati: opere a noi note e tipi perduti . . . . . . 69

Statue di divinità . . . . . . . . . . . . . . . . . . . . . . . . . . . 71

Statue di figure mitologiche . . . . . . . . . . . . . . . . . . . . . . . . . . 81

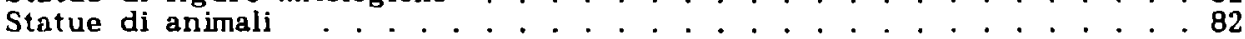

Erme . . . . . . . . . . . . . . . . . . . . . . . . . . . . . . 83

Altre figure mitologiche e umane . . . . . . . . . . . . . . . . . . . . 85

Conclusioni . . . . . . . . . . . . . . . . . . . . . . . . . 86

V: Stile e gusto . . . . . . . . . . . . . . . . . . . . . . . . . . . . . 87

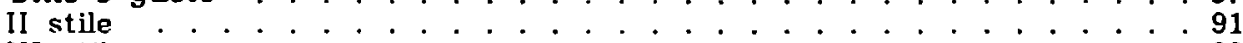

III stile . . . . . . . . . . . . . . . . . . . . . .92

IV stile . . . . . . . . . . . . . . . . . . . . . . . . 994

Rappresentazioni figurative . . . . . . . . . . . . . . . . 97

VI: Policromia e scelta del materiale . . . . . . . . . . . . . . . . . . 102

Bronzo . . . . . . . . . . . . . . . . . . . . . . . . . 103

Oro e doratura . . . . . . . . . . . . . . . . . . . . . . . . . . . 104

Argento . . . . . . . . . . . . . . . . . . . . . . . . 105

Marmo . . . . . . . . . . . . . . . . . . . . . . . 106 
Legno . . . . . . . . . . . . . . . . . . . . . . . . . . . . . . 108

Altri materiali . . . . . . . . . . . . . . . . . . . . . . . . . . . . 109

VII: Motivi scultorei nella pittura parietale postpompeiana . . . . . . . . 110

Decorazioni architettoniche . . . . . . . . . . . . . . . . . 110

Zoccolo. . . . . . . . . . . . . . . . . . . . . . . . . 110

Zona mediana. . . . . . . . . . . . . . . . . . . . . . . . . . . 110

Zona superiore . . . . . . . . . . . . . . . . . . . . . . . . . . 114

Rappresentazioni di giardini . . . . . . . . . . . . . . . . . . . 115

Scene figurative... . . . . . . . . . . . . . . . . . . 116

Conclusioni . . . . . . . . . . . . . . . . . . . . . 11

Conclusioni generali . . . . . . . . . . . . . . . . . . . . . . . . . . 119

1) Architettura . . . . . . . . . . . . . . . . . 119

2) Rilievi . . . . . . . . . . . . . . . . . . . . . . 120

3) Giardini . . . . . . . . . . . . . . . . . . . . . . . . . . . . . 120

4) Modelli scultorei . . . . . . . . . . . . . . . . . . . . . 121

5) Policromia . . . . . . . . . . . . . . . . . . . . . . . . . 121

6) Pinakes . . . . . . . . . . . . . . . . . . . . . . . . 122

7) Statue o figure vive . . . . . . . . . . . . . . 122

8) Collocazione e funzione . . . . . . . . . . . . . . . . . . . . . . 122

9) Combinazioni . . . . . . . . . . . . . . . . 123

10) Raccolta dei tipi . . . . . . . . . . . . . . . . . . . . . . . . . 123

Samenvatting ........................... 124

Inleiding . . . . . . . . . . . . . . . . . . . . . . . . . . . . . 124

Hoofdstuk I . . . . . . . . . . . . . . . 124

lloofdstuk II . . . . . . . . . . . . . . . . . . . 125

Hootdstuk Ill . . . . . . . . . . . . . . . . . . . . . . . . . . 125

Hooldstuk IV . . . . . . . . . . . . . . . 126

Hoofdstuk V. . . . . . . . . . . . . . . . . . 126

Hoofdstuk VI . . . . . . . . . . . . . . . . . . . . . . . . . 127

Hoofdstuk VII . . . . . . . . . . . . . . . . . . . 127

Conclusies . . . . . . . . . . . . . . . . . . . . . . . . . . . . . . 128

Appendice . . . . . . . . . . . . . . . . . . . . 129

Arcaismo . . . . . . . . . . . . . . . . . . . . . . . . . . 129

Quinto secolo . . . . . . . . . . . . . . . 129

Policleto . . . . . . . . . . . . . . . . . . . 129

Quarto secolo . . . . . . . . . . . . . . . . . . . . . 129

Lisippo . . . . . . . . . . . . . . . . . . . . . . . . . 129

Ellenismo . . . . . . . . . . . . . . . . . . . . . . . . . . 130

Muse . . . . . . . . . . . . . . . . . . . . 130

Hüfthermen . . . . . . . . . . . . . . . 130

Rilievi . . . . . . . . . . . . . . . . . . . 130

Periodo romano . . . . . . . . . . . . . . . . . . . . 130

Arte egizia

Parte II: Catalogo 132

Premessa . . . . . . . . . . . . . . . . . . . . . . . . . . . . . . . 133

Abbreviazioni e segni convenzionali . . . . . . . . . . . . . . . . 134

Alessandria . . . . . . . . . . . . . . . . . . . . 135

Bonn . . . . . . . . . . . . . . . . . . . . . . . 136 
-iï-

Bordeaux .. . . . . . . . . . . . . . . . . . . . 137

Boscoreale . . . . . . . . . . . . . . . . . . . . . 138

Boscotrecase . . . . . . . . . . . . . . . . . . . . . 141

Caivano . . . . . . . . . . . . . . . . . . . . . . 142

Castellammare di Stabia ....................... . 143

Catania . . . . . . . . . . . . . . . . . . . . . . 149

Cittè del Vaticano 150

Dura Europos .......................... . 151

Efeso ........................... 152

Ercolano . . . . . . . . . . . . . . . . . . . . . . . 155

Famars . . . . . . . . . . . . . . . . . . . . . . 167

Londra . . . . . . . . . . . . . . . . . . . . . . . . . 168

Luxor . . . . . . . . . . . . . . . . . . . . . . . . . . . . . . . . . 169

Luzern . . . . . . . . . . . . . . . . . . . . . . . . . . 170

Magdalensberg . . . . . . . . . . . . . . . . . . . . 171

Mariemont 172

Mérida . . . . . . . . . . . . . . . . . . . . . . . . . . . 173

$\begin{array}{ll}\text { Milano } & 174\end{array}$

Napoli . . . . . . . . . . . . . . . . . . . . . . . . 175

New Haven Conn. 195

New York . . . . . . . . . . . . . . . . . . . . . . . 196

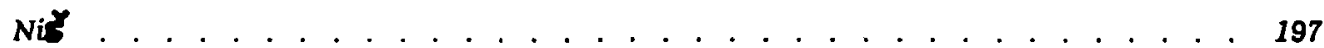

Ostia ........................... 198

Palmira . . . . . . . . . . . . . . . . . . . . . 202

Parigi . . . . . . . . . . . . . . . . . . . . 203

Pompei . . . . . . . . . . . . . . . . . . . . . . . 204

Portici . . . . . . . . . . . . . . . . . . . . . 315

Pully . . . . . . . . . . . . . . . . . . . . . . 316 
Richmond . . . . . . . . . . . . . . . . . . . . . . 317

Roma . . . . . . . . . . . . . . . . . . . . . 318

Sabratha ....................... 338

Selguk. . . . . . . . . . . . . . . . . . . . . . . . . 339

Solunto . . . . . . . . . . . . . . . . . . . . . . 340

Sparta . . . . . . . . . . . . . . . . . . . . . . 341

Sperlonga . . . . . . . . . . . . . . . . . . . . . . . 342

Svizzera . . . . . . . . . . . . . . . . . . . . 343

Tivoli .......................... 344

Torre Annunziata . . . . . . . . . . . . . . . . . . . . . . . . . 345

Treviri . . . . . . . . . . . . . . . . . . . . . . 348

Tripoli . . . . . . 349

$\begin{array}{ll}\text { Tuscolo } & 350\end{array}$

Zliten $\quad 351$

Bibliografia . . . . . . . . . . . . . . . . . . . . 352

Curriculum vitae . . . . . . . . . . . . . . . . . . . . . 372 
Questa dissertazione e ll risultato di una ricerca svoltası dall'autunno 1981 dlla primavera 1985, grazie a borse di studio della Rotary Foundation e clella Organızazione Olandese delle Ricerche (ZWO) Desıdero ringrazıare 1 colleghı dell'Istituto di Archeologia dell'Lniversita di Aapolı, dell'Istıtuto Olandese a Roma, e dell'Istituto Germanico a Roma, per la loro ospitalita e per l'assistenza durante le ricerche biblıografiche I soprintendentı di \apoli e Pompel di questl annl, 1 prof $F$ Zevl Dott ssa $M G$ Cerull Irell e Dott ssa E Pozzı Paolinı, hanno dato l permessı dı studjo e di pubblicazıone del materiale in merito Fra le persone che hanno fornito delle informazioni e delle toto ricordo A Allroggen-Bedel, A Barbet $\mathrm{M}$ Mani, V M Strocka H Wrede $H$ Mielsch $\mathrm{e}$ - soprattutto - $\mathrm{P}$ Moreno che ha letto ll testo criticamente L'italiano non sarebbe stato comprensibile se non fosse stato Inveduto a corretto dagli amici Antonella Allegrı e Andrea Vivit

Ledico l lavoro a Ma Madre che ha sempre stimolato e seguito con unteresse 1 miel stud, 



\section{PARTE I: TESTO}



La pittura parjetale dell'epoca romana e, nelle sue espression piu rieche, caratterizzata da llusione ln essa viene creato un ambito di edifici fantastici, paesaggı e graidinı al di la della realta del luogo dove sı trovano le decorazionı Lo spettatore crede che si trovi al'interno di un palazzo o nel parco di un princıpe ellenistico Tre sono 1 mezzı, peraltio molto affinı, deı qualı dispongono 1 decoratorı per oftenere una sumle llusione

In primo luogo si imitano materialı preziosı per far sembrare l'ambiente piu sontuoso di quanto permettano 1 mezzi linanziari Vari tipi di marmo tra 1 qualı alcunı rarıssimı, ornamentı metallic $\mathrm{e}$ anche pietre (semi)preziose sono stati umitati sulle pareti ${ }^{1}$

In secondo luogo si costituisce un un certo senso un nuovo spazio dietro allo spazıo reale, 1 che risulta nella presenza di porticı, giardinı con una tholos o un altro edıfıcıo o dı prospettı dı parchı e paesaggi - piu o meno coltivati ${ }^{2}$

In terzo luogo 1 pittor arredano lo stesso ambiente o quello creato con imitazionı di oggettı d'arte I quadrı, chiamatı con ll termine greco pinakes, sono gli elementı decorativi piu diffusi li troviamo in lutte le partı delle decorazion parjetali e la loro presenza trasforma 1 vani in vere e proprie pinacoteche 1 pittori pero non si lumitano a queste imitazionı 'logiche' d'altro canto vasellame piegevole, maschere teatralı e tappetı, tigure mitologiche e umane si insei iscono nelle decorazionı gia nelle tasi piu antiche Non stupisce quindı in questo mondo llusionistico la presenza di innumerevolı statue ed e proprio ad esse che e dedicato questo studio

Ognı qualvolta si incontrano figure 'a se stantı' - che rappresentano divinita o figure umane, ma anche anumali o esserı tantastici -, cl si puo domandare, come sıano state concepite talı tigure sono esserı viventı, reałmente presentı nello spazı immaginario, oppure imitazion 1 di statue o di gruppi statuari? $E^{\prime}$ evidente che in questo conlesto, pei avere una risposta, cl si debba rivolgere a quanto e rimasto della scultura antica $e$ a quanto sappiamo sull'applicazione di sculture alle costruzionı edilizie 1 datı pero di cui stamo in possesso per giudicare la ccultura antica in se stessa e la sua applicazıone all'architettura sono numericamente diversı, infattı 1 primı sono molto piu numerosi Verranno studiat parallelamente tutt e due 1 campl al fine di stabilirne la relazione reciproca $e$ di fornire una risposta alla domanda precedentemente formulata

Prima di entrare in argomento mi e parso necessario riassumere la storia della pittura parietale e ricapitolare 1 risultat 1 degli studi sulla scultura greco-romana che sono rlevanti per la ricerca attuale

1 Cf Eristov 1979, Gnolı 1971

2 Cf Drerup 1957, 1959, Fittschen 1976

3 Cf Van Buren 1938, Schefold 1952, 1972 
Nella ricerca sul campo della pittura pariotale l'interesse maggiore è dedicato allo sviluppo cronologjco e stilistico. Molti studi si occupano delle regioni dove sono state trovate ingenti quantita di pitture, in particolare Roma e la Campania, e limitatamente al periodo nol quale queste pitture sono state proclotte, e cioe i due primi secoli avanti a dopo $C$. Partendo dagli studi di A Mau dividiamo la pittura di questo periodo in quattro 'stili'. ' L'aggettivo 'pompeiano', usato da Mau e relativo al materiale della zona pompeiana, potè essere cancellato, quando si constato il valore della suddetta suddivioni per complessi in altre aree dell'Impero. Pur essendo non adat1o il termine 'stile' è bene mantenerlo finché non si stabilisca una cronologia piú raffinata secondo generazioni, parallela per esempio ai periodi di governo degli imperatori - si ricordino 'Louis $\mathrm{XV}$ ', Louis $\mathrm{XVI}$ ' ecc. Molti studiosi che seguono le sue orme hanno rispettato questa periodizzazione che sovente non lascia aperta la possibilità di un influsso mutuo e del fenomeno che vari 'stili' siano rimasti in uso contemporaneamente durante un certo periodo o che, in una fase piu recente, forme di uno stile anteriore sjano state riprese, anzi imitate. Anche dopo Mau le proposte di suddivisioni degli stili in fasi mostrano questo difetto. Finora in molti casi sembra impossibile ricostruire uno sviluppo 'logico' $e$ in tale modo stabilire una cronologia fissa, come sostengono studiosi quali W. Ehrhardt e W.J.Th. Jeters."

Il I stile (300-60,200-80 a.C. $)^{6}$ consiste in imitazioni di incrostazioni marmoree a forma di rilievi a stucco. Elementi figurativi sono scarsi.' Statue dipinte mancano: forse alcune imitazioni di rilievi figurativi, ora perdute, appartengono a decorazioni del I stile. Esempi di questo stile ad incrostazione sono stati trovati nell'intero mondo ellenistico-ronano.

Il II stile (90-10/80-10 a.C.) si caratterizza attraverso l'imitazione accurata di materiali e elementi costruttivi. In generale i pittori spendono molta attenzione negli elementi architettonici e plastici, per cui si usa il nome di stile architet tonico.

H.G. Beyen divise il II stile in due fasi, stabilite sulla base di criteri stilistici. In un determinato numero di casi Beyen potè basarsi su dati archeologici per fissare una cronologia; i criteri esterni pero rimangono scarsi e deboli. La prima fase viene suddivisa in tre subfasi, la seconda in due. Finora la cronologia di Beyen viene accettata comunemente.

La fase Ia $(90-75 ;(30-70)$ costituisce una traduzione in piano del I stile, cioe senza il rilievo a stucco." Appaiono ora diversi elementi architettonici che suggeriscono una profondità. Gli elementi figurativi, invece, sono rari e le statue dipinte non sono ancora presenti.

La fase Ib $(75-60 / 70-50)$ si sviluppa dalla fase la, ma contiene come elementi innovatori dei prospetti nella zona superiore della parete. 'L'uso di

4 Mau 1882. Riassunto tuttora valido: Beyen 1965.

s Ehrhardt 1982; Poters 1982a, 645.

- Le date indicate fra parentesi sono prese da Beyen 1965. La seconda data riguarda la Campania. Per il I stile non esiste una monografia. Cf. Mau 1882, 11-123; Laidlaw 1975; 1976; Bruno 1969. Cf. Baldassarre 1984.

7 De Vos 1977, 29-36; De Vos/Martin 1984.

- Beyen 1938, 37-60. Cf. Engemann 1967, 15-101. 
figure rimane limitato, ma si trovano esempi di sculture Un complesso importante costituisce la Vlla del misterı a Pompei (cat 306) dove ll cubicolo 4 contiene una galleria di statue esposte su finto podio 10

La tase Ic (60-50/50-40) mostra parels con larghi prospettı e con numerosi elementi architettomici 11 I prospett suggeriscono con pieno vigore uno spazio o una fuga dı spazı dietro l'ambıente reale, separati l'un l'altro da una parete divisorid (Scherwand) Nelle strutture architettoniche cresce l'uso di statue a puisa di acroters e atlanti/cariatidl Complessi importanis sono la Vlla di $P$ lannus Svnistor a Boscoreale (cat 004) e la Vula detta di Poppaed a Oplontis (cat 311)

La fase IIa (50-30/40-25) fa sempre uso di prospett 1 e di imitazioni $d_{1}$ incrostazioni marmoree, ma tutto presenta formc meno pesantı e chidre 12 L'architettura comincid a diventare piu esile e meno composta $S_{1}$ incontrano 1 prims quadrs o paesaggs suı campi centralı Per quanto riguarda la scultura prevalgono atlantı e cariatidi Inoltre si vedono figure esposte in nicchie o su podı Esempı interessantı sono la Casa del criptoportico (cat 148) e la Casa deglı epigramm (cat $185 / 2$ )

La fase lIb $(30-10 / 25-10)$ non possiede piu architetture pesantı, bensi piatte e snelle ${ }^{13}$ La parte centrale nella zona mediana non di rado e occupata da un paesaggio sacro-1dullaco o una scena mitologica I prospetti rumangono in vigore nella zona superiore A Roma la Casa di Livia (cat 317), la Casa di Augusto (cat 316) e la Villa della Farnesina (cat 319) sono importanti, a Pompel si possono menzionare la Casn di Obellius Iirinus (cat 302) e la Caserma des gladiatorı (cat 197)

Sull'origine del Il stile, e consequentemente della maggior parte della pittura dal primo secolo in poi, non esiste consenso La maggior parte delle pitture e stata trovata in Italia Beyen suppose come paese di origine dei singoh elementı l'Oriente ellenistico, ma considero il concetto un'invenzione romand ${ }^{14} \mathrm{~J}$ Engemann si oppose all'ipotesı di un'origine orientale e considero le pitture creazioni romane, sia per la composizione sia per gli elementı singolı, che sarebbero talmente diffusı nel bacino mediterraneo da non poterlı caratterizzare come 'orientalı' o 'occidentali' 15 F G Andersen e $\mathrm{G}-\mathrm{Ch}$ Picard condivisero questa opinione 16 Phyllis Wlliams Lehmann espresse una opinione simle nel suo studio sulla Villa di P Fannius Synistor a Boscoreale, ma la abbandono dopo le scoperte di pitture parietalı in complessi tombalı di epoca ellenstica in Macedonia $1 \% \mathrm{~K}$ I ittschen propose un'origine ellenistica in

- Beyen 1938, 61-88, Engemann 1967, 15-101

10 In prosieguo si menziona sempre la citta, se non e Pompel Il numero fra parentesı rumanda al catalogo, dove accanto alla descrizione si trovano 1 riferumentı bibliografici che non vengono piu citatı nel testo per non appesantire l'apparato di note

11 Beyen 1938, 89-318 Cf Engemann 1967, 102-104

12 Beyen 1960

13 Non esiste ancora uno studio approfondito Si veda Beyen 1965

14 Beyen 1938

15 Engemann 1967

16 Anderson 1977, Picard 1977

17 Lehmann 1953,1979 
base alla presenza di decorazioni del genere nell'Oriente con articolazioni e elementi simili. Nel suo contributo al colloquio di Göttingen. Hellenismus in Mittelitalien (già un titolo programmatico!), sostenne la sua ipotesi attraverso un notevole numero di esempi." Anche $K$. Schefold si è espresso a favore di questa opinione. ${ }^{19}$

Probabilmente è pjú corretto, invece di limitarsi all'una o all'altra ipotesi, supporre l'amalgama di elementi da tutte le parti della koine ellenistica, nella quale le varie regioni hanno contribuito con le loro componenti specifiche senza che si possa parlare della superiorità di un'area o città in particolare. II traffico era lalmente intenso, che i nuovi sviluppi nellarte trovavano velocemente un mezzo per diffondersi su larga scala. La produzione scultorea in merito ne è una prova. Sembra evidente che allinizio di uno sviluppo del genere, peraltro non ancora distintamente conosciuto, venissero piú elementi innovatori dall'Oriente che non dall'ambito romano, visto lo stimolo culturale dai centri greci 'classici' e ellenistici nel mondo romano iniziato nel terzo secolo in. C.

La qualita tecnica delle pitture di Il stile trovate a Pompei è considerevole, quando si tiene presente che erano già antiche al momento delleruzione del Vesuvio nel 79 d.C. Anche altrove in Campania, specie nelle ville di Boscoreale. Castellammare di Stabia (Varano), Torre Annunzlata e Portici si trovano vasti complessi. Stupisce cle ad Ercolano il numero e limitato ad un vano nelle terme della Casa dell'albergo ed a frammenti provenienti dalla Villa dei papiri. ${ }^{20}$ Dopo la pubblicazione del secondo tomo della Wanddekoration di Beyen nel 1960, sono state recuperate la grande villa di Torre Annunziata e alcune case a Pompei: tutti ritrovamenti che hanno confermato le sue ipotesi. Anche altri ritrovamenti avvenuti sia in Italia che all'estero, si possono inserire facilmente nella suddivisione di Beven. 21

A Roma le pitture di II stile si trovano sempre in vani riusati come strutture sotterranee o di fondazione per complessi costruiti posteriormente, a parte la Casa di Livia che e rimasta conservata come memoria dell'imperatrice.

A Roma $e$ in Campania abbiamo di solito a che fare con decorazioni di alto livello tecnico eseguite da pittori ben istruiti. I proprietari delle ville apprezzarono apparentemente queste decorazioni e non sentirono il bisogno di sostituirle con nuove pitture.

Il terzo stile inserisce soltanto in modo modesto brani architettonici ben articolati nei sistemi decorativi, benche la struttura dei prospetti e delle scacnae irontes non svanisca mai completamente. I prospetti architettonici scompaiono quasi del tutto e vengono sostituiti da elementi lineari. Lo stile ha inizio nell'epoca di Augusto ed è un chiaro esempio della nuova corrente classicistica stimolata dallo stesso imperatore. ${ }^{22}$ Il termine finale non e del tutto chiaro e quindi molto discusso. Cio coincide con il problema dell'inizio del IV stile. Schefold ipotizza che il terremoto del 62 costituisca la cesura fra

18 Fittschen 1976.

19 Fra l'altro Schefold 1975; 1977; 1982. Cf. anche Lauter 1971; Tybout 1979.

20 Cf. Moormann $1984 b$.

21 P.e. Ancona: EAA Suppl. (1970) tav. a col. di fronte a p. 56. Bolsena: Barbet 1971, 332-338. Brescia: Mielsch 1981, 170, 249. Sette Finestre: De Vos e.a. 1982. Glanum: Barbet 1974, 11. Massada: Yadin 1966, 44-54, 78-79. Sulmona: Van Wonterghem 1984, 247, 250, fig. 347-348.

22 Cf. Zanker 1970; 1974; Zanker/Preisshofen 1979; Art 1982. 
III e IV stale, almeno per quanto riguarda la Campania ${ }^{23}$ A Roma lo stile sarcbbe stato 'creato' per un grande inonumento dell'umperatore Nerone, dopo $d_{1}$ che si sarebbe diffuso nel resto dell'Impero. Beyen osservo 1 primi cennı del IV stile durante l'umpero dı Calıgola o Claudıo, mentre contemporaneamente sarcbbero rimaste di moda decorazion di III stue. ${ }^{24}$ La Domus Aurea tuttava signitucherebbe il termine del III stule. F.L. Bastet, in vari articol e nella sua monografia sul IIl stule, elaboro le teorie di Beyen circa una datazione alta del IV stile e tento di dimostrare che sotto Claudio L III stile avrebbe avuto termine. ${ }^{25}$ Marıa Manni ha presentato argomentı validi per un influsso reciproco e per un'esistenza contemporanea del due stzl, almeno per quanto riguarda Ercolano, la Casa del colonnato tuscanico ne sarebbe un esempio lampdnte. ${ }^{25}$

Bastet ha proposto una classificazıone del III stue analoga a quolla di Beven per 1 II stle. ${ }^{27}$ Egll presenta questa suddıvisione.

Ld fase la $(20-10$ a $C$.$) contiene ancora gli elementı della fase llb del II$ stile, ma con una scomparsa progressiva di essı 29 In linee generalı 1 sistemı sono ancora basati sull'architettura, mentre lo zoccolo e le zone mediana e supcriore costituiscono ancora un insieme. Lat parte superiore dello zoccolo mostra una certa profondita e le colonne o candelabru - elemento nuovo poggiano su membrı aggettantı Motıvı linearı e vegetalı sono applicat in numero considerevole

Aella fase Ib (10-1 a.C ) glı elementı architettonicı duventano sempre piu subordinati, perdono la loro tunzione e diventano quasi irriconoscibili 29 Le zone orizzonatalı sono sempre piu 1solate. La protondita manca quasi completamente Nella zona superiore si incontrano forme architettoniche bizzirre Girall e tirsi sostituiscono elementi architettonici

La fase lc (1-25 d C ) e il III stule nel pieno vigore la parete viene suddivisa in campl attraverso motivi lineari, non plu con colonne o canclelabri ${ }^{30}$ Solo la cona superiore contiene scaenae trontes. La predella si e sviluppata completamente e acquista una maggiore importanza. Talvolta un tregio con pinakes ripete, tra le zone mediana e superiore, la predella.

Fase IIa $\left(25-35\right.$ d $C$ ) e il cosiddetto III stile declinante. ${ }^{32}$ Entrano nello zoccolo elementı eterogenel qual prante e uccellı Nella zona mediana si presentano di nuovo candelabrı e giralı La zona superıore sembra cercare di nuovo un nesso organico con la zona mediana

La fase IIb $(35-45)$ contiene il numero maggiore di complessi e mostra una larga scala di possibulita. ${ }^{32}$ Tale diversita anticipa il linguaggio del IV stile.

23 Schefold 1962 Cosı anche Croisille 1983.

24 Beyen 1940, 1958; 1965.

25 Bastet 1964; 1971; 1972, Bastet/De Vos 1979, 12-16.

26 Mannı 1974.

27 Bastet/De Vos 1979.

2 Bastet/De Vos 1979, 24-34.

29 Bastet De Vos 1979, 42-52.

30 Bastet/De Vos 1979, 42-52.

31 Bastet/De Vos 1979, 53-61.

32 Bastet/De Vos 1979, 62-99. 
Nello zoccolo dominano element 1 fantastici Nelle zone mediana e superiore vanno integrandosı elementı architettonics in forme abbastanza realistiche Le due zone costituiscono di nuovo un'unita vella zona superiore, tuttavia, si vedono anche elementı isolat.. Svluppatisi in modo indipendente ${ }^{3} 3$

Il numero di decorazioni di III stile e poco maggiore di quello di II stile, ma ancora limitato sumumente sono rumaste conservate le decorazionı di livello superiore, custodite con cura daglı abitantı delle case Spccie le ville e alcune case a Pompel cl forniscono il materiale, ad Ercolano e tuor Campania 1 complessı sono piu rari In Francia sono statı trovatı alcunı complessı importantı di III stile, studiatı di recente da Alix Bdrbet 34

Come e stato detto sopra la tase di transizione fra ll III e 1 IV stile c problematica, altrettanto dificile e la determinazione della data piu alta 35 un inızio non esattamente collocable sotto Claudio o Verone e stato accettato da moltı, prudentemente argomentato da Mannı ${ }^{36}$ Pare sicuro che dopo al 62 non siano state prodotte decorazions di III st le

Schefold ammette un'introduzione del II stue in Campania dopo il terremoto del $62 \%$ Il periodo $62-79$ e stato suddiviso da lui in una tase neroniana $e$ in una flavia sulla base di argomentı stulıstıcı Per quanto riguarda l'inizio sono statı accertatı complessı neronıanı anterıorı a Roma Grazıe alle indaginı di Mariette de Vos un numero considerevole di decorazonı di IV stule puo essere datato prima del 62 partendo da datı archeologicı, per cuı la presenza del IV stile nel'epoca di Claudio o Nerone o tatta probabile per la regione campana ${ }^{3}$

Ln altro terremoto non menzionato nelle fontl che avrebbe colpito Pompel fra ll 62 e ll 79 non puo esserc accertato ${ }^{39}$ La suddivisione stilistica non puo nemmeno escere accottata, viste le ricerche menzionate di De Vos e le analis accurate delle decorazion nella Casa del Vettı di Heide Lauter-Bufe e Peters 40

Sulla base dı uno studio deglı schein decorativi di IV' stile Peters presume che tuttora non possa essere stabilita una cronologia "i una suddirisione analoga a quella del II e del III stule, contorme allo sviluppo della

$33 \mathrm{Cf}$ le reazioni positive di $\mathrm{A}$ Barbet, $R A$ 1981, 366-367, $\mathrm{R}$ Ling, JRS 71 (1981) $212213, \mathrm{PH}$ von Blanckenhagen, AJA 86 (1982) 307-308, con W Ehrhardt 1982 \& quale annuncid uno studio sul problema del III stile

34 Barbet 1982, 1983

35 Cf I Bragantinı, in Pompel 1748-1980, 106-118, Moormann 1983a

36 Manni 1974, 14, 49-50

37 Schefold 1962

38 De Vos, 1977, 38-43, 1982, 336-338 note 50-51 Cf Peters 1977, 1982a Strocka 1984aa cerca di provare una datazione alta per le decorazionı non finite nella Casa del sacello lliaco (cat 149), cioe prima del 62

39 Schetold 1965 Cf Strocka 1984ab, 36-37 Tuttavia la possibule esistenza di un terremoto finord ignoto non puo essere scartata in una zona ad alto rischio sismico I clanni gravi e le dunensioni dei lavori di ricostruzione "equivalgono", pero, una menzione nelle fontı

40 Lauter-Bufe 1969, Peters 1977

41 Peters 1982a, 644-645 
composizione degli schemi, non è possibile. L'una accanto all'altra si vedono pareti composte (ipotattiche) e semplici (paratattiche), mentre elementi presi da vari sistemi di base (scaenae frons e decorazione a campi) sono stati combinati dando luogo a nuovi sistemi e variazioni. Già la Domus Aurea presenta una vasta varjetà di sistemi. 42

Piú difficile ancora è uno schizzo dello sviluppo della pittura parietale romana dopo i 79.43 Non si vedono nuovi sistemi composti da elementi prima inesistenti. Le decorazioni mancano in generale di complessita e si limitano a composizioni paratattiche con campi e lesene, spesso decorate a candelabri. L'interesse per le decorazioni parietali scema a favore dell'incrostazione marmorea e della decorazione a mosaici. Elementi figurativi rimangono presenti. ma sono sempre meno frequenti.

Un problema che ostacola le nostre osservazioni è la relativa scarsità di complessi ben conservati degli ultimi secoli dell'Impero. Ostia fornisce dati per il secondo e il terzo secolo, Efeso per il quinto. A Roma invece i ritrovamenti coprono tutto il periodo, ma non sono consistenti in senso topografico e cronologico. Inoltre molto materiale e andato perduto $e$ in piú raramente documentato. Nelle provincie dobbiamo accontentarci di frammenti sporadici, raramente ricomponibili in sistemi. Le osservazioni su questo periodo, presentate in un capitolo a parte, si basano unicamente sul materiale pubblicato.

\section{LA SCULTURA GRECA E ROMANA}

Evidentemente non è possibile presentare in breve un profilo dell'arte plastica dei Greci e dei Romani e della ricerca in merito. Le osservazioni seguenti comprendono soltanto temi direttamente concernenti la presente ricerca.

Nel materiale conservato dobbiamo distinguere fra originali, varianti e copie. Nel momento in cui la richiesta di opere d'arte greche comincio a crescere nell'ambito romano, specie a causa delle spedizioni militari in Grecia ed altre parti del mondo ellenistico, non vi erano piú originali sufficienti, sicche occorreva un grande numero di copie. Tale prassi non fini se non nel tardo Impero e anche nel nuovo classicismo sotto $\downarrow$ regno di Costantino si producevano copie piú o meno fedeli. Per questo disponiamo talvolta di numerose copie di un solo prototipo. In quanto gli originali quasi sempre sono andati perduti, il materiale non è solo di importanza per la conoscenza della scultura romana, del gusto dei Romani e della funzione e del collocamento delle statue, ma anche - e per molti studiosi soprattutto - per la conoscenza della scultura greca. La ricerca si è concentrata per lo piu sulla ricostruzione degli originali in base alle copie, sulla datazione di essi e sulla attribuzione ad un determinato artista. G. Lippold ha iniziato lo studio sui modelli con varianti ed ha cosi stimolato l'attenzione per l'aspetto romano delle copie dell'epoca imperiale. Lo stesso interesse porto agli studi di $H$. Jucker e G. Becatti per quanto riguarda le opinioni dei Romani riguardo allarte greca." 4 Oggetto

42 Cf. Peters/Meyboom 1982; Peters 1982b.

43 Non soddisfano il libro di Wirth 1934 con i confronti stilistici, le monografie di Borda 1958 e Dorigo 1969 solo descrittive e lo studio di Joyce 1982 che si limita ad analisi di schemi alquanto cnsuali, costruiti dall'autrice. Cf. Mielsch 1981, 219-232, 257-258.

44 Lippold 1923, 3-14; Jucker 1950: Becatti 1951. Cf Lauter 1967, 123-128; Trillmich 1973; Zanker 1974; Raeder 1978; Strocka 1979. 
principale erano - e sono spesso - le opere di valore artistico, gli opera nobilia, equivalente latino del titolo usato dallo scultore Pasitele per un suo libro sulla scultura greca $4 \mathrm{~s}$ Le sculture decorative prodotte per l'arredamento deı giardinı, ninfel e delle facciate di varı edifıcı, venivano appena coinvolte nella ricerca visto il loro livello artistico molto modesto Nella ricerca moderna sulla decorazıone del varı generı di edificl, invece, talı sculture ricevono guustamente un interesse maggiore 46

Conosciamo innumerevoli riferimentı e descrizioni di statue e di gruppi statuari che meiltavano attenzione per il loro valore artistico o per la loro funzione, raccolte da $J$ Overbech ${ }^{4} 7$ In un certo numero dı testı sembra che le prazze, 1 santuarı, I palazzi e le ville private fossero abbellitı da un gran numero di statue Sono rimaste conservate molte statue, anche se costituiscono solo una piccola parte di quelle orıginalı Quası mai sı puo convalidare la situazione descrittd attraverso un sopralluogo o uno scavo Statue con le loro bası sono rimaste in situ in cası molto rarı, esse vennero asportate o andarono perdute 49

A parte la scultura slessa e le font scritte rappresentazion su vasi, monete e gemme possono fornurcı datı importantı Questı generı di rappresentazionı sono statı oggetto di varı studı 49

Soltanto in alcunı casi la pittura parictale e stata usata come fonte di intormazione in pochi cası nelle pubblicazıonı si trovano riferimenti a sculture antiche, poiche le pitture sono state considerate confrontı inledel Entrarono nelle discussion solo quando si discussero I rilievi e I gruppı statuarı ellenistıcı $W$ klein considero un numero di gruppı statuarı derivazionı di quadrı famosi del quarto e del terzo secolo a $C$, del qualı 1 pinakes pompeianı a loro volta sarebbero statı le copıe 50 Nonostante la loro tridumensionalita le statue arrebbero una sola 'facciata' e corrisponderebbero alla pittura e al rulievo in tale modo non sarcbbero opere - per cosi dire - coinplete e consequentemente non potrebbero essere creazionı originalı Questa opinıone venne messa in dubbio da $G$ Krahmer che nel suo articolo sulle einansichtige Gruppen cerco di provare che nell'Ellenismo si era concepito un nuovo genere scultoreo statue che fossero da ammirare da un solo lato e che si esponessero entro nicchie o darantı a paret cicche $^{51}$ La pittura, secondo Krahmer, avrebbe scelto addiritura talj opere scultoree come modell per figure dipinte su pinakes Una trontalita simile si incontra gia nel periodo classico e viene messa in evidenza come un aspetto tipico della scultura del quarto secolo a $C^{52}$

45 Plin , N.H. XXXVl 39-40 Cf Preissholen 1979, 281-282

- $\mathrm{P}$ e Kapossy 1969 (fontane), Schwingenstein 1977 (teatrı), Manderscheid 1981, Marvin 1983 (terme), hreeb 1978, 1980, Dohl 1976, Dohl/Zanker 1979, Dwyer 1982, Raeder 1983 (case)

47 Overbeck 1868

". Cf per esempio le bası ad Olımpia (Arnold 1968) e Il monumento di Daocho a Delf 1 (Borbein 1973, 68-71) Sull'asportazione Pape 1975

49 Schefold 1937 Schnelder-Herrmann 1972, Lohmann 1979, 25-38 (ceramica), Lacrolx 1949 (monete), Horster 1970 (gemme)

50 Ḱlein 1910, 1919

51 Krahmer 1927

32 Borbein 1973, 63, 73, 139 Anm 410 
Fino a poco tempo fa si è prestata poca attenzione allo studio delle parti plastiche dell'architettura, ${ }^{53}$ cioe al modo di applicare statue nell'architettura e alla funzione di esse in un tale contesto. Le architetture conservate non ci hanno tramandato molto materiale in merito, ma studi recenti su problemi dettagliati hanno fornito dati di grande valore. ${ }^{54}$ Studi sull'esposizione di statue in teatri, in complessi termali, nelle ville e case private, apparsi nell'ultimo decennio, hanno contribuito considerevolmente alla nostra conoscenza (sopra nota 46 ).

Recentemente la ricerca ha cominciato ad occuparsi della collocazione e della funzione di statue nell'antica Pompei - importante esempio di una città 'media' romana e quindi ronte essenziale per l'intero mondo romano. Nella sua inonografia Klassizistische Statuen P. Zanker fa cenno alla possibile importanza di pillure parictali. tanto quanto rilievi e altri generi di prodotti artigianali, come fonti di informazionj riguardo al gusto dei Komani. ${ }^{55}$ In parte egli stesso ci entra nel suo articolo Die Villa als Vorbild des pompejanischen Wohngeschmacks. ${ }^{56}$ Zanker conclude che la produzione di copie, specie di opere classiche e classicheggianti o classiciste, e stata determinata dai possessori di ville e che la borghesia nel'epoca imperiale si e fatta influenzare da questa corrente. Le case private pompeiane imitavano le ville in campagna, pur con un repertorio ristretto di possibilita e variazioni. ${ }^{7}$

E.J. Dwyer, nel suo Pompeian Sculpture. A Study of five Pompeian Houses and their Contents, ha descritto e analizzato tutti i mobilia - per quanto ritrovabili nei depositi del Museo Nazionale di Napoli - di cinque case." Grazie ai diari di scavo e altro materiale d'archivio egli è riuscito a ricostruire l'inventario delle cinque case al momento dell'eruzione del Vesuvio nel 79 d.C.: basandosi su questi dati ha cercato di ricostruire l'arredamento. Le decorazioni parietali e pavimentali purtroppo non sono state prese in considerazione. Benché lautore non sia riuscito a rispondere a tutte le domande e non abbia discusso tutta la problematica in merito, il libro $\dot{e}$ un contributo di grande importanza per lo studio dell'interno delle case private nel mondo romano. ${ }^{9}$

Nel suo bel libro sui giardini campani Wilhelmina Jashemski dedica molta attenzione alle statue topiarie e alle rappresentazioni di giardini su pitture parietali. ${ }^{\circ}$ Anche le statue dipinte vengono discusse brevemente. Il contributo dei De Vos alla monografia di H. Eschebach sulle Terme stabiane

53 Non intendo la pura Bauornamentik. Cf. p. e. von Mercklin 1962 sui capitelli e von Hesberg 1980a sulle cornici a mensola.

54 P.e. Schaller 1973; Schmidt-Colinet 1977; Schmidt 1982 (figure reggenti); Drerup 1957; 1959 (lusso architettonico); Hornbostel-Hüttner 1979 (nicchie); Lauter 1971; 1973; 1979; Lyttelton 1974; Bacchielli 1980; Bauer 1983; von Hesberg 1980a (ornamentazione e particolarita).

55 Zanker 1974, XVIII, citato nota 179.

56 Zanker 1979a.

57 Cf. Döhl/Zanker 1979; Döhl 1976; 1982.

5 \& Dwyer 1982 .

59 E.M. Moormann, rec. Dwyer 1982, BABesch 59 (1984) 216-217. Altre recensioni: H. Döhl, Gnomon 52 (1980) 693-694 (sul'edizione in fotocopia del 1974); R. Ling, JRS 73 (1983) 229-230.

60 Jashemski 1979, 55-87, passim. 
contiene una siliossi di tutte le rappresentazionı di giardini assieme ad un'analisi degli element, unseriti 61

Finora manca und messa a punto moderna sulla scultura pompeiana, dato che la Habilitationsschrift di 11 Dolil su questo irgomento non e ancora apparsa ${ }^{62}$ Sono infattı tuttora indispensabilı la sinossi di Mau e J Ovesbech e l brev 1 riassuntı di Dohl e Zanker ${ }^{6} 3$

\section{PITTURA E SCULTURA}

Da quanto e stato detlo fimord si puo concludere che und ricerca sulla presenza e funzione di statue nella pittura parietale e molto utle Tuttas a neglı studı sulla pitturi parıetale non si ritrovano moltı cennı in merito

Vella pruma meta del vovecento si prestava maggiore attenzione alle scene figurative specialmente al pinakes Vel caso che qualche figura mostrasse un certo paidlelismo con una statua, se ne faceva menzione $A$ volte venne discussa la questione delloriginale cioe se la figura dipinta, quasi sempre Idppresentante und dilinita o un eroc mitologico, tos ae una copia dell'originale della ccultura mon<ionate $o$ se la pitturd fosse solo l prototipo per la ucultura A Sogliano per esempio ammise che l gruppo di Dirce su un pinal nella Casa des lettil (cat 217 ) fosse ld riprodultone del Toro Earnese copic di un orjgunale ellenistico tiovato nelle Teime di Caracalla, che secondo lo scavatore della Casa clel vellil era servito da esempio per pittori e incisor Mau concor do con questa attrıbuzione Le innumerevolı differenze tra pittura e scultura vennero imputate in parte agli inadeguatı restaurı rinascimentalı e settecenteschi del Toro Iarnese, in parte alla liberta del pittore $e$ a limiti di rendere visibule la tridunensionalita in un'ummagine bidumensionale

$P$ Herrmann fa taliolta confronti fra scultura e pittura nel suol Denkealer der Maleret des Altertums, aggiungendo delle spotesı riguardantı l'archetıpo, cioe pittura o scultura ${ }^{4}$ La leda di Timoleo per minzionare un solo encmpio sarobue stata coplata alcune volte su pullhes pounpeidul ma non nella decoralione nella Casa della regina llargherita anche se essa si regge sopia un piedistallo (cat 190)

Inoltre disponiamo di numerosi studi sulle fontı del pinakes, nel qualı glı studiosi tentarono di attribuire scene mitologiche a famosi pittori classici o ellenisticl noti attraicrso le fonti scritte Le figure che mosiravano un certo parallelismo con una statua, erano impoitanti pei la datazione delloriginale 65

61 De Vos'De Vos 1979, spec 90-92

62 Dohl 1976 Cr Dohl 1982

63 Overbeck/Mau 1884, 532-563, Dohl Lanher 1979. Dohl 1982 Cf fra l'altro De Franciscis 1951, 1979, 1980, Cerull Irell 1982 (ritratts). Boyce 1937 (lararı), Ippel 1939 Adamo Muscettold 1979 (bronzettı). Castiglıone 1975, Elıa 1975 (sculturd repubblicana). Corswand 1982. Dwyer 1981, Paller 1982 (osclla), De Caso 1976 Pandermalss 1971. Sgobbo 19711972 (vلle), Richardson 1971 (casa), von Rohden 1880 (terrecotte)

64 Herrmann I-II

65 Per esempio Fuhrmann 1934 Veutsch 1940, Lippold 1951, Lauter-Bufe 1967 Cf anche Klem 1910, 1919 
Su figure in guisa di atlanti e cariatidi nella pittura parietale non si trovano osservazioni nelle ricerche pubblicate se non una breve caratterizzazione o descrizione sommaria. Beyen credette che la maggior parte di esse fossero da considerare figure fantastiche e che non potessero fornire dati importanti per la conoscenza della vera architettura ellenistica e dell'inscrimento di ligure di sostegno. ${ }^{66}$ Soltanto in pochi casi lo studioso olandese cito confronti nella vera architettura. La maggior parte degli archeologi segui questa opinione. ${ }^{67}$

Unia sola monografia e stata dedicata alle rappresentazioni di statue nella pittura pompeiana: nel 1977 Astrid Piretsghner concluse i suoi studi all'universjta di Innsbruck con la tesi Gofterstatuen auf pompejanischen Wandgem dalden. dattiloscritto mai pubblicato. ${ }^{6}$ In questo lavoro essa studia le statue di divinita in rappresentazioni figurative $e$, pur in pochi casi, in rappresentazionj architettoniche. Cerca di inventariare le figure in merito ed analizza quali dati possano fornire le pitture sulla policromia, sull'inserimento, sulla tunzione e sui modelli scultorei. Le sue osservazioni verranno discusse nei capitoli successivi. In questo momento possiamo concludere che il libro costituisce un contributo valido, ricco di materiale, ma la limitazione a scene figurative ha causato un'immagine lungi di essere completa della problematica. Proprio le categorie non inserite, cioe le strutture architettoniche, le vignette e le rappresentazioni di giardini, contengono dati indispensabili per questo argomento, come tenterò di dimostrare.

\section{PUNTI DI PARTENZA E OBIETTIVI DELLA PRESENTE RICERCA}

Lo studio presente si propone di raccogliere tutte le rappresentazioni di statue su pitture parietali $e$, basandosi sul materiale sistemato in un catalogo, di cercare risposte alle domande seguenti:

1. in quale modo le statue siano state inserite nelle scaenae frontes e nei sistemi decorativi derivati da esse e quanto questa applicazione corrisponda al collocamento di statue nelle architetture reali;

2. se le imitazioni di rilievi possano fornirci dati a proposito dell'uso di rilievi figurativi e all'origine di essi (sia greco-ellenistica o romana);

3. se le statue presenti nelle rappresentazioni di giardini corrispondano alla realta topiaria e se esse possano dare un contributo al'interpretazione di questa categoria specifica di decorazioni;

4. dove si vedano tipi greci classici, dove tipi ellenistici e dove tipi romani e consequentemente quanto siano informative per la soluzione del problema della produzione di copje a gusto dei homani per determinati periodi/stili e tipi statuari;

5. che cosa le pitture possano insegnarci riguardo alla policromia delle statue di marmo e la superficie di statue metalliche (per esempio doratura e patina);

\footnotetext{
66 Beyen 1938, 59-60, 80, 113 1960, 33-34.

67 P.e. Allroggen-Bedel 1976a; Schmidt-Colinet 1977.

6 a Pretschner 1977. Devo una xerocopia alla gentilezza di Maria Manni.
} 
6 quanto l'atteggiamento di figure mstologiche, presentı su pinakes, rifletta tipı scultore (e stata latta una scelta rappresentativa),

7 dove su debba distinguere fra figure esplicitamente dipinte come statue e tigure rappresentate come esseri viu,

8 se si possano trarre conclusioni rispetto alla collocazione e alla funzione di vere statue, per quale aspetto le scene figurative sono di rilievo particolare,

9 qualı statue si vedano combinate nelle pitture e se talı combinazionı slano o meno prese dalla realta,

10 quale sia la raccolta concreta dı tıpı notı, dı varıdentı e dı tipl non ancora conosciutı

Le figure sono state considerate come statue sulla base di uno o plu del seguentı criterı

1 lei sistem decoratıvı basatı sull'architettura le statue sono caratterizzate in maniera diversa

a dal momento che esse fungono dd sostegno nell'architettura a forma di cariatıde, atlante o erma,

b perche sorgono sopra un piedistallo entro una struttura architettonica o sı delineano su un campo, postı su una mensola,

c in quanto fungono da acroterio nelle architetture dipinte.

2 In rappresentazionı dı giardinı le figure sono ben riconoscibll come talı attraverso la scelta des colori come statue metalliche o marmoree (sia o no policrome) Uuası sempre sorgono sopra una base

3 Se si tratta di paeadggi, nature morte o scene figurative le statue sono riconoscibll in quanto svolgono una parte entro la raffigurazione sono caratterizate mediante un piedistallo e il colore del materiale umitato, marmo o metallo

Tuttı a criter menzıonatı sono statı presi in considerazıone durante la ricerca Glı acroterı sono statı catalogatı purche slano riconoscibilı distintamente come statue e non siano statı ridott a motiu decorativi schematici I rulievi non corrispondono a tuttı 1 criter 1 elencatı e saranno discussi a parte nel capitolo II

Attenzione partıcolare e stata dedicata alla Campania, regione che cl ha restituito la quantita maggiore di decorazionı parietalı nel loro contesto originale Esse inoltre per gl studiosi sono piu facumente raggiungibul e accessibili rispetto alle altre pitture, per la maggior parte non riniaste conservate completamente e/o in situ Le decorazioni di Koma, Campansa e Solunto sono state visitate e descritte dal vero, per quanto accessibili, negli annı 1981, 1982 e 1983 Le decorazionı trovate nelle altre partı dell'Impero sono state prese in considerazione soltanto sulla base delle pubblicazioni

Per quanto riguarda i limiti cronologics "s tratta di pitture del primo secolo a $C$ e $l$ periodo imperiale fino al 450 d C circa (pitture di Efeso) Le decorazioni cristiane di questo periodo, applicate nelle catacombe, non sono state inserite 
I criteri 'obiettivi' menzionati sopra servono soltanto per definire il materiale, il che quindi non significa che tutte le figure dipinte che si possono inserire in una delle categorie, costiluiscano senz'altro rappresentazioni di statue.

Un certo numero di figure oscilla fra scultura e 'vita'. Spesso si trovano in edicole figure in piedi o sedute che sono state rappresentate come esseri vivi, ma che nello stesso momento dimostrano le caratteristiche di una statua attraverso il loro atteggiamento e la loro collocazione. Alcune di esse verranno discusse. $\mathrm{Ci}$ si occupa anche di una serie di figure in scene figurative che corrispondono nel loro atteggiamento con tipi statuari. Purtroppo non e possibile essere esaurienti in merito, dal momento che per uno studio completo sarebbe stata necessaria un'analisi precisa di ogni figura presente sui pinakes.

Quando si cerca materiale di confronto nella scultura, mi pare evidente che si presti in primo luogo attenzione al materiale trovato nei dintorni dei complessi decorati con le decorazioni in merito. Statue vicine potrebbero essere state note direttamente ai pittori o ai committenti in modo da essere fonti di ispirazione. Purtroppo non è possibile ottenere un'immagine completa per le varie regioni; ciò è stato detto già a proposito della Campania. Naturalmente non significa che sja permesso limitarsi unicamente ai dirctti dintorni. E' stato incluso il materiale piu completo possibile dell'intero Impero, anche se non è sempre contemporaneo alle dccorazioni per le quali dovrebbe servire da confronto. Considerato il fatto che il materiale conservato e una cosa casuale e fortuita, in nessun senso completo, non conviene imporsi precise limitazioni. 


\section{1: ELEMENTI SCULTOREI NELLE ARCHITETTURE DIPINTE}

L'uso di elementi scultorel nell'architettura deve essere stato plu diffuso di quanto possiamo rintracciare sulld base del resti superstiti Purtroppo la nostra conoscenza dell'architettura antica, a questo riguardo, e lacunosa Le architetture dipinte crano ancora piu ricche di motivi derivatı dalla scultura I pittorl godevano di maggiore libertd quando creavano le loro composizioni Finora non e possibule tare un preciso buancio quantitativo delle vere architetture e di quelle dipinte Un certo numero di archcologi considera le architetture dipinte come costruzion immaginarie, inventale das pittori In questo capitolo si esamineranno queste ipotesi e si cerchera di lare un confronto con 1 reperti architettonici

Le statue che si incontrano nelle vere architetture posseggono nella maggior parte del cass la stessa tunzione di quelle dipinte fungono da cloment, di sostegno (caslatid) e atlant1) o da deroter oppure sono esposte in nscchie a edicole In questo contesto mi sembra utule dare una sintesi della comoscenza attuale delle figure in guisa di atlantı e dell'uso di nicchie nell'architettura

Gia in Lgitto e nel icmo Oriente gh architett adoperavano frequentemente elementi figurativ' per sostituire colonne o pldstri ${ }^{\circ}$ Il significato originale di queste figure e religioso esse vengono considerate come sostegni del tirmamento, rappresentando esseri mitici al servizio del dio universale, quello del cielo

In Grecıa atlantı e carıatıdı - per mantenere la termınologıa tradızıonale vengono in uso nel sesto secolo $A$ Schmid-Colinet distingue diversı modı di sorreggere ed e riuscito a stabulire, sulla base di questı, uno sviluppo sia stilistico che cronologico 10 l primi chempi sono le tigure temminil nel Koren-Schema che reggono con la sola testa l'architrave p cominciano nella seconda meta del sesto secolo ( $p$ e Tesoro del Cnidi a Delfi) e gh atlantı inseritı nel tempio di Zeus, il famoso Olympieion, ad Akragas (Agrigento) 71 Nell'Ellenismo si sviluppano nuove forme tigure che sorreggono larchitettura con una mano (Karyatiden-Schema, Satyr-Schema) e tigure inginocchiate (Barbaren-Schema) Tutte queste varidzion si incontrano nelle pitture

Dell'epoca romand conosciamo solo pochi esempi monumental Soprattutto ruliev 1 sarcotagi e ceidmica sono 1 nostri mezzl di iniformazione a proposito di questo aspetto dell'architettura Le Terme del foro a Pompel ( $80-60$ a $C$ ), il piccolo teatro nella medesima citta (epocd sillana) e ل ا tempio rotondo che incorona il complesso sacrale della Fortuna Primigenia a Palestrina (fine secondo secolo a C ) sono fra 1 pochı esempi di architettura nell'eta repubblicana che presentino figure umane di sostegno ${ }^{72}$ Augusto inserisce cariatidi, coplate in modo preciso da quelle dell Eretteo, nel suo Forum Augustum ${ }^{3}$ Da questo materiale si puo concludere che I Romani hanno

69 Schmidt-Colinet 1977, 4-18

70 Schmidt-Colmet 1977, 1-3, 149-156 Cf Schaller 1973

71 Schmidt-Colinet, cat W1, M1

72 Schmidt-Colınet 1977, cat M18, M19, W49, Castıglione 1975 (Pompel); LauteI 1979, 396-400, Abb 3-4, 6 (Palestrina)

73 Schmidt-Colinet 1977, cat W12 Simul figure devono essere state presentı nella Basilica Emilia e nel Foro Transitorio Bauer 1983, 164, Taf F-H 
seguito la tradizione greca senza aggiungere nuove forme

La posizione delle figure in origine e lissata dallo schema architettonico, ma a partire dal periodo classico la stessa architettura diventa meno rigida e le figure di sostegno danno l'idea di essere meno vincolate e vengono anche inserite neglı angolı degl edificl $E^{\prime}$ importante constatare che all'inizio dell'Ellenismo si suluppa la tendenza ad introdurre carlatidi e atlanti nes registri superior delle costruzion 74 ser e come quella del toro di Augusto appaiono solo nel primo secolo a $\mathrm{C}$ e le figure di sostegno. non prima

dell'epoca adrianea, vengono considerate completamente cquiralentı a plastri o colonne 75

Schmid-Colinet da l'interpretazione seguente delle cariatidi e degli atlant] 76 Atiraverso la funzione dell'edificio, il modo di sorreggere delle figure (gh 'schemi') e sulla base di fontı scritte, eglı conclude che neı monumentı sacralı e sepolcral protezione e devozione vengono espresse mediante le ligure di sostegno, mentre negli edifici pubblici - in un certo scriso politici - e talvolta anche nel complessi saciali si esprime in tal inodo l'dutorita Vel prumo caso le figure sorreggono un tetto, un baldacchino o un'altra copertura sopra una tomba o un altare Vell'Erctteo, sotto il tetto sostenuto dalle famose korai, e situata, heconclo la tradizione, la tomba del re mitico Cecrope assieme ad un altare per Poseidon e Eretteo vello stesso momento si venerd in questo luogo Atena Polias durante le sue feste. 1 cosiddetı 'Skıra' 77 Vell'Olympieion d, Ikragas invece l'elemento politico mette un accento particolare nell'interpretazione degli atlantı in quanto e la sottomissione del Cartaginesi che viene llustrata attraverso queste figure

Nell'Cllenismo le figure di sostegno esprimono l'onore e nell'epoca romana la tendenza ad esprimere ll potere del committente diventa forse l'aspetto piu importante Tuttavia non dobbiamo perdere d'occhio ll valore decorativo, specie con al gunto barocco e forse qualche volta biszarro del periodo ellenistico questa funzione non va dumenticata

Le osservazioni di Schmidt-Colnet a proposito di pitture parietalı verranno djscusse plu avant in questo stesso capitolo ( $P$ 23).

Piu prudente e l'interpretazione delle cariatıdı e deglı atlantı da parte d Evamaria Schmidt nella sua monografia apparsa nel 1982 70 Ella sostiene che l impiego di tali ligure in edificl di varia forma e funzione impedisca un'interpretazione cosi ricca di aspettı sumbolici e per tale motivo troppo 'pecante Sen dtenlese, ne riduce l'umportanza notando che un numero maggiore di argomentı puo avere svolto un ruolo in questo edificio pluritunzionale. mentre non va dimenticato l'aspetto decorativo Per quanto riguarda la terminologia, Schmidt non osa proporre una suddivisione troppo assoluta in quanto non e lecito stabilirne una sola visto che gh autori classici non sono chiari e difficilmente interpretabili - si ricordi il dibattito sine termine sulla parola

74 Schmidt-Colınet 1977, 71-72

75 Schmidt-Colınet 1977, 73, cat W12

76 Schmidt-Colinet 1977, 100-141

77 Cf invece Jeppesen 1979, 1983 egh sostiene che l'Eretteo fosse collocato altrove

7. Schmidt 1982 Cf B Wesenberg, Gnomon 52 (1980) 733-735 (rec Schmidt-Colinet 1977). B Wesenberg, Gnomon 56 (1984) 255-258 (rec Schmidt 1982) 
'cariatide' a proposito dj Vitruvio. ${ }^{9}$ Il termine 'cariatide' per Schmidt rimane tuttora valido per tutte le figure femminili di sostegno. La maggior parte del suo studio é dedicata all'analisi delle figure conservate e all'inserimento di esse nella storia dell'architettura e della scultura. Non presta attenzione a cariaticli e atlanti presenti nella pittura parietale.

Non si puó senz'altro ammettere che nelle decorazioni parietali gli atlanti e le cariatidi svolgono lo stesso ruolo che nella architettura reale. Fanno parte di un sistema decorativo e non costituiscono un elemento a sé stante.

Le nicchie vengono adoperate soprattutto nell'architettura romana, mentre in quella greca, dove questo motivo compare isolato all'interno delle pareti, l'uso rimane limitato a determinati scopi. Lina struttura composta quasi interamente da nicchie in quel periodo manca perora. ${ }^{\circ}$

Gertraut Hornbostel-Hütner, nella sua RUmische Nischenarchitektur, offre una sintesi complessiva su questo elemento architettonico e mostra le diverse possibilita presenti nell'architettura romana." La nicchia semicircolare è accanto all'edicola la forma piú frequente. Spesso appaiono serie di nicchie sia all'interno che all'esterno degli edifici. Ninfei, edifici termali, palazzi e gli immancabili teatri sono i generi che maggiormente vedono l'uso delle nicchie.

Visto l'accento particolare sulle pitture della regione campana soffermiamoci un istante su alcuni esempi di questa zona. Le facciate di alcuni edifici pubblici sul foro di Pompei, p.e. I'edificio di Eumachia e il tempio dei Lares pubblici, presentano nicchie per statue. Nelle terme ed anche nelle case private le nicchie sono state usate per la collocazione di fontane. Si pensi alle edicole a decorazione in mosaico nella Casa della fontana grande e nella Casa dolla fontana piccola, ivi adiacente, e alle nicchie per statue nei giardini della Casa dellancora e della Casa del torello. ${ }^{82} \mathrm{Si}$ incontra un esempio particolare nel peristilio della Casa di Pompeius Axiochus, di grande interesse per le decorazioni murali: nella zona superiore del muro cieco si trovano piccole nicchie nelle quali sono state trovate statuette di terracotta. ${ }^{83}$ Anche la tomba di Eumachia fuori della Porta di Nocera possiede una struttura simile con statue nel registro superiore della facciata articolata come una scaenae frons." Questa composizione corrisponde nelle sue linee generali alla disposizione frequentemente ripetuta nelle scaenae frontes della zona superiore di decorazionj di III e IV stile. $E^{\prime}$ lecito supporre che in quasi tutte le nicchie erano esposte statue, o che - come in periodi piú recenti - vi era l'intenzione di tarlo. ${ }^{5}$ In alcuni casi le vere statue venivano sostituite da figure in forma di rilievi o pitture. Ln larario lungo la strada era troppo soggetto a danni e furti di passanti perche si potessero esporre vere statue. Un larario stradale dipinto si trova sulla Via di Mercurio vicino al foro (cat. 244). Infine si noti che anche nellarchitettura a nicchie, la funzione di queste è prevalentemente

79 Cf. Plommer 1979.

" Hornbostel-Hüttner 1979, 32-52.

- Hornbostel-Hüttner 1979; rec. V. Kockel, Gnomon 54 (1982) 665-670.

- Hornbostel-liüttner 1979, 136; 81, Abb. 26; 82-83, Abb. 28.

' Von Rohden 1880, 21-22, 24, 25-26, Taf. 34; Lauter-Bufe 1975, 172 , Abb. 171; Hornbostel-Hüttner 1979, 135, Abb. 40.

- $\mathrm{S}$. De Caro, in Impegno 1983, scheda 11 OS.

is Hornbostel-Hüttner 1979, 203-212. 
decorativa.

\section{STILE}

Figure di sostegno nel II stile sono sempre facilmente riconoscibili come tali perche hanno sempre un'esecuzione plastica, quasi unicamente scultorea. Appaiono nella fase Ib sia nella zona mediana che nella zona superiore, mentre per lo zoccolo i primi esempi si incontrino nella fase IIb. Poiché le figure nella maggior parte dei casi tanno parte del sistema decorativo adoperato in una determinata zona. vengono discussi separatamente zoccolo, zona mediana e zona superiore. Per ogni elemento si segue, per quanto possible, un ordine cronologico. ${ }^{8} 6$

\section{Zoccolo}

Nello zoccolo le figure di sostegno sono spesso raffigurate in forma di uccelli. Nel reparto balneare della Casa del Menandro (cat. 163/5) e su due frammenti di una decorazione nel museo di Napoli, provenienti dall'Insula occidentalis (cat. $225 / 2$ ), si trovano sfingi e grifi come allanti; sorreggono la fascia che divide lo zoccolo e la zona mediana con le loro ali e con la testa. Sono monocrome e vanno considerate pertanto come statue, non come figure vive con la medesima funzione.

Vel tablino di una casa nell'Insula occidentalis (cat. 262/1) si vede una figura femminile con piedi a forma di tralci; essa regge con la testa l'orlo superiore dello zoccolo. Altrettanto fantastiche sono le grandi teste di donne sorgenti da calici vegetali che si delineano sulla parete opposta all'entrata delloecus della Casa di Obellius Firmus (cat. 302): in testa hanno un sostegno in lorma di calice con il quale e sorretto l'orlo dello zoccolo. Sulle pareti laterali dello stesso ambiente si distinguono delle erme schematiche che peró non hanno una funzione architettonica.

Nel cubicolo B della Villa della Farnesina a Roma (cat. 319/2) erme bianche raffiguranti dei Sileni abbelliscono le basi delle lesene laterali. I loro fusti finiscono in tralci. Il colore e il basso rilievo suggeriscono un'imitazione di appliques, forse in stucco. Nel triclinio $E$ una figura femminile fantastica e un satiro accosciato sostengono le colonne della zona mediana.

\section{Zona mediana.}

Per quanto riguarda la zona mediana incontriamo due possibilità di figure di sostegno: l'erma e la figura interamente plastica con forme dettagliatamente anatomiche.

In una pittura della fase Ic nella Casa del labirinto (cat. 208/3) si trovano centauri che sorreggono l'architrave dell'edicola centrale. Sono sistemati in modo simmetrico e sorgono sopra basi in forma di prore. Piedistalli per statue di questo tipo sono frequenti nel periodo ellenistico e nell'epoca romana. L'esempio piú antico in scultura si trova sull'Acropoli di Lindo; è datata alla

-5 Si rimanda al catalogo per la descrizione dettagliata e i riferimenti bibliografici relativi ad ogni pittura discussa nel testo. Il numero di riferimento al catalogo è indicato fra parentesi. 
metà del terzo secolo a.C. ${ }^{87}$ A causa dello stato molto danneggiato della pittura un preciso confronto con tali monumenti è difficile. Questo vale anche per j centauri che fiancheggiano la parte centrale di una decorazione nel bagno della Casa del Menandro (cat. 163/5). Essi sorgono sopra basi retlangolari aggettanti dalla scaenae frons. Hanno le zampe anteriori alzate e rivolte verso l'esterno. Con una mano reggono l'architrave mentre nell'altra tengono un timone. Il loro atteggiamento corrisponde a quello delle sculture da giardino trovate a (oplontis in numero di quattro. Di recente $\mathrm{S}$. de Caro ha dinostrato la provenienza ellenistica dej loro prototipi."

I prospetti centralj con scene mitologiche nella Casa di Livia sul Palatino (cat. $317 ; 3$ ) vengono fiancheggiati da figure femminili alate. Stanno su di una cornice in stucco $e$ sorreggono con la testa il lato superiore della trabeazione.

Quando abbiamo a che fare con erme si tratta spesso di serle distribuite davanti ad una parete a ortostati sopra un plinto o un podio, mentre reggono un tetto attraverso il quale viene suggerita la presenza di un portico nel quale i pilastri o le colonne sono stati sostituiti da erme; il basamento di tale portico è alto, l'altezza del colonnato invece limitata. Una costruzione simile non è stata trovata finora nella vera architettura, ma la facciata dell'Odeion di Agrippa sull'Agora ateniese puo darci una buona idea: giganti e tritoni stanno nella facciata di questo edificio, ricostruito nel secondo secolo d.C. nelle sue forme originali; con il loro dorso poggiano a pilastri che costituiscono i veri membri portanti. " Vella sala stessa il palcoscenico (cf. p. 21) è sorretto da una serie di ben 17 erme semplici, costituenti in tale modo una costruzione affine. Beyen propose la ricostruzione di un portico del genere basandosi sulla pittura nella Casa del Menandro (cat. 163/5).90

Nella Casa di Caesius Blandus (cat. 232/2) ogni erma sorge da foglie verdi (forse il pittore ha voluto imitare elementi ornamentali in bronzo e non solo $\downarrow$ colore naturalistico) e regge sulia testa un calice semplice sul quale poggia una trave aggettante: sta su di un podio verde. La parte superiore del corpo e sempre delineata in forma naturale come si incontra spesso presso le erme in tali costruzioni nelle fasi lc e Ila. Beven data le decorazioni della Casa di Caesius Blandus alla liase Ila. Considera le costruzioni come pure fantasie del pittore.

Nella Casa del criptoportico (cat. 148/1), datata nella stessa fase del II stile, incontriamo serie di erme nel criptoportico e nell'oecus sotterraneo adiacente al criptoportico. Nel primo ambiente vediamo erme di colore giallo con teste di satiri e menadi, che sembrano vive, sistemate davanti a lesene rosse fra ortostati gialli. Nelloecus invece le parti superiori sono rese plasticamente, mentre anche qui le figure, sia menadi che satiri, paiono figure realistiche. Esse sono monocrome in rosso. Le erme della Casa del Menandro (cat. 163/4) e dell'insula Occidentalis (cat. 262/2) mostrano la stessa caratteristica; purtroppo sono rimaste conservate soltanto parti frammentarie.

Due frammentj isolati di provenienza ignota, ora nei Musei Vaticani (cat. 012) sono gli unici esempi di erme fuori Pompei. Rappresentano teste di donne che reggono un calato ceruleo sul quale poggia la parte aggettante di una trabeazione gialla. I tratti dej volti sono vivaci e contrastano con la loro funzione.

\footnotetext{
' Jacob-Felsch 1969, 81; Ridgway 1981, 14, fig. 4.

- De Caro 1976, 198-219, fig. 5-20.

- Schmidt-Colinet 1977, cat. M175.

9 Beyen 1960, 144-14j, Abb. 61a.
} 
In luttj i casi menzionati colpisce il fatto che le figure - almeno Je parti 'umane' - sono state rappresentate come se si trattasse di figure reali presenti nel'ambiente.

Le erme nella Caserma dei gladiatori (cal, 197/2) sono meno vivaci e vive; cronologicamente vanno inserite in un periodo poco posteriore, cioè nella fase IIb. Sono rese in modo più schematico e non partecipano ad una struttura architettonica. L'erma nella Casa di Popidius Priscus (cat. 23i) infine è di tutt'altro tipo: essa occupa una nicchia aperta all'interno di un intercolumnio; sta su una base quadrangolare initante il marmo giallo antico. Dal materiale ancora presente non e possibile stabilire se vi fosse originariamente una serie di erme simili entro nicchie situate alle spalle di un colonnato finto. Sarebbe un caso unico; giá il fatto che un'erma occupa una nicchia é senza paralleli (cf. p. 18).

Le erme con elementi di persone vive presentano un particolare sincretismo di componenti fra loro incompatibili. Essi potrebbero essere ispirati dalla scultura realistica dell'Ellenismo - specje di centri come Rodi e Pergamo. Solo raramente si incontrano in edifici antichi (questo in opposizione con l'architettura rinascimentale e classicistica derivata da quella antica) erme in guisa di pilastri o colonne, sebbene non sia difficile inserirle in strutture architettoniche a motivo della loro affinita con colonne e pilastri. Solo nella monografia di Schmidt alcune frasi vengono dedicate a questo genere di figure di sostegno. 1 Per gli esempi di II stile il Portico delle erme a Cirene e di grande rilievo. ${ }^{92}$ Questo monumento datato al primo secolo a.C. contiene una serie di erme addossate a pilastri, alternamente rappresentanti Eracle e Hermes. Non le erme sono $i$ membri tettonici. ma i pilastri: generano tuttavia un effetto simile a quello delle figure nella Casa del criptoportico. Anche la tomba 228 a Cirene presenta questi elementi sulla facciata. ${ }^{3}$ Qui l'illusione di profondità è completamente inesistente visto che le figure sono rese in rilievo. Anche questa tomba daterebbe al primo secolo a.C.

Il logeion dell'Odeion di Agrippa e gia stato menzionato. Le teste delle erme in stile policleteo rippresentano sia figure maschili che figure femminili, la cui interpretazione rimane difficile. " Lungo la 'Strada marmorea' ad Efeso si vedono due Hermerakleis in una composizione architettonica, che in origine devono aver fatto parte di un edificio scomparso. Anche il piano superiore del portico che circonda il tempio di Domiziano nella stessa citta, dj recente restaurato, contiene figure umane in torma di erme. ${ }^{5}$ In piú conosciamo l'erma come sostegno di tavole e altri mobili."

Schmidt menziona infine alcune porte con sostegni in forma di erme che reggono la cornice sulla testa. ${ }^{97} \mathrm{Si}$ puo aggiungere la rippresentazione dei Rostra sull'Arco di Costantino a Roma dove le erme fungono da sostegni.

91 Schmidt 1982, 135-137.

92 Stucchi 1975, 127-131, fig. 109-110; Schmidt 1982, 136.

93 Stucchi 1975, 152-153, fig. 129; Bacchielli 1980.

94 Harrison 1965, 139, 169, pl. 59; Schnidt 1982, 136-137.

95 Barnmer 1978-1980, 81-83, 86, 88, Abb. 13-15. Cf . altri esempi: Perdrizet 1930; Baldassarre 1980 (Salonicco); von Hesberg 1983 (Corinto); Bauer 1983 (Roma).

96 Pernice 1932; Harl-Schaller 1978. Cf. le numerose statup di Attis: Tran Tam Tinh 1975, fig. 250-250; Dwyer 1982, fig.80, 183, 191.

97 Schmidt 1982, 220. 
Di recente Eleanora Windsor Leach ha proposto l'ipotesi che queste erme derivassero da un prototipo descritto da Lucrezio (II 23-26). essendo un tipo di lychnouchos. ${ }^{9}$ Il solo esempio rilevante in questo caso e l'erma che regge una lucerna nella casa nell'lnsula Occidentalis (cat. 216.2). Per le altre figure non vi sono confronti del genere.

Nelle cornici della trabeazione che chiude gli elementi architettonici della zona mediana incontriamo qualche volta figure in guisa di atlanti. Si tratta di figure che si trovano entro due serie di mensole, reggentosi su quella inferiore, oppure fra un plinto e una fuga di mensole. Questo genere particolare di cornici, i cosidetti Konsolengeisa, è stato discusso a lungo da H. von Ilesberg." Lgli descrive esempi del primo secolo a.C., datati in quel perjodo anche sulla base di confronti con pitture.

Due frammenti provenienti dall'Insula occidentalis (cat. 263) presentano cornici sopra le edicole centrali, ornate con cigni bianchi fra due plinti. Nella Casa di Livia a Roma (cat. 317/3) la doppia cornice che incorona la parete divisoria della zona mediana e abbellita con sirene bianche.

Nella Villa di P. Tannius Synistor a Boscoreale (cat. 004/1-3) centauri e figure femminili sorgono sopra mensole della cornice superiore. Sono di colore giallo e imitano membri architettonici di bronzo. In forma tridimensionale li incontriamo su urne fittili di Volterra e su un frammento architettonico in marmo proveniente da Roma. 100 Anche le cariatidi del Foro df Augusto possono essere considerate esempi di questo genere particolare di ornamentazione architettonica: sorgono sopra mensole e sorreggono le mensole della cornice. 101 Gli atleti nudi, i centauri e le figure femminili che si vedono nella Casa delle nozze d'argento (cat. 189/2) entrano nella seconda categoria. Gli allanti in terracotta nelle Terme del foro a Pompei corrispondono in senso architettonico a queste figure. 102 Il colore bronzeo e le loro dimensioni minjaturistiche (da sei a otto $\mathrm{cm}$. circa) fanno pensare che siano derivate da ornamenti in metallo anziche in marmo, terracotta o pietra. Illustrano, come i centauri nella Casa del labirinto (cat. 208:3) e nella Casa del Menandro (cat. $165 / 5)$, i centauri epistylia sustinentes disprezzati da Vitruvio. ${ }^{103}$ Sccondo l'architetto romano i decoratori hanno sconvolto $i$ limiti del possibile: non sarebbe piú il caso di parlare dell'initazione di un'architettura eseguibile nella realta, ma si tratterebbe di una fantasia bizzarra e impossibile e per tale motivo inammissibile. Von Hesberg invece mostra che Vitruvio aveva torto e che tali costruzioni esistevano realmente nella sua epoca, sia pure in quantità limitata.

Bcyen pensò alle figure fittili nelle Terme del foro a Pompei e a figure sempre in terracotta trovate a Centuripe, provenienti da una casa che sulla base delle decorazioni murali è stata datata nel primo quarto del primo secolo a.C. ${ }^{104}$ In entrambi $i$ casi $\dot{e}$ interessante osservare che le figure si trovano

98 Leach $1982,158$.

9 Von Hesberg 1980a, 131-141.

100 von Hesberg 1980a, 131-133, 135, Taf. 19.1-2.

101 Schmidt-Colinel 1977, cat. W12; von Hesberg 1980a, 136, 138.

102 Schmidt-Colinet 1977, cat. M18; von Hesberg 1980a, 139.

103 Vitr., De Arch. VII 5, 5; cf. Prop. IV 6, 49-50. von Hesberg 1980a, 133-135 dà esempi di diversi elementi decorativi.

104 Beyen 1960, 43-71; Castiglione 1975, 214, Abb. 210; Schmidt-Colinet 1977, cat. W32, M16. 
all'altezza di due terzi della parete e che quindi generano un effetto in senso ottico simile a quello delle figure dipinte.

Schmidt-Colinet invece, volendo trovare una spiegazione per questa particolarita, suggerisce una soluzione derivata dal teatro. ${ }^{105}$ Basandosi sull'idea che le pitture di II stile trovino le loro radici nelle scaenae frontes dei verj teatri, cita un passo delle Georgiche di Virgilio (III 24-25).

Vel scaena ut versis discedat frontibus utque Purpurea intexti tollant aulaea Britanni.

('Come se calasse una scena con facciata rovesciata nella quale reggono Britanni intessuti i sipari porporei.'). Il commento in merito di Servio viene menzionato altrettanto. I due passi contengono descrizioni di sipari teatrali nei quali sono state intessute piccole figure. Quando si alzano i siparj all'inizio dello spettacolo esse vengono quasi appese davanti alla scaenae frons della quale sembrano far parte. Senza entrarc nella questione problematica della derivazione della scaenae frons di II stile da quella reale e dell'origine di questo stile, il testo virgiliano non risulta sufficientemente chiaro. Certo. non possiamo aspettarci descrizioni troppo precise per quanto riguarda elementi tecnici da parte di un poeta, ma la mancanza di indicazioni precise in questo passo, oppure in altri testi antichi, non aiuta ad accettare questa ipotesi.

In alcuni casi incontriamo nelle architetture dipinte statue poste sopra basi in nicchie o su di un podio davanti ad un muro di ortostati. Proprio queste ci informano sulla collocazione di vere statue nelle case e nelle pareti a nicchie in teatri, ninfei e melle facciate di edifici di vario genere.

Nel cubicolo 4 della Villa dei misteri (cat. 306,2) figure del mondo dionisiaco sorgono sopra piedistalli gialli, a loro volta poste sopra un podio verde, davanti ad una serie di ortostati rossi. Il dio stesso, incapace di reggersi in piedi, poggia su un satiro, mentre si vedono anche un satiro danzante nell'atteggiamento dell'aposkopein, due menadi o sacerdotesse e un gruppo formato da un sileno e da un satiro. Non solo le basi, ma anche il colore, bianco con policromia parziale, caratterizzano le figure come statue. Esse sono un preludio alle scene del famoso fregio nella sala adiacente e vanno sempre considerate insieme ad esse nell'interpretazione di questa megalografia tanto discussa. 206

Statue su di un podio si incontrano anche in una casa nell'Insula occidentalis (cat. 216/2-3). Vediamo erme sopra un podio verde davanti ad una parete di ortostati rossi. I corpi son modellati plasticamente sopra il fusto. L'erma al lato destro è un lychnouchos: regge nella mano destra una luccrna appesa a tre fili o catenine sottili. La figura a sinistra molto rovinata probabilmente rappresenta una figura femminile che tiene le mani presso le tempie nell'atteggiamento della Afrodite Anadyomene. Nell'ambiente adiacente si vedono in nicchie sulle pareti est e sud le figure di un filosofo e di un poeta in forma di tipi noti dalla scultura; qui sono pensate come figure vive che simulano di partecipare alle attivita che si svolgono nella stanza. Per tale motivo essa andrebbe interpretata come uno studio o un gabinetto di lettura.

Due frammenti a Napoli si possono inserire in tale sistema. Provengono da una casa nell'Insula occidentalis (cat. 225/1), la cui decorazione è stata ricostruita in termini generali da Agnes Allroggen-Bedel sulla base delle sue ricerche negli archivi degli scavi settecenteschi. Contro uno sfondo composto da ortostati rossi (ormai scoloriti e diventati neri) si delineano statue policrome sopra basi verdi. Le due figure, Artemide e Apollo, sono state rappresentate ovviamente come statue mediante la collocazione sopra basi

105 Schmidt-Colinet 1977, 145-148. Cf. B. Wesenberg, Gnomon 52 (1980) 735 .

106 Bastet 1974, 217. 
metalliche o laminate in bronzo, ma non stanno in nessun rapporto con statue conosciute $d_{1}$ queste divinita Allroggen-Bedel riferisce alcun elementı derivatı da vari tipı Il pittore avrebbe potuto creare nuovi modell oppure copiare tipı, a noi non piu noti, dell'epoca ellenistica I visı sono caratter izzatı da trattı realisticı e mostrano le possiblita della policromia dı cuı poteva usulruire soprattutto il pittore

Lna figura isolata certamente rappresentante una statua e l'Afrodite che si slaccia un sandalo in $113,2-5$ (cat 172) Diversamente dalle numerose copse in marmo e bronzo di tipo ellenistico, clove la clea slaccia il sandalo del piede sinistro con la mano destra quella dipinta toglie 4 calzare sinistro con la sinistra Il cambio dell'atteggiamento potrebbe essere spiegato dal fatto che per $u$ pittore fosse plu facle dipingere una figura vista da un solo lato attivo' Una statuetta del tipo consueto e stata trorata nel 1970 presso la piscina della illa di Oplontis, un'altra che mostra ancora tracce della policromia originale proviene da 111,6107

Sulla base delle tracce ancora visibili non e permesso stabulire se la Giunone nella Casa di Obellius Firmus (cat 302'1) vada interpretata come statua Si trova in una posizione isolata davanti ad un ortostato forse sopra una base o una mensola, forse indipendentemente dalla tessitura architetionica

Su un Crammento 1solato della Villa della Farnesina (cat 319/7) si vedono davantı a pannell statue d donne su pied/stall $d_{1}$ verd $d_{1}$ Sono vestite $d_{1}$ chitonı blanchi e hanno sulla testa una corona vegetale Se l'una non avesse $\downarrow$ rotolo e l calamo, l'unterpietazione come Muse, proposta da Irene Bragantini, sarebbe meno plausibile a dovrebbero essere interpretate come Stagioni Lo stile e ellenistico

Nella Casa degli epigramm (cat 185/2) a sinistra e a destra del prospett centrali sulle paretı est e ovest sono state dipinte nicchie con cornici in rosso Da uno stondo chraro si staccano statue sopra basi rettangolar di colore verde, rappresentanti la stagione tutunno, una Musa e un'altra hgura femminile che probabilmente va altrettanto interpretata come Musa Sulla parete ovest la nicchia a sinistra e statc sostitusta da una porta Le figure sono rese in modo statico. ma mostrano un viva policromia I piedistall sono cardterizzat grazie al colore come bası di bronzo o di marmo coperto con lamine di bronzo e sono essi a togliere ogni dubbio concernente ll carattere e la lunzione delle figure 109 La scelta di esse non sembra stata fatta secondo un programma iconografico stabilito per questo ambiente, poiche non stanno in relazione con le scene mitologiche suı campi centralı e non hanno nemmeno una relazione specifica fra di loro, solo un nesso generico esiste con Dioniso che svolge un ruolo dominante nelle scene mitologiche Per quanto riguarda 1 tipl statuarı non disponiamo $d_{1}$ parallelı nella scultura Se non si tratta di invenzionı del pittore, possono essere tipi ormas perdutı, datantı dell'epoca tardoclassica o ellenistica

Un caso particolare e costituito dalle statue sopra candelabri o colonne esulı nella Vula della Farnesind (cat $319 / 6$ ) Si sta di fronte ad una situazione realıstica I candelabrı - in verita spesso espostı davantı a paretı - fungono nello stesso momento da lesene fra 1 campi Nell'ambiente C alternano Aurai di Stile Ricco e figure maschul barbute, torse rappresentantı Dionıso, di stule arcaizzante Negli ambientı $F$ e $G$ si tiatta soltanto di donne che trovano

107 De Caro 1976, 219-225, fig 30-35, Jashemskı 1979, fig 468, 200, Reutersward 1960, 143-168 (a proposito della doratura) Per il tipo cf Kunzl 1970

10. Per la pratica di rivestire piedistallı con lastre bronzee cf Rossignanı 1969, Dreihaus 1969 
confronto nelle tamose Danaidi della Villa dei papiri. ${ }^{109}$ Sulla testa reggono le basi delle colone della zona superjore. Nej cubicoli B e $D$ figure arcanzanti di impronta egızia, rappresentanti Iside e Giove Ammone, stanno su piedistalli vegetali. Non sono paragonabili con vare statue. Nellambiente D stingi bianche sostengono nell'Atlanten-Schema pinakes monocrom. Sono delineate come esseri viv e corrispondono ai candelabri a torma di figure umane. ${ }^{110}$

L'erma nella Casa di Popidius Priscus (cat. 237) e stata sopra menzionata a proposito delle erme nel II stile. Hornbostel-Huttner accenna che raramente vennero esposte erme all'interno di nucche, a favore di una posizione contro una parete cieca o un pilastro. Salta agl occhi la precisione con la quale il pittore ha raffigurato l'erma; l'ombra è stata indicata chiaramente. Non esiste dubbio che il modello per la figura, resa plastica dalle reni in su, proviene dalla vera scultura. Esempi di erme a mantello le incontriamo nelle Terme stabiane e nel tempio di Apollo. 211

Lna disposizione simule a quella nella Casa degli epigrammi è visibile nel Irigiclario della Casa del criptoportico (cat. 148/4). Le figure a fianco dell'edicola centrale purtroppo sono andate perdute in gran parte o svanite, ma vanno indubbiamente considerate come statue visti il colore bianco e la posizıne nelle nicchie.

Solo raramente si vedono figure tridumensionali adoperate come acroteri. Possiamo menzionare ippocampi bianchi nella Casa di Augusto a Roma (cat. $316,3)$. Specie qui il colore - particolare per questi mostri marini - accenna il caratlere plastico degli acroteri. Contronti si trovano nei tritom che meoronano il grando altare di Pergamo.

\section{Zona superiore}

Nella zona superiore ripetutamente incontriamo figure umane o fantastiche come atlanti o acroteri. Statue in nicchie sono presenti in un solo complesso, cioe nel cubicolo B della Villa della Farnesina (cat. 319/2) dove statuette dorate di tigure femminili che reggono due fiaccole e per tale ragione probabilmente rappresentano Ecate, sono inserite all'interno di nicchie.

Nel frigidario e nell'apoditerio della Casa del criptoportico (cat. 316/3, 4). sulla sommità della trabeazione della zona mediana si trovano tritoni bianchi in guisa di acroteri. Sulla cornice dell'edjcola centrale sorgono, su una parete, ligure maschili, sul'altra figure femminil vestite di poplo, e tutte quante reggono dei piatti tra le mani. Le coppie sono simmetriche l'una rispetto all'altra. Sulla testa reggono l'architrave del secondo piano della scaenae frons che inoltre viene sorretta da colonne. La monocromia bianca da maggior rilievo alle sculture; il materiale, cioe marmo bianco, viene chiaramente suggerito. Nella maggior parte dei casi precedenti abbiamo constatato un frequente uso della policromia e risultava talvolta incerto il carattere glittico delle figure; quindi, la vera intenzione del decoratore. Un tregio che chiude la struttura architettonico al lato superiore è sorretto da sfingi gialle.

Nella stessa posizione e colore sono dipinte le sfingi nella zona superiore delle prospettive architettoniche nella sala 14 della villa di Oplontis (cat. $341,3)$ e nella villa di Boscoreale (cat, 004/3). Queste figure inginocchiate sono piccole e fanno pensare alle figurine nelle cornici doppie nella Casa delle nozze d'argento (cat. 189/2) e a Boscoreale (cat. 004/1-3). Le protomi fittili

209 Sgabbo 1971.

120 Cf. Overbeck/Mau 1884, Fig. 233-234.

111 Eschebach 1979a, Taf. 52b; Overbeck/Mau 1884, Abb. 53. 
rappresentanti teste di toro nel criptoportico della casa omonima generano un effetto simule e potrebbero essere state una fonte d'ispirazione per le figure dipinte, la loro posizione angusta tra architrave e cornuce rende ancora piu accettabile tale supposizione 12

vella Casa deglı epigramm (cat 18j,2) troviamo un motivo del genere sulle paleti est ed ovest, purtroppo ora completamente crollate coppie di ligure aldte indossanti corte tumiche sorreggono con una mano la cornice, mentre hanno l'altra distesa lungo il fianco

Sono disposte in modo simmetrico Il colore giallo indica che esse sono state raffigurate come statuette di bronzo

$\mathrm{L}^{\mathrm{P}}$ a Romd che, neglı ultımı decennı del primo secolo a $\mathrm{C}$, constatiamo una crescita di elementı scultorel nella zona superıore Vella Casa di Livia (cat 317 1) e nella Villa della farnesina (cat 319/3) questa zond comincia a si lupparsi indipendentemente dalla zona mediana ed a mostrare l'articolazıone in und scdendo trons con nicchie, edicole e atlantı e cariatids Nella sala IV della Casa di Liv ıa carıatıdı classıche facumente richiamano le ligure tridumensionalı del Foro di Augusto, inserite come sostegni fra una fuga dı mensole e una cornice I conograticamente sono determinate come sacerdotesse o offerenti attraverso , loro attributı (brocca di vetro e bacchetta) Nella sala II figure femminul sorgono sopra colonne a capitellı doricı Esse sono piu fantasiose

Ancora piu vasto a 1 repertorio adoperato nella Vulla della Farnesina (cat 319). le cui decorazionı di recente sono state catalogate in modo esemplare da Bragantini e De Vos Costruzioni gid diventate irreal sono sorrette da cariatıdı, talvolta alate, talvolta riconoscibli come figure sacralı In ognı ambiente lo stile (classico arcastico e barocco) cambia All'interno di edicole - fra I 'proplei' sorrettı da cariatıdı o sopra le edicole della zona superiore sorgono figure su piedistall Raramente e possible trovare controntı precisı o affinı nella scultura Gli acroterı nel cubicolo $B$, ninfe marıne sedute su ippocampl, sono un immediato ricordo delle vereıdı di Formia ${ }_{13}$ In due cası figure maschil sono state inserite nelle architetture Vel triclinio $C$ figure barbute, indossantı lunghı mantell, $d_{1}$ Stle Severo avvicendano tigure femminli dl Ricco Stule, tutte sono dl carattere dionisiaco Nel cubicolo $E$ l'edicola centrale e sormontata da due figure maschil vestite in modo orientale che flancheggiano un mezzo busto $d_{1}$ un uomo barbuto e da tralci con protomi di pantere

Specje per l tipo di figure che sostengono un membro architettonico con una sola mano, incontriamo moltı csempı nella vera architettura Schmidt-Colinet parla, per quanto riguarda le figure femminul, di Karyatiden-Schema e per le figure maschlh di Satyr-Schema ${ }^{114} E^{\prime}$ nel periodo ellenistico che questo tipo viene introdotto Sulla lastra bronzea proveniente da Goreme in Cappadocia, ora a Berlino, databile al primo secolo a C, due Frigi in questo attegglainento fiancheggiano un'epigrafe, mentre sorgono sopra capitell corinzi $115 \mathrm{Ln}$ pendant con figure femminll dellepoca adrianea, proveniente da Pozzuolı, si trova a Napolı una tigura femminle seduta davantı ad un albero e tuancheggiata da due altre donne che sorreggono la

112 Spinazzola 1953, I, 502-503 III, tay XXIV-XXV, Mielsch 1975, 109, cat K2 (c), von Hesberg 1980a, 179 Cf anche von Mercklin 1962

113 Bieleteld 1969.

214 Schmidt-Colınet 1977, 35-43, 57-65

115 Schmidt-Colinet 1977, cat M69 
cornice del rllevo 116

Anche per queste figure Schmidt-Colinet ammette una derıvazione daj siparı teatralı 11 La sua teoria in questi casi e ancora meno sostenibile in quanto le figure sono di dimensioni maggiori. Dobbiamo ammetterc che l pittori hanno interpreiato elementı presentı nella raald, pero in modo libero, coerente all'arte pittorica

Prive di ognı elemento realistico sono le figure di dimensionı naturalı nella Villa del misteri (cat 306/3) Due gruppi consistenti entrambi in un arimaspo assalito da un grifo, sorreggono la trabedzione superiore dell'alto cubicolo 16 I) questa decorazione sono rumastı intatil solo queste parti dal momento che in una lase seguente la decorazione di II stule ha dovuto cedere ad una piu remplice di IIl atle, forse dovuta al eambiamento di funzione dell'ambiente o al degrado delle zone inleriori, mentre il solfito era stato ubbassato mediante una volta a botte, lascianclo intatte le nostre tigure

\section{STILE}

Nel periodo del III stue l'uso di statue si limita a pochi casi La minore trequenza puo essere spiegatd dall'interesse calante per la rappresentazione di elementı architetonici Le figure hanno in comume con gli altri ornainenti (per csempio I candelabri) un'esccuzione dettagliald e si staccino nettamente dalle figure di Il sile Somo pus snelle, so sl vuole piu cleganti, e per quanto riguarda l'attegguanento piu statiche' 'Tutto cio sl unserisco nel camblamento di opinionı e di gustı secondo la tendenza classicistıca di Augusto che sl vede espresso nelle decorazioni di III stle

\section{Zoccolo}

von dissumile dal II stile e la presenza ds uccell in guisa di atlantı nello zoccolo Talora fungono in questa zona della parete sumultaneamente come statue all'interno di glardinj dipintı Incontriamo predecessorı di questı animalı nella Casa di Obellıus Firmus (cat 302 1) e nella Casa del Menandro (cat $163 / 5$ ) Nel vano b d I 7,19 (cat 156/1-2) uccell sl reggono sopra basi rettangolari coll un lato superiore concavo sulle paretl est c ovest Le zampe sono divaricate Sulla testa si vede un tralcio che sorregge un capitello uguale alla base Gil animal stanno in posizione simmetrica c hanno le teste rivolte verso $\downarrow$ centro $N e$ vano c attiguo gli uccell sono piu rigidı $Q u$ si vedono aironı - o anumalı che gli assomigliano - sopra bası rettangoları. che reggono sulla testa un girale, Il pittore ha fatto tinire ll ciulfo dell'aninale in un drabesco Sulle paretı nord e sud si distinguono sfingl rigide inginocchiate, viste di fronte, che indubbiamente sono state immaginate come statue Sono dı dimensionı minor e reggono una colonnina sulla testa $E^{\prime}$ chiaro che, in questo caso, I decoratore ebbe davantı aglı occhı modellı dı sfingl marmoree, spesso usate come sostegnı d tavole e tontane o esposte nel giardim 1 is ln questa casa esse sono le uniche figure che si tanno interpretare senz'altro come statue

\footnotetext{
116 Schmidt-Colunet 1977 , cat W54

117 Schmidt-Colinet 1977,146

12 Per esempio MN inv no 6869 dalla (asa del fauno (Ruesch 1789) e senza inv no da VI 16 a (Ruesch 1704)
} 
Vella Casa di Orfeo (cat 212/1) aironi ad als dperte sl trovano in una decorazione conosciuta soltanto grazse ad un vecchio disegno Le alı sorreggono, come se tossero le mani del Koren-Schema l'or lo superiore dello zoccolo Sulla testa hanno un ornamento in torma di un calice verde che lunziona da capitello Questo calice ricorda 1 poloi e 1 kanea (canestre) che si vedono nelle cariatid, della vera architettura, per esempio nelle cinque canefore della Via Ippia, ora disperse in vari musel, e cembra ripetere 1 motivo della canestra in maniera stilizzata 119 Polos p kanos torse sono derivazionı de fustı di colonne a ornamenti in rllievo che nel periodo arcaico venrano inseriti fra testa e capitello Lnd cariatide a Dell d datald intor no al $550-540$, c1 mostra un esempio di questo fenomeno 120

Lna pittura ugualmente perduta nella lasa del vasi di vetro (cat 210/1) mostra uccell sumli, espostı in modo summetrico, entro scomparti al di sotto delle lesene della zona mediana, sono inolto piccolı rispetto agli esempi finora discuss 1

Glı uccell nel calıdarıo della Casa del labırınto (cat 208/2) e in una casa,trastormata successivamente in un panilicio, nell'Insula orientalis ad Eicolano (cat 038) sono statı delineatı Iıgidamente in manıera scultorea come le singl di 17,19 (cat $15 G^{\prime} / 2$ ) Rappicsentano statue topiarie e sono stat dipintı secondo modell realistici In questo contesto possiamo riferire agli uccell in una decorazione $d_{1}$ III stue a Vienne-l sere 121 Essi mostrano la ditfussone a larga scala di questo motivo nelle zone periferiche dell'Impero

Non si conoscono precisi confronti per le statue di uccell Le numerose statue esposte nella 'Sala deglı anmalı' des Musel Vaticanı derono dvele dvuto und collocazjone in grardino come $a$ anche $ا$ caso di alcum uccell ricuperatı in Campania, sempre con la funzione di tontand 122

Le pitture del panificio ad Ercolano (cat 038) inoltre mostrano alcune erme come elementı dellar redo topiario, mentre $H^{\bullet}$ ifthermen scparano le varie partı del giardino Esse sono cople di dumension ridotte delle crme a mezzo corpo di II stule Un frammento torse di IV stule piuttosto che di III della Collezione Ben Heller di New York (cat 126), esposto nel 1974 alla mostra su Pompei di Zurigo di provenienza ignota, ma probabilmente campana, potrebbe aver occupato la stessa posizione delle erme dell'Insula orientalis ad Ercolano ton si possono trovare buon confrontı nella scultura conosciuta per le tigure dipinte

Semplics $H^{\mathcal{U}}$ ffthermen bianche occupano gli scomparti sotto 1 candelabil della zond mediana nel vestibolo dell'Ipogeo presso la Porta lagglore a Roma (cat 320) Esse reggono attributi ora completamente svanitı $c$ sono di esecupione semplice

Uniche sono due ligure temminil che nell'ambiente $L C$ della Casa di Julius Polybius (cat 303/4) lungono da cariatidı nello zoccolo Sulle paretı nord e sud davantı ad uno sfondo nero $s 1$ vedono menadı in pannı volantı che sorgono sopra bası rettangoları e sorreggono sulla testa una colonnina che tocca il margine superiore dello zoccolo Trovano confionto con le calistidi della cona super 10 e in decorazionı di III stle da discutere piu arantı

\footnotetext{
119 Schmidt-Colınet 1977, 142-143, cat W22

120 Schmidt-Colmet 1977, cat W2

121 Barbet $1982,55-56$, fig 1

122 Amelung 1908, nos 111, 112, 122, 127, 128, Taf 31-33, Kapossy 1969, 53 , Jashemski 1979, fig 331 , Dwyer 1982, fig $44-46,70,71$
} 


\section{Zona mediana}

Carıatidı e atlantı non si incontrano nella zona mediana di decorazionı di III stule, fatto non sorprendente vista la riduzione rigorosa delle dimensioni degli elementı architettonici Anche figure su mensole nelle campiture non si trovano in questa fase della pittura parictale

Sia nella calegorid delle pitture architettoniche che in quolld dolle iappresentazion higuatue entrano le prospettive nella cisa del malde (cat 101 1), da discuterc in questo capitolo per la loso posisione cospicua sulla parete sud si vede un inuro con und porta che mette III un giardmo, al suo fianco si trovano due 'proplei' Queste costruzioni vengono sorretle da cariatidi vestite di peplo, viste di proflo e rivolte verso ll centro Lna mano sostiene l'archintrave, l'altra p distesa e regge una patera L'insiome sembra ispirato da un edificio reale e le cariatidi corrispondono al tipo classico delle horal del'Eretteo

\section{Zona superiore}

Solo nella zona superiore l'architettura e rimasta riconoscibule e in essa si meontrano frequentemente statue De Vos menziona la grande presenza di figure femminili sugh angols di edicole, specie sopra l'epistilio dell'edicola centrale della zona mediana ${ }^{123}$ Esse sol reggono talvolta una colonna o una trave in altri cası sono solo elementı decorativi o acroterı Differiscono dalle sculture dipinte nel II stile per quanto riguarda l'esecuzione e l'at to del sostenere Mon sorreggono piu di una linea esile o un tralcio sostitutivo dı una colonna Le figure come talı sono rese con movimento vivace, spesso in policromia realistica, mentre sorgono sopra piedistall composti da un plastrimo e un plinto largo, secondo un insieme spesso raftigurato a forma di $T$ La summetria non e una regola stringente Anche se non vengono sempre caratterizzate attraverso attributı specifici, possono essere etichettate in generale come menadi o baccanti Lo stule del drappi volantı e del movimento iscorda l gusto dello Stile Ricco e del periodo seguente, conosciuto da opere dı maestrı come Paionio, Timoteo e Scopa, tuttı da datare negh ultımı decennı del quinto e nella prima meta del quarto secolo a C 124 L'abbigliamento sembra mosso da un vento immaginario in modo che le forme del corpo diventano ben Wsibili Iconograficamente vengono interpretate come Nihas o Aurai, I che non e verosimule per le 1igure dipinte che, come e stato detto, entrano piuttosto nell'atmosfera bacchica I pittor possono aver avuto conoscenza diretta di queste statue gia dopo le guerre macedoni del secondo secolo a C statue vennero asportate dal templi in Grecia e trasferite come bottino a Roma dove vennero esposte sia come opere d'arte in collezioni private sia in templi o edifici pubblici come dediche alle divinita o al popolo romano 125 Le Nereidi di Forma, ora nel Museo nazionale di Aapolı, ne sono un esempio lampante 126 Anche l'influenza di lastre Campana e di rilievs neoattici non va scartata I non dı rado lappresentatı guerrierı pirrıcı sono statı derivatı da esempı

123 Bastet/De Vos 1979, 130

$124 \mathrm{P}$ e Zanker 1965 (Tindarı pezzı tardo-ellenistıcı d'impronto classicista), Thomas 1982 Delivorras 1974, 162-198 presenta una smossi di tuttı gli acroteri conosciuti

\section{Cf Pape 1975}

126 Bielefeld 1969 
neoattici 127 Dobbiamo a $W$ Fuchs l'analisı di questı elcmentı, egli mette f'accento sul tatto che oltre as maestri del quinto secolo - gia allora 1 classicsiz: - gli scultors delo Stle Ricco hanno influenzato considerevolmente l'arte des neoaticse, attraverso di loro, larte del prumi seculi a e d $C^{129}$

von solo figure in piedi, ma anche ligure inginocchiate trovano posto nelle scaenae trontes $d_{1}$ III stue, specialmente como deroterı lessuna di esse e senz'altro riconscible come statua sulla base del colore o dell'atteggiamento, ma per la loro posizione lanno parte del materiale discusso in questo capitolo

In questa tase incontriano 1 primi esempl di figure collocate sopra piedistalli nella rona superiore Riempiono cdicole o stanno tra di esse, spesso entro nicchie, costituendo in questa manierd le prime imitazioni della decorazione delle scaenae frontes teatralı Sono proprio 1 piedistalls a non permettere allo spettatore dubbi sul carattere delle tigure ul pittore pero non si sente vincolato alla regola che gli imporrebbe di mostrara le ligure sempre in afteggiamenti 'scultores' ed e libero di renderle come persone realı Aon diversamente dalla pittura dichitettonica le figure sono un ricordo di una realta esistente senza avere la pretesa di mitarla con effett lllusionistici Che unitino modell, conosciuts o non plu reperibilı, l tipl ianno cercati nel periodo ellenistico $S_{1}$ consiata una preferenza per igure come satirı, gueirieri pirrici e ejoti

Un esempio nell'architettura contemporanea che rispecchia l'esposizione di statue in nicchie nella zona superiore dela parete e tornito dalla parete cieca del peristilio nella Casa d Pompelis Axiochus a Pompes, citata nell'introduzione di questo capitolo ( $p$ 18) La struttura della parete assomuglid quella di molte decorazionı uno coccolo e una zona mediana chiusa, mentre la zond superiore e 'aperta' e ospita una serıe dl statue

In inerito vanno ricordate le paret composte da michie, delle quals, purtroppo, es e stato tramandato poco materiale Siamo ben informatı sulle decorazioni del monumento di Lrode ittico ad Atene, datato in epoca antoniniana, dove erano espostı I ritratt di membri della famiglia di Antonino Pio in ragione della tunzione della costruzione celebrativa della tamiglia impeisale, e sul monumento a forma di terro di cavallo di Perge nel quale le nicchic erano occupate da statue rappresentanti i fondator di Perge a membri della famiglia che avera tinanziato la costruzione ${ }^{130}$ Nell'edificio $M$ a Side nicchie a due pianı sovrapposti contenerano statue, le figure nel registro inferiore avevano un atteggiamento stabile, quasi in riposo, mentre quelle nella zona superiore presentavano mor umento e vivacita ${ }^{3} 3{ }^{\prime} E^{\prime}$ verosimule che l'architetto in collaborazione con lo scultore abbia fatto consaperolmente questa distinzione e stato creato un contrasto lia le due serie nel quale le statue superiori, normalmente meno cospicue vista la loro posizione, ricevono rilievo grazie al loro atteggiamento particolare Questa distinzione potrebbe spiegare il movimento delle ligure nel registri superiori delle decorazioni di III stile Nonostante 1 fatto che 1 monument 1 menzionatı datino ad epoche piu recenti delle pitture, cioe al secondo secolo $\mathrm{d} C$, si puo ammettere che le idee ed il concetto si siano siluppati nel periodo ellenistico e che all'epoca del III stile edifici con queste strutture fossero ancora visibli dappertutto Gia nel periodo arcaico si usava esporre statue su alti pledistalli si ricordino le

\author{
127 Borbein 1968, 152 n 786 Cf Tortorella 1981 \\ 12 a Pielsshofen 1979, Zanker 1979b \\ 129 Fuchs 1959, 5-7, 129 \\ 130 Lauter 1973, Vermeule 1977, 83-84, 90-91, tig 71-72, 74-75 \\ 131 Vermeule 1977, 91-93, fig 74-75
}


figure votive di Delfi collocate sopra alte colonne. Nel periodo romano le colonne dei portici lungo le strade delle città orientali, come Diocesarea. Perge, Palmira, Apamea e Gerasa, erano provviste di mensole per statue o di ligure in alto rilievo (Perge) imitanti statue su mensole. ${ }^{132}$ Statue e rilievi si trovavano sopra il livello dell'occhio a circa due terzi dell'altezza delle colonne; erano visibili solo di sotto in su. ${ }^{231}$

Le pitture che abbelliscono il portico della Villa imperiale (cat. $305 / 3$ ), presentano l'intero repertorio di possibilità. Sopra uno zoccolo e una zona mediana chiusa in nero e rosso a suddivisione in campi si trova una scaenae frons su fondo bianco. In parte costituisce la conclusione lineare delle strutture esili nella zona mediana, incoronanti le edicole e le lesene, in parte $\dot{e}$ stata costruita indipendentemente. Von sono stati suggeriti una particolare plasticità o un effetto prospettico. Alternamente si vedono edicole con un timpano o un architrave piatto e strutture fantastiche. Sui tetti dei timpani stanno acroteri di dimensioni notevoli a forma di pegasi (a sinistra) e centauri con le zampe anteriori alzate (quinto pannello di sinistra). Sul tet to piatto dell'edicola sopra il terzo pannello di sinistra gli acroteri consistono dj figure fernminili inginocchiate che reggono pintti nelle mani. vel centro delle edicole sorgono sopra basi semplici o composte da candelabri figure femminili (quinto pannello di sinistra: donna con cornucopia; altre andate perdute) e un ipollo Citaredo. Nelle architetture piu bizzarre troviamo una scelta di ligure fantastiche. Slingi e figure femminili sono state applicate come acroteri sulle 'ali' degli edifici. le donne sedute su sgabelli a forma di spirale o sulla sommita. Una di esse regge un timpano ed è cosi caratterizzata come menade. In un'altra struttura si trovano donne vestite di peplo (ora quasi invisibili) in posizione simmetrica, funzionanti come cariatidi dell'architrave curvilineo, all'altro lato sorretto da erme semplici. Tra le mani esse reggono piatti pieni di frutta. Nelle altre due architetture vediamo un Dioniso che regge un tirso - un satiro che porta su una spalla un altro giovane satiro o torse Dioniso stesso. Le figure sono snelle e allungate e sembrano ispirate da vere sculture. Il satiro si incontra anche su un frammento di III stile a Napoli (cat. 053). Le statue sono messe in posizione isolata per dare piú rilievo. Tutte le figure rientrano nell'ambiente dionisiaco.

Nella sala A della stessa villa (cat. $305 / 1$ ) troviamo sei cariatidi. Quelle delle pareti sud e nord sono dipinte in colore bronzo e hanno un carattere scultoreo. Sono viste frontalmente, indossano il peplo classico a pieghe rigide e richiamano le cariati della zona mediana nei corridoi F e G della Villa della Farnesina (cat. 319/6) e le cinque Danaidi bronzee classicheggianti provenienti dalla Villa dei papiri, ora a Napoli. Non solo le figure, ma anche l'architettura risulta piú realistica e plastica di quella del portico. Le due menadi della parete est sono viste di profilo. Mostrano uno stile diverso: l'abbigliamento e piú pesante, la policromia e l'impronta di vivacità dei volti danno l'impressione di trovarsi di fronte a figure vive. Non si conoscono confronti nella scultura.

Figure femminili sedute su basi a forma di girali si incontrano anche nella Caserma dej gladiatori (cat. 197/3). Sopra uno zoccolo nero e una zona mediana bianca, ornata con figure policrome isolate su campi, si trova una serie di figure femminili delineate davanti ad uno sfondo bianco. Non sono state inserite in un contesto architettonico; le loro basi sorgono semplicemente sopra l'orlo che separa la zona mediana da quella superiore. Non esiste piu una relazione con la realta e nemmeno si ha l'impressione di trovarsi davanti ad una galleria di statue. Ovviamente le figure stesse hanno perso ogni punto di confronto con sculture. Spicca l'esecuzione molto raffinata, specie vista in

\footnotetext{
132 Lyttelton 1974, 223, pl. 121, 157; Peschlow-Bendokat 1975, 376-377; Vermeule 1977, 95, fig. 82.

133 Jacob-Felsch 1969 non discute questo genere di basi per statue.
} 
confronto con le erme rozze sulle pareti dell'anticamera, datate nel II stile (cal. 197/2).

Alcun frammenti nel Museo Nazionale di Napoli, provenienti dalla Villa di Varano (cat. 010,2), mostrano le stesse figure temminili sedute su tralci, ora d) dimension ridotte e dipinte sottilmente. Alcune di esse hanno ali e sono state interpretate come Psiche. Occupavano una posizione simile a quella delle figure descritte qui sopra.

Il motivo si incontra nell'acroterio a risvo che incorona una stele funeraria atenese del quarto secolo a.C., ora a Berluno. Le figure femminilı sono sedute suı girali di un ornamento vegetale che esce da una palmetta nel centro del fastigio, in atteggiamento di dolore. Sono state sistemate in modo simnietrico e tengono una mano sotto il mento reclinato. Secondo C. Blumel stiarno di fronte ad una decorazione particolare per la quale non si trovano confronti nella scultura sepolcrale. ${ }^{13} 4$ Su alcuni rilievi neoattici il motivo e stato usato in contesto mitologico. ${ }^{235}$ Il carattere miniaturistico deriverebbe dall'arte metallica. Le menadi sull'ansa del cratere di Derveni nel Museo archeologico di Salonicco ne sono un buon esempio. ${ }^{136}$

Nella zona superjore delle decorazion di III stile nella Casa dj $M$. Lucretius Ironto (cat. 195,1), nella Casa del frutteto (cat. 161/2), nella Casa des quattro stilı (cat 159/3) e in I 12, 3 (cat. 169) sorgono figure femminili sulla trabeazıone superiore delle edicole centrali della zona mediana. Reggono sulla testa colonnine sulle quali poggia l'architrave che chiude la scaenae frons al lato superiore Indossano talora mantell volanti, reminiscenza - come e stato detto - delle Aurai classiche. Le cariatid nella scaenae frons della ormai svanita decorazıone della Casa dellancora (cat. 206) sono piu statiche e si inserjscono nel gruppo di figure contormı allo Stile Severo, incontrate nella Sala A della Villa imperiale (cat. $305 / 1$ ).

Delle poche decorazioni di III stle ad Ercolano, quolle nella Casa del colonnato tuscanico (cat. 035/1) e nel panificio nell'Insula orientalis (cat.038) e in IV 16 (cat. 27) danno esempi di menadi nella zona superiore. Nella Casa del colonnato tuscamico si vede una donna reggente una cornucopia di dimensıoni gigantesche inserita nel sistema come persona viva. La donna in IV 16 regge un tirso, mentre anch'essa e stata rappresentata come reale. Nel panificjo stanno ligure di Ricco Stile accanto a figure di Stle Severo. Questa decorazione, purtroppo nota soltanto in torma di frammenti conservati a Napoli, e paragonable con quella della Villa imperiale.

. ambiente accanto alle fauces un frammento di pittura, inserito in una decorazione di I stile, che proviene senza dubbio da una zona superiore di III stile: sopra una base a torma di $T$ sorge una menade nell'atteggiamento della Nike di Paionı di Mende, che regge una fraccola. Il frammento faceva parte di una decorazione peraltro perduta, inquadrato in un telaio di legno e poi inserito come pinax in un'altra decorazione. La posizione originale è paragonabile a quella delle figure appartenenti ad un altro vano della stessa casa, della quale però non ha fatto parte.

Anche nel vano I della Casa di Julius Polybius (cat. 303/1) due donne sorgono sopra basi a forma di $\mathrm{T}$, qui inserite in una struttura a edicole, sumbe a quella con il Dioniso e con il satiro nella Villa imperiale (cat. 305/3).

\footnotetext{
134 Blumel 1966, 46-47, Abb. 72 .

135 Froning 1981, 63, 64, 68-69, Taf. 12, 16.1, 17.1 (Paride su rilievi a Napoli, New York e Roma).

136 Gioure 1978, pin. 63-92.
} 
Molto manca della ricca decorazione dell"Aula superiore' nella palestra ercolanese (cat. 039/3). Nel vano 3 si vede ancora un Eros che funge da acroterio sopra un tetto a timpano. Sugli angoli sono sedute sfingi femminili alate. Si intravvedono le vaghe tracce di cariatidi: due menadi sorgono sopra piedistalli a forma di $\mathrm{T}$ e reggono in testa colonnine sottili.

Nel museo di Napoli alcuni frammenti di provenienza ignota possono essere inseriti in sistemi simili. Sul frammento 9878 (cat. 053), analizzato a lungo da $R$. Herbig, si vedono tre figure in un ambiente architettonico. Nel centro sta un Eros, a sinistra un satiro nel'alleggiamento doll'aposkopon, a destra un satiro con il giovane Dioniso sulla spalla sinistra, simile al gruppo della Villa imperiale. I frammenti 8829 e 8908 appartenenti alla stessa decorazione, che una volta decorava una casa presso il teatro di Ercolino, ormai non piú visibile, rappresentano donne vestite di peplo classico secondo lo stile delle Danaidi della Villa dei papiri.

Da Castellammare di Stabia proviene il frammento 8953 (cat. 061a): una baccante di Stile Ricco si regge sopra un candelabro. Le figure sul frammento 9372 (cat. 076) - nel centro un Eros, ai lati due guerrieri pirrici si trovano nella stessa struttura del frammento 9878. Infine vi sono da menzionare due irammenti privi di numero d'inventario, raffiguranti figure femminili sopra candelabri (cat. 123, 124a). Una di esse è una sacerdotessa di Iside, da datare nolla prime fase del III stile.

Fuori della Campania non sono state trovate figure in guisa di atlanti nelle poche decorazioni di III stile conosciute finora. Il frammento isolato di una decorazione perduta nella villa di Tiberio a Sperlonga rappresenta una figura femminile policroma sopra un pilastrino, che proviene da una scaenae frons nella zona superiore simile a quelle campane (cat. 337 ).

Raramente si incontrano erme nella zona superiore. Nel tablino della Casa di $M$. Lucretius Fronto si vedono queste figure sulle pareti nord e sud (cat. $195 / 2)$.

\section{STILE}

Come per altri elementi decorativi e 'costruttivi', nelle decorazioni di IV stile le statue sono onnipresenti e usate in ogni maniera possibile. I finora scarsi acroteri crescono di numero, cosicche quasi ogni cornice o architrave possiede una figura o un arabesco.

$E^{\prime}$ notevole la crescita del'uso di crme nei prospetti fra i campi della zona mediana e della zona superiore. Il numero maggiore si trova ad Ercolano dove si constata una predilezione specifica per questo ornamento, che fa pensare a costruzioni metallirhe. Su scala meno larga di quanto si usava nel II stile, sono diffuse le $H$ i fifhermen; nella maggior parte dei casi si tratta di erme a lusto semplice al quale di tanto in tanto sono attaccate braccia e mani soltili, spesso reggenti attributi o rese nell'atteggiamento del ballo. Le teste sono quelle di un satiro o dell'Hermes classico, forse derivato dall'erma di Alcamene.

Erme snelle di bronzo che possano servire da confronto sono state trovate in una delle due navi (quella $B$ ) affondate al tempo di Caligola nel lago di Nemi e forse proprietà privata dello stesso imperatore. ${ }^{137}$ Le erme dipinte non fanno solo parte di architetture al posto di colonne o pilastri, ma sostituiscono qualche volta vere erme.

Sulle lesene che dividevano le scene gladiatorie nell'anfiteatro di Pompei (cat. 180), decorazione conosciuta grazie ad acquerelli dell'Ottocento, erano rappresentate erme gialle che ci fanno ricordare le siepe a erme

137 Licelli 1950 220-222, fig. 241-243; wrede 1972, 121. 
(Hermenzdune). $13 \mathrm{a}$

In 116,3 (cat, 174), nel vestibolo sulla parete di fronte allentrata si trova un'erma la cui testa presenta i tratti veristici di un ritratto, come nelle orecchie, nei capelli corti e nella mancanza di barba. Ha una corona in testa e al fusto sono appoggiati un cerchio e un ramo di palma. Gli attributi collocano la figura nellambiente agonistico e il rifratto trova contronto nelle erme a ritratto cosi frequenti in Campania, come per esempjo quella del banchiere Caecilius Jucundus. ${ }^{39} \mathrm{Ci}$ trovjamo probabilmente di fronte a] proprictario della casa, uomo attivo nel mondo sportivo, ancora giovano o almeno rappresentato come tale.

Quattro erme adornano i quattro piedi di una tavola, posta come cartibulum nel'atrio della Casa dei quattro stili (cat. 158,1). Forse il proprietario non ha potuto permettersi vere erme come piedi, pratica peraltro ben nota, e ha dovuto scegliere questa soluzione più economica perché la tavola avesse ancora qualche pregio.

Ie erme dipinte sui pilastri di una pergola del portico nella Casa di Octavius Quartio (cat. 177/3) sono dello stosso genere: non c'era la possibilità di acquistare vere erme e per tale motivo si decoravano $i$ pilastri con erme finte. Anche la parete nord di questa pergola, decorata con paradeisoi e con un Orfeo in stile grossolano, ha una serie di erme sulle lesene. La pratica di erme dipinte fra scene di caccia, paradeisoi e rappresentazioni di giardini e presente unche altrove, per esempio in I 12, 11 (cat. 171).

L'Hermenzaun quale siepe di un giardino o di uno stagno si incontra per la prima volta nel primo secolo d.C., sia in lorma vera che in forma dipinta. Le erme di Nemi costituivano il parapetto della nave. Nella Casa dell'efebo e stata trovata una serie di quattro erme a teste fittili. facenti parte della siepe che separava il triclinio estivo del resto del giardino. 340 In uno dei giardini della villa di Oplontis quattro erme a ritratto facevano probabilmente parte di una tale siepe. 14 A parte le pitture menzionate dell'epoca si ricordi la pittura funeraria di Alessandria, dove un giardino edenico viene circondato da un Hermenzaun (cat. 001). Durante l'Impero l'uso di Hermenzäune cresce di numero. Dobbiamo a $\mathrm{H}$. Wrede una trattazione e un catalogo di questo aspetto del'arte romana nella sua pubblicazione del'Hermenzaun di una villa tardoromana a Welschbillig presso Treviri. ${ }^{142}$ La pittura parietale aggiunge al suo catalogo alcuni esempi che si fanno inserire facilmente nello sviluppo tracciato da Wrede.

Pergole sorrette da erme non sono state conservate nei giardini di Pompei. Se ne vede un esempio nella Casa di Achille (cat. 283/2): i prospetti della zona superiore a sinistra e a destra della parte centrale contengono vedute di giardini con pergole sostenute da erme. In I 17, 2-3 (cat. 175) un portico sorretto da erme è distinguibile su di una vignetta miniaturistica (cf. capitolo III, p, 62).

130 Wrede $1972,121-141$.

139 De Franciscis 1951 (Pompei); 1979; 1980 (Oplontis); Cerulli Irelli 1982 (Ercolano); Dwyer 1982, 115.

140 Wrede 1972, 132-133, Taf, 76, 77.1-3; Jashemski 1979, fig. 146 .

141 Jashemski 1979, fig. 458-461; De Franciscis 1979; 1980.

142 wrede 1972 . 
Un complesso che proprio neglı zoccolı presenta un alto numero di atlantı e la Casa di Meleagro (cat 202) In quası tuttı glı ambientı tigure dell'atmostera dionisiaca sorreggono l'orlo superione dello $L$ occolo Nel vano 29 le figure sono alternamente satiri nuds e menads vestite di un chitone reggenti l'orlo con una mano Nell'altra tengono un oggetto qualsiasi Le tiguic maschli ripetono tipi scultore del quarto secolo, in particolare opere di Lisıppo, che tiovano variazom nell'Ellenismo Sono evidents in particolare le lunghe gambe, la suellezza del torso e ll forte contrapposto Nel vano 24 l satir cono dipinti in rosso nel vano 27 in glallo-marrone, tuttı derivatı da tipı cllenisticı qualı saturo danzante della Casa del fauno Sono sempre disposte a coppie simmetriche, soprattutto per quanto I iguarda l'atteggiamento di braccia testa e gamba d'appoggio Il iano 26 presenta figure ingmocchiate, nella pittura meno irequenti di quanto si trox 1 nella vera architettura sostengono con una mano l orlo dello zoccolo, mentre l'altra poggia sul ginocchio secondo l Barbaren Schema 143 Anche in queste figure ritroviamo tipl ellenistici In nessun'altra casa incontriamo un'uguale accumulazione di atlanti nello zoccolo $\Gamma^{\prime}$ stato determinante il gusto del commitiente per la scelta del motivo

Atlanti che tiancheggiano un pannello o un'edicola nella decorazione della zona interiore non sono rare Il pannello o l'edicola corisponde quası sempre ad un campo nella zona mediana, mentre gli atlant sembrano sorreggere le lesene 01 prospetti della zona mediana vel cubicolo della Casa di Paquius Proculus (cat $153 / 1$ ) solo le figure sulla parete est sono rimaste conservate al modo deglı atlantı ingmocchiatı nella Casa di Meleagro (cat 202/5) due satiri sorreggono l'orlo che chiude lo zoccolo al lato superiore Il colore giallo indica il materiale bronzo o doratura Spicca ll contrasto fra l'elaborazione dello zoccolo al contronto con la semplicita della zona mediana, ornata con candelabrı esli incoronatı da ligure temminuli miniaturistiche su uno sfondo bianco

La stessa suddivisione si presenta su di un frammento a vapolı, inv 9688 (cat 106), proveniente da una decorazione peraltro ignota Fra cassette ornate con animalı mar inı sorgono due erme a mezzo corpo incoronate con una corona di foglie e reggenti una patera nell'una e una clava nell'altra mano

Nell'ala 6 della Casa della caccia antica (cat 24j 2) due cariatidı sorreggono il baldacchuno dı un'edicola sotto la scaenae trons della zona mediana, con una mano sostengono $\downarrow$ baldacchino, nell'altra tesa reggono una patera Sumlı sono le cariatıdı nel Macello (cat $252 / 1$ ) Sono dipinte in stule classico e mostrano uno schema che cl e gia noto dalla Casd del marale (cat $301,1)$ )

Sfingl come sostegnı della fascia supcriore dello zoccolo erano dipinte in una decorazıone perduta nella Casa delle Vestalı (cat 198/1) Glı uccell tantastici stanno sotto le lesene accanto al campo centrale della zona mediana Sono similı alle sfingi di il stile nell'Insula occidentalis (cat 225/2) e verranno poi discusse a proposito delle rappresentazıonı di giardinı, dove le incontriamo come sostegni di fontane

Centaurı, altrettanto frequentı come sostegnı $d$ f fontane, sono adoperatı una sola volta nello joccolo nell'atrio della villa di S Marco (cat 009/1) s1 veciono vighe tracce di esse sulle pareti nord e ovest, mentre su quella sud solo la ligura di sinstra e ancora leggibile Davantı al centauro danneggiato di destra si trovava una cassa forte, Ivi collocata qualche tempo prima del 79, in una situazione uguale a quella nella Casa del Vetti 244 Con una certa prudenza si puo accettare una datazione della decorazione dell'atrio anteriore

\footnotetext{
143 Schmidt-Colınet 1977, 55-57

144 Cf Peter's 1977, 99, pl 61, fig 5
} 
alla ristrutturazione della v lla dovuta al terremoto del 62

Statue esposte dasantı ad un podio sono visibilı nell'atrio - se e lecito chiamare il grande ambiente in tale maniera - della Casa del palestrita (cal 267) Solo qui vediamo un'intera scaende trons con figure di soggetto agonistico, completate da un podio simile a quello del teatro di Pomper telle nicchie di esso e davantı sorgono tigure in giallo scuro, riconoscibuli come statue, peraltro tutte da collegare con tipi notı nella scultura La pittura c1 informa in modo chidrissimo sull'allestumento del podi nel teatri romani Le quattro ligure di fontane. cloe una coppla di ninte a bacmo e ll gruppo con il fanciullo che strozza un'oca, mostrano la relazione con l'sconografia des nintel 145 Le altre tigure, Hermes, un arbitro delle gare $e$ un paio di atleti, protengono dal mondo sportivo e stanno in relazsone con le figure nella zona modidna

A proposito di higure che occupano nicchie o edicole nello zoccolo non e sempic possibile decidere se si abbia a che fare o no con statue La monocromia di innumerevolı figure, certo da l'umpressione di statue, ma non dobbiamo dimenticare che anche ll contesto a spesso di un solo colore La tavolizza era, per quanto i1guarda lo zoccolo sobria e contrasti di color troppo dcutı andavano evitatı, forse per motivi di composizione cioe per non dare tioppo risalto a guesta parte della parcte

Nella Casa di Meleagro (cat 202 4) incontriamo 1igure monocrome collocate entro le edicole nello soccolo dell'oecus corinzio In si vedono anche altri ornamentı, per esemplo paesaggi idilliac1, in un solo colore cioe rosso scuro su uno stondo rosso chiaro vella Casd dell'orso terito sl incontrano talı tigure, ora policrome nelle tauces ${ }^{146}$ llcun vanı della Villa di Arianna a Castellammare di Stabia (cat 010/4) hanno uno zoccolo con niccie monociome, occupate da figure di sacerdotesse filosolı o figurı simlı La maggior parte di esse e stata staccata e trasferita d museo di vapolı. ma si deve a Allroggen-Bedel la ricomposizione di queste decorazion

Per quanto riguarda la presenza di nicchie nello zoccolo non disponiamo di esempi nella verd architettura a parte 1 podi di teatri e ch ninfel, costruzionı pero di tutt'altro ordine, poiche la loro altezza contenuta non permette un'articolarione in piu zone Le nicchie vengono sempie separate dal suolo mediante un plinto o un piede di zoccolo in modo che si trovano sempre in una posizione piu o meno elevata L'effetto creatosi in tale mamera si difterenzia da quello delle nicchie dipinte

In opposizione alle statue presenti nella zona inferiore di decorazions di III stule quelle di IV stile rivelano caritterı puu redistici e sembra che corrispondano d modell notı nella scultura La mancanza, per esempio, di uccellı flabeschi in guisa di atlantı ne e una prova chiara

Figure che reggono elementı nella zona inleriore si trovino talora nella vera architettura Nel teatro di Segesta, datato nel secondo secolo a $C$, due satir sorreggono con una mano la cornice del podio nel Satyr-Schema iel pulpito del teatro di Dioniso (sempre del secondo secolo d C ) troviamo inselitı del Sileni inginocchidt nel Barbaren-Schema litre fonti offrono 1 Satil dell'altare dı Dioniso a Delo e 1 Silenı e una figura interpretata come barbaro nel teatro dolla stessa isola i4 Vel piccolo teatro di pompei ai lati estremı della cavea Sileni nell'Atlanten-Schema sorreggono la cornice della scala 14 Anche alcunı monumentı funerarı dell'epoca imperiale sono ornatı con

${ }^{145} \mathrm{Cf}$ il pulpito del teatro di Amman (Giordania) dove le nicchie contenerano statue in guisa di fontane (El Fahharanı 1975)

146 Schetold 1957, 174

147 Schmidt-Colmet 1977, cat M163-1166 
atlantı nello zoccolo si ricordi in merito il sarcofago imponente di Velletri, datato nel secondo secolo a C $149 \mathrm{La}$ preferenza per tigure dal mondo di Dioniso non e casuale Schmidt-Colinet osserva che queste figure sono presenti nel tiaso bacchico come inservienti del dio del teatro 150 La loro presenza su sarcofagi rimane collegata con quella di Dioniso nella sua veste di dunimia funerarid Vel periodo ellenistico le ligure cionisiache entrano nell'ambito prisato e perdono il loro contenuto religioso, mentre diventano elementı puramente decorativi Le decorazıonı parietalı convalidano questo siluppo completamente, un accento troppo pesante sulla loro presenza - s1 pensi alle interpretazioni concernenti 1 gidrdinı - e insostenibile ${ }_{51}$

\section{Zona mediana}

Sono tre le maniere in cui le statue si presentano nella zona mediana Iclle scaenae frontes e nelle architetture derivate da esse le statue sono state adoperate come elementi decorativi combinati con figure vive che mettono in scena temi derivatı dal vero dramma 252 Incontriamo inoltre statue su paretı chiuse interamente o parzialmente, composte da campi, suddivise o meno tramite prospettive o lesene La terza categoria consiste nelle figure che si reggono su mensole delineandosi davantı a campı

Restano poche tracce delle picche scaenae frontes nella Domus Aurea di Nerone a Roma per poter concludere sul carattere delle figure esposte in esse (cat 310) 153 Sulla base di vecchie incisioni e disegni si ha l'impressione che le figure che riempiono le edicole e le michie, quasi sempre siano raftigurate come esseri viventi forse attori, solo in alcuni casi si trovano confronti con statue

Alcune partı sono runaste visıbll dl una decorazıone neroniana sotto la Basilica di $S$ Giovanni in Laterano (cat 313) che va inserita in questo genere di decorazioni Nelle nicchie della scaenae trons si distinguono statue marronı, ora svanite per quanto riguarda 1 dettaglı Sono ancora visibili un Hermes, un guerriero nudo (forse Ares) e una figura femminle (?) vestita di un lungo mantello von e possibile stabilire una relazıone iconogratica fra queste figure

Solo due scaenae frontes pomperane contengono statue Nella Casa dell'ara massima (cat 221/1) statue verdı di Poseldon e di Nike sorgono sopra basj aggettantı al lianco degh hospitalıa. Le statue non hanno una relazione jconografica o mitologica Ira di loro e nemmeno con ll resto della decorazione, ma sono elementi puramente decorativi La pittura, composta di elementi eterogene1 (paesaggio sacro-idilliaco, sipari, prospetti), ha perso ogni connessione con il teatro ed e unicamente decorativa

\footnotetext{
140 Castighione 1975, Abb 220-221, Schmidt-Colınet 1977, cat M19 (cf cat 173, M80)

149 Andreae 1963, Schmidt-Colınet 1977, cat w19, M74

150 Schmidt-Colinet 1977, 144-145

151 Schmidt-Colinet 1977, 145-148 Cr P 17

152 Moormann $1983 b$

$153 \mathrm{Cf}$ Peters/Meyboom 1982, Peters 1982b
} 
Sulle pareti est e ovest del triclinio di I 3, 25 (cat. 146) statue verdi di guerrieri, due per ogni parete, sorgono sul plinto aggettante a fianco dell'aula regia. Se le figure nelle edicole centrali delle tre pareti rappresentano Afrodite, Dioniso e Adone, si potrebbe trattare di Ares, rivale di Adone nella relazione amorosa con Afrodite. Le statue derivano da modelli classici del quinto secolo a.C.; l'Ares Borghese è il tipo usato per la figura sulla parete est, ora sparita a causa di un furto.

A volte si incontrano statue in prospetti fra pannelli derivati dalle scaenae frontes, memoria delle grandi facciate teatrali. L'effetto che generavano queste sontuose costruzioni a nicchie non si lascia ricostruire attraverso queste prospettive e sotto questo aspetto esse non hanno grande valore come fonte per la conoscenza del'architettura teatrale e della sua decorazione scultorea. Il fatto che le prospettive non contengono un grande numero di statue $\dot{e}$ spiegato in primo luogo dalla loro ampiezza ridotta e in secondo luogo dalla struttura dell'architettura delineata. Quest'ultima infatti rappresenta soltanto una fuga di colonne, nel quale non vi é posto per statue. (Juando si tratta di hospitalia, per esempio nella Casa dell'ara massima (cat. 221/1), figure vive occupino il posto di nllori su di un vero podio o sembrano scendere dal podio per presentare il loro ruolo. Le architelture danno solo luogo a ljgure di atlanti e cariatidi. Erme sostituiscono spesso figure tridimensionali; la preferenza per tali figure nelle decorazioni di Ercolano, e già stata menzionata ( $p$, 33).

Nella seconda categoria si tratta di architetture leggere e pareti semichiuse. Talvolta incontriamo crme e atlanti nei prospetti. Vella Casa delle nozze d'argento (cat. 189/1) donne munite di piatti fungono da carintidi di edicole che a loro volta hanno centauri come acroteri. le figure sono di piccole dimensioni e si inseriscono armoniosamente nelle architetture semplici di questo ambiente bianco, noto soprattutto grazie ai tregi con Pigmei nelle predelle sotto le architetture. ${ }^{154}$

Nel triclinio della Casa del poeta tragico (cat. 198i'3) incontriamo satiri nudi di colore giallo nei prospetti. Sorreggono con una mano una colonna sulla testa. Nei prospetti della decorazione nel vano e della Casa dei Vettii (cat. $217 / 3$ ) sono inseriti erme $e$, in guisa di acroteri, centauri. Le erme rapresentanti satiri nella sala $q$. famosa per i fregi con gli amorini, sono molto particolari: hanno tra le mani pedum. lepre, piatto o un altra oggetto, mentre portano sulla testa la colonna elaborata fra $\mathbf{j}$ campi della zona mediana.

Nell'edicola che circonda it Narcisso nella Casa dell'ara massima (cat. $221 / 2$ ) sono state inserite erme che reggono la trabeazione; si tratta di uno dei pochi esempi di satiri resi in modo plastico sorgenti sopra un fusto lungo, che ancora corrispondono alle erme di II stile. Erme di questo tipo sono altrettanto presenti nella diaeta della Casa di Apollo (cat. 198h/4), nota per le sue scaenae frontes con le storie di Marsia e Fetonte. Nella Casa di Octavius Quartio (cat. 177/2) infine un'erma di questo tipo occupa il prospetto centrale del vano $d$.

Nei prospetti sulla parete est dell'esedra della Casa della caccia antica (cat. $245 / 4)$ si trovano statue equestri in monocromo rosso sopra la trabeazione; $i$ cavalieri sono ritratti nel gesto della salutatio. Statue di questo tipo sono state trovate a Pompei e Ercolano, esposte originariamente sul foro e presso edifici pubblici e quindi note ai pittori. ${ }^{55}$ Le pitture nei Praedia di Julia Felix (cat. 179/1) rappresentano la situazione originale sul foro pompeiano prima del 62. Statue equestri su pitture nell'atrio delle Terme del Sarno (cat. $265 / 1)$ sono andate perdute. Anche nella Domus Aurea statue equestri sono

154 Schefold 1962, Taf. 121.

155 Per esempio Döhl/Zanker 1979, 185; Cavalli 1981; Adamo Muscettola 1982a. In generale Roques de Maumont 1958. 
state inserite nel contesto di una scaenac frons (cat $310 / 3,11$ ) In nessuno del casi e chiaro se le statue avessero un significato particolare o se fossero solo acroterı o membri decoratıvı

vella Casa della cacela antica (cat 215/3) si trovano statue dorate nel tablıno, purtroppo molto roinate Allantı sorreggono la trabeazione con entrambe le manı nell'Atlanten-Schema, questo atteggiamento e abbastanza raro nella pittura, mentre anche a Pompel, cioe nelle Terme del foro. lo schema, prima usato nel tempio di Zeus ad Agrigento, e adoperato per le figure tittli inserite fra le nicchie adibite a deposito per gh indumentı 156

vella Casa della parete nera (cat 247) le pitture, di notevole qualita, presentano architetture fra l pannell, quası di carattere metallico sullo stondo nero si distinguono statue dorate Dietro a tramezzi semiaperti, sopra bası parzialmente visibul, sorgono statue di Eros e di Dioniso Figure temminlı con 1 puedi a forma di tralcio, centaur e satirs sor reggono 1 varı pianı della struttura architettonica

I satirı del rano 24 e le cariatidı del vano 12 nella Casa di Fabıus Rufus nel'Insula occidentalis (cat 261/5, 2) equivalgono a quellı della Casa della pareto nesa Sono dipinte in colore dorato e mantestano una plasticita realistica I satiri sembrano protendersi oltre la trabeazione aggettante in cui sono statı sistematı, Ia gambe sono delineate nell'attegglamento della corsa

Non e del tutto chiaro se le figure in rosso chiaro poste nei prospetti delle frucer della Casa di Mcleagro (cat 202,1) siano da considerare come statue Le pilture di questa parte della casa sono interamente eseguite in rosso chiaro su uno stondo cupo iosso a la monociomid delle figure per tale motivo non e di grande peso In questo contesto valgono le osservazioni fatte a proposito delle decorazionı oligocrome di zoccoll ( $p$ 36)

I pannell nel recinto del tempio di Iside (cat $275 / 2$ ) renivano separatı da candelabrı sormontatı da statue in gialo Una serse di quattro trammentı con tigure dı sacerdotesse, o magarı dı Isıde stessa, e rumasta conservata a Vapoli Il resto e andato perduto Jon hanno una funzıone dl carlatids, ma sono espostı daranti al muro del recinto come verı candelabri, componentı dell'arredamento templare Sono controntabli con le figure des satiri nella Casa del poeta tragico (cat 198/3) Ga nella Vulla della Iarnesind (cat $319 / 6$ ) si trovano candelabri incoronatı da figure fra 1 campı

Ad Ercolano 1 decoratori sembra avessero una predilezione particolare per le architetture aeree sottilı per quanto riguarda le strutture nes prospettı Come e stato detto, un'influenza da parte della toreutica e verosimile Le erme inserite entro di essi sono sempre monocrome e dello stesso colore della struttura e percio talvolta difficumente riconoscibili

Ses delle otto pitture con elementi architettonici studiate in merito posseggono erme Nell'ambiente l della Casa dello scheletro (cat 022,1) si vede una prospettiva nell'abside Nel centro sorge un candelabro incoronato dalla statua di un pavone, mentre la tholos che circonda ll candelabro ha acroterı a forma di ippocampi Neı prospettı sulle paretı lateralı si distınguono erme a braccia sottli in rosso, quasi interamente andate perdute

Veglio conservate sono le erme nel rano 10 della Casa del cervi (cat 029 1) Qui il colore delle strutture e giallo, quello dello sfondo rosso vell'ambiente 16 della medesuma casa si vedono erme rosse su di uno sfondo ceruleo Ivı si trovano anche centaurı rossi sopra le cornici delle edicole, dipinte in modo deltaglsato con un pennello sottile Hanno contemporaneamente la funzione di atlanti e sostengono con la testa le esli colonne di un piano superiore Bighe con Nihal incoronano le edicole come acroteri

vella Casa dell'atrio a mosaico (cat $025 / 2$ ) le erme nell'esedra hanno teste dettagliatamente eseguite Nella Casa del bicentenario (cat 032 2) incontr lamo erme simll Nella Casa del bel cortule (cat 031), per finure,

156 Schmidt-Colinet 1977, 31-35 Cf p 16. 
cáriatidi plastiche sorreggono l'edicola centrale.

Le erme che ravvivano le architetture dipinte del portico della villa romana di Pully in Svizzera (cat. 308a) sono l'unica prova linora di una diffusione del motivo oltre la regione resuviana.

Vel cubicolo della Casa del mobilio carbonizzato (cat. 030) sfingi e sirene fungono da acroteri sulle edicole centrali della zona mecliana. Vella Casa dellalcova (cat. 026) i prospetti sulle pareti nord e sud del vano 19 ospitano tholoi con un tetto a forma di pagoda. Due frammenti a Napoli provengono dalla parete sud, mentre la parete nord si presenta ancora intatta. Sul tetto sorgono piccole statue di figure femminili vestite di peplo a chitone - il formato miniaturistico non permette una determinazione precisa - simili alle Danaidi della Villa dei papiri.

tumerose sono le figure che fungono da acroteri. Mi limito a menzionare alcuni esempi particolarmente evidenti. La scelta si è basata sul criterio che essi debbano essere considerati concrctamente come elementi plastici, non schematizzati in forma di tralci e girali o di figure riconoscibili solo per le protomi. Esiste una preferenza per i centauri, spesso muniti di una codal di pesce (ippocampi) o di ketea, come si vede fra l'altro nella Casa di Pinarius Cerealis (cat. 182/1). I centauri corrispondono a modelli ellenistici, gia citati. conosciuti per esempio da quattro copie trovate ad Oplontis. ${ }^{25} 7$ Alcuni di essi sono stati menzionatj di passaggio. Nel periodo ellenistico questi anunali diventano accompagnatori di innumerevoli divinita ed eroi e fanno il loro ingresso nel tiaso bacchico. Specialmente come meinbri del corteo di Lioniso hanno acquistato un'enorme popularita nel repertorio pittorico. La loro Cunzione è puramente decorativa $e$ in nessun caso un rapporto iconografico tra essi e il resto della decorazione puo essere stabilito.

Nella Casa di Sirico (cat. 232) vediamo gruppi gialli composti da centauri e pantere inseguite dai mitici cacciatori. Nel museo di lapoli si trovano alcuni tramunenti di prospetti (cat. 059) dove capre di bronzo stanno su di una trave accanto a figure femminili o maschili, spesso interpretate come sacerdoti e sacerdotesse per j loro attributi. Il signilicato di queste capre non e chiaro. Per il tipo statuario disponiamo di un bell'esemplare in bronzo proveniente da uno degli scavi borbonici in Campania. ${ }^{158}$

Nelle decorazioni a campi incontriamo un grande numero di figure isolate, sia volanti sia stanti in atteggiamento rilassato. Per quanto riguarda le ligure in volo bisogna constatare che esse non sono mai state oggetto di studio sistematico. La monografia di Ellen Schwinzer non entra nella questione dell'origine del genere. ${ }^{59}$ probabilmente l'idea di decorarc campi con ligure isolate in volo deriva dal costume, nolo dallepoca cllenistica in poj, di appendere statuette di terracotta a fili, e di modellarle noll'atteggiamento del volo: nei musei giustamente le figurine fittili di questo tipo vengono esposte in tale maniera. ${ }^{160}$ In origine l'uso si limita a Psichi alate e ad Eroti, esseri per qualita naturale capaci di volare, piu tardi si modellano anche altre figure in questo modo. Per corroborare questa ipotesi. sarcbbe necessario un confronto approfondito delle figure dipinte con le terrecotte. Parte delle figure stanti $\dot{e}$ indubbiamente riconoscibile come statue, in quanto si reggono su mensole o piccole macchie che equivalgono a mensole. Il pittore le rafifigura in modo tale da mostrare le mensole e le figure di sotto in su anche se si

257 Schäfer 1972, 164-192; Lattimore 1976, 58. CC. Bielefeld 1969 (Nereidi di Formia) e De Caro 1976, 198-219, fig. 5-20 (centauri di Oplontis).

158 MN inv. no. 4903 (Ruesch no.827).

159 Schwinzer 1979.

160 Cf. Borbein 1973, 171 nota 525; Hill 1981, 87. 
trovano all'altezza normale. Ciò si spiega attraverso la pratica, sopra ricordata (p. ×0), di esporre statue in alto. A parte gli esempi già menzionati di mensole su colonne, si ricordino le mensole nella cella del tempio di Iside a Pompei collocate nella zona superiore delle pareti laterali. Non si puó piú stabilire se esse servissero proprio per collocare statue oppure lucerne o oggetti di culto; $\dot{e}$ possibile che si tratti di elementi costruttivi reggenti la trabeazione del soffito. Anche nel muro che circonda il Foro triangolare a Pompei si vedono mensole, probabilmente inserite per l'esposizione di lucerne. Nel macello di Pozzuoli, il cosiddetto Serapeo, si trovano mensole in alcune dolle botteghe, mentre solo nella Casa del fabbro a Pompei una mensola in un muro cieco sembra aver avuto la funzione di sostegno per una statuetta. ${ }^{16} 1$ L'inserimento di figure stanti sopra mensole davanti a campiture è pratica comune nei rilievi. Si pensi alla base atenese, attribuita a Prassitele, con su ogni lato una figura sopra una mensola 'per obbligarci a considerare le figure come statue. 1162 Le decorazioni delle mensole ogni tanto dettagliatamente raffigurate, per esempio nei Praedia di Julia Felix (cat. 179/2), si ritrovano su mensole in vari musei, dove esse - repertl di vecchi scavi il cui contesto e funzione non sono piú ricostruibili - sono inscrite nelle pareti per sostenere statue o elementi architettonici. Nel Museo Nazionale Romano un esemplare mostra una somiglianza sorprendente con le mensole dipinte; la datazione proposta nel nuovo catalogo della collezione, che lo pone in età flavia, verrebbe convalidata dalle mensole dipinte di IV stile. ${ }^{163}$

Il repertorio di figure su mensole è limiato. Divinitả e figure mitologiche sono quasi completamente assent1. La maggior parte delle figure rappresenta guerrieri nudi e Muse. Eroti si incontrano in una sola casa, cioe nella Casa di $M$. Lucretius (cat. 282) e su un frammento a Napoli (cat. 074), essendo parodie di guerrieri.

Incontriamo serie di Muse delineate semplicemente su campi e non sostenute da mensole nella Casa di Fabius Rufus (cat. 261/5) e nella casa adiacente nell'Insula occidentalis (cat. 262/8), nella Casa delle Muse (cat. 286/3). Nella Casa di Sirico (cat. 232) e nei Praedia di Julia Felix (cat. 179/2) le ligure sono poste su mensole. La Casa di Sirico contiene otto Muse su mensole su altrettanti campi fiancheggianti l'entrata dell'ambiente e i campi centrali delle altre pareti, mentre per ragione di completezza la nona Musa e Apollo Citaredo sono stati dipinti nelle prospettive sulla parete di fronte all'entrata accanto al campo centrale. Che queste due figure siano state aggiunte per completare la serie $\dot{e}$ dimostrato dal fatto che nelle prospettive delle altre pareti non sono state inserite figure. Il decoratore, pero, non si $\dot{e}$ orientato a favore della caratterizzazione delle figure come statue, ma le ha raffigurate come persone vive.

Le otto Muse e l'Apollo Musagete dai Praedia di Julia Felix (cat. 179/2) sono stati scoperti nel 1755. Una nona Musa apparentemente mancava: nel vano 92 non vi è posto per essa. La collocazione dei frammenti da parte di

161 Jashemski 1979, fig. 193.

162 Rizzo 1933, 29-30, tav. 44-45; Fuchs 1979, 530-531, Abb. 623. Un altro esempio è un rilievo di Cirene con quattro divinità: Beyen 1951, pl 14, fig. 6; Paribeni 1959, 34-35 no. 49, tav. 49. Cf. Gabelmann 1977, 201-202, Abb. 1, 6-9, 11-12, 16, 26-27, 29.

163 CatRoma I/2, cat. 65. Cf. le mensole nelle Terme di Vedius ad Efeso, secondo lo scavatore J. Keil adoperate per le statue ivi trovate (OeJh. 24 (1929) Beiblatt, 37); Manderscheid 1981, 26, invece, sostiene che esse portassero candelabri. La piccola mensola nella Casa dell'ara massima avrebbe potuto servire da sostegno per una lucerna o una statuetta(cosi La Rocca/De Vos 1976, 282). Altri esempi non mi sono noti. 
Maiuri all'ambiente 97 , dove credette di poterne vedere vaghe tracce, è insostenibile: queste presunte tracce mancano completamente, inoltre non vi $\dot{e}$ posto per i nove frammenti del Louvre. Le mensole reggono un'epigrafe in grcco che esprime nome a funzione di ogni figura. Per tale motivo le figure hanno un valore particolare nello studio delliconografia delle Muse; “. infatti si tratta della prima testimonianza di una fino a quel momento non ancora fissata attribuzione di oggetti e qualità a determinate persone. Nel primo secolo d.C. si è sviluppata questa iconografia fissa, che è rimasta valida durante tutto l'Impero. Apparentemente l'iconografia, spiegata attraverso le epigrafi, non era ancora familiare al committente.

L'applicazione di iscrizioni sui piedistalli di statue era frequente, ma solo raramente esse riportavano il titolo del lavoro o un'indicazione del soggetto rappresentato, cose senz'altro chiare per chi le guardava; in quasi tutti i casi si tratta della firma di uno scultore o di una dedica. ${ }^{165}$

Anche nella scultura disponiamo di serie di Muse. Secondo Doris Pinkwart si distinguono tre gruppi di base creati tra 150 e 120 a.C. Lino di essi viene attribuito a Filisco, artista menzionato da Plinio, il cui gruppo venne esposto nel lempio di Apollo presso il Portico di (Ittavia a Roma. ${ }^{166}$ ina ricostruzione completa di questo gruppo non e possibile, ma si puo ammettere che la maggior parte delle statue di Muse tramandateci in forma di copje romane derivino da questa serie. 167

Purtroppo le pitture non danno nemmeno una soluzione per questo problema. Ogni complesso è diverso e le tigure singole derivano, se mai un confronto è possibile, da statue appartenenti ai vari gruppi stabiliti da Pinkwart. Non vi sono esempi di vere e proprie copie di statue. Un tipo frequentemente copiato come la Musa appoggiata (la cosiddetta aufgelehnte Muse), idontificala come Polinnia, si osserva nelle pitture mafliguranti liodialo c learo, dove la figura funge da Akte, cioe da ninfa locale, mentre non lo si incontra mai nelle serie di Muse dipinte. ${ }^{260}$

Anche nella pittura postpompeiana si conoscono serie di Muse. Anticipando il capitolo dedicato a queste pitture, presento un complesso interessante, cioc quello della Casa delle Muse ad Ostia (cat. 130.1), decorita nellepoca adrianea. Bianca Felletli Mal e P. Moreno, nella loro pubblicazione accurata e dettagliata, non hanno osservato che le 'strisce di terreno' sulle quali si reggono le Muse sono rettangolari e hanno orli a rilievo, due caratteristiche proprie alle mensole. ${ }^{169}$ Soprattutto la mensola di Erato esclude ogni dubbio. Solo in questa casa l'ordine stabilito da Esiodo e stato rispettato, sempre che quello delle otto Muse nei Praedia di Julia Felix non fosso uguale. In testa portano una piuma, premio della gara con le Sirene, motivo non ancora presente nelle pitture pompeiane. 1701 sarcofagi del secondo e del terzo secolo

164 Della bibliografia abbondante si citano i lavori piú recenti: Pinkwart 1965; Pinkwart 1967; Felletti Maj/Moreno 1967; Wegner 1969; Paduano Faedo 1981; Marabini Moeus 1981; Pucci 1981; Fuchs 1982; Theophilidou 1984.

165 Peschlow-Bindikat 1975, 377-379; Kron 1977, 148-162.

166 Pinkwart 1965, 91-168.

167 Cf. il gruppo del teatro di Pompeio sul Campo Marzio: Coarelli 1972; Fuchs 1982 .

169 Una statuetta di questo tipo è stata trovata nella Casa di Octavius Quartio: Spinazzola 1953, I, fig. 462. Cr. p.e. cat. 161,2.

169 Felletti Maj/Moreno 1967, 21, tav. V. 
mostrano la stessa piuma e l'ordine esiodeo; $i$ tipi solo in alcuni casi corrispondono a quelli pompeiani.

La scelta di queste Muse $\dot{e}$ stata fatta volutamente sulla base di un interesse culturale del committente e non solo per motivi decorativi: gruppi di Muse venivano esposti in teatri, terme, biblioteche, ginnasi e giardini di ville per esprimere personificazioni delle belle arti. ${ }^{27}$ Stranamente non sono stati trovati gruppi di Muse in santuari dedicati ad esse: conosciamo solo il famoso gruppo di Ambracia, asportato da Fulvio Nobiliore e dedicato nell'Aedes Herculis Musarum a Roma, ${ }^{17}$ Nelle ville venivano esposte per suggerire l'atmosfera dell'Elicone, la loro sede tradizionale, o dell'Accademia. Cicerone, in una delle sue lettere (Fan. VII 23, 2), considera le immagini delle Muse adattissime come abbellimento della sua biblioteca. Le statuette nella Casa di Cotavius Quartio - di un gruppo di sei o sette solo quolle di Polinnia e Erato si sono conservate - costituiscono un rispecchiamento modesto di questa moda. ${ }^{173}$

Le piture parietali sono derivate dalla stessa idea e devono clare all'ambiente il carattere di un semplice Ifouseion che simbolizzi la formazione spirituale del padrone di casa. Sulla base del materiale a disposizione non è possibile rintracciare la precisa funzione dei vani in merito. Non sono stati trovati armadi o scaffali che indichino l'uso degli ambienti come biblioteche, ma si puo ugualmente pensare a un gabinetto di lettura o a uno studio, come avviene nell'ambiente di II stile nell'Insula occidentalis (cat. 262/3). 174

Serie di guerrieri nudi si incontrano nella Casa dei Dioscuri (cat. 204:1), nella Casa di (Octavius Quartio (cat. 17T/1), nella Casa delle Vestali (cat. 198,1), nella Casa delle Muse (cat. 286,2) e nella Casa della gemma ad Ercolano (cat. 036). Nelle Terme suburbane ad Ercolano (cat. 024) si è conservata una serie di guerrieri in rilievo a stucco. Nei prospetti nella Casa del poeta tragico (cat. 198/3), nel giardino della Casa dell'efebo (cat. 15j;4) e nella scaenae frons in I $3,2 \bar{j}$ (cal. 146) si vedono coppie o esemplari isolati. Nel museo di Napoli si trovano alcuni pezzi staccati con tali figure, provenienti con una certa probabilita da tali gruppi (cat. 070, 124 sopra candelabri).

Si tratta sempre di figure nude, armate di elmo, clamide, lancia, spada e scudo, rappresentate in atteggiamento di riposo sopra mensole o basi ovoidali. L'esecuzione spesso è priva di dettagli e non permette un confronto preciso con la scultura, ma in generale i guerrieri dipinti sono stati modellati secondo tipi di statue di atleti del quinto secolo a.C.; specialmente le statue canoniche di Policleto come il Doriforo e il Discoforo sono state di grande importanza nello sviluppo del corpo nell'arte glittica non solo nell'epoca classica, ma anche oltre. ${ }^{175}$ Nelle pitture se ne constata l'influsso nella posizione delle gambe, nel leggero contrapposto, nell'armonia di gambe, torso e testa. Ció non

170 Esiod., Theog. 77-78; Felletti Maj/Moreno 1967, 25-26.

171 Teatri (Schwingenstein 1977, 55-57), spec. il teatro di Pompeio a Roma (Coarclli 1972; Fuchs 1982); biblioteca di Celso ad Efeso (Eichler 1953, 59-60, 81-84, Abb. 102; cf. Callmer 1944); terme (Manderscheid 1981, 34); ville (Zanker 1979a, $476 \mathrm{n}$. 54; Hill 1981, 87-89, fig. 3-5).

172 Marabini Moeus 1981.

173 Spinazzola 1953, I, 405-406, fig. 461-462; Pinkwart 1965, 91; Zanker 1979a, 476, Abb. 8; Döhl/Zanker 1979, 205, fig. 115.

174 Cf. Callmer 1944; Leach 1982.

173 Arnold 1969; von Steuben 1971: Borbein 1973; Zanker 1974, spec. XVIII. 
significa che solo esempi policletei siano stati adoperati. L'Ares Borghese di Alcamene si incontra talvolta, per esempio in I 3, 25 (cat. 146), cosi corne copie di figure quali l'Agias di Lisippo o di figure neoattiche o classicistiche del primo secolo a.C. possono aver influenzato i pittori. Il fatto che proprio a Pompei sia stata trovata la copia piú fedele del Doriforo di Policleto, esposta in una palestra presso $i$ teatri, serve come testimonianza di una conoscenza diretta e come prova della preferenza per il maestro classico. ${ }^{276} \mathrm{Si}$ puo concludere che nel primo secolo a.C. e in quello successivo, specie nell'epoca augustea, il modello per l'uomo nudo(j cosiddetti 'Achilleis') era il Canone di Policleto e che la domanda di copie di statue in questo stile cresceva. ${ }^{2}$, Anche nel tempo di Adriano, forse sotto l'influsso dei ritratti di Antinoo, si verifica un revival dell'ideale policleteo. Dobbiamo a P. Zanker vari studi su questo tema: egli ha dimostrato che l'interesse per statue del genere va spiegato con la tendenza ad ideali, certo poco concreti, come giovineza eterna, bellezza perfetta e armonia. ${ }^{17}$ Le statue dipinte possono essere inserite in questa tradizione e corroborano le osservazioni di Zanker. ${ }^{179}$ Purtroppo esse non permettono osservazioni accurate concernenti la critica di copie e lo studio di varianti neoclassiche; per tale scopo sono troppo libere e non dettagliatamente eseguite. Le caratterizzazioni generali qui schizzate sono per lo meno ben distinguibili.

Non è detto che le statue dipinte databili nel periodo del IV stile abbiano ancora il significato delle statue dell'cpoca augustea, quando gli eroi fungono da simbolo della giovinezza e servono da esempio per la vita. Il fatto che quasi sempre appaiano in serie di piú di due indicherebbe una scelta imposta da una certa filosofia. Tuttavia il valore decorativo non cleve essere stato meno importante. R. Herbig ritenne che si trattasse dei Sette contro Tebe. ${ }^{180}$ Stupisce peró che in nessun caso abbiamo a che fare con serie dj sette figure. Naturalmente dobbiamo essere prudenti con questo argomento, in quanto la tradizione di molti complessi decorativi è debole. Ln secondo punto di opposizione esiste nel fatto che la saga tebana non figura quasi maj in pitture campane. Né su pinakes ne in forma di figure isolate incontriamo Edipo, Antigone o il duello di Eteocle e il suo fratello Polinice. ${ }^{21} 1$ Nel caso che i guerrieri rappresentassero determinate figure mitologiche, queste sarcbbero individuate per mezzo di attributi specilici.

176 Zanker 1974, B. Taf. 5.1. Cf. la testa policletea dalla Villa dei papiri: ibid. , 8, Taf. T.1, 31.2.

177 Zanker 1974, XVIIII, 41-45.

17в Zanker 1974: Sie ist Ausdruck einer allgemeinen Sehnsucht nach Erneuerung, die Augustus mit der Programmatik des saeculum aureum politisch frilchtbar machen versuchte. Cf. Zanker 1979; 1981; Preisshofen/Zanker 1970-1971.

179 Zanker 1974, XIX: Entsprechende Untersuchungen an Monumenten, die von Bev Blkerungsgruppen der 'Mittelschicht' in Auftrag gegeben wurden (z.B. Graburnen, Grab- und Weihreliefs, Malereien und Skulpturen der entsprechenden pompejanischen H^ausern), $m^{\prime} v s$ sen zeigen, wie weit der Zeitgeschmack sich hier entwickelte, wo Eigenheiten und besonders auch zeitliche Verschiebungen festzustellen sind.

100 Herbig 1962, 16.

181 VII 6, 28 (Niobidi, funerali di Eteocle. Eteocle e Polinice, Amfiarao); III 4. 2 (Edipo $e$ locaste). Solo $\mathrm{i}$ Niobidi si incontrano piú frequentemente (c1. Schefold 1957, registro s.v. Niobiden). 
L'importanza del valore decorativo avrà prevalso col passare del tempo. Gli Eroti in veste di guerrieri nella Casa di $M$. Lucretius (cat. 282), dove sono visibili in ben tre vani, imitano e sono parodie nello stesso momento di tali serie. Nel vano $\mathfrak{G}$ vediamo Eroti vestiti come Apollo, Hermes, Dioniso e Eracle. Nei vani 7 e 9 reggono armi e sono indeterminabili in senso mitologico. L'Eros diventa nell'Ellenismo la figura piú adatta per esprimere la relativitá di molti soggetti e nell'epoca romana questo fenomeno si è diffuso su larga scala. ${ }^{132}$ Un frammento isolato a Napoli (cat. 074) raffigurante un Eros che regge una fiaccola davanti al petto potrebbe provenire da una serie simile.

Anche altrove incontriamo figure in riposo isolate su campi. Mi limito a menzionare alcuni esempi. Nella Casa di Octavius Quartio e nel tempio di lside sono stati raffigurati sacerdoti di Iside. Von sembra che siano state statue i modelli di queste figure come nel caso delle Muse. Anche qui vale il fatto che il committente e 11 decoratore hanno compiuto una determinata scelta nella quale il valore decorativo non viene al primo posto. Nel caso delle figure egizie si tratta dell'interesse generale per l'Egitto ed in particolare per il culto isiaco. 183

Un carattere scultoreo è leggibile nelle figure dipinte in edicole isolate; queste imitano vere nicchie in uso per statue. In I 13, 16 (cal. 173) in un inquadramento rosso, molto grezzo, si trovano tre statue su piedistalli in forma di colonne. A sinistra sorge Afrodite che regge con la sinistra i suoi capelli e nella destra uno specchio. L'atteggiamento delle braccia e delle gambe è preso dall'Anadyomene, anche se in modo libero. Anche il contrapposto è derivato da questo modello. La figura è di colore bianco, mentre i capelli. gli occhi, il drappo attorno al corpo e lo specchio sono stati dipinti in colori vivi. Accanto sorge una base bassa con la figura di un pavone in blu, raffigurato come animale reale. A destra si trova la statua verde di Priapo visto di profilo e rivolto verso Afrodite. L'insleme è reso in modo schematico e rozzo. Le tre statue stanno su di una striscia rossa forse presente come indicazione in prospettiva della nicchia. Il telaio che circonda le tre figure non ha nessuna traccia di profondita. Il significato del gruppo non deve essere altro che erotico, trattandosi di una camera da letto.

Lo stesso modello di Afrodite, ma ora rappresentato come figura reale, si incontra sulla scaenae frons della Casa di Pinarius Cerealis (cat. 182/1) e nella fullonica di Vesonius Primus (cat. 213/1). In quest'ultimo caso Ermafrodito è modellato secondo questo tipo. Sullo sfondo si distíngue la statua di Priapo.

In VI 8, 20 si trovava un'immagine di Afrodite posta in un'edicola, frammento ora conservato a Napoli (cat. 199), probabilmente proveniente da un pannello nella zona mediana. Non si puo stabilire se si tratti di una statua - di una figura viva.

Una pittura ora perduta nella Casa della regina Margherita (cat. 190) mostra un'edicola nel centro della parete occupata dalla statua di leda e il cigno. Il gruppo sorge sopra una base rettangolare e rappresenta la famosa creazione di Timoteo in situazione inversa. Secondo Herrmann qui non è lecito parlare di una copia di quest'opera, datata attorno agli anni 380-370; scultura che egli riconobbe piuttosto su pinakes di altre case dove la Leda viva sarebbe stata modellata secondo questo tipo. Già M. Moreau nel 1961 e piú recentemente la specialista in merito Anita Rieche menzionarono la pittura come una copia della statua. Rieche aggiunge all'elenco di ben 28 copie in

102 Stuveras 1969, 70-71.

193 Risale alla Repubblica questo interesse (mosaico di Palestrina, pitture nilotiche di II stile nellatrio della Villa dei misteri), ma è soprattutto nell'epoca augustea che questa 'cineseria' trova grande diffusione (Aula isiaca, Villa della Farnesina). Cf. De Vos 1981 . 
mirnio anche I punakes in VIII 2, 38 (cat. 269/4) e nella Casa della caccia antica (cat 245/1), ricordati in precedenza anche da Herrmann. Nel nostro caso proprio il piedistallo e un'indicazione precisa dell'intenzione del pittore di rappresentare una statua. Che la figura non mostri tutte le fattezze della scultura non diminusce l'importanza della figura come esemplare da inserire nella serie delle copie Herrmann non prendeva in considerazione una figura a meno che non rispondesse a tutte le caratteristiche, come se fosse una fotografia dell'originale. Questo punto verra discusso a proposito di altre figure plu avanti (p.Ss).

\section{Zona superiore}

Vumerose sono, come nel III stile, le scaenae frontes nella zona superıore, spesso prive di connessioni con la zona mediana e con le prospcttive inserite tra 1 pannelli ciechi, essendo presentı come entita indipendentı ${ }^{4}$ " Le figure in queste architetture dumostrano differenze maggiorı per quanto riguarda la collocazione e l loro numero e per tale motıvo il ventaglio dei modellı usatı e maggiore di quello impiegato nel III stule. Imitano in modo modesto le vere scaenae frontes di teatri romani imperiali. Spesso le edicole centralı sono occupate da figure, quasi sempre con pendants sulle paretı opposte. Emerge l Latto che sovente solo due delle pareti dipinte in un vano presentano tali edicole: in tale caso si tratta sempre du pareti che si fronteggiano Spesso le figure non sono state caratterizzate come statue attraverso 1 colore o la collocazione su di una base. Alcune di queste figure 'clubbie' sono state inserite nel catalogo. L'mteresse maggiore e stato dedicato a ligure che sulla base del criteri stabiliti possano essere distinte chiaramente come statue.

Le Auraj, cosı caratteristiche per ll III stlle come acroteri, cedono ora quası completamente a favore di centaurı, bighe (soprattutto ad Ercolano), sirene, sfingl e le forme meno articolate di acroteri.

Figure di sostegno sono presenti in numero minore. La scaenae frons in I 3,25 (cat. 146) e composta di due pıanı, nel superıore del qualı - nonostante l'altezza ridotta del sano considerato come zona superiore - I igure in piedi e tigure inglnocchiate iungono da atlanti. Sulla parele sud tigure ammantate ricordano le carjatidi classiche vestite di peplo, ma sono ralfigurate come figure armate Lo stato di conscrvazione pessimo non permette un'unterpretazione precisa di esse. Sulla medesima parete e su quelle lalerali si vedono naophorai, figure che entrano nell'ambiente egizio introdotto nell'epoca augustea e presenti anche nel IV stlle. Sopra l'aula regia della parete sud sorge una biga guidata da una Nike, mentre sopra quelle delle altre pareti statue bronzee di Artemide sono esposte come acroterl. Tutte le figure sono monocrome in colore bronzo e gia per questo caratterizzate come statue. Come per ll III stile non e possibile trovare un esempio nella realta finora conosciuta La posizione delle figure a un livello superiore ricorda gli atlanti delle Terme del toro o le cariatıd del foro di Augusto a Roma. I tentativi dell'architetto $G$. von Cube di tradurre le scaenae frontes dipinte in ricostruzioni tridimensionali e, sulla base di esse, i contront con monumenti esistenti non sono convincenti. iof Gia ll punto di partenza, cjoe che modelli concreti siano stati la base per le scaenae frontes dipinte, suscita dubbi. 197

\footnotetext{
104 Cf. Peters 1982a.

185 Per quanto riguarda i centauri cf. p. 40, nota 157.

186 von Cube 1906.
} 
Un frammento a vapol (cat 052) presenta una suonatrice di lira sopra un elemento architettonico a due volute, forse la cornice dell'edicola centrale du una zona mediana non plu esıstente Sopra di essa si vede una cornıce doppia a mensole nella quale sono inserite le figure gialle di una menade $e$ di centaurı Sono quası imitazionı delle figure nella Villa di P Fannius Synıstor a Boscoreale (cat 004/1) e nella Casa delle nozze d'argento (cat 189/2, of p 000)

Acroter che si situano ancora nella tradicione del III stule sono usibil nella Casa della Venere in bihmi (cat $166 / 1$ ) dove sulle pareti nord, sud c est sono disposte tigure circondate da trales a torma di miandorla L'assoluta simetria di molte pareti e andata perduta poiche su ognu parete una figura temminle fiancheggia una figura maschile Non si puo stabllire se avessero un significato particolare a causa del cattivo stato di conservazione Sembrano delineate come figure vive, datı, lineamentı del viso e l'atteggiamento del corpo Von $v 1$ sono confrontı nella scultura conosciuta

Cariatidı indossantı ll classico peplo si incontrano nelle Terme del Sarno (cat 266/2), dove esse reggono la cornice con una mano secondo il Karyatiden-Schema Nella Casa dell'atrio a mosaico ad Ercolano (cat 025 2) le edicole centralı hanno acroterı a torma di figure femminlı gialle $d_{1}$ stule classico che sorreggono con la testa un'esile colonna von differiscono dalle figure in guisa dı car atıdı del IIl stule

Alcune volte incontriamo erme nella zona superiore $D_{1}$ purticolare interesse per la conoscenza del motivo delle pergole nel giardinı sono le prospettive della Casa di Achulle (cat 283/2), menzionate prina Von costituiscono un'unita con l resto della decorazione, ma si trovano all'interno di finestre neglı angolı superjori della parete In IX 1, 7 (cat 276) nel prospetto della zona superiore sorge un'erma, attorno al cul fusto sono stati avvolt con offerte votive La decorazione spicca per la raftinatezza dell'esecuzione del dettagh, soprattutto del centauro armato di scudo e lancia che sorregge la trave

Nell'aula absidiata della palestra ercolanese (cat $039 / 1$ ) si trovano scarsı resti di un'architettura complicatıssima nella quale sono inserite erme funzionanti come plastri Di recente Allroggen-Bedel e riuscita a stablire che ll noto frammento inv no 9731 del museo di Napolı sul quale si vedono due erme, proviene dalla decorazione di questa sala, probabilmente da una delle ante a sinistra e a destra dell'entrata La struttura e paragonabile con le prospettive esli in altre case ercolanesi, dove la propensione per l'erma come motivo architettonico e cosı frequente una raffinata costruzione con erme si rede per esempio anche nella draeta della Casa del gran portale (cat 034/2), dove e eseguita in blu su uno stondo ceruleo

I framment unv 8572 e 9859 a Napolı (cat 051,111 ) e un frammento sporadico a Castellammare di Stabıa (cat 010/7) provengono da decorazıonı simulı Sul primo frammento si distingue un colonnato sorretto da erme dorate che hanno teste di figure femminil], mentre la cornice e incoronata da sfing grigio-nere poste su piedistalli rettangoları Il secondo frammento mostra un'erma con la testa di un uono barbuto secondo ll famoso tipo dell'Hermes Agoraıs dl Alcamene Anche in questo caso deve trattarsi di una struttura sontuosa, la cui posizione non e ricostruibile ll colore dell'insieme e rosso, ora scolorito e divenuto in parte glallo, in parte rosa

Piu frequenti nell'ultima fase pittorica di Pompei sono le figure esposte in nicchie 0 in edicole Non rarı sono 1 frammentı con figure entro nicchie a Vapoli, provenientı da tall decorazioni Dionysos, Afrodite e Apollo sul frammenti inv 8947, 9542 a 9277 (cat 061) stanno a cavallo fra figure vive e slatue Potrebbero provenire da una struttura nella zona superiore della Casa deı Dioscurı (cat 204/1, 5), al disopra di campı bianchı con guerrierı su

2:7 Cf Moormann 1983b 
mensole (vano 35) e nel vano 43. Le tugure non sono state caratterizzate come statue attraverso bası o color Come e stato detto sopra ( $p$ 000), un caso dubbio e anche I'Atrodite Anadyomene di VI 8, 20 (cat 199)

Jel vano 1 della Casa del sacello llaco (cat 1492 ) l'edicola della parete est c quelle lateralı della parete sud sono occupate da guerrier nudi La decorazione dell'ambiente non lascia un'impronta omogenea sui campi della zona mediana si vede una combinazione di figure poste su mensole, crifterizate soltanto grazie a questo clemento come siatue, e di ligure hodute e stanti prive di questa aggiunta Nella zona superiore la situdzione non c diversa un soldato romano, riconoscibile per la sua divisa dettagliatamente delineata, non si lascia combinare con le altre figure mitologiche o di genere

Fuor d'ogni dubbio e l carattere scultoreo delle figure gialle egizie nel cubicolo della Casa dell'efebo (cat 155/1) Due donne munite di un sistro sono interpretablı come sacerdotesse di Iside o come la dwinita egizia stessa. Si trovano di fronte l'una l'altra sulle paretı nord e sud e mettono di nuovo in evidenza l'amore deı Romanı per I pendants

Anche nell'ambiente d della Casa della piccola fontana (cat 200 1) le figure della zona superiore sono caratterizzate come statue per $\downarrow$ loro colore giallo Apollo e Eros si trovano sulle parets lateral, mentre sulla parete di tondo vediamo la statua di una divinita femminule in trono Diversa rispetto alle altre figure, essa e dipinta in marrone lo sbiadimento ne rende difficile l interpretazsone, forse si tratta di Demeter o di Tyche Il modello corrisponde in linea generale a quello di statue ellenistiche di figure sedute Ina specificazione e impossibile Nell'ambiente 24 della stessa casa uomini nudi, forse del medesimo genere del guerrierı su campı, sorgono sopra candelabrı; purtioppo manca la maggior parte dell'mtonaco

vella Casa del naviglio el troviamo dainntı ad una delle poche opera nobilia della scultura classica inserite in una struttura architettonica (cat 2072 ) nel'edicola centrale della zona superiore della parete nord si vede l'Hermes di Olimpia, opera di Prassitele per il tempio di Hera, menzionato da Pausania (V 17, 3) Regge nella destra alzata un grappolo d'ura, presentandolo al giovanissimo Dionso seduto sul braccio sinistro L'unica variazione e la costituzione della testa $d_{1}$ Hermes con quella di un satiro l che non toglie niente all'importanza della raffigurazione Per $\downarrow$ pittore si tratto di una copia immediata, volutamente scelta dal famoso pezzo Sulla base della pittura si puo con quasi assoluta sicurezza ricostruire $l$ braccio destro della statua di Olimpia I critici che si sono opposti dlle differenze di stle ed esecuzione in contronto alla statua prassitelica, la cui dutenticita non e tuttora certa non hanno riconosciuto ll carattere particolare della pittura, mentre nello stesso tempo non si sono resi conto delle difficolta che incontra un pittore che voglia copiare un corpo tridmensionale su di un plano Quante volte una fotogralla e inesalta Il colore rosso del satiro dipinto e spiegabile cucarattere della figuia

Altrı gruppı con satırı e Il giovane Dionıso, forse derivatı dall'Hermes prassitelico, ma di stule ellenistico, sono plu frequenti in breve sono stati discussı gli esempi della Villa imperiale (cat 305 '3) e su di un frammento a Vapolı (cat 053) Solo attraverso una descrizione si conoscono esemplari di IV stule nella Casa dı Sallustio (cat 191/2) a nella Casa degli amorini doratı (cat 22 1), nell'ultimo caso il gruppo avrebbe avuto una funzione nella struttura architettonica Nella Casa di Giasone (cat 288) la situazıone e simile a quella della Casa del naviglio Il frammento con un satiro e un Dioniso, ora nell'Antiquarium di Pompei, si trovava originariamente in una posizione jsolata nella zona superiore di una decorazione orn quası completamente svanita Secondo A van Buren ll gruppo copia in modo piu tedele la statua di Prassitele Come nella Casa del naviglio il pittore ha scelto una collocazione isolata della figura suggerendo una nicchid (solo un semplice telaio) per dare alla figura un maggiore rilievo. 
Solo le sagome rimangono delle figure dorate appartenenti alla fastosa decorazione nella zona superiore della già discussa decorazione della Casa di Sirico (cat. 232). Ivi le statue sorgono su piedistalli rettangolari; sulla parete nord, secondo le descrizioni, si vedrebbe un Ares.

Solo mediante il colore giallo l'Hermes seduto nel registro superiore dell'ala 6 della Casa della caccia antica (cat. 245/2) è caratterizzato come statua. Probabilmente si tratta di una copia dell'llermes gigantesco creato da Zenodoro nell'epoca claudiana su committenza degli Averni in Gallia; la statua è conosciuta attraverso bronzetti, fra i quali l'esemplare di Montorio Veronese spicca per la sua qualitá. 18 Il modello potrebbe essere preso da tipi lisippei, per escmpio dell'Herakles Epitrapezios (si veda l'atteggiamento delle braccia e delle gambe). Le pareti laterali dell'ala, altrettanto ornate con scaenae frontes, sono prive di sculture nella zona superiore; cio potrebbe corroborare l'impressione che la divinita non sia stata scelta per i soli motivi decorativi, ma in dipendenza dalla scena di Achille nella zona mediana.

Durante lo scavo delle Terme del Sarno sarebbe stata ritrovata la raffigurazione di un atleta nella zona superiore del tepidario, forse un Apoxyomenos del quale mancano dati concernenti atteggiamento, colore e collocazione (cat. 266/3). Proprio in questo caso sarcmmo felici di poter disporre di descrizioni piu dettagliate. Ora non è possibile stabilire se si tratti di un tipo policleteo o lisippeo, entrambi noti grazie a diverse copie. Naturalmente non possiamo nemmeno scartare la presenza di un modello scionosciuto.

In VIII j, 15 (cat. 272) si regge sopra una base rettangolare un satiro munito di due flauti, forse rappresentante Marsia. Anche in questo caso $\mathbf{j}$ dati sono troppo scarsi per sapere se esso sia il satiro del gruppo mironiano di Marsia ed Atena.

. Vel vano $t$ della Casa dell'Ercole cbbro (cat. 284/3) le pareti est e ovest presentano ricche scaenae frontes con statue su piedistalli, in parte coperti da drappi appesi nelle edicole. La figura centrale su entrambe le pareti è feminile, nelle altre nicchie si distinguono figure maschili. Di nuovo constatiamo una simmetria assoluta nella composizione. Se le figure nel loro insieme costituiscano una determinata iconografia, non è del tut to chiaro. Sulla base delle tracce ancora visibili, specialmente la veste degli uomini, la toga, desta interesse, in quanto essa è raramente presente nella pittura parietale. Si ricordino le figure isolate su campo giallo trovate nella Villa di $S$. Marco (cat. 009/4), secondo Olga Elia rappresentazioni di membri della dinastia giulio-claudia.

Delle figure nelle edicole centrali sulle pareti del vano a della Casa delle Muse (cat. 286/1) restano solo tracce bianche: una di esse è descrivibile come donna seduta.

Ricca di motivi statuari è la decorazione nel tablino della Casa del bicentenario ad Ercolano (cat. 032/3). Purtroppo la zona superiore in particolare è andata perduta. Si intravvedono le figure di statue su piedistalli e solo sulla parete sud alcune di esse sono ancora leggibili: si tratta di statue clorate su basi alternamente basse e alte. Le due figure rimaste intatte rappresentano satiri, quello di destra nell'atteggiamento dell'aposkopon.

Nella Casa del colonnato tuscanico la zona superiore di due ambienti è articolata da edicole con statue (cat. $035 / 2,4$ ). Nel vano 11 le edicole centrali sono occupate da satiri e da un Apollo Citaredo, mentre quelle laterali contengono statue di menadi. L'Apollo della parete nord assomiglia alla figura sulla parete sud dell'ambiente 5 , provvista degli stessi attributi, della stessa veste e delineata nel medesimo atteggiamento. Solo i seni rendono impossibile

18 Besch1 1962, 31-60; 1974: Braemer 1978, 195-1977, fig. 6. Una statuetta del tipo e stata trovata nella Casa degli amorini dorati (A. Sogliano, NSc $1907,570-571$, l'ig. 16). 
un'interpretazione della figura come Apollo; deve quindi essere una menade o una Musa.

Nelle nicchie della parte inferiore dell'atrio nella Casa del rilievo di Telefo (cat 037), satiri e menadi sono statı inseriti allo stesso modo nella struttura dchitettonica. Lo figure sono quasi completamente svanite e non permettono una descrizione dettagliata.

Le tigure nelle edicole centrali della zona superiore dell'ambiente 19 della Casa dell'alcova (cat. 026) non sono caratterizzate come statue attraverso il colore e la collocazıone sopra pıedistall, esse sı inseriscono nel genere delle tıgure menzıonate nella Casa del Dioscurı (cat. 204/1-2). 
La discussione delle imitazionı di rulievi in un capitolo speciale e spiegata dal latto che essi si sottraggono alla problematica delle statue indipendents o delle figure in guisa di atlantı. Le questions nn $1,4,5$, presentate nell'introduzione ( $p$ is) valgono mutatis mutandis, i $\mathrm{n} i$ completamente in piu si presenta il problema delle originı del genere del rlievo paesaggistico, cioe se provenga dal mondo ellenistico (specialmente dall'ambito alessandrino) o se sid un'invenzione romana cl si puo servire del tinti rlievi per trovare una soluzione a questa domanda?

I verı riljew sono sempre statı oggetto di studio entro ll quadro della scultura in generale, ma costituiscono anche una categoria speciale viste le loro particolarita $19^{\circ}$ Vengono adoperatı in due modi Applicatı su stelaı, lekythol, altarı e sarcotagl I rilievi sono espostı come monumenti funerarı o sacrals indipendents, specie nelle necropols, in templi e luoghi sacri e in ambientı pubblici Inoltre formano un elemento obbligatorio dell'ornamentazione d) editici, nella quale il loro valore puo variare da und puramente decorativa a una tunzionale Nel'cpoca greca l'uso si lumita a tumpani, metope e fregi di 1ompli e a cornicioni e antelisse - spesso in terracotta - in edifici di vario genere Nel periocio ellenistico 1 rllevi cominciano a siolgere un ruolo nella propaganda politica e sono inseriti in monumentı pubblicı, qualı monumentı di trionto, in teatrı e in edificı statalı I soggettı raffiguratı nell'epoca classica 51 Limitano alla mitologia, dall'Ellenismo in pol si aggiungono temi storici non di rado in forma allegorica I Romani si luppano il rlievo storico come genere piu importante e glı danno un Juogo da non sottovalutare nella propaganda umpcriale Diventano un elemento scmpre presente nell oruamento di edifici pubblici e su archi triontalı e 1 tregi su monumentı trionfalı in torma di colonne ne sono l'espressione piu chlara

von e completamente chiaro quando $e$ in quale modo $l$ rilievo si sia acquistato un posto nell'ambiente privato Heide froning, nella sua monografia sui r'llevı a soggettı initologici del primo secolo a $C$, suppone che 1 pinax rotuvo nell'Ellenisino abbia perso la sua funzione originale e che in quel periodo 1 primı rilievi siano stati introdottı nell'interno di case private igo von e prima del primo secolo a $\mathrm{C}$ che disponiamo di datı piu concret $\mathrm{d}_{1}$ tutt trovatı in un contesto romano Cicerone ordino del rlievi per l'atriolum di una sua $\checkmark$ lla che fungessero da ornamenta ${ }^{91}$ In Campana rlievi sono stati ricuperatı nel loro contesto originale Nella Casa del ilievo do Telefo ad Ercolano un rluevo con ll mito di questo eroe si trovava in un oecus spazioso, riccamente ornato con lastre di marmo, nel quale tirava l'attenzione come objet d'art o Sammelstelek vella Casa degli amorini dorati iliev con rappresentazionı di satirı sono stati incastratı nel muro del peristilıo, la loro posizione e meno umportante e la loro qualita e scadente in confronto con ll rllievo ercolanese ${ }^{29} 2$ Simile era la posizione delle pitture su lastre di marmo. ora nel museo di Napolı, provenientı da luoghi ancora sconosciutı, ${ }^{9}{ }_{3}$ gli

109 Maaskant-Kleıbrinh 1982 da una sınossı della letteratura sul genere e discute alcunı deı problemı in merito

190 Froning $1981,33-47$

191 Cic.,Att I 10,3 (cf. I 1, 5, I 6, 2, Quint III 1, 5).

192 Froning 1981, 14-15, Taf. 33-34 1.

193 Froning 1981, 21-26, Taf 2-3, Moormann 1984b, 646 nota 52 
intagli marmorei, ${ }^{194}$ le plaquettes di ossidiana e cosi via. ${ }^{195}$ Le pitture monocrome in tecnica ad encausto su marmo potrebbero, a loro volta, essere initazioni di veri rilievi. Secondo Froning dobbiamo supporre che rilievi in avorio lossero la fonte d'ispirazione, ${ }^{196} \mathrm{ma}$ il formato fa piuttosto pensare a imitazioni di rilievi in marmo o stucco. Questi ultimi rappresentano un'altra specie di decorazione che si è sviluppata su larga scala nel mondo romano.

Nei giardini della Casa degli amorini dorati $e$ in molte altre case pompeiane dei rilievi di dimensioni ridotte venivano esposti come parte dell'arredamento topiario. Negli intercolunni dej portici intorno ai giardinj erano appesi piccoli rilievi a forma di oscilla, pelte e maschere. Il loro valore artistico e la tecnica d'esecuzione non sono eccellenti ed abbiamo a che fare con prodotti locali, provenienti da botteghe piu o meno industriali. ${ }^{197}$ Froning considera la maggior parte di essi come oggetti puramente decorativi che non hanno una posizione ben precisa nei giardini e nei peristili. ${ }^{198}$ Raramente si è di fronte a pezzi da collezione come nel caso del rilievo di Telefo ad Ercolano.

Il solo esempio di imitazionj di rilievi dell'epoca ellenistica presenta la facciata di una tomba di un ufficiale macedone a Lefkadia (Grecia), datata al primo quarto del terzo secolo a.C. ${ }^{199}$ Un'architettura di ordine dorico contiene nel'epistilio un fregio di triglifi e metope in stucco colorato. Le metope sono ornate con episodi clolla lotta fra $i$ lapiti e i Centauri. Per ottenere la suggestione del rilievo sono statj dipinti tratteggi e sfumature in lilla attorno alle ligure. V.J. Bruno ha dimostrato in modo convincente che in questo caso il pittore ha copiato un archetipo famoso, cioe le metope del Partenone. 200

Nel mondo romano l'inserimento di initazioni di rilievi in decorazioni parietali è abbastanza limitato. Nel I stile si incontrano pochi esempi di finti rilievi. Soprattutto nel II stile rilievi fanno parte del repertorio dei pittori. Nel III e nel IV stile essi si trovano quasi esclusivamente in rappresentazioni di giardini.

\section{STILE}

Nelle decorazionj di I stile nella Casa del fauno (cat. 209) Mau vide ancora paesaggi su lastre sopra le entrate. Dati piú dettagliati della sua descrizione sinottica non sono disponibili. Nel vano $3 i$ si trovava l'imitazione di un rilievo di cui di recente e stato ritrovato un disegno. Su di una lastra di marmo rosso con vene gialle si vede la rappresentazione di un gruppo di centauri e una menade, tutti rịvolti verso sinistra. Non è possibile stabilire se il

194 Dohrn 1965; Froning 1981, 28-30.

195 Froning 1981, 21. Sull'inserimento di oggetti nella parete Maiuri 1940; Allroggen-Bedel 1983.

196 Froning 1981, 27; cosí Fittschen 1976.

197 [)wyer 1981. Cf. Dwyer 1982, 129-134: local hands. Altri studi sono Corswand 1982: Pailler 1982.

190 Froning 1981, 8-9, 14-15.

199 Petsas 1966, 100-107, 179-182 (datazione); Lehmann 1979; Bruno 1981.

200 Bruno 1981. 
disegno copil l'untero rilievo o meno

Per rendere chıaro l'effetto del rluevo il decoratore ha curcondato le tigure con tratteggi sottlli Il modello potrebbe essere un rilievo neodtico

Anche in VI 14, 40 imitazionı di rlievi sono stati visibilı (cat 215 1)

\section{STILE}

Solo nella fase Ib del II stue cresce 1 numero di imitazionı di riliev, specie su ortostati o su bugne nella zona mediana I ruliew sono riconosciblli per la piesenza di un orlo con proflo, reso attraverso linee bianche, rosse e nere, e dell'uso modesto deı color Le raffigurazıonı sono quası sempre monocrome e l'applicazione del solo colore scelto in varie stumature scure e chiare rende visible l'effetto delle partı basse e alte del rulievo In generale l'esecuzione non e tanto precisa quanto sulla facciata $d$ l Lethadia, vediamo delle rappresentazioni semplicemente monocrome su lastre marmoree, pardgonabli con 1 sol ramenziondt dipintı encaustici su marmo Specie nel caso di paesaggi non e sempre charo a quale effetto mirasse $\downarrow$ pittore $S_{1}$ ricordi in questo contesto un passo nel De institutione oratoria di Quintiliano (XI 3, 46)

ut qui singulis pinxerunt coloribus, alia tamen eminentiora, alia reductiora fecerunt, sine quo ne membris quidem suas lineas dedissent

('Come anche pittor dipingentı con un solo colore, facevano apparire certe partı piu alte e certe partı piu basse poiche altrimentı, senza tale tecnica, non sarebbero in grado di dare 1 contornı necessarı per delerminate figure ')

I pittorı servono da esempio per gli oratorı anche questi devono, pur avendo una sola voce (cf monocromia), non pronunciare le loro orazioni in modo monotono, ma inserire slumature melodiche Per $R$ Bianchi Bandinellı e $S$ de Marinis il termine singolis coloribus non necessariamente pertenerebbe alle monocromie 201 Sulla base del contesto, ma soprattutto grazle al significato grammaticale di singuli non esiste dubbio e non puo trattarsi di altro che monocromia ogni pittore adopera un solo colore per volta ed ll testo quindi costituisce un buon confronto per le pitture ora oggetto di studio

In un solo caso si tratta di temı mitologıcı Nella Casa del lararıo o di Achlle (cat 149/3) Marsia e Olumpo e una menade assalita da un satiro sono delineatı su due rilievi

Quası sempre siamo di fronte a raffigurazionı di paesaggı sacro-ıdillaci glı esempi a Boscoreale (cat 004/3), nelle ville di Oplontis (cat 341/2) e Portici (cat 308) e nella Casa di Livia a Roma (cat $317 / 2$ ) sono chiarı esempl del genere Nel suo libro sui paesaggi nella pittura romano-campana Peters accenno per primo ed in maniera esplicita al nesso fra rllievi veri e dipinti 202 allroggen-Bedel riprese $\downarrow$ i suggerimento nella sua discussione di un frammento di pittura provenente dalla Villa del papirı (cat 039A) e Fittschen indipendentemente menziono le imitazionı di rlievi come uno degli elementi derivatı dall'Ausstattungsluxus ellenistico 203

201 Branchı Bandınellı, EAA 2, 549 s.v. chioroscuro, S de Marınıs, ibid. 5, $164 \mathrm{s.v}$. monochromata Cf le traduzioni einfarbig (H Rahn, Darmstadt 1976, II, 626-627), peintres qui n'ont employ qu'une couleur ( $\mathrm{J}$ Cousin, Paris 1979, VI, 234 (Bude) Per ll significato ora OLDict s.v. siguli.

202 Peters 1963, 20-22, 38, 60 
Queste imitazionj di rilievi paesaggistici, tutte eseguite nel primo secolo a.C., sono i primi specimina ben databili del genere rilievo paesaggistico e proprio essi possono contriburre alla soluzione del problema della loro origine. 284

Th. Schreiber fu il primo ad occuparsi dei rilievi paesaggistici. ${ }^{2}$ s Mise alla luce gli elementi provenienti dall'Cllenismo e credette di individuarne l'origine nell'ambiente alessandrino. A. Adriani segui questa opinione e aggiunse argomenti a favore di essa in varı studi, specie nelle sue Divagazioni intorno ad una coppa paesaggistica del Museo di Alessandria. F. Mat $L$, in una recensione di questo saggio, si oppose in termini chiari contro l'ipotesi di Adriani e ritenne che gli elementi da lus presentati come cllenistici fosscro per la maggior parte romani e che inoltre i monumenti discussi non potessero essere datatj nel terzo o secondo secolo a.C. ${ }^{206}$ Margarete Bieber nel suo libro sulla scultura ellenistica entra appena in questo dibattito: accenna solo alla presenza di elementi paesaggistici nell'arte cllenistica, antecedenti del genere tanto popolare nell'epoca romana. Fittschen, mediante il suo rimando all'arredamento ellenistico, cerca implicitamente un'origine nell'Uriente. Strocka, seguendo $1 \mathrm{latz}$, si esprime contro l'attribuzione del genere ad Alessandria. ${ }^{2} 7$ Nel suo saggio sui rilievi Grimani egli considera il genere come un'invenzione puramente romana, nella quale, certo, sono stati inseriti elementi ellenistici, ma dove composizione e contenuto sono innovazioni. Le coppe presentate da Adriani pro Alexandria provengono quasi tutte da un contesto romano e per tale motivo non sono normative. Strocka presenta un confronto strano, visti gli argomenti usati, con delle rappresentazioni di paesaggi e con le architetture dipinte di IV stile nelle quali le forme curvilinee sarebbero ritrovabili. Rileva un interesse calante per decorazioni parietali a favore di rilievi durante l'epoca flavia e soprattutto nel periodo adrianeo. Tale suggerimento sembra alquanto esagerato: certamente non si trovano piú paesaggi e paradeisoi di grande formato dopo il 79 circa, ma rimangono presenti ingnette con paesaggi. Solo in complessi opulenti ll rilievo e stato preferito al posto delle decorazioni in stucco e dipinto. 200

I rilievi dipınti di II stile, non menzionati da Matz e Strocka, non si presentano come invenzioni originali del decoralori romani, ma fanno parte dell'intero repertorio legato al sistema decorativo. Neanche $\mathrm{j}$ rilievi a stucco che abbellirono le volte della Villa della farnesina possono essere considerati creazioni puramente romane; mostrano troppl elementi ellenistici per essere stati concepiti indipendentemente. Clementı paesaggistici si trovano indubbiamente gia su rilievi ellenistici.

I rillevi funerari rupestri in Licia, datati nel quarto e nel terzo secolo a.C., contengono rappresentazioni di citta e di elementi paesaggistici. Naturalmente essi non servono come immediati contronti per $j$ rilievi paesaggistici in generale, ma provano l'esistenza di tali rappresentazioni in età

203 Allroggen-Bedel 1976b, 88; Fittschen 1976.

204 Le osservazioni seguenti sono state parzialmente presentate prima (Moormann 1984b).

205 Schreiber 1888; 1894.

206 F. Matz, Gnomon 32 (1960) 289-297: recensione con sinossi dello status quaestionis e rimandi biblıografici.

207 Bieber 1961b, 152-155; Fittschen 1976, 552; Strocka 1965. Sui rilievi Grimani anche Kampen 1979.

208 Strocka 1965, 93-98; cf. Joyce 1981, (ii-88. 
cllenistica. ${ }^{209}$ Phyllis M. Carroll discute nella sua dissertazione i rilievi classici e ellenistici con motivi paesaggistici, un contributo di grande valore nela presente discussione. 210

.ion e possibile paragonare i finti rilievi ai rilievi conosciuti finora. ${ }^{211}$ Le raftigurazioni spesso sono schematiche e miniaturistiche e paiono derivate piuttosto da esempi dipinti. In tale contesto si ricordi anche la composizıone in tregi l'uno sopra l'altro in pitture da Portici (cat. 308) e ad Oplontis (cat. 3412 ) dove, come ha proposto Bastet, ${ }^{2} 12$ gli esempi di rotoli, i Bilderb tacher, sono quasi tagliati in pezzi. Il tregio giallo nella Casa di Livia (cat. 317/2) sarebbe l'unico esempio di un Bilderbuch interamente coplato. Solo il rilievo della Villa dei papıri (cat. 039A) ha forme piu marcate, ma anche qui un modello dipinto è verosimile. Riassumendo si puo dire che imitazioni di rilievi con scene paesistiche sono state possibili solo quando esisteva gla una tradizione consolidata: il genere quindi potrebbe avere una radice ellenistica, forse alessandrina e farebbe parte del repertorio di elementi generalmente noto nella koine mediterranea.

I rilievi mitologici nella Casa del larario o di Achille (cat. 149/3) potrebbero essere derivati da veri rilievi dello stesso genere. La composizione della scena di Marsia e Olumpo e simile a quella di pinakes di IV stile con lo stesso soggetto. Froning lo menziona come aufschlussreiches Vorbild dei rilievi ordinati da Cicerone. 213 Non e completamente da escludere che anche rilievi a stucco servissero da modello. I rilievi stuccati nel larario della stessa casa mostrano la combinazione di figure bianche su sfondo rosso, per creare cosi il medesimo eftetto della pittura. Altrove si vede uno sfondo blu.

Rilievi si incontrano anche come elementi decorativi nelle architetture dipinte. Semplici e basate su esempi ellenistici sono le bosse sulle colonne nella Villa di P. Fannius Synistor (cat. 004/3), i cui antecedenti sono stati recentemente raccolti da $\mathrm{H}$. Lauter, ${ }^{214}$ Anche le columnae celatae si basano su esempi nella vera architettura. Sulle colonne nella Casa di Obellius Firmus (cat. 302/1) e ad Oplontis (cat. 341/3) sono state applicate figure femminili stilizzate che somigliano alle figure sulle colonne lungo la strada colonnata di Perge. Sopra le aulae regiae delle scaenae frontes di Boscoreale (cat. 004/3) e Oplontis (cat. 341,3). i timpani mostrano figure femminili con piedi a forma di girali in bianco su uno sfondo rosso. Esse si ritrovano fra l'altro sull'Ara Pacis. $215 \mathrm{Si}$ andrebbe troppo oltre i termini della presente ricerca se si analizzassero tutti gli elementi plastici presenti nelle architetture di II stile. Beyen, Engemann e anche Margaret Lyttelton hanno raccolto molto materiale in

\footnotetext{
209 wurster 1977 .

210 Carroll 1983. Il rilievo di Archelao con l'apoteosi di Omero è significativo in merito. In generale $i$ rilievi dj periodi precedenti non sono stati presi in esame nel dibattito nonostante la presenza di elementi importanti. Cf. per esempio il rilievo di Pitodoro da Cleusi e ll rilievo Torlonia (Ridgeway 1981, 134-137, fig. 99-100).

211 Cf. Schreiber 1888, 63 nota 12 (citato Moormann 1984b, 647 nota 54).

212 Bastet/De Vos 1979, 24-25. Diversamente Peters 1963, 51-52.

213 Froning 1981, 20.

214 Lauter 1983.

215 Lyttelton 1974, 2, pl. 20.
} 
merito 2 :5

\section{E IV STILE}

Imitazıonı di rilievı sono meno frequentı in queste fası della pittura parietale e nello slesso tempo meno importantı vell'ornamentazione architettonica rimane l'applicasıone dı elementı plastıcı, ma pinakes e pannellı con effetto plastico non si ritrovano nei ustemi decorativi Froning sostiene che 1 pinahes mitologicl in I 7,19 , dipint 1 su di uno stondo glallo imitano pezzl da collezıone qualı il rulıevo di Telefo da Ercolano e ll rlıevo di Arıccsa, ora a Copenhaghfn 217 Manca pero una cornice in rlievo, come mancano pure altri indizi di plasticita Lo slondo monocromo come tale non e un'indicazione sufficiente per l'ipotesi di Froning

Solo le rappresentazionı dı glardinı contengono numerosı esempl di pinakes sorrettı da plastri o erme tra le piante e di oscilla, pelte e maschere sospese entro 1 telai o, come nella Casa del frutteto (cat 161'1), in un portico stlizzato I pitton avevano davantı agh orchi una situazione reale Le scene raffigurite sono simil a quelle suı verı pmakes e hanno quasi esclusivamente un contenuto bacchico 218

Sı vedono talrolta ımıtazıonı dı craterı neoattıcı Verı esempları sono statı trovatı fra laltro nelle v lle di San Marco a Castellammare di Stabia e di Uplontis 219 tnche in questo caso e evidente la volonta di riprodurre sulle parets der veri giardinı

\section{CONCLUSIONI}

I rilıevi dipintı ci forniscono datı importantı per la conoscenza di quell verı Vediamo dove e come crano espostı nel gidrdinı o come erano incastrati nelle paretı I fintı rilievi di II stule cl impongono una collocazione ellenistica dell'origine e della provenienza del veil rulievi paesistici Purtroppo non possiamo fare confronti con rlievi esistenti se non per gli oscilla topiarı Lo stile puo essere caratterizzato in linee general come 'ellenistıco-romano', cioe affine allo siluupo artistico contemporaneo

von diversamente da un certo numero al figure caratterizzate come statue, ma nello stesso momento in possesso dı elementı eterogenel, I rlievı stanno a meta tra ld tantasia e il realismo jon e possıbule stabilure quali di essi sıano derivatı da verı modellı e qualı siano statı inventatı dai pittorı localı Talvolta le caratteristiche di rilievo sono state ridotte al minumo e solo nei rilievi topidri si constata un evidente realismo

\footnotetext{
216 Beyen 1938, 1960 Engemann 1967, Lvttelton 1974, 17-25 R A Tybout sta preparando una monogratia nella quale questo argomento verra discusso

217 Froning 1981, 14-15, Taf 25, $261,27,292$

21. Sugli oscilla Dwver 1981, Pallier 1982, Corswand 1982

219 Jashemskı 1979, fig 131, 480.
} 
E' soprattutto in Campania che - grazıe alle circostan 2 fa forevol - possiamo finord constatare quanto grande tosse l'amore del Romall per ll giardino Sla come parte di case private sia come terreni indipendenti essi sl trouano dappertutto nell'areale della citta di Pompes Aumerose sono do case nello qualı si possono vedere ilcostrulionı di aluole con plante e albeil nel peristll o nel cortll, qualche volta un lotto dietro una casa e ddoperato come orto o giardino La Casa di Octavius Quartio cl da un chiaro esempio di questo uso I terreni plu grand spesso indipendenti da case o tabbriche, dentro le mura della citta venivano struttatı come ort o vignetı Sulla planta pompeiand disegnata da H Eschebach, che mostra l'urbanistıca neglı ultımı annı prims del 79 , questı lottı sono ben riconoscibil 220

Disponıamo di tre generı di font per lo studio del glardino nell'antichita tracce in situ. rappiesentazioni di glardinı in pitture par letali $p$ testumonianze letterarie Queste ultune costituiscono la base di Les jardins romains, Libro molto ricco, pubblicato nel 1943 da $P$ Grimal 221 Il conoscitore francese della cultura antica non dedica molte parole all'ordinamento del giardino reale, ma schizza un modo imponente la storia della recezione e spicga chiaramente quale ruolo importante wolgesse il giardino nella vita dei Romani La maggior paite del dati e le conclusionı tratte da essı sono valide tuttora, benche manchino gli elementı urcheologicı che avrebbero potuto 'rianımare' gli stessi giardinı Osservazionı attraent1, allo stesso modo basate sulla lettura de testi antich1, si trovano nel lavoro di Zoja Pavlovshis che mette l'accento sul carattere artificiale della 'ndtura' nell'ottica del Romani 222

Soprattutto in Cimpana semi carbonizzati $c$ nltri restl di piante, assieme a cavı di tronchi, ceppl e ramı d'albero. sono venutı alla luce durante gli scavi a Pompes La bıo-archeologı, scienza perfezionata neglı ultumı decennı, permette agl studiosı di estrarre numerosi datı preziosi da questo materiale apparentemente cosı povero Whlmelmina Jashemsk1, nel suo The Gardens of Pompeii, Herculaneum and the Villas Destroyed by Vesuvius presenta $I$ primo sommario di talı ricerche in Campania 223 In questa monografia l'autrice americana llustra datı importantı concernentı l'impianto del giardino Colpisce l fatto che ll giardino di citta venisse usato nello stesso tempo come orto e come giardino A parte gli aspettı biologicı, altrı soggettı, fra 1 qual le pitture, oggetto di studio in questo capitolo, vengono discussi in modo interessante nel libro d) Jashemski 224

Nell epoca ellenistica parchi paesaggistici venıvano creatı presso ville e palazzı secondo la maniera del cosiddett paradeisol 225 parchı pieni di alberı e plante esotiche, e qualche iolta occupati da aninals selvatici, nell'ultimo caso 1 parchi funzionavano da terrenı d'esercizio e di caccia per l giovanı principl I parchı artıficialı contenevano non solo piante e anımalı ma anche pergolatı, tontane e ognı tanto verı programm conograficı in forma di statue Achille

\author{
220 Appendice a NF 1975 \\ 221 Grimal 1943, 1969 (edizione rivista) \\ 222 Pavlovskis 1973 \\ 223 Jashemski 1979 \\ 224 Cf Burr Johnson/Griswald 1963 (Atene). Ancient Gardens 1981 \\ 225 Grumal 1969, 76-87, ()ch, RE 71 (1910) 768-841 s.v. Go\&rtenbau
}


Tazio descrive una fontana nascosta nei cespugli in un giardino a Tiro nel

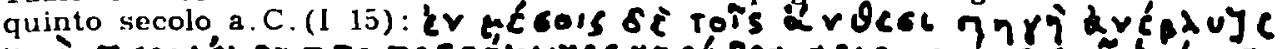

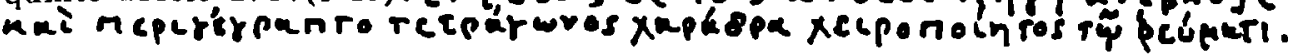

('Nel mezzo dei fiori zampillava una fontana che era circondata da un bacino artificiale in forma quadrata per l'acqua cadente.').

Arrjvando gli influssi dell'Oriente a Roma nell'epoca tardorepubblicana, essi venivano adattati e trasformati secondo il gusto romano. Gli Horti Luculliani a Roma, sorti a meti del primo secolo a.C. costituircbbero il primo esempio di un parco paesistico nell'ambiente romano.226 Contemporaneaunente il giardino lorse perdeva il suo carattere 'naturnle' e assumeva il valore di una lincriata, costruita come ornamento e veniva tuttavia considerato una miscela del giardino coltivato e della natura selvatica. ${ }^{227}$ tin parco quale quello della Domus Aurea di Nerone ne $\dot{e}$ un esempio chiaro. Grimal ammette che l'aspetto teatrale abbia svolto una parte importante: ${ }^{229}$ solo cosi si capisce la struttura del giardino modesto nella Casa di $M$. Lucretius dove le statue stanno, davanti ad un ninfeo, in una messa in scena volutamente costruita; i satiri e gli animali non sono disposti come esseri vivi nella natura nella quale si muovono, ma stanno fermi come una collezione di bric-a-brac. ${ }^{230}$ La Villa Adriana e stata un esempio significativo di questa tendenza a creare effetti teatrali, considerata la disposizione raffinata degli edifici e delle statue nell'ambionte. ${ }^{23} \mathrm{Di}$ parchi simili presso ville del primo secolo a.C. e del suecessivo non si e conservato nulla. La Villa dei papiri ci avrebbe potuto informarc sull'allestimento topiarjo proprio di questa epoca, se non fosse solo diventata conosciuta attraverso gli scavi settecenteschi tramite gallerie e non completamente ricostruibile sulla base del materiale presente negli archivi. Le statue raccolte in essa rappresentano un programma iconografico sofisticato, che esprime in tal modo la mentalità del proprietario. 232

Cosa indiscussa per i Romani era la presenza di divinita della fertilita nei giardini. ${ }^{23}$ Specie nella Repubblica la roligiosita costituiva una componente influente sullarredamento e sulla composizione del giardino. Non è piú possibile stabilire fino a quale livello essa sia rimasta importante nell'epoca imperiale. Plinio ancora spiega la presenza di statue di satiri dal punto di vista religioso (N.H. XIX 50).

Quam ob rem comitata est et religio quaedam hortoque et foro tantum contra invidentium effascinationes dicari videmus in remedio saturica signa, quamquam hortos tutelae Veneris adsignante Plauto.

226 Grimal 1969, 126-127, 428-430; Kaster 1974 .

227 Grimal $1969,84-87,438$.

220 Cf. Peters 1982b, 62.

229 Grimal 1969, 240-242.

230 Dwyer 1982, 38-54, ill. 1, fig. 111-11i. Per un'analisi of. Zanker 1979a. Cf. anche Lauter-Bufe 1975.

231 Cf. Salza Prina Ricotti 1982; Raeder 1983.

232 Pandermalis 1971; Sgrobbo 1971; 1972; Zanker 1979a; Sauron 1980; 1982; Moormann 1984b.

233 Grimal 1969, 41-56. 
('Per tale ragione si è aggiunto un aspetto religioso e vediamo come sia nel giardino che sul foro vengono esposte statue di satiri come remedio contro gli influssi negativi dei nemici, anche se, testimone Plauto, Venere ì la patronessa del giardini.'). Al contrario, i visitatori di un giardino in una lettera del retore Alcifrone non sono toccati da sentimenti religiosi quando vedono statue delle ninfe e di Pan: queste sembrano intensificare l'atmosfera sensuale che è presente nel descritto giardino erotico. ${ }^{234}$

Per quanto riguarda l'arredamento del giardino ci interessano sopratutto le statuc. ${ }^{235}$ Solo Pompei, Ercolano e Oplontis danno occasione di studiare i giardini e le loro statue in situ. $\mathrm{Di}$ Delo e altre località uon si sa ancora molto. ${ }^{36}$ Le osservazioni seguenti si basano sul materiale campano. Le statue nei giardini rappresentano per la maggior parte figure dell'ambito di Afrodite e Dioniso: la divinità dell'amore stessa, ognitanto accompagnata da Ares, satiri, menadi e Eracle come membro del tiaso dionisiaco esprimono nello stesso tempo la fertilità della natura. Una divinita cosí specifica come Priapo è rara; altrettanto Silvano e Pomona, divinità unicamente romane. Posso menzionare solo una statuetta bronzea di Pomona nella Casa dell'efebo e il Priapo di marmo nella Casa dej Vettii. ${ }^{23}$ ?

L'acqua costituisce un altro elemento immancabile, connesso in modo strettissimo con quello della fertilità $e$ in un certo senso facente parte di essa. Gli autori greci citati, già menzionano fonti e fontane; nell'epoca romana i giochi d'acqua devono aver svolto la parte di un soggetto necessario. Lurante l'impero si costruivano dappertutto ninfei e edifjci con fontane. ${ }^{23}$. Nel clima mediterranco l'ucqua vale come simbolo di fertilità; fa crescere piante. esseri umani e animali. La facoltà biogenetica era, secondo alcuni, adoperata come espressione dell'ottica verso la vita, una Lebensbejahung. ${ }^{23}$; filosolia che sarebbe stata presente anche nei complessi termali.240 L'introduzione di un acquedotto centrale a Pompei nel periodo augusteo ha favorito il collocamento di fontane nei giardini privati. ${ }^{241}$

Le rappresentazioni di figure dellambiente culturale sono derivate dall'Ellenismo: ${ }^{242}$ Apollo citaredo, le Muse, poeti e filosofi devono introdurre il visitatore del giardino sull'Elicone $o$ in un Mousaion. Statuette di Apollo, per

234 Alciphron, Fragm. VI 1-9. Cf. K. Dilthey, AZ 36 (1878) 48; Olck cit. nota an. 810

235 In generale Hill 1981.

236 Kreeb 1979; 1980; 1984. Nel giardino del 'palazzo' a Fishbourne, uno dei pochi esempi fuori d'Italia, non sono state trovate statue, che tuttavia originariamente crano presenti nelle 'alcove': Cunliffe 1971, 128-148, pl. $36-42$, IJ-IV, fig. 30; Cunliffe 1981 .

237 Jashemski 1979, fig. 147.

23 Neuerburg 1965; Kapossy 1969; Becatti 1971. Grimal 1969, 424 cita Stazio, Silv. I 16-18 come testimone per la collocazione di statue di ninfe; Pavlovskis 1973, $19 \mathrm{n}$. 57 interpreta nymphas come metonimia dell'acqua.

239 Michel 1980.

240 Manderscheid 1981, 30-31.

241 Dwyer 1982, 113

242 Zanker 1979a. Cf. Lorenz 1965; wrede 1972. 
esempio, sono state trovate in VII 2, 15 e nella Casa del citarista. ${ }^{243}$ Dalla Casa di Octavius Quartio conosciamo due statuette di Muse, facenti parte di una serie di sette, ${ }^{244}$ Ritratti di Pittaco e Menandro sono stati trovati respettivamente nei Praedia di Julia Felix e nella Casa degli amorini dorati. ${ }^{245}$ Ritratti del poeta pjú famoso della Commedia Nuova sono frequenti in particolare, come ha dimostrato Th. Lorenz; si pensi al Menandro dipinto nella casa omonima a Pompei. ${ }^{246}$ Altre gallerie con personaggi di questo ambito sono state recuperate nella Villa dei papiri e nella villa di welschbillig (Germania).247

Ritratti di principi, generali e politici si incontrano fra l'altro nella Villa dei papiri e nella villa di Oplontis. 240 Non solo per la Villa dei papiri vale

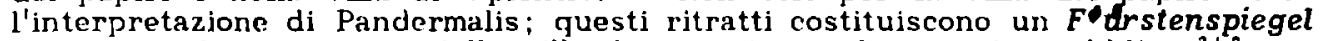
e servono al proprietario della villa da esempio per la sua vita pubblica. ${ }^{49}$

$\mathrm{Si}$ desiderava in certi casi creare atmosfere esotiche esponendo statue egizie e costruendo opere acquatiche nominate 'canopi' o 'euripi' ed etichettando certe zone, come nella Villa Adriana, con nomi stranieri, quali 'Stoa Pecile' e 'Valle di Tempe'.

Alla fine dellepoca repubblicana comincia a svilupparsi, su larga scala, il genere della pittura di giardino che si sarebbe sviluppato durante l'intero periodo imperiale. Queste rappresentazioni di giardini ci forniscono, come è stato detto, molti dati concernenti giardini reali: la vegetazione, gli uccelli, le statue e fontane, tutti soggetti che richiedono studi indipendenti. I pittori lavoravano secondo la natura conosciuta o secondo modelli veristici provenienti dall'Oriente. Specic in questo genere l'illusionismo rimane di gran rilievo fin nel tardo impero.

Rappresentazioni di giardini possono occupare l'intera parete, il solo zoccolo, la predella sotto l'edicola centrale nella zona mediana e la zona mediana stessa. Del periodo del III stile conosciamo alcuni vani, nei quali ora tutte le pareti, ora alcune sono affrescate con tali pitture. Qualche volta elementi irreali fanno la loro entrata in queste rappresentazionj naturali. In una sala, arredata come una grotta, nella villa di Livia a Primaportasso vediamo su tutte le pareti un orlo di rocee al disopra delle rappresentazioni di giardini. In tal modo si produce l'impressione che questa sala sia aperta su tutti e quattro i lati, mentre ovviamente una sola parete avrebbe dovuto rappresentare l'entrata della grotta. In un ambiente nella Casa del frutteto a Pompei le piante si delineano su di uno sfondo nero, altrove si vede uno

243 Dwyer 1982, fig. 207, 127; Zanker 1974, Taf. 54-55.

244 Spinazzola 1953 , fig. 461-462. Cf.p.0.

245 Jashemski 1979, fig. 84; de Franciscis 1951, fig. 9; Dwyer 1982, fig. 212,215 .

246 Lorenz 1965.

247 Pandermalis 1971; Wrede 1972.

24 I Pandermalis 1971; Jashemski 1979, fig. 458-461; de Franciscis 1979; 1980; A. Lagi De Caro, Pompeii Herculaneum Stabiae 1 (1983) 365, fig. 35. Forse un ritratto nella Casa degli amorini corati rappresenta un membro della gens Poppaea: de Franciscis 1951, 23-24, fig. 7-9; Döhl/Zanker 1979, 196, fig. 107.

249 Pandermalis 1971, 196-197.

250 Gabriel 1955; Peters 1971; Helbig ", no. 2486 (B. Andreae). 
sfondo giallo o rosso In tale contesto le piante e le statue si trovano davantı ad una parete cieca che sembra respinta indietro Lo sfondo quindi indica ll recinto del gjardino o 1 muri del vano (ipetrale)

vel IV stile pitture di giardino si presentano piu frequentemente in ambientı ipetralı, per esempio sulla parete di fondo in un vero giardino, o -quando si tratta dı un peristilio - sulla parete senza portico la tecnica e meno precisa e una conservazione a lungo termine non e garantita a causa della loro posizione rulnerdbule Percio la maggior parte des complessi in merito va datata agl ultumı ann pruna del 79 Giardinı nello zoccolo sono rarı - per esempio nel tablino della Casa di $M$ Lucretius fronto -, ma runangono in uso piante singole, corne si vede per esempio nella Casa del Vettil 251

lelld ricerca sui giardinı romanı le pitture sono sempre state adoperate come fonte Gia Grumal ${ }^{252}$ attinse un certo numero di dati utill e anche Jashemskl253 dedica un capitolo al genere Peters e Michel ${ }^{254}$ discutono 1 genere come tale, l'analizzano e accennano il parallelismo fra realta e pittura Michel mette l'accento sul carattere diomsiaco e considera queste pitture come espressionı della dionysische Diesseitsfreude I De Vos, 255 nella loro sınossı d) un numero cospicuo di rappresentazioni di giardini, latta a proposito di quelle nelle Terme stabiane, non entrano nella discussione sul loro significato

Nel II stule non appaiono pitture di giardinı con statue

\section{STILE}

In uno dei due ambientı nella Casa del frutteta, completamente decorati con rappresentazionı $d_{1}$ giardinı, si vedono fra 1 cespugli statue di figure egizie (cat 161/1) Le figure monocrome bianche sorgono sopra basi, composte da un pilastro $P$ un plinto - lorma caratteristica per ll III stule, spesso schemalizzato in lorma di $T$ - davant 1 dd uno stondo ceruleo Ogni parete presenta due statue, che costituiscono sempre una coppla simmetrica Della loggla - resa con colonne snelle, ornate con elementı di candelabrı - sono sospesi oscilla e stanno esposti riliev rettangoları policromi con 11 bue Apıs, un satiro con una mende e Dioniso Siamo di tronte ad un unicum nella pittura romana il cui significato e tuttora dibattuto France Le Corsu e V Tran Tam Tinh considerano entrambi gli ambienti (e questo con le statue in particolare) come oratorres per funzioni religiose di culto egizio Peters e Jashemski accettano l'interpretazione del dettagh, ma contestano la conclusione 1 vani lanno parte di una casa ll cui pioprietario, certo, puo essere stato adepto $d_{1}$ un culto egizio 256 Secondo $H$ Sichtermann le pitture non alludono che a un senso religioso in generale, anche lus non crede nella tesi di Le Corsu Michel considera le pitture allo stesso modo delle altre rappresentazionı di giardinı, cioe come fenomeno di un atteggiamento positivo

\footnotetext{
251 Bastet/De Vos 1979, tav XXXI, 57 (Fronto), Peters 1977, pl 77, fig 31-34 (Vetti1)

252 Grimal 1969, 229-244, 443-458 Ivı si trovano riferimentı a tutte le ricerche anteriorı

253 Jashemsh1 1979, 55-87

254 Peters 1971, Muchel 1980

255 De Vos/De los 1979, 90-93

256 Si ricordino anche 1 lettı in questı ambienti'
} 
verso la vita. De Vos, infine, nella sua monogralia sulla cosiddetta egittomania, non dedica nessuna parola al dibattito finora svoltosi e non da nemmeno una sua interpretazione. Osserva soltanto che le statue non corrispondono a modelli conosciuti della scultura egizia, sia per quanto riguarda i tipi che per l'iconografia. E' per questa ragione che si puó constatare - anche se con una certa prudenza, poiché altri esempi possono essere andati perduti - che l'intenzione a creare un'atmosfera egizia, o magari un'atmosfera interpretata come egizia, aveva maggiore importanza della volontà di imitare una situazione reale. Anche gli oscilla e i pinakes a temi dionisiaci. ognitanto interpretati come trasposizione di Horus, si aggiungono al carattere inmaginario del complesso. I soggetti raffigurati non permettono un'interpretazione religiosa o un'associazione a figure egizie, a parte il bue Apis. In un ambiente di culto ci vorrebbero statue e immagini ben riconoscibili di divinità, assieme ad un'jconografia adatta per quanto riguarda gli altri elementi. Solo la situla e l'idria nel cubicolo nero adiacente sono oggetti sacri nel culto di Iside. ${ }^{25}$ ?

Sulla parete sud del viridario della Casa di Cerere (cat, 162/2) si trova una pittura ora illeggibile che, vista l'esccuzione e la posizione, precede il IV stile. Nel centro, a sinistra e a destra di una fontana, sono esposte sfingi bianche in posizione simmetrica. Nella Casa di Julius Polybius (cat. 303/2) si incontra la medesima disposizione. Secondo Michel gli animali fungono da guardiani presso l'acqua. $E^{\prime}$ vero che la sfinge spesso veniva adoperata a questo scopo, specie in un contesto funerario, ma piú verosimilmente costituiscono elernenti egittizzanti da confrontare con la stinge di marmo presso l'euripo nella Casa di Octavius Quartio. ${ }^{258}$ Il Joro carattere ornamentale si vede espresso anche nel fatto che vengono adoperate frequentemente come sostegni di fontane.

In tre ambienti adiacenti, probabilmente adoperati come ninfei, al piede dell'Insula occidentalis, di recente sono state trovate rappresentazioni di giardini di dimensioni considerevoli, parti delle quali debbono essere state tagliate nel Settecento (cat. 262/6). Il loro restauro è ancora in corso e non è ancora possibile ottenere un'immagine completa di questo complesso importante. Le lunghe pareti sono divise in tre campi tramite finestre di legno che guardano verso dei giardini. Su di uno sfondo ceruleo si delineano piante, uccelli e statue, tutto dipinto in uno stile realistico con tocchi di pennello molto sottili. Allorlo superiore dei telai sono sospesi oscilla, peltae e maschere e tra le piante sorgono erme a testa di satiro, reggenti piccoli pinakes. Tutto rinvia all'ambiente dionisiaco. Fusti e rilievi sono di colore bianco-bigio ad imitazione del marmo, mentre le teste sono realizzate in colori di grande effetto realistico. Come nella Casa del criptoportico constatiamo una contaminazione di due mondi illusionistici.

Alcune rappresentazioni di giardini in zoccoli sono già state discusse nel primo capitolo a proposito degli atlanti (p.34). Nelle pitture della Casa del labirinto (cat. 208/2), della Casa di Orfeo (cat. 212/2), di I 7, 19 (cat. $156 / 2$ ), del pistrino ercolanese (cat. 038) si vedono statue di uccelli che sostengono lorlo superiore dello zoccolo. Ad Ercolano e nella Casa del labirinto gli animali sono raffigurati indubbiamente come statue di marmo, visto il loro colore bianco. In merito si ricordino le statue di uccelli esposte nei giardini come fontane, per esempio il corvo della Villa di San Marco e il pavone della Casa del camillo. ${ }^{259}$ Le sfingi nell'ambiente c di 17,19 ed il

257 De Vos 1980, 19-20, tav. XVIII-XIX.

25. Spinazzola 1953, I, fig. 451-453. Sulla sfinge: Demisch 1977, spec.76-116.

259 Jashemski 1979, fig. 531; Dwyer 1982, fig. 70. Cr. Amelung 1908, nos. $111,12,127,128,7$ af. $31-33$ (Sala degli animali). 
resto del gruppo rappresentano solo uccell fantasticı, in parte resı cone se fossero vivi.

Delle altre decorazıoni dı zoccolı e dı predelle un frammento a Napolı ( $\mathrm{MN}$ 9638, (at 101) merita qualche parola $I$ isto 1 formato il pezzo proviene da uno zaccolo sumile a quello nel tablino della Casa di $\mathrm{M}$ Lucretius Fronto 260 Un giardino dipinto in giallo su di uno stondo nero e dl composizione simmetrica Nel centro vi e una pergola occupata dalla statua di una ligura femminule. Statuc di cani sono esposte sugli sleccatı in aranti Le pergole a sinistra e a destra vengono sorrette da erme ln una pittura, tuttora quası completamente svanita, ma conosciuta da disegni, nella Casa di Orfeo (cat $212 / 2$ ), si trova un glardino simule, come anche in VI 1j, 2 (cat 211), a giudicare dal disegno che ne e l'unica documentazione

\section{STILE}

In ben 24 rappresentazion du glardinı si incontrano statue, chiaramente riconoscibll attraverso ll colore bianco (ognitanto con una policromia parziale) e l'esposizione su di una base Le pitture decorano sempre la parete di fondo di un giardino in modo che si crel l'ulusione di un giardino plu largo, ricco di piante esotiche e opere d'arte Proprio in questo genere della pittura antica e chiara l'intenzione di imitare vere statue

In questo contesto si ricordino anche le statue che sl vedono in rappresentazionı di paradeısol Essı vengono spesso abbinatı a rappresentazionı di gidrdinı e svolgono lo stesso ruolo di questı Vanno menzionatı 1 paradeisol nella Casa di Romolo e Remo (cat 251), nella Casa del Cen (cat 152,3) e nella Casa del re di Prussia (cat 253). Nel primo complesso la statua di una sfinge - l paradeisos intattı e combinato con l prospetto di un giardino sulla parete adiacente - e l sumbolo del luogo lo spettatore si trova in un paesaggio egizio Nelle due altre pitture si trovano statue di Priapo usate come sumbolı di sacell semplici per questa divinita o come sumbolo della tertilita Gia 1 De Vos hanno presentato un elenco di element che si incontrano in rappresentazioni di giardini 261 Essi catalogano fontane, sfingı e altre statue nella sınossı citata. In quası tuttı 1 giardinı si vedono fontane, spesso poste sopra plastrı, ma anche con sostegn a forma di sfingi o centauri Altre statue in guisa di fontana sono le ninfe che reggono, davantı al grembo, con entrambe le mani un bacino Nion si trova in torma di pittura ll ricco repertorio di figure che fungono da fontane raccolte dd $B$ hapossy 262 Cio vale invece per la vera scultura riscoperta nei giardini pompelanı

Le ninfe acquatiche corrispondono a vere statue che nell'Ellenismo sono state introdotte secondo l'esempio di statue di Afrodite del quarto secolo come la Pudica e specie la cosiddetta Landolina nel Museo Archeologico di Siracusa ${ }^{263}$ Le donne sono nude oppure coperte da un mantello sulla parte inferiore del corpo, quasi mal un vestito copre l'intero corpo Reggono un bacino con tutte e due le manı al posto della conchiglıa di alcune tigure del tipo originale Figurano sempre in copple simmetriche von sempre viene raffigurato il carattere scultoreo, dato che alcune figure sembrano

260 Bastet/De Vos 1979, tav. XXXI, fig 57.

262 De Vos, De Vos 1979, 90-93.

262 Kapossy 1969.

263 Becatti 1971. 
rappresentate vive, presenti nel giardino, nonostante il fatto che siano esposte su basi La finzione si è sviluppata in modo opposto: non è la statua che viene trasfigurata in un modello vivo, ma la figura viva che viene ridotta a una statua. Cosi la distinzione fra statua e figura viva sembra completamente sparita. Tale fenomeno è una netta espressione del concelto ellenistico sul livello veristico delle raffigurazioni: si mira alla realizzazione piú veristica possibile di un oggetto e risulta evidente come i pittori avessero raggiunto un livello artistico molto alto per rendere in pittura gli stessi effetti della scultura. Certo manca la terza dimensione, ma stanno a disposizione mezzi diversi. Un caso unico - nel suggerire questa realtà - è fornito dalla Casa della fontana d'anore (cat. 278) dove al disopra del bacino della ninfa dipinta é immesso, nella parete, un tubo dal quale esce acqua che viene raccolta in un vero bacino, esposto nella nicchia davanti alla linta fontana: la ninfa dipinta funziona da vera fontana. Esempi di ninfe raffigurate vive con bacini gettanti si incontrano nella Casa di Romolo e Remo (cat. 251), nella Casa delle Vestali (cat. 198/3), nella Casa di Caecilius Jucundus (cat. 186/2), nella Casa dei Ceii (cat. 152/3) e nella Casa di Sallustio (cat. 198b/3). Nella Casa della fontana d'amore (cat. 278), nella Casa della Venere in bikini (cat. 166/1), nella Casa degli archi (cat. 176), nella Casa di $M$. Lucretius Fronto (cat. 195/3) e nelle Terme stabiane (cat. 231/1) le ninfe sono dipinte come statue. Si ricordino in questo contesto le statue dipinte in colore bronzo davanti al podio della scaenae frons nella Casa del palestrita (cat. 267): anche esse reggono bacini in cui zampilla l'acqua. Una prova particolare della contaminazione di realta e finzione si vede sulla scaenae frons con Attis nella Casa di Pinarius Cerealis (cat. 182,1): ninfe vive che rappresentano il fiume Sangarios, luogo dell'avvenimento, reggono brocche dalle quali esce acqua; si muovono sul podio come veri attori. 264 una copia in marmo o bronzo della ninfa con conchiglia o bacino non è stata trovata a Pompei. La statuetta menzionata della Pomona è una variazione su questo tema: la dea regge una conchiglia colma di frutti davanti al grembo. 265

Nella Casa di Romolo e Remo (cat. 251), nella Casa delle Vestali (cat. 198;3) e nelle Terme stabiane (cat. 231/1) tali ninfe fiancheggiano divinità fluviali maschili rappresentate secondo lo schema fisso del Sileno sdraiato sul suo lato sinistro. ${ }^{266}$ Nella Casa degli epigrammi (cat. 185/1) si trova una tale figura isolata. Come per le ninfe non si puó distinguere chiaramente se si tratti di una figura viva o di una statua. I Sileni non sono caratterizzati attraverso attributi particolari come fiumi determinati; normalmente vengono etichettali 'Sarno', cioe il fiume vicino a Pompei, ${ }^{26}$ ' ma probabilmente non si è voluta rappresentare una precisa località. Mediante il cantaro, il mantello sciolto a la corona in testa le figure rimangono dei Sileni, facendo parte del tiaso bacchico.

$\Gamma^{\prime}$ già stato detto che ninfe e divinità fluviali, nella loro funzione di metafora dell'acqua, simboleggiano fertilità e felicità. Questa doppia qualità entra bene nel carattere dionisiaco del giardino. La loro combinazione potrebbe essere presa dalla decorazione scultorea dei ninfei. Nella Casa di Romolo e Remo (cat. 251) una nicchia o una schola finta occupata da una fontana a forma di vaso neoattico e circondata da due ninfe, mentre sopra il vaso, sul muretto della nicchia si vede esposta la statua della divinita fluviale. La nicchia costituisce un elemento frequentemente adoperato nell'architettura dei

\footnotetext{
264 Bastet 1974, 224, Abb. 2; Moormann 1983b.

265 Jashemski 1979, fig. 145, 147.

266 Bastet 1974, 220-224, Abb. 8-14.

267 Cf. O Höfer, Roscher IV (1909-1915) 387, s.v. Sarno.
} 
ninfei il ché va spiegato - secondo Hornbostel-Hüttner - come imitazione delle grotte che a loro volta sono gli antecedenti dei santuari e dei monumenti artificiali, eretti in onore di divinita di fonti o fiumi. ${ }^{26}$ La decorazione della Casa delle Vestali (cat. 198/3), ora nel Louvre, è diversa, in quanto lo sfondo è completamente bianco e vuoto e non presenta alcuna indicazione di giardino o architettura. Inoltre, la pittura è l'unica che provenga da un ambiente chiuso dove ornava lo zoccolo.

Nella Casa del gran portale ad Ercolano (cat. 034/2) la diaeta possiede uno zoccolo con elementi architetionici. Nel mezzo di ogni parete si trova uno stretto prospetto a fondo blu nel quale, dietro un cancello, si distingue una ninfa a fontana. Sullo sfondo si delineano degli alberi.

Nella Casa della Venere in conchiglia (cat, 178) una statua di Ares sorge nel giardino a sinistra del campo centrale che ha dato il nome alla casa. Per quanto riguarda la sua funzione la divinita non ha una relazione con il giardino; la sua collocazione su questa parete si spiega con la presenza di Afrodite, la sua amante, con cui Ares in altri contesti viene raffigurato frequentemente. 269 Quest ultima era presente in forma di una statua marmorea in diverse case, per esempio nella Casa della Venere in bikini, in 111,12 e nella Casa del camillo. 270 Dubbiosa è una pittura ora perduta nella Casa di Apollo (cat. $198 \mathrm{~h} / 2$ ) che avrebbe rappresentato Artemide; sarebbe I unico esempio della sua presenza in raffigurazioni di giardini. Dalle descrizioni si conclude che si tratta di una Artemide cacciatrice, forse derivata dall'iconografia delle Amazzoni o dell'Artemide di Gabii. ${ }^{2}>1$ Una fotografia scattata nel 1900 circa, invece, mostra le gambe nude e il corpo snello di un' Afrodite Anadyomene. Il documento fotografico forse rappresenta una pittura diversa, sia nella stessa Casa di Apollo sia altrove, mentre l'Artemide era già sparita completamente.

Troviamo satiri dipinti nella Casa della fontana d'amore (cat. 278)e nella Casa di M. Lucretius Fronto (cal. 195/3). Dioniso stesso non figura mai nelle rappresentazioni di giardini se non in forma di erma. Eracle ebbro, anche lui un membro del tiaso, è presente nella Casa della fontana d'amore (cat. 278) dove forse - per questa decorazione sparita mancano le fonti illustrative funziona da fontana, reggente un cantaro o una brocca. Il suo modello originale, come quello dell'Eracle sulla scaenae frons delle Terme stabiane (cat. 231/3), è dell'epoca ellenistica; una statuetta di marmo nella Casa dei cervi ad Ercolano ne è una copia. ${ }^{2} 72$

I due guerrieri accanto alla rappresentazione di un giardino nel giardino della Casa dell'efebo (cat. 155/4) si intrecciano nelle serie di guerrieri su campi, modellati secondo tipi policletei (cf. p.43). Per quanto si possa stabilire, non hanno una relazione iconografica con il giardino, se non come rappresentanti della palestra o - nel caso che si tratti di Ares - In relazione ad Afrodite. Si vedono delle erme su lesene fra le pitture di giardini o di paradeisoi nella Casa della Venere in bikini (cat. 166/1), in $I 12,11$ (cat.

260 Hornbostel-Hüttner 1979, 17-19, 66-86. Cf. Neuerburg 1965; Muthmann 1975.

269 Dwyer 1982, 124 spiega la presenza di statue di Ares anche come elemento della palestra.

270 Jashemski 1979, fig. 200, 199; Dwyer 1982, 63-64, fig. 79.

271 Zanker 1979a, 482 n. 67; Dwyer 1981, 265.

272 Non pubblicata se non in guide turistiche. Cf. anche $\mathrm{j}$ sileni nella Casa di M. Lucretius e nella Casa del Granduca di Toscana (Dwyer 1982, fig. 51, 186). In generale anche Nicholls 1982. 
171), nella Casa di Octavius Quartio (cat. 177/3), in V 2, 15 (cat. 193) e nella Casa delle quadrighe (cat. 238).

Lna menzione particolare meritano due gruppi che sono copie in forma di pittura di statue ellenistiche. Nei giardini dipinti delle Terme stabiane (cat. 231\%1), ricchi di statue, si trovava un gruppo di una menade e un satiro, conosciuta solo attraverso descrizioni. Un esemplare in marmo e stato trovato presso la piscina nella villa di Oplontis. ${ }^{273}$ Ben conservate sono le pitture nella Casa dell'Adone ferito (cat. 198f), dove due gruppi sono dipintí come pendants davanti ai linti pilastri tra la scena di Adone nel centro, un paradoisos a sinistra e un giardino a destra. Essi, anche se nei detlagli differiscono considerevolmente, possono essere considerati come copie assai libere di un esempio ben noto. Il modello scultoreo forse è il noto gruppo, conosciuto solo grazie alle fonti scritte. dei Saepta a Roma, del quale anche la pittura della cosiddetta basilica di Ercolano (cat. 019) sarebbe una riproduzione. Nel caso della pittura della 'basilica' le figure sono dipinte come persone vive, sia in atteggiamento rigido, davanti alla parete riccamente ornata dell'interno dei Saepta, mentre nella Casa dell'Adone ferito non vi è alcun dubbio sul carattere scultoreo dei gruppi. Non si puó comprendere quale dei tre corrisponda meglio alla statua dei Saepta, ma il fatto che $i$ gruppi nella Casa del'Adone ferito siano rappresentatj cosi chiaramente come statue, potrebbe essere un elemento a favore di essi. Qui. inoltre, è evidente che sulla medesima parete sono mesi assieme due gruppi quasi identici. $E^{\prime}$ un chiaro esempio della preferenza per i pendants nel caso che le figure dovessero avere una prescritta esposizione. Il committente non si interessava al soggetto o al valore artistico, ma mirava solo ad una composizone armoniosa, adatta al suo gusto. ${ }^{274}$ In nessun altro luogo si puó constatare questo fenomeno tanto chiaramente quanto in questo giardino. Certo, troviamo ognitanto coppie di ninfe, ma cio non suscita tanta meraviglia, considerando il tipo usato e la funzione: sono elementi fissi della decorazione topiaria senza che abbiano un significato iconografico specifico. In piú, non si presentano molte possibilità di variazione sul tema di base.

Anche l'Eros dormiente nella Casa dell'Adone ferito (cat. 195) potrebbe essere una statuetta da giardino. Si conoscono varie copie di un pezzo del genere, fra le quali una nella Casa della fontana piccola. L'esemplare di Cirene possiede pressappoco la stessa policromia della figura dipinta. $2 f_{5}$

Incontriamo un altro esempio della preferenza per la simmetria nella villa di Oplontis (cat. 341/9). Adiacente al portico con la grande piscina si stencle un ambiente ipetrale nel quale si trovano quattro cubicoli privi di porte, ma forniti di finestre, forse usati come uccelliere. La posizione assiale di queste aperture genera un effetto di grande profondita. Le pareti dei vani sono affrescate con rappresentazioni di giardini su sfondo giallo. Ogni parete cieca presenta un paio di fontane, sorrette da una sfinge o da un centauro di colore bianco, delineate con una certa accuratezza nei dettagli. Le decorazioni sono composte simmetricamente l'una rispetto all'altra e in ogni giardino le figure di una parele sono immagini rillesse di quelle dolla parete opposta. Nel piccolo giardino (20) vicino all'atrio (cat. 341/4) si nota la medesima composizione su entrambe le pareti: due sfingi sorreggono bacini nei campi laterali; purtroppo queste pitture sono quasi completamente scomparse.

Tre frammenti appartenenti ad una collezione privata svizzera (cat. 338) non sono mai stati compresi nella ricerca e meritano un maggiore interesse per la loro oscurita. Il luogo di ritrovamento è sconosciuto ma si puó ammettere

\footnotetext{
273 Jashemski 1979. fig. 480. Cf. per il tipo: Marconi 1924.

274 Jucker 1950, 7-10. Cf. Lauter 1967.

275 Paribeni 1959, cat. 308, tav. 147.
} 
una provenienza campana I tre pezzi, costituenti certamente l'intera decorazıone di una stessa parete, mostrano del giardinı su uno slondo ceruleo, inquadratı da telaı dı legno delle finestre simli a quelle dell'Insula accidentalis (cat 262/6) Due frammentı simli presentano una palma da datteri alta nel mezzo di cespugls e uccell volant - per quanto sia possible constatare attraverso le Hustrazionı pubblicate - paragondbli aglı uccellı nella Casa della Venere in bikını (cat 166/1) Al tumpano del telaso sono appese maschere policrome Il frammento piu largo, in origune quello centrale. possiede gli stessi elementı piu un bacino di marmo nel centro sorretto da una stınge femminule dlata La larga colonnd sulla testa dolla sfinge si incontra uguale nelle pitture di Oplontis (cal 3419 ) con altre atfinita anche nelle plante e nelle slingi Vel bacino zampllid acqua, un uccello posato sull'or to di esso fa pensare al lamoso mosaico di Sosos $276^{\prime}$ Al lato superiore vediamo un pinax marmoreo che rappresenta due maschere Con un certo grado di prudenza si puo ammettere che entrdmbi 1 complessi decoratiu sidno stat dipint i dalla medesima bottega

\section{CONCLUSIONI}

I pittorı rappresentavano le statue attraverso l'uso di colorı suml al materiale e collocandole sopra una base Le figure che sono raffigurite come esseis vivi si ispirano a motivi noti nella scultura antica, mentic, nello stesso tempo, trovano dei parilleli nella pittura parietale Le tigure bianche sono scmpre ravvirate con giolell, vestitı e attributı a colorı, dandocı un'immagine ottuma della vera policromia parziale, particolarita che non si incontra spesso in altre statue dipinte, anche la chioma e 1 trattı del viso sovente sono dipinti a colori naturali Reutersward ${ }^{27}$ sostiene che soprattutio lo statue da glardino lorniscono informazioni valide sull'uso di dornture e di colori Paragonanclo ligure dipinte con statue trosate in Campania, per esempio la Venere il bihini e l'Afiodite di Oplontis. ${ }^{27}$ non si trovano differenze nell'effetto della policromia parziale 1 pittorı hanno avuto davantı aglı occhi statue del genere quando eseguivano le loro decorazionı

Rispetto al tipı, le pitture contermano la conoscenza gla raggiunta attraverso la scultura La maggior parie cl e nota in lorma di sculture $e$, come e stato detto, il repertor 1o e limitato Gruppi come 'Achlle e Chrrone', Iisto che forniscono dati concretı, sono di giande rlievo rispetto a lavori artistici finora conosciutı solo grazıe a fontı scritte

La collocazione di statue non difterisce dalla realta, almeno per quanto sı puo riconoscere neı verı giardinı pompeianı L'esposizione di statue davantı alle lesene fra le prospettive corrisponde all'usanza di esporre statue nel peristilı a ridosso di pilastri o di colonne anzic che negli intercolunni Troviamo esempl in situ nel tempio di Apollo, nella palestra presso il teatro (ul Doritoro) e nelle numerose erme a ritratto in case private, per esempio nella Casa di Caecilius Jucundus In tutt 1 casl si tratta di figure che vanno guardate da un solo lato, secondo un concetto venuto in uso a partire dal quarto secolo a C La terminologia einansichtige Gruppen, introdotta da G

276 Cf Parlasca 1963, K Parlasca, Helbig " no 1256 Anche 1 suddettı ambientı dell'Insula occidentalis sono ornati con bacinı e piccionı essi decorano le lunette sopra la porta del lato ovest

277 Reutersward 1960,60-68, 181-191

270 Reutersward 1960, 18j-186, Abb 28, Jashemshi 1979, [1g 200, 468 
Krahmer nel 1927, esprume completamente questa tenclenza 279

Le statue sembrano esposte senza ordine fra le plante I glardinı pompelanı presentano un insieme disordinato in cus non si e miralo ad ottenere una simmetria o un'assialsta, ammesso che le statue siano stato trovate nella loro collocazione originale I guardini dipintı anvece risultano composti secondo un ordine plu preciso tontane. munte a bacino e crme sl pichintano n coppse o nel centro des campl, mentre le altre ligure sono esposte in modo piu libero Queste statue non sl trovano sempre nel centro nid a volte sono nascoste dictro una fontana o fra l cespugli purtroppo le pitture delle Ierme stabiane (cat $231 / 1)$ che a questo riguardo sono tanto important Jeggibll

Relativamente al programma iconografico si constata una riduzıone tematica al mondo dionisiaco o all'ambiente delle divinita fluvialı in corrispondenza con

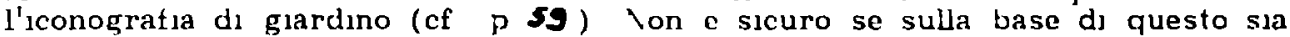
possibile attribuire un significato religioso al giardini in gencrale e a quell in paiticolare e se essi abbjano avuto und tunzione saciale Bisogna tonor presente ll concetto diverso riguardo alla religiosita del Romani Il cardtere decorativo sembra essere stato sempre di maggiore importanza, anche nes giardinı egizi nolla Casa del frutteto (cat 161 1) I lararı nei giardinı non hanno aruto influsso sulla scelta di particolari tipl per le statue dipinte, tipi scultores adopelat nel lararı non si incontrano ne nel veri glardinı ne nel giardini dipinti ll latto che questi grardins dipintı venissero combinats con paradeisol, conferma ll loro carattere decorativo 1 paesaggi con animali esotici certamente non possono cssere inseriti in un'atmosferd religiosa

Se gli elementı dionisiaci andassero consideratı come espressionı di un alteggiamento positıo verso la vita, di und dionysische Diesseitsfreude, come dice Vichel, 280 cio sarrebbe per quasl tutte le pitture murali dove si incontrano ininterotlamente elementi dell'atmosfera dionisiaca, sia in torma di motivi decorativi, sia nel pinakes mutologici Cosi l'accentuazione di tali caliteristiche in un solo genere di pittura, aflunche sl dia un signiljcato specifico a tale genere perde il suo senso Le rappresentazionj di giardinı sono 'dionisiache tanto quanto le altre pitture In esse prevale, come nel resto della pittura parietale, la funzione decorativa

$E^{\prime}$ difficle un puragone fra l III e ll IV stle, visto ll numero ridotto di rappresentazionı di giardinı dı III stule Sulla base del materlale discusso non si incontrano grandi difterenze fra le pitture del due periodi per quanto riguarda le statue II repertorio adoperato e uguale, solo nel III stile si incontrano eleinentl egizı, cioe nella Cosa dol frutteto (cat 16] 1), e statue di uccell, nello zoccolo

\footnotetext{
279 Krahmer 1927, Jucher 1950, $11 \mathrm{Cr}$ P 10.

200 Michel 1980
} 


\section{IV: MODELLI SCULTOREI ADOPERATI: OPERE A NOI NOTE E TIPI PERDUTI}

Sono state accennate le complicazıonı incontrate da chı cerchı per le figure dipinte del confront bidumensionale, con la tigura posta trontalmente, sulla quale non si leggono facumente la posizione delle membra. l'equalibrio e le torsion del corpo L'incarnato non puo essere studiato al modo in uso per la scultura e cro iale ancora meno per la stoffa e le pieghe delle vesti vella maggior parte de cası 1 pittori non hanno prestato molta attenzione al dettagli Bisognd di nuovo domandarsi se essi abbiano aruto davero la pretesa di coplare esatidmonte des modell scultores o se abblano soltanto voluto renclere le figure in atteggiamentı e posizıonı simlı L'accuratezza sı constatd dı rado, nernmeno quando siamo di Ironte a tigure indubbiamente interpretabilı come statue di dimensioni non ininiaturistiche Se si studiano altre tontı per la conoscenza dei tipl scultores, cioc le rappresentazioni su vasi, monete e pietre preziose 281 incontriamo lo stesso problema Schelold amuise nel suo saggio sulle rappresentazıon di sculture su vası, che le statue eraro riconoscibili soltanto per la presenza di piedistall Aon sarebbero slate dipinte con lo scopo di umitaic pezzi notı. Le statue di divinita sarebbero determinate dalla loro funzione e non rispecchierebbero una concreta situazione, solo in pochi casi si troverebbe l'unmagine di un'opera d'arte famosa 2.2 Per quanto riguarda le monete e le pietre preziose la situazione e diversa Gli artisti miravano compre al'unitazione di opera nobilia La statua si trova in una posizione isolata o occupa l'edificio dove stava esposta anche in realta La scelta delle figure e stata determmata dalla tunzione del mezzo sulle monete da ragionı politiche o topografiche, sulle gomme dal desiderio di avere, insıeme alla pietra prezıosa, la copia di un'opera d'arte in forma minaturistica In certi casi lo pitture sono paragonabli con la ceramica Quando non si trovano contronti cro non vuole dire che la figurd dipinta non copil und statua Il materide ancora presente e solo una trazione del repertorio originale

Aon si deve trascurare la crealione di variazionı su temı a loro notı Il fenomeno delle copse e delle varianti nella scultura e stato studiato dettagliatamente, anzi per decenni lo studio della scultura si era concentrato su questo aspetto Rumane difficle stablire qual elementi sono dovuti alla mano dello scultore-copista i" qual appartengono all'origmale Lo stesso vale per le sculture dipinte, torse ancora su scala maggiore vista la liberta de pittor nellapplicare elementi scultores e considerati gli argoments sopramenzionati concernentı l'dustralione di statue sul plano in questo contesto si pensi all'assenca del problema statico l pittore non doveva occuparsi della ponderazione del corpo, dell'applicazione di accessorı necessarı qualı sostegnı e puntoll fra corpı e meinbrı, ne di una resa particolare deı drappi Conseguentemente $l$ numero di archetıpı riconoscibuli e ristretto Possiamo tuttavia inserire le statue dipinte nella tipologia e nella cronologia stulistica, benche sid ignoto lo scultore dell'archetıpo Se si tiene conto di quanto e difficle attribuire statue a determinati maestri o botteghe, si puo essere soddisfattı deı datı recuperatı nello studio delle statue dipinte

\section{Cf risp Schefold 1937, Lacrow 1949, Horster 1970}

$2: 2$ Schefold 1937 Cf Lohmann 1979, 4-5 (grande realismo), 20-24 (polıcroma), $2 j-38$ (13 statue funerarie, $p$ e l'ifrodite di Alcamene, cat $\mathrm{H} 196$, Tal 82 ) 
Pfretschner discute la complessita di cuestı problemı nel capitolı III e IV

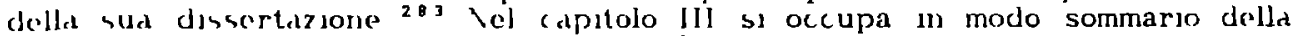
ponizione e dell aspetto delle statue L'inserimento di statue in costruzioni architettoniche (cui discusso nel prumo capitolo, $p$ 16 -50 ) e simile all'csposizione di statue in ambientı prilatı di paldzzi ellenistici Stavano sotto 1 portici come opere d'arte, prive di un significato specifico (religioso), ma talvolta secondo un precsso programma konogratico (cf $p$ ( ) vel mondo romeno sarebbe cinentata di moda l'esposizjone dl statue davanti d paretı cicche Cosi non e chiaro come, nell argomentazione di Ptretschner, ${ }^{284}$ sla da spiegare l'inserimento di statue entro l sistem decoratil esse stanno in nicchie o intercolunni, di rado davanti a pareti divisoric (per esempio nella Villa del misteri, (at 305 1) Le figure esposte sopia mensole potrebbero testimoniare questo sriluppo ma non sono state discusse da Pfretschner

Le statue che 4 iedono nelle rappresentazion figurative sono state coprate msıeme al quadrı (cf $p$ 97) Quinds non e necessar so, come sostsene Pfretschner, che 1 pittorl conoscessero queste statue dal vero

Nella seconda parte del capitolo III Ptretschner discute la maniera d'apparenza come con qualı attributi e in quale parte della decorazıone si trovano le statue di divinita I tipl statuari vengono discusai in alcuni casie non in modo sistematico A causa di und divisione delle tiguro sulla base della loro posizione nel sistema decorativo questo capitolo non e ben strutturato e contiene diverse ripetizion di osservazioni tatte nel ca,itolo precedente sui tipl singoli

Secondo Piretschner gli escmpl per le sculture nel sistem decorativi in parte sono statı presı da repertor (Musterb acher) in parte tormiti da tipı notı agli stessi pittoil Fsprimerebbero ein $H^{\circ}$ óhepunkt einer Folge von Szenen aus threm Mythenkreis oder Ausdruck einer bestumnten Geisteshaltung des Besitzers $285 \mathrm{D}_{1}$ consequenza specialmente le statue noto sarebbero state esempl per 1 pittor 1 Questa conclusione puo essere sostenuta solo in alcuns casi, come cerchero di demostrare nel prossuno capitolo

Finalmente Ptretschner osserva che e impossibule suggerire datazion sulla base del piedistall delle statue (Uuasi sempre 4l usano 1 piedistalli normalmente presenti nel periodo romano ${ }^{2 B 6}$

Sono statı compostı gruppl in base all'sconografia (divinita e altrı soggettı) e all atteggramento (in predi, sedute) lella ricerca di inateriale di contronto al e mirato a cercale per quanto possible statue trovate nel medesimo ambito delle pitture un parallelo dalla Campania ha plu autorita rispetto alla familisita del pittore con un certo archetipo che un esemplare trovato in und zona distante dell'Impero, dove anche la datazione puo essere un elemento importante Ciononostante non dobbiamo omettere la ricerca du esempi nel mondo ellenistico da dove provengono I cartons e I modell pittories (et p 97) Von si propone in questo capitolo una discussione sugli esempi singoli, ma si offre una panoramica del tipi piu comunı o importantı I dati concernentı le singole pitture sono inseritı nelle descrizion del catalogo L'appendice presenta un elenco del potenzialı tipı statuarı e delle variazion per le qualı non sono conosciutı controntı precisı

vel capitolo "Stile e gusto" (p 87 ) verranno discusse le pieferenze per quanto riguarda gli stilı e 1 tipl Inevitablenente alcune pipetizion saranno la consequenza di questa discussione articolata in varı capitoli Sostanzialmente

\footnotetext{
283 Pfretschner $1977,83-112$

204 Pfretschner 1977,83

2 в 5 Pretschner 197791

286 Pfretschner 1977, 91-92 Cf Jacob-relsch 1969
} 
i tipi scelti sono d'origine greca o ellenstica. Rari sono j tipi romani: le statue equestri sulle scene del loro nes Praedia di Julja Felix (cat. 179/1) e nclla (asa della caccia antica (cat. $24 ; 4$ ) e le figure vestite di una toga nella Cassa di Ercole cbbro (cat. 2843 ) c nella Villa dj San Marco (cat. 009 1) dove, secondo Olga Llia, si tratterebbe di ritratti di membri della dinastia grulio-claudia - costituiccono l'intera raccolta. Gli esempi erano reperibili nelle vicinanze. Le raffiguralioni del foro sono state dipinte contormi all'attualita, per le altre figuro 1 pittori avevano davanti agli occhi numerose statue esposte sul toro e negli edifici pubblici. In prosieguo quindi si parlera esclusivaniente di modelli greci e ellenistici.

\section{STATUE DI DIVINITA}

I personaggi sono discussi in ordine alfabetico. 207

Afrodite/Venere

Statue di Afrodite si incontrano quasi esclusivamente in rappresentazioni di giardini. Quando si tratta di quadri con temi mitologici la dea e spesso delineata secondo un tipo statuario. Quasi sempre è nuda. I tipi usati piú frequentemente si trovano a Pompei in forma di statuetle topiarie. Le statue dipinte e quelle vere corrispondono alla produzione di cople nell'intero Impero: vediamo sempre la Pudica, l'Anadyomene e 1 modelli chramati Arles e Frelus. 2" Dei tupi noti e popolari manca soltanto la Kallupyge.

Singolare e la Venere pomperana. In senso iconografico essa unisce elementi di varie divinita. La reste e l'atteggjamento sono simili a quelli di figure femminili dell'epoca ellenistica. Gli attributi sono statl presi da Tyche Fortuna (timonc), Felicita (ramo d'oliva) o ttrodite (Eros, Priapo). Hancano completamente dei contronti nella scultura, a parte una statuetta fittile. ${ }^{2 a}$ La slatua di culto del tempjo di Vencre non e stata ritrovata, ma non e improbabile che fosse stata asportata durante o dopo il distastro del 79 Forse la stessa sorte subirono le statuette del larari privati. 290

\section{Apollo}

Mancano quasi completamente paesaggi sacro-idilliaci con santuari dedicati a Apollo, dove talvolta un tripode simboleggia la sua presenza. La drinità appare in rappresentazıoni mitologiche - quasi sempre come figura viva in atteggiamento scultoreo - $p$ in un contesto architettonico I modelli Irequentemente adoperati sono il Liceo e il Citaredo, entrambe creazioni del quarto secolo. Sorprendente $\dot{e}$ il grande numero di varianti, ottenuti con elementi presi dai due modelli e talvolta con Hggiunte eterogenee. Non si incontra mai un 'vero' Liceo. La caratterizzazione delle figure da Pfretschner come durchweg hellenistisch è corretta. Tali considerazioni stilistiche valgono anche per le statue trovate a Pompel.

\footnotetext{
287 Non si cutano confronti scultorei per tutte le singole rappresentazioni che sono state analizzate e citate nel catologo.

28 Brmkerhof 1978 discule $i$ vari tipi.

209 Spinazzola 1953, 1. 218, fig. 244-245. Cf. Göttlicher 1981, 68-70.

290 La statua di Venere (MN 6294) e quella di Ermafrodito (MN 6352) trovate nel tempio di Apollo probabilmente provengono dal tempio di Venere e erano state collocate provvisoriamente qui (Dohl Zanker 1979, 183).
} 
Fa eccezione l kouros dipinto nella Casa del centauro (cat 203 1), conosciuto solo attraverso un disegno Il modelo originale va datato negli ultum decenn del sesto secolo a C una testa di kouros, prodotta probabilmente nel primo secolo d $C$ Cl fa capire come fossero noti alcuni modell in quel momento 291 Chiaramente differiscono dell'Apollo arcastico trovato nella Casa cel Menandro

\section{Ares/Marte}

Ares non sl incontra in paesaggl sacro-idlllacl la sud prescriza si lumita a ralfigurizions architettoniche e pinakes mitologici, spesso in combinazione con Alrodite lela Casa dell'alcora ad Ercolano (cat 026) incontriamo l unico esempio di un tres seduto Questo atteggiamento e raro anche nella scultura st pensi all tres Ludorisi ora nel Vuseo Vazıonale Komano che usale ad un originale cired del 300 a $\mathrm{C}$ e che si inserisce nella serie di drimita sedute 292

In piedi ll dio si presenta sempre nudo, armato con una lancia un gladio e taliolta con un elmo $C^{\prime}$ scmpre simule al tipi policletel usatı ugualmente per 1 guerrieri nudi (cf $p$ 43) Soltanto ll Doriforo e l'ties Borghese sono individuabli, il resto moutra soltanto tadti generici

\section{Arpocrate}

In und pitturd proveniente dal templo di Iside (cat 275 1) ll figlio di Iside e Osiride. Plconoscibile dal gesto dell'indice presso le labbia sta in un'edicola dipmta Latleggiamento ripete quello consueto, noto dalla scultura e in altre produzioni artiglanal

\section{Artemide/Diana, Artemide-Ecate}

trtemide non appare spesso in veste di cacciatrice Lo stule di questi tipi e tardoclassico o ellenistico lad dea indossa un chitone corto mentre e munita di taretra ed axco talsolta anche di una lancld Il grande trammente di II stile dell'Insuld occidentalis (cat 225 1) la mostra come Voriforo A parte una statuptta proreniente dalla Casa del moralista non ponsono essere indicatı precisi confront 1293 Inche l'Artemide lersalles e l'lirtemide di Gabil sono state coplate benche in maniera assal libera

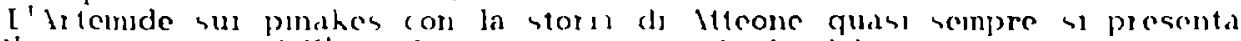

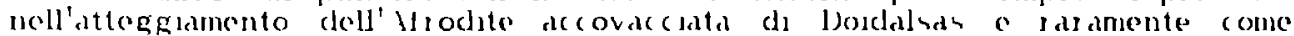
Pudica 294

Dopo Priapo Artemide-Lcate e la dirinita plu frequentemente presente nes paesaggi sacro-iduliacl Indossa un peplo e regge in entrambe le manı una traccola Talvolta la dea ha sulla testa una corona rachata o un numbo Lo stule e sempre dicalzzante e suggerisce un'alta vetusta lon possiamo trorare controntı ummediati nella scultura, una statuetta pompeidna la mostra in chitone corto e in stile ellenistico 295 Originale e il rimando di Ptretschner all'Artemide dı Efeso questa figura, o un tıpo simule quale l'Afrodite $d_{1}$ Afrodisia, axrebbe potuto seri ure da prototipo Risaltano l'atteggiamento delle

\footnotetext{
292 MN 109621 trovato in VII 3, 40 (CatLondon 1976, no 96)

292 CatRoma I 5, 115-121 no 51

293 Spinazzola 1953, II, 759761 , fig 740-741, Zanker 1979, 488-489, Abb $19-21$

294 Cf Lulles 1954 Linfert 1969 (contesta l'atiribuzione a Doidalsas), Brinkerhof $1978,35-5 j$

295 Spinazzola 1953 fig 466
} 
braccia e la rigidita del corpo. La diffusione della divinità e con essa la conoscenza dell'iconografia rimane un lattore incerto, ma con qualchecerta prudenza si può ipotizzare la presenza di un elemento comune nell'ambito ellenistico.

Il tipo molto diffuso dell'Ecate triforme è quasi assente pitture: l'Ifigenia nella Casa di Pinarius Cerealis (cat. 182'1) regge uno xoanon di tale schema.

Devono essere menzionate in merito gruppi di tre figure vestite di un peplo che stanno su una sola base Se reggono delle fiaccole l'identificazione con Ecate è senz'altro l'unica possibile: in altri casi il gruppo potrebbe rappresentare le tre Grazie in veste di ninfe acquatiche (vedi sotto Divinita acquatiche).

Su pinakes con la storia di Ifigenia in Tauride si vedono idoli semplici, quasi ridotti ad erme che sembrano essere scolpite in legno.

Senza confronti resta la potnia theron nella scaenae frons nelle Terme stabiane (cat. 231,3).

\section{Atena/Minerva}

Atena $\dot{e}$ presente quasi esclusivamente in scene milologiche e non si trova entro sistemi decorativi o corne opera d'arte indipendente. Per quanto si possa stabilire, essa non valeva come ligura decorativa, priva di un contenuto religioso, in contrasto con la maggior parte delle divinita. In Campania il suo culto e accertato a Pompci, dove Atena veniva adorata nel tempio sul foro come inembro della Trias Capitolina e nel tempio di Zeus Meilichios presso i teatri. Un grande numero di bronzetti proviene dai larari privati.295 La prudenza nell'uso decorativo di questa dea forse va spiegata da ragioni del genere.

Solo in 1 2, 6 troviamo la l'arthenos (cat. 140), rarila assai strana, quando si pensa alla frequenza di bronzetti del genere. Ben nove volte invece incontriamo la Promachos, spesso eseguita in stile arcaizzante. L'atteggiamento assomiglia a quello dell'Artemide arcaistica scavata nella Casa di $M$. Ifolconius Rufus. ${ }^{29} 7 \mathrm{Va}$ ricordato a tal proposito l'Atena che stava esposta nel tablino della Villa dei papiri. ${ }^{29}$ La preferenza di questo tipo si spiega attraverso il contesto: i temi dei pinakes sono la caduta di Troia o Teseo e Il Minotauro. In entrambe le storie Atena entra in veste attiva, partecipe, in un ruolo che si esprime bene sotto la lorma della Promachos combattiva. Forse i pittori hanno copiato la statua insieme agli interi pinakes senza conoscere la Promachos o una copia di essa dal vero.

Sono sei le rappresentazioni del Palladio secondo il tipo abituale Le terrecotte recuperate " Lavinio sono fra gli esempi piú recenti. ${ }^{2} g^{\mathrm{Si}}$ avvicinano al tipo della Parthenos di Fidia conosciuta fra l'altro attraverso copie trovate a Pergamon e nella vicina Sperlonga.

L'Atena Ergane, patrona degli artigiani, si trova in scene ove sono rappresentate attivita artigianali. Sulla facciata della bottega I 8,10 (cat. 157) era rappresentata un officina ceramica, da datare nel periodo sannitico. In VI 7, 8 (cat. 194) sono rimasti conservati solo uno scudo rotondo e l'orlo inferiore del peplo, nentre in VI 14, 22 (cat. 213/2) il modello è piuttosto quello di Ecate. In nessun luogo si è di fronte alle raffigurazioni normali,

296 P.e. Dwyer 1982, fig. 168-169.

297 Döhl/Zanker 1979, 205; Fuchs 1979, 241-242, fig. 261-262; Dwyer 1982, fig. 208

298 Comparetti/de Petra 1883, 277, tav. XIX d: Pandermalis 1971, 183, 205; von Hesberg 1981, 1130-1131 no. 37.

299 Enea 1981, 190-195. 
proveniente dall'Asia Minore. 300

Demeter/Cerere, Cibele. Tyche/Fortuna

Queste tre divinita vencono rappresentate allo stesso modo sono sedute su un trono e differiscono soltanto per quanto riguarda gli attributi. Specie per Cubele puo essere servita da archetıpo la statua esposta nel Vletroon sull'Agora d Atene scolpita da Agoracrito Di questa statua si conoscono copie di varıe dimensioni. ${ }^{30}$ Anche per Demeter e Tyche ss puó upotizzare il medesmo modello La tainosa Demeter di Cnido, ora nel I3ritish Museum a Londra, non e stata riprodotta in forma dipinta. Della Tvche di Antiochia si trorano due copie esatte in una pittura del tempio dj Bel a Dura Luropos (cat. 013). La Demeter nella Casa del Dioscurı e e'seguita in stile classico (cat 204'2).

\section{Dioniso/Bacco/Liber}

Le rappresentazioni dı Dioniso si dividono in tre gruppi rispetto al contesto decorativo e al modello.

1. In paesaggi sacro-jdilliacs e in un certo numero di scene mitologiche Dioniso si presenta sollo forma di un uomo anziano barbuto, vestito di un chitone e/o un himation Regge un cantaro c un tirso nelle mani Il dio o raffigurato in questa maniera upecie nell'epoca arcaica e classica Lunitandosi alla scultura si pensi al cosiduetto Sardanapallos nei llusej laticani. ${ }^{302}$ Per la testa si deve menzionare l'erma di Alcamene. ${ }^{303}$ Talrolta indossa un chitone corto La statua attribuita a Calamide vale come contronto soprattutto per l'atteggiamento

Il glovalle I) joniso nel trammento di Portics (cat. 308) si inserisce nella serie di questo tipo. In csso si vede ripetuto il motivo policleteo del pirdi, normale per l'ultimo quarto del quinto secolo a.C. L'interpretazione, sostenuta cla Ptretschner, della tigura dipinta come Lubera non e corretta

Su un pmax nella villa della rarnesina (cat. 319/2) una statua del Dioniso anziano e presente ad un glovane Dionlso: essa sembra custodire l'allattamento del Dioniso neonato da Leucotea.

2. Vella maggior parte delle scene mitologiche e nelle rappresentazioni architetionicho Dioniso e un giovane nudo, in pied,, con nella mano destra il cantaro e nella sinistra ll tirso. In tutti 1 casi la tigura ripete un modello del quinto o del quarto secolo a.C, copiato molte volte nell'epoca romana. Lo schema policleteo del Doriforo rieccheggia in queste statue, per esempio nel Dioniso trovato nella Villa adriana di Tivol. ${ }^{304}$

300 Cr. Di Vita 1952-1954.

301 Xaumann 1983, 159-169 non considera la statua Doria Pamphli come una copia della statua di Agoracrito (cosi Calza 1977, 35-37). Le statuette delle tre dirimita nel larari a Pompes sono sumili, p.e. un bronzetto (D)wer 1982, fig. 88). Anche delle variantı ellenistiche possono essere state usate (Naumann 1983, 247-253)

302 Helbig 4, no 496; 1726 (rephica Museo vuovo Capitolino), 2333 (erma Museo Nazionale Romano; cl. CatRom I 5, 159-162 no. 68).

303 Harrison 1965, 129-134.

304 CatRoma I 5, 70-72 no. 58 . 
Infine sl incontrano erme con la testa barbuta in paesagg sacro-idillaci Lo stue delle teste e sempre arcaizzante e rumanda

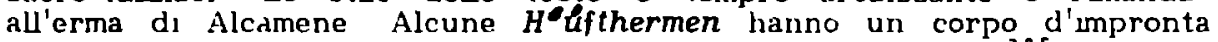
classica, ma sono ispirate a tipi ellenistici, non plu esistenti 305

\section{Divinitd acquatiche}

Anche se 1 pittorı disponeiano di una vasta scelta di tipı statuarı per le iıgure inserite nelle rappresentazion di giardin, ne usavano pochi Arevano gli esempı davant agl occhi nelle case pompelane I tipl statuarı e l'iconogralia topiaria sono ugualı in entrambı l cası, cioe sia per l giardinı vers che per quellı dipint 1 ( $c$ i $p$ o00)

In quindici case a Pompel si vedono nunfe acquatıche che reggono, dalanti al grembo, un bacino nel quale zampilla dell'acqua Come e stato detto precedentemente ( $p$ 63) ll prototipo va cercato nel quarto secolo a $C$, specie nella Venere Landolina 306

lel Castellum aquae a Pompel (cat 221) una figura del genere si trova in compaenia di altre due ninle l'una imita l'Inadyomene di Apelles. l'altra icgge und brocca dalla quale esce acqua daranti al guembo Le tre ligure probdbulmente vanno interpietale come le tre Grazie (ci infra p 76 ) in guisa di protettrici dell'acqua Inoltre e presente und divinta fluviale maschile, anch'essd modellata secondo un tipo noto, si pensi al famoso vilo nei Muse1 Vaticanı 307 Questo 'Vlo', a Poinper etichettato come 'Sarno' o 'fiume', si vede anche altrove, solo o in compagnia di ninfe acquatiche

\section{Ecate ( Artemide/Diana; Artemide-Ecate)}

\section{Eracle/Ercole}

Sono 1requentı le Immaginı dell'eroe Eracle, la maggior parte delle qualı lo rappresenta modellato secondo notı tipi statuari Raramente, pero, le figure sono dipinte esplicitamente come statue I modellı usati sono soprattutto creazioni di Lisippo, lo scultore che ha contribuito in modo decisiso all'iconografia di tracle Dobbiamo a Voreno un conbiderevole numero di onservasion sul'minlusso di opere lisippee sull'ale e sull'artigianito ellenistico-romino 300

Per l'Eracle seduto l pittore copiava l'Epitrapezios o l'Tracle di 'laranto, statue note attıaverso molte copie, ira le qualı alcune trosate in Campania Anche altre figure sedute sono state modellate secondo questı tipı Ricordiamo soltanto l'atleta nella Casa del Menandro (cat 163/5) e l pugde nella Vlla di S Marco (cat 009 6)

Quando si tratta di Eracle in piedl. sl incontra l'Eracle Farnese a l'Eracle di Sicione Due volte rediamo l Cracle Lansdowne di Scopa nel Macello e presente su un pinas mitologico, mentre nella Vila di L Crassius Tertius a Torre Annunziata (cat 340) una vignetta con l'eroe abbellusce una decorazione semplice Inoltre conosciamo alcune rappresentazıon dell'Eracle orinante o

305 Cf l'erma vestita nel Museo Nazionale Romano (CalRoma I 5, 159-162 no. $68)$

306 Becattı 1971

30 ' Helbig ", no 440

308 Cf ultumamente Moreno 1981, 180-185, 190-195, 199-20, 201-202, 1982, 1983-1984, 1984

jog $\dot{\mathrm{P}}$ e MN 136683 ( $R$ Parıben, NSc 1902, 574-575, Jashemshı 1979. 320. fig 496) 
ebbro, tıpi ellenıstici In questo caso il numero di statuette trovate in Campania supera qucllo delle ummagini dipmte. ${ }^{310}$ [na sola rolta la statua dipinta si trova in un contesto topiario, cioe nella Casa dela fontana damore (cat 278 ).

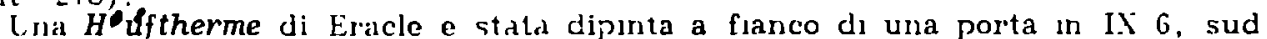
4-j a Pompel (cat. 289) Probabilnionte l'archetipo e uguale a quello del noto Teseo Ludorisi nel Vusco lazionale Romano 311

\section{Eros/Amor}

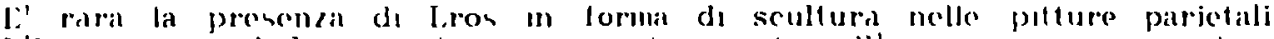

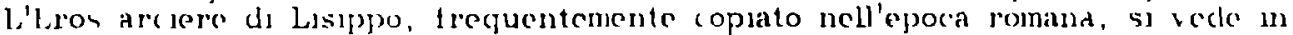
VI J, I (cat 19,1) L'liros che strozza loca di Bocto a presente in due esempları come statua di lontana nello zoccolo della scaenae trons nella Casa (de) palestrita (cal. 2(6i). L'Cros dormiente, statuetta di gonere ellenistica, e dipunto nella Lasa dell' luone Ierito (cat. 19j).

Quattro volte Eros regge una Laccola davanti al petto. Visto il contesto non vi e motivo di interpretarlı come genu lunerari, ipotesi proposta da $S$. Remach nel wo repertorjo Bsogna pensare a lampadophoroi o lychnouchoi ispiratı da tipı cllenistic 1 ora non ritrovabul ${ }^{312}$

Nolla Casa di M. lucretius (cat. 282) vediamo Trotı sotto forma di guerrers o divinta, poggiantı sopra mensole Anch'essi rispecchiano modelli policletel rifatt nel periodo ellenistico

In solo Hermeros si trova in una decorazione ercolanese, ora a Napoli (cat, 038).

L'Lros con il cervo su MN 9175 (cat. 067) sembra ispirato ad una creazione tardoclassica o ellenistica quale il gruppo bronzeo rappresentante Eracle a la Cerva di Cerinia trovato nel 1797, forse a Torre del Greso, ora nel Museo Archeologico dı Palermo

\section{Le Grazie}

Le diumita acquatiche, chamate le Charites, si incontrano in due vesti. In paesaggi sacro-idillaci gruppl di tre statuette di donne vestite di un peplo, spesso schematicamente delineate, stanno presso una fonte Sono simill alle rappresentazionı di Ecate triforme (cf. P 73).

Plu famosi sono 1 gruppl de tre donne nude che si abbracciano. si tratta di una creazione ellenistica molto dillusa nell'Impero. La lunga e inefficace discussione sul cardtere dell'archotipo (scultura o pittura) e stata caratterizzata chiaramente da Trillmich che conclude: Lang und fruchtlos ist die Diskussion um die Frage, ob ein plastisches oder gemaltes Vorbild anzunehmen sei und wann und von wem diese "Urgruppe" geschaffen worden sein mag. ${ }^{34}$ Egll ricorda ll dubbio espresso da Pausania $(1 \mathrm{~A} 35,6)$.

\section{Hermes/Mercurio}

$310 \mathrm{Cf}$. Nicholls 1982.

312 CatRoma I 5, 170-172 no. 72.

312 Cf. una statuctta di un genio o un Eros trovata ad Crcolano (Mauuri 19.58, $375)$.

313 Inv. no. 8364. Johnson 1927, 190, 197; Bieber 1961b, 37, fig. 78; 123. L'attribuzione tradizıonale a Lisippo e stata messa in dubbio con ragion valide da Kunzl 1968, 140-147, Abb. 20; Moreno 1981, 190-195, spec. $194-195 ; 1984,147-152$, fig, f, 23-24, 26.

314 Trillmich 1983,323 
Sporadicamente Hermes si presenta sotto forma di statua nella pittura parietale La figura nella Casa del palestrita (cat 267) riprende un tipo trequentemente copidto nell'epoca romand Risalta il cambiamento degli oggetti la borsa nella sinistra presso ll hanco e l caduceo nella destra distesa Apparentemente il pittore ha sfruttato al massino le posibilita offertegli dalla pittura, cosa spesso negata allo scultole legato a problemi statici 315

L'llermes di Olimpia si vede tre volte, le copie dipinte sono umportanti visto l'esiguo nuinero di copie d) questa statua prassitelea (ct p 88 nota 362 ) Grazıe alle figure dipinte si puo ricostruire della statua di Olumpia la posizione della mano destra che e alzata e regge un grappolo d'uva A parte (le copie del)la statua del quarto secolo a $C$ bl conoscono alcune statue modellate secondo 1 medesimo schema, ma di stile ellenistico ${ }^{316}$ l unca ditferenza fra le statue vere e quelle dipinte $e$ che queste rappresentano dei satiri

L'Hermes seduto nella Casa della (accia antica (cat 2lj 2) copia la statua colossale di Lenodoros, scolpita per incarico degh drein sotto l'umpero di Claudio o Nerone e nota attraverso und scrie di bronzett l La maggior paste e stata trovata nelle province un bell'esemplare venne trovato nel larario della Casa degli amorinı doratı ${ }^{317} \mathrm{E}^{\prime}$ interesunte constatare che la riproduzione e di poco successiva all'originale, il che significa una rapida dirulgazione del tipo lorse gracle all'invito che Nerone tece allo scultose di imnanzargh una giandiosa iminagine divenuta celebre come 1 Colosso ${ }^{318}$

\section{Iside}

Le rappresentazioni di Iside non sono tanto Irequentı quanto Schefold $e V$ Tran Tam Tinh hanno voluto suggerire Solo nell. I llla della Farnesina a Roma (cat 319) ld ded egizia e onmipresente, come nel fregio nulotico che adorna L trichnio estivo nella Casa dellefebo (cat 15j 3) Si incontrano invece anche se in quantita minore, sincretismi o adattanenti con la tigura di Afrodite Le tre statue dipinte conosciute in (ampania e quelle nelle pitture delia Casa di Livia a Roma (cat 3173 ) rafígurano iside nell'iconografia abituale, nota fra l'altro dalla statua del tempio di Iside ${ }^{319}$ Non di rado le figure sono molto schematiche, specidmente quando si tratta di statue collocate in sacellı rusticı neı paesaggı sdcro-ıdlllidcı

\section{Muse}

Precedentemente ( $p$ 42) e stato discusso 1 f fatto che solo pochi modell scultores usats per le Vuse siano ritrovabil nelle pitture parietalı a Pompes, Ostia e Eteso Lo stule delle figure dipinte e ellemistico e in tale senso coincide con la data degli origmali dei gruppi statuari incostruits da Pinhwart 320 Il tipo della aufgelehnte Muse e stato usdto per alcune thtal in

315 Cf Boucher 1976, 89-90, 105-106, 370-371

316 Erum 1974

317 Beschı 1962, 31-60, 1974, Leibundgut 1984 (tıpo) $\&$ Soglano NSc 1907 570-571, fig 16, Boyce 1937, j7 no 221 (Casa degh amol inı dorat 1 ) Il tıpo e stato usato, fra l'altro, per l ritratto di Uemetrios II Vicator in veste di Herines, trovato nella Casa del camulo (de I ranciscis 1951 adamo Vuscettola 1979, 9091 , tav 38 fig 16, Dnyer 1982, 62, fig 76-77)

31: Plin, N.H. \\\V 45 Cf $\mathrm{P}$ Moreno, EAA VII, 1259-1260, $s v$. Zenodoros

319 Tran Tam Tmh 1964, 156 no 81 pl XIII CatLondon 1976, no 202, Dohl Zanker 1979, 183, fig 24 
vedute marine.

Una figura fernminile che regge una cetra, prosente nella scaenae frons della Casa degli epgrammi (cat.1852), probabilmente va interpretata come Nusa. Anch'essa non puo essere paragonata ad una dello statue note. Non e sıcuro se la tugura lomminile (?) che si trova nella Casa del colomnato tuscanico (cat. 035,2) che regge in una mano una cotra, debba essere interpretata come Musa, come sostiene Manni: la veste che non copre il petto e anomala nell'jconogratia delle Muse.

Le Kuse che si vedono sur sarcofagi del secondo e del terzo secolo d.C. non sono simili, se non in rarj casi. alle luse dipinte. Anche gli scultori di sarcolagi si permetteranu una certa liberta e non copiavano (tutte le) figure statuarie. Questo porta a concludere che i gruppi origmali erano noti e venivano copiati sia in forma statuaria che in altrı modi, ma che se ne taceva uso soltanto in modo eclettico. ${ }^{32}$

\section{Nike/Vittoria}

In generale si incontra il tipo della Vittora romana, statua diventata popolare dopo il trasferimento da 'Taranto alla Curna a Romil da parte dj Augusto. ${ }^{22} \mathrm{E}^{\prime}$ presente due volte su pmakes. In una scona di genere nolla Casa della parete nera essa viene venerata da un coro di psiche (cat. 24i). In V 2, 9 (cat. 192/1), in un quadro con Pasifae nella bottega di Ledalo si trova una Nike vista di profilo, apparcntemente inserita come uno dei prodolti dello scultore. Il modello ricorda vagamente la Vittoria di Brescia. ${ }^{323}$

Le altre Nikai fanno sempre parte di strutture architettonsche o fungono da vignette su campi. Vella Casa del bicentenario (cat. 032 1) glı acroteri sono stilizzati in modo che si puo parlare di erme. La posizjone delle Nikai nei campi e nelle architetture a abbastanza importante. S1 pensi alla statua posta su un blocco aggettante della scaenae trons nella Casa dollaria massima (cat. 221 1) o alla tıgura sopra il candelabro nel tablino dolla Casa del mobilio carbonusato ad Freolano (cat. 030) M sembra, pero, alzardato concludere da queste osservazion che l comittentı abbiano fatto una scelta precisa per una tale posizione e per questo tıpo di figura in base al significato politico dato alla Vittorıa Romana da parte du Augusto.

Lna categoria diversa e costituita dalle bighe e quadrighe guidate da Nikai. La biga dorata in I 3, 25 (cat.no. 14) incorona l aula regia che ottiene, in tale modo, il carattere di una porta trionfale. Anche altrove i gruppi fungono da acroteri. ${ }^{324}$

\section{Pan e satiri}

ll dio rurale Pan si vede soltanto raramente in paesaggi sacro-idilliaci, specie se si contronta con il numoro di statue di Lcate, Priapg e Dioniso. Nella scena di Atteone nella Casa del frutteto (cat. 161,2) una $H^{\circ}$ eftherme dettagliatamente eseguita sembra persa nel paesaggio selvatico. Come oggetto di venerazione

320 Pinkwart 1965. Dalla vasta bibliografia sulle Muse ricordiamo alcune opere importanti: Pinkwart 1967: Felletij 1aj Moreno 1967; Pucci 1981; Marabini Moeus 1981; Fuchs 1982. Theophilidou 1984.

321 Sui sarcofagi esiste, di nuovo, una vasta biblogralia. p.e. Wegner 19G6; Panclla 1966-1967, 1968; I’aduano Iacdo 1981; hoch Sichtermann 1982, $197-203$.

322 Holscher 1968.

323 Hölscher 1970.

324 Si confronti Dunbabin 1982 per altre immagini del gencre su nosaici. 
Pan e in posizione centrale su un pinax nella Casa degli epigramm (cat 185 '2) La statua presenta l'atteggiamento del'aposkopein cioe 1 satiro nell'atto di spiare con una mano sopra le ciglia, gesto stucilato da Inez Jucker 325 Lna decorazione perduta in VIII 7,3 (cdt 274) arrebbe contenuto, secondo Helbig, una figura simile

In alcuni casi non si puo stabllire se si trattl di Pan o di un satıro anonumo Il gesto dell'aposkopon sembra riservato per il dio stesso, almeno si ottiene questa idea valutando il materiale raccolto da Jucker I catir s si vedono in alcune scaenae frontes di Ill stule, per escmpio nel portico della Vulla umperiale (cat 305/3) e su II 9878, 9123 e 9125 (cat 053), tre frammentı che tanno parte di una sola decordzione non piu accessibule nel sotterraneo di Lrcolano L'na delle lesene trd I paradesoo nel gardino della Casa di M Lucretius Fronto (eat 1953 ) e decorata con la statud dipinta in bianco di un satıro aposkopon Lnd statua marmorea del tipo e stata 1 rorata nel giardino della Casa di $M$ Lucreisus ${ }^{326}$ Essa si inserisce tacilmente nell'xonografia 'seltatica' c 'idullica' delle figure topiarie animali, dcqua e altre figure dionssiache

Il kriophoros nella Casa del larario (cat 149/2) si inserisce bene nell'atınostera dionssiacd Una statua topidrid recuperata nella V'lla del pastore in contradd Petraro a Castellammare di Stabia ci da 11 modello ellenistico 327 a sua iolta variante su un tema gia presente nell'arte arcalca Vella Casa del criptoportico (cat 148,3 ) \& satiro nella micchia destra delld scaenae frons nel frigidario, segue lo stesso modello della statua di foniana nella Casa di ()bellıus I irmus 32: I satiri danzanti che si incontrano l'a l'altro nella Casa di Velcagro (cat 202) sono paragonabli con il fauno danzante nella Casa del tauno e con ll bronzetto trosato nella Villa del papirı 329

Per quanto riguarda, satirı modellatı secondo l'Hermes di Olumpia sı vedano le considerazioni a proposito dell'Herines ( $p$ 88)

\section{Poseldon/Nettuno}

If ic dol mare contituisce un elemento immancabile nolle vedute marme o nella magfiol parte del punahes con la caduta di lcaro, stranamente non mseiti nel lavoro di Ptretschner ln questı quadri il dio e sempre sciduto su uno icoglıo, con le gambe incrociate, e regge con una mano stesa in avantı ll tridente L'archetıpo va cercato nell'ambito lisippeo 330

Poseidon in piedi ripete uno del due modell creatı da Lisıppo Il ritratto di Demetrio di Falero si ritrova nella scdenae frons nella Casa dell'ara massima (cat 221,1) e nella Casa deglı epigramm (cat 1852$)^{311}$ La statua sul molo

325 Jucker 1957

326 Dwyer 1982, fig 43 a-b

327 Jashemshi 1979, fig 535 Per l genere of. Laubscher 1983

32. Spinazzola 1953, I, 387-388, fig 383, 386, Kapossy 1969, 37, Dohl Zanker 1979, 202

329 Fauno Ippel 1939, 350-362, Abb 1-6 da l'analısı migliore. Bıeber 1961b, 39, fig 95-96, Dohl/Zanker 1979, 202, Fuchs 1979 137, f1g 127-128 Papirs Comparett1, de Petra 1883, 270-271 no 51. tav IVI 6

33 a Cf per esempio l'Hermes in riposo della Villa del papirı, dove la roccia sulla quale il dio e seduto sorve ad ambientare ll personaggio in un'atmostera pdesistica (Comparettı de Petra 1883, 268 no 33, tai IIII 2) 
di Kenchreai (Corınto) si rivede nelle vedute di mare su $11 N$ 9491 (cat. 092), a Varano (cat. O10 5) e nella Villa della Farnesina a Koma (cat. 319/6). 332 Nolla cisa dei Dhoscurı (cat. 204/1) Poscidon sta in un'eclicola isolata e scrubra esposto appositamente come copia di un capolavoro di Lisippo Viclla Villa di San Marco (cat. 009, 10) e nella Casa dell'orso ferito (cat. 239) i ninfei sono abbelite da figure sımilı in stucco e mosaico.

\section{Priapo}

La divinita protettrice della natura e della fertilita si incontra nella maggior parte dej paesaggi sacro-ıdillaci e in un gran numero di pinakes con soggetto mitologico. In generale le ligure non sono altro che una sagoma o una striscia, di rado si vede una statua rappresentata dettagliatamente.

Innumerevols sono le erme itifalliche Spesso sono iste di profilo - per mostrare meglio il fallo - e hanno il dormo inclinato indictro nella chordosis. La tipologia precssa, stabilita da ll. Herter nel suo De Priapo, e applicabile soltanto in pochi casj alle figure schematiche che si trovano nelle pitture. ${ }^{33}$ Statue e statuette del tipo devono essere state molto popolari ovunque nell'Impero, ma rispetto a questa considerazione il numero trovato e abbastanza ridotto lorse per l'uso di materıali facilmente deteriorabil come il Jegno, la terracotta e il prombo. Nella Casa des Vettii un Priapo di formato assal grande ornava il glardino. ora a celato nel cubicolo decorato con scene crotsche posto presso la cucsna. ${ }^{334}$ I na piecola erma di legno trovata nella Casa del bel cortile ad Ercolano illustra l'esistenza di talj erme in contesto privato, erme che si vedono, Ira l'altro sul pinax nel cubicolo 4 della Villa dei misteri (cat. $306^{\prime} 2$ ). 335

Meno trequente $\dot{e}$ il Priapo vestito di un mantello che vene sollevato per far vedere il sesso; il grembo della veste e sempre colmo di frutti. Le gambe talvolta sono state sostituite da un fusto di erma. Di recente If. Blanck ha raccolto gli esempl scultores a proposito di una statua a Formello. ${ }^{336}$ Il Prrapo, che solfre (o gode?) di un doppro fallo, nel bordello di Pompei (cat. 255) e la copia piu tedele in torma dupinta di questo tıpo Anche in scultura si incontrano escmpı a l'ompel, soprattutto statuette fittill

Il colore in genere c rosso, conforme l'abitudine di dipmgere le statue di Priapo con minio o altre tinte naturali. Lon indica, qund,, il materiale che può essere marmo, bronzo o legno.

Fuori del contesto paesistico si vede Priapo accanto ad Afrodite, per esemplo in I 13, 16 (cat. 173) e in V 2, d (cat. 187) dove un'erma di Priapo funge da appoggio per la dea, o con la Venere pompeiana. ${ }^{33} 7$

331 E. Wbdt, Poseidon, RE XXII 1 (1953) 539-540 cita 67 esemplari.

332 Per il tipo Moreno 1976. Per la posizione originale cf. Walde 1978; Walde-Psenner 1979.

333 Herter 1932. Cf. H. Herter, Priapos, RE XXII 2 (1954) 1914-1942.

334 Soghano 1898, 269, Kapossy 1969, 29.

335 L'erma ercolanese, ora nell'Antıquarıum di Ercolano, non è stata pubblicata.

336 Blanck 1979.

337 Cf. anche la statua di tufo rappresentante un gladiatore che si poggia su Priapo, trovata in I 20, 1 e ora nel'Antıquarium di Pompei (inv. 11734): CatEssen 1973, no. 200; Elia 1975, 121, 124-135, fig. 11: Jashemski 1979, fig. 264; Dohl/Zanker 1979, 179-180. 


\section{Saturno}

J'unica rappresentalione conosciuta del dio romano Saturno si vede nella Casa deI Dioscuri (cal 204/2) lvi e rappresentato come flamen con la testa coperta, reggente una talce nelld destra

\section{Zeus/Giove}

Lnica e la rappresentazione di una statua dorata di Zeus nel larario stradale lungo ld Via di Mercurio a Pompes (cat 244) ll dio e modellato come un Doriforo ed e ben i iconoscibule grazie al tulmine che tiene nella mano destra slena in asduti

Zeus seduto 51 trova in alcune scaende trontes di IV stle Le figure sono irriconoscibul circa ll loro carattere scultoreo 1 pittor non facevano distinzione fra una higura viva e una scultorea Pfretschner fa cenno ad un trammento nel Vuseo Vazionale di Vapolı, proveniente dalla Casa del Dioscuri. c ad und figura nella wisd dei vetti il rilıevo a stucco nella palestra delle lerme slabiane (cat 2313 ) contuene al lato destro sopia la porta, un Leus seduto che imita 1 modello ellenistico delle divinita sedute Ln confronto plu o meno preciso non puo essere presentato

\section{STATUE DI FIGURE MITOLOGICHE}

\section{Arimaspi}

lella Vllla del misteri (cat 306/3) un gruppo composto da un Arimaspo e un grifo tunge da elemento reggente nelle stiuttura architettonica ${ }^{\prime}$ diffic le che esso abbia un archetipo nella vera scultura Puo essero icoidato ll Ganimede rapito dal'aquila che adornata l'entrat. della grotta di Sperlonga e si troraia in una posizione alta, sımile a quella del nostrı gruppi dipıntı

\section{Chiron e Achille}

$F^{\prime}$ stato discusso gia alcune volte 1 gruppo $d_{1}$ Achulle e Chirone (cf $p$ ) Le rappresentazion clipinte nella Casa dell'Adone ferito (cat 195) e nella cosiddetta Basilica di Lrcolano (cat 019) con ognı probabilita devono essere considerate copıe del gruppo statuario esposto nel Saepta a Roma, distruttı da un incendio L'origmale sarebbe da datare nel secondo secolo a $\mathrm{C}$, come ha dimostrato in modo valido $\mathrm{H}$ Sichtermann

\section{Enea}

Sulla facciata di una casa lungo la Via dell'Abbondanza a Pompei si vede Enea che regge su una spalla suo padre Anchise, mentre conduce ll figho Ascanio per la mano (cat 304) Si tratta di una copia assal fedele al gruppo statuario presente sul foro di Augusto a Roma, del quale anche sul toro di Pompel era esposta una copld A parte questı gruppl marmorei non conservatı, conosciamo copie piu piccole in forma di statuette fittilı

\section{Paride}

Ind rola volta Paride e presente fuor da un contesto mitologico Nella Casa dell'alcova ad Lrcolano (cal 026) eglı si trova nell'edicola di una scaenae frons, seduto sopra un blocco L'atteggiamento corrisponde a quello delle statue di divinita e principi ellenistici

33 Iacopl 1963, 114-117, fig 109-111 


\section{Psiche}

La statua di Psiche su un pinax nella Casa di M. Lucretius (cat. 282/7) non puo essere correlata con un tipo scultoreo ben noto. Per Psiche non esiste una particolare iconografia. La l'igura si schiera tra la serie di statue cllonistiche di donne vostite. ${ }^{339}$

\section{Romolo}

Romolo che regge un trofeo, costiuisce il pendant dell'Enea sopracitato (cat. 304). Anch'egli ripete una statua esposta sul foro di Augusto e sul foro di Pompei.

\section{Scilla}

Nella Villa di Arjanna a Castellamnare di Stabia si vede un gruppo con Scilla nella posizione di un acroterio (cat. 010/3). La composizione ricorda il gruppo marmoreo trovato nella grotta della villa di Sperlonga, forse una copia di una creazione ellenistica da Rodi.

\section{STATUE DI ANIMALI}

Statue di animali sono frequentemente in uso come acroteri o per sostituire, anche se in rari casi, l'immagine antropomorfa di una divinita. Esempi scultorei sono conosciuti per la maggior parte di essi.

Solo una volta si incontrano un pavone, in $I$ 13, 16 (cat. 173), un elefante, su MN 8555 (cat. 050), mentre le capre su MN 8900-8902, 8908 (cat. 058) hanno un solo riscontro nella Casa di Giuseppe II (cat. 269/5). I cervi si vedono nella Casa del marinaio (cat. 259) e nella Casa di Sallustio (cat. 198b:1). Il bue Apis è presente nel fregio nilotico che adorna il triclinio estivo nella Casa del'efebo (cat. 155/3); ll colore giallo indica che si tratta di una statua di bronzo dorata. Nella Casa del frutteto due pinakes policromi contengono il bue con il disco solare fra le corna (cat. 161/1). Nella Casa dei Pigmei (cat. 285) un coccodrillo di bronzo è esposto sopra una colonna a forma di un sarcofago di Osiride; esso rappresenta il dio egizio Sobek. Coccodrilli come custodi di templi sono visibili su MN 8512 (cat. 049).

Nelle rappresentazioni di architetture si vedono grifi, stingi e centauri in guisa di acroterio. Vccelli come elementi reggenti sono presenti in alcuni zoccoli di III stile (cf. p. 27). Solo presso gli uccelli bianchi nella Casa del labirinto (cat. 208/2) e sull'nsula Orientalis Il A1, la ad Ercolano (cat.038) si può rilevare un certo carattere scultoreo.

Sfingi reggenti bacini di fontane appaiono in quindici rappresentazioni di giardini. Copiano sempre il medesimo modello: sono sedute sulle zampe posteriori, viste di fronte o di scorcio, mentre sorreggono con la testa, talvolta anche con le ali, la vasca. Conosciamo soltanto pochi esemplari del tipo, apparentemente una volta molto popolare come figura topiaria. Nella Casa di Sallustio o di Pansa (la collocazone originaria è clubbiosa) è stato trovato un esemplare in marmo bianco; nel Museo Nazionale di Napolj si vede una fontana simile, sorretta da una tritonessa, di 'rosso antico' che fa parte della collezione Farnese e che $\dot{e}$ forse databile in un periodo piu recente, quando i marmi policromi e rossi divennero materiali di moda. ${ }^{340}$

\footnotetext{
339 Cf. Linfert 1978 .

340 Ruesch no. 13 (inv. 6866). Ruesch 68; Gnoli 1971, fig. 40 (inv. j821).
} 
Sfingl come statue topıarıe sono meno trequentı Spesso sono del tipo egizio sono sdraiate con le zampe anteior stese in avanti e mostrano uno stule arcaizzante Lna statuetta del genere e stata trovata nella Casa di Octar us Quartio 342

A parte le stingl, centaurı sono in uso come sostegnı di vasche dd fontana Nella Vlla di Poppaea a Torre Annunziata (cat 341,4 , 9) e nella Casa deglı archı (cat 176) ne troviamo alcunı esemplari nella medesima decordzione Le figure del centaurı, poggiate sulle zampe posterıorı, derivano da modeli ellenistici, probabilmente di origine pergamena come ha dimostrato chraramente $S$ de Caro in un saggio su alcune statue toplare trovate nolla stessa Villa di Poppaea ${ }^{34}$ In generale sl osserva un uso di figure mitologiche, umane o fantastiche come sostegno di taiole ? altri mobili I cenlauri e le stingl si inseriscono facilmente in questa abitudine ${ }^{34} 3$

I centauri fungono irequentemente da dcroteri Quasl sempre rappresentano il tipo delle statue da fontana $G_{1 a}$ l tempio di Asclepio a Epidauro era abbellito con centauri In origine sono figure che custodiscono l'edificio nel quale sono presentı, ma nell'Ellenismo ricevono un carattere puramente decorativo, mentie nello stesso momento entrano nel tiaso bdcchico

Cavallı marını, tritonı e altrı mostrı marını sono altrettanto frequentı come acroter Anche in questo caso le tigure possono essere derivate da modell scultores Glı acroterı del tempıo dı Poseidon ad Ermione in Grecia sono stati portatı a Roma nell'epoca repubblıcana L'acroterio centrale ora sı trova a Copenhaghen, quell lateralı, le 'Nereidl di Formia' si trovano nel Museo vazıosiale di Nipolı 34" I gruppl dı centaurı marını che hanno una deresde sul dorso $e$ un tanciullo sulle spalle nella vlla di Varano (cat 010/8) possono essere copie libere L'originale del gruppo $d_{1}$ un centauro che insegue un leone di colore bronzeo nella Casa di Sirico (cat 232) deve essere ellenistico

\section{ERME}

Volle pitture murali incontriamo delle erme in diverse posinoni e con diverse funtioni Ord cono figure elaborate, ord figure schemntiche composte da un lusto rettangolare o und lesta Nes paesaggi sacro-idلliacl sono schematiche e non hanno una funzione particoldre, ogn tanto rappresentano Priapo e sono ilconoscibli grazie a un tallo Talvolta raffigurano Dionso, Ecate e. nella Lasa del fruttoto (cat 161'2), Pan Lo crme inscrite nol sistemi architeltonic in generale non hanno tratti iconografici specilicı, mentre le tigure distinte per mezzo di oggettı entrano nell'atmosfera bacchica

Per quanto riguarda le erme scultoree manca uno studio recente Il libro di $R$ Lullies del 1931 rimane la monogralia principale, mentre un capitolo nel libro sulle sculture classiche e classicistiche dell'Agora atenese da parte di Evelyn Harrison aggiunge datı importantı Anche Wrede, nel suo libro sulla galleria ds erme a ritratto nella villa romana di welschbllig, fornisce delle osservazionı di gran valore 345

${ }^{341}$ Spinazzola 1953, I, fig 451.3 Cf in generale Demisch 1975

342 De Caro 1976, 198-219, tig 5-21

343 $C f$ pe Attis come sostegno (Tran Tam Tinh 1975) e il Sleno (Overbech/Mau 1884 Abb 233) e altrı tipl (Gnolı 1971, fig 48-50) Cf Harl-Schaller 1976 1977, Schmidt 1982, 120

344 Bieleteld 1969 
La forma piu antica e quella di un fusto con una testa Pruma questa composizıone strana veniva usata unicamente per le ummaginı di Jermes, al'inizio del quarto secolo ligure umane ricevevano la stessa forma Creazioni ellenistiche sono le erme con un torso tidumensionale, da distingueje fra Mantelhermen e Hofthermen 346

vella iera architettura le erme hanno sostituito le colonne e i pldastri Purtroppo non conosciamo esempi dellepoca arcalca e classica, durante la quale l'uso di tali figure non deve essere mancato vel capitolo sulle figure inserite nell'drchitettura sono stati menzionati alcunı complessi del periodo tardo ellenistico e romano con erme ( $p 2 /$ ) Si osserva che spesso le erme erano appoggiate contro una lesena o un pulastro che, in realta, costiturva l'elemento portante Dd menzionare sono fra l'altro, la facciata dell'Odeion sull'Agord atemene la 'Porta dei prigionieri' a Corinto $\downarrow$ portico del tempio dı Domıziano ad Líeso e 'las Incantadas', parte dı un portico a Salonicco, ora nel Louvre a Parigi ${ }^{34}$ t Nelle monografie sulle figure reggentı di Schmidt-Colinet e Schmidt le erme non vengono discusse ${ }^{34}$

Materıale dı confronto sı tiova anche nel mobll e neglı arredı, quals bracierı, lampadarı e tavole trovatı a Pompes e altrove 3 “9 Spuca la taiola marmorea nela Casa des quattro stll (cat 158'1), 1 cul pedi lises sono stat decorati con erme dipinte le bronzer snelle Hobfhermen d Erot 1 che escono da motivi vegetali, possono darci un'idea del modellı usati dal pittori per le architetture acree gidlle ad Ercoldno, caxatterizzate sopra come artigianato metallico ( $\mathrm{P}$ 000) Iiederike Harl-Schaller hd studiato alcuni esemplari del genere 350

$H^{\&}$ ifthermen e Mantelhermen si incontrano nel II stule in numero considerevole Beven accentuo 1 loro carattere pittorico (malerisch) e credette che non si trattasse di copie di vere erme nonostante ll parallelo fatto da lui stesso con le sette erme nel Vluseo Nazionale Romano 351 I pittori, secondo la sua opinione, avrebbero inserito le erme come elemento fantastico nelle architetture peraltro redistiche ( cf la discussione a $p$ (2/)

Mentre origindriamente erme a ritratto erano esposte soltanto negli edifici pubblici e nelle plazze come memorje di personaggl importanti, dal quarto sccolo in poi trovarano collocazione nel doininio prisato Jel Palazzo II ad Eretrid e stato liovato un ritratto del quarto secolo a $C^{352}$ Limitandoci alla Campanıa possiamo menzıonare gli esempları studiatı da A de Franciscis a

345 Lullies 1931, Harrison 1965, 108-176, Wrede 1972

346 Lullies 1931 Questı terminı tecnici si usano sempre nelle descrizionı per evitare espressionı meno chiare qualı 'erma a mant(ell)a' e 'erma a corpo/torso'

347 Schmidt-Colinet 1977, cat M75, von Hesberg 1983, non pubblicato (cf due erme di Cracle lungo la 'strada marmorea' ad Cfeso e un'erma nel museo dı Selçuh) Pedrızet 1938, Baldassarre 1977

340 Schmidt-Colmet 1977, Schmidt 1982

349 e Gnols 1971, fig 44-47

350 Harl-Schaller 19761977

351 Beyen 1960, 33-34 Per le erme Catkoma I 5, 156-167, 170-172, 175-177, 181 , cat $68,68,70,72,7476$

352 Gard 1974 Cf Harrison 1965, 124-129 
Pompei e a Torre Annunziata e alcune erme ad Ercolano. ${ }^{353}$ Le pitture parictali ci presentano alcuni esemplari. Purtroppo molte erme non possono piu essere interpretate con certezza, poiché sono diventate illeggibili. Soltanto nella Casa del panettiere (cat. 241) e in I 16. 3 (cat. 174) indubbiamente abbiamo a che fare con erme dipinte a ritratto. Ln Hermenzaun dipinto si incontra in tombe ad Alessandria (cat. 001), Nis (cat. 127) e Roma (nelle Catacombe di San Sebastiano, cat. 323).

Per la maggior parte delle erme non possiamo ricostruire il tipo della testa. Helbig credette di riconoscere un Uoriforo nell'erma menzionata nella Casa del panettiere (cat. 241), da confrontare con la famosa replica bronzea, firmata da Apollonios, della Villa dej papiri ad Ercolano. ${ }^{354}$ Teste di Dioniso o Hermes barbute potrebbero essere copie del limoso Hermes Agoraios di Alcamene. Harrison tuttavia è contraria a questa attribuzione troppo semplicistica: per le teste infatti esistevano un numero elevato di tipi piu di quanto conosciamo finora. ${ }^{35} 5$

Per le erme semplici conosciamo, come è stato detto, un numero considerevole di esempi nelle città campane. Gli esemplari di Mantelhermen invece sono rari: nelle Terme stabiane e nel tempio di Apollo sono stati trovati esemplari marnorei. ${ }^{35} \mathrm{c}$ L'unica $H^{\circ}$ iftherme trovata a Pompei, quella nella Casa di M. Lucretius, ricevette un giudizio aspro di Ovcrbeck: Jene Herme z.B. im genannten Hause, welche einen jungen Satyr darstellt, der eine Ziege, man begreift bei seiner Angewurzeltheit in den Boden nicht wie -, ihr Junges genommen hat, und an dessen Schaft nun die alte Ziege mit voller Nat"urlichkeit emporspringt, ist geradezu eine Geschmackslosigkeit, und das in diesem Falle ganz Unurganische des aus Akanthusblottern aufsteigenden Pfeilers tritt uns auffallend und peinigend entgegen. ${ }^{357}$

\section{ALTRE FIGURE MITOLOGICHE E UMANE}

La maggior parte delle figure rappresentano divinita e personaggi mitologici, in parte vagamente clichettate come menadi, baccanti (lemminili e maschili) o Frigi. In senso iconografico esse entrano nell'atmosfera dionisjaco onnipresente nelle decorazioni parietali. Queste figure non bene definite appaiono soprattutto nelle strutture architettoniche. Nel capitolo $v$ verra discussa la provenienza dei modelli dall'epoca classica. Le figure femminili coperte da un peplo, cariatidi o meno. copiano le korai classiche e trovano confronti contemporanei nelle Danaidi della Villa dej papiri e nelle cariatidi del foro di Augusto a Roma. Le aurai con le vesti agitate da un vento immaginario sembrano concepite nella bottega di uno scultore dello Stile Ricco quale Scopa o Timoteo.

1 Frigi nella Casa del bell'impluvio (cat. 159/2) sono senza confronti.

Per le statue di atleti dobbiamo soprattutto cercare fra le opere di Lisippo. Si pensi agli atleti seduti nella Casa del Menandro (cat. 163/5) e nella V'illa di S. Marco a Castellammare di Stabia (cat. 009/6), modellati secondo l'Eracle di

353 de Franciscis 1951; 1979; 1981; Cerulli Irelli 1982.

354 Comparettl/de Petra 1883, tav. VIII 3; Zanker 1974. 8-9, Taf. T.1, 31.2.

355 Harrison 1965, 129-134.

356 Eschebach 1979. Taf. 52b; Manderscheid 1981, 81 no. 27, Taf. 23. Overbech/Mau 1884, 191, Abb. 53; von Hesberg 1981, 1076 no. 14.

357 Overbeck/Mau 1884, 539. Per l'erma Dwyer 1982, 44, fig. 42a-b. 
Taranto, allapoxyomenos nella Casa del palestrita (cat 267) che copia l'apoxyomenos di Lisippo e cosl via.

Lo rappresentazion di donno amuantate seguono sempre tipl ellenistici

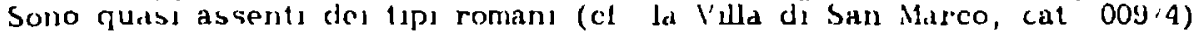

\section{CONCLUSIONI}

Rlassumendo si puo dire che ll repertorio di modell non e stato molto vasto, bensi privo di raricta Donne coperte da un peplo o un mantello copiano tipi classici o eliemstici, mentre gli atlet policletel o lisippes non rappresentano una ricca scala di possibilita Statue di tigure sedute copiano sempre modelli del quarto secolo, anche uaite come prototipl per le statuctte bronzee nel larari Soltanto raramente si puo fare un contronto esatto. Afrodite e Dioniso sono le unche disinita a essere state delmeate secondo tipi scultore ben precisl

'Tuttava la pittura paretalo cs presenta talvolta malcridle concernente tipi perdutı o notı solo in parte, inentre motle in evidenza le possibul varianti. Trovidmo del datı anche prr guanto riguarda gli attributi inancant nelle sculture, si ricordi ll grappolo d'ura dell'hermes di Prassitele ad Olimpia.

I pittori non hanno auto l'untenzione di copiare determinate statue, per quanto si possa concludere dal inateriale conservato 'Tutto cio non stumolo di consequenza uno studio accurato del modellı scultorel boltanto nel caso di alcune opere d'arte famose si puo constatare un'attenzione particolare

Inoltre le statue inserite nelle architetture dipinte sono sempre solo elementi decoratix e mas oggettı a se siantı L'attenzione prestata ad esse quindı non e stata mal molto grande 


\section{V: STILE E GUSTO}

La consultazione del catalogo permette di constatare che le pitture parietali contengono raramente riproduzioni di opere famose, di opera nobilia. ${ }^{358}$ Lo spettatore non riesce a trovare copie piú o meno fedeli del Discobolo di Mirone o dell'Atena di Fidia; non sono nemmeno presenti opere ellenistiche quali l'Apollo del Belvedere, il Laocoonte o i Galli. Solo in pochi casi le statue dipinte sono state immaginate proprio come copie di famosi oggetti d'arte.

La ragione di questa mancanza di opera nobilia va cercata nel carattere decorativo della pittura parietale in generale $e$ nella funzione delle statue all'interno di essa in particolare. La pittura parietale non $\dot{e}$ che decorazione, cioè un genere d'arte dipendente, un artigianato che attinge da un repertorio sviluppatosi nell'arco del tempo, ricco di sistemi e elementi decorativi, nel quale non esiste la necessità di eccellere per originalità o senso artistico.

L'elemento artistico quasi unico consiste nel genere dei pinakes, benché anch'essi siano copie o variazioni di quadri ellenistici, dei quali nulla ci è stato tramandato. Essi spiccano per la loro qualita tecnicamente piu alta, la loro posizione particolare entro i sistemi decorativi e poiché non hanno una relazione jconografica con le decorazioni attorno ad essi. ${ }^{359}$

Le statue inserite nei sistemi parietali corrispondono completamente al carattere decorativo delle pitture. Non saltano agli occhi mediante un'articolazione cospicua, mentre appaiono, nella loro sintassi di cariatidi e atlanti o di figure entro edicole e nicchie, come elementi ravvivanti della struttura architettonica.

Se una statua occupa una posizione determinante su una parete, siamo sicuramente di fronte all'imitazione di un'opera d'arte; verosimilmente il pittore ha voluto imitare, su richiesta del committente, una determinata opera. Alcuni esempi chiariscono queste osservazionj

Nel vano c della Casa della regina Margherita (cat. 190) la parete di fondo era occupata da una decorazione a campi semplici di IV stile, ora perduta. Nel pannello centrale una nicchia, dipinta a strisce grossolane in rosso, e occupata da un gruppo statuario (sopra una base rettangolare) ralfigurante Leda e il cigno. E' una copia riflessa della famosa Leda di Timoteo conosciuta attraverso ben 28 copie marmoree. ${ }^{360}$ Dal momento che il resto della decorazione contiene solo rari elementi figurativi che possano distogliere lo sguardo, la statua attrae in modo particolare l'attenzione dello spettatore. Al posto del consueto pinax ora sorge, dentro una nicchia, un capolnvoro dipinto. La presenza della copia della famosa Leda è certamente il prodotto di una scelta volontaria. L'opinjone di Herrmann lascia perplessi: egli negò la dipendenza della figura dipinta dallopera di Timoteo in contrasto con Lede 'vive', riprodotte su altri pinakes, da lui messe in relazione con la statua originale (cf. P. 12 ; cat. $245 / 1,269 / 4$ ). Benché la raffigurazione non possegga molta plasticità e mostri alcune irregolarità rispetto all'originale, quesro non nuoce all'intenzione del decoratore per essere piuttosto atribuita al suo scarso talento ed alle modeste pretese artistiche.

$E^{\prime}$ simile la situazione in 113,16 (cat. 173) dove nel triclinio si vede sulla parete nord una modesta decorazione architettonica di IV stile su uno sfondo bianco. Nel campo centrale e delineato un rozzo telaio per suggerire un'edicola

358 Sul termine ef. Preisshofen 1979; Zanker $1979 b$.

$359 \mathrm{Cf}$. lo stato economico migliore dei pictores imaginarii:I. Calabini Limentani, EAA VI (1965) 156-159, s.v. pictor; Blanc $1983,320$.

360 Rieche 1978. 
o una micchia All'uterno vi sono tre statue su piedistallı La statua maggiore rappresenta Afrodite che solleva 1 capell con la sinıstra, mentre nella destra regge uno specchio nel quale si oscerva Accanto stanno un pavone e un Priapo itıfallico L'Afrodite e una variazione della Anadyomene e rientrd nella seric di statue del genere, trovate a Pompei, qualı ld venere in bihini e l'Afrodite che si slaccia il sandalo 361 lonostante la scarsi qualita artistica della decorazione $e$ ben evidente che il decoratore ha inteso una micchia riempita di statuette

Atrodite che si slaccia 4 sandalo e un tema dipinto in I 13, 2-5 (cat 172) in und pittura a campi di II stule La statuetta si delined daranti a un campo veıde senza indicazione di un'edicola, ma spicca proprio per ld semplicita dell'insieme

I'Hermes di Olumpia e stato recuperato in due esemplari dipinti a Pompel Nolla lasa del naviglio (cat 207/2) occupava l'edicola centrale nella zona superiore di una decoldzione architettonicd di IV stile su uno sfondo bianco, priva di ligure von e dissimule la disposizione originale dell'Hermes nella Casa di Gidsone (cat 288), staccato e ora custodito nell'Antıquarium di Pompel In entrambi 1 cası la ligura dell'Hermes a stata trastormata in un satiro di colore marrone con capell 1 ricci e orecchie a punta Regge un grappolo d'uva nella mano destra alzata Ora dato che la figura occupa una posilione secondaria nella zona superiore e lecito domandarsi se vi losse l'inten opus nobile II v uoto sul resto della parete potrebbe esserc un argomento a favore e inoltre la zona superiore potrebbe essere cosiderata come un secondo, altrettanto importante registro C10 duminuirebbe la subordinazione accrescendo al contrario l'umportanza della statua $E^{\prime}$ strano che a parte le pitture pompeiane non siano atate trovate copie $d_{1}$ questa statua, considerata da molti come una creazione originale di Prassitele 363

L'Afrodite e ll Dioniso nella Casa del principe di lapolı (cat 219) sono modellatı secondo sculture conoscıute, cıoe l'Anadyomene e 1 l Dioniso dı Tivolı, ma sono raffigurate ive lella maggior parte del cası prevalgono modellı del periodo tardoclassico e ellenistico per le immaginı di Ifrodite e Dioniso La Casa del pi incipe di Vapolı e uno degli innumerevolı esempi

Abbiamo osservato nell'introduzione che la discrepanza fra 1 restı superstitı e quanto wene descritto nelle fonti letterarie e notevole ( $P$ 10) Inoltre c1 devono essere state molte statue non menzionate dagli autor antich 1 L'idea che cl siamo creatı deglı opera noblla e stata fuorviata da questı testı, soprattutto dalla storja dell'arte plinsano e dalla teorıa artistica del Settecento e dellotlocento Certamente molti lavori di alto livello artistico erano sconosciuti o non stumati da Plmio - si ricordi l'assenza dell'arte arcaica e ellenistica, campi interamente neglett ${ }^{363}$ Il periegeta Pausanıa expressis

361 De Caro 1976, 219225 , fig 30-34, Jashemskı 1979. fig 197-200, 468, Dwyer 1982, fig $79,89,190$

362 In questa sede non si puo entrare nel dibattıto sul'originalita della statua dı Olumpıa Jecisamente positivo fu Rizzo 1932, 6673 , tav 99-104 (ivi anche riproduzioni in altru medi 102, tav 106), mentre Blumel 1948 espresse un'opinione diametralmente opposta Antonsson 1937 credette che si trattasse di un originale greco, rappresentante Pan con Dioniso, rielabordto come Hernies nell'epoca Iomana Cook 1977 presenta la testa di Aberdeen come originale, la statua olimpica sarebbe una copia romana (cf Rizzo 1932, 74-75 tav 111) Cf G Becatt1, EAA VI (1965) 430, s.v. Prassitele, Lulles Hirmer 1979, 116-117

Figure simul nella Casa degl amorini doratı (cat 2201 ), nella Villa umperiale (cat 3053 ), nella Casa di Sallustio (cat 198b 2) e sul MN 9878 (cat 0j3) mostrano variantı ellenisticı 
verbis dice che non sara (o non potra esserc) esaurionte nel catalogare le opere d'arte nelle citta wsitate

In altro elemento che disturba la wione consiste nell'incerta collocazsone originaria delle statue, ora custodite nei muse Cosa fosse esposto nelle case prilate e quale tosse il livello artistico, possiamo solo ipotizzarlo Il gusto corrente sembra fosce determinato dalla clisse dirigente che dal secondo secolo a C entro in contatto con opere d'arte greche, ne asporto un numero considerevole senza coscienza critica \& pour épater le(s autres) bourgeois. le espose nelle case 364 il pubblico ne ebbe conoscenza soltanto per mezzo del trionfi nel qualı trovarono posto le opere d'arte, ${ }^{365}$ le vide esposte su pidzze e in edifici pubblici e sacralı Grazie a talı stimoli la gente comincio ad acquistare cople 0 , se 1 mezzi finanziar erano insufficientı adattamentı plu modest in forma di bronzett o terrecotte Gli opera nobilia rumanevano ilscriati alle case a ville des ceti nobll 366

Lo studio di Magrit Pape sul bottino dei condottieri nella Repubblica romana ha messo in luce che nei primi due secol la maggior parte delle opere predate venne collocata nei templi come offerta lotiva (come le lamose lluse du Tulsco, esposte nel tempio di 4pollo Sosiano) o rumasero nelle collezions private degli stessi generalı o del loro amici (ad esempio gls enormı tesorl di Verre) 367 Ispirati dall'arredamento esclusivo di quel palazzi l benestanti a Roma fecro importare le opere originali - qualora tosse stata possibile - del mondo greco, o le fecero riprodurre sia nel luogo d'origine che a Roma 360

$E^{\prime}$ solo alla tine del primo secolo a $C$ che le opere d'arte vennero esposte in pubblico come mezzo propagandistico, si pensi alle idee di Agrippa in merito egh insisteva per una proprieta prisata modesta a larore dell'ampliamento e dell'abbellumento del monumentı pubblicl ${ }^{369}$ Se Roma prima exa und citta sobria. da quel momento divenne una selva di statue ammirabili da tuttı e paragonabilı d gran numero di statue pubblsche o votive presentı in santudri qualı Delfi e Olimpia Torse questa tarda possibulita di conoscrere le opere d'arte greche fu la ragione del notevole salto che subi la produzione di cople durante 1 prim due secolı dell'Impero Allora la borghesia comincio ad imitare ll decoro delle vlle del ricchı che gia da tempo godevano al loro interno della presenza dolle opere d'arte greca cosı stuntate 37

Il ritardo che su constata anche in Campania e per lo piu doruto al fattors descrittı Sarebbe tıoppo banale attribuire alla distanza da Roma la causa del ritardo rispetto alle mode in voga nella capitale la comunicazione era veloce e efficiente e I propistar delle vlle piu tastose certamente ebbero un influsso

\footnotetext{
363 Cf Plin , N.H. XXYIV 19 (Zanker 1979b, 302-304)

364 Cf la tradizione del testı letterarı (Lesky 1973, 15-20)

365 Cf anche la pompa per 1 theol soteres allestita da Ptolemeo II Fuladelfo e Berenice nel 279 o 270 , descritta da Callissino (Ateneo V 196a, of Caspar1 1916, Nilsson 1961, 159).

365 Cf Jucher 1950, Becattı 1951

367 Pape 1975, 206-208

368 La nave di Mahdıa e le statue recuperate nel Pıreo ne sono chrarı esempı Forse clo vale altrettanto per i bronzı di Riace
}

369 Pape 1975, 76-80 Cf anche la polıtica dı Augusto (Suet, Aug 28)

370 Zanker 1974; 1979a, 1979b 
determinante ${ }^{371}$ Scarsd e la nostra conoscenza circa 1 ruolo che svolgeva Napolı nella Repubblica e nel primo Impero Pozzuol, citta la cui umportanza crobbe nell'epoca flava, e l'esempso dell'umportanza di un tale trait d'union ${ }^{372}$ Per quanto riguarda la tradizione di motivi decoratıvi nella pittura parıetale, pergamene o papirı llustratı non possono essere scartatı come mezzo dı comunicazione Finche non si trovano degli esemplari, la loro esistenza non e sicura e puo soltanto essere considerata probabile 373

Di grande rlieso fu ll cambianento della struttura socule a Pomper dopo il terremoto del 62, quando 1 ricelu il ritirarono, molte ville vennero abbandonate e numerose cahe signorih nella citta rennero suddivise in appartaments piu piccolı. ${ }^{3 / 4}$ Il ceto medio occupo 1 post i unportant 1 , finora non aperti a tale classe ${ }^{375}$ In questo processo l'umitazione culturale delle classi bencutinti is und loged conseguental 376

Ld pitlura parjetale conterma l'ummagme ora tracciata Visto l suo carattere decorativo essa non accoglie l'inserimento di nuovi elementı. diventatı di moda. Sı ricordino I motıvi egill e egittizzantı che gia nel II stule, ma soprattutto dopo la conquista dell'egemonia da parte di Ottaviano prendono voga e sono paragonabil alle cineserie del Settecento 377 Gli innumerevoli paesaggi, rappresentazionı di vlle, paradeisol e prospettı di glardini rispecchiano ll bisogno di una casa in campagna, di preterenza una villa, nello stesso modo le ımitazionı di materiali e ornamentı costosi e rars (specialmente nel II stule) esprimono la voglid di crcare su scala modesta un palazzo ellenistico, fenomeno analizzato in maniera limpida da Fittschen 37. I pittori non tendevano subito all'imitazione di lavori tamosi. la totalita della decordzione avrebbe clovuto creare un determinato etfetto e I dettagh erano subordinati a quello scopo. Dobbiamo tener presente che il decordlore godeva di tanta liberta quanta lo scultore nel rumaneggiare un modello o nel farne una variazione sccondo il suo gludizio In base a tutto questo e possibule che vi slano plu 'copie' (nel senso piu Jargo dela pirola) di quanto linora sia stato constatato Invero non stamo capaci de distinguere fra cople cotte e libere per inancanza delle originali, ma nondimeno pare accertato uno siduppo del genere Tuttora solo in poche occasioni le statue dipinte corrispondono a statue o copie contemporanee Esempi ne sono I guerrierı polıcletel che riflettono le numerose copıe del primı due secoll del Principato, l'Hermes nella Casa della Caccia antica (cat 245 2), copia dell'Hermes $d_{1}$ Zenodoro latto nell'epoca

371 Cf D'Arms 1970, 1977; 1979; Allroggen-Bedel 1982, Moormann 1984b, $331-334$

372 D'Arms 1977; Sommella 1980, spec 77-84, e diversı saggi nella rivista Puteoli. Cf anche Von Hees-Landwohr 1982.

373 Cf. Mielsch 1981, 189-194, 252-254, Schefold 1982, 2 (positivo).

374 Dohl/Zanker 1979, 178, Allroggen-Bedel 1982, 526-528

375 Castren 1975.

376 Zanker 1979a Cf Gigante 1979, 3.3-44

37 De Vos 1981, spec 75-95, C1v ilta 1983, 53-78 (articolı di S. De Caro, M. de Vos e V Sanpaolo) La termmologia Egittomania contiene un'intenzione negativa rispetto a questo fenomeno, non spiegata e peraltro ingiusta Nel caso si voglia introdurre un nuovo termine, Egittofilia sarebbe meno discutibile, in ogni caso parola non adatta.

378 Fittschen 1976 
claudı-neroniana e ll cervo nella Casa del marineso (cat 259) nel quale sı ritrova la moda di intaglı di pietre multicolori in marmo (cf p. 108 ).

Gls elementi presentati e le tacolta limitate delld pittura, anch'esse gia menzionate nell'introduzione ( $p$ \&7), non faclitano l'illustrazione della produzione di copie attraverso le statue dipinte, perche contribuiscano alla conoscenza di tale produzione e del tipl (cosiddetta Kopienkritık ${ }^{39}$ ) Tuttavia l'mportanza delle statue dipinte non va sottovalutata, esse derono essere sempre considerate nella ricerca sulla scultura romana, specie quando sl tratta di nuovi tipl trovatı durante deglı scavı o di pezzl finora non pubblicati e nascosti nei depossti musedi Infattı lo siluppo nella scultura del gusto per determinati stali e per jodi puo essere riletto at1inerso un paragone con le statue appartenenti alla pittura par ietale I tre itll pomperani mostrano ognuno una chiara preferenza per un proprio modo di modellare le statue - in questo contesto sl potrebbe intattı parlare di 'stıl' che sono paralleli al cambio del gusto nel primo secolo a $C$ e nel successivo Le statue nelle scene tigurative si sottraggono a questo sviluppo $e$ di questo parleremo in un paragralo alla line dell copilolo.

\section{STILE}

Le statue dipmte nel II stile dimostrano una fortc plasticita e cono carattelizzate come statue in tutti l casi attraverso l'esposizione su basi $\varepsilon$ o entro micchie o l'applicazione come atlanti o acroter in contrasto con l'intenzione del II stule di raffigurare architetture ancora costruibili, le statue non corrispondono ad esempi a nos not 1 ma la loro posizione non e tale da tarei pensare che tossero solo fantastiche (ct $p 2$ ) Sembra che il desiderio di fingere la presenza di statue dentro nicchie o davanti a und parete di ortostatı fosse abbastanza modesta quası come se fossero mancate nel materiale ellenistico disponibule al pittori del periodo Le tigure nel cubicolo 4 della $V$ llla del misteri (cat 306,2) imitano una collezione di sculture, riconoscibli non solo dtraverso la monocromid bianca, ma anche dalla collocazione sopra piedistallı glallı Le tigure nelle micchie a tianco del prospettı centralı nella Casa degli epigramm (cat $18 ;$ 1) e nella Casa del criptoportico (cat 148,3-4) sono benz'altro statue isti 1 piedistall. ma attraverso la policromid realistica danno l'impressione di personnaggi WII C10 vale altrettanto per l'Apollo o l'Artemide dell'insula occidentalis (cat 225/1) L'effetto realistico cercato dagh scultor ellenistics era raggiunto piu facilmente attraverso la pittura

La maggior parte delle figure presenta una ritmica forte e ha unimpronta barocca Benche sia possibile in pochi casi trovare controntı, si pensa che 1 pittori si siano fattı ispirare soprattutto da opeie ellenistiche lorse specialmente l'arte pergamena e rodia e creazioni contemporanee servirano da fonte d'ispirazione, se pur per vid indiretta 310

Questa conclusione non desta meraviglia dal momento che - come e stato dimostrato ancora do recente da Fittschen ${ }^{31}$ - innumerevoli elementi del II stile sono statı derivatı dall'Ellenismo Confutando Engemann, Fittschen presume l'origine dello stue ad Alessandria, dove I palazzi realı presentarano decorazionı e temı appartenentı al repertorio del II stile La roglia di ambientarsi entro un'atmostera sontuosa quale quella della corte ptolemaica

39 Studio di base rimine Lippold 1923 Cf Zanher 1971 introduzione

380 Per l'influsso dı Rodı of Gualandı 1976, 15-17

381 Fittschen 1976 
trova echi in forma dipinta nelle case di coloro che non erano in grado di imitare tutto 1 materiale alla medesima scala Non vi e dubbio che le statue avevano una grande importanza nell'arıedamento palazıale, ma cıo pare non rispecchiarsı nelle pitture parietalı

Le decolazioni murali di ll stle non contengono mal statue areatiche e neinneno irequentemente pezzi del'epoca classica L'assenza dssoluta di esempi arcaici sı spiega nel carattere decorativo della pittura l'arte arcaica ieniva considerata severa, seratica adatta per le immaginj delle disinita 32 Nell arte ellenistica in generale non trousamo rimandi ad essa, mentre si rimane sempre collegati con l'arte classica ${ }^{38} 3$ L'arte neoattica e L classicismo del primo secolo a $C$ sono quasi assentı nel linguaggıo del II stule Solo alla fine del periodo, per esemplo nella Casa di Livid e nella Casa di tugusto sul Palatino e nella Vula della Farnesina, si incontrano innovazioni d'umpionta classicheggiante, ma questı complessi gia precorrono il III stule (cat 316317 , 319)

\section{STILE}

Indubbiamente ll III stule e stato determinato dal classicismo entra nel nuovo mondo mentale stimolato, se non iniziato da Augusto $30^{4}$ Riprende il quinto secolo periodo fiorente ad Atene che gld dal Romani ieniva considerato il periodo (lassico' 385 Soprattutto Zanker ha prestato la sua attenzione a questo fenomeno e ne ha potuto charire rail aspetti complessi ${ }^{36}$ Il toro di Augusto esprime per la prima volta questo nuovo linguaggio tormale, insieme a un programma conogratıo sotisticato di contenuto romano, ma realizzato in forme greche, classiche 387 Le cople di statue ideal del primo Impero riproducono quası tutte statue del quinto secolo con una netta preferenza per Policleto e 1 suor seguaci 38 a

Le sculture inserite nelle pitture parietah mostrano un carattere classicistica si inseiscono nello suluppo indicato Le cariatidi nelle zone superiori (cf p $29-34$ ) sono quasi eclusildmente classiche e contrastano con le ligure barocche del II stule Anche se come nel periodo precedente non e possibile applicare il metodo dolla Kopienkritik alle statue dipinte e giungere a una datazione degli csempl seguiti, a chidro che lo Stile Severo e lo stile Ricco provalgono Le I Jandids delld Vila des papirı, nuove creazioni in Stle Severo delld tarda Repubblica, sono presents in numerose decorazion in funzione di cariatidi ${ }^{36}$ La Villd imperide (cat 3053 ), la Casa di M

\footnotetext{
302 Cf Preisshofen 1979

383 Per l'influsso di Lısıppo, per esempıo, sı vedono Moreno 1978, 1981, 1982

384 Cf Zanker 1972, 1974, 1979b, 1983, s d

325 Bowersoch 1979, Pieisshofen 1979

386 Zanher 1974, 1979b, 1983, s d, Zanher/Preisshofen 1971, Dohl 7anker 1979

307 Zanker s d Cr Zanker 1965

3: Zanker 1974, 1-45

399 Sgobbo 1971 riassume la bibliografia anterıore e stabilisce in modo convincente ll significato Diversamente pensa Pandermalis 1971, 181-18?,
} 
Lucretius Fronto (cat. 195/1) e la Casa del frutteto (cat. 161/2), per menzionare soltanto alcuni esempi, hanno nelle scaenae frontes della zona superiore figure fenminili simili, indossanti un peplo o un chitone, vestiti classjci e tradizionali e non piu in uso. vella discussione di queste figure nel primo capitolo ( $p .29-32$ ) e stato fatto il confronto con le cariatidi del foro di Augusto, imitazioni precise delle Korai dell'Eretteo atenese: la loro influenza è stata tale da coinvolgere non solo le decorazioni parietali, ma anche lo stile e la forma, benché questi aspetti non siano sempre ben distinguibili nel materiale conservato. Anche in questo caso peró dobbiamo tener conto della presenza di esempi a noi sconosciuti. ${ }^{390}$

Non si incontrano repliche dipinte di opera nobilia copiate nel senso proprio della parola: in tutti $\mathrm{j}$ casi vediano variazioni o imitazioni libere che riprendono piuttosto lo stile che il modello. Cio non vale soltanto per le cariatidi, ma ugualmente per gli acroteri a forma di Aurai e Nikai: un archetipo qualunque dello Stile Ricco è servito da modello senza che un lavoro determinato sia riconoscibile. La caratterizzazione non va oltre termini generali come 'la maniera di Timoteo' o 'lo stile di Scopa'. Il fat to che gli acroteri dipinti certamente copino veri elementi architettonici, può essere ricercato nelle importazioni di queste figure che venivano asportate da edifici greci come bottino $o$, in seconda istanza, di copie e varianti. Le famose Nereidi di Formia, per esempio, hanno fornito il modello a una statua romana trovata in una villa a Posillipo, l'antica Pausilypon presso Napoli. ${ }^{39}$

Di nuovo constatiamo l'assenza assoluta di statue di stile arcaico a parte le rappresentazioni figurative. Nella scultura, invece, si incontrano creazioni nuove di questo stile. Siano ricordati l'Atena e la testa di un kouros nella Villa dei papiri, l'Apollo nella Casa del Menandro, l'Artemide nella Casa di M. Ilolconius Rul'us, la testa di VII 3,40 e l'Iside nel tempio di Iside. ${ }^{392}$ I soggetti sono quasi sempre religiosi: le divinita sono ralfigurate come esseri arcaici e venerabili." ${ }^{31}$ Consequentemente si stabilisce una discrepanza fra questi aspetti e il carattere decorativo della pittura parietale che impedisce un inserimento di tali statue nei sistemi decorativi.

II linguaggio barocco dell'Ellenismo non sparisce complelamente, ma continua su scala minore. E' evidente che statue esposte entro nicchie e su cornici non di rado sono state modellate in questa maniera. Ne e chiara testimonianza il portico della Villa imperiale (cat.305/3): l'Apollo con la cetra, il Dioniso con il tirso e il satiro con il Dioniso fanciullo sulla spalla sono stati modellati in stile ellenistico, anche se $\mathrm{i}$ primi due potrebbero avere archetipi tardoclassici del quarto secolo.

Taf. 85.1-2, 86-88, 89.3 (in base alla collocazione sbagliata).

390 Anche la Basilica Emilia presumibilmente possedeva ligure del genere. come sono anche presenti in edifici piú recenti (Bauer 1983)

391 Bielefeld 1969, spec. 62.

392 Comparetti/De Petra 1881, 273 no. 66, tav. XX 1: Pandermalis 1971, 198; Maiuri 1933, 407-419, fig. 153-158; Overbeck/Mau 1884, 543-544, fig. 281 , CatLondon 1976, no. 94, Fuchs 1979, 241-242, fig. 261-262, Döhl Zanker 1979, 205 (da VII 6, 3 secondo Jashemski 1979, fig. 210; Uwyer 1982, fig. 208); CatLondon 1976. no. 96; Tran Tam Tinh 1964, no. 81, pl. XIII, DöhliZanker 1979, fig. 24.

39 Maiuri 1933, 418-419. L'esposizione dell'Apollo nel portico meridionale del peristilio della Casa del Menandro deve essere stata provvisoria (ibid. 407-408, fig. 153). 
In contrasto al IV stile le figure si presentano piu chiaramente come statue, mentre piedistalli e colori non sono elementi altrettanto significativi. Questa impressione si ha non soltanto attaverso il grado di 'classicita', ma anche mediante l'atteggiamento, la simmetria sottolineata dalla posizione delle gambe e delle braccia. e la raffigurazione delle vesti. La mancanza delle basi corrisponde all'assenza di una forte tridimensionalità e di una rappresentazione fedele degli elementi architettonici.

Le rapprentazioni di giardini contengono unicamente figure d'impronta ellenistica: pinakes, oscilla e statue sono da associare raramente ad esempi noti nella scultura, ma innegabilmente sono riconoscibili per quanto riguarda lo stile e la modellazione. Cio non contrasta la scultura topiaria, ma questa conclusione va usata con prudenza dato che $\mathbf{j}$ giardini scavati nell'area vesuviana presentano un arredo degli ultimi anni prina del 79 cosicché possono essere confrontiti solo con grande riserva. Il grande peristilio della Villa dei papiri probabjlmente è un esempio di un giardino dell'inizio del primo secolo d.C. ${ }^{394}$ Le statue ivi esposte furono scelte per motivi iconografici, raggruppate in una determinata maniera, nella quale persino lo stile dei pezzi - arcaico, severo o classico - potesse essere un fattore significativo per l'iconografia, cioe un rimando alla storia che servisse da specchio moralizzante. ${ }^{395} E^{\prime}$ l'unico complesso che dia un'eco indubbia dei palazzi ellenistici, i cui repertori si riflettono altrove in Campania su scala modesta. ${ }^{396}$ La Villa dej papiri quindi supera di gran lunga i giardini pompeiani e i giardini dipinti.

\section{STILE}

Nel IV stile non si puó rilevare una predilezione ben precisa per una corrente artistica. Ció corrisponde al carntterc eslettico del IV stile. Cala l'interesse per il periodo classico purche si tratti di copie di opere d'arte famose, come è stato detto sopra.

L'unico escmpio di una statua arcaica era l'Apollo, ora purtroppo completamente perduto, nella Casa del centauro (cat. 203:1). Per quanto si possa vedere nell'unico disegno conosciuto, il dio è raffigurato come un kouros puramente arcaico della seconda metà del sesto secolo con le braccia lungo i fianchi e la gamba destra davanti alla sinistra. Il contesto, pinax o vignetta, non e ricostruibile. Maiuri prudentemente propose l'ipotesi che esso, come nel caso della statua arcaistica della Casa del Menandro, simboleggiasse l'inunagine di culto del tempio di Apollo sul foro. Ora non é chiaro se egli pensasse a una copia fedele della statua; se cosi fosse, bisogna opporsi vista la notevole differenza di stile fra la statua marmorea e la pittura. Gia il confronto con la statua e piuttosto azzardato, poiché essa è una creazione nuova. Confronti piu o meno ledeli sono pressoche assenti in Campania. ${ }^{39}$

394 Pandermalis 1971, 188-196 (la conclusione si trova a p. 196); Sgobbo 1972, 283; Sauron 1980, 281. I lavori di ristrutturazione nella villa forniscono almeno un terminus ante quem (forse il 62): cf. Sgobbo 1971, 61 con bibl.

395 Pandermalis 1971, 187; contra Sauron 1980, 282-285.

396 Zanker 1979a; Dölnl:Zanker 1979.

397 Si pensi alla testa arcaica della Villa dei papiri (Comparetti/De Petra 1883, tav. VII 1), alle erme arcaizzanti nella Casa di M. lucretius (Dwyer 1982, [ig. $3(i-41,54)$ ( le opere menzionate sopra $p .93$ notin 392. 
Sopra sono stati oggetto di discussione 1 guerrieri nudi: rappresentazioni di Achille, Ares o dei Sette a Tebe, ora presenti in scrie, ora in posizione isolata ( $p$. 43-44). Essi confermano lo sviluppo della produzione di copie stabilito da Zanker, anche se le copie dipinte sono di alcuni decenni piú recenti. ${ }^{39}$ All'inizio del Principato la produzione si sriluppa su scala quasi industriale anche per soddisfare la forte domanda. I gessi trovati in una cantina delle cosiddette Terme della Sosandra a l3aia nel 1954, sono un'indicazione unica di una bottega di riproduzione e presentano un canpione del repertorio prediletto. ${ }^{39}$ La scelta addiritura di guerrieri policletei sarebbe secondo Zanker eine Ausdruck einer allgemeinen Sehnsucht nach Erneuerung. die Augustus mit der Programmatik des saeculum aurewn politisch fruchtbar $z u$ machen versuchte. ${ }^{400}$ In un complesso termale come le T'erme suburbane ad Ercolano (cat. 024) le figure - qui eseguite in rilievo a stucco - si inseriscono indubbiamente nel contesto e rispecchiano la funzione delledifjcio; nell'ambiente privato questo tipo rappresenta l'interesse per l'ideale estetico espresso nel Canone di Policleto (ci, p. 43). Le ligure di Ercolano fecero pensare C.C. Vermeule ad imitazioni di rilievi neoattici." 02 Questi pero contengono solo di rado figure policletee in atteggiamento rilassato. I confronti - esatti o meno - vanno piuttosto cercati nella scultura contemporanea. Esse clecorano ambienti speciali, forse etichettati con i nomi di Ares o Achille, cosi corne esistevano anche Hermaea e Apollinea. J'er quanto riguarda la Campania varie opere possono essere menzionate, tra lepiu importanti si ricordi il Doriforo esposto nell'antica palestra presso il tempio di Iside. ${ }^{402}$ Nell'ambito privato vi sono fra l'altro le erme e le Danaidi della Villa dei papiri l'Apollino della Casa del citarista, l'Apollo di VI 2, 15 e l'Hermes di V' 3, 10.403

Proprio nel IV stile risulta evidente la preferenza per la simmetria. Ancora piú della scultura la pittura tende a produrre pendants o figure speculari aí modelli originali. Le ninfe acquatiche nelle rappresentazioni di giardini appaiono sempre in coppia e sono sempre simmetriche. Gli acroteri senza eccezione sono sistemati allo stesso modo. Nicchie dipinte si trovano sulle due pareti di fronte e le figure dentro di esse forse non sono identiche o simmetriche, ma rispettano un concetto simile, per esempio una figura seduta di fronte a un'altra figura seduta, un uomo nudo dirimpetto a un altro uomo nudo. Se manca la decorazione di una parete, essa puo essere ricostruita in base a quella dell'altra.

La scultura topiaria non copre un repertorio molto vasto. Tutti i tipi derivano da archetipi del quarto sccolo o dell'Ellenismo. Le Afroditi di Prassitele sono state popolari sia in forma tridimensionale che in pittura, viste le numerose repliche e variazioni. Lo stesso vale per l'Anadyomene di Apelles.

La nostra conoscenza dell'arredamento delle case pompeiane e scarsa nonostante $i$ ritrovamenti in situ, dal momento che spesso non siamo bene informati sulla posizione originale attraverso i giornali di scavo. Il libro di E.J. Dwyer, nel quale tutti i mobilia di cinque case pompeiane sono stati

39. Zanker 1974, 1-45.

399 Von Hees-Iandwehr 1982.

400 Zanker 1974, 43.

401 Vermeule $1977,10-11$

402 Zanker 1974, 7-8, Taf. 5.1, 7.3 con bibl.; Fuchs 1979, 86, Abb. 79; Lullies/Hirmer 1979, 82-83, Taf. 132-133.

403 Comparetti/De Petra 1883, tav. VII 3-4, VIII 1.3, XIV, XIX 2; Iwyer 1982 , fig. 129, 207, 209. 
oggetto di studio, fornisce alcunı datı in mersto "o" Glı abitantı preferivano sculture decorative: possederano raramente opera nobilia, naturalmente prima di tutto a causa della loro preziosita All'interno delle case si collocavano, per quanto sı puo ricostiuire, i ritrattı di antenati e le statuette delle divinita nei lararı 405 Sul cartibula spesso riccamente scolpiti stavano esposti oggett d'arte e vasellame sontuoso La maggior parte delle statuc trovava un posto nel glardino, preferibilmente raggruppate presso una fontana che, a sua $\checkmark$ olta imitava un ninfeo 406 Mentre Dwyer ferma $L$ suo stimolante studio dopo una descrizione accurata dei ritrovamenti e una nota conclusiva, Zanker presenta nel suo saggio Die Villa als Vorbild des pompejanischen Wohngeschmacks ulteriori datı 407 Discute l'origine degli elementı e cerca di interpretarli sisteminticamente Anch'egli constata che nella scelta dell'arredamento privato preiale il valore docorativo, forse anche funzionale degh oggetti, non un amore per 1 valor artisticl L'arredo nel suo insieme rispechid quello delle vile repubblicane Sia le case modeste che quelle grandı con caratterıstiche dı ville (tra l'altro la Casa di Octavius Quartio con il suo euripo che occupa quası un'intera insula) mostrano gli stessi elementi e questo nemmeno con grandı differenze di qualita $S_{i}$ confrontı l'inventario de lla vasta Casa del citarista con quello della piccola Casa del camillo 408 In entrambe le case siatue bronzec costose stavano accanto a statue di marmo e oggetlı fittilı, solo la quantitd di materiale nella Casa del citarista supera notevolmente quella dell'altro complesso Le decorazioni parietalı e musive nelle case studiate da Duyer (del resto non discusse nel libro) confermano 1 tatt che emergono dal materiale moble $E^{\prime}$ scorretto che nelle case ricche prevalgono decorazion ricche (cioe con sistemı complicatı, di alta qualita tecnica e $\cos 1$ via) mentre in quelle piccole vi sarebbero solo pitture nello stule del Nebenzimmer 409 I na casa di piccole dimensioni quale quella di Pınarıus Cerealıs, con le sue scdenae trontes complicatissime, non e inferiore a complessi maggiorı, decoratı nel medesumo periodo come la Casa di Meleagro - la Casa del Dioscurı

In sintesi si puo dire che queste osservazioni sono anche valide per le stritue inserite nei sistemi decoratih. Le sculture si incontrano in pitture $\mathrm{d}_{1}$ varia qualita e in tutti l tip 1 di case $C_{h}$ poteva permettersi vere sculture, non ometteva l'applicazione di statue dipinte, facendo esse parte fissa del repertorio Vondimeno rispecchiano lo sviluppo del gustı e la produzione di copie nel periodo contemporaneo

\footnotetext{
404 Dwyer 1982

405 Dwver 1982, 13-116, 120, 121

406 Dwyer 1982, 116-118, 123-126

407 Zanker 1979d Cf E M Moormann, BABesch 59 (1984) 216-217 (rec Dwyer 1982), Dohl Zanker 1979, Dohl 1982

400 Dwyer 1982, 79-108, fig 119-181, 57-67, fig 67-84

409 Il termine proviene da Strocha 1975
} 


\section{RAPPRESENTAZIONI FIGURATIVE}

In generale le rappresentazioni figurative sono state inserite nella ricerca sullo sviluppo dei sistemi decorativi solo in modesto grado. Mau. Beven e Bastet non le considerevano pilevanti nelle loro classificazioni degli stidi pompeiani."10 Ottenevano invece un posto negli studi di G. Rodenwaldt, Schefold e Heide Lauter-Bufe." ${ }^{11} \mathrm{E}^{\prime}$ gencralmente stato accettato che si tratta nella maggior parte dei casi di copic di quadri e pitture parictali create nell'ambiente greco, soprattutto nel periodo ellenistico. Tentativi di attribuire pinakes con lo slesso tema, che ovviamente devono avere la medesima origine, ad artisti menzionati nelle fonti scritte, vennero fatti soprattutto nella prima metà del secolo ancora sotto l'influsso della filologja, allora dominatrice del'Altertwonswissenschaft, ma fallirono non di rado a causa della mancanza di dati archeologici o risultarono in un numero di ipotesi piú o meno plausibili, basale su osservazioni spesso di carattere speculativo e soggettivo."12 'lemi romani sono rapi: soggetti storici (in gencrale) sono eccezioni e nuovi argomenti mitologici non paiono inventati in grande numero. ${ }^{43}$

Il problema dell'origine vale ancora di piu per le rappresentazioni di paesaggi sacro-idilliaci. Vonostante gli studi di $M$. Kostowzew. Ch. Lawson, Peters e Susan Silberberg non e possibile stabilire quali clementi provengano dal mondo greco e quali siano stati inventati successivamente. "/4 Il passo sul pittore Studio o Ludio ha suscitato un dibattito con molti argomenti pro e contra che qui non $i$ il caso di ripetere. ${ }^{45}$ Rispetto ai ridievi il dibattito per il quale si veda il capitolo sulle imitazioni di rilievi (p.s/-s6) - non finisce positivamente a favore dell'origine greca a scapito di quella romana o vice versa. Solo puo essere stabilito che nell'arte romana l'interesse per il paesaggio è maggiore di prima.

Una conoscenza maggiore sullorigine dei pinakes sarebbe utile per la domanda sulle fonti delle statue che vi appaiono dipinte. Le statue sono accessori, copiate simultaneamente all'insieme e per tale motivo il loro valore a proposito del tema discusso in questo capitolo non può essere sopravialutato.

4. Si vedano le considerazioni in Mau 1882, 448, 460: Bastet/De Vos 1979, 7 . Cf. Bragantini/Parise Badoni 1984.

412 Rodenwaldt 1909; Schefold 1952; 1962; 1972; Lauter-Bufe 1967.

412 Fra l'altro a parte i lavori citati nella nota precedente Fuhrmann 1931; Neutsch 1939; Lippold 1951. Moreno 1982, 416 menziona Apelles a proposito della 'Eracle e Telefo' della cosiddetta basilica ad Ercolano; Schefold 1982, 19-20.

423 Sulla base di criteri stilistici vengono ammessi maestri locali da Gabriel 1951; Beyen 1951; Richardson 1955; Kagghianti 1963; Lauter-Bufe 1967; cf. Bianchi Bandinelli 1977; Mielsch 1981, 189-193, 252-253. Non parlo di pitture repubblicane quali la famosa tomba di C. Fabius e la tomba Arieti (cf. Affreschi 1976, 13-28, tav. A, I-V; La Rocca 1984). Soggetti romani sono Romolo e Remo nella casa omonima (Helbig 1384, ora perduto) Sofonisba e Massinissa (cat. 269/3); Enea e Romolo (cat. 304). L'iconografia ovidiana di alcune rappresentazioni di Piramo $e$ Tisbe viene dimostrata da Baldassarre 1981.

414 Rostowzew 1911; Dawson 1944; Peters 1963; Silberberg 1981.

415 Ling 1977 e la reazione di Schefold 1982, 2. Cf. Mielsch 1981, 194-197, 254 . 
Si notano differenze nel delineamento dj figure e sfondi fra le copie di III e di IV stile: in questo paragone svolgono un ruolo anche il formato, la scelta dei colori e la posizione entro il sistema decorativo. I quadri di III stile in generale sono dipinti in una tecnica più sottile e con un equilibrio coloristico piu raffinato, in tutto conforme al carattere del III stile.

Statue di stile arcaico si incontrano quasi esclusivamente in rappresentazioni figurative. Le vesti sono delineate con pieghe precise e le forme dei corpi sono snelle e rigide. Citazioni dirette di statue a noi conosciute non si ritrovano in forma dipinta; spesso si tratta di modelli perduti, in alcuni casi di figure inventate dai pittori. La chioma, gli occhi e il viso, per quanto riconoscibili, viste le dimensioni ridotte, frequentemente mostrano tratti classici e sono privi delle caratteristiche dell'arte arcaica quali il sorriso e gli occhi a forma di mandorla. Le figure conseguentemente sono arcaizzanti o arcaistiche. ${ }^{45}$

Lo stile delle fjgure è stato scelto accortamente. Quando l'oggetto della rappresentazione è di carattere mitologico, la divinita che svolge un ruolo nella storia assiste solto forma di statua, per mezzo della quale vanno accentuate la vetusta e la venerabilita del potere divino e probabilmente, nello stesso tempo, l'antichita del sacello presso il quale la scena si svolge. L'arcaismo veniva considerato un mezzo per esprimere l'antichità e non disponiamo di indicazioni che esso fosse stimato come stile artistico."17 Nel dipingere non si aveva di fronte una determinata figura, ma l'idea di rappresentare una divinità antica. Nei santuari gli xoana venivano custoditi e venerati scrupulosamente. Sono visibili nei pinakes con Ifigenia in Tauride dove la sacerdotessa e rappresentata nell'atto di asportare la statua di culto di Artemide. Nella scaenae frons nella Casa di Pinarius Cerealis (cat. 182;1) dietro Ifigenia, all'interno del tempio. sorge una seconda statua di Artemide, anch'essa delineata in forma arcaizzante, che apparentemente non è la statua di culto, ma caratterizza soltanto l'architettura come tempio, sottolineandone nello stesso momento la vetusta. Un altro esempio di uno xoanon si vede in un pinax nella Casá del Menandro (cat. 163,1). Vi è rappresentata Atena alla quale si aggrappa Cassandra al momento della conquista di Troia da parte dei Greci. La statua lignea, di coiore marrone, assomiglia alle terrecotte che sono state trovate alcuni anni or sono nel santuario di Atena a Lanuvio, fondato secondo la tradizione da Enea." 1.

Mentre le statue in questo tipo di rappresentazioni svolgono un ruolo importante. nella maggior parte dei casi esse non simboleggiano che la località - la presenza spirituale della rorza divina. Talvolta il copista romano potrebbe aver aggiunto all'originale la statua per evidenziare la scena o per ravvivarla. Il Teseo che uccide il Minotauro nel grande pinax nella Villa imperiale (cat. 305,1 ) sembra assistito da Atena in forma di una statua bronzea. Ia scena non si svolge nel Labirinto, ma in un paesaggio roccioso presso un santuario rustico di Atena: vicino alla statua sono state poggiate piccole offerte votive, l'rá l'altro una statuetta in miniatura della stessa Atena.

Nel pinax con Ifigenia in Aulide nella Casa del poeta tragico (cat. 198i/2) si vede sulla spiaggia una colonna sormontata dalla statua bronzea di Artemide in veste di potnia theron. Nell'aria si avvicina la divinita in volo. La presenza della statua non è determinante per la storia e la figura non è un elemento

416 Sulla terminologia G. Becatti, EAA I, 537-540, s.v. Arcaistico, stile/Arcaizzante, stile; Harrison 1965, 50-67.

$417 \mathrm{Cr}$. per esempio Zanker 1972, 25, $A b b$. 44: il rilievo che jappresenta $M$. Curtius e stato eseguito con un criterio conservativo, alquanto rozzo, un die Ehrw Ádigkeit des Monuments zu betonen.

410 Enea 1981 
logico, organico nella composizione, ciò che avviene invece nel caso dell'Atena nella Villa imperiale. L'Artemide potrebbe essere un accessorio immesso dal copista che volesse situare la storia in templ remoti.

Anche paesaggi sacro-idilliaci non di rado contengono un sacello con una statua arcaistica o arcaizzante. Inizia con i prospetli di Il stile, dove statue di Afrodite occupano tholoi o statue di Artemide stanno davanti a case, come per esempio ad Oplontis (cat. 341/1, 3) e Boscoreale (cat. 004/4). Di nuovo vale l'intenzione di rappresentare un antico luogo sacro.

Irequentemente si vede Ecate (magari Artemide-Ecate) munita di due fiacrole nelle mani stese in avanti. Il corpo è coperto da un mantello rigido e talvolta sembra piuttosto un'erma. mentre la testa is incoronata da un modio. L'unica statuetta nota da Pompei rappresenta un tipo ollenistico e certamente non e stata la fonte di ispirazione per i pittori, come e il caso con le statue triforme. ${ }^{49}$ Per le ligure dipinte i pittori hanno avuto a disposizione un modello che non ci è stato tramandato.

Mentre le statue nei pinakes sono d'impronta arcaica, le figure vive che possono essere paragonate a modelli scultorei, mostrano quasi esclusivamente tratti tardoclassici o ellenistici. Non si possono notare differenze fra figure su pinakes di III e di IV stile. In merito vengono presentati alcuni esempi.

$P$. Moreno, in diversi contributi, ha evidenziato l'influsso imponente di Lisippo su tutti i generi d'arte e sull'artigianato ellenistici e romani."20 Nellarte pittorica incontriamo diverse volte figure che scmbrano ispirate da opere di questo scultore. I due tipi dell'Eracle seduto, cjoe l'Epitrapezios e l'Eracle di Taranto, spesso sono stati usati per gli eroi o gli atleti in riposo, specie per Eracle in persona, ad esempio nella Casa di Epidio Sabino (cat. 277/1) e nella Casa delle nozze d'argento (cat. 189/3). L'Eracle Farnese si ritrova sulla scena di Eracle e Telefo proveniente dalla cosiddetta basilica ercolanese (cat. 019). I due tipi di Poseidon sono presenti in paesaggi sacro-idilliaci nella Casa di Livia (cat. $317 ; 2$ ), nella villa di Varano (cat. 010,5 ) e nella Casa dell'ara massima (cat. 221/1). Non bisogna meravigliarsi della frequenza di opere lisippee visto il suo influsso onnipresente.

la maggior parte delle figure è di stile ellenistico. L'Afrodite, in generale attribuita a Doidalsas, si vede usata per l'Artemide che si lava sui pinakes con Attcone. "21 Dioniso viene modellato non di rado secondo il tipo del Dioniso di 'Tivoli. munito di un cantaro e un tirso e con una pantera ai piedi, 0 in modo arcaizzante. simile al famoso Sardanapallos. Pezzi appartenti a questo genere sono lo Spinario, l'Eros che strozza J'oca di Bocthos e le tre Grazie. I famosi gruppi di Achille e Chirone e Marsia e Olimpo, di datare probabilmente nel secondo secolo a.C., sono soltanto conosciuti attraverso le pitture della cosiddetta basilica di Ercolano (cat. 019), della Casa dell'Adone ferito (cat. 198f) e di alcuni pinakes (p.e. cat. 181.1, 198i/3).

possiamo domandarci se si puo riuscire ad ottenere una conoscenza maggiore circa il gusto dei pittori di queste scene e del gusto dei committenti. Siamo in grado di datare gli archetipi sulla base dei dati ottenuti in questa ricerca sui pinakes? Le due questioni sono collegate strettamente l'una con l'altra. Il gusto dei committenti e dei copisti romani non è facilmente ricostruibile. Ia scelta del repertorio sembra limitata. Di consequenza si era costretti a prendere tali rappresentazioni, se si voleva inserire un membro figurativo entro il sistema decorativo. Si puo solo constatare che nel II stile la

419 Spinazzola 1953. I, fig. 456 (dalla Casa di Octavius Quartio): in generale Kraus 1960; Harrison 1965, 86-107; Wrede 1972, 156-15i. Lin altro tipo, nemmeno presente su pitture parietali, viene discusso da Gualandi 1969.

420 Fra l'altro Moreno 1978; $1981 ; 1982$.

421 Contra l'attriburione Linfert 1969). 
maggior parte del tipl manca ancora Cio e dovuto allarticolazione e al carattere di questo stule e forse non e determinato da un gusto specifico per (l'assenza di) pinahes Ie case con decorazioni di III e di II stile, invece, in geneiale sono ricche di quadrs, anche so sl osserva un und casil un amore maggiore che non nell'altra von e casuale che in und I Illa lipo Oplontis non

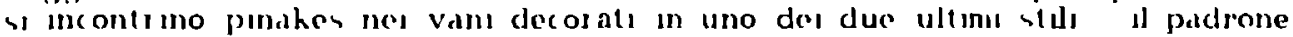
lia pictersto una sintass parjetale costitusta da pannellı e modestı prospeti, mentre ha tenuto in onore le vecchie decorazioni che datano dalla costruzione della illa repubblicana 422 Coricentrazioni di pinahes si trovano in case che sono state arredate nello stle della wlla benestante (cf sopra p s/l), come per esempio nella Casa di Meleagro e nella Casa des Dioscurı Sarebbe utule indovinare attraverso una ricercd statistica come sia la relazione quantıtatıa (e Gualitatıva) II a case rucche c modeste

Quanco si notano andlogie fra una scultura e una ligura dipinta, Iatfigurata come escere vivo, emerge la domanda se la decorazione possa essere datata mediante 1 modello scultureo la cul data di concepimento e quası sempre conosciuta La soluzione di questo problema viene ostacolata dal iatto che non e sempre chiaro se la figura sia derivata dalla statua, dal momento che essa puo essere stata modellata indipendentemente e mostra solo lo stosso attegelamento per ragioni di conografia o logica Una bagnante, per eseinpio, vicme raffigurat nell'Ellenismo scguendo uno schema lisso, noto dalla sopramenzionata statud di Doidalsas inche quando sidmo sicurl che un pittore ha seguito un modello scultoreo, non e facle darc una datazione sicura. l'archetipo puo essere stato antico gld allora, eventualmente noto attraverso copie, e in tale caso ll periodo al quale data l'originale costituise solo un terminus ante quem non per la decorazıone Le statue dipinte senza attenzıone per 1 dettagli non ci formiscono indicazioni che facilitino la datazione di cople romane $E^{\prime}$ chiaro che moltı archetipi sono ellenistici prodottı parzialmente ancora nel primo secolo a C Il II stle non ha fatto uso di questo repertorio, come e stato detto fenomeno per il quale non esiste ancord una spiegazione E'inverosumile che 1 decorator non conoscessero 1 pinakes e la loro assenza quindi sembra una questione di moda

In questo contesto rientra la vecchia questione se di determinatı modelli, conosciuti sia in forma dipinta sia sotto forma di rulevo o statua, una pittura, una statua o un rllievo siano statı l'archetıpo vell'introduzione ll problema e citato bicvemente Si ricordi I'esempio doll'originale delle tro Grazie (p 76 )

A proposito del symplegma, u gruppo del satiro e la menade, di cui si conoscono due escinpl pompeian dipint] (cat 117, 118) e una statua a Oplontis, la discussione e stala conclusa a favore di un archetipo scultoreo La statua viene datata da Fede Bertı nel terzo secolo, le pitture sarebbero cople tarde di essa 423

In tuttı questi studi sı incontrano riflessioni come la 'mancanza di qualita' d) fronte all'ottıma qualita' e la 'mancanza di rilievo' contro la 'plasticita' soltanto argomentı arbitrarı sembrano applicablı alle opere d'arte discusse

Si puo concludere che 1 pinakes non sono un'indicazione fedele per rintracciare lo svluppo del gusto del Romani per quanto riguarda specialmente la scelta del tipı scultores Costıtuiscono una certa fonte per la conoscenza del tıpı statuarı in uso nell'ambiente relıgıoso Glı esempı sono prevalentemente ellenistici e sono statı adoperatı quası esclusivamente nel III e nel IV stle Il II stile adopera raramente talı tıpı

422 Cf Bragantını, Parise Badonı 1984, Moormann 1984b, 672-674

423 Bertı 1972-1973 Cf Marconı 1924, Schmıdt 1925, 96-102 La statua di Oplontis si vede in Jashemshl 1979, hig 180 
Le statue visıbli suı pinakes sono d'umpronta arcaizzante, J che non va tanto spiegato con ll gusto del copistı o del committenti quanto con l'untenzione di ratligurare un luogo sacro antico e venerabile In alcun casi la staturi $c$ stata afgiunta dal copista romano 
La maggior parte delle statue pervenuteci dall'antichita conserva poco o mente del colori con 1 qualı in origine erano coperte Le intemperie e le condizionj nelle quali si trovano da secoll hanno causato la loro scomparsa Tuttora e difficle ricostrume lo stato originale e l'elfetto creato dalla policromia, mentre non possiamo nemmeno escludere l'esistenza di un certo numero di statue non colorate

Le statue raltigutalc nelle pitture parietali $\mathrm{cl}$ formscono informazion notevolı a proposito delle varje applicazionı $d_{1}$ colorı, na non va dunenticato che I pittori cercavano di assecondaie ll gusto contemporaneo alle tecniche acquisite senza rincorrere fedelmente la policromia piu antica, e in particolare quella del quinto secolo alla quale si rifacevano piu spesso

Gia $P$ Reuterswald adopero la pittura pompeiana come lonte di maggiore interesse nelle sue ricerche sulla policromin, apparse nel 1960 sotto il titolo, Studien zur Polychrome der Plastik. Griechenland und Rom 424 lnoltre si avvalse largamente delle fonti scritte I risultati del suo studio ralgono luttora e di recente sono statı aggiuntı solo contributi su aspetti particolari 425 Ridssumlamo in breve 1 dati plu importanti per questa ricerca contenuti nel testo di Reutersward

Mentre il periodo arcilco e caratierizzato dal trequente uso di colori vivaci, quello classico adopera colori piu veristici di modo che l'eftetto di realismo a cui si tende, viene accentuato Dettagl come 1 bordi e le frange dei lestiti, corregge di sandali giolell, occhs e chioma vengono eseguit i in modo preciso 426 Glı sfond, del rilievi in questo periodo sono blu o rossi e vi appaiono talvolta elementı figuratis qualı piante e foglıame dı alberı eseguitı a rilievo Ld pittura paiziale e ul trattamento delle parti umane del corpo con la ganosis divengono plu diftusi lendenza che continua fino all'epoca romana I colorl sono ivac1, ma hon e sicuro se vemissero quelli pantollo preteriti Le terrecolte rispecchiano fino a un certo giado le mode usate nella scultura maggiore, ma for se nello stesso tempo hanno subito un influsso della ceramica policioma contemporanea come quella di Centuripe e Canosa Probabilmente 1 drappı e l suddettı dettagh come trattı del viso e glı elementı metallici venuano sempre accentuati mediante colors, mentre l'incarnato rumaneva privo di colore o coperto da una modesta ganosis Cresce la combmazione di bianco e oro 427 Tale procedimento sarebbe stato inventato - a almeno per la pruma volta applicato su larga scala - dd Prassitele Benche risalga all'epoca classica l'uso di oro e doratura - si ricordino le statue criselefantine - ll fenomeno aumenta nel'Lllenismo La doratura completa era inizialmente limitata alle statue di divinita, poi nel periodo romano si estese anche alle statue degli imperatorı e di ditre persone importants Per quanto riguarda 1 rilievi si nota una policromia dipendente dall'elletto ottenuto dallo stesso rihevo lo

\footnotetext{
424 Reutersward 1960, spec 181-191

425 Pehary 1968, Langlotz 1968, 1969, Wunderlich 1975, Dimitriou 1982 (= 1947)

426 Cf per esempio le considerazions sulle cople della leda di Tirnoteo le piume dell'oca della statua origmale non exano rese in ruevo, ma accentuate attraterso policromia (Rieche 1978, 44-50)

427 Si veda per esempio la Venere in bikını da Pompel (Reutersward 1960, 185-186, Abb 28, Jashemshi 1979, fig 200 Dohl Zanher 1979, 179, tav $\mathrm{Va})$
} 
sfondo o alcuni detlagli altrimenti sfuggenti potevano essere accentuati con l'ajuto di un tocco di pennello. L'inserimento di elementi in materiali dirersi, spesso di grande pregio, andava crescendo nell'epoca imperiale. E' allora che si comiciarono scolpre statue in marmi policromi o fare composizioni con pezzi d marmo diverso, tecnica nella quale ll marmo bianco veniva usato per l'incarnato e marmi colorati per i drappi. 420

Ptretschner, nella sua dissertazione, riassume $i$ dati presenti nella monografia di Reutersward e dimostra che le statue di divinita presenti nelle decorazionj parietali corrispondono all'immagine della pratica antica schizzata da Reutersward."29 Le statue dipinte presentano quasi l'intera scala di possiblita del trattamento della superficie. Sorprende invece che ne Reutersward ne Ptretschner facciano menzione della mancanza di colori pastello nelle pitture, colori tanto di moda nell'Lllenismo. I colori delle statue dipinte sono sempre vivaci e privi di sfumature sottil.

Ora analizziamo in modo articolato 1 materiali e le tecniche di colorazıone.

\section{BRONZO}

Il colore delle statue bronzee originariamente era giallo scuro o marrone chiaro (color tabacco) ottenuto dalla lavorazione del bronzo con bitume per proteggere il materiale dalle intemperje e nello stesso tempo per poterlo facilmente lustrare. ${ }^{30}$ Tuttavia questo accorgimento non impeduva lo scolorire delle statue o la tormazione di una patina verde. I colori cosi prodotti non venivano tolti o restaurati dai Romanı che preferivano una condizıone che evidemziasse l'antichila e la preziosita di esse. Molto interessante a questo proposito e un passo nella Naturalis Historia di Plinio (XXXIV (63) a proposito di una statua di Lisıppo.

\section{Fecit (scil. Lysippus) et Alexandrum Magnum multis operibus a pueritia eius orsus quam statuam inaurari iussit Nero princeps delectatus admodum illa: dein, cum pretio perisset gratia artis, detractum est aurum, pretiosiorque talis existimabatur etian cicatricibus operis atque consisuris, in quibus aurum haeserat, remanentibus.}

(Lisippo scolpi molte statue di Alessandro Magno, dalla sua puerizia in poi, e all'imperatore Nerone piacque talmente quella statua del glovane che la fece dorare: dopo invece, poiche la grazla dellarte era andata perduta a causa del materiale costoso, fece togliere l'oro e la statua cosi venne considerata piú preziosa benchè vi fossero ancora rimaste tracce della procedura e graffi là dove l'oro era stato attaccato.)

Le statue di bronzo nelle pitture sono monocrome di colore giallo, rosso, marrone o verde, La pratica dell'insermento di dettagli in materiale diverso come occhi, labbra o attributi non e stata imitata nelle pitture.

Pfretschner elenca ben 28 esempi di statue marroni eseguite in bronzo, collocate in un contesto mitologico su pinakes." 12 Accentuando cosi l'importanza del colore che esprimerebbe l'antichita delle statue. l'archeologa

\footnotetext{
428 P.e. Gnoli 1971, fig. 48, 154.

429 Pfretschner 1977. I, 117-134.

430 Reuterswärd 1960, 10j-142.

431 Pf retschner 1977, I, 118-122.
} 
austriaca conclude: Schon allein die Farbe solle ein Beweis foll ihre Altert fimlichkeit, four ihre griechische Herkunft sein. Che le statue fossero proprio greche e anticho mi sembra una conclusione ardita. Anch'esse fanno parte delle scene mitologiche derivate da fonti greche (forse ellenistiche, una distinzione per i Romani inesistente) e non vanno considerate in modo isolato. fuori del loro contesto. La Altert Uumlichkeit forse era già stata scelta appositamente dal pittore greco volendo mettere in evidenza che allora. nella sua realtà, la storia rappresentata era successa in un'epoca remota, mitologica. Per questo motivo lo stile della statua - non di rado arcaistico o arcaizzante - è un mezzo piú importante (dell'imitazione) del colore (cf. p. 98 ).

Lapplicazione del colore bronzo potrebbe essere un'indicazjone per la conoscenza dell'originale o di una copia bronzea da parte dei pittori. La scelta quindi pare che sia stata suggerita dal materiale dell'originale. Pfretschner cita come esempio due Poscidones dipinti del tipo Kenchreai. ${ }^{32}$ La statua nella Villa della Farnesina (cat. 319;6) è di colore bronzeo, mentre quella nella Villa di Arianna a Castellamare di Stabia (cat. 010.5) è bianca, cioe eseguita in marmo. Pfretschner crede che la differenza di colori sia da attribuire piu al livello del modello adoperato che ad esempi concreti di marmo o di bronzo.

La patina verde - talvolta si incontra rossa o blu - é, secondo Pfretschner, un elemento importante per indicare l'antichita della statua, ${ }^{43}$ Abbastanza raramente si incontra questo colore. La statua dell'efebo con il vassoio in un pinax proveniente dalla Casa del triclinio (cat. 191/1) era gia allora un pezzo antico da collezione che faceva parte dellarredamento della sala per banchetti di un ricco personaggio. Nella Villa imperiale (cat. 305/1) Atena Promachos veglia su Teseo, mentre egli uccide il Minotauro: la statua non è un oggetto prezioso, ma il simbolo della divinità protettrice, venerabile fra l'altro per la sua antichita. Nel pacsaggio sacro-idilliaco proveniente da una villa a Porticj (cat. 308) la statua di Dioniso presso una tomba unisce entrambe queste qualita. Risalta l'esecuzione precisa degli effetti della luce sul materiale liscio.

\section{ORO E DORATURA}

Il colore giallo può indicare sia bronzo chiaro, nuovo e appena lustrato, sia oro o doratura. "34 Non e possibile distinguere fra questi due materiali in tutti i casi. In generale oro non veniva usato per statue grandi, visibili a tutti. Per tale motivo non e verosimile che si incontrino statue di oro nelle pitture parietali. La doratura invece è abbastanza frequente, soprattutto per statue di divinità. Anche le statue equestri nelle rappresentazioni del foro pompeiano provenienti dai Praedia di Julia Folix (cat. 179/1) sono dorate. Dal primo secolo a.C. si adoperava la doratura per i ritratti di personaggi importanti, sia di morti che -nell'epoca imperiale - di persone ancora vive. ${ }^{3} \xi$

Pfretschner ha collegato quindici esempi di statue di divinità tutte inserite in contesto mitologico. $\$ 36$

\footnotetext{
432 Pfretschner 1977, I, 121.

433 Pfretschner 1977, I, 122-123.

434 Pfretschner 1977, I, 123-125.

435 Pekáry 1968; Lahusen 1978.
} 
In un certo numero di architetture dipinte del II stile sono inseriti elementi scultorei di colore giallo o verde. Essi devono essere considerati come appliques o membri di bronzo, sia dorati o meno, poiché le rappresentazjoni di architelture dj questo perjodo rispecchiano in maniera piu o meno ledele le costruzioni reali. Come esempio puó servire la testa di pantera verde su di un frammento proveniente dalla Villa dei papiri che trova riscontro in appliques della stessa villa $e$ in altri luoghi. ${ }^{437}$ Gli elementi melallici sono quasi inesistenti, essendo sempre stati oggetto di saccheggio c riuso.

Le prospettive del periodo del IV stile presentano una preferenza per gli elementi in giallo. $E^{\prime}$ difficile stabilire a quale scopo mirassero i decoratori; se volessero creare delle imitazioni di costruzioni metalliche (dorate o meno) o se scegliessero il colore solo pensando allo siondo e alla composizione coloristica. In innumerevoli casi acroteri, atlanti e appliques hanno il medesimo colore delle architetture nelle quali sono inseriti.

Nelle rappresentazioni di giardini le statue di bronzo sono inesistenti. Solo in pochi casi. cioè nella Casa dei Ceii (cat. 152/3) e nella Casa delle Vestali (cat. 198/3), il bacino di una ninfa acquatica è eseguilo nel colore del bronzo, ma figure completamente gialle o verdi non si incontrano. Per questo aspetto le pitture non rispecchiano la prassi normale conosciuta attraverso i ritrovamenti campani. Sono state trovate numerose statue di bronzo topiarie, specialmente in guisa di fontana, materiale diffuso anche per questo genere. ${ }^{439}$

\section{ARGENTO}

L'argento come materiale per le statue viene menzionato raramente da Reuterswärd, mai da Pfretschner." "4o Infatti il materiale sembra usato solo raramente. ${ }^{4} 1$ La sua presenza nelle pitture è difficilmente reperibile, dal momento che esso non si distingue molto dal marmo visto il colore grigio/bianco. Attraverso macchie lucide si capisce l'intenzione del pittore di rapjresentare una statua argentea. Non incontriamo l'uso di accentuare parti anatomiche come labbra, sopracciglia o capezzoli in argento, pratica conosciuta per molte statue.

Una delle due statue che abbelliscono l'interno del palazzo di Massinissa nel noto pinax della Casa di Giuseppe II (cat. 269/3) deve essere d'argento: mentre il Dioniso di colore verde apparentemente è una statua bronzea, l'Apollo bianco a macchie grige non puó essere composto da altro che argento. Solo su questo quadretto si osserva l'effetto del contrasto di materiale fra due statue, entrambe considerate come pezzi da collezione nel ricco ambiente del principe numida. I soggetll non sono stati scelti appositimente; ancora offi è inpossibile fare dei confronti validi tra queste figure classicheggianti.

436 Pfretschner 1977, I, 123-126.

437 Moormann 1984b, 642, fig. 7.

438 Cf. Drerup 1957; Rossignani 1969.

435 Si pensi al numero di 27 bronzj della Villa dei papirj (Pandermalis 1971) e della preferenza per questo materiale in alcune case pompeiane (Döhl/Zanker 1979, 179; Dwyer 1982, 135).

440 Reuterswärd $1960,152-153$ nota 399.

44. Si pensi alle statuette di Artemide ad Efeso (Atti degli Apostoli 19, 24). 
Nelle tholoi sui prospetti di II stile nella Villa dei misteri (cat. 306/4) e nella villa di Oplontis (cat. 341/3) e sul frammento dell'Insula occidentalis a Pompei (cat. 263) si intravedono statuette di colore bianco-grigio, quasi sempre rappresentanti Alrodite. Nell'armadio sul fregio degli amorini orefici nella Casa dei Vettii (cat. 217/9) si trova la statuetta argentea di Afrodite e nell'ala destra della medesima casa una natura morta contiene la statuetta argentea di un atleta del tipo policleteo westmacott.

\section{MARMO}

Le statue di marmo per quanto riguarda la policromia si dividono in tre gruppi. Statue interamente bianche, prive di colore, non appaiono nella pittura, cosa non sorprendente visto che l'utilizzazione del materiale tale quale cominciò non prima del periodo antoniniano. 42 Il secondo gruppo, statue che presentano una policromia parziale, appare molto spesso, ma il maggiore numero è costituito dalle statue completamente policromate.

La policromia si incontra già frequentemente nel II stile quando si tratta di statue esposte in nicchie, come per esempio nella Casa degli epigrammi (cat. $185 / 2)$. Particolarita da sotlolineare è che queste statue spesso erano collocate sopra piedistalli verdi o gialli; il verde in tal caso e simile a quello delle statue bronzee. "43 l colori vivaci fanno sparire la distinzione fra figure vive e statue, anche se i piedistalli e la collocazione entro il sistema architettonico non lasciano dubbi sull'intenzione dei decoratori. Cio vale anche per le erme che sono provviste di volti con un'espressione vivace: un'crma cosi non puó essere altro che una statua, ma la sua testa sembra appartenere a una figura viva, presente nell'ambiente. Studiosi quali Beyen e Allroggen-Bedel hanno tentato di trovare una soluzione attraverso un compromesso: le figure si troverebbero a cavallo tra la scultura e la realta. Essi considerano le raffigurazioni come lantasie bizzarre e paiono ignorare le possibilità di una decorazione a molti colori. Come abbiamo visto (p. 20), costruzioni come i portici con le erme sono riferibili a degli esempi concreti, nei quali la policromia deve aver svolto una parte importante, pur su scala ridotta. Non va dimenticato in proposito che $\mathrm{i}$ pittori probabilmente avevano a disposizione mezzi piú espressivi per creare costruzioni realistiche, completamente conformi ai criteri artistici sviluppati durante l'Ellenismo.

Nel III e nel IV stib la policromia rimane molto importante nelle architetture dipinte, ma i pittari sviluppano nuove vie delineando architetture in un solo colore e con forme lineari. Anche le statue vengono colorate in maniera piú modesta. Purtroppo non si può stabilire se questo sviluppo stilistico sia avvenuto sotto l'influsso del Classicismo del primo Impero. Reuterswärd non distingue fra opere nconttiche e copie di statue greche e definisce una stessa policromia vivace per entrambe le categorie.

Rispetto alle rappresentazioni di statue policrome di divinita Pfretschner ritiene che esse non fossero mai state considerate come statue di culto: la policromia sarebbe stata applicata su ornamenti e statue decorative, mentre per le statue di culto il bronzo e il legno sarebbero stati i materiali preferiti. ${ }^{44}$ La Venere pompeiana avrebbe una policromia realistica essendo una divinità popolare che nicht durch eine kultbildhafte goldene Farbung den

\footnotetext{
42 Reuterswärd 1960, 218-219.

443 Cf. Rossignani 1969; Dreihaus 1969.

444 Pfretschner 1977, I, 128-129.
} 
Anbetenden entroúckt.45 Rumane l dubbio se la dea sia stata rappresentata in forma di una divinita iva, reale, oppure come una statua di culto Le scene nelle qualı e presente, potrebbero essere un indizıo per la seconda ipotesı vella pittura lungo la Via dell'Abbondanza essa si trova sopra un podio nel mezeo der fedcli, nella Casa del labirinto (cat 208 1) sta trd alcune divinita domestiche in un lararjo, nella Casa delle nozze di Ercole (cat 254) occupa l'entrata del suo lempio, mentre nella Casa del Mendndro (cat 163 3) l'ainbiente e un bosco sacro

Iina suddivisione delle statue di divinita in policrome e monocrome risulta troppo studiata $\mathrm{Cl}_{1}$ si puo domandare se 1 pittori abbiano 1atto consapevolmente distinzione tra statue di culto e sculture decorative A proposito del pinahes si giunge alla medesima conclusione di quanto $c$ stato detto rispetto alle statue bronzee e dorate le loro originı vanno cercate nel mondo greco-ellenistico e hanno trovato, senza modifiche specitıche, un loro posto nel repertorio des decordtor romani lnoltre e diticule provare se la scelta des material, losse un preciso atteggiamento upecitico verso una divinita Nel materiale conservato - nel quale purtroppo non si trovano statue d) culto - non se ne trova prova e nemmeno dalle tonts scritte possiamo ricasare dati in merito Nel caso della Venere pompciana non disponiamo nemuneno di una statuetta che fornisca dati concernenti 1 colori originali 446

I color delle statue dipinte nella maggior parte des casi sono lantastici ton si incontra und scelta $d$ i color fissl per determinate figure, un'iconografia di colori' non puo essere stablita, il che non sembra nemmeno Il caso per le vere statue, eccetto l'uso dell'oro

Statue bianche con una policromia parziale sono rare secondo Pfretschner Quattro delle dodici statue da les elencate appaiono su rappresentazioni di glardini 447 Specie in quel contesto si vede una preferenza per statue pressocche bianche Questa predulezione $e$ in contrasto con l'abbondante applicazione di color constatata da Reutersward $44 \mathrm{~g}$ Le statue recuperate in Campania - materiale conclusivo per Reutersward - spesso hanno tracce di una vilace polıcroma Si presentano due soluzionı per questa discrepanza fra realta e imitazione pittorica Le statue policrome exano sempre esposte al raggi solail, alla ploggia $p$ all'umidita $d_{1}$ piante e stagn cosicche taclmente perderano 1 loro colori Il proprietarıo probabumente non faceva ognı rolta ridecorare le sue statue, ma le lasciavd diventare bianche I pittori ar rebbero unitato questa realta nella pittura Ln'altra risposta si poticbbe cercare invece anche nelle stesse pitture $A_{1}$ pittorı, quasi mal molto dolat,. mancara la tecnica di esprimere volume e profondita Erano costretti a scegliese colors chırı, afinche potessero far spiccare le statue sul piano e davanti alle prante $E^{\prime}$ evidente che in queste situazioni l colore piu adatto era ll bianco

Senz'eccezione le figure blanche piesentano una policromia parziale, specie per chioma, occhi, labbra, mantell, attributı e altrı dettagli L'Ares nella Casa della Venere in conchiglia (cat. 178) ne e un chiaro esempio

Nelle rappresentazion architettoniche di II stule incontriamo talvolta statue bianche L'alcova 4 della Villa deı misterı (cat 306,2 ) contiene una serıe di sculture bıanche sopra bası gialle su di un podıo verde davantı ad ortostati rossı Glı acroterı nella Casa di Augusto (cat 316,3) risaltano per la scelta del bianco Le figure bianche nella zuna superiore delle scaenae frontes nei bagnı della Casa del Menandro (cat 163'3) e della Casa del criptoportico

445 Pfretschner 1977, I, 129

44 G Spinazzola 1953, I, 218

447 Pfretschner 1977, I, 126-128.

4\% Reutersward 1960, 192-193 
(cat 148/3-4) sono immaginate come statue di marmo

Diffic lumente si puo stablire se nel caso di policromia o monocromia in un colore diverso del bıanco sı intendessero imitazıonı di pjetre e marmi coloratı L'erma nella Casa di Popidius Priscus (cat 237) sorge sopra un piedistallo di colore giallo con vene rosse, chuaramente imitante il guallo antico. Lo stesso materiale potrebbe essere stato imitato nel cubicolo 4 della Villa dei misterı Le erme rosse nell'oecus sotterraneo della Casa del criptoportico (cat 148'1) forse sono imitazioni di opere in poifido rosso, granito rosso o rosso antico, mater all largamente diflusi durante I'Impero $4 \mathrm{~s}$

In generale 1 marmi colordti vennero in uso fin dal primo secolo d C per cui la loro presenza nelle opere del IV stule non sorprende un esempio molto interessante si rede su un pinax nella Casa del marindio (cat. 259) in un parsaggio selvatico dove ha luogo la strage delle Viobidi vi e un sacello con la statua di un cervo in giallo e rosso Il modello e sumlle al ceru di bronzo e marmo conosciutı come elementı topiarı I colorı imitano un intaglio di pezzi di marmo rosso o di pietre (semi)preziose in un corpo di marmo giallo Ia lecnica dell'mtaglio sarebbe, secondo Plinıo (N.H. XXXV 1, 3) venuta in voga durante l'impero dı Claudio vella Sala deglı anumalı nei Vuseı Vaticanı sı trovano una pantera e un cervo eseguitı con questa lecnica 4bo L'jpotesi presentata a proposito della pittura vene convalıdata dal tatto che il giallo e I rosso non imitano 1 verı color della pelle macchiata di un cervo e dalla presenza di esempl sumb

\section{LEGNO}

In generale e umpossibile riconoscere statue di legno attraverso ll colore Anch'esse erano dipinte Generalmente le statue de Prapo prano scolpite in legno e dipinte in rosso, pratica riconoscibule nelle pitture 451 lella Casa del bel cortule ad Ercolano e stato trovato un Priapo ligneo, purtroppo privo del colore originale 452

Quası sempre l legno era un materiale adoperato per le unmaginı di culto. eidola, breté. o xoana Come gia dsce l'ultuna parola, le ummaginı di culto spesso erano di legno, materiale che valeva da indicazione per la vetusta della dirinita e del luogo sacro Le figure di colore rosso a marrone scuro sono sempre di stule arcaizzante o arcaistico per cul sono dd considerare oggetti antichı Si pensı as pmakes con $1 \mathrm{l}$ Palladio (cat 140,1631 ) e Ifigenia in Tauride (cat $186^{\prime} 1,217,9$ )

\footnotetext{
449 Gnolı 1971, 98 (cf Delbrueck 1932), 119, 162, fig 136, 140-141 (per 1 color1)

450 Amelung 1908, 340, Taf 33, Helbig * 175 Gnol 1971, 192-193, fig 287

$451 \mathrm{H}$ Herter, RE YXII 2 (1954) 1922, s.v. Priapos

452 Nion pubblicato
} 


\section{ALTRI MATERIALI}

Statue criselefantine non appaiono nelle pitture romane. L'avorio sarebbe stato imitato nell'absicle del caldarıo della Casa del Menandro (cat.163/6) secondo Fittschen. I rilıevi di figure femminili, eseguiti in colore bianco-crema, sono stati citati dallo studioso tedesco come esempi dell'inserumento di questo materiale costosissimo nel palazzi ellenistici dei Diaclochi, ma e stato detto sopra ( $p$. ) che una imitazione di rilievi a stucco e piu verosimile. 
Questo capitolo raccoglie un sommario piú completo possibile delle statue che si incontrano nella pittura parietale del periodo dopo il 79 d.C. Purtroppo lo studio del materiale vicne ostacolato dal fatto che mancano pubblicazioni di buon livello sulla maggior parte dei complessi. Inoltre si osserva che, specialmente fuori d'ltalia, molte pitture non sono rimaste conservate in situ, $e$ in forma di frammenti sono state usate come materiale di fondazioni o buttati come rimasugli. Le monografie di F. Wirth, M. Borda e $W$. Dorigo contengono molto materiale e sono indispensabili, ma non tengono conto dei nuovi ritrovamenti, per non parlare degli svijuppi nella ricerca sullarte romana in generale. 453 In piú, la presentazione del materiale è disordinata, non completa e con osservazioni spesso troppo soggettive. Nello studio di Hetty Joyce, apparso alcuni anni or sono, gli clementi ligurativi non ricevono molta attenzione dato che l'autrice si concentra sui sistemi decorativi. 44

Si osserveri che nel prosieguo Ostia e Efeso saranno trattate a lungo, dal momento che queste due citta sono diventate ben note per quanto riguarda la pittura parietale grazie ai volumi di vari autori nella collana Monumenti della Pittura Antica e la vasta monografia di V.M. Strocka nolle Forschungen in Ephesos (VIII 1). Per gli altri complessi pittorici mancano pubblicazioni del genere oppure se ne la menzione in modo superficiale, magari in un capilolo a parte.

Le decorazioni verranno trattate nell'ordine presentato nell'introduzione: strutture architettoniche, rappresentazioni di giardini e scene figurative.

\section{DECORAZIONI ARCHITETTONICHE}

Le figure verranno discusse per zona orizzontale, seguendo l'ordine del capitolo I.

\section{Zoccolo}

In generale lo zoccolo è privo di elementi figurativi. Prevalgono imitazioni di incrostazioni marmoree, motivi geometrici quali losanghe e cerchi, e semplici piani monocromi.

Soltanto lo zoccolo di una decorazione nella Casa dell'attore tragico a Sabratha (cat. 334) contiene elementi scultorei, ricostruiti sulla base di frammenti. La zona è composta da lastre marmoree e edicole occupate da sirene e sfingi femminili che stanno sopra mensole. La suddivisione fa pensare al podii dei teatri e dei ninfei del periodo imperiale. Ne per gli zoccoli ne per la disposizione degli animali esistono esempi simili.

\section{Zona mediana}

\footnotetext{
453 wirth 1934; Borda 1958; Dorigo 1966.

454 Joyce 1981.
} 
La zona mediana contiene due generi di figure scultoree: vignette su campi lisci e figure inserite in scaenae frontes o sistemi derivali da esse. Le architetture complicate sono rare in confronto con il periodo precedente il 79, ma quelle conosciute non sono prive di figure.

Ln'incisione del Settecento nella ricca collezione di Eton mostra una parete che avrebbe decorato un ambiente nella Villa di Adriano a Tivoli e che si può ancora completamente inserire nella tradizione del IV stile (cat. 339/1). Nella nicchia centrale a forma absidale e nelle due edicole adiacenti vi sono tre statue, raffiguranti Hermes, Afrodite e una seconcla figura maschile, probabilmente Ares, posti sopra piedistalli rettangolari. Le figure sono policrome - almeno per quanto si puo concludere in base all'incisione - e sembrano concepite come figure vive nonostante le basi. Mancano completamente dei riscontri nella scultura.

Non molto diverso da questa decorazione $\dot{e}$ il complesso decorativo di una casa trovata nel 1777 nella Villa Negroni, abbastanza ben documentato grazie ai disegni colorati di Raphael Mengs e Anton von Maron e studiato di recente dettagliatamente da Joyce (cat. 314). L'elemento scultoreo in questo caso si limita ad acroteri di colore bianco in forma di tritoni e grifi. Un bollo laterizio del $134 \mathrm{~d}$.C. ci fornisce un terminus ante quem non per la costruzione della casa e per le sue decorazioni che vista l'affiniti con le suddette decorazioni della Villa adriana possono essere state applicate contemporaneamente.

Sotto la Basilica di S. Giovanni e il Palazzo Papale in Laterano sono stati trovati $i$ resti di una casa neroniana, rimasta in parte conservata, poiché costituiva le fondamenta di una caserma del periodo severiano (cat. 313). La datazione della casa e accertata dalla struttura muraria. Parti di una scaenae frons appartengono a questo periodo e non differiscono dalle strutture architettoniche nella Domus Aurea (cat. 310). Sono rimaste visibili soltanto le sagome di alcune figure che popolano le edicole.

In una villa romana presso Tor Marancio, costruita nella seconda meta del secondo secolo d.C. sono stati recuperati nel Settecento del frammenti di campi abbelliti con figure. alcune volanti, che ora si trovano nei Musei Vaticani. Una parete, purtroppo nota solo attraverso una incisione settecentesca, conteneva candelabri che sorgevano sopra un podio stretto davanti ad una serie di pannelli (cat. 330). I candelabri erano sormontati da figure femminili che ricordano la fuga di candelabri in un corridoio della Villa della Farnesina (cat. 319/6).

Le decorazioni nella Casa dell'attore tragico a Sabratha (cat. 334) sono composte allo stesso modo delle scaenae frontes di Tivoli e della casa sotto la Villa Negroni. Le forme architettoniche abbastanza pesanti sono da associare con le scaenae frontes del tardo II stile, specie quelle nella Casa di Augusto a Roma (cat. 316), mentre i dettagli e gli ornamenti, per esempio i putti volanti, e l'articolazione dello zoccolo ( $v$. sopra) apparentemente rispecchiano sviluppi piú recenti. S. Aurigemma, in una monografia sulle pitture trovate nella Tripolitana, non propone delle datazioni in base al materiale dello scavo e sostiene che una datazione secondo criteri stilistici non puó essere fissata. Il parallelismo con le pareti discusse prima giustifica una datazione nel secondo secolo, specie nel periodo antoniniano. Le edicole centrali delle 'pareti I e III' vengono incoronate da figure umane che sorreggono l'epistilio della decorazione al lato superiore. Le $H^{*}$ dfthermen sulla parete I copiano esempi ellenistici e rjcordano le numerose erme di II stile. Finora esse sono uniche. Questa constatazione e rilevante soprattutto quando si pensa alla moda di adoperare figure umane nel'architettura proprio nel secondo secolo (cf. sotto p. 1t5). Le figure femminili che abbelliscono la parete III, invece, sono vestite di un peplo classico e rjentrano nella tradizione classicista conosciuta dalle cariatidi nelle scaenae frontes di III stile.

Conosciamo un notevole numero di decorazioni tombali, pitture che non si inseriscono nelle schematizzazioni degli stili. Contengono raramente elementi 
architettonici a favore di rappresentazionı figurative qual nature morte, paesaggi sacro-idlliaci e - di rado - scene con figure umane e mitologiche, piuttosto schemi lineari e vignette Specie le lesene fra glı cosolia o l loculi richiedono la nostra attenzione

La tomba del \asonil a Roma (cat 326) viene datata a meta del secondo secolo d C Ad essa e stato dedicato uno studio dettagliato di B Andreae velle fasce fra gli arcosolia si vedono edicole occupate da bel figure maschil nude che fanno pensare al guerrierı nudi presentı tante volte a Pompel (c1 $p$ (43) Potrebbero essere anche loro un ricordo all'ideale estetico che avera successo durante il classicismo adrianeo Stranamente mancano nell'andisı dı indrede osseriazionı riguardantı el significato e l'jconografid degh uominı che reggono cestine colme di frutta Intatti e difficle trovare una soluzione il numero di ses impedisce l'interpretazıone come Stagioni maschul e nello stesso momento le figure non possono essere genil funerari, polche manca l'abituale flaccola rovesciata

vella tomba phi nella necropoli vatıcana (cat 325), datata nel perıodo de Severı. H^ufthermen dı Silenı adornano le lesene tra glı arcosolia Copıano tıpı not gia nel Il stule qual le erme nella Casa del criptoportico (cat 148 1-2) In senso iconografico si inseriscono senza problemi nell'ambiente dionisiaco e quind non turbano 1 contesto funerario

Per quel che riguarda le provincie possiamo menzionare solo due tombe a Palmira in Siria (cat 138, 139), nelle qualı si delinedno rappresentenzioni della Vittoria Romana fra le nicchie con i sarcotagl Le tombe possono essere datate con precisione l'una nel 249 , l'altia at torno al 259

Immagin di strutture architettoniche sono rase nelle provincie e non sono note nenumeno architotture abbellite con statue $S_{1}$ incontrano in generale decoralsonı a camps che sono separatı l'un dall'altro per inc $\angle 20$ di lesene con tralcs o candelabri, questi ultumi taliolta incoronati da tigure umane A Treverı queste figure, non ancora pubblicate, sarcbbero state sostituite da sculture (cat 342 )

Il maggior numero di statue e costituito da figure che si reggono sopra una mensola o sopra un segmento a forma di trapezio o parallelogramma, oppure ancora davantı ad un campo semplice Mentre a Pompel le mensole sono spesso dettagliatamente eseguite, le pitture piu recenti non tormiscono esempi del genere Soltanto le Tuse nella casa omonuma ad Ostia (cat 130,1) stanno su mensole rettangolari con orli scolpitı La questd Lorma, gle appiatita, sono state dervate le 'mensole' geometriche

Ad Ustia, Sparta c Eteso sono state trovate serse di Vuse sumli a quelle che conosciamo cosı bene da Yompei (ct p 000) 11 complesso pittorico della Casa delle Muse ad Ostıa (cat 130\%) e gia stato discusso in breve nel pi uno capitolo ( $p$ (r)

Il contesto archeologico della tomba, scavata parzialmente nel 1930 sull'acropoli di Sparta, non e del tutto chiaro come non lo e la datazione (cat 336) Dentro la camera tombale sono state osservate decorazionı a campi suı qual un Apollo Musagete e sette Muse fungono da vignetta L'Apollo puo essere associato all' $\Lambda$ pollo Liceo di Prassitele, pur con vallazion she gls danno un'impronta classicisticd A Adamantiou menziono come confronto valido l'Apollino negli Uffizi Delle sette Muse soltanto due vennero ritrovate, una delle qual e nota per una fotografia appartenente alla relazione di scavo Dall'umagine e dalla descrizione non sl puo concludere nulla rispetto all'ıconografia e as tipi unitatı. mentre non e nemmeno chiaro se esse lossero sistemate secondo l'ordine esiodea, come si vede nella Casa delle Muse ad Ostid (cat $130 \%$ )

Le decorazionı piu recentí - anche in senso assoluto nel quadro del presente lavoro - sono quelle trovate in un quartiere sul pendio al lato sud della strada principale (la "strada marmorea") di Efeso Esse sono state 
applicate nel quinto secolo Un'epigrafe greca undica sempre ll nome della ligura rappresentata La presenza delle epigrafı probablmente testumonia la scarsa conoscenza delle singole Vuse, come nel Praedia di Julla Felix a Pompel (cat 179/2) Iunzionarano da didascale di una nuova conografia (ci $p$ 42) Cosı osserv lamo 1 due polı di un tema molto popolare durante l'Impero Quando un vano conta puu campl del nove necessarl, le Vuse si troiano in compagnia di altr' personaggı culturalı in $\mathrm{H} 2 / 12$ (cat 015 ) bl vedono Apollo Musagete e Salfo, in $\mathrm{H} 2 / 7 \mathrm{a}$ (cat 014/1) il flosolo Socrate Strocka suggerisce assal prudentemente che la ligura di Socrate poteva essere una copia dipinta del ritratto di Socrate scolpito da Lisippo, lunico ritrat to che rappresenta il fllosofo seduto Egli nello stesso $10 \mathrm{mpo}$ rleva notevolı diflerenze con ll torso nella Iy Carlsberg Glyptotek a Copenhaghen che viene considerato la copia plu importante della statua lisippea Voreno a questo proposito si e rilevato scettico ed ha ricostruito ll Socrate di Lisippo sulla base della pittura

Nella sua discussione delle Muse nella Casa delle Muse Moreno tenta di trovare des posubli controntı nella scultura Certamente le Muse ad Ostia, non diversamente dalle figure pompelane, mostrano forti similitudin con le statue ellenistiche, ciononostante e impossibile tare del contronti lon si ritrovano modell che tanno parte del gruppi ricostruitı dd Pinkwart (ci p 42.)

Vella Casa dı Giove e Ganımede ad Ostra (cat 130/1), datata nell'epoca dı Comnodo, filosof 1 e glovam offerentı sono stat tıgure stanno sopra segmentı a torma dı parallelogranma Fdto singolare e che la testa del flosofo taglia l'orlo super lore del suo pannello il pittore sembra aver sbaghato 11 rapporto dimensionale tra la figura del fllosoto e ll pannello Le altre figure invece hanno le proporzioni adatte ai loro campi

Molte decorazioni nelle tombe della necreopoli du Isola Sacra ad Ostra sono state ravvivate con vignette caratterizcate da Borda come statuarie figure isolate Dobbiamo a G Calza una pubblicazione della niaggior parte di esse apparsa nel 1910 e luttora indispensabule kardmente possiamo fare confionti scultore per le singole tigure, ma l'mtenzione di mutare vere sculture, esposte entro le nucchie tombalı, e cudente Nella lomba 11 (cat 131) le tre Parche avvicinano le statue ellenistiche di donne amunntate Atropo che somigha a Clio reggente un volwmen davantı al grembo,non stonerebbe in una serie di Muse von sono state trovate statue o statuette delle Parche talvolta esse sono presentı in rlievi tardo-ellenistici e in sarcofagi imperiali is5 La similitudine con 1 suddettı tıpi non suscita meraviglia, se si tiene presente che l pittore non ebbe a disposizione un esempio concreto per le stesse Parche

Nelle tombe 15 (cat 132) e 70 (cat 134) le figure sono identificabll come sculture grazie al loro colore Purtioppo Calza non si sofferma a lungo su queste statue, tace completamente sulla moncroma e tralascia le lllustrazion delle decorazıonı ora svanite completamente lella tomba 70 sarebbe stato visibile Eracle, nella lomba 15 due uomini nudi torse dssociabil al guerrieri eseguitı secondo modell polıcleteı

vella Tomba deı Voconı a Merıda (cat 047) le paretı sono state affrescate con architetture che assomigliano as sistemi $d_{1}$ II $s$ tule, ma che sono da datare alla fine del primo secolo $d \mathrm{C}$ Le finte nucchie sono occupate dai ritrattı del membri deluntı della tamiglia deı Vocom Il tipo statuario e quello delle statue tunerarie normalı

Le tre Grazie sono visibli nella tomba omonima (cat 135), raffigurate nell'atteggiamento consueto La loro presenza nell'ambito funerario e stata oggetto dı studio da parte di Trillmich che di recente ha pubblicato un rllevo

455 Cf $P$ welszacher, Roscher II 2 (1894-1897) 3084-3102, s.v. Moira, C Caprino, EAA 5 (1963) 143-144, s v. Moira con bibl 
con le tre Grazie 456

Ignoto e $\mathrm{l}$ contesto archeologico di un frammento d'intonaco trovato a Zliten nella provincia 'I I spolıtana (cat 344) Si vede la statua dı Ares dipınta in lerde, colore che apparentemente indica la patina del bronzo (cf $P \quad 000$ ) Il falto che la ligura sl delinea su un campo bianco ta pensare che essa costituica una vignetta in una decorazione a campl Proporie una datazione in base a questo solo frammento e impossibile

Quattro dusinita, Cerere, Iortuna, Ercole e Vittoria, appasono di quattro latı di un altare irorato a Mlano (cat 018) Riproducono ceitamente modellı scultorei piu o meno a rilievo La composizone delle figure non differisce dagli altari scolpitı ad ogm lato sl stacca una figura appogglata sopra und mensola Le mensole a loro volta copiano und pratica frequente in architetlura 457

\section{Zona superiore}

La zona superiore contiene soltanto di rado delle statue Questa osservazione e relativa, poiche e legata al numero molto ridotto di zone superiori di cul disponiamo Le paretı ad Ostıa ed Efeso costıtusscono l pochi esempl du decorazions conservate quasi completamente Nella I lla di Tor Marancio (cat 330), testunonı le incısionı, le zone superior ospitasano carlatıdı che reggrvano la trabeazıone superıore della decorazıone nel Karyatiden-Schema

Sia nella Casa di Giove e Ganmede (cat 128) che nolla Casa delle volte dipinte (cat 129) ad Ostia, entrambe da datare nell'epoca antoniniana, si vedono tigure statuarie Le lunette dei vani II e IV della Casa delle volte dipinte tanno pensare a quelle della Domus Aurea (cat 310) scaenae tiontes snelle e elegantı sono occupate da figure poste sopra piedistall Glı archetipı sono da cercare nel perıodo ellenıstıco vel vano YII tigure fomminll policrome che stanno su mensole, rupetono s inodellı delle terrecolte landgrine Abbiamo a che lare con uno dej sarı esempl di tigure sopra mensole nella zona superiore

Nolla Casa di Giove e Ganimede ad Osl la (cal 128) vediamo horal che stanno su elementi aggettanti di una trabeazione ['n'edicula nolla parete est e occupata da un'Afrodite Anadyomene, I appresentata come persondggio vivo

In frammento nol Vusel Vaticani pioveniente da Ostia (cat 137) mostra un guerriero romano entro un'edicola Esso si regge sopid und base a torma di calice vegetale e vene interpretato come statua, anche se la ligura sembra uva Lo stile pitiorico e ل piedistallo dipinto in modo cosi preciso non sono differenti dal frammento $M N$ 9646 a Vapoli (cat 102) che dov rebbe provenire da Pompel Anche $\downarrow$ trammento osticnse potrebbe aver fatto parte di una decordzione di I' stule, ma e pru verosimile che costistussca la parte centrale della zona superıore dı una scaenae trons del tipo che conoscıamo da Tor Marancio (cat 330) e dalla Villa Adriana (cat 339/1)

Un trammento tros ato a Tuscolo e ora nel Louvre deve essere datato a meta del secondo secolo (cat 343) Una tigura temminle vestita dı un peplo classico sorregge con la lesta un arco che sembra tar parte di un'arcata clie incorona la parte superiore di una decorazıone tnche glu altri archi probabumente ienivano sorretti da cariatidi La costruzione assomiglua alla fuga di carıatıdı lungo l canopo della Vlla Adriana ed e senza confrontı nella pittura parietale, specie quando l discorso riguarda le arcate

\footnotetext{
456 Trullmich 1983 Cf $p$ 76.

45. Cr Gabelmann 1977
} 
Anche ad Efeso sono rumaste conservate delle statue nella zona superiore In $\mathrm{H} 2 / 16 \mathrm{a}$ (cat 016) una donna assomiglia in modo preciso alle Danaidı della Vulla deı papırı e alle cariatıdi nelle scaenae frontes di III stule. In $\mathrm{H} 212$ (cat 01j) si vedono guerrierı nudi di colore grigio, espostı come statue entro edicole strette e semplici. Il colore indica il marmo. Purtroppo le figure sono in uno stato trammentario.

Le architetture dipinte rispecchiano solo modestamente la pratica edulızia dell'epoca imperiale Proprio nel secondo e nel terzo secolo le facciate des teatrı, ninfes. portıcı e porte monumentali vengono abbellıte con numerose statue che, in parte, sostituiscono colonne e plastri, quando si tratta di costruzionı as piu pian, specie al piano noble licordo, di nuovo (ct p. 000), l porticato altorno al tempio di Domiziano ad Cleso e 'lus Incantadas' a Salonicco. Non sono le statue a reggere, ma 1 plastri contro 1 qual esse si appoggiano e che sono nascostı in modo tale che le tigure sembrano degli atlantı Schmidt-Colinet non discute in modo dettagliato questa categoria marginale delle figure reggent, ma accenna agl eventual, antecedentı ellenistici, mentre non prende in considerazione le decorazioni parietalı che in questo contesto sono molto llustrative $e$ che sono state discusse altrove (cf p. 21$) .450$ Von Hesberg, nella discussione della Porta del prigionieri a Cormto, dimostra la presenza di figure reggents nella porta costruita nel periodo augusteo - precedentı quindı le higure consersato " datate nel secondo secolo - e cita contronti contemporanei e piu antichı, tra l quahl bl Santuario della Fortuna Primigenıa a Palestrina 459 Bisogna concludere, anche se con una certa cautela, sulla base del materiale discusso che la ricchezza di figure umane inserite nelle strutture architettoniche delle pitture di Il e di IV stile corrisponde molto di piu alla pratica nella vera architettura che non la decorazıone parıetale contemporanea aglı edificı menzıonatı

\section{RAPPRESENTAZIONI DI GIARDINI}

Il genere delle rappresentazioni di glardini rimane popolare durante l'intero Impero. I pittorı continuano ad imitare in modo piu o meno fedele piante, poste tra siepı o cancell, talvolta ravvivate con uccell voldntı. Tanto in Italia quanto nelle province incontriamo esempi del genere, ird l'altro ad Efeso e Nimega 460 Una grande differenza ruspetto ai glardını dipunti in Campanıa costituita dalld mancanza di figure umane e di statue tra 1 cespugli velle Terme di Buticosus e nelle Terme di Vottuno ad Ustia sono stati dipintı giardinı con Iontane di colore giallo - torse umitante sl bronzo - nelle quah zampilla dell'acqua $4{ }_{1}$ I bacini non sono plu decoratı con rillevi al modo del crater neoattic e non sono nemmeno sorretti da sfingı o centaurı (cf $p$. 63)

Le uniche figure statuarie sono le erme, presenti in pitture tomball, che fanno parte della siepe di un giardino come elemento di un Hermenzaun Il giardino in questo contesto rappresenta l'Eliseo dove $v$ ivono 1 defunt 1 Nel catalogo della monografia di Wrede su Welschbillig, citata precedentemente ( $p$.

458 Schmidt-Colınet 1977, 95-97.

459 von Hesberg 1983

460 Strocka 1977, 88-101, Abb 207-211, 216-219; Peters 1965-1966, 131-136, fig. 9-10, pl. XIV-XV; Peters 1979, 396-397 Cf Joyce 1981, 57.

462 Baccins Leotardi 1978, 15-17, fig 4-7, tav. I-IV 
(39) glı esempı fmord notı sono statı elencatı und tomba ad Alessandria (cat 001), datatd nel prino secolo d C , e tre decorazioni del quarto secolo a Nis (cat 127) e Roma (cat 322, 323) Vessuna delle erme e stata eseguita in modo dettagliato cosicche non s1 puo concludere se esse siano 1 ritratt del mort 1 o meno Vella tomba alessandrina un'erma e caralterizzata come immagine dı Pan, Pridpo o Hermes mediante la presenza dı oggeltı appesı al fusto

\section{SCENE FIGURATIVE}

Si osserva che nell'epoca imperiale la presenza di scene figurative, specie nei pinakes cosi indispensabul as sistemı 'pompeiani', diminuisce in modo notevole, riselarido cosı un calo dı interesse per tah elementı figurativi I paesaggı rimangono presentı sotto forma di vienette e quadretti, non diveral dal pinakes, ma di lado essi hono di dumension grand, mentre 1 singoli elementi qualı edifici, alber e figure umane si presentano privi di dettagli e in modo schematico Anche le statue spesso sono solo delle strisce

luove nel secondo e nel terzo secolo sono le rappresentazioni figurative che coprono un'mtera parete o la maggior parte du essa cenza tener conto della suddivisione tiddizionale in tre zone la ben nold decorazione in una casa sotto la chiesa di SS Giovannı e Pdolo sul Celıo a Roma llustra chiaramente questo tipo ${ }^{462}$ Molte delle decorazioni discusse in prosieguo decorano camere tombalı

L'unica scend mitologica importante per la nostra ricerca proviene da una tomba dell'epoca severiana trovata presso la Basllica di $\mathrm{S}$ Paolo tuori le mura a Roma (cat 327) Prometeo mostra ll bozzetto fittule di un uomo nudo ad Atena La figura del'uomo " stata modellata in un atteggiamento rigido, quasi di un kouros in questa scena abbiamo una bella umpressione della bottega di uno acultore antico come si vede ugualmente sul pinax con Vedalo e Pasilae a Pompel (cat 192 1)

$E^{\prime}$ controversa la datalione $d_{l}$ un pinas $d_{l}$ provenienza ignota, ora nel Museo Vazionale Rommc (cat 333), nel quale si vede un atleta del tipo westmacott che si incorona Benche la figura sia rappresentata viva, si constala una netta sumlarita con l'archetipo scultoreo, usato anche su rappresentazionı di tıgure sportive a Pompel (cat 26i) Borda, Tellettı Mal e Mor eno pensano ad un'esecuzione della pittura nellepocd adrianea, mentre Mielsch preferisce una datazione piu alta coe nel IV stule, suggex mento che eglı I jesce a corıoborare con alcunı buoni confrontı

vell'I seo sull'Aventino a Roma, costruito intorno al 200 c stato trovato un pinax con una scena rituale (cat 318) Iside si presenta nelle vestı di Fortuna - come si rede gia a Pompel - e regge una cornucopia nella destra e una patera nella sinistra

Due decorazionı tombalı dell'inizio del terzo secolo fanno vedere bambinı nell'Elıseo Sulla scend proveniente da Ostia, ora nei Musei Vaticanı (cat 136), essi presentano offerte ad Artemide che e stata rappresentata come l'Artemide nel Louvre attribuild a Leochares La dea avanza, mentre prende con la destra una freccia dalla faretra posta sul dorso e porge l'arco con la simstra In un roveto nella pittura nella Tomba degli Octavil a Roma (cat 32J) I bambinı stanno presso la statua di Lcate triforme

Per quanto riguarda I paesaggı sacro-ıdulhacı dobblamo menzionare alcunı esempi dell'epoca imperiale La Iomba del Pancratı lungo la Via Latina (cat 328) e stata oggetto di studıo da parte di Janına Kacena Darling Le lunette sono state abbellite con papsagg pienı deglı element 1 consuetı, analizzatı 
scrupulosamente da Dirling Le statue in generale sono schematıche ma si riconoscono una aufgelehnte Muse, tipo usato per una Akte, e un Poseidon lisippeo Un frammento nell'Antıquario Comunale a Roma (cat 332) del quale la provenienza e la data $d_{i}$ esecuzione sono ignote, potrebbe datare nello stesso periodo Contielie la statua di una figura maschule che regge due flaccole e non differisce molto dalla statua di Ecate lon e chiaro quale divinita sia stata rapppresentata

Ia tomba di Calvano presso Arpolı (cat 007) contiene due pareseggi abbastanza grandı e dettagliatamente dipmts che decorano le lunetle, e alcum quadrettı al lato inferiore della volta Le statue 11 presentı sono sottul e rappresentano tra l'altro Priapo La datazıone del complesso nell'ultumo quarto del primo secolo, pioposta da Elia nella prima pubblicazione, sembra troppo alta I confront presentati non riescono a convincere ll lettore Piu veroumle e la datazione nell'epoca adrianea accettata da Wirth, Borda e Suberberg

La cosiddetta Ded Barberini, ora nel Museo lazıonale Romano (cat 312), deve provenire dalla Domus Faustap nel Laterano, dove nel 1962 sono state trovate tracce di altre partı di decorazıonı eseguite nel medesumo stule e delle stesse cimensionı monumental $S_{1}$ tratta $d_{1}$ una composizione figurativa, purtroppo non del tutto chiara, con la statud di culto della clea Roma esposta nel tempio di Venere e Roma dopo l'incendio del 307 Vella tigura sono stati combinatı elementı sconografics di Venere (puttı, delfins) e Roma (armi) La tigura ieratica fd ricordare le enormi statue cultuali di fidid ad thene $e$ Olimpia

Intine va ricordata la decorazione nel pronao del tempio di Bel a Dura Europos (cat 013) Nel 239, o poco dopo, l'ufficlale Julius Terentius a i suol mulitarl hanno dedicato questa decorazıone che commemora una loro offerta alle divinita protettrici della citta A destra si vedono 1 soldati, nel centro Terentius e a sinistra le divinita, sistemate in due registri lella rona inferiore si trovano due statue delle. Tychal di Dura Luropos e Palmira, riconoscibli grazje a epigrafi greche Sono state modellate secondo il tupo classico della Tyche di Antiochia di Eutichide Molto precisı sono 1 dettagli qualı il fiume, la posizione delle gambe e la corona a torma di citadella Vellepoca imperiale questa iconograila diventa quella comune, specie in Griente Conosciamo una serie di statuette che imitano taliolta in inamera liberd, talvolta precisamente, la creasione di Lutichide 463 lel registro superiore $v$ sono tre statuette di dir mita maschili, vestite come soldati romanı che trovano riscontro in statuette di Marte

\section{CONCLUSIONI}

Benche non esista una netta divisione fra la pittura parietale pre- e postpompeiana - 1 Romani naturalmente non l'hanno sentita in tale modo - vi sono argomentı valıdı per una discussione separata del due periodı Dopo la fase tlavia (in complessi rappresentativi e palaziali) si constata un interesse minore per la pittura parietale come genere decorativo I decoratori si basano sul repertorio del IV stıle senza aggiungere nuovi elementı Tra le plu usate troviamo decorazionı a campl e siatemi ripetitivi dettı motivi di carta da parato Pochı elementi figurativi e pinakes ravivano le decorazioni completamente dommate dalla geometria e dalla simmetria il parte 1 pinakes le figure isolate fungono da vignetta o come decorazine di candelabri posti sulle lesene fra 1 pannell Soltanto di rado figure reggentı o acroterialı abbelliscono le architetture dipinte, peraltro ridotte in numero $e$ volume. Alcune rappresentazionı di giardinı contengono delle erme

${ }^{463} \mathrm{Cf}$ Spatantike 1983, 481-483 no 84 
Per quanto riguarda i tipi deve essere chiaro che le decorazioni postpompeiane non aggiungono molto al vasto repertorio del periodo precedente. Interessanti sono le divinita di citta a Dura Europos (cat. 013) e Roma (cat. 312), quella ultima raffigurante la statua di culto nel tempio di Venere e Koma. Le statue delle Parche nelle tombe ostiensi (cat. 131. 133) rimangono senza conf ronti. 
Le rappresentazioni di statue nella pittura parietale romana finora non sono state oggetto di studio. La presente ricerca ha cercato di colmare questa lacuna. Obiettivo è stabilire in quale modo la pittura possa fornire dati per la nostra conoscenza della scultura antica. I vari aspetti della scultura sono stati discussi, se presenti nelle statue immaginarie delle pitture parietali. Riassumiamo i risultati seguendo l'elenco di obiettivi presentati nel'introduzione ( $p .13$ ), cosi da vedere quali di essi siamo stati in grado di concretare.

\section{1) ARCHITETTURA}

La categoria piú grande é costituita dalle figure plastiche inserite in strutture architettoniche (cap. l). E' stata accostata al materiale finora noto nella vera architettura. Due sono i gruppi principali che richiedono il nostro interesse: le figure reggenti e le statue esposte entro nicchie o edicole o sopra mensole - su un podio. Negli stili II, III e IV si adoperano su larga scala figure umane e fantastiche in guisa di atlanti e cariatidi.

Il II stile, quello chiamato architettonico, fa vedere l'intero repertorio dell'architettura ellenistica. I pochi edifici di quest'epoca conservati mostrano una presenza abbondante di elementi plastici fra i quali statue, erme e rilievi. Le decorazioni di ll stile, che peraltro contengono imitazioni molto precise di elementi architettonici quali capitelli, colonne, e presentano sistemi decorativi quali la scaenae frons, anche in questo caso sono una fonte di valore inestimabile. $E^{\prime}$ stato messo in evidenza il parallelismo fra la pittura e l'architettura, ció in netta opposizione con le opinioni finora accettate.

Durinte i perjocli dol II, del IV stilo o nella pittura postpompeiana i pittorj seguono in modo meno ledele questa tendenza. Il linguaggio lormale si adatta al gusto contemporaneo, mentre al tempo stesso non si abbandona completamente la tradizione. In tale modo le decorazioni di questo periodo forniscono pochi dati concreti, pur arricchendo la nostra conoscenza su aspetti poco noti. Ricordo a questo proposito l'affinita lia le decorazioni ercolanesi e la produzione metallica, notoa attraverso mobili e utensili. A differenza dell'aschitettura reale, nella quale va crescendo l'uso di elementi plastici - parallelanente alla tendenza barocca -, la pittura dopo il 79 contiene sempre meno frequentemente elementi scultorei. Questo si spiega con lo sviluppo generale della pittura che si semplifica nel suo insieme, attraverso soprattutto dei sistemi parattatici, costituiti da pannelli, o da scene figurative che coprono l'intera parete.

Per quanto riguarda le varie zone orizzontali (zoccolo. zona mediana, zona superiore) si osserva una fedele riproduzione della pratica edilizia. Lo zoccolo e adornato in maniera sobria con figure reggenti. Vediamo degli uccelli. delle sfingi e delle figure satiresche che sorregono l'orlo superiore dello zoccolo, categorie che trovano riscontro nella vera architettura.

Nella zona mediana la figura reggente piú diffusa é l'erma, Lalvolta a nezzo corpo. Si incontrano imitazioni di portici, controntabili con esempi contemporanei. Le figure completamente plastiche sono meno popolari.

La zona superiore è sempre riccamente ornata con ligure statuarie. $E^{\prime}$ evidente il parallelismo fra pratica edilizia e pittura, dal momento che le statue sembrano staccarsi dallo sfondo perche i pittori vogliono imitare l'effetto delle statue che si inclinano verso lo spettatore per ottenere un maggiore rilievo. 
Le figure non inserite in una strutturd architettonica mostrano la pratica nornale di esporre statue In generale tutte le nicchie e le edicole sono occupate da statue, sla nel vero sia nel mondo immaginarıo della pittura

La pittura parietale e la fonte principale per la nostra conoscenza della collocazione di statue sopra mensole $E^{\prime}$ da notare che il repertorio delle ligure esposte in tale modo e lumitato Muse e guerrieri nudi del tipo 'ideale' policleteo sono piu numerosı di altrı soggettı Gli ambientı decorati in tale modo sono da interpretare come musaea e rispecchiano gli interessi culturali e forse anche morali a proposito des guerrieri, nel senso di sunbolo dell'inmagine ideale - des committenti

\section{2) RILIEVI}

I fints rlievı erano trascurat, quası completamente in precedenza (cap II) $E^{\prime}$ stata messa in evidenza la loro importanza per lo studio deı ver r ruevi Glı esempi dipintı informano in modo preciso sull'inserimento entro le strutture murarie Lastre marmoree a rulievo costituiscono un genere prezioso di oggettı da collezione Cio si constata non solo sulla base del rulievi conservatr, del quali raramente si sa la collocazione originale ma anche dalla posizione del finti rlievi entro l sistemi decorativi Si tiovano sempre nella zona mediand da considerare la zond principale - o in una tascia fra le zone mediana e superiore Talvolta adornano le cornici di una porta tinta Purtroppo le rappresentalioni Visıbl, sono troppo schematiche e prive di dettagli per essere controntate con des veri rulievi - che inoltre sono abbastanza rarı L'analisı dei fints julev a soggetto paesistico a permette, pur con una certa prudenza, di concludere che essi si trovano in una tradizione ellemistica, torse gia classica $e$ che non costituiscono un'innovazione puramente romana Il genere del rllevo paesaggistico deve essere stato noto gia pruma del I stule Queste rappresentazionı rattorzano le ipotesi tinora discusse a proposito di un origine orientale Non si puo slablire Il luogo d'origine

\section{3) GIARDINI}

Le rappresentazionı dı giardını, genere popolare nella pittura murale romana. rispec chiano completamente la pratica reale (cap III) le statue esposte nci verl flasdini appartengono alle medesume critegorie si tratta di figure dell'ambito dionisidico, deçuatico e di divinita che custodiscono la fertilita Le tontane rappresentano sia le divinita acquatiche che l'amore per l glochi acquatici I datı che possiamo recuperare, valutando le fontı scritte, vengono convalidatı dalle pitture, come e l caso del materiale trovato nei veri giardini, specie in Campania

Le statue dipinte non sono la chiave per un'interpretazione conclusiva del genere, tanto discusso negli ultum decennı Rimane aperta la questione del significato religioso o meno dell'iconografia topiaria Riguardo alle numerose figure dell'ambito dionisiaco non possiamo supporre in modo troppo assoluto una flosofia popolare che si sarcbbe espressa in modo positilo sul ialore della ita attuale, la cosiddetta dionysische Diesseitsfreude, in quanto la presenza di motivi bacchicl presenti ov unque nella pittura non permette un'interpretazione specifica per le figure del genere nel contesto topiarıo 


\section{4) MODELLI SCULTOREI}

Lin soggetto complesso e di non facile soluzione $\dot{e}$ la determinazione dei prototipi che sono serviti da modello ai pittori (cap. IV). La bidimensionalita propria della pittura che poteva ostacolare una riproduzione fedele, specie per un pittore meno abile, e la possibilita di non dover copiare la figura nei clettagli permettevano ai pittori di interpretare liberamente il modello scelto. Inoltre il fatto che tuttora non disponiamo di tutto il materiale che era a disposizione dei pittori, non consente di esprimere giudizio sul carattere fantastico delle statue dipinte. Tuttavia si riesce facilmente a determinare $i$ modelli scultorej di molte statue dipinte. Nello stesso momento i possibile, anche per le figure non determinabili, distinguere tra i periodi storici (arsaico ecc.) e talvolta specificare una corrente quale il classicismo o lo stile di un particolare scultore (Policleto, Lisippo). In un'appendice si trova un elenco delle statue note, delle varianti e di possibili altri tipi, divise per periodo storico.

La categoria di statue romane è ristretta a un piccolo numero di casi. Statisticamente l'Elenismo supera in assoluto gli altri periodi. I tipi arcaici sono scarsi, ma nello stesso tempo si constata un grando numero di ligure arcaistiche o arcaizzanti. Il quinto secolo, il perindo classico, o presente con opere d'impronta policletea: si osserva una prelerenza di ligure maschili nude 'ideali'. Solo di rado uno scultore puó essere determinato nel dettaglio. Vel III stile lo Stile Ricco è prediletto: le cariatidi nella zona superiore spesso sono simili alle Aurai e Nikai di Timoteo, Scopa ed altri maestri del periodo. Un altro scultore del quarto secolo che ha ispirato $\mathbf{j}$ pittori è Lisippo di cui numerose opere possono essere rintracciate nella pittura parietale. Questo notevole influsso e parallelo a quello in altri generi dell'arte e dell'artigianato. Per il resto non e lecito legare nomi di scultori a determinate figure dipinte.

La raccolla di una grande quantita di figure per ogni stile che costituisce il catalogo, permette di comprendere quale fu lo sviluppo del gusto dei Romani in alcuni periodi artistici (cap. V). Si constata una preferenza legata ai tipi ellenistici durante il II stile, mentre il III stile è caratlerizzato da una forte tendenza classicista ispirata dallo stesso imperatore Augusto. Talvolta tipi arcaizzanti o arcaistici sono alle spalle di figure classiciste. Nel IV stile un amalgama di tutti i periodi impedisce una determinazione precisa del gusto.

Meno chiara, a questo proposito, è la situazione della pittura postpompeiana (cap. VII) che ci ha tramandato una quantita notevolmente minore di statue dipinte, anche se i complessi pittorici considerati, sia in relazione al periodo precedente che in assoluto, sono pochi; inoltre tutto il materiale copre un periodo di quasi cinque secoli. Certamente si puó riconoscere, in alcuni casi, lo stile piú seguito e il prototipo, ma la particolare situnzione di ogni complesso non permette considerazioni generali.

\section{5) POLICROMIA}

La questione della policromia delle statue antiche non puó essere risolta soltanto attraverso il materiale statuario a nostra disposizione. La pittura si presenta come una fonte indispensabile per la conoscenza dei vari lipi di policromia e del trattamento della superficie di statue bronzee (cap. VI). Specie le statue topiarie rivelano la preferenza della policromia parziale, forse per la loro posizone nel mezzo delle piante. Le figure inserite nei sistemi architettonici spesso sono completamente colorate fornendo effetti di grande realismo, riproducendo persone presenti nellambiente reale, mentre si trovano altrettanto facilmente figure grige o bianche indubbiamente imitazioni di statue marmoree. In alcuni casi si puó osservare l'uso di marmi colorati secondo un 
gusto che teste Plinıo ( $\mathrm{NH}$ XXXV 1, 3) avrebbe avuto inizio nel regno di Claudio a che e diventato veramente popolare nell'ultumo quaito del secondo secolo

Per quanto riguarda le siatue di bronzo si possono distinguere tre modi per ll trattamento della superficie doraturd (molto frequente), patina verde e vernice rossa II rosso corrisponde all'antıca ganosis, Il verde invece indica un'ossidazione che deve dare una prova dellantichita della figura - forse elemento di umportanza nell'interpretazione Dopo ll marmo ed 1 bronzo $\downarrow$ l legno appare I mateisale preferito Questo si usa pei determinate categorie di statue, cioe per immagini di culto, gli xoana, e per lo statue di Prapo l'argento deve essere itato un materiale molto raro, prezioso oppure poco popolare, in quanto il numero di statue argentee e iddotto Altri materiali, come l'dsorio, sono stati raramente imitatı

\section{6) PINAKES}

Le figure vive nel pinakes non sono state analizzate tutte quante, ma e chiaro che in non pochi casj incontriamo famosi tipl scultorel Specie Afrodite e Dioniso rupetono tipı statuarı, ma anche Artemide bagnante e Leda con 1 cigno sono da associare con statue note da molte copie Puo darsi che un'andisi plu approlondita e completa posia fornire del risultati importanti, visto che 1 pochs casi collegatı linos a hanno dato esito positivo

\section{7) STATUE O FIGURE VIVE}

Una risposta al problema della distinzıone fra figure 'vive' c figure statuarie e in pruno luogo legato alla necessita di stabulire del criter ( $p$ ), quali la presenza di piedistall, la lunzione del reggere e ll colore Pero, se le figure sono, rispetto a (uno dı) questı criterı, da considerare statue cjo non ruole dire che esse siano concepite esplicitamente come statue spesso si vedono elementı eterogenes alla scultura nclle tigure esposte sopra mensole o piedistall, inserite nell'architettura e cosi via Esempio lllustrativo costituiscono le erme in guisa di atlantı che sono state raffigurate come esseri vil a mezzo corpo, uscenti da un fusto quadrangolare La distinzione fra statua e figura 'viva' inoltre e difficie, poiche c1 sfuggono moltı elementı come nel caso di figure non conservate o non riconosciute fmora nel repertorio statuario Dobbiamo tener conto della conoscenza maggiore della nostra che avevano 1 pittorı circa la sculturd Su quale scala essı abbiano fatto uso di modell scultore 1 o di fontı diverse, non e nemmeno ben chiaro $S_{1}$ entra nel delicato problema delle fontı iconogratiche des decoratorı in generale, dibattito inutule e senza fine in guanto non abbiamo a disposizione datı concretı a forma di cartonı. Bilderb acher e testı che lllustrino l'antica pratica

\section{8) COLLOCAZIONE E FUNZIONE}

Le decorazion architettoniche risultano importantı per quanto riguarda la collocazione e la funzionaluta delle statue Questo aspetto e stato discusso nel primo capitolo I giardinı dipintı rispecchiano in modo fedele la pratica topiaria (cap II) Le rappresentazioni figurative si dividono in tre categorie scene mitologiche, paesaggi sacro-ıdلliaci e vedute di ville, nature morte velle scene mitologiche le statue possono cardterizzare l luogo dell'avvenmento o 
sostituire la div unita che svolge una parte nella storia Talvolta fanno parte dell'arredamento degli internı di templi, palazzı e case

I paesaggi sacro-idilliaci e le vedute di ville marine rendono visıbil santuarı, tombe $d_{1}$ erol e vlle arredati con delle statue Non ritroviamo edifics concretamente individuabili, ipotesi da controllare in una ricerca dettagliata, ma su tratta sempie du generalizzazioni e schematizzazioni che cl danno un'idea piu o meno realistıca della pratica consueta

\section{9) COMBINAZIONI}

Discutendo ll significato delle varıe tigure scultorce e stata posta l'attenzione anche sulle combinazioni delle tigure $b_{1}$ constata un accostamento voluto ds figure con un atteggiamento simle, sia come pendants su und parete che come riscontro su piu di una parete La posizione seduta o in piedı, la posizione delle manı e della gamba di appoggio sono statı determinatı da regole di simmetria e parallelismo Una tale antitesi non puo sempre esseie osservata a proposito dell'iconografia (maschule'temminle, divinita in coppia e cosi $v i a)$, ma non puo nemmeno essere scartata completamente

\section{0)RACCOLTA DEI TIPI}

Un'appendice contıene un ınventarıo sistematıco, ordınato seguendo i perıodı storici, del tipı noti del modell con sariazionı e del tipl tinora sconosciutı, ma possibilmente importantı per la ricostruzione dı tipı notı dalle fontı

Insomma, la ricerca ha dumostrato che ld pittura murale costituisce una fonte di non poca importanza che non puo runanere mosservata da ch s studia la scultura greco-romana Specialmente a proposito di problemi qualı l gusto, lo stule e la tipologia, essa fornisce dati umportanti 
In deze studie wordt nagegaan in hoeverre de wandschilderkunst uit de Romeinse tijd te gebruiken is als bron voor de kennis van de Grieks-Romeinse beeldhouwkunst. Ook de als levend voorgestelde figuren waarvan de houding aan de sculptuur is ontleend, zijn in het onderzoek betrokken. Het feit dat de Romeinse wanddecoraties worden gekenmerkt door de nabootsing van materiaal, suggestie van ruimte en initatie van kunstwerken, is voor deze vraagstelling een belangrijk gegeven.

\section{INLEIDING}

In het kort wordt de geschiedenis van de Romeinse muurschilderkunst geschetst. Dit is van belang in verband met de chronologische ontwikkeling van het gebruik van geschilderde beelden en reliëfs. De vier 'stijlen' waarin de wanddecoraties uit de eerste eeuw $v$. en de eerste eeuw $n$. Chr. worden onderverdeeld (Mau 1882), zijn van belang voor de veranderingen in de toepassing van beclden in de decoratiesystemen. Ten aanzien van de beeldhouwkunst wordt in het kort dat onderzoek gemenoreerd dat van belang is voor het onderhavige onderwerp. Alleen de dissertatie van Astrid Pfretschner (1977) gat in op een gedeelte van de hier gepresenteerde problematiek, namelijk de godenbeelden. In vroegere publicaties werd slechts incidenteel op de relatie tussen beeldbouwkunst en schilderkunst gewezen; een alles omvattend onderzock werd, voor zover uit publicaties bliikt, tot dusverre niet verricht.

\section{HOOFDSTUK 1}

De geschilderde toneelfaçades en de daarvan afgeleide architectuursystemen bevatten een groot aantal figuren die als beelden zijn bedoeld. Soms fungeren zij als decoratie van nissen en aediculae. Meestal zijn zij onderdeel van de bouwkundige structuur in de vorm van dragende figuren (atlanten en karyatiden), hermen en acroteria. In alle stijlperioden zijn dergelijke figuren aanwijsbaar. Wat betreft de drie zones waarin de wanden in de regel zijn ingedeeld, kan men per periode verschillen constateren. In de sokkel komen plastische figuren zelden voor. In de derde stijl vallen vogels als dragende elementen op. De hoofdzone telt talrijke hermen in de imitaties van zuilengalerijen (met name in de tweede stijl) en in de prospecten tussen de panelen (vierde stijl te Herculaneum). Volledig plastisch uitgewerkte figuren zijn vrijwel uitsluitend in de bovenzone te vinden. Daarbij is de frequentie van dergelijke figuren in de verder zo lineaire scaenae frontes van de derde stiil opmerkeliikk.

Met name in nissen in de hoofdzone van decoraties van de tweede stijl zien we beelden op sokkels. Voorts treften we deze aan in aediculae in de bovenzone van schilderingen van de vierde stijl. Waar de hoofdzone in velden is onderverdeeld, tekenen zich daarop soms figuren af die op plastisch weergegeven consoles staan. Opvallend is bij deze figuren de voorkeur voor twee categorieën: Muzen en naakte krijgers naar voorbeelden uit de vijfde eeuw v. Chr.

Voor nagenoeg alle geschilderde situaties zijn parallellen in de echte architectuur aan te wijzen. Helaas zijn die voorbeelden niet talrijk en zelden 
goed geconserveerd Het thans beschikbare materiaal, hoe schaars het ooh is, stelt ons toch in staat de geschulderde voorstelingen als imitaties van bestaande architectuurvormen te harahteriseren H G Beyen (1938, 1960) en andere archeologen hadden tot nu toe anngenomen dat de geschulderde figuren uitsluitend aan de fantasie van de schldders waren ontsproten Het is dus mogelijh om zich met gebruihmaking van de geschulderde constructies een beeld te vormen van de rijh uitgevoerde pronkfaçades en nissenarchitecturen

\section{HOOFDSTUK $I I$}

Imitaties van reliefs vindt men ingevoegd in systemen ian de tweede still Datarnaast worden ze gebrulht als ondhanhelipk opgestelde urerstuhken in tunnvoorstellingen van de derde en de vierde still Le eerstgenoemde calegorie betrett met name afbeeldungen van sacradl-idylische landschappen in een of twee hleuren op panclen die met een unitatie-relieflijst zijn omgeven Deze voorstollingen hunnen cen schakel vormen in de geschiedenss $v$ an de ontwhkeling van het landschapsreliet dat we uitsluitend van Romemse exemplaren kennen, maar dat volgens sommigen een Hellenistische wellicht Alevandrinnse oorsprong heeft Aangezien de geschulderde reliefs geen nabootsıngen zijn ran ons bekende stukken - de afbeeldingen ziln reelal schetsmatig en summer -, hunnen zuj nict dienen on met zeherheid adn te tonen dat zil teruggaan op echte reliefs wel wordt duidelijh dat de geschulderde relsefs een tot nu toe net in de discussie betrohken, vroege groep vormen

De geschulderde relsefs laten voorts goed zien boe de echte reluefs in de wandstructuur en in de tumen en zulengalerijen werden ingevoegd, c $q$ opgesteld

\section{HOOFDSTUK III}

De in de eerste eeuw $v$ en de eerste eeuw $n$ Chr zo populaire tuinschulderingen sluiten in alle facetlen aan bil de echte tuinen in Campanie, die sinds hort systematisch onderzocht worden wat de beelden betreft constateren we een overeenstemming met het materiaal dat ter plaatse gevonden is De geschlderde figuren behoren tot een beperkt repertorre Aphrodite, Dionysos en ziln kring, Herahles en watergoden komen het meest voor Zelden rinden we beelden van dieren in geschulderde tuinen, en dan gaat het meestal nog om de dragers van fontembekhens wel ziln er soms beelden van dieren in lachtparken ( $\mathrm{g}$ paradeiso1) en landschapsvoorstellingen, meestal als aanduiding van een exotisch landschap lconogratisch leveren de beelden, zoals gezegd woinig nieuwe types Voor de interpretatie van de tuinvoorstellingen kunnen zij van belang zin De religieuze interpretatıe door geleerden als Schefold $(1962,1972)$ en Michel (1978) komt zwakker te staan, wanneer men bedenkt dat echle vegetatiegoden ontbrehen Hadden de Romeinse schulders een religleuze dumensie willen uitbeelden, dan zou de keuze eerder gevallen ziln op echte Romeinse liguren als Pomona en Sivanus 
In dit hoofdstuk zijn de voorbcelden die voor de verschllende categorıeen ran beelden goden, menselilke tiguren en dieren, zijn gebrusht geinventariseerd Vooropgesteld $71 \mathrm{j}$ dat een precieze vergelilhing moeilih is omdat de figuren op het platte v lah zijn weergegeven en omdat de schulder de wruheid genoot om ziln voorbeeld on te werken

Ondanks deze problemen is een annzienlipk aantal prototypen herhenbaar Hel blılht dat Aphiodite en Dionysos irllwel steeds naar voorbeelden uit de verde eeuw i Chr ziln weergegeven, dat geldt evenzeer voor de geschulderde beelden als voor de als levend voorgestelde tiguren Ten aanzien van andere goden is een zo duidelijke voor heur niet te constateren

Uit alle hunsthistorische periodes ziln modellen gecopreerd liet meest talrık tiln types uit de Hellenistische beeldhouwhunst, slechls incidenteel zien we archarsche modellen In zeldzame gevallen kan er verband worden gelegd met bil name bekende beeldhouwers werken van Polyhleitos en ziln school ziln in de talrilke voorstellingen van naakte hrijgers en atleten herkenbaar Het omvangrilke ocuvre van Lysippos is in de wandschlderkunst in verschillende typen terug te vinden

De houding van de figuren is net wlleheurig the hunnen een duidelipke vooi heur aflezen voor symmetrie en pendants hanneer twee figuren op een wand te zien zijn, worden hum armen en benen v rujwel altijd in symmetrie weergegeven Figuren op tegenoverliggende wanden vertonen steeds dezelfde houding in spiegelbceld Uok in detals blijkt de sterke voorheur van de Romeinse hunstenaars roor symmetrie, die men ook elders, vooral in de architectuur, han aantreften

In een appendix is cen lipst gegeven van de uit de beeldhouwkunst bekende typen en hun varianten en van de in de schulderkunst als beelden geharahteriseerde typen waarian in de beeldhouwhunst geen exemplaren zijn aan te hilzen

\section{HOOFDSTUK $V$}

Op grond van de inventarisatie van tvpes die in de schilderkunst zijn gebruiht, 15 het mogelilk na te gaan welke stujlen de Romemen bij voor heur hebben nagevolgd Weze probleemstelling is van belang voor het onderzoek van de Romeinse hopicen van Griekse originelen en voor dat naar de kunstoprattıngen van de Romeinen

Elke stijlperiode in de wandschilder kunst vertoont een eigen voorkeur voor zehere periodes in de Griekse beeldhouwhunst De tweede still stoelt op de Hellenistische traditie en laat met name types uit deze periode zien De derde stıll geeft blilk van een voorkeur voor figuren uit de vilfde eeuw v Chr. vooral uit de Strenge Stıl $(480-450)$ en de Rilke Stıl $(420-400)$ Dit is geheel in overeenstemming met het door keizer Augustus gestımuleerde klassicisme De verde stıl tenslotte is, wat de beelden betreft, evenzeer een amalgaam als ten aanzien van de andere elementen waaruit de decoratiesystemen zijn samengesteld, er is geen periode van de Griekse beeldhouwkunst die duidelijk domineert Archaiserende en archaistische invloeden zinn terug te vinden in figuren ian zowel de derde als de vierde stıl

De variatie in heuze san beroemde hunstwerken de opera nobilia, is niet omvangrilk wanneer een figuur op een opvallende plaats op de wand is aangebracht, mogen we aannemen dat men de bedoeling heeft gehad een hunstuerk na te bootsen Jergelipke figuren komen orenwel in geringere dantallen voor dan de 'secundar gebruste figuren 
Zeer belangrık is de schulderkunst als bron voor onze hennis van de polvchrome en van de materiadkeuze en -behandeling in de sculptuur wat deze aspecten aangaat heeft $P$ Reutersward $(1960)$ reeds de belangruhste gegevens verzameld Het blıkt dat nast volledige beschudering parucle polychromie veelvuldig voorkomt bij marmeren beelden Vooral tumbeelden werden vaak slechts ten dele van hleur voorzien Architectuurschuderingen bevatten, tenzij ze zelf monochroom zijn, voornameliyk iolledig gepolychromeerde beelden

De opperilaktebehandeling van brons is niet beperkt gebleven tot vergulden, al komt dat het meest voor Rode beelden rormen een bewijs voor de behancteling ran beelden met was (zg ganosis) en bitumen, die ze beter beschermen moet tegen invloeden van het weer Groen is een hleur die vooral bil bronzen beelden op sacrdal-ıdyllische landschappen te zien is Zeker wanneer archalsercnde liguren deze hleur hebben kan hieruit worden opgernatekt dat do klour iconografische betekenis heeft op deze wilze lat de schilder de ouderdom en eerbiedwaardigheid van een godenbeeld uitkomen

Hout treft men voord aan bij oude godenbeelden (xoana) en bll beelden van Priapos Zlver homt zelden voor vog zeldzamer zijn andere materialen 'Tenslotte kan blechts op een voorbeeld gewezen worden van een beckl in verschillende marmersoorten (cat 259) Dit is interessant in verband met een contemporaine mededeling van Plinıus (Naturalıs Hıstorıa $\mathrm{KXXV} \mathrm{1,3)}$

\section{HOOFDSTUK VII}

In een afzonderlılk hootdstuh zıjn de schulderıngen besprohen die dateren uit de tıld na de uitbarsting van de Vesurius in $79 \mathrm{n}$ Chr len han vaststellen dat de postpompeiaanse schlderingen geen meuwe ontwikhelingen te zien geven, maar dat de schulders voortgaan met hetgeen in de eerste eeuw $v$ en de eerste eeuw $n$ Chr is geconcipieerd.

Een duidelijhe voorstelling $v a n$ de geschlderde beelden kan men zich niet vormen. omdat er zoveel minder bewaard ziyn gebleven Bovendien betreft het schilderingen die uit con periode van vier ecuwen en ust alle delen ian liet Homeinse rolk stammen Met name itatistisch is daardoor de waarde van het materiaal in kwestle gering

Buiten de reeds uit de voorgaande perıode bekende toepassingen komen in de latere schlderhunst geen nueuwe voor Het valt op, dat de geschilderde pronhfaçades zo spaarzaam gebruık maken van beeldien, terwill toch de contemporaine bouwhunst zo rujh is aan sculptuurelementen Ook in deze perıode zien we talrujhe figuren op consoles, geschlderd tegen een neutrale achtergrond

Imitaties van relief $2 u$ in beperkt tot een paar pronhiazen in Neo-Attische trant op tuinschilderingen, die overigens voorts geen beelden bevatten De incidentele figuratieve voorstellngen zin zelden voorzien van beelden Op de sacraal-ıdyllische landschappen zin zowel de stoftagefiguren als de beelden nauwelips uitgewerkt 
Uit het voorgaande moge blıken dat het onderzoeh talruke gegevens oplevert die van belang tiyn voor de kennis van de Griekse en Romeinse beeldhouwhunst De wijze van opstellen van beelden in architectonisch verband en in tumen kan aan de hand van schilderingen duidelilk worden gellustreerd Nieuw zijn gegevens met betrekking tot de landschapsrehefs die in een rumer kader kunnen worden geplaatst door vergelijhing met de imitaties van dergelybe reliets in de muurschiderkunst van concreet belang voor de hennis van typen en varianten ziln de schulderingen op verschllende manieren. $Z_{1 j}$ geven de modellen compleet weer en verschatfen dus aanwijzingen voor de reconstructie van ontbrekende delen van bewaard gebleven beelden Een groot aantal tot nu toe nuet uit de echte sculptuur bekende typen kan worden geinventarıseerd, sommige llustreren kunstwerken die in de schriftelijke bronnen worden genoend, andere zijn mogelilk verloren gegane typen De ordening naar still en of beeldhouwers werpt een licht op de voorkeur van opdrachtgevers en schulders uit de Romeinse tijd voor bepaalde kunstperiodes Voor de kennis van polychrome en materiadbehandeling tenslotte blıjken de geschilderde becken een onmisbare bron 
Sı presenta una sinossı del modell scultorei trovatı nella presente ricerca [ssi sono statı divisi in tre categorie modell notı, variantı e modell nuovi (cioe modell non conosciutı finora) La suddivisione cronologica non e stata fatta in modo dettagliato, cosa umpossibule per le statue dipinte Le correntı arcaizzanti, arcaistiche e classiciste sono state inserite rispettivamente nei perıodı arcaici e classici Le opere di Polıcleto e di Lisippo. le statue di Muse. le erme a mezzo corpo e 1 rulievi sono statı elencatı a parte vista la loro quantita. I numerı rimandano al catalogo

\section{ARCAISMO}

1) Opere note $203 / 1$

2) Variant $0101,016,038,140,152 / 2,159 / 1,161 / 2,163 / 1,194,195 / 1$, $266^{\prime} 2,269,2,305 / 1,317 / 3,319 / 3,329,337$

3) Opere nuove $0044,009 / 3,009,5,009 / 7,157,182,1,198 / 2,231 / 3,269 / 3$, $275 / 2,3194,3413$

\section{QUINTO SECOLO}

1) Opere note $006,2,054,091,200 / 2,204 / 2$ (tutte di Agoracrito)

2) Variantı $059.060,138,144,148 / 4,163,5,187,245 / 2,252 / 1,301,1$, $3051,319,7$

3) Opere nuove $010 / 4,038,128,158 / 2,158 / 3,305 / 1,317 / 1$

\section{Policleto}

1) Opere note 121. 197/1, 238, 267, 333

2) Variant $1024,070,102,119,146,155 / 4,177 / 1,178,197 / 1,198 / 3,204 / 1$, $204 / 6,207 / 1,253,286 / 2$ (guerrierı nud1), 48, 191, 209/3

\section{QUARTO SECOLO}

1) Opere note 008, 048, 066, 072, 128, 130/2, 146, 154/1, 166/1, 169, 190, $1912,192,197 / 2,215 / 2,217 / 9,219,263,288,340$

2) Varıantı o $77,146,147,4,146,173,187,199,207 / 2,213 / 1,220 / 4,234 / 2$, $245,1,267,269 / 4,303 / 3,326,335$

3) Opere nuove $303 / 1,308,319 / 4$

\section{Lisippo}

1) Opere note. 009/9, 010/5, 014/1, 092, 189/3, 193, 204/1, 221/1, 239, $282 / 7,319 / 6,328$ 
2) Varianti. $009 / 6,039 / 2,067,098,156 / 3,161 / 2,163 / 5,164,261 / 3,267$, $269 / 5,295 / 6$

\section{ELLENISMO}

1) Opere note. 004/5, 011, 019,031, 032'3, 035/4, 040, 014, 053, 114, 115, $117,118,135,148,3,149 / 2,151,152,3,160,1612,166 / 2,171,172$, $181 / 1,181 / 2,1821,185 / 1,185 / 2,186 / 2,188,1953,198 / 3,2201,224$, $227.231 / 1,231 / 2,231 / 3,239,245 / 5,251,261 ' 2,2623,262 / 8,267,2695$. $274,275 / 1,278,2793,282,7,2827,284 / 2,284 / 3,292,294,305 / 3,3061$, $316 / 5,328330$

2) Varianti. $009 / 6,009 / 8,010 / 3,0354,048,066,106,128,153,1,202 / 4$, $202 / 5,202 / 6,2027,207 / 2,217 / 9,261 / 6,267,295,1,309,319 / 2,326,336$

3) Opere nuove $010,1,010,5,026,053,070,098,121,1 \mathrm{G} 1 / 2,179 / 2,185 ' 2$, $197 / 3,204 / 6,2083,225 / 1,267,3053$

\section{Muse}

1) Opere note. $161 / 2,262 / 8,294,328$

3) Opere nuove $014,1,014 / 2,015,017,018,130 / 1,232,261 / 5,262 / 8$, $286 / 3,305 / 3,306^{\prime} 1,317 / 1,319 / 7,336$

\section{$H^{\circ}$ ufthermen}

1) Opere note. 238

3) Opere nuove $012,038,106,126,148 / 1,148 / 2,1634,180,197 \% 2,233,2$, $237,262 / 2,262,7,2811,289,325,334$

\section{Rilievi}

3) Opere nuove $0042,04 / 3,004 / 4,148 / 3,149 / 3,152 / 3,161 / 1,163 / 6$, $209 / 1,209 / 2,220 / 2,262 / 6,277 / 2,308,316 / 1,316 / 3,317 / 2,341 / 2,341, j$

\section{PERIODO ROMANO}

1) Opere note. $139,179 \prime 1,180,221 \% 1,245 / 2,245 / 5,2624,304,312$

2) Varianti. $009 / 4,013,138,204,2,284,3$

3) Opere nuove. $043,046,047,131,133,160,163 / 3,174,208^{\prime} 1,235 / 2,254$, 259 
ARTE EGIZIA

2) Varianti. 146

3) Opere nuove. 155,1, 155/3, 161/1, 212,1, 315, 328 

PARTE II: CATALOGO 

Il catalogo segue l'ordine alfabetico per citta (indicate con 1 nom italiani) In ognı citta dove esiste un ordinamento del complessı in regiones, insulae e case (Eteso, Ercolano, Ostıa, Pompel e Roma), questo e stato rispettato Glı eventualı (sopran)nomı degh edificı sono statı indicatı, I nomı del pelsonaggı storici sono statı (itati in ortografia latind In ogni complesso l'ordine c la numerazione del vani sono quelli esistentı per Pompes per esempio si segue Il Repertorio, per le altre citta le pubblicaziom archeologiche pincipali Dove posibule, le parets sono state mdicate secondo l'orientamento geogratico altrimentı sı citano le indicazions delle pubblicazionı ( $p$ e 'parete di tondo'. 'parete $C^{\prime}$ ) I trammentı di pittura custoditı nel museı sono statı discussi nell'ordine del numerl d'inventario Per l framment 1 del qual la provenienza e nota, si trovano rinandi as complessı dove sono statı inseriti ( $p$ e Inv no $9551>$ Pompes VI 9, 6, cat 204,2) Inche gruppl di trammenti che fanno parte della medesima decorizjone, ma di provenienza sgnota. sono statı descrittı insieme, per I singolı [rammentı si trovano sempre rimandi al numero rispettivo ( $P$ e lnv no $9542>\operatorname{lnv}$ no 8947 , cat 060 )

Ognu parete viene descritta secondo l sistema consueto, cioe dal basso verso l'alto Per quanto possible e stato indicato ll colore della figura e degli attributı Sono sempre statı citat 1 controntı scultore per rigjonı di brevita non i jene ripetuta ognı volta la pubblıcazıone rıguardante il modello, se questo appare frequentemente, $m a$ ci si riferisce ad un'altra descrizione nel catalogo Per lo stesso motivo' si tiene presente che 'base piedistallo a forma $d_{1} T^{\prime}$ indica una base composta da un plastro e una lastra orizzontale non rappresentata in modo correttamente prospettico, ma schematico Cosi segmento ovoidale/a forma di parallellogramma/trapezıo' indica una mensola schematizzata

(Igni descrizione consıste di tre partı

1) numero di catalogo, collocalione (vano fra parenlesi), datazione $e$, eventualmente, indicazione che la decorazione e andata perduta (+),

2) descrizione con eventualı commentı e riterimentı a controntı,

3) bibliografia

La datazione se non argomentata nel testo, e basata su quella proposta dalle pubblicazioni usate La descrizione puo essere letta indipendentemente dalla discussione generale e lornisce tutt l dati specificl Velld descrizione si segue, quando e possibile, l'ordine logico di posizione, atteggiamento delle gambe e manı, veste e attributi, interpretazione (per figure non direttamente riconoscibli)

I riferimentı biblıograficı sono ll piu possibule completı per 1 complessi non pubblicati di recente in modo esaustivo Quando, invece, disponiamo di un catalogo descrittivo con una bibliografia completa, si rimanda ad esso, citando soltanto le pubblicazıonı mancantı e apparse succesıamente, pubblicazıonı precedenti non sono piu state citate purche non siano rilevanti per le statue dipinte Per la Villa della Farnesina a Roma, per esempio, si rimanda a Bragantini/De Vos 1982, per Pompel a Schefold 1957 e Repertorio Von sono state citate opere generalı se non per specialı motiv qualı llustrazionı mighor o osservazioni rulerantı 
ABBREVIAZIONI E SEGNI CONVENZIONALI

inv.no.: numero d'inventario

MN: Museo Nazıonale Aapoli (, inv.no.)

s.n.: senza iumero d'inventario

t: decorazione ora perduta 
Musee greco-romain

Cat. 001. Inv. no 27030; epoca flavia

La parete esterna nord di una tomba e stata decorata con una rappresentazione di un glardino circondato da un cancello Un palo e stato sostituito da un'erma la testa di un uomo barbuto puo rappresentare Pan, Priapo o Hermes. Il fusto e stato ornato con un tallo

Riad ha proposto una datazione nel primo secolo a $C$, mentre glu altrı studiosi preteriscono una datazione piu bassa, cioe nellépoca tlavia, il che sembra preferıble vista l'esecuzione clella decorazione

Bibl : Von Blanckenhagen 1963, 146; Riad 1964; Schefold 1965, 120-121. Riad 1967, 95, pl III, B; Wrede 1972, 132 no C1; Carroll 1984, 216-217 
Akademisches Kunstmuseum

Cat. 002. Inv. no. B 275; IV stile

Frammento di una rappresentazione di un'architettura, proveniente dalla Campania, forse da Pompei. Una sfinge grigia con chioma gialla sormonta un architrave. Sulla testa poggia un candelabro vegetiale. Il frammento costituisce la parte destra di un pannello o edicola.

Bibl.: Melsch 1971, 32-33 no. 44, Abb. 43. 


\section{BORDEAUX}

Allees de Tournay

Cat. 003. Frammento sporadico; III stile

Paesaggio sacro-idilliaco nel quale sorge la statua rossa itifallica di Priapo. Il dio sta nell'atteggiamento della chordosis.

Questa rappresentazione viene separata da un'altra mediante un candelabro incoronato da un grifo. Solo i piedi sono rimasti conservati.

Bibl.: Barbet 1983, 135, 137-138, fig. 16; Barbel/S Avault-Debbich 1983, 22, pl. A, fig. 18-19. 


\section{BOSCOREALE}

Villa de $P$ Iannus Symistor

Cal 001,1 (posistlo) 'I utte le pareti, zona superiore, Il stle, lase Ic +

Una cornice doppla consiste di due fughe di mensole tra le quals sono stati applicatı alternamente elementı a forma $d_{1} S$ e centaurı verdı I centaur sono poggiatı sulle zampe posteriori e sorreggono con una mano la mensola sopra la loro testa

Bibl Beven 1938, 334, Abb 77, 102, Lehmann 1953, 9-10, fig $7-8$.

Cat 004/2 (3) Parete ?, zona superiore, II stle, fase Ic (ora Mariemont, Musee de Mariemont inv. no. 98, 98bis)

Al lato superiore della zona mediana corre un fregio violaceo con figure blanche di tritonı, nereidı e amorinı, che umitano un fregio in rilıevo, sumle a quello nel vano $F$ \& $G$

Sopra la cormice su vede un paesagglo monocromo in rosso, di nuovo usı'imitazione di una lastra marmored scolpita in rilievo. Su un tempio tetrastuo stanno tre statuette schematiche $\mathrm{Ln}_{n}$ altro edificio viene sorretto da una cartatide classica

Bibl. Beyen 1938, 223-227, 244-245, Abb 92, Lehmann 1953, 15-16, fig. 12-13, $M$ Kenard in Marlemont 1952, 149 no $R 59-60$, pl 54, Peters 1963, 11, fig 4-5, Mariemont 1966, 31-32, pl 47 (a color1), Slberberg 1981 , no 3 , fig 2

Cat 004/3 (F e G) Tutte le pareti, zona superiore, II stle, tase Ic (ora MN $s$ Il Marjemont. Vusee de Mariemont inv no 96)

Lna cornuce a doppie mensole separa la zona mediana e la zona superiore. Figure gialle stamno sulle mensole interıorı e sorreggono quelle superior Nel vano $G$ alternamente menad vestite di chitonı cort 1 , satirı danzanti e centaurı che si reggono sulle zampe posterior ravivano la cornice. Vel vano $F$ si vedono solo centaurı con pledı a torma di tralcio

La porta nel centro della zona mediana del trammento a lapoli e incoronata da una lastra nera con l'imitazione di un rilievo Su uno sfondo viola si dolineano guerrier dipinti in colori vivaci. Il rulievo entra nel genere del pezı da (ollezione come anche 1 fintı rilıevi nella Casa del tauno (cat 209) e

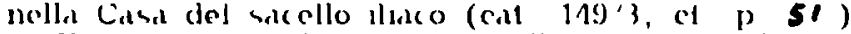

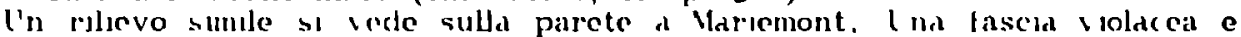
ornata con tritonı, nereid $e$ amormi bianchi. Sono simll alle ligure nel vano (3)

Sopra la porta centrale del trammento napolitano, fra due pilastri, si trova un pilastro con un capitello cormzio sul quale sta un Eros, che sembra volare nel cielo Solo la parte inferiore del corpo e una parte delle alı eccessivamente lunghe e di una clamide sono rimaste conservate. Il peso del corpo poggıa sulla gamba destra

Bibl Beyen 1938, 94-95, 114, Abb. 22, 203; Lehmann 1953, 19, 21, fig. 17-18, $M$ Renard in Mariemont 1952, 148-149 no R. 56 , pl. 54: Schmidt-Colınet 1977, 145-146, 266 no P3, Von Hesberg 1980a, 134, Taf 20 .

Cat 004/4. (M) Tutte le pareti, zona mediana; II stile, tase Ic (ora New York, Metropolitan Museum) 
I prospett 1 contengono vedute di citta e dl un paesaggio Il campo centrale della parete sinistra contiene un paesaggio con una porta sacra occupata dalla statua gralla di Ccate Indossa un chitone senza maniche e regge nelle mani, distese per meta und llaccola La figura e stata eseguita in stule arcalzzante

Nei due campi laterali con vedute di una citta $e$ di un bosco vi sono due statur sumli sopra un pilastro, ora di folmato ridotto

La parete di fondo contiene a destra della finestra un paesaggio sacro-ıdilliaco Presso una fonte e una grotta sta una statuetta di Ecate simule alle tre clella parete sinistra

Il lato destro delld finestra vene fiancheggiato dall'imitazione di un rilievo guallo circondato da una cornice a rilievo $S_{1}$ vede un paesaggio schematico sumite a quelio nella $V$ illa di Oplontis (cat 341 , 2 , of $p$ 53)

Il pannello centrale della parete destra contiene un bosco sacro In una porta sucra e la statua glalla di una tigura femminile vestita di un chitone senza mansche Regge nella sinistra un piatto con fruttı davanti al petto e ha ul braccio destro disteso L'epistulio della porta sacra consiste di una cornice doppld con grif 1 bianchı

II campo destro mostra la veduta dl un bosco In primo plano sorge una colonna sormontata dalla statua gidlla di Artemide, vestita di un chitone senza maniche Ha una corona sulla testa Regge nella destra stesa uno scottro, nella sinistra und pateid

Sul campo sinistro si vede davanti ad una veduta di case una colonna dorica sopra la quale sta la statua glalla di una figura temminile Indossa un chitone sonza moniche c regge melle morm stese in avant und benda

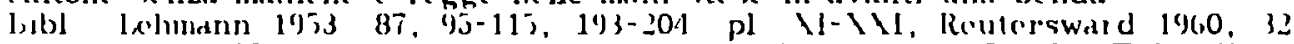
nota 325 Peters 1963, 13-19, Schetold 1965, 117-119, Taf 483 , Lvttelton 1974, 18-21, 291, pl 17-19, Muthmann 1975, 30-31, Tat 73 , Plretschner 1977, no 37, Jashemski 1979, 133, Suberberg 1981, no 4-5, fig 3-4, Leach 1982, 141-142, fig 3 Moormann 1984b, 645

Cat 004/5 (H) Parete di tondo, zona mediana II stule, fase Ic (ora MN s n ) +

vel campo centrale e seduta Afrodite A sinistra vi sono due statue bianche sopra una base comune rettangolare Quella davantı e Fortuna Regge una cornucopia nella sinistra e ha la mano destra lungo l fianco La figura dietro che indossa un chitone e andata perduta per la maggior parte e non puo essere individuata

Il campo destro conteneva un gruppo delle tre Grazie, andato perduto al momento dello scavo Probabumente le tre figure erano modellate secondo il tipo consueto per cui si rimanda all'esemplare del'Insula occidentalis (cat 227)

Bibl Lehmann 1953, 29-30, fig 247, Beyen 1960, 220, Peterc 1963, 12, fig 3. Schefold 1964, Engemann 1967, 124, nota 523-525, Schefold 1975, 55, Andreae 1975, 78, Abb 59, Schefold 1977

Villa di Popidius Florus

Cat 005 (4) Parete sud, quadro centrale. III stule (ora Richmond, Virgina Museum inv no 66-35)

Paesaggio sacro-ıdlliaco con una porta sacra Ina costruzıone adiacente al lato destro viene sorretta da una cariatıde marrone Essa indossa un peplo e regge una bacchetta con la destra lungo il fianco e ha la sinistra stesa 
Il timpano della porta sacra e incoronato da una ligura femminile gialla, vestita di un peplo lla le mani disiese

Bibl $M$ della Corte, visc 1921, 48, 11g 13, CatRichmond 1973, 120-121 no 137 
Villa di Agrippa Postumus

Cat. 006/1. (15) Pareti nord e est, quadro centrale; III stile (ora New York. Metropolitan Museum inv.no. 20.192.1, 10)

Il pinax della parete nord contiene una lorre e due porte sacre. A destra sta una statua bianca sopra un piedistallo altissimo. La ligura ha la destra in riposo lungo il fianco, mentre la sinistra e distesa. La presenza di uno scudo $e$ una lancia come offerte votive potrebbe essere un'indicazione per l'interpretazione della ligura come Ares o Atena.

Il pinax della parete est mostra una porta sacra sormontata dalla statua verde di una figura che indossa un lungo mantello. Nlza un lembo della veste con la sinistra all'altezza della coscia e ha la destra stesa verso il basso.

Bibl.: Von Blanckenhagen/Alexander 1962, 18-19, pl. 29, 31, A: Bastet De Vos $1979,45-46$, tav. XV.28; Silberberg 1981, no. 26, fig. 27-29.

Cat. 006/2. (16) Pareti nord, est e ovest, quadro centrale; III stile (ora MN $147501-147503)$

Sul paesaggio della parete nord si vede la statua giallo-arancione dj Cibele presso un albero sacro. $E^{\prime}$ seduta su un trono sopra una base rettangolare. Regge nella destra uno scettro c poggia con la sinistra su un timpano. Corrisponde al tipo normale che sarebbe stato concepito da Agoracrito.

A destra in posizione avanzata sorge l'erma itifallica rossa di Priapo sopra una base rettangolare.

Nel pinax della parete est stanno due erme di colore bigio sulle scale di una porta sacra. Ovviamente si tratta di offerte votive, lorse di legno.

Il quadro della parete ovest ha al lato sinistro tre statuette schematiche di colore grigio-marrone sopra una base comune. Le tre ligurine costituiscono un hekataion. A destra vi e un'erma.

Bibl.: Von Blanckenhagen'Alexander 1962, 21-23, pl. 32-39; Schefold 1962, 62. Taf. 8; Peters 1963, 69-70, fig. 52-54; Schefold 1965, 116, Taf. 48.1; Andreae 1973, Abb. 47: Muthmann 1975, 33, Taf. 8.1-3; Pfretschner 1977, no. 85: Vermaseren 1978, 12 no. 3; Hastet/De Vos 1979, 46-47, tav. XVI. 29: Sichtermann 1984, 297, Taf. IV.

Cat. 006/3. (19) Parete ovest, quadro centrale; III stile (ora New lork, Metropolitan Museum inv.no. 20.192.17)

Polifcmo e Galatea. A destra sul primo piano sorge una colonna bianca con la statua grigio-marrone della lortuna vestita di un lungo manicllo. lla una lunula sula testa. Regge nella destra stesa una patera, nella sinistra lungo il fianco una cornucopia.

Bibl.: Dawson 1944, 100-101, 145-146 no. 42, pl. XVI-XVII; Von Blanckenhagen/Alexander 1962, 38, pl. 40-43, D; Ling 1977, 8; BastetiDe Vos 1979, $4 \overline{7}$.

464 CE. Calza 1977, 35-37, Vedi anche Naumann 1983. 
Cat 007. Tomba, $130-160$ (ora $\mathrm{MN}$ s.n )

Le lunetie delle pareti 'A e $B^{\prime}$ contengono paesaggi sacro-ıdillacı.

Parete A. A destra sta un edificio rosso con una tetloia che vene sormontata dalla statua marrone di una sfinge femminile alata, rivolta verso destra

Parete B. Su un'isoletta sorge una colonna presso la quale si vede un'erma itilallica di Priapo di colore marrone. Sulla testa ha un cappello.

Lin fregio separa la zona superiore del soffito $S_{1}$ osservano due registrl con nature morte e parsaggi sacro-idillaci Sul 'lato $C^{\prime} u n$ pacsaggio conijene una statua marrone molto schomatica dj una divinsta che ha un braccio steso.

Bibl.. Elia 1931, 438-441, 115-44T, fig 5-6, 8, tas. I-III. VI-VII: Wurth 1934, 87, Ab 43, Tal. 18; Borda 1958, 96-97, Suberberg 1981, no. 146, fig. 123 . 
Villa di Petraro

Cat. 008. (2) Parete ?, zona mediana; IV stile (ora Castellammare di Stabia, Antiquarium inv. no. 2521)

Su uno stondo rosso si delinea Afrodite che si lava. Sta a destra di una vasca. Ia mano sinistra attinge l'acqua, mentre l'altra i davanti al petto. Le ganibe sono unito e quella sinistra regge il peso del corpo. Liatteggiamento è simile a quello della ludica. La scena potrebbe contermare l'ipotesi di Ch. Blunkenberg che la statua originariamente fosse esposta accanto a una vasca. 465

Bibl.: Giordano. Pelagalli 1957, 189, tav. IV. 16.

Villa di San Marco

Cat. 009:1. (44) Pareti sud, nord e ovest, zoccolo e zona mediana; IV stule (ora Castellamnare di Stabia, Antiquarium inv. no. 2455) +

l,orlo superiore dello zoccolo vene sorretto da una coppa di centauri marinj accovacciati, dipinti in colori naturali. Le figure sulle pireti nord e est sono andate perdute, di quelle sulla parete sud il centauro destro e quasi illeggibile. Era nascosto dietro una cassaforte.

La figura a sinistra del larario e seduta sulle zampe posteriori e sostiene con la sinistra un blocco quadrangolare sulla testa, mentre la destra e stesa in avanti.

Uno dei paesaggi della parete ovest contiene una porta sacra e un tempietto con una statua schematica.

Bit).: Llia 1957, 52-53, tav. XXVIII; Peters 1963, 153; Silberberg 1981, no. 70, lig. 66 .

Cat.09/2. (57) Pareti sud e est, zona mediana; IV stile

Ina vignetta sulla parete ovest contiene una natura morta. Accanto ad un vaso sta una Mantelherme di cui e rimasta conservata la sagoma.

Le vignette sulla parete sud sono sormontate da sfingi femminili di colore giallo, viste di fronte.

Bibl.: inedito.

Cat. 009/3. (30) Parete ovest, quadro centrale: IV stile

Sopra l'orlo fra lo soccolo e la zona mediana vi e una donna che regge nella sinistra il palladio. L'Atena è resa in stile arcaizzante. Ha un elmo a pennacchio sulla testa. Lino scudo rotondo copre il braccio sinistro. Forse si tratta di Cassandra.

Bibl.: Elia 1957, 45-46, tav. XVIII.

465 Blinkenberg 1933, 56-57, Abb. 13.

465 Questo fatto puó essere un indizio per una datazione della decorazione nell'epoca claudio-neroniana prima del 62. Cf. la situazione nell'atrio della Casa dei Vettii a Pompei (Peters 1977, 99, pl. $61 \mathrm{fig} .5$ ). 
Cat. 009/4. (64-65) Parete ?, zona mediana; IV stile (ora MN)

Sei personaggi romani stanno su mensole o strisce ora invisibili su uno sfondo giallo. Ogni numero d'inventario contiene due figure.

MN 8890. A sinistra sta una donna in veste vercle e con un drappo sulla testa e le spalle. Presso il fianco regge un piatto con la sinistra, sul quale poggiano una cassetta e un ramo d'olivo. Li destra è sotto $i$ seni.

A destra sta un uomo vestito di una tunica rosa e una toga verde. Ha una corona sulla testa e regge una patera nella mano destra che è stesa per metá, montre l'altra è davanti al petto.

MIN 8891. A sinistra si vede una donna vestita di una veste trasparente e un mantello bianco. Ha un velo sulla testa. Regge nella sinistra distesa una pisside. mentra il braccio destro e in riposo Jungo il fianco.

La figura a destra e un uomo vestito di una loga rosa che ha una brocca nella destra e un piatio con frutti nella sinistra. Avanza verso destra.

MN 8966. A sinistra $\dot{e}$ un uomo che indossa una loga bianca $e$ ha una corona sulla testa. Le braccia sono avvolte nella veste. La gamba sinistra e di appoggio.

A destra sta una figura femminile vestita di una veste diafana e un mantello verde. Ha nella sinistra lungo il fianco una brocca; con la destra regge un piatto o un costino sula testa.

Elia interpreto queste sei figure come ritratti di menbri della famiglia giulio-claudia senza, pero, poter individuare personaggi precisi, anche se le facce mostrano espressioni individuali e le figure difteriscono da molte altre per il loro carattere romano. Non si puó invece trovare alcun confronto nell'arte ritrattistica contemporanea. Non va scartata la possibilita che cqui siano stati ralfigurati antenati o membri vivi della familia che abitava in questa villa.

Bastet propose una datazione nel secondo quarto del primo secolo d.C.

Bibl.: llelbig 1774. 1783, 1786, 1797; Coscnza 1907, no. 7.1-76; RP 229.2-4, 6; Elia 1932, 217; Elia 1938; Elia 1957, 44, 46-17; Bastet 1972, 83.

Cat. 009/5. (16) Parete sud, zona mediana; IV stile (ora Castellamare di Stabia, Antiquarium inv. no. 2748; deposito scavi)

I due prospetti fra i campi contengono ognuno una statua grigia simmetrica di Apollo arciero. Lal liguril e rivolta verso il centro $p$ tende l'arco. Ila una corona di lauro sulla testa e una faretra sul dorso. Regge nolla destra una freccia lungo il fianco. Il viso mostra elementi arcaizzanti (occhi e labbra), ma anche la rigidita dei muscoli e delle membra è determinante in merito. Il colore indica argento, materiale raramente usato. Si confronti l'Apollo sul pinax con Sofonisba e Massinissa nella Casa di Giuseppe II (cat. 269/3; cf. p. 105).

Attualmente i frammenti vengono ricomposti da un gruppo di archeologi francesi diretto da Alix Barbet.

Bibl.: Elia 1957, 44, tav. XV; D'Amore e.a. 1983, 922, fig. 10-11.

Cat. 009/6. (25) Parete ?, zona mediana; IV stile (ora Castellammare di Stabia, Antiquarium inv. no. 4813-4814)

Su campi rossi si delineano atleti vivi che sono stati modellati secondo modelli statuari.

Su inv.no. 4813 si vede un atleta con le gambe divaricate e le mani alzate. Si allena con i manubri. Il modello si ritrova anche nella Casa del palestrita (cat. 267) e nella Casa di Fabius Rufus (cat. 261/6) e puó essere paragonato ai pugili della Villa dei papiri.

Su inv.no. 4814 un atleta è seduto su un cilindro giallo. Poggia la testa sulla mano sinistra e ha la destra in riposo nel grembo. Il modello è simile all'Eracle di Taranto.

Bibl.: inedito. 
Cat. 009/7. (1) Parete est: zona superiore; IV stile (ora deposito scavi)

Vel centro dell'architettura sorge la statua gialla di Apollo sopra un candelabro. Il piede sinistro sta un po' in avanti. Ha un mantello e una faretra sul dorso, sulla testa ha una corona. Regge nella destra stesa per metà un ramo d'olivo e preme con la sinistra un tripode contro il fianco.

Ia figura è stata modellata in stile arcaizzante, non dissimile dall'Apollo nel vano 16. Di nuovo la faccia e l'atteggiamento delle gambe sono gli elementi piú evidenti.

Bibl.: Elia 1957, 39-40, tav. XIl; Pfretschner 1977, no. 49; D'Anore e.a. 1983, 914, fig. Gb.

Cat. 009/8. (50) Parcte est, zona mediana: IV stile

lel prospetto a sinistra del campo centrale sorge una colonna con la statua gialla di Eros. La gamba destra è di appoggio. Regge una faretra verde con la mano sinistra e ha la destra presso le tempie, atteggiamento simile all'Agon di Mahdia. 467

Bibl. : inedito.

Cat. 009/9. (ninfeo) Zona mediana; IV stile

In una scaenae frons eseguita in rilievo a stucco colorato si trovano figure vive modellate secondo tipi statuari. Nella seconda nicchia da ovest $\dot{e}$ un Poseidon del tipo kenchreai (cf. la Villa della Farnesina, cat. 319/6). L'Afrodite alla sua destra e la Fortuna (?) alla sua sinistra sono troppo danneggiate per essere analizzate accuratamente.

Bibl.: Mielsch 1975, 48, 131-132 K35; D'Amore e.a. 1983, 924.

Cat. 009/10. (caldario) Parete ?; zona superiore; IV stile (ora Castellammare di Stabia, Antiquarium inv, no. 282)

Scena figurativa che dimostra la costruzione di una villa. A destra sorge un tempietto bianco e una statua bianca di una figura maschile. Ha la mano destra distesa: non si distinguono dei dettagli.

Bibl.: Adam/Varene 1980, 216, fig. 1-2.

Cat. 009/11. Frammenti di provenienza ignota; IV stile

Castellammare di Stabia, Antiquarium inv. no. 3705

Un'architettura contiene un satiro giallo che funge da atlante. Ha un tralcio sulla testa. Con la destra tiene le zampe di una capretta che si trova sulle spalle. Il braccio sinistro $\dot{e}$ in riposo lungo il fianco. Non è chiaro se il frammento provenga dalla decorazione del soffito dell'ambiente 1, conosciuto attraverso un disegno. ${ }^{468}$

Bibl.: inedito.

Napoli, MN 9408

Quattro tondi raffiguranti vedute di ville marine provengono da campi nella zona mediana. Sul frammento nell'angolo sinistro superiore si vede un pilastro sormontato da una statua gialla. Una divinità è seduta su di un trono. Ha un cappello sulla testa che potrebbe essere il petaso di Hernes. Ora il frammento

467 Fuchs 1962, 12-14, Taf. 1-8.

460 Elia 1957, 22, Grafica B. 
e illeggibile

Bubl Ruggiero 1881. IY-גII, tav I-II, Cosenza 1907, 193 no 60, Herrmann I, 22T. Taf 1643 , RP 3834,1 , Llia 1932, 276, Peters 1963, 157, fig 148

Napolı, M\ 9479

Due vedute di ville marine provenientı da campi nella zona mediana. Il frammento a smistrd contiene la statud di un uomo su un predistallo rettangolare La figura rossa siede su un trono e ha la mano sinistra nel greinbo e la destral distesa

Bibl Ruggiero 1881, 16 Cosenza 1907, 194 no bi Rostowzew 1911, 73, Tat 81 , Crosslle 1969,164 nota 3

Vlla di Varano/ detta di Irianna

Ca1 0101 (Webe1 24) 469 Parete?, zona superiore, Ill stle (ora MN)

Lo ligure lanno parte della scaende irons nolla zond superiore che deve essere stata simile a quella della Villa umperiale (ef $p$ 000)

I1 8910 Lnd donna vestita di un chitone ceruleo e un mantello rosso sta con 1 piedi uniti sopra l'architrave $\mathrm{Ha}$ un loto sulla testa Regge con la sinistra un lembo del chitone presso la vita, mentre solleva con la destra un jembo del mantello presso la spalla L'atteggiamento e simule a quello delle Danaidı della Villa de papirı

MN 9763 Sopra un plastro fra linee sottili (forse di edicole) sta un Eros dipınto a colorı naturalı La gamba sinıstra e di appoggio Ha una sciarpa verdastra sulla spalla sinistra Regge nelld destra lungo l fianco un cestino e nell altra una patera

M) 9664 mostra quattro figure femminll alate che sono sedute su tralcı Sono simul alle figure acroterialı del portico della Villa imperiale (cat 3053 ) e a quelle nella Caserma dei gladidtor (cat $197 / 3$ ).

Bıbl , Allroggen-Bedel 1977, 39-40, Taf 42,5

Cat 010'2 (Weber 28) Parete ?, zona superiore, IlI stile (ora MN 8840)

Due figure sono state inquadrate nello stesso telaio A destra sj vede una donna che sta, con 1 pledi uniti, sopra un architrave Indossa un peplo verdastro e un mantello vola, del quale solleva un lembo con entrambe le manı $E^{\prime}$ sumule a VIN 8910 da Weber 24

Bibl Allroggen-Bedel 1977, 34, Taf 23

Cat 010'3 (3, antisala) Parete ovest, zona mediana, IV stule (ora MN 8860) Sull'architrave aggettante del prospetto a sinistra di un pannello vi $e$ un acioter 10 rosso a forma di un gruppo statuario di Scilla Scilla anguipede brandisce un tumone sopra la testa e minaccia due uomini l cul corpl sono parzialmente visibili sotto il mostro Il gruppo vivace mostra elementı parallel con l gruppo marmoreo della Vllla di Speilonga 470 Le due tigure sono simli a1 due tritonı su MN8879 a MN8881 (cat 055) per quanto riguarda lo stile

Bibl Allroggen-Bedel 1977, 62, 64, Abb 6, Taf 342

469 I numeri del wanı sono quellı di Allroggen-Bedel 1977

470 Conticello 1974,28-36, fig 4-4a, 18-19, tav. 24-37, Andreae 1974, 81-87 
Cat. 010/4. (E) Tutte le pareti, zoccolo; IV stile (ora MN e in situ)

Le edicole sotto le lesene della zona mediana sono occupate da tigure di colore bianco. Su ogni parete si vedono due figure femminili classiche vestite di un peplo. Sono sempre disposte in modo simmetrico.

Parete est ( $\mathrm{VN}$ 8912. 8914). Hanno un lembo della veste nella mano esterna all'altesca dello coscie (" surreggono un calice sulla testa.

Parete nord (MN B.511, in situ) Keggono nella sinistra all'allezsa dolla spalla un piatto e nella destra stesa verso il basso un ramo. Anch'esse hanno un calice sulla testa.

Parete ovest. Ia figura a sinistra ha un lembo della veste nella destra presso la vita e regge con l'altra un piatto. La figura a destra regge un festone duvanti al grembo.

Le ligure sono escguite in stile classicheggiante. Non e chiaro se esse siano state concepite come statue. Lo stesso dubbio valc per figure simili nei vani 9 e 12 .

Bibl.: Allroggen-Bedel 1977, 74, Ab. 8-9, Taf. 44.

Cat. 010/5. Frammenti di provenienza ignota (ora MN a Castellammare di Stabia, Antiquarium): IV stile

11 ง $9396,9401,9402,9403$

Quattro paesaggi sacro-ddillaco provenuenti da campi rossi nella zona medıana. Sono state eseguite in grallo e branco.

M) 9396 contiene un pilastro sormontato dalla statua di Ecate. La divinità regge una fiaccola in entrambe le mani.

Su MN 9401 sl vede una statua di Poseldon modelato secondo il tipo henchreai, di colore buanco Nella Villa della Farnesina (cat. 319/6) si trova un esemplare verde (cf. p. 79). A destra vi è una statua delineata schematicamente. posta sopra un pilastro.

Su MN 9402 si vede a sinistra la statua delineata schematicamente di una figura ammantata.

MN 9403 presenta al lato destro una statua priva di dettagli sopra un pilastro.

Forse MN 9405 (cat. 079) proviene dello stesso complesso visti i colori, le misure e lo stile.

Bibl.: Allroggen-Bedel 1977, 41-43, Taf. 6.3, 7.3-4, 8.1.

Castellammare di Stabia, Antiquarium inv. no. 3843

L'edicola centrale di una zona superiore contiene un uomo giallo che ha le spalle coperte da un mantello. La gamba destra e di appoggio. Regge una faretra nella destra e solleva un lembo del mantello con la sinistra. Potrebbe trattarsi di Apollo o Ares.

Bibl.: inedito.

Castellammare di Stabia, Antiquarium inv.no. 4830

In un'architettura glallo-bianca si trova un'erma con testa barbuta. Proviene da un prospetto fra due campi nella zona mediana.

Bibl.: Inedito.

Castellammare di Stabia, Antiquarium inv, no. I.G. 160027, 160029

Due frammenti smmetrici di un'architettura, forse parte della zona superiore; altrumenti provengono da prospetti fra campi nella zona mediana. Su travi aggettanti sono seduti centauri che hanno una persona sul dorso.

I.G. 160027 mostra ancora la trabeazione. Il centauro. forse a coda marma, regge un f'anciullo sulla spalla destra e s'aggrappa una menade che e seduta sul suo dorso. 
Su 1.G. 160029 mancano l'elemento architettonico e la parte inferıore del centauro Esso regge un oggetto abbastanza grande, ma non identificable sulla spalla sinistra, mentre rivolge lo sguardo a una menade sul suo dorso.

Forse I frammenti provengono dallambiente 3, l'antisala, dove si trovava il gruppo di Sclla. La posizione originale e tale che 1 centauri sono rivoltı verso il lato esterno Non si trovano controntı esattı, ma del gruppi come le vereidi di I orma possono essere serı itı da lonte di ispirazione

Bıbl.. Elia 1957, 69, tav XiLIV.

Frammento perduto

Due fanciulli sı trovano presso un'erma di Priapo. Alle estremità destra e sinistra del quadretto si vedono piccole erme.

Bibl : Helbig 575; Cosenza 1907, 183 no 16; RP 119.2

471 Bielefeld 1969. 
Cat 011 (") Parcte? zona mediana; IIl stle

Nelledicola centrale si vede un paesaggio id山liaco con le tre Grazie dasanti ad una grotta. Esse sono modellate secondo il gruppo noto ellenistico $\mathrm{Si}$ confrontı Pompei, VI insula occidentalis (cat. 227) per la discussione dell'originale von si conosce nulla circa la posizione originale e la datasione della pittura catanese.

Bibl F Maler, AdI o (1837) 60-61, F Maler, MonInst 1837, tav. YLVI, E Braun, AdI 9 (1837) 177-182, RP 158.1. 
Musel Vaticanı

Cat 012 Inv no $2577-2600$, Il stile, tase IIa

Due frammenti, entrati nel museo nel 1836, mostrano le rappresentazioni simmetriche di teste di figure femminil lieggono sul cranio un calato azzurro sul quale poggia la parte aggettante di una trave gialla Le donne hanno una chioma ricciuta che scende sulle spalle Lo sguardo e riolto lateralmente; originariamente erd rivolto verso ll centro Lo stondo e formato da quattro fa-cie colorate in nero, giallo, rosso scuro e nero

Lo stle delle figure e smile a quello dolle erme nella Casa del criptoportico (cat 148/1-2) e nella Casa di Caesius Blandus (cat 233/2) e debbono provenire da un complesso uguale Lon ${ }^{\circ}$ chiaro se la parte inferiore delle iıgure tosse tridimensionale o semplificato a forma di erma Come nel suddetti complessı l contrasto Ira la funzione architettonica - quindi l carattere scultoreo - e la viracita des tratti del riso $c$ noterole

Bibl Nogara 190t, 61, tas VLVII a-b, RP 333.2-3.

Inv no $\rightarrow>$ Ostaa, Porta Laurentina (cat 136)

Inv no? >stia, Proventenza ignota (cat 137).

Int no? > Roma, Regio IV, lia Cavour (cat. 311). 
Tempio di Bel

Cat. 013. (pronaos) Parete nord, zona mediana, 239 d.C. curca (ora New Haven Conn.. Yale Unuversity Art Gallery, unv no. ?)

Il sesto pannello mostra l'ofterta di un gruppo di mulitarı direttı da Julıus Terentıus alle divinita locali di Palmira e Dura.

vel registro inferiore si vedono due statue sumil delle personificazioni di Palmira e Dura, con epigrafi greche Tyche Palmyron e Tyche Douras Le divinita sono sedute su un trono Palmira poggia con l piedı sulla personificazione femminle del fiume Orontes. Doura su quella femminule dell'Eulrate. Indossano vestitı bianchi con bordı glalli e hamno sulla testa una corona a torma di un bastione, attorno al quale vi e un numbo Lad Iortuna di Dura poggia la mano simistra sulla lesta di un fanciullo nudo, forse simbolo della citta stessa Al $_{\text {i }}$ pied della Fortuna palmirena si vede un leone Sono imitazion della famosa Tyche di Antiochia tatta da Eutichide, la cul copia migliore si trova nel Vaticano 472

$\mathrm{Nel}$ registro superiore stanno tre statuette di divinita sopra piedistalli bassı e rotondi. Sopra una tunica blanca hanno una corazza marrone e una clamıde, stıvalı ai piedi e attorno alla testa un numbo Lella mano destra alcata reggono una lancia La figura a sinistra ha il fianco sinistro coperto con uno scudo Le due tigure a sinistra hanno ld gamba destra di appoggio Le tre tıgure sono state interpretate da F. Cumont come Iarhibol, Aghbol e Maldkhbêl. Ann Perkıns, seguendo altri, non accetta questa interpretazione, perche l'iconografia non e quella corretta Ella non da nom alle tigure. Secondo J. Teividor, mvece, la trias rapresenta Bel, I arhibol e Aglibol.

Sono modellate secondo lo schema policleteo del Doriloro, usato frequentemente per statue corazzate dell'epoca imperiale - s1 pensi per esempio all'Augusto di Prumaporta. Le due statue a sinistra si possono controntare con una siatuetta bronzea che e stata trovata sull'acropoli di Pergamo e che data dalla seconda meta del primo secolo a.C. sta sopra un pledistaLo uguale 4- $^{-3}$

Bıbl : Cumont 1926, 89-114, pl. XLIX-LI, Dohrn 1960, 12, Taf 71 , Perhins 1973, 42-45, pl 12, Telvidor 1979, 93-94, pl AXX, Horig 1979, 174-176, Taf 8a, Parlasca 1984, 167-168, Abb. 1-2.

472 Dohrn 1960; J G Szlagyi, EAA VII (1966) 1038-1041, s.v. Tyche; Heidenreich 1966, Spatantike 1983, 481-483 no. 84.

${ }^{473}$ Pinkwart 1972, 131-138, Abb. 2-28. 
$\mathrm{H} 2 / 7 \mathrm{a}, 2 / 14$

Cat. 014/1. Pareti nord e est, zona mediana; 60-80 d.C. (ora Selçuk, Efes Müzesi inv.no.1574)

Vignette su uno sfondo rosso. Sulla parete nord si trova Socrate seduto su uno sgabello che sta sopra un piano a forma di parallelogramna. Indossa una tunica bianca che lascia scoperto il petto. Regge nella sinistra un bastone e poggia con la destra sul banco. Sopra la testa si legge un'epigrafe greca che reca il nome del filosofo. La figura viva corrisponde a un modello scultoreo di cui la copia migliore si trova a Copenhaghen. Forse il ritratto é stato fatto da Lisippo.

La figura della parete est è Urania che indossa un mantello azzurro senza maniche del quale un lembo è avvolto attorno al braccio sinistro. Ha una piuma sulla testa. La gamba destra è di appoggio. La mano destra si trova davanti al petto.

Bibl.: Bammer/Fleischer/Knibbe 1974, 66-67 (con bibl.): Strocka 1977, 103, Abb. 194-197.

Cat. 014,2. H2:7a, 2:14

Parete ovest, zona mediana; 400 d.C. (ora Selçuk, Efes Muzesi inv. no. 1572, 1573)

Lin panncllo contiene una vignetta rappresentante Clio. Indossa un chitone marrone e un mantello grigio-blu. Regge un dittico nella sinistra e ha la destra davanti al petto. La gamba sinistra è di appoggio.

Bibl.: Bammer Fleischer Knibbe 1974, 63-64 (con bibl.); Strocka 1977, 97, Abb. 200-201, 203.

H $2 / 12$

Cat. 015. Tutte le pareti, zone mediana e superiore; 440-450 d.C.

I campi della zona mediana sono state abbelliti con vignette che rappresentano Apollo, le Muse e Saffo. Sorgono sopra piani a forma dl parallelogramma e sono modellati secondo tipi statuari dellepoca ellenistica; tuttavia non si possono trovare confronti esattl. Sopra le teste si vedono $i$ nomi, scritti con caratteri greci.

Parete sud, campo sinistro. Saffo indossa una tunica bianca e un mantello grigio che copre le sue mani. La gamba sinistra è di appoggio.

Parcte sud. campo centrale. Apollo sta nell'atteggiamento dell'Apollo Liclo: il braccio destro poggia sulla testa, mentre il gomito sinistro trova sostegno in una cetra che sta sopra un piccolo piedistallo. Regge nella sinistra un ramo d'olivo. La gamba destra è di appoggjo.

Parete sud, campo destro. Calliope è sinile a Saffo. Una piuma è il suo attributo specifico (cf. p. 42 ).

Parete ovest, campo sinistra. Della figura di Lirania e rimasta conservata solo la testa.

Parete ovest, campo destro. Polinnia indossa una tunica verde. La maggior parte è andata perduta.

474 Richter 1965, fig. 556-558; cf. Moreno 1981, 174, fig. 1-3. 
Parete nord, campo sinistro. Erato ripete l'atteggiamento di Saffo e Calliope. Indossa una tunica verde con maniche lunghe e un mantello grigio. Regge nella sinistra una lira che viene suonata con un plettro nella destra. La gamba sinistra è di appoggio.

Parete nord, campo destro. Melpomene è andata perduta.

Parete est, campo sinistro. Talia ripete il modello di Saffo. Differisce solo la mano destra distesa che regge una maschera. Ha nella sinistra un pedum.

Parele est, campo centrale. Eutcrpe indossa un chitone violaceo con maniche e un mantello. Regge un aulos nella sinistra distesa e ha la destra nel fianco. La gamba sinistra è di appoggio.

Parete est, campo destro. Clio ripete l'atteggiamento di Saffo. Indossa un chitone viola con maniche e un mantello grigio-verde. Regge nella destra stesa una matita e nella sinistra un dittico.

Nei pannelli stretti della zona superiore, sopra i campi laterali della zona mediana, vi sono guerrieri nudi di colore grigio che purtroppo sono rimasti conservati frammentariamente. Entrano nel genere dei guerrieri policletei ( $\mathrm{cf}$. p. 431). Il colore potrebbe indicare l'imitazione dell'argento (cf. p. 1es).

Parete est, campo sinistro. Il soldato tiene stretto uno scudo con la sinistra che sta sulla terra. La destra è stesa per metà. La gamba destra è di appoggio.

Parete sud, campo sinistro. La figura assomiglia quella precedente. Regge una lancia nella destra stesa verso il basso.

Parete sud, campo destro. La figura si vede di spalle. Regge uno scudo con la mano sinistra e una lancia con la destra. La gamba sinistra è di appoggio.

Parete ovest, campo destro. Ripete la figura della parete est.

Bibl.: Strocka 1977, 126-137, Abb. 312-343, 347-348.

Cat. 016, H 2/16a

Parete sud, zona superiore; $440-450$ d.C.

Nel campo sinistro si vede, sopra una base a forma di calice, una figura femminile alata di colore giallo. Indossa un chitone senza maniche del quale solleva il kolpos con entrambe le mani. L'atteggiamento e simile a quello delle Danaidi della Villa dei papiri ad Ercolano.

Bibl.: Strocka 1977, 124-125, Abb. 302, 304.

H $2 / 18$, H $2 / 12 \mathrm{a}$

Cat. 0i7. Frammenti sporadici; $440-450$ d.C. (ora Selçuk, Efes Müzesi)

Sul primo frammento si vede Polinnia che indossa una tunica bianca e un mantello rosso, mentre sulla testa ha una corona. Regge un timpano nella sinistra alzata e un lembo del mantello con la destra. Sopra la testa si legge il suo nome, scritto in caratteri greci.

Sul secondo frammento si vedono la testa e il petto di Calliope che regge un volumen nella destra. Indossa un mantello marrone.

I due frammenti appartengono a una decorazione a campi simile a quella di H $2 / 12$.

Bibl.: Strocka 1977, 140, Abb. 457-459. 
H 2:SR 19-20

Cat. 018. Tutte le pareti, zona mediana; 400-410 d.C.

l campi sono decorati con le Muse che stanno sopra strisce. I loro nomi, scritti in greco, si leggono sopra le teste. Non tutte le figure sono rimaste conservate.

Parcte est, campo sinistro. Talja indossa un chitone senza maniche $c$ un mantello con motivi violacei. Il peso del corpo poggia sulla gamba sinistra. La mano destra è stesa verso il basso; nella sinistra si trova un pedum, ora non piu visibile.

Parcte est, campo centrale. Melpomene indossa un lungo mantello e cothurnae. La gamba destra è di appoggio. Regge nella sinistra una maschera, mentre la destra $\dot{e}$ in riposo lungo il fianco.

Parete est, campo destro. Euterpe rispecchia Melpomene. Ha una cetra nella mano sinistra e un plettro nella destra.

Parete sud. campo sinistro. Erato indossa un clitone. Suona con la mano destra una lira che tiene con la sinistra sopra una colonnina.

Parete sud, campo destro. Polinnia e andata perduta per la maggior parte. Si intravede un volumen nella destra.

Parete ovest, campo sinistro. Urania indica con una bacchetta nella destra stesa verso il basso un globo sopra un pilastrino. La mano sinistra è in riposo lungo il fianco. La testa e una parte del corpo sono andate perdute.

Bibl.: Strocka 1977, 74-75, Abb. 131-139, 142. 
Cosiddetta basilica

Cat. 019. Parete nord, nicchie; IV stile (ora MN 9109, s.n., 9008, 9825)

Davanti ad una parete ornata con un'incrostazione marmorea, appliques e rilieri finti si trovano, sui frammenti 9109 e s.n., due gruppi statuari che costituiscono dei pendants. La posizione originale dei trammenti non e conosciuta. A. Little li inserisce nello zoccolo delle nicchic laterali, ppotesi difficilmente accettabile a causa della loro forma absidale. Von e nemmeno chiaro se decorassero lo zoccolo o la zona mediana.

Su MN 9008 si vede Churone che msegna ad Achille a suomare la cetra. II centauro e seduto sulle zampe posteriorj c abbraccia il lanciullo stante che ha, davanti al petto, la cetra. Lo stesso motivo si vede in due copie nella Casa dell'Adone ferito (cat. 198f) e corne applique sullo scudo che vene impugnato da Achllle sui pinakes che rappresentano la sua scoperta a Sciro.

Su MN s.n. Marsia insegna ad Olumpo a suonare i due flauti. Marsia e seduto su un blocca o una roccia e stende le mani verso Olimpo che sta in pied accanto al satıro. Questo gruppo si incontra su vari pinakes pompejanı, spesso con un'ambientazione paesistica ( $p$. e. cat. 181,1,198i/3).

Il primo gruppo é una copia di un gruppo ellenistico esposto nej Saepta a Roma distrutti da un incendio nell' 80 d.C." 75 Lo slondo potrebbe imitare l'ambiente originale. L'archetipo è stato datato da Sichtermann nell'epoca tardoclassica. Il solo confronto scultoreo e fornito da un sarcofago nel Museo ৯azionale Jomano. ${ }^{46}$

Il gruppo di Marsia e Olimpo non e stato menzionato nelle fonti, ma sembra il diretto pendant visti lo sfondo architettonico e l'iconografia. K. Hadaczek e E. Langenskiold pensarono che il Torso Belvedere avesse fatto parte di questo gruppo. Si controntino anche i gruppi di Pan e Dafne. 47

la combinazione dei gruppi in un edificio pubblico potrebbe significare la paideia come stimolo morale. Ton esiste una relazione diretta con le altre scone figurative. ${ }^{4} 78$

L'Eracle su MN 9008 è stato modellato nell'atteggiamento dell'Eraclo Farnese di Lisippo, di recente analizzato da Moreno. La ponderazione sarebbe d'impronta policletea. presente nel tipo Copenhaghen-Dresda. ${ }^{79}$

MN 9825 mostra la zona superiore della nicchia centrale. In un'architettura rossa si vedono due cariatid vestite di un peplo, simmetriche. Reggono con la mano esterna un piatto davanti al petto e sorreggono con la mano interna la trave sopra la testa. La figura destra e andata perduta.

Sula cornice sono seduti tritoni che reggono un pedum con la mano esterna presso la spalla, mentre l'altra e alzata.

Bibl.: Helbig 1291; Hadaczek 1907, 312-317, fig. 92; kelsey 1908; Herrmann I, 101-104, 109-111; Langenskiold 1930, 136-137, Abb. 6; Jucker 1950, 31-34; Sichtermann 1957; Peters 1963, 147; Little 1964; Martini 1969; Bastet 1974, 231-232, Abb. $18-19$ (s.n. considerato perduto);

45 Plin., NH XXXVI 29: Mart. II 14, 5-6; Juv. IIl.

476 Sichtermann 1957, 10. Taf. 15; CatRoma I 2, 90-93, Ala II no. 6.

47 CatRoma I 5, 90-94 no. 38.

47. Teseo liberatore (MN 9049); Eracle e Telefo (MN 9008); Medea (MN 8976); Zeus nel cielo (MN 9j53). Cf. per l'iconografia Allroggen-Bedel 1983.

479 Moreno 1982, 406-411. 
hemp-Lindemann 1975, 18-24; Vogel 1978, I, 9-11, fig. 1; 1I, 579-580; Moreno 1981, 201-202, fig. 28; Moreno 1982, 415-419, fig. 31; Allroggen-Bedel 1983, 148, 150-153, AAb. 14; LIMC 149 no. 55.

II 2 Casa di Argo

Cat. $020 \% 1$. (3) lareto nord, quadro contrale: IV stile

Paesaggio sacro-idilljaco con un tempio corinzio. A destra del tempio vi é. sopra una base rettangolare, una biga guidata da un uomo. La pittura è rovinata. E' l'unica ralfigurazione di una biga come statua indipendente.

Bibl.: Maiuri 1958,366; Peters 1963, 152 .

Cat. 020/2. (?) Parete ?, zona ?: IV stile +

Helbig menziona una statua marmorea di Ares. Ha una clamide sulle spalle e un elmo sulla testa. Regge uno scudo e una lancia nella sinistra e una spada nclla destra. Forse il modello è d'impronta policletea come la maggior parte di tali rappresentazioni.

Bibl.: ilclbig 271.

II 26 Collegio degli augustali

Cat. 021. (cella) Pareti est e ovest, zona superiore; IV stile

Nei prospetti laterali si vcdono sopra basi rettangolari bighe rosse che vengono condotte da Nikai. Sono rivolte verso l'esterno. Il gruppo al lato destro della parete est ha cambiato colore a causa del fango bollente ed è diventato rosso.

Gli angoli dell'edicola centrale vengono sormontati da tritoni gialli. Hanno un timone sulla spalla esterna e tengono la mano interna davanti al petto.

Bibl.: Andreae 1973, Abb. 73-74; Guadagno 1983, fig. 4; Moormann 1983a.

III 3 Casa dello scheletro

Cat. 022/1. (10) Pareti est. nord e sud, zona mediana; IV stile

Nell'abside della parete est sorge una tholos nel quale sta un candelabro sormontato da un pavone. Esso é visto di fronte con la coda alzata. Una statua bronzea, usata come fontana, è stata trovata in VII 12,17 a Pompei. 400

Sull'architrave si vedono tritoni simmetrici che reggono nella mano interna una clava e hanno l'altra mano davanti al petto.

l prospetti fra i pannelli sulle parcti nord e sud contengono erme rosse con teste barbute. Ai fusti sono state attaccate delle braccia che reggono un pedum e un piatto con frutti. Sulla parcte sud l'erma è priva della testa, quella sulla parete opposta è priva del fusto.

Bibl.: Maiuri 1958, 271-280, fig. 215; Cerulli Irelli 1969, tav. 23.

4 \& Dwyer 1982, fig. 70. 
Cat $022 / 2$ (viridarıu) Parete sud, zona mediana. IV stile Lal rappresentazione di un giardino e circondata da un telaio. Al lato superiore si vede un oscullum

Bibl Jashemsh1 1979, 61

IV Area sacra

Cat 023 (1) Parete est, quadro centrale; IV stile (ora Casa del bel cortule)

Sileno porge un'offerta ad Apollo. La statua gialla di questa diwinita sorge sopra un'alta base rettangolare Apollo suona con la destra una cetra che tiene nella sinistra. La gamba sinistra e alzata e serve da appoggio per lo strumento.

Bibl. Maluri 1958,178 , fig 145

'Terme suburbane

Cat. 024. (E) Paretı nord e est, zona mediana: IV stile

Decorazione in rllievo a stucco. I campl sono decorati con guerrieri nudi che stanno sopra mensole $E^{\prime}$ l'unico esempio di guerrierı del genere eseguitı a stucco (cf. p 000) Nella descrizione si da tra parentesi il numero dellillustrazione in Vauri 1958.

Parete nord, campo destro (fig 124). Regge uno scudo nella sinistra e una lancia nella destra Il peso del corpo poggia sulld gamba sinistra

Parete nord. campo sinistro (fig 125) Tiene con la mano sinistra uno scudo presso l tianco e una lancia nella destra. La garnba sinistra e di appogg10.

Parete est da sinistra a destra Regge uno scudo nella sinistra presso ll flanco, ma in modo tale da mostrare linterno (fig 125) Regge una spada nella destra stesa verso il basso. La gamba destra e di appoggio

Regge uno scudo nella smistra in parto sul dorso c una spada nella destra stesa verso il basso (lug. 126) La gamba destra sostiene ll peso del corpo La faccid volta indietro esprume sofferenza.

Regge uno scudo nella sinistra e una spada nella destra stesa verso ll basso (fig 127) Il peso del corpo poggia sulla gamba sinistra.

Regge uno scudo nella sinistra cosi che si vede l'interno (fig 128). Nella destra tiene una lancia. Il peso del corpo poggia sulla gamba sinistra.

Regge an elmo nella sinistra distesa e una lancia nella destra lungo il fianco (fig 130). La gamba destra e di appoggio Le gambe sono leggermente dir aricate.

Bıbl Maiurı 1958, 159-162, lıg 124-130, Mielsch 1975, 57-59, 141-142 K53; Vermeule $1977,10-11$

IV 1-2 Casa dell'atrio a mosajco

Cat. 025/1 (j) Parele est, zona superiore; IV stile

L'edicola centrale viene sorretta da donne vestite di un peplo dj colore rosso. Ilanno un tralcio sulla testa c reggono nella mano interna un piatto, mentre sollevano con l'altra un lembo della veste vicino alla coscia. 
Su di un tripode al disopra sorge una menade della quale sono rimaste visibilı soltanto le gambe.

Bibl : Cerull Irell 1971, 15-19, tav. II

Cat 025 '2 (9) Tutte le paretı, zone mediana e superiore, IV stule (ora anche M.N 9516-9517)

II quadro centrale della parete nord rappresenta il supplizio di Dirce In fondo sorge una statua dipinto in modo schematico di colore marrone Peters l'interpreta come elemento di un sacello dionisiaco.

Sul pinax con Atteone sulla parete sud Artemide e modellata secondo $l^{1}$ Af rodite di Doidalsas

I prospettı di tutte le paretı contengono snelle erme $d_{1}$ colore marrone. Nolle man attaccate al fust r reggono un pedum o un piatto. Quelle della parete est sono quasi lleggibll.

Nella zona superiore delle paretı est c ovest e un'drchitettura leggera su stondo ceruleo con tigure che sorgono sopia plastri rossi Solo quella al lato sud della parete ovest e rimasta conservata, le altre si trovano nel museo a Napolı

La figura rimasta in situ ha le gambe chiuse. Un mantello copre il dorso. La destrid e presso ld spalld, l'altra e distesa

IA $9 J 16$ Quattro figure femminili La donna a sinistra indossa un chitone senza maniche del quale solleva un lembo con la mano sumstra all'altezza dei reni. Le gambe sono chiuse Regge nella destra un cestino o un piatto.

La figura al centro indossa lo stesso chitone. La gamba sinistra e di appoggio Regge nella sinistra un cestmo e solleva con la destra un lembo della veste presso la coscia

La terza tigura rispecchia la prima

La figura a destra rappresenta Atena vestita di un peplo e con un elmo a pennacchio sulla testa Regge uno scudo nella sinistra e una lancia nella destra alzata. Il tipo e la Promachos

VN 9517 Tre tigure Quella a sinistra e un guerriero nudo che regge nella smistra alzata uno scudo e nella destra stesa verso l basso un gladio. Le spalle sono coperte da un mantello, la lesta da un elmo a pennacchio Le gambe sono chiuse sopra un segmento circolare Il tipo e quello del guerrierı pirrici, simile a quellı su un rilievo nei Musei Vaticani. 4 .

La figura centrale e uguale alla terza di MN 9516. La mano destra è leggermente alzata

La figura a destra e simmetrica a quella di sinistra. La mano destra e distesa e il punto del gladio e rivolto verso l'alto. Lo scudo e rivolto verso il basso

Bibl : Helbig 260, 1804, 1839, RP 20 3, 58 3, 234.2; Schefold 1957, 349; Maluri 1958, fig. 238; Paar 1962, 156, Peters 1963, 127, fig. 103; Cerulli Irelli $1971,27-31$, tav. VIII-X (senza MN).

Cat. 025/3. (14) Paretı nord, sud e est, zona mediana, IV stule

II quadro centrale della parete nord contiene un paesaggio sacro-idilliaco.

Delle erme sorreggono una schola

Le prospettive tra 1 pannell delle pareti nord e sud contengono erme marronı ugualı a quelle nel vano 9.

Nella prospettiva della parete est sı distingue una figura femminile alata di colore grigio Regge sulla testa una colonna snella e nelle mani distese oggettı non distinguibul, forse corone. In tal caso potrebbe trattarsi di una Nike. Bıbl.: Peters 1963, 149; Cerulli Irellı 1971, tav. XVI.2.

$4 \cdot 1$ Lippold 1936, 4-7 no 489, Taf. 28. 
Cat. 025/4. (portico) Parele ovest, zona superiore; IV stile

L'unica parte della decorazione conservata mostra una biga dorata guidata da una Nike, usata come acrolerio. I cavalli sono rivolti verso sinistra e hanno le zampe anteriori alzate. A sinistra, nello stesso prospetto, sta un Eros con i piedi a forma di iralcio.

Bibl. : inedito.

Cat. 025/5. (esedra) Pareti nord e est, zona mediana; IV stile I prospetti rossi sono dati da sostegni a forma di erme semplici. Hanno teste di satiri e assomigliano a quelle nei vani 9 e 14 .

Sulla parete nord si vede un Eros inginocchiato, rivolto verso destra, in guisa di acroterio. $E^{\prime}$ di colore rosso. Regge nella mano destra distesa una brocca e nela sinistra distesa un piatto.

Bibl.: inedito.

IV 3-4 Casa dell'alcova

Cat. 026. (19) Tutte le pareti, zone mediana e superiore; IV stile (ora anche $\mathrm{MN}$ 9865)

Nei prospetti marroni delle paretj nord e sud sono stati inseriti tholoi a forma di pagoda. I tetti sono incoronati da donne vestite di un chitone e con una corona radiata attorno alla testa. Reggono nella mano esterna un piatto. Due sollevano con la mano interna un lembo del mantello presso la coscia, altre due hanno la mano vicino alle tempje. Le due figure della parete sud sono state staccate e sono ora nel museo di Napoli.

Le edicole centrali della zona superiore di tutte le pareti sono occupate da figure che forse sono delle statue.

Parete nord. Afrodite è seduta su una sedia a spalliera. $E^{\prime}$ vestita di un mantello blu che lascia scoperto il petto. Regge un ventaglio nella mano destra e ha la sinistra presso la spalla.

Parete sud. Ares siede su una seggiola a spalliera. In mantello gli copre le gambe, un elmo la lesta. Le mani, che erano poggiate in grembo, sono andate perdute. Le statue di Ares seduto sono rare. Si pensi alla statua trovata nelle terme di Leptis Magna." 2

Parete est. Paride é seduto su un blocco o una sedia senza spalliera. Un mantello copre la parte inferiore del corpo, un berretto frigio la testa. Regge nella destra un pedum e poggia con la sinistra sul blocco.

Parete ovest. Dioniso sta nell'atteggiamento del Doriforo. La gamba destra è di appoggio. Lna clamide copre le spalle e attorno alla testa si vede una corona. Regge nella sinistra alzata una lancia e ha la destra presso la coscia. Bibl.: Maiuri 1958 , fig. 326 .

IV 16

Cat. 027. (9) Parete ovest, zona superiore; III stile

4 z Manderscheid 1981, 107 no. 319, Taf. 3, 40. 
La scaenae frons contiene una figura femminule che sta appoggrata con le gambe unite soprd ad und base $E^{\prime}$ vestita $d_{1}$ un peplo verde e ha una corona sulla testa Regge nella sinistra un tirso lungo l fianco e sollera con la destra un lembo del mantello presso la coscia Lo stule e classico, sumble a quello delle Danaidı della Villa deı papirı

Sull'architrave sopra la donna si trova un tritone che regge un pedum nella sinistra contro la spalla e ha la destra alzata $E^{\prime}$ rivolto verso sinistra Vel III stle acroter dı questo tipo sono ancora rarı, precede chiaramente le innumerevolı figure del genere del IV stule

Bibl Malurı 1958, 435, fig 376

IV 17

Cat 028 Parete sud, zona mediana, IV stle

In uomo sta presso un dolium e un'erma rossa itıfallica di Priapo che e rivolta verno destra La scend e stata eseguita secondo lo stule dell arte plebea Ormal e ditficlmente leggible

Bıbl Malurı 19j8, 437438 , fig $382-383$

IV 21 Casa del cervi

Cat 0291 (10) Paretı nord e sud, zona mediana, IV stule I prospettı grallı contengono erme con funzione di plastri Le teste sono modellate secondo l'Hermes Agoralos di Alcamene

Bibl inedito

Cat 029'2 (16) Tutte le paretı, zona mediana, IV stule (ora anche MA 8615)

IN 8615 prow iene dalla parete est o ovest In un paesaggio sono statı espostı un cantaro, la testa di una pecora, una brocca e una statuetta marrone di Dioniso Il dio indossa un chitone e ha una barba $E^{\prime}$ rivolto verso destra e regge nella sinistra un tirso e nella destra stesa in avant un cantaro Sulla base del colore e della dimensione (in contronto con gls altri oggettı) la statuetta sembrd talta in terracotta questo sarebbe l'unico esempio evidente di una siatu(et1)a di questo materiale linitata in und pittura parietale

I prospetti delle paretı est e ovest contengono erme marroni, sumli a quelle nel vano 10 Solo sulla parete ovest sono rimaste visibulı Sulle edicole da esse sostenute stanno bighe condotte da Muka

Nel prospettı delle paretı nord e sud sono statı mseritı del centaurı marroni Sorreggono con la testa una colonna snella Reggono nella sinistra un piatto daranti al petto e nella destra un pedum sopra la testa Sulla parete nord sono rivolte verso destra, sulla parete opposta nella stessa direzione

Bibl Helbig 580, RP 118 6, Beyen 1928, 11, 32, Elıa 1932, 321, Peters 1963, 154. Croisulle 1965, 31 no 15 , pl Cl photo 191-192, Alloggen-Brdel $197 \mathrm{ib}, 102$ Pfretschner 1977, no 61a

V 5 Casa del moblio car bonızzato 
Cat. 030. (4) Pareti est e ovest, zona mediana; IV stile

Sopra colonne davanti a campi bianchi sorgono le statue policrome di Nike e Afrodite. Sulla parete est Afrodite indossa una veste rossa che lascia scoperto il petto. Regge nella sinistra distesa un ventaglio e nella destra stesa verso il basso un lembo del mantello. Sulla testa si vede una corona. Le gambe sono chiuse.

La Nike della parete opposta indossa una veste simile. Solleva un lembo della veste con la sinistra e ha la destra presso la spalla. Le ali sono state delineate dettagliatamente in colori vivaci $e$ hanno dimensioni esagerate rispetto al corpo. Le gambe sono andate perdute.

Bibl. : inedito.

\section{8 Casa del bel cortile}

Cat. 031. (2) Pareli nord, est e ovest, zona mediana; IV stile

Nei prospetti che fiancheggiano i campi a sinistra e a destra sorgono colonne con le statue di menadi. Sono vestite di un mantello rosso che lascia scoperto il petto. Nella mano esterna tengono un lembo del mantello presso la testa, nel'altra reggono un thymiaterion.

Le edicole centrali delle pareti est e ovest sono sorrette da cariatidi vestite di un peplo rosso a bordo azurro. Le figure sono simmetriche e reggono nella mano esterna un'oinochoe, nell'altra un festone che scende dalla cornice dell'edicola.

Bibl. : inedito.

V 15-16 Casa del bicentenario

Cat. 032/1. (3) Parete nord, zona superiore; IV stlle

Gli angoli dell'edicola centrale della zona mediana sono sormontati da Nikai stilizzate. Il corpo consiste di un'erma azzurra al quale sono state appese ali. Reggono nelle mani dei festoni.

Bibl.: inedito.

Cat. 03\%/2. (17) Parete ovest, zona mediana; IV stile

Nel prospetto fra i campi si trovano erme snelle nella posizione di colonne.

Bibl.: inedito.

Cat. 032/3. (tablino) 'Tutle le pareti, zona superiore; IV stile

Nelle scaenae irontes, che vista la semplicità e rigidità delle forme architettoniche fanno pensare a scaenae frontes di III stile. si trovano statue sopra basi rettangolari nelle edicole. Purtroppo la maggior parte della decorazione e svanita e difficilmente leggibile.

A destra sulla parete nord si vede ancora chiaramente un satiro nell'atteggiamento dell'aposkopon. La destra e alzata, nella sinistra regge un gladio presso la spalla. Il piede sinistro poggia a quello destro che è di appoggio.

Alla sua destra sta una figura maschile nuda che sembra avere le mani davanti al petto. Le macchic presso le spalle potrebbero essere ali, cosicchè la figura andrebbe interpretata come Eros. La gamba destra è di appoggio. 
Le altre due figure della parete nord e le tre sulle due altre paretı sono diventate lleggibil Quella all'estrema sinistra ha le gambe nello stesso at1e'g gramento dell'aposkopon

Bibl inedito

V 31 Casa del larario del sacello

Cat 033 (5) Parete sud, zona superiore, III stile

Un tregio contiene paesaggı sacro-ıdilliacl di colore rosso su uno stondo bianco Il pannello a destra della finestra contiene un tempio con la statua di und figura temminule vestita $d_{1}$ un chitone Sembra che regga una traccola nella sinistra Un modio sulla testa la caratterizza come Isıde o Artemide

Sul pannello a destra del precedente si vede un edificio con, sopra una mensola al lato destro, una sfinge femminule accovacciata Davantı ad essd sta un'erma itıfallica dı Priapo

Bıbl Malurı 1958, 254-25j, Peters 1963, 114, Slberberg 1981, no 51

V 34-35 Casa del gran portale

Cal 034,1 (1) Parete nord, quadro centrale, IV stule

Dioniso, Arianna e Sieno stanno in un pdesaggio Su una colonna in fondo sorge la statua bianca di Priapo La parte superiore del corpo e coperta da un mantello Regge in entrambe le manı una bacchetta.

Bibl Malurı 1958, 379-380, fig 311

Cat 034/2 (6) Tutte le paretı, zoccolo, IV stule (ora anche MN 8595)

Nel centro di ogni parele si vede una prospettiva con un giardino Dietro un cancello basso sorgono statue di ninfe acquatiche che reggono un bacino davanti al petto Tutto e dipinto in toni azzurri Il modello delle figure e consueto e ripete la Venere Landolina (cf $p$ 63.

Lo zoccolo della parcte sud si trova attualmente a Napolı La ninfa ora presente su questa parete proviene dalla parete ovest La testa della figura sulla parete est sembra essere quella di un satiro

Bıbl Masurı 1958, 383, fig 314, Cerull Irell 1969, tav 19-20, Moormann $1983 \mathrm{~b}, 101$ nota 109

VI 17, 23 Casa del colonnato tuscanico

Cat $035 / 1$ (3) Parete est, zona superiore, IV stlle

La statua policroma di Fortuna sta, nel prospetto a sinistra, sopra una base rettangolare $E^{\prime}$ rivolta verso destra Indossa un chitone verde e ha una corona sulla testa Regge una cornucopia nella sinistra e un tirso nella destra Sulla testa si vede una colonna che sostiene una trave La trave vjene sormontata da una sfinge femminule alata sdraiata Anche il prospetto a destra, ora perduto, deve aver contenuto una costruzione simile

Bibl Manni 1974, 14, tav I 2, III 1 
Cat. 035/2. (5) Parete nord, zona superiore; IV stile

Nell'edicola centrale sta, sopra una base rettangolare verde, una figura a colori naturali che ha un mantello ceruleo diafano attorno alle reni. La gamba destra è di appoggio. Regge una cetra nella sinistra presso il fianco e un plettro nella destra distesa. Non è chiaro se si tratti di un uomo (e quindi Apollo) o una donna. Se dobbiamo ammettere una figura femminile (forse l'accennare di seni ne è un'indicazione), rimangono strani il colore dell'incarnato e l'iconografia. L'interpretazione come Musa data de Manni sembra difficile per mancanza di paralleli contemporanei: solo nel secondo secolo appaiono delle Muse nude e il loro numero è sempre ristretto. "3 Menadi che reggono una cetra sono altrettanto rare. Quest'ultima denominazjone, però, potrebbe essere la piú plausibile vista la presenza di oggetti dionisiaci in questa scaenae frons.

Bibl.: Manni 1974, 22, tav. VII.2.

Cat. 035/3. (7) Parete ovest, zona superiore; III stile

Lna scaenae frons contiene quattro statue di menadi sopra basi a forma di $T$. Le due figure accanto all'edicola centrale sono simmetriche. Indossano vesti violacee, ora non determinabili, delle quali alzano un lembo con la mano esterna, mentre reggono nell'altra un tirso. Le altre, altrettanto simmetriche, indossano un chitone. Reggono con la mano esterna un tirso e con quella al lato interno un canestro rotondo sulla testa.

Bibl.: Manni 1974, 17, tav. IV.3.

Cat. 035/4. (13) Tutte le pareti, zona superiore; III stile

In scomparti quadrangolari nel fregio sopra le lesene della zona mediana si vedono delle figure verdastrj delle quali e rimasta visibile solo un'erma nella parcte ovest. Si tratta dell'H deftherme di un satiro del tipo Formello. " 44 L'erma di una Nike sulla parete nord ricorda le erme di Nikai nell'anfiteatro di Pompei (cat. 180).

La scaenae frons su tutte le pareti contiene statue, parzialmente conservate.

Parete ovest. A destra sorge un satiro che sta con le gambe unite sopra una base rettangolare. Regge dei frutti in entrambe le mani. Ha una corona sulla testa e una nebride sopra le spalle.

Parete nord. Nell'edicola centrale sta Apollo Citaredo che ha una cetra nella sinistra e un pleftro nella destra stesa per meta. Il peso del corpo poggia sulla gamba destra. Un mantello copre la parte inferiore del corpo.

Le edicole laterali vengono sorrette da cariatidi simmetriche che indossano un chitone grigio-azzurro. Con la mano esterna reggono un piatto sulla testa, sulla quale poggia, a sua volta, una colonna. Con l'altra distesa sollevano un lembo del mantello. La gamba esterna sta un po' in avanti.

Parete est. A sinistra sorge un satiro danzante che ha la gamba destra dietro quella sinistra. Regge un pedum nella sinistra presso il flanco e un piatto nella destra stesa.

Nel centro sta Dioniso le cui spalle sono coperte con un mantello. Ha una corona sulla testa. Regge nella sinistra un tirso, nella destra un lembo del drappo. La gainba destra è di appoggio.

Bibl.: Manni 1974, 29-34, tav. XII-XV; Pfretschner 1977, no. 59; Vermeule $1977,10-11$, fig. 8 .

\footnotetext{
43 P. e. Woburn Abbey (Wegner 1966, 90-91 no. 231, Taf. 3b).

494 Cf. Blanck 1979.
} 
Insula orientalıs I A, 1 Casa della gemma

Cat 036 (atrio) Paretı nord, sud e ovest, zona medıana, IV stle

Davantı a campl lossi stanno sopra mensole guerrierı nudı d'umpronta policletea Ora sono severamente danneggiatı e quası lleggıbli

Sulla parete nord sono rimaste conservate due delle quattro figure Di quella sul secondo campo da smistra si vede la parte inferiore, la gamba destia o dı appoggio Sul campo adracente ll guerriero regge una spadd nella destra stesa verso ll basso e un arco (?) nella sinistra alzata La gamba sinistra c di appoggio

Sulla parete sud e isibile solo la figura a destra Regge uno scudo e una lancid nella mano sinustra alzata e una spada nella destra stesa verso 1 basso Ia gamba sinistra e di appoggio

Sulla parete orest, sul campo a destra dell'entıata, sl distungue una sagonin

Bibl Malurs 1958, 36-312, fig 267

Insuld orientalis I A 2 Casa del rihevo di Telefo

Cat 037 (atrio) Nicchie al lato sud, zona superiore, IV stue

Sulle parcti est $\varepsilon$ ovest delle nicchie le edicole sono occupate da uomini nudi dipintı a colorı natural sulle paretı nord e sud sopra 1 candelabri vi sono delle donne

Nicchid sud, parete est $\mathrm{L}_{n}$ uomo con una clamide sulle spalle regge una lancia nolla mano sinistra alzata, mentre il braccio destro e in posizione di riposo lungo ll fianco Il peso del corpo poggia sulla gamba sinistra $\mathrm{S}_{1}$ distinguono chidramente I muscolı

Sulla parete ovest l'uomo regge un oggetto con entrambe le manı poste a sinistra La gamba sinistra e di appoggio Anche qui i muscoli sono ben visibili L'atteggiamento particolare si incontra anche nella Casa di Veleagro (cat 202/3) e su M\8957 485

La parete sud mostra una figura femminule su un candelabro, vestita di un chitone verde senza maniche Regge un pratto nella sinistra distesa e solleva un lembo della veste con la destra all'altezza del petto Lo stule della figura e classicheggiante

Nicchia est, parete nord La donna rispecchia la figura di fronte Sulla testa $s_{1}$ distingue una corona $\mathrm{E}^{\prime}$ rumasta conservata meglio della precedente

Sulla parete ovest l'uomo avanza verso destra Suona una siringa La figura della parete est e andata perduta

Bibl Majurı 1958, 345-360 (quası nulla sulle decorazıonı parıetalı)

Insula orientalis II $A$ la

Cat 038 (D) Tutte le paretı, zoccolo e zona superiore, III stile (ora anche MN)

485 Helbig 1872 
Lo zoccolo contiene la rappresentazione di un giardino su stondo nero Erme semplicı e statue di uccell, tuttı dı colore bianco, sorgono fra le piante Glı scompartı che corrispondono al campi nella zona mediana sulle paretı nord e sud vengono separntı da $H^{d}$ ffthermen di Eros e satirı

Parete est Erme con teste $d_{l}$ satirı stanno al lat Sorreggono l'orlo superiore dello zoccolo

Parete ovest La decorazione e dndata perduta, ma deve essere stata sumle a quelld precedente

Parete sud Al lato sud si trova un'erma simle alle precedentı Segue $\mathrm{MN}$ 9755 che contiene un'erma $d_{1}$ un satıro che sorge da una calice di acanto Ha un drappo attorno alle reni e una corona sulla testa sulla quale poggı l'orlo dello zoccolo Regge un'oinochoc nella destra lungo ll flanco e un platto con una cassetta nella sinistra presso la spalla

Segue una parte quası lleggibile in situ, una porta e M 9769 che forma $\downarrow$ pendant di MN 9755 Adesso 1 l satıro regge un cestino con fruttı nella sinistra presso la spalla e una brocca nella destra lungo il fianco

Parete nord Al lato destro deve essere collocato 119756 Un Hermeros sorge da un calice vegetale $\mathrm{d}_{1}$ colore vorde $\mathrm{Ha}$ due ah verdl $\mathrm{e}$ und corona sulla testa Regge con entrambe le manı, davanti al petto, un cantaro Da questa parete provengono anche MV 8758 e 8763, due trammenti del glardino, quası simmetrici Su tuttı e due si distingue un airone bianco, visto di profilo che regge con un piccolo calice sulla testa l'orlo 1 ra zoccolo e zona mediana Su MN 8763 si vede inoltre un'erma semplice

La zona superiore, totalmente perduta in situ, si puo ricostruire in generale grazıe ad alcunı trammentı conservatı nel museo di vapolı

Su MN 8938,9670,9674 e s n donne stanno sopra bası a forma d $\mathrm{T}$ e reggono colonnine sulld testa Indossano chitonı senza maniche Su MN 9674 si vedono una hore classica e una Aura di Stule Ricco La hore regge con la destra un lembo della veste presso la spalla, con l'altra un lembo presso la coscia L'Aura ha un lembo nella destra, mentre tiene dritta la colonna con l'altra IN 8938 rispecchia l'Aura

Su MN 9677 un Eros sorge sopra un candelabro vegetale fra due alberell, tonsj Ha alı vere e una clamide rossd e regge una lancia nella sinistra, mentre la destra e stesa verso ll basso Le gambe sono chiuse Glı alberı sorgono da bacinı sorrett dd sfingı bianche alate

Bibl Helbig 1877, RP 131 1, Schefold 1957. 351, Allroggen-Bedel 1983, 154 nota 81, Moormann 1984d.

Insula orientalıs II A, 4 Palestra

Cat $039 / 1$ (aula absidiata) Parete nord e ante nord, zona superiore, IV stule (ora anche MN 9731)

Una scaenae trons dı colore marrone copre la parete nord, la parete sud deve aver aruto una decorazione simile Sul frammento runasto conservato si distinguono $H$ dfthermen di satiri come sostegni Le due figure summetriche hanno un drappo attorno alle renı e sulla spalla interna Reggono nella mano esterna lungo il fianco un oggetto non determinato, mentre la mano interna e alzata l muscolı, la barba e 1 trattı del viso sono stati delineati in modo dettagliato

A sinistra del satiro siede un tritone sopra und trave Ha la mano sinistra alzata e la destra davantı al petto

Su MN 9731 si vede nella prospettiva un'erma grigia con la testa di una figura maschule barbuta La posizione del frammento non e precisamente ricostrubule, ma $v i$ si puo arrirare considerando la fascia nera sul lato destro posta tra due campı nella zona mediana o super 101 e 
Bıbl Elıa 1932, no 384, Drerup 1957 12-13, Taf 1, Schefold 1957 352, Vlalurs 1958, 113114 , Lyttelton 1971 2, pl 26, Alloggen-Bedel 1983, 141. Abb 3,5

Cat 039,2 (III) Parete ?, quadro centrale, III stile (ora MN 9141)

Marsia e Olımpo Marsia e seduto nell'atteggiamento dell'Lracle Epitrapezios di Lisippo Olimpo sta un po' in disparte I due quindi non copiano il gruppo del Sacpta (cl cat 019)

Bıbl Helbig 229, RP 32 6, Elıa 1932, no 186 , Schetold 1957 338, tllroggen-Bedel 1983 i44, Abb 6

Cat 039/3 (aula superiore 3 ) Tutte le parcti, zona superiore, III stle Sul tumpano dell'edicola centrale della zona mediand sorge un Eros nudo alato danzante Solo la sagoma e rimastd conservata II plede simistro sta in arantı Cros regge con la destra una benda mentre ha la sinistra distesa Sulle estremita del timpano si vedono stingi temminul alate

Le edicole semplici della scaenae frons sulla parete ovest contengono figure femminul simmetriche sopra bası a forma $d_{1} T$ Sono rivolte verso il centro Indossano un chitone verde e un drappo rosso La figurd a sinistra e andata perduta quella a destra sembra reggerc una colonna sulla testa con la destra, mentie ha la smistra in posizione di riposo lungo ll lianco Bibl medito

Villa del papiri

Cat 039A (atrio) Parete ovest, zona mediana, II stule lase Ic (ora M 9123 e +)

In una fuga dı umitazıonı dı lastre dı marmo sono statı inserıtı del fintı rulevı, dei qualı nel Settecento almeno due sono statı staccatı e depositati nel museo Solo uno, MN 9423 e ancora esistente un paesaggio sacro-idilliaco con un tempietio ed un albero sacro sono dipinti in glallo scuro

Bıbl Allroggen-Bedel 1976, Voormann 1984b,643, fig 8 (con bibl) 


\section{FAMARS}

Jardin a Pois

Cat 040 Frammento sporadico, secondo secolo d C

Il trammento mostra la parte sinistra di un'architettura nella zona mediana Sopra la trave si vede la statua bianca-grigia di una divinita iluviale maschule $E^{\prime}$ sdiarata sul lato sinistro e poggla l gomito sinistro bu uma biocca rovesciata II tipo e quello consueto delle statue $d_{l}$ fiums, irequentemente presentı nelle rappresentazion di glardıu per esempso nolla Casa dı Romolo e Remo (cat 251)

Sull'epistilio di una colonna, essendo parte della trabedzıone sta un'umago clipeata abbastanza grande, delineata in modo dettagliato Entro un cerchio giallo si delinea, davantı ad uno stondo rosso, ll busto griglo di un uomo calvo e magro Macchie e strisce bianche danno un po di rilievo I colori indicano un imitazione d'argento Si puo pensare al lamoso psemplare bronzeo con l ritratto di Tralano nel museo di Anhara 4 es

La datalione non si puo stablire in base al dati di scrio ma va fatta con criter stlistici Belot conclude prudentemente, elencando una serie di confrontı validı, per un'esecuzione della decorazione nella seconda meta del secondo secolo d C o piu tardi

Bibl Bclot 1984a, 40-41, Belot 1984b, 17-26, fig 10 pl Ic, Belot 1984c, 41,43

406 Budde 1965 Cf in generale Winkes 1969 Solo questo esempio e stato inserito nel catalogo mentre altrı, per esempio a Torre Annunziata, sono statı esclusi Soltanto qu la plastıcita e l'umitazione di toreutica sono cospicuamente presentı 
British Museum

Cat. 041. Hunks 1933, no 28, IV stile

Un pinax della zona mediana, proveniente da Pompei, mostra Dedalo e Icaro. A sinistra sorge un tempıo. Suglı orlı della rampa si vedono due leonı o sfingı

Bibl. Helbig 1210; RP 183.6; Hinks 1933, 15-16 no 28, pl. XII; Dawson 1944,109 no 58 , pl. XII; Schetold 1962, 196. Paar 1962, 33-34; Peters 1963, 131-132, 208 nota 459, Von Blanckenhagen 1968, 113, pl. 39.2 .

Cat. 012. Itınks 1933, no 49, IV stile Un pinax della zona mediana, proveniente da Pompei, conticue un pacsaggio sacro-ıdiliaco Vicino ad un albero sacro vi e l'erma itifallica di Prilpo che per meta e andata perduta.

Bibl., Hinks 1933, 26 no 49, fig. 29.

Hinks 1933, no. 32: > Pompei, VI 9, 6 (cat.202/2). 


\section{LUXOR}

Templo dl Ammon

Cat 043 (vano dı culto) parete sud, abside, zona mediana, circa $300 \mathrm{~d} C$ I quattro Tetrarchı sono statı dipintı come figure luve, ma secondo tipı statuarı "87 Indossano una toga e hanno al pledi del sandalı doratı o delle scarpe rosse Le teste sono curcondate da numbl giall Vel centro sta Diocleziano che regge lo scettro nella destra alzata $c$ una sfera ceruleo nclla sinistra davanti al petto A destra si vede una traccia di Massimiano 1 due augusti sono trancheggiatı dal caesares Galerıo e Costanzıo Cloro Sempre la gamba esterna e di appoggio in modo che s] raggiunge un certo grado di simunetrua

Le tigure sono maggiori delle altre nello stesso ambiente La posizione cntro una nicchia rispecchia la pratica normale durante l'Impero. La forma e stata scelta conforme a gruppi statuarı o gruppi eseguitı altrove a mosdico

Bibl Deckers 1979, 640-644, 650, Abb 27-29, 33-34, J. Deckers, in Spatantike 1983, 272-273, 277, 280, Abb. 102-103

4.7 Cf Niemever 1960, 54-63 sulle Idealdarstallungen, non sı trovano confrontı per , mantelli 


\section{LUZERN}

Cat. 044. Già Collezione A. Ruesch

Sopra una mensola sorge un satiro nudo sopra le dita dei piedi davanti ad un pannello bianco. Regge nella sinistra una coppa e ha sulla testa una corona di pigne e una colonmma. La mano destra e modellata nell'atteggiamento dcllaposkopon. Il traminento sarcbbe stato acquistato a Pompei. L'attuale luogo di collscriazione non e noto.

13ibl.: Kuesch 1936, Tal. 40; Jucker 1957, 70-71. 


\section{MAGDALENSBERG}

AA $15 f$

Cat. 045. Frammento sporadico; II stile, fase IIb

lina vignetta su un campo rosso repprosenta Ifigenia cho rogge con la sinistril davanti al grembo l'idolo verde di Artemide. Lit parte inturuore ha la lormil di un erma. La testa e coperta da un velo e da una corona di togli dorati.

Bibl.: Kenner 1962, 436, 444-445 (datazione), Taf. I-II; kenner 1966; Kenner 1967 ; Kenner 1970. 


\section{MARIEMONT}

Musee de Mariemont

Inv. no. B. 96, 98, 98bis: > Boscoreale, Villa di P. Fannius Synistor (cat. $004,2-3)$. 
Casa del teatro/basulica

Cat 046 (tablino) Abside, zona mediana, 320-350 d C

Fra I campı, sopra bası rettangoları dı colore glallo, v's sono quattro tıgure umane vestite dl tunishe bianche ornate con motwi tardompcrialı, qualı orbiculi a segmenta di colore rosso l'altezzd e poco inferrore al quella naturale la figura grande, rumasta conservata $\mathrm{f}$ in alle spalle misura $130 \mathrm{~cm}$

Della prima figura di destra e rumasta conservata solo la parte interiore I piedı sono in posizıone di equulibrio e sono copertı con sandalı rossı

La seconda tıgura poggia sulla gamba sinistra $\Gamma^{\prime}$ l'esemplare megho conservato Molta attenzione e stata data aglı ornamentı sulla tunica de $q$ qualı si distingue nettamente il ricamo. I piedi sono scalzı

Della terza figura si vede ancora la meta inferiore I piedi sono in posizione di equilibrio La tunica mostra di nuovo del ricamı preziosı

La quarta ligura corrisponde alla prima, ne a rumasta conservata solo una parte difficilmente leggibule

Le figure sono state interpretate come orantı, sulla base della denommarione della casa come basuluca cristiana beconclo L Abad Casal l'upotesi e insostenibule, bisogna pensare a figure che offrono degli oggetis I vestitı lorniscono degli elementı per l'inserimento cronologico Abad Casal presenta una serie di contronts, tra l'altro 1 mosaicı dı Pıaza Armerina, che contengono ornamenti sumll Il sesso delle figure rumane incerto forse le figure lateralı sono maschil. quelle centralı femminu

Solo I predistall caratterizzano le tigure come statue Non si trovano confronti nella scultura conosciuta Per quanto s1 possa concludere sulla base della pubblicazone di lbad Casal, le figure sono rappresentate cone persone vive, presenti nell'ambiente L'archeologo spagnolo non u esprime a proposito del carattere scultoreo e non discute ll significato des piedistalli

Bibl Abad Casal 1982, 40-46 (con bibl), 356-35i, fig 22-26

Cat 047 Tomba del Voconi1, seconda meta del prumo secolo d C

l ritrattı delle persone seppellite sono raffigurate in nicchie Le figure stanno sopra pledistall quadrangoları e sono modellatı secondo 1 tip s statuarı normalmente in uso per monumentı tunerarı Indossano una tunica e una toga e poggiano sul piede sinistro

Sulla parete $d_{1}$ fondo stanno $C$ Voconius e Caeculıa Anus Il loro atteggiamento e uguale a parte la posizione della testa la donna ha lo sguardo ritolto verso d marito Ella regge nella sinistra stesa due cembalı, egli ha la sinistra sul dorso della moglie La destra e sempre davantı al petto

Sulla parete sinistra si delinea C Voconius Proculus, colui che fece costruire la tomba e tiglio de sunnominatı Regge nella simistra un volumen sul quale si legge AVG. EMER. (Augusta Emerita) ll liso mostra tratts somatici ben precisi

Alla fine della parete destra fine sorge la statua di Voconia Marıa, la figlia des due primi II suo atteggiamento e uguale a quello della madre inchiessa regge cembali nella sinistra

Abad Casal non descrive le figure come statue, ma segnald la presenza del piedistall Quelli potrebbero sumboleggiare l'eroizzazione delle tigure morte II visitatore della tomba capisce in tal modo di non trovarsi di fronte a ritratti di esserı umani, ma davantı a persone entrate nell'ambito delle disinita

Bibl tbad Casal 1982, 79-81 no $1.82,334-351$, fig 106, 108-109 
Museo Archeologico.

Cat. 048. Inv no 220; epoca adrianea

Altare dipinto. Sui quattro lati s] vedono le rappresentazions di quattro divinita romane: Cerere, Fortuna Ercole e Vittoria. Mielsch crede che 1 loro modell derivino da tipi statuari, ipolesi plausibıle, quando si controntano con esempi in rilievo dello stesso perıodo." La Fortuna poggia sulla gamba sinistra. Regge nella sinistra una cornucopia e nella destra un timone Il modello sarebbe quello della Fortuna, tıpo Loggia deı Lanzı. ${ }^{49}$

Cerere e sdraiata e poggia il gomito sinistro su un oggetto non adentil icable. Regge nella sinistra tre spighe e solleva con la mano destra un lembo del mantello che copre le gambe $\mathrm{Ha}$ una corona sulla testa. L'atteggiamento non e quello consueto di Cerere, ma ricorda le ninfe sdraiate nella Casa di Pinarius Cerealis (cat. 182'1)

Anche la ligura di Vittoria non corrisponde al consueto modello. Il peso del corpo poggia sulla gamba destra Jia un drappo sul clorso e regge nella mano destra distesa una corona e nella mano sinistra una lancia L'atteggiamento e quello del Doriforo. Se non vi fossero gli attributı ben riconoscibli, la donna sarebbe da interpretare come Afrodite.

Eracle poggia sulla gamba destra. Jiegge un ramo di palma nella sinistra e una clave nella destra. La leontide copre l'avambraccio sinistro. Ripete il modello dell'Eracle Lansdowne di Scopa.

Bibl.. Borda 1958, 278; Melsch 1981, 224, Tat. XVII 23.

4. Cf. Gabelmann 1977.

$4: 9$ lippold $1950,335$. 
Museo Nazionale

lnv. no. 1265: > Pompei, VIII 8, 28 (cat.275;1).

Cat. 049. Inv. no. 8512; IV stile

Paesaggio egizio con la lotta fra un coccodrillo e un asino proveniente da Ercolano. Sul tetto di un tempio prostilo posto a sinistra vi e un serpente bronzeo con funzione di acroterı. Sugli appoggiatoi della rampa si vedono due statue bronzee di coccodrilli che fungono da custodi o da raffigurazioni del dio Sobek come nella Casa dei Pigmei (cat. 285). In una piccola edicola a destra sta la statuetta aniconica di lside. smile a quelle nei paesaggi del tempio di Iside (cat. 275,2 ).

Dei serpenti di bronzo sono stati trovati nella palestra di Ercolano e nella Casa del citarista." 90 Dei coccodrilli sono stati trovati, fra l'altro nella V'illa adriana. 49

Bibl.: Helbig 1568; RP 377.2; McDermott 1938, 281-282 no. 484; Schefold 1957, 325; Elıa 1974, 161, tav. IV.4;

Inv, no. 8517-8520, 8527-8529, 8536: > Pompei, VIII 8, 28 (cat, 275/1).

Cat. 050. Inv. no. 8555; IV stile

Parle di una scaenae frons o di un prospetto della zona mediana di una decorazione a Pompei, di colore giallo su uno sfondo nero. Su una base aggettante vi è un elefante con un suo cucciolo, tutti e clue resi in modo naturale. Le orecchie grandi, la curva del dorso e le zanne della madre caratterizzano glı animali come elefanti africani. Siccome l'intera decorazione e gialla, non si puo stabilire se il pittore veramente abbia voluto rappresentare un gruppo statuario. Statue di elefanti sono anche conosciute dalle fonti scritte: una biga dorata eretta da Augusto e quattro statue di ossidiana esposte nel tempio di Concordia. Poi vi sono le statue marmoree da Iivoli (forse dalla Villa adriana) e Leptis Magna e delle statuette provenienti da Pompei. ${ }^{492}$

Bibl.: Helbig 1600; Niccolini IIl, tav. 25; RP 357.9; Scullard 1974, 282 nota 161 , fig. 2 .

Inv. no. 8570: > Pompei, VIII 8, 28 (cat. 275/1).

Cat. 051. Inv. no. 8572; IV stile

Prospetto policromo su fondo bianco di una zona superiore. Un portico contiene due erme gialle che fungono da pilastri anteriori. Le teste sono quelle di satıri umberbi. La figura sinistra è diventata lleggibile.

490 Jashemski 1979, fig. 24i; Dwyer 1982, fig. 139.

491 Roullet 1972, $127-128$ no. 254-259.

492 Scullard 1974, 177, 244, 258, 283-284 pl. Xa, XIXa; Toynbee 1974, 43, 51-52; Lunsingh Scheurleer 1975; (per Tivoli) Helbig 4362 Roullet 1972, 50; Raeder 1982. 
Sul tetto vi sono, sopra piedistalli rotondi di colore rosso, sfingi femminili alate grigie in guisa di acroteri. Sono sedute sulle zampe posteriori e viste di fronte.

Bibl.: Schefold 1957, 326.

Inv.no. 8574-8575: - Pompei, VIII 8, 28 (cat.275/2).

Inv. no. 8593: > Portici, Villn sotto l'Escuderia Real' (cat. 308).

Inv. no. 8594: - Pompei. VII lnsula occidentalis (cat.263).

Inv. no. 8595: : Ercolano, V 34-35 (cal.no.034'2).

Cat. 052. lnv. no. 8605; IV stile

la parte superiore di una decorazione, provenjente da Pompei, di colore giallo su sfondo nero. Un architrave viene sorretto da una figura fomminile e da due centauri. La cariatide indossa un peplo. Regge nella mano sinistra posta lungo il fianco un piatto e alza un lembo della veste all'altezza delle reni.

I centauri alla sua sinistra o destra hanno le zampe anteriori alzate e rivolte verso sinistra. Reggono nella inano sinistra stesa per meta un pedum, nella destra presso la spalla un ritone. Ricordano le statue topiarie e gli atlanti di II stile a Boscoreale (cat. 004:1-3).

Bibl.: Schefold 1957, 327; De Franciscis 1963, tav. XXIX.

Inv. no. 8615: > Ercolano, IV 21 (cat.029:2).

lnv. no. 8691: > Pompei, VI insula occidentalis (cat. 225\%).

Inv. no. 8715: > Pompei, VII Insula occidentalis (cat. 9263).

Inv. no. 8817: > Pompei, VI lnsula occidentalis (cat. 225/2).

Inv. no. 8758, 8763: "Ercolano, Insula orjentalis II A la (cal. 038).

Cat. 053. Inv. no. $8829,8908,8924,9123,9125,9267,9878$; IIl stile

Questi frammenti, piú $M X$ 8919, 9265, 9267, 9538, 9539, e lirammenti a Monaco di Bavaria, Parigi e Tokio, provengono da una casa presso il teatro di Ercolano, scavata dai Borboni.

MN 8924 è il quadro centrale di una parete, rappresentante un'offerta a Iside. Sugli appoggiatoi del tempio sono sedute due sfingi temminili con un modio sulla testa. Il pendant di questo pinax e MN 8919.

Fra le zone mediana e superiore si trova un fregio con piccoli pinakes (MN 9265, 9267, 3538, 9539 e i frammenti fuori Napoli). MN 9267 contiene tre dome vicino alla statua rossa di Priapo. Il dio indossa un mantello, con un lembo del quale regge dej frutti. Regge nella sinistra un tirso e nelia destra stesa un cantaro. La gamba destra è di appoggio. Sulla testa si vede una corona.

MN 9538, sempre parte del fregio, mostra Ifigenia in Tauride. Oreste sta presso un tavolino con, entro un armadietto, l'idolo di culto di Artemide. La dea è vestita di un peplo e ha una corona sulla testa. Armadietti simili si vedono nella Casa di Caecilius Jucundus (cat. 186/1) e nella Casa dej Vettij (cat. 21T/9). Secondo Parlasca il quadretto sarebbe stato fonte d'ispirazione per 'Goethe in campagna' di Tischbein.

I frammenti superstiti fanno parte di una scaenae frons nella zona superiore, non completamente ricostruibile. MN 9878 costituisce Ja parte centrale; si vede ancora la cornice dell'edicola centrale dela zona mediana. Nel centro vi è un Eros con le ali azzurre e una clamide azzurra appoggiata sulle spalle. Ha con le gambe unite e regge con entrambe le manj uno scudo rotondo sopra la testa. L'atteggiamento ricorda i guerrieri pirrici, simili a quelli nella Casa dei Dioscuri (cat. 204/7) e su MN 0372 (cat. 076). 
Ai suoi fianchi vi sono dei satíri dipinti a colori naturali che stanno con $j$ piedi uniti sopra colonne sottili. Reggono sulla testa una colonna simile a un candelabro vegetale. La figura a sinistra e modellata come l'aposkopon: regge un pedum nella sinistra stesa e ha la destra sopra la testa. La figura a destra regge un Dioniso fanciullo sulla spalla sinistra e ha un pedum nella destra. $E^{\prime}$ una variazione ellenistica dell'Hermes di Olimpia." 93

MN 9123 e 9125 mostrano aposkopontes simili al precedente.

Su MN 8829 e 8908 si vedono cariatidi vestite di un peplo e di un mantello dj colore bigio-bianco, poste sopra piedistalli simili. Reggono nella mano sinistra un piatto all'altezza del fianco e sollevano un lembo della veste con la destra presso la coscia. La cariatide di MN 8829 regge una colonna con la testa. Lo stile é quello delle Danaidi della Villa dej papiri.

L'insieme entra nel genere popolare di scaenae frontes nella zona superiore delle decorazioni di III stile (cf. p. 2g).

Bibl.: per frammento

8829: Helbig $1876 \mathrm{~b}$.

8906: Helbig 1876b; Schefold 1957, 331.

8924: Helbig 1111; Elia 1932, no. 103; Schefold 1957, 331; Tran Tam Tinh 1964, 27-28, pl. 23: Tran Tam Tinh 1971, 29-38, 83-84, fig. 40; Müller 1971, tav. $X$ : Malaise 1972, 251-252, pl. 35; A. Allroggen-Bedel, Gnomon 50 (1978) 429; Jashemski 1979, 139, [ig. 218; LIMC 1, 418 no. 38 , s.v. Aithiopes.

9123, 9125: Helbig 428; Herbig 1949, 45-46, Taf. XVIIl.2; Jucker 1957, 70; Schefold 1957,337

9267: Helbig 570; RP 119.3; Elia 1932, no. 61: Schefold 1957, 341; Tran Tam Tinh 1974. 51-54; Allroggen-Bedel, Gnomon cit.: Pfretscliner 1977, no. 63.

9538: Helbig 1334; Herrmann I, 159, Taf. 117.1; RP 170.2; Philippart 1925, 18 no. 26; Elia 1932, no. 74; Schefold 1957, 350; Tran Tam Tinh 1974, 52; Parlasca 197i, 231-236; Pfretschner 1977, no. 18.

9878: Mau 1882, Taf. XIX; Beyen 1938, 121-122, Abb. 40; Schefold 1957, 353; Herbig 1962, 26-29, Taf. 53-54; Borbein 1968, 152 nota 786; Allroggen-Bedel 1977, 34 nota 25; Allroggen-Bedel, Gnomon cit.; Bastet/De Vos 1979, 41, tav. XII. 22.

Inv. no. 8836: - Pompei, IX 3, 5 (cat. 282/7).

Inv. no, 8837: > Pompei, VI 9, 6 (cat. 204/2).

Inv. no. 8840-8841: > Castellammare di Stabia. Villa di Varano (cat. 010/2)

Cat. 054. Inv. no. 8845; III stile

Pinax centrale di una zona mediana rappresentante un paesaggio sacro-idilliaco. Sileno fa un'offerta alla statua gialla di Cibele che è seduta su un trono. Regge una lancia nella sinistra e poggia con il gomito sinistro su un timpano. Il modello deriva da una statua di Agoracrito.

La schola è sorretta da una cariatide bianca che liene con la destra la trave sopra la testa, mentre ha la sinistra in riposo sul fianco. La gamba destra e di appoggio; quella sinistra sta un po in avanti."95

493 Cf. Erim 1974.

494 Cf, Naumann 1983; Calza 1977, 35-37.

495 Helbig e Reinach, basandosi sull'illustrazione di un dettaglio in Zahn, considerano queste due figure come pitture diverse. 
Sul muro del recinto e seduta una sfinge maschile barbuta che ha sulla testa un bicchiere, attributo comune di Serapide

Bibl Helbig 421, 1877b, Herrmann 1. Tal 148, RP 1207,232 2, Elıa 1932, no 179, Spmazzold 1953, I, 239-240, Scholold 1957, 330, Schetold $1962,41,48$, Taf 22, Peters 1963, 72-73, fig 55, Schetold 1965, 116-117, Schmidt-Colinet 1977, 266 no P1 (collocazione erronea), Vermaseren 1978, 20 no 45 , pl XIA

Int no $8846>$ Pompe1, VI Insula occidentalis 10 (cat 2251 ).

Ins no $8849>$ Pompel, VII 4, 51 (cat 246).

Inv no $8860>$ Castellummare di Stabıa, Vulla dı Varano (cat 010/3)

Cat 055 Inv no 8879.8881 , IV stule

Partı di una rappresentazione architettonica proveniente dalla zona mediana o superiore Due tritonı rossi sorgono quali acroter su una trave Sono simmetrici Reggono un tumone con una spalla, mentre con una mano tengono davanti al petto, un piatto Lo stile delle figure, vivace e realistico, ricorda la Sclla della Villa di Varano (cat 010/3)

Bibl Helbig 1065, RP 44 2-3, CatLondon 1976, 153-154

Inv no 8890-8891, Castellammare di Stabıa, Vlla di San Marco (cat

009 4)

Cat 056 Inv no 8893 , IV stule

Da Ercolano provene questa parte di un campo nella zona mediana Su un campo rosso si delinea una ninfa acquatica gialla che regge, davanti al petto, una conchiglia Sta sopra una striscia o und mensola semplice La sua veste e un peplo E' l'unico esempio di una ninfa dipinta che regge una conchiglia 496 Bıbi Helbig 1054, RP 48 4, Elıa 1932, no 250, Schefold 195T, 330

Cat 057 Inv no $8899,8906,9369$; IV stule

Tre framments con edicole, probabumente provenients da una decorazıone ad Ercolano Su un capitello ionico vi sono satırı nudi di colore marrone che hanno le gambe unite e reggono una colonna sulla testa. Una clamide copre le spalle, una corona si vede sulla testa Reggono un piatto in una mano (quello su MN 8899 nella sinistra) vicino al fianco e nell'altra mano distesa un ramo d'olivo IL formato snello potrebbe essere un'indicazıone per la collocazıone del frammentı nel prospettı della zona mediana; anche la zona superiore, pero, non e da scartare

Bibl Helbig 1790, RP 68 2, Herrmann II, 64-65, Taf 243, Schefold 1957, $330,331,334$

Cat 058 Inv no $8900-8902,8908$, IV stule

Prospettı con offerentı, da Ercolano Sulla trabeazione accanto agli offerent $1 \mathrm{v} 1$ e una capra marrone in guisa di acroterıo, rivolta verso la figura umana Una statuella topiaria di bronzo e stata trovata a Pompel Von e chiaro se gli animalı siano da collegare, in senso iconografico, con gli offerenti Simuls acroterl il vedono nella (asa di Giuseppe II (cat 269/5)

${ }^{496}$ Cf la statuetta della Casa dell'efebo (Jashemskı 1979, fig 147). 
Bibl.: Helbig 1784, 1788, 1795; RP 229.10. 12, 230.2, 231.7; Elia 1932, no. 388; Maiuri 1953, 4; Schefold 1957, 331.

Cat. 059. Inv. no. 8904; IV stile

Sopra una trave ornata con una imago clipeata, proveniente da Ercolano, vi è un uomo che regge una fiaccola con la sinistra, mentre tiene la destra appoggiata al fianco. Ha i capelli ricci. Forse si tratta di Apollo.

Li figura contiene elementi di varje opere. Herbig confronta la testa con l'Apollo di Kassel. L'atteggiamento del corpo assomiglia all'Adonis di Capua. ${ }^{4} 7$

Helbig menziona Gegenst lacke che non ho potuto rintracciare. La datazione nel III stile da Herbig non è corretta.

Helbig 1792; RP 68.3; Schefold 1957, 331; Herbig 1962, 15 no. 11. Taf. 19; Peters 1963, Stelling VIII.

Inv. no. 8906, 8908: > Inv. no. 8899 (cat. 057).

Cat. 060. Inv. no. 8947, 9277, 9542; IV stile

Tre figure di una zona superiore in posizione simile a quella delle divinità nella Casa dell'alcova (cat. 026), provenienti da Ercolano. Su piani rettangolari stanno figure divine, dipinte a colori vivaci.

Su MN 8947 si vede Afrodite vestita di una veste diatana e un mantello rosso. Poggia con il gomito sinistro su un pilastro e regge con la destra un lembo del mantello presso la spalla. L'atteggiamento é simile a quello dell'Afrodite in I 3, 25 (cat. 146). Schefold pensa ad un'imitazione libera dell'Afrodite di Alcamene. "9iggins, discutendo terrecotte simili, attribuisce il modello originale a Prassitele. 49

Dioniso, su MI 9277, sta nel medesimo atteggiamento. La destra è presso le tempie. Un mantello bianco copre le gambe delle quali quella destra è di appoggio. Regge un tirso nella sinistra.

Su MN 9542 si vede Apollo Citaredo, rivolto verso destra. Suona la cetra che sta su un pilastrino, Le spalle sono coperte con una clamide rossa. Le proporzioni allungate sono d'impronta lisippea.

Bibl.: Helbig 1869, 387, 180; RP 233.9, 108.1, 24.2; Herrmann II. 30-31, Taf. 218; Schefold 1957, 342, 350.

Inv. no. 8910: > Castellammare di Stabia. Villa di Varano (cat. 010/1).

Inv. no. 8912: > Pompei, VIII 8, 28 (cat. 275/2).

Inv. no. 8913: > Castellammare di Stabia, Villa di Varano (cat. 010/4).

Inv. no. 8914: > Pompei, VIII 8, 28 (cat. 275/2).

Inv. no. 8924: > Inv. no. 8829 (cat. 053).

Inv. no. 8926, 8928: > Pompeí, VIII 8, 28 (cat. 275/2).

Cat. 061. Inv, no. 8953; III stile

Un frammento di una scaenae frons proveniente da Ercolano è simile alla struttura del portico nella Villa imperiale (cat. 305/3). Sopra un piedistallo a forma di $\mathrm{T}$ vi è una figura femminile di colore bianco che indossa un chitone

497 Zanker 1974, Taf. 80.4.

498 Cf, ora Delivorrias 1968.

499 Higgins 19j4, 194. 
corto. La gamba destra sta un poco in avanti. Ha la mano destra presso la spalla e la sinistra alzata nellatteggiamento di Artemide che prende una freccia dalla sua faretra. A causa del pessimo stato di conservazione non si puo stabilire di quale figura si tratta; l'interpretazione della figura come Artemide sarebbe possibile visti l'atteggiamento e la veste.

Bibl.: Helbig 1874 (o 1876 ?).

Inv. no. 8966: > Castellammare di Stabia, Villa di San Marco (cat. 009/4).

Cat. OG2. Inv. no. 89967; IV stile

In un solo telaio si trovano due frammenti. La figura sinistra ì una donna vestita di un chitone senza maniche che ha la testa coperta da un velo e una corona. Regge un piatto con entrambe le mani davanti al petto. Sopra la testa si vedono tracce di un arco, forse parte di un'edicola. Non è chiaro se la figura rappresenti una statua.

A destra si vede una figura maschile gialla che sta su una trave rossa, forse parte di un'edicola. Le gambe sono chiuse. Regge nella mano destra stesa verso il basso un ramo d'olivo e tiene nella sinistra presso la spalla un cestino.

Bibl.: Helbig 1781, 1813; RP 229.1, 23.6; Schefold 1957, 332 (forse assieme a MN 8890-8891 da Castellammare di Stabia, Villa di San Marco, cat. $009 / 4)$.

Inv. no. 8968: > Pompei, VIII 2, 38 (cat. 269/3).

Inv. no. 8975: , Pompei, VIII 8. 28 (cat. 275/2).

Cat. 0G3. Inv. no. 8990; III stile

Il giudizio di Paride sul quadro centrale di una zona mediana, ora molto svanito. Dietro Atena. Afrodite e Hera si vede la statua rossa itifallica di Priapo. La statua non ha una funzione nella storia rappresentata, ma appartiene all'ambiente paesistico.

Bibl.: Ifelbig 1283; Herrmann II 28, 1 bb. 5, Taf. 216; Schefold 1962, 333; Paar 1962, 123 no. 6.

Inv. no. 8991: > Pompei, VI 7, 8 (cat. 194)

Inv. no. 8992: > Pompei, IX 3,5 (cat. 282;7).

Inv. no. 8998: > Pompei, VI 9, 6 (cat. 204/7).

Cat. 064. Inv. no 9010; IV stile

Il cavallo di Troia. pinax centrale proveniente da Pompei. A sinistra sta la statua bianca di Atena Promachos, rivolta verso sinistra. Indossa un peplo e ha un elmo sulla testa. Regge nella sinistra lungo il fianco uno scudo e nella destra stesa una lancia.

Bibl.: Helbig 1326; RP 171.6; Davreux 1942, 134-135, fig. 33; Dawson 1944, no. 13; Schefold 1957, 334: Peters 1963, 134-135.

Inv. no. 9018: > Pompei, VI 1, 10 (cat. 190/2).

Inv. no. 9059: > Pompei, II 4, 3 (cat. 179:1).

Inv. no. 9061: > Pompei, IJ 4, 3 (cat. 179:1).

Inv. no. 9067-9070: : Pompei, II 4, 3 (cat. 179/1). 
Cat 065. Inv. no. 9090, IV stile

Pigmel in lotta su un pinax da Pompel La seconda ligura da destra regge noll sinistra distesa una piccola statua rossa di una figura con le braccia distese La lunzione della statuetta non e chiara, come non e nemmeno noto l significato della scena.

Bibl Helbig 1534; RP 161.7, Schefold 1957, 336

Inv. no 9111. "Pompei, I 4, 5 (cat. 1475).

Inv no 9112 . > Pompei, VI 8,3 (cat. $198^{\prime} 2$ ).

Cat. 066 Inv. no. 9120, 9153, IV stile

Figure marroni stanno su una trabeazione rossa davanti ad uno sfondo bianco su due frammenti provenienti da Ercolano. Probablimente appartengono ad una scaenae trons nella zona superiore.

Su MN 9120 si vede un Dioniso dj Tivoli che regge un tirso con la sinistra alzata e un ramo nella destra lungo ll fianco. La gamba destra e di appoggio Il contrapposto accennato potrebbe essere un elemento della scuola policletea.

Su MN 9153 si vede un saturo danzante Ha la gamba destra alzata Regge una siringa nella destra alzata e con la sinistra un pedum presso la spalla. Sulla testa ha una corona e attorno alle spalle una clamide. Una figura simule si vede nella Casa del colonnato tuscanico ad Ercolano (cat 035,4). 1.o stile di quel pezzo e uguale, ll che potrebbe essere un elemento per attribuirlo ad una bottega comune

Bibl Helbig 389, 431, Schefold 1957, 337

Inv. no 9123: > Inv no. 8829 (cat. 053).

Inv. no 9123 : > Pompel, VI 10, 11 (cat 207/1). soo

Inv no.9125 > Inv. no. 8829 (cat 053).

Inv no $9132>$ Pomper, VI 10,11 (cat. 207/1)

Inv. no 9141: > Ercolano, Insula orientalıs II A, 4 (cat 0392 ).

Inv. no. 9153. > Inv no. 9120 (cat. 066).

Inv no. 9154., Pomper, III 3, 7 (cat 181,1).

Inv. no. 9165: > lnv no 9183 (cat. 068).

Inv. no 9171: , Pompel, III 3, 7 (cat 181/2).

Cat. 067. Inv. no. 9175; IV stile

Pinax da Ercolano Eros prende un cervo presso l'armatura. Il gruppo corrisponde al gruppo bronzeo di Eracle e il cervo, trovato nella Casa di Sallustio e ora a Palermo 501 ll gruppo dipinto certamente non e stato concepito come statua.

Bibl.: Helbig 796, RP 837 , Schefold 1957. 338.

Cat. 068 Inv. no 9183 , III stile

500 Apparentemente nell'Inventario e stato fatto un errore cosicche due pezzi diversi recano il medesimo numero.

501 Inv. no. 8364 (Jashemski 1979, 123). Cf. Moreno 1984, 147-152, IIg. f, $23-24,26$. 
Pinax ds un fregıo fra zone mediana e superiore, insieme a MN 9165, proveniente da Pompes Ln'erma dl Priapo sorge fra figure di baccantı

Helbig 569, RP $1194-9$, Llia 1932, no 382. Schefold 1957, 338, 339, Schefold 1962. 66-67, Taf 72 , Ilerbig 1962 21-26 'Taf 35-50, Bastel, De los 1979, 73-74, Taf XXXVIII 69, Architects 1981, 254-255, 270-272

Inv no 9206-9208 > Pompe1, IX 3, j (cat 282 i)

Inv no $9231>$ Pomper, VI Insula occidentalis (cat

Cat 069 Inv no 9244, II stule, fase Ic

Pinas provemente dalla zona superiore di una decorazione ad Lrcolano, con una posizione simule a quella dei pinahes nel criptoportico della casa omonima a Pompes I na mendde viene assalita da un satıro presso und lonte La statuctta gialla di Priapo che e rivolta cerso sinistra, mostra l lallo ai due Regge nella mano sinıstra un pedum a tiene l braccio destro steso

A destra vi sono tre statuette gialle sopra una base rettangolare La figura centrale e leggermente piu alta e regge un piatto $S_{1}$ tratta di un hekataion o un gruppo delle tre Grazic, in entrambi 1 casi divinita acquatiche

Bibl Helbig 1053, RP 124 3, Beven 1960, 361, Abb 139, Grumal 1969, 306-307, Allroggen-Bedel 1974a, 75-79, Fig 1

Inv no 9245 > Pompel, V 2, 9 (cat 192 2)

Cat 070 Inv no 9253 , IV stule

Sopra un piedictallo sta un guerriero nudo di colore giallo che regge nella smistra uno scudo e nella clestra stesa per meta un elmo la gamba destra e di appoggio Lna clamide copre le spalle Il frammento e stato oggetto di restaurı settecenteschi La figura entra nella serie dei guerrierı policletel (cf $p$ 43) ma appartiene, come le due figure nella Casa dell'efebo (cat 1554), al pochı esempl che mediante il colore cono indubbiamente statı concepiti come statue

Bibl Schetold 1957, 311

Inv no $9255>$ Pompe1, $1 X 3,5$ (cat 282 7)

Cat 071 Inv no 9258 , IV stule

Paesaggio sacro-ıdillaco di colore marrone su un pannello della zona mediana, proveniente da Pompei Su un altare presso un albero sacro e un'erma con una testa barbuta incoronata Sulla base delle vecchie incisioni sembra che II siano tracce ritrattistiche Il cantaro a sinistra potrebbe essere un indizio a favore di un'interpretazione delld figura come Dioniso

Peters data 1 Irammento nel III slule visto the clear cut drawing

Bibl Helbig 572 RP 117 1, Ela 1932, no 83, Schetold 1957, 341, Peters 1963,72 , fig 57

Cat 072 Inv no 9261, IV stule

Un quadro centrale proveniente da Ercolano, mostra Dioniso che porge un ritone ad un satiro Sopra un piedistallo rettangolare a destra sta la statua marrone itıfallica di Priapo $E^{\prime}$ ilvolto verso destra e regge nella mano destra una bacchetta 
Dioniso e stato raffigurato nell'atteggiamento del Dioniso di Tivoli Poggia sulla gamba sinistra e regge un tirso con la sinistra, mentre stende con la destra ll ritone sopra ll satiro

Bibl Helbig 403, RP 109 1, Herter 1932, 138 no 39, Elıa 1932, no 78, Her big 1949, 84, Taf 341 , Schetold 1957, 341

Inv no 9267 , Inv no 8829 (cat 053 )

Cat 073 Inv no 9275 , IV stule

Tre nature morte su sfondo rosso, provenuenti da Pomper Nel frammento a sinistra si vede una sfinge femminile alata posta sopra un blocco rettangolare L'acqua che esce dalla bocca cade in una vasca che sta davants al puedistallo Questa posizione fa pensare alle table fountains 502 Secondo Demisch l'uso dı sfingl come fontane e raro

vel trammento centrale vi e un'erma bianca presso un bacino, un rullo e und palma La testa mostra un uomo barbuto, probabilmente ll vincitore della gara sportiva o Eracle come patrono dello sport

Il terzo trammento contiene l'erma du Sleno ammantato posta sopra und base rettangolare Tutto e di colore bianco Sulla testa si vede una corona Secondo Bianca Maiurı egli regge un piccolo Dioniso in un lembo del mantello Questo, pero, non e piu visibule

Bibl Helbig 1758, 1777, RP 117 6-7, 348 10, Malurı 1950, 187-188, fig 9 , Lehmann 1952, 112, fig 62, Spinazzola 1953, I, 292, fig 328 Croisulle $1965,51-52$ no 89, pl CXV ph 217, Ling 1971, 268, pl 49a, Demisch 1977, 116, Abb 334

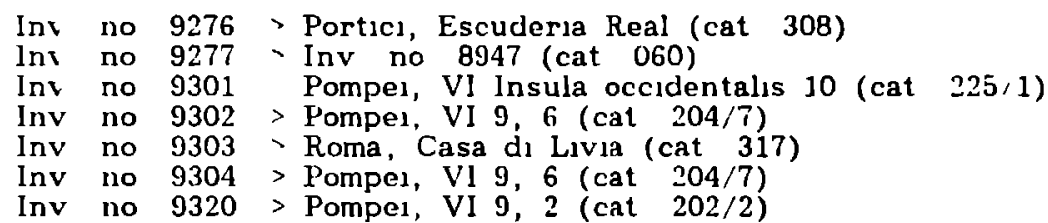

Cat 074 Inv no 9336 . IV stile

Il frammento, che prov iene da Ercolano mostra un campo bianco della zona mediana sul quale si delinea un Eros sopra una mensold $E^{\prime}$ giallo e ha un mantello verde sulle spalle, mentre anche le alı e una corona sulla testa sono di questo colore La gamba destra e di appoggio Regge con entrambe le mani davantı al petto una fraccola

La figura entra nelle serie delle figure sopra mensole che ornano ambienti con una decorazione a campi La Casa di $M$ Lucrezıo e l'unico esempio con numerl di Erotı (cat 282) S Reınach descrisse la figura come génie func raire, interpretazione soltanto possibile in un contesto funerario, ma improbable in una casa privata

Bibl Helbig 610, RP 69 4, Schefold 1957, 344

Inv no $9369>$ Inv no 8899 (cat 057 )

502 Dwver 1982, 117 Cf la fontana della Casa di Sallustio (Kapossy 1969, j3) 
Cat 075 Inv no 9370 , IV stule

Il I rammento proviene da Ercolano Su uno sfondo bianco si delinea sopra un candelabro un uomo vestito di una tunsca bianco-gralla con una corona sulla testa Le gambe sono chiuse Regge nella destra posta lungo Il franco una corona, mentre l'altra e avvolta nella tunicd La posizione originale non e sicura un prospetto nolla sona mediand o una seaellu Iroms nella cona uuper lore

Bibl Holbig 1799

Cat 076 Inv. no 9372 , III stule

Alcunı framment 1 provenient 1 da Pompei sono stat 1 incastratı in un solo tela A sinistra e a destra si vedono guerrierl pirrici che stanno con le gambe unite sopra elementi architettonici Sull'elmo a pennacchio poggia una trave Nella sinıstra stesa reggono uno scudo ovoudale, nell'altra stesa verso il basso hanno un gladio la cul punta sembra conficcarsi nel petto Le spalle sono coperte da una clamide 503

Si inseriscono nel genere di figure usate nelle scaenae frontes nella zona superiore $d_{1}$ III stule Lo stule di dipingere a strisce sottli per indicare l'incarnato e l'allungamento del corpi fa pensare alle figure su MN 8829 (cat 053) Forse provengono da un altro ambiente del medesimo complesso, probabilmento sono opere di una sold bottega

Vel centro si vede un Eros sopra un candelabro La gamba destra e di appoggio Lna clamide copre le spalle Eros regge con entrambe le manı una cornucopia davantı al petto La posizione originale del trammento non e chiara Don e nemmeno sicuro se provenga dalla decorazione con 1 guerrieri

Bibl Helbig 604, RP 69 1. Schetold 1957, 344, Borbein 1968, 152 nota 786

Inv no $9378>$ Pompe1, VI 14, 42 (cat 216)

Cat 077 Inv no 9385 , IV stule

Quadro centrale con varciso, proveniente da Torre Annunziata Il fanciullo sta nell'atteggiamento dell'Apollo Saurohtonos di Prassitele, ma in modo simmetrico poggia con la mano destra contro un albero e tiene la sinistra nel fianco Poiche la maggior parte delle rappresentazionı mostra Narciso seduto, in questo caso e probabile che $1 \mathrm{l}$ pittore abbia scelto volontariamente 1 modello prassiteleo

Bıbl Helbig 1358, Elia 1932, no 133, Herrmann II, Taf 231; Schetold 1957, $34 \overline{5}$

Cat 078 Inv no 9398 , IV stule

Paesaggio sacro-idiliaco in grallo su uno sfondo rosso, proveniente da Castellanmare di Stabia A sinistra si vede un'erma bianca, probabulunente rappresentante Priapo Come MN 9405 (cat 078) mostra una certa affinita con MN 9401-9403 della Villa di Varano (cat 0105 )

Bubl Cosenza 1907, 213 no 145, Schefold 1957, 345

Inv no 9401-9403 > Castellammare di Stabıa, Vlla di Varano (cat. 010'5).

503 Cf Scarpl 1979 
Cat. 079. Inv. no. 9405; IV stile

Paesaggio sacro-idilliaco in giallo e azzurro su uno sfondo rosso. Dentro una torre sorge un pilastro sormontato da un'erma. Forse il frammento proviene dalla Villa di Varano a Castellammare di Stabia, appartenendo ad un solo complesso con MN 9401-9403 (cat. 010/5).

Bibl.: Rostowzew 1911, 87; Schefold 1957, 345.

Cat. 080. Inv. no. 9409: IV stile

Quattro tondi da Castellammare di Stabia. Sul tondo nel registro inferiore. a sinistra, si vedono due statue sopra una base presso una villa marina. A sinistra vi e una figura femminile che ha la mano destra appoggiata sulla testa; un mantello copre la parte inferiore del corpo. A destra una figura fermminile nuda stende la mano destra e ha la sinistra in riposo sulla testa.

Bibl.: Cosenza 1907, 193 no. 61; Rostowzew 1911, Taf. $T .2$; Herrmann I, 227 nota 2; Elia 1932, no. 276; Schelold 1957, 345; Peters 1963, 157, fig. 148.

Cat. 081. Inv. no. 9410; IV stle

Paesaggio sacro-idilliaco su uno sfondo verde entro una fascia rossa attorno alla quale si vede un pannello bianco. In un tempietto su un'jsoletta sta una statua amorfa. Sopra un portico a destra sorge un'erma bianca.

Bibl.: Rostowzew 1911, 85 nota 2; Schefold 1957, 345; Silberberg 1981, cat. 62 .

Cat. 082. Inv, no. 9411; IV stile

Paesaggio sacro-idilliaco, forse il quadro centrale della zona mediana. Sopra un piedistallo rotondo sorge una statua ora illeggibile. A Jestra si vede un trofeo giallo. Forse la statua puo essere interpretata come Ares.

Bibl.: Rostowzew 1911, 93; Herrmann 1, 227 nota 2; Schefold 1957, 345; Peters 1963, 163.

Inv. no. 9413: Portici. Villa soto l'Escuderia Real' (cat. 308).

Cat. 083. Inv. no. 9414; IV stile

Veduta marina da Castellammare di Stabia, costituente in origine il quadro centrale di una parete documentata attraverso un vecchio disegno. A sinistra in primo piano stanno, sopra una base rettangolare tre statue rosse di figure femminili. Reggono in entrambe le mani una fiaccola e sono vestite di un peplo. Ovviamente stiamo di fronte a un hekataion.

Bibl.: Cosenza 1907, 213 no. 147; Rostowzew 1911, 83, Abb. 49; Elia 1932, no. 258bis, fig. 34 ; Spinazzola 1953 , II, 859-860, fig. 862; Schefold 1957 , 346; Peters 1963, 151-152; Silberberg 1981, cat. 61.

Cat. 084. Inv, no, 9418; IV stile

Paesaggio idilliaco da Pompei, il cosiddetto Ariete smarrito. A sinistra sorge sopra uno scoglio l'erma gialla scura di Priapo o di un'altra divinita rurale, il cui busto è reso in maniera molto plastica. In cappello copre la testa. Regge una bacchetta nella sinistra e tiene la destra distesa.

Il frammento sarebbe da attribuire alla bottega della Casa dei vettii secondo Beyen e Peters. 
Bibl.: Helbig 1564; Rostowzen 1911. 87, fig. 55; RP 386.3; Elia 1932, no. 258; Bcyen 1951, 53 nola 5; Schelold 1957, 346; Potcrs 1963, 118-119, fig. 140; Ptretschner 1977, no. 86; Silberberg 1981, cat. 51, fig. 57.

Inv. no. 9423: > Ercolano, Villa dei papiri (cat, 039A).

Cat. 085. Inv, no. 9427; IV stile

Paesaggio sacro-idilliaco della zona mediana. In un tempio che sorge nel centro si vede una statua sopra una base a forma di $\mathrm{T}$. La figura indossa un lungo mantello e ha la mano destra nel fianco e quella sinistra alzata.

Bibl.: Overbeck Mau 1884, 573, fig. 297: RP 383.6.

Inv. no. 9454: > Pompei, VI 9, 6 (cat. 204/2).

lnv. no. 9456-9457: 'Pompei, VI 10, 11 (cat. 207/1).

Cat, 086. Inv. no, 9459; IV stile

Paesaggio sacro-ıdillaco rovinato, da Castellammare di Stabia. Fa parte di una zona mediana. Navanti ad un tempietio sta una statua schematica alla quale un uomo porge un'offerta.

Bibl.: Cosenza 1907, 194 no. 66; Schefold 1957, 347.

Inv. no. 9476: > Pompei, VI Insula occidentalis 10 (cat. 2253 ).

Inv. no. 9479: > Castellammare di Stabia, Villa di San Marco (cat. 009/11).

Cat. 087. Inv. no. 9482; III stile

Veduta di mare dalla zona mediana. Un portico e una tholos sono sormontati da statue schematiche di colore bianco.

Bibl.: Helbig 1574; Rostowzew 1911, 227, Taf, 165: Schefold 1957, 347; Von Blanckenhagen/Alexander 1962, 36, pl. 56; Peters 1963, 117-118, fig. 98.

Cat. 088. Inv. no. 9484; IV stile

Veduta di mare dalla zona mediana di una decorazione a l’ompei. Sul molo nel centro stanno due arch triontali sormontati da tre statue dorate per arco, Sulle sei colonne che sorgono ai due lati del molo vi sono delle statue, delineate in modo schematico. Si confronti MN 9514 (cat. 097).

Bibl.: RP 382.8; Schefold 1957, 348; Peters 1963, 158.

Cat. 089. Inv, no. 9486; IV stile

Paesaggio sacro-ıdilliaco dalla zona mediana, proveniente da Pompei. Nella micchia di una schola e la statua bianca di una figura temminile che indossa un peplo. Tiene le mani incrociate davanti al petto. La stessa nicchia è sormontata da un centauro. A destra vi e un'erma.

Bibl.: Rostowzew 1911, 83; Herrmann I, 236-237. Taf, 172a; Elia 1941, 32, fig. 29; Schefold 195i, 348; Peters 1963; 150, fig. 141; Silberberg 1981 , cat. 58 , fig. 58 .

Cat. 090. Inv. no. 9488; IV stile 
Paesaggıo sacro-ıdullaco dalla zona mediana, provenıente da Pompel Presso un fiumicello si vede sopra uno scoglio la statua di tcate, la cur parte inferiore consiste in un'erma. Regge in entrambe le mani stese in avanti una traccola Il tusto e alzurro, la parte superiore, invece, bianca a macche rosse

Bibl Rostowzew 1911, 86, fig 53, Herrmann I, 236, Taf 71, Schefold 1957, 348. Peters 1963, 158, Pfretschner 1977, no. 42, Suberberg 1981, cat 88 , fig 76 , Swinkels $1984,41-42$, fig. 1 .

Cat 091 Inv no 9489 , IV stule

Papsaggıo sacro-ıdıllıco dalla zona mediand, provenıente da Pompeı Darantı ad una porta sacra e und tholos si vede la statua gialla di cibele, modellata secondo \&l tipo consueto, attribuito a Agoracrito (cf M. 8845, cat 054)

A sinistra della tholos si trova un tavolone con la statua bianca di un androsfinge alato Sulla testa regge un calice. Von e chiaro se abbia o no la barba

Bibl Helbig 1558, Rostowzew 1911, 44 nota 2, Herrmann I, 236-237, Taf $172 \mathrm{~b}$, RP 388 4, Elia 1941, 33, tig 30, Schefold 1957, 348, Peters 1963,150, fig 142, Schefold 1965, 117, Vermaseren 1978, 20-21 no 46, Suberberg 1981, cat. 50, fig 59

Cat 092 Inv no 9492, IV stule

Paesdggio sacro-ıdilliaco in bianco su un campo rosso della zona mediana, proveniente da Castellammare di Stabia A destra si vede la statua di Poseidon sopra un piedistallo rotondo $\Gamma^{\prime}$ modellato secondo ll tipo henchreal regge il tridente nella mano sinistra alzata e un delfino nella sinistra 504

Nel centro presso una tholos si trova la statua schematica di una ligura femminile vestita di un mantello lungo Regge una patera nella destra stesa e ha la sinistra in riposo lungo il fianco

Bibl Cosenza 1907, 213 no 149, Rostowzew 1911, 41 nota 1, tbb 19. Elia 1932, no 259. Noack/Lehmann Hartleben 1936, 218, Schefold 1957, 348, Peters 1963, 163, fig 160, Suberberg 1981, cat 93, fig 84.

Cat. 093. Int no 9494, IV stule

Veduta di una villa dalla zona mediana A destra sı trova un'erma bluastra a forma dı una pedina sopra una base alzata

Bibl inedito

Inv no. $9496>$ Pompel, I 4,5 (cat. 147/1).

Cat 094 Inv no 9508 , IV stule

Parıde sull'Ida, quadro centrale di una decorazıone a Pompeı Davantı ad una porta sacra e una schola si distingue la statua gialla di Priapo sopra una base rettangolare $d_{1}$ colore buanco Nel mantello tenuto in alto regge truttı Corrisponde al tipo Formello, analizzato da Blanch

Bibl Helbig 1279, Rostowzew 1911, 41. Herrmann 1, Taf 8; RP 163 1, Elıa 1932, no. 95. Uawson 1944, 8, pl 6: Schefold 1952. Taf 25. Schelold 1957, 348, Beyen 1960, 443, Schefold 1960, 92-93, Taf 10, Paar 1962, 120-121, Abb 23, Peters 1963, 128, fig 104, Pfretschner 197i, no 35 . Blanck 1979,342 nota 18

$504 \mathrm{Cf}$ Walde 1978, Walde-Psenner 1979 
Cat 095 Inv no 9510 , IV stule

Quattro paesaggi sacro-ıdlliacı clalla zond mediana, provenientı da Crcolano La seconda da sinistra contiene un gruppo di edifici su un'isoletta Accanto sta la statua rossa di un uomo nudo che regge con la destra alzata una lancia a uno scettro, mentre tiene stesa in avantı la mano sinustra Ilauna conora sulld testa

Bibl Schetold 1957, 349

Inv no $9512>$ Pompel, VI Insula occidentalıs 10 (cat 2253 )

Cat 096 Iny no 9513 , IV stule

Veduta di ville lungo il vilo in un quadro della zona mediana, proveniente da Ercolano In primo piano un asino pascola presso la statua rossa di Dioniso barbuto che e vestito di un mantello lungo Regge un tirso nella sinistra alzata e un cantaro nella destra stesa

A sinistra sul ponte si vede l'erma rossa itıfallica di Priapo che sta presso un editicio

Bibl Helbig 1581, Rostowzew 1911, 92, fig 58, Schefold 1957, 349, Peters 1963,163

Cat 097 Inv no 9514, IV stule

Veduta dı mare dalla zond mediana, trovata a Castellammare di Stabia Sul molo nel centro sorge un arco trionfale incoronato da due tritonı giall postı summetricamente che reggono un tumone sulla spalla $A$ destra dell'arco vi sono due colonne con statue, accennate soltanto con strisce bianche In fondo si distinguono quattro altre colonne alte e una bassa con statue sumli

Il pinax a volte e stato interpretato come una veduta del porto dı Pozzuol Gli elementı corrisponderebbero d element 1 su bottıgliette dell'epoca imperiale Seconclo $J$ holendo sl vedrebbe Alessandrı Gia Peters accenno alla schematıcita della rappresentazıone e alla scelta non precisa degls elementı topografici S Ostrow, nella sua analisı accurata delle bottigliette, ha posto fine al dubbio su questo problema e hd dimostrato che il quadro non e altro che la veduta di un pos to qualsiasi

Bıbl Helbig 572d, Cosenza 1907, 195 no 70, RP 3794 , Llia 1932, no 277, Schetold 1957, 349, Picard 1959, Peters 1963, 152, fig 146, Ostrow 1979,78 nota 2 , kolendo 1982

Inv no 9516-9517> Ercolano, IV 1-2 (cat 025 2)

Cat 098 Inv no 9540 , IV stle

Tre raffigurazionı glallo di Apollo su uno sfondo bianco, vignette della zona mediana di una decorazione da Castellaminare di Stabia Sono nudi e hanno una corona sulla testa Sorgono sopra un segmento o mensola non piu visıble L'Apollo a sinistra regge un ramo d'alloro nella destra distesa e poggia con l gomito sinistro su un plastrino coperto da un drappo La gamba destra e d) appoggio Contro ل $\downarrow$ pulastro e appoggiata la cetra

La ligura centrale suona una cetra che sta su un plastro alla sua sinistra Il piede sinistro sta daianti a quello destro In terra un piccione e appoggiato su un oggetto rotondo, probabulmente raffigurante l'omphalos L'atteggiamento 
dell'Apollo è simile a quello di una statuetta di bronzo trovato a Pompei. ${ }^{50 s}$

La terza figura assomiglia alla prima. Ora la gamba sinistra è di appoggio.

Il dio regge una cetra nella sinistra e un ramo dalloro nella destra.

Le tre figure non copiano statue a noi note. ma csprimono l'influsso di modelli tardoclassici e ellenistici. Le proporzioni e l'attegriamento delle gambe si devono a tipi lisippei.

Dal momento che il contesto originale manca, non è sicuro se il pittore abbia voluto dipingere copje di statue o se le figure facciano parte di una decorazjone monocroma gialla.

Bibl.: Helbig 185; RP 23.1; Elia 1932, no. 41; Schefold 1957, 350.

Inv. no. 9541: > Pompei, VI Insula occidentalis 10 (cat. 225/1).

Inv. no. 9542: > Inv. no. 8947 (cat. 060.

Inv. no. 9550: > Pompei, VIII 2, 38 (cat. 269/4).

Inv. no. 9551: > Pompei, VI 9, 6 (cat. 204/2).

Inv. no. 9555: > Pompei, VI 9, 1 (cat. 201).

lnv. no. 9558: > Pompei, VIIl 8, 28 (cat. 275/2).

Inv. no. 9559: > Pompei, VI 8, 3 (cat. 198/1).

Cat. 099. Inv. no. 9561

Pittura encaustica su una lastra marmorea, trovata a Ercolano, nota come 'Il Sileno stanco'. Su una colonna vi è la statua di Atena vestita di un peplo e con un elmo a pennacchio sulla testa. Kegge una lancia nella destra e tiene uno scudo rotondo con la mano sinistra.

Bibl.: Helbig 1405; RP 121.5; Elia 1932, no. 53; Schefold 1957, 350; Mielsch $1979 b, 240$; Von Graeve 1984, 95, Abb. 6, 8.

Cat. 100. Inv. no. 9605; III stile

Paesaggio nilotico in un quadro centrale. Nella porta di un tempio presso il fiume si trova la statua di lside. Indossa una veste stretta $e$ ha un modio sulla testa. Regge nella destra alzata un sistro o un loto e alza con la sinistra un lembo della veste.

Sul tetto dell'edificio vi sono due Eroti nudi di colore marrone, resi in modo simmetrico. La gamba al lato interno è di appoggio. Probabilmente reggono con entrambe le mani davanti al petto un festone.

L'insieme è diventato quasi illeggibile e i colori sono svanitt.

Bibl.: Schefold 1957, 351.

lnv. no. 9608, 9610: > Pompei, I 4, 5 (cat. 147/1).

Cat. 101. Inv. no. 9638; III stile

Rappresentazione di un giardino di colore giallo su uno sfondo nero, parte dello zoccolo di una decorazione ad Ercolano. La pergola centrale è occupata da una statua ora quasi invisibile. Sui cancelli a sinistra e a destra vi sono cani o leoni.

Le pergole laterali sono sorrette da erme snelle con braccia e mani che reggono un pedum e una patera.

Bibl.: Grimal 1943, pl. XIX.3; Schefold 1957, 351.

505 Dwyer 1982, fig. 207. 
Cat 102 Inv no 9646 , IV stule

Edicola centrale di una zona superıore, proveniente da Pomper Dasanti ad un'edicola absidale std sopra un piedistallo giallo la statua bianca di Ares La gamba sinistra e di appoggio Regge una clamide, una lancia e uno scudo con la simistra contro ll fianco e tiene nella destra stesa verso l basso una spada La testa, rivolta ierso sinistra, e coperta da un elmo a pennacchio Gli attributi sono dipinti a color veristici, giallo e rosso, pritica visibule anche presso f'Ares della Cand della venere in inchegla (at 178)

La ligura non copid in modo disetto un esempio noto, ma esprime unimpronta classicheggiante L'atteggiamento delle gambe f della testa tanno ricordare glı atletı di Policleto, le braccia sono sumlı a quelle dell'Ares Borghese

Bibl Helbig 270 RP 58 2. Schefold 1957, 351, Reutersward 1960. 182 nota 509, Pfretschner 1977, no J3a

Cat 103 Inv no 9659, IV stule

Frammento di una zona superiore da Ercolano $S u$ uno stondo rosso si delinea una can latide bianco-gialla che regge sulla testa un piccolo segmento quadrangoldre sul quale poggia una trave Sta sopra l'elemento aggettante di una trave lndossa un peplo e una solravveste e ha una corona sulla testa Regge nella destra itesd verso ll basio un ramo di palma e nella sirustra, presso la spalla, un lestone che continud verso destra la gamba destra e di appoggio

Bibl Helbig 1878, Schefold 1957, 351

Cat 104 Inv no 9660 IV stile

Tre vignette di campi nella zona mediana provenientı da Pompel a sinistra e a destra si vedono nature morte a soggetto sportivo, nel centro sta un Eros (non 1 levante al finı della nostra ricerca) In entrambe le rappresentazioni si vedono erme gialle con una testa maschule barbuta Stanno su blocchi quadıangolar A sinistra davanti all erma si vede un bacino a destra un'idrid, una patera e un ramo di palma

Bıbl Helbig 1757, Maiurı 1950, 188 no 41, Schefold 1957351

Inv no 9670-9671 > Ercolano, Insula orientalıs II A, 1a (cat 038)

Cat 105 Inv no 9672 , IV stule

Quattro vignette dı campı nella zona medıana, provenıentı da Castellammare dı Stabia La seconda dd sinistra contiene un paesaggio sacro-ıdilliaco con una statua rossa schematica

Bıbl Cosenza 1907, 196 no 72, Schefold 1957, 351

Inv no 9674,9678 Ercolano, Insula or jentalis Il $A, 1$ d (cat 038)

Cat 106 Inv no 9688 , IV stule

Zoccolo nero con cassette riempite da anumalı marmi, divise da due erme gialle Da calici stulizzali sorgono figure ammantate del tiaso di Dioniso A sinistra si distingue un satiro incoronato che regge nella sinistra distesa una patera e nella destra una fiaccola o una clava sulla spalla A destra $v$ e un Suleno con la testa tasciata con una benda Regge una patera nella destra stesa verso l basso e una clava o una fiaccola nella sinistra sulla spalla 
L'atteggramento delle erme è simile a quello dell'erma di Teseo nel Museo Nazionale Romano. ${ }^{306}$

Bıbl. Helbig 1120, 506 (?; 1075, 1541, 1543 a proposito deglı anumalı marinı).

Cat. 107. Inv. no. 9515; IV stile

Candelabro su uno sfondo branco sulla lesena fra due campi gialll. Sotto il candelabro si vede un paesaggio sacro-idlliaco con una statua bianca schematica presso alcuni edifici.

Bibl inedito.

Inv. no. 9731. > Ercolano, Insula orientalis, II A, 4 (cat. 039/1).

Inv. no. 9755-9756 > Ercolano, Insula orientalis il A, la (cat. 038)

Inv. no 9763: > Castellamare di Stabia, Vla di Varano (cat 010/1).

Inv. no. 9769: > Ercolano, Insula orientalis II A, 1a (cat. 038).

Inv. no. 9774: > Pompei, VI 8, 20 (cat. 199).

Cat. 108 Inv. no. 9775 ; IV stule

Paesaggio sacro-ıduliaco e natura morta in un solo telaio. Sulla tholos nel paesaggio sorge un acroterio a forma di sfinge.

La natura morta con soggetto sportivo contıene un'erma grezza dietro una vasca.

Bıbl. inedito.

Cat. 109. Inv. no. 9777; IV stile

Figure gialle si delineano su uno sfondo nero molto rovinato; il frammento proviene da uno zoccolo. Ln cervo viene inseguito da una pantera. A sinistra sarge una colonna con la statua di una figura ammantata. Le mani sono stese verso destra. Probabumente si tratta di Priapo che costituisce l'elemento paesistico.

Bıbl.' Ineduto.

Cat. 110. Inv. no. 9787 ; II stile (?)

Frammento con elementı architettonici dalla parte superiore di una zona mediana Su un architrave aggettante di colore grigio siede un gruppo giallo composto da un tritone e una vereide poggiata sul suo dorso. Manca la parte superiore del gruppo.

Bibl. . inedito.

Cat. 111. Inv. no 9859; IV stile

Frammento di un'architettura gialla, diventata rossa a causa del fango bollente, proveniente da Ercolano. Sopra un pilastro sorge l'erma di Sileno. La testa dipinta con molta cura presenta una barba ricciuta e orecche a punta. Una benda cinge la fronte. Non e possibule capire se la figura avesse funzione di atlante.

Bibl.: RP 108.9.

Inv, no 9878: > Inv. no, 8829 (cat. 053).

s06 CatRoma I 5, 170-172 no 72. 
Cat. 112. Inv. no. 9891; IV stile

Quattro vignette su uno sfondo rosso dalla zona mediana. Sulla seconda da sinistra si vede una Mantelherme posta sopra una base rettangolare presso un'idria. Due peda sono appoggiati sia alla base che all'erma. Probabilmente si tratta di Priapo.

Bibl.: inedito.

Cat. 113. Inv. no. 9934; IV stile

Frammento architettonico dalla zona superiore di una decorazione a Pompei. In un edicola, forse quella centrale, vi è una biga. Il carro è stato raffigurato come un blocco quadrangolare. I due cavalli sono rivolti verso l'esterno. Il conduttore indossa un peplo e ha una corona sulla testa. Regge nella destra alzata un ramo di palma e nella sinistra la briglia. Nonostante la mancanza di ali sembra trattarsi di una Nike. ${ }^{507}$

Bibl.: incelito.

Cat. 114. Inv. no. 9970; IV stile

Tre frammenti in un solo telaio. Il frammento centrale contiene la trabeazione di un'edicola centrale della zona mediana. Sopra di essa sta un atleta nudo di colore giallo che poggia su un'erma. Tiene la mano sinistra vicino alle tempie, mentre la destra è appoggiata sulla testa. La gamba destra è di appoggio. La testa dell'erma, coperta da un ctrappo, raffigura il Dioniso di Alcamene.

Alle estremitá della trabeazione si vedono dei cigni bianchi che fungono da acroteri.

L'atleta e una copia simmetrica dell'igon di Boethos di Calcedone, trovato nella nave di Mahdia, come ha osservato giustamente Schefold. Differisce la posizione dolle mani e delle gambe. 500

Bibl.: Schefold 1957, 353.

Cat. 115. Inv. no. 10004; primo secolo d.C.

Le tre Grazie fanno parte di un paesaggio, del quale sono rimaste visibili solo alcune tracce in un mosaico parietale. L'atteggiamento delle figure copia il gruppo ellenistico: sj veda la discussione a proposito di Pompei, VI Insula occidentalis (cat. 227).

F. Sear confronta tecnicamente il mosaico con quelli trovato nelia Casa di Apollo e lo colloca nella prima metá del primo secolo d.C.

Bibl.: Sear 1977, 71 no. 31 , pl. 16.2 .

Cat. 116. Inv, no. 10010

Mosaico parietale, forse di un ninfeo. Nel registro superiore si vede sopra una base bianca rettangolare la statua di un pugile dipinta a colori naturali. Le mani, avvolte con bende di cuoio per la boxe, sono in riposo lungo il corpo. La gamba sinistra è di appoggio.

Bibl.: Sear 1977, 87 no. 57 (con bibl.); Architects 1981, 255 no. 110; 277.

Inv, no. 12514: > Pompei, Fondo Barbatelli (cat. 307).

507 Cf. Dunbabin 1982 per l'iconografia delle bighe.

500 Per la statua Fuchs 1963, 12-14, Taf. 1-8. 
Cat. 117. Inv. no. 27693; IV stile

Una menade inginocchiata che regge un tirso viene assalita da un satiro. Il gruppo copia una composizione ellenistica. Agli esempi elencati da Berti si aggiunga il gruppo trovato presso la piscina di Oplontis. 509

A destra vi é un'erma marrone di un uomo ammantato che sta sopra una base rettangolare. Helbig I'interpreta come Dioniso.

Bibl.: Helbig 553; RP 125.9; Schefold 1957, 354; Berti 1972-1973, 455-456, fig. 4.

Inv. no. 27700: - Pompei, VI 9, 6 (cat. 204/2).

Inv. no. 109751: > Pompei, I 2, 6 (cat. 140).

Cat. 118. Inv. no. 110878; IV stile

Rappresentazione identica a MN 27693 (cat. 117).

Bibl.: Schmidt 1925, 99-102; Schefold 1957, 354; Berti 1972-1973, 455-456.

Inv, no. 111439: > Pompei, V 1, 26 (cat. 186/1).

Inv. no. 111477: > Pompei, VI 13, 2 (cat. 210/2).

Inv. no. 111479: > Pompei, VII 15, 2 (cat. 259).

Inv. no. 113195: > Pompei, VIII 5, 24 (cat. 273).

Cat. 119. Inv. no. 116086; IV stile

Su uno sfondo bianco si vede un guerriero nudo dipinto con 11 colore della pelle. Sta sopra una mensola rettangolare. La gamba destra è di appoggio. Le spalle sono coperte da una clamide e la testa da un elmo a pennacchio. Regge nella destra una lancia e nella sinistra uno scudo. Is figura appartiene a una serie di guerrieri d'impronta policletea (cf'. p. 43). Sicuramente non è di III stile, come sostiene Herbig.

13ibl.: Schefold 1957, 355; Herbig 1962, 15-16.

Inv. no. 120030: > Pompei, V 2, 4 (cat. 191/1).

Inv. no. 120033-120034: > Pompei, I 4, 5 (cat. 147/2-3).

Cat. 120. Inv. no. 120176; III stile

Pinax centrale con il cavallo di Troia. A sinistra sul quadro ora quasi illeggibile vi è la statua di Atena munita di uno scudo e una lancia nella mano sinistra e con un elmo a pennacchio sulla testa.

Bibl.: Elia 1932, no. 10; Dawson 1944, pl. 4; Schefold 1957, 356.

Inv, no. 147501-147503: > Boscotrecase, Villa di Agrippa Postumus (cat. $006 / 2)$.

Cat. 121. S.n. 3; IV stile Atleti in una palestra su un frammento della zona mediana o dello zoccolo. Vel centro si trova un'erma inarrone sopra una base rettangolare che scmbra triangolare a causa della prospettiva resa maldestramente. La testa ha una

509 Jashemski 1979, fig. 480. 
barba ricciuta e una corona. Forse rappresenta Eracle.

L'atleta a destra si pulisce la fronte con una strigile e assomiglia all'atleta in VIII 2, 24 (cat. 267), entrambi probabilmente modellati secondo il tipo dell'atleta Westmacott.

A sinistra vi sono due atleti che si allenano con i manubri, simili alle figure nello zoccolo dolla stessa casa VIIl 2, 24 (cat. 267).

Bibl.: Llia 1932, no. 84; Maiuri 1950, 177-178, no, 11; Schetold 1957, 357.

Cat. 122. S.n. 19; IV stile

Sopra un capitello vi é una donna che regge una brocca sulla testa con la sinistra, mentre la destra e in riposo lungo il fianco. Indossa un peplo. Forse si tratta di una ligura in una scaenae frons della zona superiore.

Bibl.: Helbig 1874; RP 234.1.

Cat. 123. S.n. 22; III stile

Sopra un candelabro $v i$ c la statua di Iside vestita di un chitone giallo e di un mantello violaceo. Sulla testa si vede una corona e una colonna sormontata da un disco solare. Regge nelle mani stese delle bende. Una figura simile si vede nella I'illa dei Misteri e su un frammento nel Allard Pierson-Museum a Amsterdam. ${ }^{510}$ Il frammento forse costituisce la divisione fra due campi nella zona mediana.

Bibl.: Schefold 1957, 357.

S.n. 33: > Pompei, VI 9, 6 (cat. 204/7).

Cat. 124. S. n. 112 e s.n.; IV stile

Due frammenti di lesene rra campi nella zona mediana. Sopra candelabri vi sono dei guerrieri nudi dipinti in giallo. Hanno un elmo a pennacchio sulla testa e un mantello sulle spalle. Reggono nella sinistra uno scudo rotondo e nella destra una lancia. La testa del guerriero su s.n. 112 è incoronata da un candelabro con elementi egittizzanti. La gamba sinistra è di appoggio. Nell'altro frammento il peso del corpo poggia sulla gamba destra: manca peró il candelabro sopra la testa.

Bibl. : inedito.

Cat. 125. S.n.; IV stile

Prospetto della zona mediana. In un'architettura policroma vi è un'erma con una testa femminile. Sulla testa poggia l'architrave che incorona la zona mediana. Un'erma sinile si vede su MN 857 ? (cat. 051).

Bibl. : inedito.

S.n.: > Boscoreale, Villa di P. Fannius Synistor (cat. 004/5).

S.n.: > Ercolano, Basilica (cat.019).

S.n.: > Frcolano, Insula orientalis II A, 1a (cat. 038).

S.n.: > Caivano (cat. 007).

510 De Vos 1980, tav. C, VI, XXIV. 
NEW HAVEN CONN.

Yale Lniversity Art Gallery

Inv. no. ?: > Dura Europos. Tempio di Bel (cat. 013). 
Ben Heller Collection

Cat. 126. Inv. no. ?; IV stile

Frammento di una rappresentazione di un giardino, proveniente dalla Campania. Fra piante davanti ad uno sfondo ceruleo si delinea la $H^{\text {ll }}$ ftherme marrone di un satiro. Il fusto esce da un calice vegetale. Il satiro regge un ramo di palma nella sinistra lungo il ljanco e un piatto nella destra all altezza della spalla. Ha una cintura attorno alla vita e una corona sulla testa. Non è chiaro se il frammento appartenesse alla decorazione di uno zoccolo o di una zona mediana. Non si può quindi stabilire se l'erma svolgesse il ruolo di atlante come nella decorazione di III stilc dell'Insula orientalis ad Ercolano (cat. 038). Una striscia rossa al lato inferiore potrebbe essere un indizio a favore dello zoccolo.

Bibl.: CatZürich 1974, 212-213 no. 368.

Metropolitan Museum of Arts

Inv. no. 20.192.1, 20.192.10, 20.192.17: > Boscotrecase, Villa di Agrippa Postumus (cat. 006:1,3).

S.n.: > Boscoreale, Villa di P. Fannius Synistor (cat. 004:4). 
Cat. 127. Tomba; seconda meta del quarto secolo d.C.

Le pareti nord e sud mostrano una rappresentazione di un giardino. In cancello attorno alle piante contiene una serie di erme al posto di pali: sulla parete nord cl sono sei erme, sulla parete sud quattro. L'unica nota attraverso una fotografia ha la testa di un glovane umberbe. Si tratta dell'imitazione di un Hermenzaun, genere studiato in dettaglio da Wrede (cf. p. 84 ).

Bibl.: Wrede 1972. 133 no. C4, Taf. 77.4. 
I 4,2 Casa di Giove e Ganimede

Cat. 128. (14) Tutte le pareti, zone mediana e superiore; epoca antoniniana I campi laterali nella zoni mediana contengono figure sopra segmenti a forma di parallelogramma, usati come mensole. Solo alcune figure sono rimaste visibili.

Parete est, campo destro. Un uomo barbuto vestito di una tunica poggia sulla gamba sinistra. Il braccio sinistro è avvolto nella veste. l'altro pende in riposo lungo il fianco. Si osserva che la testa taglia l'orlo superiore del campo. Il tipo della figura es quello dei ritratti di oratorj e f'ilosofi. ${ }^{11}$

l'arete ovest, campo destro. Un uomo vestito di una toga avanza verso sinistra e tiene le mani nell'atteggiamento di un oratore: la destra è stesa per metà. mentre la sinistra è avvolta nella veste. Di nuovo il tipo corrisponde a quello usato per oratori e filosofi.

Sopra i campi centrali della zona modiana si vedono campi quadrangolari nella zona superiore, occupati da figure femminili. la figura sulla parete est imita l'Afrodite Anadyomene. La gamba destra è di appoggio.

Nei pannelli rossi. a fianco dei campi centrali, si delineano, sopra elementj architettonici aggettanti, korai vestite di un peplo. Le figure simmetriche reggono un piatto nella mano esterna. Il piede destro sta in avanti. Soltanto sulla parete est la donna a sinistrae rimasta conservata.

Bibl.: Wirth 1934, 109-115. Taf. 25-26; Borda 1958, 103. 105-109, 291; Dorigo 1966, 66-67, fig. 46-47; Packer 1971, 136, fig, 42-43; Andreae 1973, Abb. 537-538; Joyce 1981, 52-53, l'ig. 51.

III 5,1 Casa delle volte dipinte

Cat. 129/1. (III) Parete nord. lunetta; epoca antoniniana

L'edicola centrale viene occupata da una rigura femminile, seduta su un trono sopra una base rettangolare. Solo la sagoma è rimasta visibile.

A sinistra e a destra stanno figure maschili nude sopra mensole. La figura a sinistra è andata perduta. Quella destra regge un piatto con frutti nella sinistra presso il finnco e una bacchetta nella destra stesa verso il basso. la gamba sinistra $\dot{a}$ di appoggio. Sulla testa si vede una corona di pini. Si potrebbe trattare di una rappresentazione di una stagione, cioe la primavera; le figure mancanti sulla medesima parete e su quella di fronte completerebbero la serie. La figura seduta in questo contesto probabilmente é Demeter. La composizone della parete con le figure sopra mensole è simile a quella con le korai nella Casa di Giove e Ganimede ad Ostia (cat. 128).

Bibl.: Felletti Maj 1961, 8, tav. I, II 1-2.

Cat. 129/2. (IV) Tutte le pareti, lunetta; epoca antoniniana

Le architetture sono ravvivate con figure umane di colore bianco, ora quasi illeggibili. Una figura maschile nuda ha la destra lungo il fianco e l'altra sopra la testa. Secondo Felletti Maj le figure copiano statue famose.

Bibl.: Felletti Maj 1961, 11, fig. 5.

511 Cf. Sgobbo 1972. 
Cat. 129/3. (XII) Tutte le pareti, zona superiore; epoca antoniniana Accanto alla parte centrale della decorazione vi sono figure forminilj sopra mensole. Sono vestite di una tunica o di un mantello " ricordano lo figure littili ellenistiche quali quelle di Tanagra.

Parete est. A destra $\dot{a}$ una donna che porge una bacchetta con la destra e regge un piatto con la sinistra presso il fianco. La gamba destra $a$ di appoggo. La figura a sinistra poggia sulla gamba sinistra. Tiene la destra nel flanco e la sinistra davanti al petto.

Parete nord. Le donne sono state sostituite da pantere. In un'edicola a sinistra della pantera destra si vede una donna che regge una fiaccola.

Parete sud. Al lato destro la figura femminile ha la destra davanti al petto e la sinistra lungo il fianco La gamba smistra è di appoggio. L'altra figura liene una bacchetta nella sinistra lungo il fianco, mentre la destra è clavanti al petto. 11 peso del corpo poggia sulla gamba destra.

Nella parte centrale un candelabro viene fiancheggiato da due donne sopra candelabri. Indossano tuniche corte e hanno le gambe unite. Sono di colore bigio-bianco. Entrambe le figure hanno la mano destra distesa e la sinistra in riposo nel fianco.

Bibl.: Felletti Maj 1961, 25-27, fig, 10-17, tav. VIc, VII-VIII.

III 9,22 Casa delle Muse

Cat. $130 / 1$. (V) Tutte le pareti, zona mediana; epoca adrianea

Statue di Muse e di Apollo sorgono sopra mensole quadrangolari davanti al pannelli. La serie segue una tradizione iniziata a Pompei nel IV stile che potrebbe esprimere un interesse culturale del proprietario In pochi casi le figure singole possono essere confrontate con modelli scultorei. noti dall'iconografia delle Muse (cf. p. Yis).

Parete nord, campo sinistro. Talia inciossa un chitone bianco c un mantello verde. La gamba destra è di appoggio. Regge nella destra distesa una maschera e tiene la sinistra in riposo lungo il fianco.

Parete nord, campo centrale. Euterpe indossa un chitone bianco senza maniche. Avanza verso destra. Ha due flauti nella destra distesa, mentre la sinistra è lungo il franco.

Parete nord, campo destro. Melpomene è vestita come Talia. Nella destra regge una clava, nella sinistra una maschera.

Parete est, campo sinistro. Tersicore tiene una cetra davantí al petto. La parte inferiore del corpo manca.

Parete est, campo destro. Erato indossa un chitone giallo. La gamba sinistra è di appoggio. Regge una cetra nella sinistra contro il flanco $e$ un plettro nella destra lungo il fanco. Il tipo è quello della Piccola Ercolanese a Dresda.

Parete sud, campo centrale. Apollo non è raffigurato in veste di Citaredo, ma come arciere. Prende con la destra una freccia da una faretra sul dorso e regge un arco nella sinistra stesa. La gamba sinistra e di appoggio.

Parete sud, campo destro. Úrania indossa un chitone ceruleo e un mantello bianco. Il peso del corpo poggia sulla gamba destra. Regge nella sinistra un globo e nella destra una bacchetta con la quale indica qualcosa sul globo.

Parete ovest, campo destro. Clio e vestita di un chitone verde senza maniche e dj un mantello azzurro. Ha nella sinistra stesa un ditlico o tienc la sinistra davanti al petto. La gamba destra e di appoggio.

Bibl.: Felletti Maj/Moreno 1967, 20-30, tav. II-VII 2.

Cat. 130/2. (IX) Pareti sud e ovest, zona mediana; epoca adrianea 
I campl centralı contengono figure che stanno sopra segmentı a forma di parallelogramma Vella parete sud si trova un satıro nudo cle regge un pedum e una clamide nella sinistra lungo l tıanco e una siringa nella destra distesa Sulla palete ovest un Dioniso indossa un chitone rosso e un mantello verde Regge un tirso nella sinistra alzata e con la mano destra rersa vino da un cantaro Il modello e quello del Dionıso dı Tivolı

Bibl Fellettı Maj/Moreno 1967, 36-37, tax $\mathrm{L}-\mathrm{X}$

Necropolı sull'Isola Sacra

Cat 131 (11) Tomba del Hollotigen,, epoca adrianea (ora Vuseo Ostiense inv no ?)

Le tre Parche anımano la nicchia La prima, Atropo, sorge sopra una base a due gradinı Indossa un chitone senza maniche e regge con entrambe le mani, davantı al petto, un volumen aperto Il modello e quello della Venere Landolina e delle ninfe dcquatiche 512

La seconda figura $f$ Cloto che indossa un chitone senza maniche Regge nella sinistra alzata und fuseraiola e nella destra lungo il Lianco una rocca

La terza, Lachesi, regge nella mano destra una blancia lungo il fianco, mentre la sinistra e davanti al petto La buancia non si trova frequentemente, piu spesso si vedono le sorti

Statue di queste divmita non sono state trovate finora e non sono nemmeno conosciute attraverso le fonti scritte Gli esempi per le figure dipinte sono di stule ellenistico e sono sumul alle statue di Vluse 513

Bibl Calza 1940, 120-123, fig 53-5j Borda 1958, 279, 287, Pellegrino 1984, 61

Cat 132 (15) Tomba di Veria Zosıme, prima dı $135 \mathrm{~d}$ C

Due figure maschilı nucle di colore metallico si delineano su uno sfondo bianco L'una 1igura poggia sulla gamba destra, ha la destra alzata e la sinistra in riposo lungo il tianco parzialmente coperta da una clamide L'altra regge una lancia nella mano destra alxata e tiene con la sinsstra una clamide lungo l

franco La gamba sinstra e di appoggio

Bibl Calza 1940, 121 122, fig 51-j2 Pellegrino 1984 b1

Cat 133 (16), curca 110

Atropo una delle Parche e stata modellata in modo sumle a quella nella tomba 11 (cat 131) Ln'altra Paica non descritta da Calza e menzionata brevemente da Pellegrino e andata perduta

Bıbl Calza 1940, 127-128, 139, fig 57-58, Pellegrıno 1984, 62

Cat $134(70), 123$ d C o plu tard

trcole regge nella mano destra le mele delle Esperid, mentre pogga con l'avambraccio destro sulla clasa II braccio smintro e coperto dalla leontide $E^{\prime}$ di colore rosso che, secondo Calza, starebbe ad indicare un'imitazione di bronzo patinato

\footnotetext{
512 Becatt 1971

513 Cf $P$ weiszacher in Roscher II 2, 3084-3102, s.v. Moira, C Capıno, EAA 5 (1963) 143-144, s.v. Moira
} 
Bibl : Calza 1940, 117-118.

Cat 135. (77) Tomba delle tre Grazle, epoca antoniniana (ora Museo Ostıense inv no. 10033)

Nella nicchia si vede il gruppo delle tre Grazie su un campo. Non sono presentı elementı paesaggistici. Il modello e quello consueto per il quale si rimanda a Pomper, VI Insula occidentalis (cat. 227).

Bıbl Calza 1940, 135-136, Borda 1958, 287; Helbıg ${ }^{4}$, no 3041 (H von Steuben), Calza Florianı Squarciapino 1962, 39 no 2, Pellegrmo 1984, 70.

Porta Laurentina

Cat 136. Tomba; 209-211 d C. (ora Muses Vatıcanı)

Fanciulli porgono ofterte ad Artemide La statua di questa divinita sorge sopra una colonna di porfido Indossa una tunica corta e ha una faretra sul dorso Regge un arco nella sinistra stesa e prende con la destra una freccia dalla faretra La gamba sinistra sta in avantı Il modello originale entra nella tradizione del quarto secolo, per esempio dell'Artemide $d_{1}$ Versailles, ora al Louvre

La datazione della tomba si basa sulle rappresentazıonı dipınte di vexilla con ritratt 1 di Settimio Severo, Caracalla e Geta su un altro frammento. Le scene fanno parte di un ciclo calendario

Bıbl. Logara 1907, 68,72-78, tav XlVII; Borda 1958, 314, 316, Helbig 4 , 467 (B. Andreae) Andreae 1973, Abb. 566, Stern 1975; Mielsch 1981, 233.

Cat 137 Provenienza ignota (ora Musei Vatıcani)

Un trammento che deve essere stato trovato nel 1868 ad Ostia rappresenta un guerriero romano soprd una base gialla Indossa una tunica bianca, una lorica gralla un mantello rosso e ha un elmo a pennacchio sulla testa Regge una lancia nella destra alzata e uno scudo nella sinistra stesa La gamba destra e di appoggio.

La figura assomiglia all'Ares su MN 9646 (cat. 102) per quanto riguarda lo stile e potrebbe provenıre dalla zona superiore di una decorazıone di IV stile. Bibl . Nogare 1907, 65, 78-79, tav XLX. 
Cat 138 Ipogeo di Magharat-e-diedideh, circa 259 d C

Le quattro lesene sono decorate con Vittorie che sorgono un globo Reggono con entrambe le manı alzate sopra la testa imagines clipeatae, rappresentantı probabilmente 1 ritrattı del defuntı L'attegglamento ricorda 1 portatorı di specchi nell'arte greca arcaica e classica

Bibl Borda 1958, $126 \quad 331-332$

Cat 139 Tomba di Atenatan, esedra del Maqqal, 229 d C

Una Vittoria si regge sopra un globo $\mathrm{Ha}$ una palma nelle manı II modello e quello della Vittoria Romana

Bubl Borda 1958, 331 


\section{PARIGI}

Musée du Louvre

Inv. no. P2: > Pompei, VI 1, 7 (cat. 198/3).

Inv. no. P4-9, 11-12: > Pompei, II 4, 3 (cat. 179/2).

Inv. no. P5j: > Tuscolo (cat. 343). 
I 2,6

Cat. 140. (m) Parete sud, quadro centrale, III stile (ora MN 109751)

Ratto del Palladio. Odisseo regge nella mano sinistra il Palladio di colore guallo. L'Atena e resa in modo dettagliato, vestita di un peplo classico. Regge uno scudo rotondo con il braccio sinistro e con la mano destra brandisce una lancia sopra la testa. La testa e coperta da un elmo a pennacchio Raffigura cosi l tipo consucto. conosciuto fra l'altro dalle terrecotte trovate di recente a Lavinio. "514 Piretschner crede che visto 1 colore della statua - non si trattı di uno xoanon, ma $d_{1}$ una statua bronzea, sla o no dorata. Invece o plu verosumile che sia rappresentata una statua di legno coperta di lamine bronzee, in quanto il suo peso nol puo essere troppo grande, vista la maniera nella quale Odisseo la regge. Inoltre, secondo la tradizione il Palladio sarcbbe una statua lignea e difatti sulle altre rappresentazioni della stessa scena essa risulta scolpito in questo materiale.

Bibl.: Sogliano 580, Herrmann I, 205-206, Taf. 149; Elia 1932, no. 38; Davreux 1942, 128 no j0, fig 28, Schefold 195i, 9; Schetold 1962, 89-90, Taf. 11.1, Ptretschner 1977, no. 27.

I 2,17

Cat. 141;1. (c) Parete nord, quadro centrale; III stile +

Solo da un disegno di Discanno si conosce questa pittura che raffigura Endimione addorinentato. Vicino a una colonna e un albero sacro vi e la statua bronzea di Ecate posta su una base rettangolare. Indossa un peplo e porta sulla testa und corona a forma di modio Regge nelle manı stese due torci. Si tratta del tipo consueto Forse Ecate rappresenta nello stesso momento Selene, la divinita innamoratd di Endimione. Cosi almeno ipotizzano Lawson e Peters; Sichtermann non esclude che si tratti di una statua di Iside o Artemide.

Bibl.: Soghano 456, Herrmann 1,186, Abb. 54, Dawson 1944, 91 no $24, \mathrm{pl}$. IX, Schetold 1956,212-213,215, Schetold 1957, 10; Schefold 1960a, 89, Peters 1963, 86-87, t1g 72, Ptretschner 1977. no. 8. Repertorio 1981,4 , Sichtermann 1984, 291-294, 295, Taf. 1 .

Cat 141/2. (?) Parete ?, zona ?; ? stile +

Veduta di mare con la statua di una divinita.

Bibl.: A. Mau, BdI 1873, 238-239, Mau 1882, 140; Schefold 1957, 10; Pfretschner 1977, no. 2.

I 2,24

Cat. 142. (k) Parete nord, quadro centrale; III stile +

Paesaggio sacro-1dillaco, conosciuto solo da vecchie descrizıoni. Nell'interno di un tempio si distıngue la statua di Dioniso che regge un tirso e un cantaro. Forse si tratta del tipo del Dioniso di Tivoli.

514 Enea 1981. 
Nel paesaggio si trovano un'erma itifallica di Priapo e una statua di una figura lemminile, vestita di un chitone lungo.

Bibl.: A. Mau, BdI 1873, 246; Sogliano 247; Herter 1932, 138 no. 33; Schefold 1957. 10; Bastel'De Vos 1979, 139: Repertorio 1981, 4.

\section{2, 28 Casa della grata metallica}

Cat. 143. (i) Farete sud, quadro centrale; III stile +

paesaggio sacro-idilliaco. In un tempio si trova la statua di una figura femminle vestita di un chitone senza maniche. Ha sulla testa un modio e regge. davanti al petto, un piatto con truttı. Lin cimbalo e un pedum. posti sulle scale del santuario, potrebbero identificarlo come tempio di Artemide.

Davanti al tempio si trova un'erma Itifallica di Priapo.

Nel fregio che separa la zona mediand dalla zona superiore, secondo Mau, sarebbero dipinte alcune statue, delle quali non abbiamo descrizıoni piú precise.

Bibl.: A. Mau, BdI 1873, 244; Sogliano 684; Rostowzew 1911, 40; Herter 1932, 158-1.59 no. 181, 190; Schefold 1957, 11; Bastet, De Vos 1979. 139; Repertorio 1981, 5 .

I 3,3

Cat. 144. (u) Parete?, quadro centrale; III o IV stile +

Paesaggio sacro-idillitco. Presso un albero sta una statua dj una divinita maschile sopra una base. Ha un modio sulla testa e regge nelle mani un vassojo.

Secondo Mau e Rostowzew la decorazione $\dot{e}$ di III stile "a candelabri", di IV stile invece secondo Schefold e Repertorio. Ura non se ne puo piú distinguere una traccia.

Bibl.: A. Mau, BdI 1873, 237-238; Sogliano 685; Rostowzew 1911, 41; Schefold 1957, 12; Repertorio 1981, 6 .

\section{3,18}

Cat. 145. (?) Parete ?, quadro centrale; IV stile (ora MN 9015)

Convivio sotto una tenda. Sullo sfondo al di la della tenda si distingue la statua verde, ora svanita, di una figura maschile barbuta, vestita di un chitone lungo. Regge nella sinistra un bastone. Helbig e Herter la descrivono come Priapo, ma visti il modello e il contesto essa va interpretata come Dioniso.

Bibl.: Helbig 1445; Herrmann II, 25, Abb. 3, Taf. 210; Herter 1932, 130 no. 8; Schefold 1957, 12 .

I 3,25

Cat. 146. (h) Tutte le pareti, zone mediana e superiore; IV stile 
Le scaenae frontes nelle zone mediana e superiore costituiscono un'unità organica. Statue verdi di guerrieri nudi stanno su basi aggettanti sulle pareti est, ovest e (probabilmente) nord. Su tutte le pareti acroteri e telamoni adornano l'architettura del registro superiore. Alcune parti sono andate perdute a causa di un furto nel 1977 (qui indicate con: $(+)$ ).

Sulle pareti est e ovest guerrieri nudi in verde monocromo fiancheggiano le aulae regiae. Hanno un elmo sulla testa e reggono tra le mani scudo, spada e/o lancia. Le gambe sono disposte summetricamente, cosicche il peso del corpo poggia su quella esterna. I modell dal qualı dipendono sono d'impronta policletea. La figura destra della parete est $\left(^{+}\right.$costitusce una copia assai tedele dell'Ares Borghese di Alcamene. Delle statue sulle lesene accanto alla porta nella parete nord restano solo le sagome delle quali ovviamente non si puo dare una descrizione accurata. Nelle pubblicazıonı precedenti esse non sono state menzıonate o raffigurate.

L'aula regia della parete sud vene sormontata da una quadriga giulla, guidata da una Nike (+). E' raffigurata trontalmente $e$ in modo schematico.

Sull'arco delle edicole fra l'aula regia e gli hospitalia stanno figure di guerrier vestiti di una tunica e di donne armate vestite di un chitone in guisa dı cariatıdi. Sono disposte in ordine simmetrico.

Le edicole nell'angolo estremo vengono sormontate da figure femminils inginocchiate d'impronta egizia. La figura a sinistra regge, davanti al petto, un platto e un'urnetta, quella a destra un sistro nella destra stesa.

Le aulae regiae delle pareti est e ovest sono ornate con acroteri a forma di statuette gialle di Artemide. Indossano un chitone corto, stivali da caccia e reggono sulle spalle una faretra e un arco nella mano stesa verso il centro della composizione. Rappresentano copie assai libere dell'Artenude di Versatles.

Gll hospitalia hanno, di nuovo, figure egizıe ingınocchiate che reggono l'orlo superiore della decorazıone sulla testa. Indossano un mantello lungo e sono rivolte verso 2 centro. Con entrambe le manı reggono, davantı al petto, una cassetta o una tavoletta. Si tratta des naophoroi eg1zi, introdotts a Roma nell'eta imperiale dove svolgevano un ruolo puramente decorativo in quanto non se ne conosceva piu il loro contenuto sacro originale. ${ }^{5}{ }^{5}$

La datazione della decorazione a prima del 62 da parte di De Vos non e accettable: la mancanza dello zoccolo non da prova di un restauro, ma indica soltanto che la decorazione non era tinita nel 79; strisce al piede della zona mediana, dovute alle modeste capacita del pittore, sarebbero state cancellate al momento del completamento della parte intersore della pittura.

Le figure di Dioniso sulla parete sud e di Alrodite sulla parete ovest sono delineate secondo modelli scultorei. La prima corrisponde al Dıonıso di Tivoli, la seconda copıa in modo libero la Venere de Fréjus.

Bibl.: A. Trendelenburg, BdI 1871, 171-182; Von Cube 1906, 21-27, Taf. II-III; Schetold 1957, 13; Bieber 1961a, 232, lig. 777-778; Schefold 1962, 126 , Taf. 90-91; De Vos 1977, 41, 72, fig. 32-33; Schmidt-Colınet 1977, 146, 271-272 no. P12; Ling 1978, 155; De Vos 1980, 65-66, tav. XLVIII; Pompei 1748-1980, 65, tig 32-33; Repertorio 1981,7 .

I 4, 5 Casa del citarista

Cat. $147 / 1$. (18) Tutte le pareti, zona superiore; IV stile (ora MN)

Paesagg nilotici nel fregi della zona superıre.

535 Roullet 1972, 111-115. 
MN 9496. A sinistra di una grande tholos si vede la statua di una figura maschile resa in modo schematico che sta seduta. $E^{\prime}$ nuda e ha un semplice panno sulle spalle. Davanti alla tholos vi è sopra una base rettangolare una coppia di statuette di colore marrone, delle quali non si puó dare una descrizione dettagliata.

MN 9608 (nel museo considerato perduto). Sul lato destro, vicino a un tempio, vi sono due statue rosse rese schematicamente, erette su di unfa base rettangolare.

MN 9610. Tre grifi bianchi fungono da acroteri su un tempio al lato sinjstro. Nel centro sorge una colonna corinzia sormontata da una statua bianca resa sommariamente. A destra e un'erma rossa su una piccola isola nel Nilo. In fondo si distingue una villa a pianta semicircolare, davanti alla quale sta un'erma o una statuetta. Secondo F. Kakob l'edificio rappresenta una villa di età flavia nell'Africa settentrionale.

Bibl.: Herrmann I, 234-235, Taf. 170; Elia 1932, no. 270-272; Schefold 1957, 14; Peters 1963, 165-166, fig. 158-159; Rakob 1969, 300, fig. 28; CatLondon 119, fig. 8; Silberberg 1981, no. 108, fig. 94-95.

Cat. 147/2. (20) Parete sud, quadro centrale; III stile (ora MN 120034)

Cosiddetta scena rituale: storial di incerta identificazione che secondo alcuni rappresenta Leda, secondo altri Nemesis. Su piedistalli, ai lati della donna con l'oca, sono due statue dipinte in giallo: a sinistra una figura femminile indossa un chitone senza maniche e regge un alabastro nella sinistra; a destra una figura femminile nella stessa veste regge un piatto nella sinistra e un uccello nella destra. Entrambe le figure sono di Stile Severo e ricordano le Danaidi della Villa dei papiri. Pfretschner ritiene che la donna con l'uccello (che sarebbe un piccione) sia una statua di Afrodite.

Bibl.: Helbig 152; Herrmann I. 147-148, Taf. 111; Elia 1932, no. 71; Elia 1937, 11, tav. IV; Schefold 1957, 15; Reuterswärd 1960, 136; Schefold 1962, 160-161; Pfretschner 1977, no. 2; Bastet/De Vos 1979, 26-27.

Cat. 147/3. (21) Parete nord, quadro centrale; IV stile (ora MN 120033)

Giudizio di Paride. Dietro l'eroe troiano $a$ Hermes si distingue la statua gialla di una ligura femminile vestita di un peplos. Regge nella mano destra stesa un piatto o un fiore, nella sinistra una bacchetta. Non è chiaro di quale divinita si tratti; Pfretschner sostiene che ausgehend vom Mythos sia rappresentata Eris.

Bibl.: Helbig 1286; Herrmann I, 152-154, fig. 43, Taf. 113: Elia 1932, no. 4; Elia 1937, 12, tav. 5; Schefold 1957, 15; Reuterswärd 1960, 132 nota 326; Pfretschner 1977, no. 7.

Cat. 147/4. (23) Parete est, quadro centrale; III stile Apollo sta in un paesaggio ornato di un albero sacro, un tripode e un corvo. Il peso del corpo poggia sulla gamba sinistra. La mano destria $c$ in riposo sulla testa, l'altra si appoggia ad una cetra posta su un piodislallo. Ovvianente il pittore si $\dot{e}$ ispirato all $\Lambda$ pollo Licio di Prassitele.

Bjbl.: Helbig 183; Elia 1937, 13-14, 1ig. 7 ; Schefold 1957, 15; Repertorio 1981,11 .

Cat. 147/5. (35) Parete est, quadro centrale: IV stile (ora MN 9111)

Ifigenia in Tauride. La sacerdotessa di Artemide regge nel braccio sinistro lo xoanon di colore marrone, attualmente non piú visibile. Secondo Herrmann la statuetta schematica era gialla. 
Bibl Helbig 1333 Herrmann I, 158-159. Taf 115, Phulippart 1925, 20-21 no 30, fig 4 Elia 1932, no 75 , Clia 1937, 17 , tav 6 schefold 1957, 16 , Pfretschner 1977 , no 22

$16,2-4$ Casa del criptoportico

Cat $148 / 1$ (22) Paretı nord est e sud, zona mediana, II stue, fase IIa Su una base verde rotonda che sorge sopra un podio ${ }_{1}$ sono delle erme $d i$ colore rosso, cmque sulle paretı nord e sud, due su quella est Sulla testa portano calici verdi sui quali poggia un drchitrave aggettante in modo che le erme tunzionano da plastrı di un portico La parte superiore delle erme e rappresentata in forma tridimensiondle, assai realistica e dinamica, con l suggerunento delle tigure come fossero vive nonostante ll colore la forma di erma e la funzione Sono rimaste conservate solo sel figure alternamente satirı e menadi del tuaso dionisiaco

Sulla parete sud si distinguono da sinistra a destra la sagoma molto iaga di un satiro, una menade vestita di un mantello rosso che regge una cornucopia gialla, un satiro barbuto she tiene due tlautı tra le mani, una figura femminile con un piatto nella sinıstra, nel quale versa vino dd una broccd e un satiro munito di und siringa nella sinistra e con ll braccio destro coper to da una nebride

Sulla parete est le due tigure gia risultavano perdute al momento dello scavo

Sulla parete nord si distinguono ancora due figure al lato ovest sorge un satiro con una piccola lira davantı al petto, modellato secondo un tipo ellenistico del quale e stata trovata una copia marmorea nella Casa di il lucretius, ${ }^{515}$ sul lato est e rimasta visible la parte superioro di una menade con una corona sulla lesta e un panno attorno alle renı che batte con la destra un tumpano rotondo

Fatta eccezione per il satıro con la lira non si conoscono confrontı nella scultura tinora trovata per queste $H^{e}$ efthermen

Bibl Spinazzola 1953, I, 483-525, fig 569-583, tav 21-32, Schefold 1957, 2022 , Beyen 1960 99-106, Allroggen-Bedel 1974d 134-13j Repertorio $1981,22-26$, Leach 1982, 158

Cat $148 / 2$ (17) Tutte le paretı, zona mediana, II stule, fase IIa

Su un podio verde davanti ad un muro ripartito da lesene rosse vi sono delle erme gialle con teste policrome di menadı e satırı Formano un portico nel quale 1 plastrı sono statı sostituitı da erme Sulla testa reggono calic verdı sui qualı poggia un architrave aggettante I fustı sono ornati con oflerte votive in torma $d_{1}$ siringhe, corone $e$ bastoni Per la maggior parte le decorazioni sono dndate perdute e quelle ancora presents sono gravemente danneggidte dall'umidita e non piu leggibilı Per tale ragione le illustrazioni nell'opera di Spinazzola sono di grande imiportanza

Beven sugger che l'erma nella Casa del Menandro (cat 163/4) fosse della stessa bottega, spotesi non piu controllable a causa del deperimento $d_{1}$ entrambe le pitture

Bibl Spinazzola 1953, I 454-462, II, 905-970, fig 558 565, Schefold 1957, 18, Beyen 1960, 106-108, Schelold 1962, 48-49, Fittschen 1976, 553, Repertorio 1981, 16-19, Leach 1982, 158

516 Dwver 1982, fig 42 
Cat 148/3 (20) Paretı nord e sud, zone mediana e superiore, II stle, tase

Ila

Scaenae frontes nei cul scomparti si vedono statue, atlanti a forma di Eroli e animali fantastici Le due zone costituiscono un insieme organico

Parete sud velle nicchie accanto alla porta che mette nel vano 21 si distinguono tigure in bianco monocromo che sorgono sopra piedistallı reltangoları di colore gıallo La figur-d a sinıstra a scomparsa, quella a destra rappresenta un satiro nudo poggiante con il piede sinistro su di un piccolo dado Ddd una brocca nella sinistra versa del vino in un ritone rosso tenuto con la destra Una statua dı questo tıpo e stata trovata nella Casa dı Obellus Firmus, dove funzıonava da fontane 517 L'esempio orıguale deve essere concepito nel pieno Elenismo e potrobbe essere ispirato da tupu guali l Giasone Lansdowne e l'Alessandro Rondaninı

Sulla trabedzione dell'edicola che circonda la porta si vedono ligure di rolore bianco vestite di una corta tunica, andate perdute per la parte superiore II peso del corpo poggia sulla gambd esternd Vella mano esterna reggono un oggetto ininterpretabile, l'altia e alzata Sulla trave a destra e rimasto visibile un tritone bianco in guisa di acroterio Il suo pendente a sinistra e andato perduto

Appliques in rilievo a forma di teste di Medusa sono state sistemate nella trabeazıone gialla della porta Sopra di essa sulle cornjel si vedono iting femminll alate ingmocchiate Sono messe nella stessa posizione dello ligure nella Villa dı Boscoreale (cat. 004 1-3) e nella Casd Jelle nozze d'argento (cat 185/2), croe come piccolı atlantı fra due cornici a mensola 51 a

Parete nord La struttura architettonica e simle a quella della parete sud von restano tracce delle statue nelle nicche Al momento dello scavo era ancora visibile la sagoma di una figura lemminule ammantata che regge una brocca con entrambe le manı davanti al petto, rappresentante una statud dı fontana

Al posto delle figure con tuniche si vedono Eroti nudi alatı di colore bianco La gamba al lato interno sta un po' in avantı e e di appoggio Con la mano interna reggono plattı davantı al petto, con l'altra und brocca lungo $\downarrow$ fianco Il loro sguardo e volto verso il centro

vel prospetto si distingue un trupode sopra una base rotonda bianca. ornata ad alto rlievo Vagamente si intravede un tiaso bacchico, Il cui esempio e da cercare nell'ambito neoattico

La trabeazione viene sormontata dd due stingl alate dl lati esternı e da due grifı sopra gh trotı Questi anumal sostengono l'orlo superiore della decorazione

Bibl Jucker 1950, 8, Spinazzola 1953, I 472-486, fig 533-545, tav agg 7; Schetold 1977, 19, Bryen 1960, 89-97, Jbb 25b, Schefold 196!, 41, 48-52, Taf 6 (micostruzıone fallace composta da eleniont 1 di entrambe le paret1), 34, Peters 1963, 23-24, Engemann 1967, 172, Taf 672 : Allroggen-Bedel 1974a, 18-23, Fittschen 1976, 533 , Schmidt-Colmet 1977,146-147, 268 no P7, Repertorjo 1981, 19-21, Leach 1982, $155-156$

Cat 148/4 (21) Paretı nord e sud, zone mediana e superıore, II stile, fase IIa

517 Spinazzola $1953,1,387-388$, fig 383,386 , Kapossy 1969,37

51. Von Hesberg 1980a 
Scaenae frontes con statue in micchie e atlantı sulla trabeazıone della zona mediana

Parete sud Le nicchie accanto alla porta che metin nel vano 20 sono occupate da tigure in una posizione simile a quella delld statur in 20 I colorl sono troppo svaniti perche possano essere distintı \on stanno su un pjedistallo, ma su una striscia semplice A sinistra si vede una tigura femminile vestita di un mantello che lascia scoperto ll petto Il peso del corpo poggia sulla gamba destra Regge nella sinistra, dasantı al petto, un ventaglio e tiene ll braccio destro mezzo disteso Lo sguardo e rivolto al centro Nell'altra nicchia si trova un uomo nudo che regge, davantı al petto, una lancia La gamba sinistra e di appoggio ll braccio sinistro e coperto da un panno Lo sgualdo e rivolto verso 1 centro Probibilmente vi sono raffigurate Ares e Atrodite, sistemalı in forma di pendants sid per quanto riguarda l'atteggiamento del corpo sia per ragionı d'iconografia

La trabeazione intorno alle nicchie e sormontata da tigure gialle che nello stesso momento servono da atlantı reggentı l'orlo superıore della decorazıone Sono disposte in ordine sumetrico Al latı esternı sı vedono figure femminilı vestite di un peplo che reggono una brocca nella destra lungo l Iianco e un piatto nella sinistra all'altezza della spalla Il peso del corpo poggia sulla gainba destra il lati interni si trovano figure maschli nude che sostengono con la mano al lato interno la trabeazione, mentre reggono un piatto nell'altra all'altezza della spalla Le gambe sono und dictro l'altra

Le figure fernminili corrispondono al tipo classico delle horai quelle maschil si confrontino con le figure di Pan nel liusel Capitolinı e su una lastra di bronzo a Berlino 519

Von sono piu visibilı le figure bianche, volte verso ll centro, alle estremita della decorazione Stesano su plintl o tenevano tra le mani un drappo della loro tunica corta

Parete nord Quasi nulla e rumasto conservato della cecorazıone du questa parete lel campo centrale dell zona mediana sı trovava un paesaggio sacro-idlliaco nel quale era, subito dopo lo sterramento, visıble una statua dı AI temide

A sinistra dell'edicola si vede una sfinge maschle barbuta sopra und bdse che sorge su di un podjo $E^{\prime}$ volta verso $l$ centro $\rho$ resa di proflo Rappresenta l tipo egizio del quale $s i$ conosce un piccolo escmplare della Casa di Octavius Quartio

Bıbl Spınazzola 1953. I, 486-492 fig 356. Schefold 1957. 20. Schetold 1962, 30-51, Taf 35 (ricostruzione gratica), Peters 1963 2j-24, Repertorio $1981,21-22$, Silberberg 1981, no 8, tig 7, Lcach 1982, 15j-156

I 6, 4 Casa del sacello liaco del lararıo di Achulle

Cat $149 / 1$ (h) Paretı nord e sud, zona superiore, IV stule

Negli scomparti compresi fra le edicole si trovano dei tritoni di colore rosso, due per ogni parete, che reggono un con la mano esterna un timone appoggiato sulla spalla e un ritone nella mano interna La figura a destra sulla parete nord e dndata perduta

Si notı che la pittura e databile nell'ultima tase di Pompel, posche mancano completamente le zone mediana e inferiore Strocka invece, propone per l'intero complesso pittorico una datazione anteriore al 62

\footnotetext{
519 Schmidt-Colınet 1977, M76, M69.
} 
Bibl.: Spinazzola 1953, I, 448, fig. 515; Schelold 1957, 23 (vano d); Barbet,' 1 llag 1972, 975, fig. 17; Repertorio 1981, 29; Strocka 1984, 125-126, Taf. 50,1 .

Cat. 149/2. (I) Pareti nord e sud, zona mediana; IV stile

Sul campi rossi si delineano figure sopra mensole rese in colori vivaci e come persone reali. Ie mensole peró le caratterizzano come statue.

Parete sud, campo ovest. Un uomo nudo regge un agnello sulle spalle e nella mano sinistra lungo il fianco un mantello. Il peso del corpo poggia sulla gamba destra. Rappresenta il tipo del kriophoros, del quale si conosce un esemplare della Villa di Petraro a Castellammare di Stabia. 520

Parete sud. campo est. Lna donna vestita di un mantello ceruleo regge nella mano sinistra un mazzo di spighe. nella destra distesa una falce. 11 peso del corpo poggia sulla gamba smistra. Gli attributı caratterizzano la figura come una stagione: l'estate.

Parete nord, campo ovest. Una donna vestita di un drappo viola e di un mantello azzurro ha una corona sulla testa. Le mani sono aviolte nel mantello all'altezza dei seni.

Parete nord, campo est. Perduto.

Bibl.: Spinazzola 1953, I, 542. fig. 597; Schefold 1957, 2 (vano b); Repertorio $1981,30-31$; Strocka 1984, 127-128, Taf, 53, 1-2.

Cat. 149/3. (q) Parete est, zona superiore; II stile, fase Ila Fregio in rosso con quattro imitazioni di rilievj in rosa e bianco. Nel comparto sud il rilievo di sinistra non è piú leggibile, su quello di destra si distinguono appena due figure sedute e una in piedi.

Nel comparto nord il rilievo a sunistra mostra un ermafrodito aggredito da un satıro modellato secondo il tipo dell'aposkopon. Il riliev'o a destra mostra Marsia e Olimpo. Quest'ultima scena è conosciuta anche attraverso quadri di IV stlle. Al di sotto dej rilievi si distingue un fregio a mensola doppia ornata da uccelli fantastici. ${ }^{521}$ I rulievi imitano pezzi da collezione.

Bibl.: Spinazzola 1953, I, 549-550, fig. 609-612; Schefold 1957, 24 (vano g); Beyen 1960, 109-110, Abb. 54-55; Pompel 174-1980, 141, fig. 5; Froning 1981, 19-20; Repertorio 1981, 32; Moormann 1984b, 645, 649.

I 6, 12 Bottega di Junianus

Cat. 150. (m) Parete sud, zona mediana; IV stile

Il campo est contiene la statua di un guerriero che indossa una corta tunica di colore giallo scuro. Sta su una mensola a forma di parallelogramma. La gamba destra è di appoggio. Regge un uccello nella destra all'altezza della spalla, mentre uno scudo ovale copre la spalla sinistra. Dietro le spalle si vede una lancia piu lunga del guerriero stesso che sembra sostenuta con la sinistra coperta dallo scudo. Non si puo dire se si tratti di un'immagine di una divinità. La pittura è eseguita secondo lo stile dell'arte plebea.

\footnotetext{
520 Jashemski 197. fig. 535. Cf. per l'iconografia Laubscher 1983; J. Engemann in Spätantike 1983, 257-259.

522 Cf. cat. 263; Von Hesberg 1980a; qui p. 22.
} 
Bibl.: A. Maiuri, NSc 1929, 428, fig. 42; Schefold 1957, 26; Repertorio 1981, 39.

I 6. 13 Casa di Stallius Eros

Cat. 151. (13) Parete nord, zona mediana: IV stile

Pittura di giardino. Nel campo centrale sorge, tra le piante, una fontana che ha come sostegno una sfinge femminile alata. L'insieme di colore bianco ad eccezione degli occhi e dei capelli resi in colori naturali. Nel bacino a forma di cantaro zampilla dell'acqua. Lesecuzione risolta nei piú piccoli dettagli ricorda la sfinge in VII 2, 38 (cat. 249). La combinazione di un cantaro e una sfinge non ha altri riscontri.

Nel campo destro si trova la statua bianca di una sfinge femminile alata sdraiata sopra una base rettangolare. $E^{\prime}$ raffigurata di profilo, rivolta verso sinistra. Le ali sono delineate in modo dettagliato.

Bibl.: A. Maiuri, NSc 1929, 434; Grimal 1943, 480 pl. IV fig. 3-4; Schefold 1957, 26; Grimal 1969, 446 no. 3; De Vos 1977, 39; De Vos; De Vos 1979, 92, Taf. 66d; Jashemski 1979, 67; Repertorio 1981, 39.

I 6, 15 Casa dei Ceii

Cat. $152 \% 1$. (a) Parete est, zona superiore; IV stile

Nel tratto nord si vede un paesaggio sacro-idilliaco. A sinistra di un albero sacro sorge una statua resa in modo schematico.

Bibl.: Spinazzola 1953, I, fig. 287; Schefold 1957, 26; Peters 1963, 116; Repertorio 1981, 39.

Cat. 152/2. (f) Pareti nord, sud e est, zona superiore; IIl stile

Figure femminili di Stile Severo reggono la trabenzione curvilinea oppure occupano edjcole sopra o accanto ai pannelli centrali della zona mediana.

Parete nord. Le figure stanno nelle edicole al di sopra delle prospettive della zona mediana. Reggono un piatto sulla testa con la mano interna e hanno il braccio esterno in riposo lungo il fianco. Corrispondono alle cariatidi del tipo Albani. 522

Parete sud. Decorazione identica alla precedente.

Parete est. Due cariatidi sostengono l'architrave semicircolare del'edicola centrale. Ia mano esterna regge un drappo del mantello all'altezza della spalla, l'altra è in riposo lungo il fianco. Il tipo si ritrova nella Villa imperiale (cat. $305 / 3$ ).

Bibl.: Spinazzola 1953, I, 269-271, [ig. 300-301; Schefold 1957, 27; Bastet/De Vos 1979, 98; Repertorio 1981, 42-43.

Cat. 152:3. (h) Pareti nord, est e sud, zona mediana; IV stile

Parete nord. Un paradeisos viene inquadrato da una fascia rossa ornata con elementi topiari. Lavanti a uno sfondo con cespugli si distinguono fontane bianche sorrette da sfingi femminili alate che poggiano su basi rettangolari.

522 Schmidt 1982, 99, Taf, 23, 25. 
Al du sopra delle fontane si vedono lastre bronzee di colore giallo ornate du figure femminul che indossano un peplo in stile classico e un mantcllo Reggono nella mano al lato esterno una pisside vicino al petto, nell'altra un drappo del peplo Linee scure accentuano l'effetto del rilievo. Non sono state trovate finora lastre a rihevo sumil a quelle delle nostre pitture, ne per quanto riguarda la raffigurazione, ne di un tormato simle 523

Parete est Sul pulastro al lato sud si vede una ninta acquatica dipinta a colorı naturalı con un mantello sulle gambe. Regge, davantı al grembo, un bacino di bronzo lucido, nel quale - contro l'abitudine - non zampllla acqua. Il tipo e quello della venere Landolina

Parete sud. Vel tratto ovest del paradeisos con figure egizie e la statua rossa it ifallica di Priapo, che e rivolto verso destra e visto di proflo

Bibl Spinazzola 1953, I, 275-280, lig 306-307, Schetold 1957, 28; Reutersward 1960,182-183; Schefold 1962, 141, Peters 1963, 172, Peters 1971, 11. Jashemskı 1979, 69-70, fig. 111-114, Yichel 1978, 399; Repertorio 1981, 43-44, Suberberg 1981, no. 127, fig. 105.

I 7. 1 Casa di Paquius Proculus/Cuspius Pansa

Cat. $153^{\prime} 1$ (6) Parete est, zoccolo: IV stule

Accanto al pannello centrale con ippocampi giall $v$ vi sono due suleni composti simmetricamente che reggono con la mano esterna l'orlo superiore dello zoccolo Il peso del corpo poggra sulla gamba esterna Hanno un drappo attorno alle renı e una corona sulla testa L'oggetto che reggono nella mano interna distesa lungo il fianco non e identificable: forse si tratta di un askos L'atteggiamento dei sulenı fa ricordare glı atlanti su MN 9688 (cat. 106) e sul rilievo marmoreo di Goreme a Berlino $524^{5}$

Bibl Spinazzola 1953, I, 297-314; Schetold 1957,29; De Vos/De Vos 1975, 36, 121 nota 32, fig. 32; Repertorıo 1981 , 48-49.

Cat. 153/2. (9) Parete ovest, vignetla sul 5. pannello da sud; III stue Paesaggio sacro-1dilliaco in bianco A sinistra di una colonna vi e la statua di una ligura ammantata sopra una base rettangolare. A destra di un tempietto si trova una figura sumle, posta sopra una base piu alta. Tutto e delineato in modo schematico

Bibl - Spinazzola 1953, I, 301-302, f1g 341; Schetold 1957, 28-29; Peters 1963, 113; Repertor1o 1981, 50; Suberberg 1981 , no. 50, fig. 43 (falsa indicazione).

I 7, 7 Casa del Sacerdos Amandus Cat. 154/1. (b) Paretı sud e nord, quadro centrale, III stıle

Sulla parete sud si vede un pinax con Polifemo e Galatea Sul davanti a destra si distigue un'alta colonna sormontata dalla statua itıfallica di Priapo in rosso. Ha un cappello in testa; I corpo e piegato nell'attegiamento della chordosis. Appartiene all'ambiente paesistico e non siolge una parte nella

523 Cf. I rulievo votivo di Delo (Marcade 1969, 215, pl. XXXIX).

524 Schmidt-Colmet 1977, M69. 
storld rappresentata

Il quadro della parete nord mostra Eracle nel giardino delle Esperidi L'eroe e rappresentato secondo ll modello dell' Lracle Lansdowne ds Scopa, come ha osservato giustamente A Stewart Differisce soltanto il fatto che la leontude manchi e l'eros abbia una corona di foglie di vite sulla testa

Bibl 4 Malurı, NSc 1927, 24, lig 6-7. Herter 1932, 159 no 195. Malurı 1938, 5-12, Schefold 1957, 31, Schefold 1962, 87, Paar 1962, 85-87. Abb 16, Von Blanckenhagen'Álexander 1962, $4851, \mathrm{pl} 551$, I'eter's 1963, 91-92. fig 78, Stewart 1977, 139-140, pl 30-31, Bastet De Vos $1979,81-83$. Repertorio 1981, 58-59

Cat $154 / 2$ (d) Parcte sud, quadro centrale, III stule

Paesaggio sacro-idlliaco sulla rive di un liume si trovano un tempio e due statue dl'interno di un recinto Peters confronta queste statue, rese in modo schematico, con monumentı funerarı fuor della Porta di locera a Pompel Anche in questo caso l'insieme potrebbe raffigurare un monumento funerario Bibl Malur1 1938, 13, Schefold 1957 31, Peters 1963, 111, Bastet/De Vos 1979 , 46, Repertorio 1981,59 , Suberberg 1981 , no 37

I 7,10 Casa dell'efebo

Cat $155 / 1$ (2) Pareti nord e sud, zona superiore, IV stile

L'edicola centrale e occupata da una figura temminle di colore giallo, vestita di un peplo classico Regge un sistro nella destra alzata e un lembo della veste nella smistra L'atteggamento e quello delle Landidi della Villa del papirs ad Ercolano Di entranbe le figure si distinguono solo le sagome Secondo Tran Tam Tinh sono d'impronta egittizzante

Bibl A Malurı, ISc 1927, 36, Malurı 1938, 14, Jucker 1950, 8, Schefold 1957, 32, Tran Tam Tinh 1964, 123 124, Repertorio 1981, 61-62

Cat 155/2 (17) Parete ovest, zona superiore; IV stule

L'edicola centrale e ilancheggiata da figure femminil vestite di un peplo, che reggono un festone Siccome esse sono conservate frammentariamente non s] puo piu stabilire se avessero anche la funzione di cariatidi per la trabeazione dell'orlo superiore Non si puo nemmeno distinguere ll loro colore Lo stile e arcazzante

Bıbl $\rightarrow$ Malurı, \Sc 1927, 45-49, Malurı 1938, 19, tig 15, Schetold 1957, 33-34, Repertorio 1981, 67-68

Cat $155 / 3$ (23) Triclinio estivo, IV stile

Un paesaggio nulotico copre interamente il triclinio Sul lato corto di sinistra s1 vede un tempietto circondato da un recinto In esso si trova una statua gidlla di Iside-Fortuna, seduta su un trono, che regge nella sinistra una cornucopia e nella destra stesa una patera Corrisponde alle statuette che si trovano nei lararı Sul tumpano del saccllo si distungue un dcroterio a forma di slinge femminule dlata $\rightarrow$ destra sta una nicchid ogivale occupata dalla statua gidlla di una figurd temminule, probabilmente di nuovo Iside-fortuna

Sul tratto in fondo si vede la statua glalla del bue Apis esposto su una base rettangolare che sembra triangolare a causa della propettiva poco accurata In prumo piano si trova un'edicola ad arco occupata dalla statua di una figura femminle restita di un peplo, simle a quella descritta sopra 
Sul tratto destro dell'interno si vede presso un edificio e una statua di una figura maschile un banchetto all'aperto La statua e resa in modo schematico e non permette un'identificazione Al di sopra si trova un'isoletta con un'edicola nella quale si vede una statua, di nuovo molto schematica

Bibl A Mamrı, ASc 1927 j3-60, Malurı 1938, 23-27, tal III-IV, Schefold 1957, 34, Peters 1963, 181-182, fig 179, Tran Tam Tinh 1964, 124 no 4. Whitehouse 1977, 63-64. Repertor10 1981, 69-70

Cat 155/4 (23) Parete sud, zona mediana, IV stule

Sulle lesene accanto alla rappresentazıone di un giardino si vedono del guerrieri dipunti in giallo che si reggono sopra una mensola $\ln$ semplice drappo ne ricopre le spalle Hanno un elmo sulla testa e reggono nella sinistra uno scudo rotondo La figura a sinistra regge con la destra distesa una lancia, la figura a destra una spada Corrispondono al guerrierı d'umpronta polucletea (cf p. 43)

Bibl A Malurı, ISc 1927, 60-61, fig 29, Malurı 1938, 27, fig 30, Schefold 1957, 34, Repertor10 1981, 70

I 7,19

Cat 1561 (b) Paretı est e ovest, zoccolo, parete sud, quadro centrale, III stule

tyronı bianchı l guisa di atlantı si delineano su uno sfondo nero, molto rovinato, nel mezzo dl cespuglı Stanno su bası verdı a forma d rettangolı il cus lato superiore e concavo e reggono sulla testa un corno su cul poggla un segmento uguale alla base e una fascia che diride lo zoccolo dalla zona mediana Su ognı parete vi sono due uccellı dispostı in ordine simmetrico, ralfiguratı come anımalı vivı

Sulla parete sud si trova un paesaggio sacro-ıdilliaco nel quale statuette schematiche pogfiano contro 1 plastri di un piccolo santuario Tutto e reso in modo sommario

Bıbl A Malurı, ISc 1929, 361-362, fig 3, Malurı 1938, 28, fig 21. Schefold 1957, 35-36, Bastet/De Vos 1979, 35-96, Repertorio 1981, 73-74, Slberberg 1981, no 36, fig 40-42.

Cat 156/2 (c) Paretı nord, sud e est, zoccolo, III stule

Uccellı branchi reggono l'orlo supersore dello zoccolo come nel vano b Stanno su bası rettangoları verdı e hanno un elemento vegetale sulla testa Sono piu snell degli uccell s suddettı Sulla parete est sı vedono auronı, sulle paretı nord e sud invece sono raftigurate stingl alate femminll, viste di fronte

Bıbl Schefold 1957. 36, Bastet, De Vos 1979, 95-96, Repertorio 1981, 75

Cat 156/3 (f) Parete est, quadro centrale e zona superiore, III stule Paesaggio sacro-ıdillaco Presso un gruppo di alberı a destra sta la statua itıfallica rossa di Priapo A sinistra in tondo si vede la statua gialla di Eracle che regge una clava nella sinistra $\Gamma^{\prime}$ seduto e imita 4 tipo dell'Herahles Epitrapezios

Il tumpano dell'edicola centrale e sormontato nella zona superiore da figure femminul in giallo che funzionano da cariatidı reggentı le colonne della scaenae frons Sono vestite di un peplo classico 
Bıbl. A Maiurl. NSc 1929, 372-373, tav. XX1; Maiur 1938, 32, 11g 25. Schetold 1957, 36, Von Blanchenhagen/Alexander 1962, 36, Peters 1963, 68-69; Bastet, De Vos 1979, 62, fig. 3, Kepertorto 1981, 76, Slberberg 1981, no 27 , fig. 30

Cat. 156/4 (g) Parete sud, zona mediana, III stile

Pittura di glardino Al centro davantı a una siepe bassa e piante verdi si vede la statua bianca ds ttrodjte che sorge sopra una base rotonda Soltanto le gambe sono rumaste visibil. Il peso del corpo poggia sulla gamba sinistra 525

Bıbl A Maıurı, NSc 1929, 370, tav XX, Malurı 1938, 34, fig. 27, Grimal 1943, 480-482, pl VI.1: Schetold 1957 36, Schetold 1962, 152, Grumal 1969, 445 no 5; Peters 1971, 8. I'fretschner 1977, no. 1, Jashemsh1 1979, 57-58, Bastet/De Vos 1979, 95-96, Michel 1978, 394-395; Repertorso 1981. 76.

I 8, 10 Taberna vasaria

Cat 157 Facciata, zona mediana (ora Antıquarıum Pompeı, dove irreperıbile) Bottega di vasellame A sinistra vi e la statua di colore porpora di Atena Ergane La dea e vestita di un peplo e vista di profilo, rivolta verso destra. Regge una lancia nella sinistra stesa in avanti La parte superiore, sopra il petto, e andata perduta La statua si presenta senza una rorma precisa, e grezza e fa pensare a uno xoanon o a una statua fittue Si ricordino le terrecotte di Lai inio 526

Ln'altra Atena Ergane sl vede in una pittura in VI 7, 8 (cat. 194) La figura corrisponde piu o meno a una statua (?) di Atena in rlievo su una cista di plombo. ${ }^{52} 7$

La decorazione e stata eseguita secondo l'arte plebea e difficulmente datable 52B Lo scopritore Maiuri non oso proporre una data precisa; B. Fellettı Maj suggerı una data alla fine dell'eta repubblicana o nel primı annı del Principato. Vero e che la pittura era coperta da altri strati d'intonaco.

Bibl. Maiuri 1953-1954 90-92, tav 31 , Schefold 195i, 37, Folletti Maj 1977,241 , lig 105b, Repertorı 1981, 80, Zummer 1982, 42.

I 8,17 Casa del quattro stilı

Cat. 158/1. (1) Cartibulum presso l'impluvio

1 pledi del cartibulum sono copertı d'intonaco bianco ornato con erme in rosso monocromo Attorno as loro fustl si vedono delle bende Esse imitano le erme

\footnotetext{
525 Nillo stesso viridario e stata trovata una statuetta marmorea di Afrodite in una cassetta di legno (Jashemskı 1979, 125).

526 Enea 1981.

527 Adamo Muscettola 1982b, 710-711, fig. 15.

52. Ci Ziminer 1982, 89-91 sulla terminologia.
} 
usate frequentemente come piedi nell'arredamento romano. ${ }^{529}$

von è necessario interpretarle -come suggerisce la didascalia in Pompei 1748-1980 - come segni contro it malocchio. La pittura ancora abbustanza fresca sembra che sia stata applicata nell'ultimo decennio prima del 79 .

Bibl.: Pompei 1748-1980, 152, fig. 9c3; Repertorio 1981. 82.

Cat. 158/2. (4) Parete ovest, quadro centrale; III stile

In una decorazione di I stile si vede inserito un frammento di III stile. Sopra una base a forma di $\mathrm{T}$ vi è una menade vestita di un mantello verdognolo che lascia scoperto il seno sinistro e le gambe. La gamba destra sta in avanti. Con la destra regge una fiaccola gialla sopra la spalla sinistra (non e un tirso, come sostiene Maiuri), mentre il braccio sinistro è in riposo lungo il fianco. Io sguardo e rivolto verso destra. La figura e una variazione sulle Aure che funzionano da acroteri, preferite specialmente nello Stile Ricco (cf. p. 29).

II frammento faceva prima parte di una scaenae frons nella zona superiore di un sistema di III stile (cf. vano 18) ed è stato ovviamente inserito dopo il 62 nella parete attuale, forse per ravvivare la decorazione monotona. Questa pratica non era insolita in Campania. 530

Bibl.: Maiuri 1940, 158, fig. 7; Repertorio 1981, 83.

Cat. 159/3. (18) Parete ovest, quadro centrale e zona superiore: IIl stile Il quadro centrale, non piú leggibile chiaramente, rappresenta Pan e le ninfe. Nel centro sorge una colonna sacra presso uno scoglio sul quale sta la statua marrone di Ecate. Solo gli avambracci che reggono le flaccole e il piedistallo a forma di segmento di colonna sono visibili attualmente.

La scaenae frons della zona superiore è ornata da quattro cariatidi ruppresentate come persone vive. Tutto i di colore grigjo-bluastro. Nel mezzo sopra il pannello sinistro si vede una donne rivolta verso destra, vestita di un chitone senza maniche. Regge una bacchetta nella destra mezzo stesa e una pisside nella sinistra lungo il fianco. Il suo pendant sopra il campo destro è andato perduto.

Gli angoli del timpano dell'edicola centrale sono sormontati da baccanti vestite di chitoni senza maniche. La figura a sinistra regge un ramicello nella destra stesa a metà e una cornucopia nclla sinistra accanto alla spalla. L'altra figura é andata perduta per la maggior parte, ma risulta asimmetrica.

Bibl.: Peters 1963, 89; Bastet/De Vos 1979, 47; Pompei 1748-1980, 150-155, fig. 10b; Repertorio 1981, 8i-89.

I 9, 1 Casa del bell'impluvio

Cat. 159/1. (11) Pareti sud e est, zona superiore; IV stile

Le figure bianche che occupano le edicole centrali sono forse da considerare delle statue. Sulla parete sud si distingue la sagoma di una figura femminile vestita di un peplo classico che regge con la mano sinistra un lembo della sua veste all'altezza della coscia. Essa è paragonabile alle Danaidi della Villa dei papiri ad Ercolano.

529 Harl-Schaller 1976-1977.

530 Maiuri 1940. Cf. Allroggen-Bedel 1983, 144-145. 
Sulla parete est si distingue la sagoma di un Eros nudo alato che regge un alabastro nella destra mezzo stesa e un piatto nella sinistra davanti al petto. Bibl.: Schefold 1957, 38; Bastet/De Vos 1979, 40; Repertorio 1981, 91.

Cat. 159/2. (7) Parete est, zona superiore; III stile

$\mathrm{Nel}$ centro si trova un pinax con un paesaggio sacro-idilliaco sopra una base a forma di $\mathrm{T}$. Presso una tholos su un'isoletta si distingue, sopra un'alta base rettangolare, la statua gialla di una figura seduta. E'vista di profilo, rivolta verso destra. La mano destra è distesa e le gambe sono tese in avanti. Forse si tratta di un Poseidon presso un suo tempio; mancano peró indicazioni precise per un'identif́jcazione esatta della figura.

A sinistra e a destra del quadro centrale vi sono delle figure maschili sopra basi a forma di $\mathrm{T}$. Sono raffigurate vive, viste di profilo, in movimento verso destra. Il loro abbigliamento, costituito da scarpe gialle e tunica corta, pantaloni e un berretto li caratterizza come Frigi. La figura a destra porta vestiti bianchi e un berretto marrone, quella a destra pantaloni bianchi e una tunicr gialla-cerulea. La sua parte superiore manca.

La parete ovest con ogni probabilita era decorata alla stessa maniera. Non ne sono state trovate tracce.

Bibl.: Fuchs 1956; Schefold 1957, 38; Bastet/De Vos 1979, 40; Repertorio 1981,90 .

I 9,3 Casa di Successus

Cat. 160. (5) Parete est, quadro centrale, pareti ovest e sud, zona superiore; IV stile

Un fanciullo seduto regge con entrambe le mani un piccione bianco davanti al petto. A destra si trova un'oca e sul davanti si vede un melograno. La ligura del fanciullo imita in modo libero il fanciullo che strozza l'oca del famoso Boethos. ${ }^{31}$ Secondo Jashemski il frutto simboleggia la morte e percio il quadretto sarcbbe il ritratto del Successus defunto L'insiene sembra molto rielaborato, per cuj lo stile differisce dai quadretti consucti. Tutlavia non credo che vi siano tratti particolari che permettano l'interpretazione del quadro come ritratto; il viso e schematico e privo di caratteristiche.

Le edicole nella zona superiore delle pareti ovest e sud sono occupate da figure femminili policrome. Sulla parete est si vede la sagoma di una venere poinpeiana, fiancheggiata da un Eros di cui resta soltanto la testa.

Sulla parete sud si trova una menade vestita di un chitone senza maniche che sorregge nella destra. Tiene un timpano lungo il fianco e ha nella sinistra un tirso. Il peso del corpo poggia sulla gamba sinistra. Sulla testa si distingue una corona d'edera.

Le teste di entrambe le figure sono dipinte con un accento particolare per i dettagli. Specie la Venere pompeiana sembra avere tratti veristici che fanno pensare a un ritratto in forma d'immagine di una divinità. ${ }^{532}$ Non è chiaro, inoltre, se le figure rappresentino statue o figure vive. Il gruppo della Venere pompeiana corrisponde a quello della Via dell'Abbondanza.

531 Per il tipo si vede Andreae 1974.

532 Cf. i ritratti di un fanciullo e di una fanciulla in guisa di Hermes e Afrodite nella Casa di M. Lucretius Fronto (vano 6): Pompei 1748-1980, 173 no. $35 \mathrm{D}$. 
Bibl.: Schefold 1957, 38; Jashemski 1979, 102, fig. 160; Repertorio 1981, $92-93$.

I 9, 5 Casa del frutteto/dei cubicoli floreali

Cat. 161/1. (8) Pareti nord, est e sud, zone mediana e superiore; III stile Rappresentazione dj giardino con elementi egittizzanti. In un porticato stilizzato si delineano fra le piante statue bianche di figure maschili egizie sopra basi a forma di $T$. Nell'intercolunnio centrale non si trova una statua, ma un pinax sopra un pilastrino. Anche sopra l'architrave si vedono pinates.

Parete nord. Due statue di uomini seduti su seggiole a spalliera bassa, disposte in ordine sinmetrico, sono delineate di profilo con il viso rivolto verso il centro. Indossano un drappo attorno alle reni e reggono nella destra tesa in avanti una bacchetta, nell'altra, in riposo sul grembo, il ka. Non corrispondono a modelli desunti dalla scultura egizia finora conosciuta. ma si confronti il rilievo della Porta di halabsha, ora nell'Egyptisches Museum a Berlino, dell'epoca di Augusto, dove si trovano figure simili nel registro superiore. Le nostre figure non esprimono un'iconografia specifica e risultano ininterpretabili.

Il pinax nell'intercolunnio centrale rappresenta il bue $A$ pis bianco a macchie nere, con una cintura dorata attorno al fianco e con il disco solare fra le corna. Avanza verso sinistra. Lo sfondo è di colore viola.

Parete est. Due statue di uomini che avanzano verso il centro, rese in ordine simmetrico, sorgono sopra le consuete basi a forma di $T$. I loro attributi non sono piú discernibili, il che non permette un'interpretazione precisa - se maj possibile. Anche in questo caso un confronto con la scultura egizia si presenta inattuabile.

Sul pinax centrale si delinea un Dioniso sdraiato che poggia sul gomito destro. Alla sua destra si vede una menade danzante con un panno attorno alle reni. Regge un tirso nella destra e alza la sinistra sopra la testa. Lo stondo del pinax è viola.

Nella zona superiore sopra i pilastri stanno due pinakes con scene di tematica egizla. A sinistra un uomo stante porge un'offerta a un uomo seduto. Regge contemporaneamente un $k a$ nella sinistra. Sul pinax a destra un uomo si inclina davanti ad un uomo seduto. Fra di loro si trova un tavolino giallo. Lo sfondo è pure di colore giallo.

Parete sud. Due statue di figure maschili identiche a quelle della parete nord sono meglio conservate e permettono una descrizione degli ornamenti. Si distinguono linee gialle di gioielli che ricordano la policromia parziale di statue, per esempio della lamosa Venere in bikini.

Sul pinax nel centro una menade o un ermafrodito viene denudata da un satiro che avanza da destra. Esso regge un pedum e una clanide nella sinistra. Lo sfondo è di colore viola. Anche su questa parete un pinax con il bue Apis incorona l'architrave. Avanza verso sinistra, quindi non nella medesima direzione dell'altro.

Per l'interpretazione della decorazione si vedano pp. $61-62$.

Bibl.: Schefold 1957, 38; Reuterswärd 1960, 182; Schefold 1962, 140-141; Peters 1963, 179; Tran Tam Tinh 1964, 197-200; Le Corsu 1967; Peters 1971, 6-8; Tran Tam Tinh 1971, 9; Sichtermann 1974; Kater-Sibbes/Vermaseren 1975, 22-23 no. 30, pl. 5i-58; Michel 1978, 386-390; Bastet, De Vos 1979, 74-76; Jashemski 1979, 74-79, fig. 117; De Vos 1980, 15-20, tav. XII-XIIl; Froning 1981, 12-13, Frontespiz: Repertorio 1981, 95-96. 
Cat. 161/2. (11) Pareti est e ovest, quadro centrale e zona superiore; III stile

Il quadro della parete ovest rappresenta Atteone divorato dai suoi cani in un paesaggio silvestre. A destra vi e un'erma bianca di Pan: dalle reni in su il corpo é delineato in modo dettagliato e plastico. Il dio regge una siringa o una patera nella mano destra tesa in avanti, un pedum nella sinistra presso la spalla. Le corna sono messe in evidenza paticolare: se ne vedono chiaramente le righe. L'interpretazione della statua - sostenuta da Peters e da Pfretschner - come immagine di Artemide è spiegata da queste corna che sono state interpretate come $i$ denti della consueta corona della dea.

Il quadro della parete est mostra Dedalo e Icaro. A destra su uno scoglio si vede la statua gialla di Poseidon. Egli e seduto su di una base rotonda di colore rosso. La gamba sinistra è leggermente protesa in avanti. Regge un celfino nella destra distesa e un tridente nella sinistra all'altezza della spalla. La statua e identica a quela sul pinax dello stesso tema in $V$ 2, 9 (cat. 192/2). L'atteggiamento e derivato da tipi ellenistici. a loro volta ispirati dall'Herakles Epitrapezios di Lisippo. Specialmente il braccio disteso e la posizione delle gambe sono identiche.

In avanti a sinistra si vede un'Akte viva, vestita di un mantello giallo-blu, resa secondo il tipo statuario della aufgelehnte Muse, in uso per Polinnia. ${ }^{33}$ Anche in altri paesaggi e scene mitologiche si trovano Aktai di questo tipo.

Paesaggi sacro-idilliaci si vedono in un fregio fra zona mediana e zona superiore. In quello sopra il prospetto della parete est si distinguono statue schematiche in una composizione con torri.

Alcune cariatidi fanno parte della scaenae frons nella zona superiore. Indossano chiloni verdi senza maniche i cui lembi reggono con le mani, l'uno all'altezza del fianco, l'altro presso la spalla. I piedi stanno accostati su di una base a forma di $T$. Sulla testa reggono un pilastrino sottile. Incontriamo cariatidi di questo tipo nell'arte glittica, per esempio sul sarcofago di Velletri e su un rilievo a Napoli. 534

La decorazione è stata eseguita dalla stessa bottega che ha decorato la Casa del bell'impluvio (cat. 159/1).

Bibl.: Peters 1963, 85-86; Von Blanckenhagen 1968, 112, pl. 37-38; Pfretschner 1977, no. 12; Bastel; De Vos 1979, 74-75, tav. XXXIX; Berard/Hofstetter 1979, 123; Repertorio 1981, 97-100; Silberberg 1981, no. 44; LIMC J (1982) 463 no. 95; Ehrhardt 1982, j85. Sichtermann 1984, 295-296, Taf. III; Bragantini/Parise Badoni 1984, 120-121, fig. $3-5$.

I 9, 13 Casa di Cerere

Cat. 162/1. (n) Parete ovest, zona superiore (ora staccato e posto nel tablino); III stile

Paesaggio sacro-idilliaco. A sinistra in avanti presso una schola si vede un'erma gialla di Priapo. Piú a destra si distinguono le vaghe tracce di un'altra statua gialla.

Bibl.: Peters 1963, 60; De Vos 1976, 49, pl. 53 fig. 30; Bastet/De Vos 1979, 91; Repertorio 1981, 108-109.

533 Pinkwart 1965, 187-192. Cf. la statuetta trovata nella Casa di Octavius Quartio (Spinazzola 1953, I, fig. 462).

534 Schmidt-Colinet 1977, no. W19, W54. 
Cat 162/2 (o) Parete sud, zona mediana, III stile +

Pittura di giardıno L na fontana biancd e fuancheggiata da sfingl femminul alate che sono rivolte verso 1 centro Il pessimo stato di conseridzione unpedisce una descrizione dettagliata Una situazione simule si vede nella Casa d) Julıus Polybıus (cat 303,2)

Bibl De Vos 1976, 50, pl 54 fig 32, De Vos 1979, 92. Bastet De Vos 1979, 91, Ropertorio 1981, 109

I 10, 4 Casd del Menandro

Cat $163 / 1$ (4) Parete nord quadro centrale, IV stile

Il punax unserito nella prospettiva fra I due pannell rappresenta 1 ratto del Palladio, l'architettura runziona da cavallet to La protetessa Cassandra alubraccia la statua marione di Atena la dea e vestita di un peplo e ha un elmo con pennaccho sulla testa Regge nella destra una lancia sopra la spalla e copre la spalla sinistra con uno scudo rotondo Lo stle arcaistico della statua contrasta con la vivacita ellenistica delle altre figure Il colore indica l materidle, cioe dl legno, non e verosimule che si trattı d una statua bronzea, come sostjene Pfretschner, perche la statua di culto era abitualmente eseguita in legno e, oltre a c1o, tale colore era convenzionalmente in uso per la finzione del legno Il tipo e quello origmale conosciuto fra l'altro dalle terrecotte $d_{1}$ Lavinıo e da una statueta marmorea $d_{1}$ Sperlonga che una valta faceva parte di un gruppo con lo stesso soggetto 535

Bibl Marurı 1933, 49-71, lav VI, Ghali-kahl 1955, 247 no 201, pl 712 , Schelold 1957, 10, Reutersward 1960, 136. Perotichner 1977. no 28, Gigante 1979, 181-183 Repertorio 1981, 115. LIMC 1 (1982) 346 no 83

Cat $163 / 2$ (18) Farete sud, zona mediana, IV stule +

In un Iregio si vedono amorini che incoronano un'erma J colori e le sagome sono quası completamente svanitı e non ne esiste una fotografı o un disegno

Bıbl Maıurı 1933,174

Cat 163/3 (24) Parete sud, zona mediana, IV stule

Paesaggio sacro-idiliaco In un bosco sacro si trova un'edıcola di legno occupata da un gruppo statuarıo di Afrodite e Eros La dea indossa una veste giallo-viola e ha una corona sulla testa La mano destra e nascosta nella veste, con la sinistra Afrodite si appoggia ad un plastrino Alla sua sinistra Eros ne sostiene il braccio inferiore con la destra, mentre regge un globo nelld smistra $E^{\prime}$ nudo e ha al in rosso scuro Ia posizione delle gambe non e pru visibile

Il gruppo rappresenta la Venere pompejana conoscıuta quası esclusivamente da pitture pompelane $P$ in un solo caso come statuetta 536 ld composizione $d_{1}$ un'edicola con una statua in un bosco sacro si vede qualche volta copiata nei gıaı dını pompeianı. nella Casa del moralısta, per esempıo, e stata trovata una statuetta marmorea dı Irtemide, nella Casa deglı scienziatı una dı Ares e

535 Enea 1981, 160, 193-194, Conticello 1974, 39, 44-45, tav. 4, Andreae 1974, 96-103, tav 44 Cf Chevalier Lemaitre 1969

536 Spinazzola 1953, I, 218, fig 244-245 
altrove $s i$ incontrano situazioni simili. ${ }^{537}$

Bibl.: Maiuri 1933, 94, fig. 45-46; Schefold 1957, 42; Beven 1960, 176; Peters 1963, 179-180, fig. 180; Repertorio 1981, 128; Silberberg 1981, no. 129, fig. 106 .

Cat. 163/4. (46) Pareti nord e est, zona mediana; II stile, lase IIb Erme bianche stamo sopra plinti davanti agli ortostati. La decorazione e difficilmente leggibile. Dalla vita in su sono rese in forma naturale. Sulla parete nord si é conservata la testa di un'erma, sulla parete est si vede il torso dj un satiro, coperto con una nebride gialla. Le due mani, davanti al petto, reggono un festone.

Secondo Beyen la decorazione era opera della bottega che aveva lavorato nella Casa del criptoportico (cal. 148), ipotesi non piu controllabile a causa del pessimo stato di entrambi $\mathrm{i}$ complessi.

Bibl.: Maiuri 1933, 128, fig. 58; Schefold 1957. 44; Beyen 1960, 144-145, Abb. 59, 61a; Schmidt-Colinet 1977, 14i, 267 no. Pjb; Repertorio $1981,130$.

Cat. 163/5. (47) Parete est, zoccolo e zona mediana; II stile, fase Ila

Scaenae frons molto rovinata e attualmente illeggibile. Jue uccelli bianch sostengono l'orlo superiore dello zoccolo con le loro ali aperte. Le teste sono rivolte verso l'esterno. Si tratta di uno dei pochi esempi di uccelli con questa funzione nello zoccolo di II stile; un'altro caso si vede sull'Insula occidentalis (cat. 225/2). Liccelli in altre zone si incontrano gia nella fase Ib, per esempio nella Villa dei misteri (cat. $306 ; 2$ ).

L'architrave dell'edicola centrale della zona mediana viene sorretto da centauri simmetrici di colore bianco. Le zampe anteriori sono alzate e volte verso l'esterno. Trovano riferimenti nella scultura ellenistica.53a Non si conoscono confronti nell'architettura, ma si deve pensare al passo vitruviano (VII 5,5$)$ : centauri epistylia sustinentes, descritti come ornamenti realistici, a lui bell noti. In pittura se ne conoscono vari esempi (cf. p. 220.

Nelle nicchie a sinistra e a destra dell'edicola centrale si trovano ligure di atleti rappresentate come figure vive, ma delineate secondo modeli scultorei. Beyen disse giustamente: Haben die Gestalten auch noch alle etwas Statuarisches, so sind sie dennoch als auf Felder gemalte Bilder gedacht.

A sinistra si vede un discobolo con il disco nella destra lesa verso il basso e con la sinistra dietro le spalle. Le gambe sono divaricate. Corrisponde genericamente al discobolo mironiano.

A destra si trova un atleta in riposo, seduto su di un segmento di colonna. Le mani sono in riposo nel grembo, la testa guarda a sinistra e le gambe sono incrociate. $E^{\prime}$ simile al famoso pugilista nel Museo Nazionale Romano. Il loro modello comune è l'Eracle di Taranto di Lisippo. ${ }^{539}$

Bibl.: Maiuri 1933, 139-142, fig. 65; Schefold 1957, 45; Beven 1960, 120-198, Abb. 58; Ling 1971, 267; Schmidt-Colinet 1977, 147, 267 no. P5a, nota 585; Repertorio 1981, 130; Von Hesberg 1983, 233 nota 136.

Cat. 163:6. (48) Abside, zone mediana e superiore; II stile, fase IIa

\footnotetext{
537 Dwyer 1982, 118.

530 Cf. De Caro 1976; Jashemski 1979, fig. 466.

539 CatRoma I 1, 194-198 no. 123. Cf. Moreno 1978.
} 
Sulle fasce a sinistra e a destra dell'abside si distinguono erme bianche con teste di figure temminilı incoronate Queste sono rese in modo dettagliato Guardano verso ll centro Sopra di esse s1 trovano Erolı bianch1, disposti in ordine simmetrico La gamba esteriore e di appoggio vella mano rsterna reggono una brocca, l'altra e in riposo lungo il fianco Particolarmente evidentı sono le alı lunghissume

La stessa abside ha una serie di unitazioni di rulievi bianchi incastratı in una parete rossa La loro profondita e ottenuta mediante sottli pennellate rosse Fittschen suggerisce che essi imitino avorio, ipotesı improbabule in quanto il tormato delle lastre e troppo grancle e la protondita dello stesso rilievo e troppo alta Quest'ultimo aspetto impedisce nello stesso momento l'ipotesi di umitazionı di rilievi in stucco, come, inoltre, e tatto evidente dalla presenza della cornice Deve quindi trattarsı di rliev di marmo

Del sette rilievi sel sono rimastı intattı, raffiguranti, da sinistra a destra 1 seguentı soggettı

1) Donna alata vestita di un peplo che regge una palma davantı al grembo Il peso del corpo pogela sulla gamba destra l capell sono raccoltı in un nodo simile all'dcconciatura della Cnidia

2) Donna vestita di un peplo, che regge nella sinistra alzata una lancia e nella destra uno scudo rotondo accanto al tianco La gamba sinistra e di appoggio Lo sguardo e rivolto verso sinistra

3) IJonna vestita $d_{1}$ un peplo e $d_{l}$ un mantello che copre la testa, avanza verso destra In entrambe le manı tese in avant regge una paterd Probabilmente appartiene a una scena di culto su di un rilievo oblungo nel centro dell'abside ora in gran parte perduto

4) Donna vestita di un peplo, che regge nella mano smistra alzata un kalathos all'altezza della spalla, mentre l'altro braccio e in riposo lungo il fianco La gamba destra sostiene il peso del corpo Lo sguardo e volto a destra Anch'essa potrebbe far parte del rllievo suddetto

5) Donna vestita di un peplo, che ha l braccio destro disteso per meta, l'altra e in riposo lungo il fianco La gamba destra regge il peso del corpo Sulla testa ha un cosiddetto cappello ionico, che si incontra spesso nelle figurine fittil ellenistiche Guarda verso sinistra

6) Donna vestita dı un peplo, che regge con entrambe le manı, davantı al petto, un drappo

I rlievi sono separatı l'uno dall'altro per mezzo di figure femminili alate di colore giallo, 1 cui corpi inferior terminano con ornamentı florealı $S_{1}$ tratta di imitazionı di appliques di bronzo

Nella zona superiore si trovano scene cultuall e balnearı in bianco su sfondo rosso. Vediamo di nuovo imitazionı di rulievi Sul rilievo a sinistra alcune donne stanno in piedı presso una fonte, in fondo si trovano due statuete schematiche di divinita fluvialı

Bibl Maiur1 1933, 152-158, fig 72-78, Schefold 1957, 45-46, Beyen 1960, 153-154, Ab 65. Farbtaf I, Fittschen 1976, 352 nota 99, Muthmann 1975, 29-30, Taf 71, Repertorio 1981, 131-132, Moormann 1984b, 649 .

I 10,7 Casa del fabbro

Cat 164 (9) Parele ovest, quadro centrale, III stlle

Iedalo e Icaro A sinistra si distingue, sopra uno scogho, la statua gialla di Poseidon Il dio marino e seduto sullo scoglio e regge un tridente nella sinistra alzata, mentre ha la destra in riposo sul grembo Il tipo - derivato dall'Herakles Epitrapezios - si ritrova su altrı pinakes con lo stesso tema ( $p$ is e cat $161 / 2$ ) 
Ciò vale ugualmente per la Akte che sta in avanti, modellata come l'aufgelehnte Muse.

Bibl.: Schefold 1957, 46; Schefold 1962, 191; Peters 1963, 90-91, fig. 76; Von Blanckenhagen 1968, 111, pl. 36.2; Bastet/De Vos 1979, 87-88; Berard/Hof stetter 1979, 123, pl. 78 fig. 10; Repertorio 1981, 137.

I 10, 11 Casa degli amanti

Cat. 165/1. (1) Parete nord, zona mediana; IV stile

Tondo con un paesaggio sacro-idilliaco nel tratto ovest. Su uno scoglio si trova la statua itifallica rossa di Priapo. Sta nella chordosis. Non e chiaro se l'oggetto a sinistra del Priapo raffiguri una statua o un ceppo.

Bibl.: Schefold 1957, 47; Peters 1963, 159; Croisille 1969, 170 nota 1, pl. LVIII fig. 3; Jashemski 1979, 130, fig. 208; Silberberg 1981, no. 114; Repertorio 1981, 139.

Cat. 165:2. (4) Pareti nord e sud, zona mediana: IV stile

Centauri a coda di pesce in giallo fungono da acroteri sull'cdicola centrale $e$. nello stesso tempo, da atlanti per la trabeazione nella zona superiore. Reggono, davanti al petto, un piatto con la mano esterna, mentre il braccio al lato interno, leggermente piegato, è in riposo lungo il fianco. Dettagli come occhi, bocca " chioma sono stati resi in giallo: cio potrebbe significare l'imitazione di intagli di diverso materiale.

Bibl.: Schefold 1957, 4i; Repertorio 1981, 141.

Cat. 165/3. (8) Parete ovest, quadro centrale e zona superiore; III stile Il quadro centrale rappresenta Didone abbandonata. Sullo sfondo vi e una statua rossa ora illeggibile, forse rappresentante un Priapo che fa parte dei consueti elementi paesistici senza svolgere un ruolo nella scena raffigurata.

Sull'edicola centrale si trovano figure femninili, vestite di un peplo e di un mantello, in guisa da acroteri. Sostengono una colonnina snella sulla testa, parte della scaenae frons della zona superiore. Reggono in entrambe le mani distese per metà dei piatti (ora illeggibili). La figura a destra è svanita, l'altra e andata perduta completamente.

Bibl.: Schefold 1957, 48; Repertoro 1981, 141.

I 11,6 Casa della Venere in bikini

Cat. 166/1. (4) Pareti nord, est e sud, zona superiore; IV stile

L'architrave dell'edicola centrale viene sormontato da figure in colori naturali. Sulle pareti nord e sud esse snno circondate da un festone sottile a forma di mandorla e stanno su di globi rossi.

Parete nord. A sinistra si vede una figura maschile nudo con un solo panno sopra la spalla sinistra. Il peso del corpo poggia sulla gamba destra. Regge davanti al petto nella sinistra un piatto con un serpente e agita uno scettro sopra la testa con la mano destra. Lo sguardo è rivolto verso il centro. Potrebbe trattarsi di Apollo Pithios.

A destra è una figura femminile vestita di una tunica agitata da un vento immaginario. Il peso del corpo poggia sulla gamba sinistra. Regge un piatto 
nella sinistra e una brocca nella destra Il vestito e la presenza dell'Apollo sull'altro lato caratterizzerebbero la figura come Artemide, ma gli attributı non sono normali per questa divinita. L'atteggiamento e sumule a quello delle Artemidi in I 3, 25 (cat 146).

Parete est. I simstra si trova un uomo nudo con una clamide sopra le spalle Il peso dol corpo poggia sulla gamba destra Regge un pidto o un cestino nella sinistra, mentre la destra e presso la spalla.

A destra sta una figura femmunule vestita di un mantello verde (solo di questa tigura si possono distinguere chiaramente 1 colori), il cul torso e la cola parte ancora visibile

Parete sud A sinistra si vede la sagoma di una tigura che regge un cestino nella sinistra La figura a destra e andata perduta

Bibl Repertor10 1981, 150-151

Cat. 166/2. (8) Pareti sud e ovest, zona superiore; IV stle

Sulla parete sud la tascia rossa che divde in due la rappresentazione di un glardino, contiene la statua bianca di Afrodite Solo le gambe, delle qualı la destra e di appoggio. sono rumaste visibulı sopra una base rotonda.

Su una fascra nell'angolo destro della medesuma parete sorge un'erma con una testa maschule dietro un cespuglio verde. Solo la sagoma e rumasta visibile. Il colore originale probabilmente era bianco.

Sulla parete ovest il centro o occupato dalla statua dı una ninfa acquatica in color naturalı, ora quası svinita La gamba destra sorregge il peso del corpo Un mantello rosso copre le gambe e le reni La nunfa regge, davanti al grembo, un bacmo gidlo nel quale zamplla dell'acqua. Copia ll modello della Venere Landolina ( $c f$ p 000 ).

Bibl Reutersward 1960, 182. Jashemshi 1979, 125, 128; Repertorio 1981, $153-154$

Cat. 166/3. (10) Parete est zona superıore; IV stile

L'edicola centrale e occupata da un Eros nudo ad alı grandi. La gamba simistra e di appoggio Il torso ruota verso destra. Nella sinıstra alzata regge un tirso, con la destra un lembo di una clamide che copre le spalle.

Bıbl. . Repertorio 1981, 154.

I 11,15 Casa del primo piano

Cat. 167. (24) Parete nord, zona mediana; IV stile

Un centauro impennato giallo sta su un plinto nel prospetto a destra del campo centrale. La parte superiore del corpo e rivolto verso destra. Il braccio destro e disteso e quello sinistro poggia sulla spalla Regge nella destra una baccheta. Corrisponde a tipl ellenistici, noti fra l'altro da Oplontis.

Bibl. Repertorio 1981, 163.

I 11,16

Cat. 168, (6) Parete est, zona mediana; IV stile 
Rappresentazione di giardino molto rovinata. Fra le piante si vede una fontana sorretta da una stinge femminile alata di colore bianco. Dietro la fontana si trova la sagoma bianca di una figura, forse rappresentante una scultura.

Bibl.: Packer 1978, 23-24, fig. 11; Jashemski 1979, 73-74, fíg. 265; lepertorio $1981,164$.

I 12,3

Cat. 169. (3) Parete sud, quadro centrale e zona superiore; III stile

Sul quadro centrale si distingue una figura maschile in piedi che imita l'Apollo Licio di Prassitele: la destra è in riposo sulla testa e la sinistra e distesa. $E^{\prime}$ simile ad un pinax nella Casa di M. Lucretius Fronto (calt. 195/1), prodotto dello stesso pittore.

L'edicola centrale della zona superiore è occupata da una figura femminile su di una base rettangolare. Avanza verso destra. $E^{\prime}$ vestita di un mantello verde a orli viola che lascia scoperto il seno. Con entrambe le mani regge un tirso davanti al petto. La figura i aftine alle statue dello Stile Riceo che incontriamo frequentemente nelle scacnae frontes della zoná superiore come cariatidi o acroteri.

Bibl.: Bastet/De Vos 1979, 84-85, tav. XLIV fig. 78: Repertorio 1981, 169; Bragantini/Parise Badoni 1984, 123-124, fig. 14-16.

I 12.9

Cat. 170. (3) Paretj est e ovest, vignetta centrale; III stile

Paesnggi sacro-idilliaci in verde su uno sfondo bianco come vignette, delineati in modo schematico. In entrambi i paesaggi si vedono statue schematiche. Sulla parete ovest si distinguono piedistalli a forma di $\mathrm{T}$.

Bibl.: Bastet/De Vos 1979, 44-45, tav. XV fig. 27 (erroneamente sottoscritto "cubicolo 15"): Repertorio 1981, 175-176.

I 12,11

Cat. 171. (8) Parete nord, zona mediana; IV stile

Sulle lesene rosse al fianco di un paradeisos si distinguono statue di colore bianco. A sinistra si vede un'erma con testa maschile. quasi completamente perduta. A destra sorge la Mantelherme di una figura femminile vestita di un mantello. Regge con entrambe le mani, davanti al grembo, un bacino rotondo pieno d'acqua. E' una variazione sulle ninfe consuete del tipo della Venere Landolina. Erme a mantello sono state trovate, per esempio, nel tempio di Apollo e nelle Terme stabiane.

Bibl.: De Vos 1979, 92; Jashemski 1979, 31-32, [ig. 50; Repertorio 1981, 178.

I $13,2-5$

Cat. 172. (6) Parete nord, 7.ona mediana; II stile, fase IIb 
Sul campo centrale di colore verde si distingue la figura isolata di Afrodite che si slaccia con la mano destra il sandalo destro. La mano sinistra è davanti al petto. Accanto a lei si trovano i suoi panni. Non è chiaro se il pittore abbia voluto dipingere una statuetta sopra una mensola o una figura viva come vignetta.

Il tipo è popolare dall'Ellenismo in poi. Ad Ercolano e stata trovata una statuetta di bronzo, a Oplontis una copia in marmo. ${ }^{540} \mathrm{Su}$ di un mosaico a intaglio da I 2, 10 (ora Museo Nazionale s.n.) si vede un'Afrodite che compie lo stesso gesto mentre si appoggia ad un pilastrino. ${ }^{543}$

Una figura isolata su un campo e ancora rara nel II stile. Si pensi alla figura nella Casa di Obellius Firmus. La datazione di questa decorazione in questo periodo, proposta da De Vos, è, con argomenti validi, basata sulla raffigurazione illusionistica dej drappi appesi nello zoccolo. ${ }^{542}$

Bibl.: De Vos 1976, 64, fig.49-50, pl. 60-61; Repertorio 1981, 183.

I 13,16

Cat. 173. (3) Parete nord, quadro centrale; IV stile

In una nicchia rossa, delineata in modo rozzo, si trovano tre statue. A sinistra si vede un'Afrodite bianca nuda con una sciarpa svolazzante at torno al corpo. Le gambe stanno unite l'una contro l'altra. Regge con la sinistra uno specchio all'altezza dela testa, mentre alza i capelli con la destra. La ligura rappresenta in modo narrativo l'atto della venere allo specchio. Si tratta di una variazione dell'Anadyomene di cui quattro copie sono state trovate a Pompei. ${ }^{543}$ In pittura si incontra talvolta questo tipo, per esempio nella Casa di Pinarius Cerealis (cat. 182,1).

Alla sua sinistra si trova la statua di un pavone ceruleo con una coda in forma di ventaglio sopra una base bassa. Un esemplare in bronzo é stato trovato in VII 12,17 .

Pure a sinistra di Afrodite sta sopra un pilastro la statua gialla di Priapo itifallico, che volge lo sguardo sulla dea. Tali statuette non sj conoscono da Pompei, ma si puo pensare al Priapo grande di marmo trovato nella Casa dei Vettii. La combinazione Afrodite-Priapo si trova in una statuetta marmorea proveniente da I $11,6.545$

Il pittore ha voluto suggerire una nicchia piena di bric a brac in una parete quasi interamente bianca. La preferenza per Afrodite e Priapo si vede espressa anche nei falli collocati nella zona superiore della stessa parete e nella scena erotica sul pannello a destra della nicchia finta. Lo stile è quello dell'arte plebea.

540 CatLondon 1976, no. 209 (MN 5133); De Caro 1976, 219-225, fig. 30-34; Jashemski 1979, fig. 468; ibid. fig. 200 (da I 11, 6).

541 Dohrn 1965, 130-131, Taf. 56.2.

542 Cf. Centuripe: Libertini 1927, 58, tav. IV.

543 Jashemski 1979, fig. 197-198; Dwyer 1982, fig. 16, 79, 189, 190.

544 Dwyer 1982, fig. 70.

545 Jashemski 1979, fig. 200. 
Bibl Jashemskı 1979, 128, fig 202, Pompel 1748-1980, 160 no 15a, Repertorio 1981, 191

I 16,3

Cat 174 (1) Parcte sud zona mediand, IV stile

Sulla lesena ciestra iccanto alla porta che mette nell'atrio di fronte alla porta principale della casa, sorge un'erma bianca con una testa maschile Sembra un ritratto data la precisione del dettagli come le orecchie la corta acconciatura e la mancanza di una barbd La testa e incoronata da una benda l cul nastri pendono lungo le tempie Il fusto dell'elma tunziona da appoggio per un cerchio e una rame di palma

Forse si tratta del ritratto del padione di casa, ralligurato coms un vincitore di gare sportive (sl controntimo le nature morte con attributi sportivi) Erme a ritratio si trovano normalmente nell'atrio di fionte allentrata Se ne conoscono varı esempi 546 La pittura entra nell'ambito del ritrattı di vincitor che venivano spesso prodott in questa forma Il tipo e, viste le bende. quello clel cosidetto Anadvomenos Casparrı, di cul si conosce una copia a Schloss Erbach in Germania 547

La pittura non entra nella suddivisione di Mau ma deve essere datata nell'ultuma fase sulla base della tecnica pittorica

Bibl Repertorio 1981, 197

I $17,2-3$

Cat 175 (4) Parete nord, quadro centrale, III stule

Paesaggio sacro-idilliaco Un portico viene sorretto da cariatidi rosse, molto snelle a rese sommariamente All'interno del portico si alza sopra una base la statua rossa di una disinita non distinguibule

A destra dell'albero sı vede la statua rossa dı Priapo, raffigurato nell'itteglamento della chordosis Accanto "I sono tre statuctte schematsche, ov lamente rappresentantı un hekataion triforme

Bibl Bastet/De Vos 1979, 137, Repertor1o, 1981, 203

I 17, 4 Casa deglı archi

Cat 176 (1) Parete nord, zona mediana, IV stule

Pittura di giardıno, diviso in quattro partı da lesene rosse In ogni scomparto vi e una fontana bianca sorretta da anumal fantastıcı Le due esteriori vengono sorrette da centauri simmetricı, sedutı sulle zampe posteriorı, le due interne da sting, femminul alate viste di fronte 1 bacinı del centauri sono

546 De Franciscis 1951 (p e case di Caeclius Jucundus, Cornelius Rufus, Vesonius Primus, Citarista e Bancario a Pompei e la Casa dell'erma di bronzo a Ercolano)

547 Fittschen 1977, 9-11, Taf I, Bell I Informazione ottenuta du dott $R$. de Kind 
rettangoları, quelli delle sfingı rotond In ogni fontana zamplla dell'arqua I centaurı reggono una scodella nella mano al lato interno presso la spalla

Bıbl Jashemski 1979, 58-59, fig 95, Repertorio 1981, 203-204

II 2, 2-5 Casd di Octavius Quartio

Cat $177 / 1$ (b) Tutte le parntı, zona mediana, IV stue

Figure gialle di guerrierı nudi sı delinedno su campl rossı. Sorgono sopra piccolı dischı ovoidalı di colore verde Cinque delle sette figure originalı, tutte armate con elmo a pennacchio in testa, sono runaste conservate

Parete sud, campo est Il guerriero regge nella mano destra una lancia, l fianco simistro e coperto da una clamide e da uno scudo rotondo La gamba destra e di appoggio L'atteggiamento della figura e quella del Doriforo policleteo A una cintura attorno al torso e appeso un gladio

Parete ovest, (ampo sud Il guerriero guarda a sinistra e corrisponde all'Ares Borghese Il braccio sinistro e coperto da uno scudo e una clamide, l'altro braccio e in riposo lungo il fianco

Parete ovest, campo nord Il gucrriero poggid sulla gamba destra Non rappresenta un tipo noto dalld scultura Regge und lancia nella mano sinsstra, un gladio nella destra distesa verso ll basso L'avambraccio sinistro e coperto da un drappo Dietro la spalla si vede l'orlo di uno scudo circolare

Parete nord, campo ovest L'atteggiamento del guerriero e sumile al Doriforo policleteo Le gambe sono andate perdute Lungo il fianco sinistro si vedono uno scudo e una clamide Regge una lancia nella mano destra.

Parete nord, campo est Il guerriero poggia sulla gamba simstra. Sorregge con la destra l'orlo superiore di uno scudo rotondo che sta decanto a

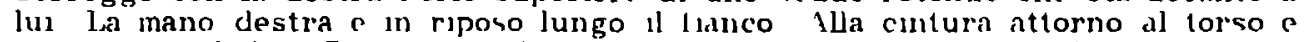
appeso un gladio Per questa tigura non si puo trovare un contronto preciso nelld scultura.

Nei prospetti che dividono I pannelli sulla parete est erme snelle $d_{1}$ colore gidlo funzionano da plastrı

Bibl Spinazzold 1953, I, 378, fig 427, Schefold 195i, 50-51, Repertorio $1981,213-214$

Cat 177/2. (d) Paretı nord e est, zona mediana, IV stıle

I campl sono separatı da prospett glall su fondo bianco L'edicola sulla parete est e occupata dalla $H^{d}$ Uftherme d, un satiro $E^{\prime}$ nudo, soltanto un panno ne copre le renı Regge un pratto nella sinistra e un canestro nella destra distesa Un ramicello e una corona. attributo priapel secondo Schefold, sono appesi al fusto

L'edicold sulla parete nord e sorretta da due $H^{\mathcal{L}}$ Lfthermen vestite di una tunica corta Reggono nella mano esterna un pratto, mentre l'altra e distesa per meta. Lo sguardo e rivolto verso l'esterno La figura di sinistra e andata perduta

Bibl Spinazzola 1953, I, 379, f1g 430, Schefold 1957, 51, Pomper 1748-1980, 130, fig 30 , Repertorio 1981, 214-215

Cat 177/3. (1, k, 1) Plastrı della pergola e parete nord, zona mediand, IV stule

l plastrı della pergola e le lesene fra i paradeısol sulla parete nord sono ornati - alternamente - con plastrı sormontatı da pigne, e con erme a testa maschile barbuta In modo semplice si suggerisce una pergola sorretta da talı 
sostegnı Non sono state trovate pergole dı legno o cannd con erme in guisa di pilastrı, ma si puo avere un'ummagine di tali costruzionı con le pitture in $J$ 17,2-3 (cat 175) e nella Casa di Achille (cat 2832)

Bibl Schefold 1957, J3, Repertorio 1981, 218

II 3,3 Casa della venere in conchiglia

Cat 178 (8) Paretı sud e est, zond mediana, IV stile

Rappresentazıonı di giardino fiancheggiano la tamosa immagine sulla parete sud Maschere policrome adornano l'orlo superjare. tagliato in torma triangolare, del telaso che circonda le prospetive a giardino Sono elementı comunl a portici, trovati in gran numero a Pompel 5

Davanti al cancello che. in un certo modo, separa 1 glardino dipinto dal glardino reale, sorge al lato sinistro ld statua bianca di Ares sopra un piedistallo quadrato La gamba destra e di appoggio Il dio e nudo a parte una clamide rossa distesa sopra le spalle In testa ha un elmo a pennacchio Regge nella destra alzata una lancia e nella sinistra lungo l flanco uno scudo

Corrisponde alle statue d'umpronta policletea senza copiare un tipo particolare Vella loro pubblicazione di un rlievo trovato a Maria Saal (Austria), Neutsch e Walde lo confrontano con l'Hermes di Andros nel Vuseo Nazionale di Atene con cui condivide lo stule classicistico del due secol prima e dopo Cristo Reutersward accenna l'umportanza della figura per la conoscenza della policroma parziale, che si siluppa specie nell'Ellenismo Forse dobbiamo, in questo caso, prendere in considerazione l'ambientazione in un giardino 1 marmo bianco si distingue plu facumente tra 1 cespugli e produce cosı un effetto migliore di quanto puo fare una statua policroma

Stritue di Ires nel contesto da un glas dino sono rasc visto che questa divinita non ba un nesso speciale con esso $Q$ ui il dio della guerra e stato scelto solo come compagno di Venere, presente nel quadro centrale In merito sı ricordi un passo in und lettera di Ciccrone, in cul esprime 1 suo disgusto per la presenzd di Ares in un ambiente pacifico come ll giatino Martis vero signum quo mihi pacis auctori? "Ma a quale scopo serve a me, fautore della pace, una statua di Marte" ") s49

Sulla parete est e raffigurato un altro giardino $\lambda e l$ centro $v$ e una fontana ovoidale ornata da tigure a rlievo di colore bianco $S_{1}$ distingue un tiaso di Ligure femminll alate, elemento conosciuto da rasi neoattici alcunı esempları del qualı sono statı recuperatı anche in Campanid 550

Bibl Schefold 1957, 53, Reutersward 1960, 190-191, frontesp , Grimal 1969, 233 n $5, \mathrm{pl} 29,31$, Peters 1971, 10, Andreae 1973, 175, I arbtat 23. Neutsch halde 1978 , $146 \mathrm{n} 41$. Ptretschner 1977, no 54 . Michel $1978,399-400$, 1 at 751 , Jashemsk 1979, 63-64, 128-129, hg 101-10s, 204, Von Hesberg 1981, 1140-1141 no 41a, Repertorio 181, 222-224

548 Dwyer 1981, Corswand 1981

549 Cicero Fam VII 232 ['na statua du Ares e stata trovata in un'edicola nel giardino della Casa deglı scienzıatı (Dwyer 1982, 118, $124 \mathrm{n}$ 2)

$550 \mathrm{P}$ e Jashemsh1 1979, fig 131, 480, 530,535 
II 4, 3 Praedia di Julıa Felix

Cat $179 / 1$ (24) Paretı est e ovest. zona superiore, IV stle (ora M\9059 $9061,9067-9070)$

Kappresentazion della vita quotidiana sul foro di Pompei delineate in modo realistico secondo lo stue dell'arte plebea Davanti al portico che circonda la pıazza si vedono statue equestri di colore glallo erette su basi rettangolari I cavalierı sono raltiguratı nell'atteggiamento dell'adlocutio con la mano destra alzata mentre 1 calall hanno una delle zampe antersor alzata Con ognı probabulita abbiamo a che tare con la situazione sul toro pi ima del 62 Dopo 1 terremoto tutte le statue vennero rimosse e portate in deposito, fra l'altro nel tempıo di Giove sullo stesso toro, prima dell'inızio del lavorl di restauro 551 Ion sı puo stablire se anche le pitture datino al periodo precedente ll terremoto

A Pompel e stata trovata una sola statua equestre mentre gli scar 1 borbonici a Ercolano ne hanno restituito un numero considerevole sia in bronzo che in marmo, in parte allo stato di frammento Rappresentano sempre lo stesso tipo In torma dipinta si contronti la statua nella Casa della caccia antica (cat $\left.245^{\prime} / 5\right)^{552}$

Bibl Schefold 1957, 54, Roques Maumont 1958, 85-86, Reutersward 1960, 132 n 326, Allroggen-Bedel 1979, 142, Dohl'Zanker 1979, 185, Repertor1o 1981,233

Cat $179 / 2$ (97) Tutte le paretı, zona mediana, IV stule (ora Louvre ins $P$ 4-9, 11-12)

Su uno sfondo giallo sı delinedno otto Muse e Apollo Citaredo sorgenti sopra mensole Le inensole recano epigrafi greche che indicano il nome e la funzione dolla Musa Le ligure sono rappresentate vive in color vivaci e sono caratterizzate come statue solo dalle mensole Per lo studio dell'iconografia dolle Muse l gruppo e di grande importanza Per la prima volta sembra stabilito un ordinamento delle figure secondo uno schema che limarra in uso durante l'Impero Certo, gia su coppe aretıne al trovano Muse con epigrafi, ma mal sl incontra un gruppo completo 553

Le igure non si lasciano confrontare con sculture di Muse e non rappresentano un del gruppl notı soltanto dalle font 1554

1) Apollo e seduto su dl un trono marrone Indossa un mantello verde che lascia scoperta la parte superiore del corpo Sulla testa ha una corona Con la destra regge una cetra di dunensionı considerevoli, l'altra mano e in riposo sulla lesta Al plastro a sinistra del dio e appoggiato un ramo d'olivo

2) Calliope Indossa un chrtone verde a un mantello dello stesso colore ma piu chraro Regge, dasanti al petto, con entrambe le manı un volumen Li gamba destra a dı appoggio

551 Cf Dohl/Zanker 1979 Sembra che questi lavor non si svolgessero su scala ampia poiche l'importanza della zona era diminuita a favore della Via dell'Abbondanza e della zona adiacente

$552 \mathrm{Cf}$ Roques Maumont 1958, Cavalli 1981, 27-57, 272, no 180, Adamo Muscettola 1982, 1Lroggen-Bedel 1983

553 Puccl 1981, 107, fig 4-5

554 Cf Pinhwart 1965, qui p.41. 
3) Clio e seduta su una seggiola, vestita di un chitone verde Nella destra me $\angle z o$ distesa regge un volumen aperto, con l'altra poggia sulla sedia Accanto al piedı si vede una capsa piena di volumina

4) Eiato poggia sulla gamba sinistra $E^{\prime}$ vestita di un chitone rosa senza manthe $e$ dı un mantello verde Con entrambı le manı regge, davant 1 al petto una piccola lira

5) Tersicore indosid un chitone rosa e un mantello verde La gamba destra e di appoggio Suona con la mano destra una cetra che regge nella sinistra lungo il tianco

6) Melpomene ha la gamba destra dı appoggio E' sestita dı una tunica verde e di due mantelli, iosa e blu lella sinistra mezzo distesa regge una maschera tragica, nel'altra, lungo il fianco, tsene und clava Questo attributo orramente immancable per Liacle e stato scelto a motio dell affinita di questo eroe con ll teatro in genere e per la relazione intima con le Muse in particolare

7) Talia poggid sulla gamba sinistra Indossa un chitone verde e un mantello giallo Della destra mezzo distesa regge una maschera comica, nell'altra, clavanti al petto, un pedum

8) Polmma poggia sulla gamba destra lndossa un chitone verde e un mantello dello stesso colore Presso le labbra tiene l'indice della destra nell'attitudine $d_{1}$ chi voglia parlare Con la sinistra solleva un lembo del mantello darant 1 al guembo

9) Lranıa e seduta su di una seggiola a gambe tese Indossa un chitone rosa e un mantello blu vella sinistra regge un globo, mentre con una bacchetta nella destra indica qualcosa su di esso

Bıbl Schetold 195 $\overline{\text {, }}$ j3 Pinkwart 196j, 79, Wegner 1966, 98-99, Bel 1 , Tran Tam Tinh 1971, 25-34 no 1-9, fig 1-9, Bastet 1974, 217, Abb 5. Allroggen-Bedel hammerer-Grothaus 1980, 201, Pompel 1748-1980, 58, $61 \mathrm{fig}$ 6, Repertorio 1981, 241

\section{6 Anfiteatro}

Cat 180 Davanzale attorno all'arena, IV stule +

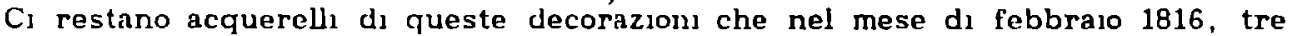
annı dopo la loro scoperta, andarono perdute Pannellı con scene di lotte gladiatorie e e di venationes e con imitazioni di incrostazioni marmorei sono separate da lesene rosse ornate con erme candelabri e Vittorie su globi di colore giallo Le Vittorie corrispondono al tipo della Victoria Romana sorgono sopra un globo, sono vestite di un ampio manto, reggono una corona e una palma 555

Le erme sono di due tıpı La maggior parte consiste di erme semplici a testa di atleta, incoronata con una benda o una corona mentre al rami del fusto sono appese altre corone, scudı sono statı post $H^{*}$ Ufthermen rappresentano satirı che reggono nell'una mano un pedum presso la spalla e nell'altra tesa verso il basso una situla La loro presenza nel contesto di un anfiteatro non e del tutto chiara L'insieme delle erme imita un Hermenzaun 55

\footnotetext{
555 Cf Holscher 1968 Lna statuetta di bronzo e stata trovata a Pompel (Dwyer 1979 fig 7 , Dwyer 1982 122, n 77)

556 Cf Wrede 1972, qui p g4).
} 
Su una delle scene a ludi gladiator la lotta viene flancheggiata da due $H^{*}$ ifthermen gialle rappresentanti Vittorie Reggono una palma nella sinistra e una corona nella destra stesa Scudi stanno appoggiatı contro 1 fustı

von si limita a Pompes l'uso di clecorare l'interno di un anfiteatro con rappresentazion che hanno stretta relazione con la tunzione delledificio A Lapud e a Lecce glı anfitatri posseggono rlievi con quesio toma, a Lecce originariamente nella stessa posizione delle nostre pitture Gli antiteatrı di Ptolemaide, Corinto a I lippi avevano decorazioni dipinte, ormal perdute 557

Bibl Helbig 1j1j, 1j19, Niccolini III, tav IIl RP 288 1, Spano 19j3, 43-65, tig 7-14, Schetold 1957, 54, Mchel 1978, 395, I'omper 1748-1980, 36-37, fig 4-6 183-185 no. ADS 81-89, 196-199, fig 1-9

III 3, 7 (?) o nord

Cat 181/1 (protıro) Parete ?, quadro centrale, IV stule (ora MN 9154)

Marsia e seduto su di un blocco e regge in entrambe le mani un flauto Suona su quello tenuto nella destra Latteggiamento e simile a quello del Marsia della cosiddetta Basilica di Ercolano (cat 019) e potrebbe per tale ragione, rappresentare la statua del Saepta a Roma Manca ll tanciullo Olumpo

Bibl Helbig 224, RP 32 3, Elı 1932, no 185, Schefold 195i, 55

Cat 181/2 (a sin del protiro) Parete?, quadro centrale, IV stue (ora MN 9171)

In Eros e seduto su di un blocco e si toglıe una spina dal piede L'atteggiamento e quello dello Spinario, di cul la mighore copia si trova nes Musel Capitolinı 55 Nel nostro caso la figura viva e modellata secondo un típo statuario

Bıbl Helbig 629, RP 692 ; Elıa 1932, no 72, Schefold 1957, 55

III 4, 4 Casa di Pinarius Cerealis

Cat $182 / 1$ (a) Tutte le paretı, zona mediana, IV stule

Scaenae frontes con tems teatralı Alcune delle figure vive sono modellate secondo tipı statuari Inoltre, le architetture sono ornate con acroteri plastici

Parete nord Itigenia in Tauride Nel tempio, dietro la sacerdotessa, si vede la statua gialla di Artemide, vestita du un chitone senza maniche Regge nelle mani distese delle traccole e corrisponde all'Ecate Lo stule della figura e arcaistico

- Ifigenia regge un hekataion triforme nella mano destra, ovviamente l'ummagine di culto La parte inferiore consiste di un fusto, mentre quella superiore e composta da tre corpl con sel braccia reggenti cldscuna una fiaccola L'hehataion di colore giallo e modellato probabilmente in Ingno,

557 Cf Corchia 1980, Stullwell 1952, 86-94, fig 76-83, Collart 1937, 383-384, pl LXVII 13 Cf anche l'anfiteatro di Tarragona (Abad Casal 1982, $250-251$ no $311,337-338$, fig 412

55. Fuchs 1958, Bastet 1966, Zanher 1971, 69-74 
coperto con lamine d'oro o bronzo Il tipo e noto dalla scultura marmorea 559

Centaurı tungono da acroter sopra l'aula regia Sono sedut s sulle zampe posterior 1 e reggono nella mano minerna una bacchetta, noll'altra un platto piesso la spalla Si puo pensare alle statuette marmoree irotate a (Oplontis 560 Inoltre sfing 1 femmuli alate ravvivano la trabeazıone a smistra a a destra dell'aula regia

La sacerdotessa a sınıstra dı Ifigenıa assomiglia alla Fanciulla di Anzıo nel Museo Jazionale Romano, come la baccante nella Vlla del mister (cat 306 2)

Parete est Attis e raffigurato nell'atteggiamento consueto, noto dai trapezophorol pompeiani 561

Anche su questa parete e sulla parete ovest si incontrano acroter a forma d) centaurd

Palete sud A destra della rinestra si vede la sagoma di un'Afrodite, raftigurata secondo ll tipo dell'Anadyomene

Bibl Curtius 1947, 106-120, Spmazzola 1953, II, 693-707, fig 661-671, Schefold 1957 59, Bieber 1961a 231 fig 774, Schefold 1962, 113-115, Taf 78-79 Bastet 1974, 210, Abb 2, Schmıdt-Colınet 1977, 147, 272 no P14, Gigante 1979, 124, Repertorio 1981, 252-253, Moormann 1983b, $76-84$, fig $1-6$

Cat $182 / 2$ (b) Tutte le paretı zona superiore, IV stile

Le edicole centralı sono occupate da figure stantı, delle quals non e chraso se siano statue o meno Sulla parete est bl lede una figura lemmmle di colore rosso, vestita di un peplo Regge una corona nella destra tesa in diantı, nella sinistra una bacchetta

Sulla parete nord si trova un Dioniso nudo con le spalle coperte da una clamide Regge nella destra stesa una siringa e nella sinistra alıata un tirso la gamba destra e di appoggio

Sulla parete ovest al distingue la figura di un Eros nudo con una clamide sopra le spalle Regge una lancia corta o und freccia con entrambe le manı darantı al petto La gamba destra e di appoggio

Bibl Spinazzola 1953, 1, 708-709, Schefold 1957, 60, Repertorio 1981, $253-255$

V 1, 7 Casa del toro

Cat 183 (b) Parete?, zona mediana, II stule, tase Ic o Ila +

Centauri di colore bronzeo sostengono la trabeazione della zona superiore, e funzionano contemporaneamente da acroterı dell'edicola centrale Le fjgure. delle qualı non sı possiede un'lllustrazione, vennero gludicate wenig $k$ unstlich da Beven Lo stesso autore data la decorazione nella tase Ila nel suo primo volume, nella fase Ic nel suo secondo volume della Wanddekoration, aggiungendo all'ultimo giudizio l'osseriazione che le figure sono molto snelle e allungate

Bibl Yau 1882, 145, 252-253, Beyen 1938, 107, 114, Schelold 195i, 62, Beyen 1960, 75

\footnotetext{
559 Cf Kraus 1960

560 De Caro 1976, 198-219, fig 5-21, Jashemskı 1979, fig 466

561 Cf Tran Tam Tinh 1975
} 
V 1,15

Cat. 184. (d) Parete?, quadro centrale; IV stile +

Paesaggio con due cervi, di cui non si conosce un'illustrazione. Sullo sfondo sorge un'erma di Priapo.

Bibl.: A. Mau. BdI 1877, 134; Sogliano 720; Herter 1932, 159 no. 191; Schefold 1957, 63.

$\mathrm{V} 1,18$ Casa degli epigrammi

Cat. 185/1. (k) Parete est, zoccolo; IV stile

Rappresentazione di giardino su sfondo rosso. Al centro si dolinea la statur bianca di una divinjtà lluviale maschile. L'uomo è sdraiato sul lato sinistro, in appoggio sul gomito sopra una brocca dalla quale esce dellacqua. Regge nella destra alzata un cantaro. Un drappo copre la parte inferiore del corpo. Segue l'iconografia tradizionale delle divinita fluviali, esempi clelle quali si trovano nella Casa delle Vestali(cat. 198/3), nella Casa di Romolo e Remo (cat. 251) e nelle Terme stabiane (cat. 231/1). Il colore e il modello tolgono ognj dubbio sul carattere glittico della figura, ma Michel assume la presenza viva di Sileno nel giardino come rappresentante del tiaso dionisiaco e conseguentemente come espressione del dionysische Diesseitsgl ${ }^{\circ}$ ick nel giardino. sécondo l'interpretazione da lei sostenuta dei motivi dionisiaci nella rappresentazione artistica.

Bibl.: Presuhn 1878, pl. I-II; Sogliano 170: Schefold 1957, 66; Grinal 1969, 445-446 no. 6; Michel 1978, 397-398, Taf. 74.2.

Cat. 185/2. (y) Pareti ovest, nord e est, zona mediana e superiore; ll stile, fase IIa

Le scaenae frontes con nicchie, i pinakes nelle edicole centrali e gli atlanti posseggono molti elementi scultorei. Purtroppo la decorazione $\dot{e}$ in pessimo stato di conservazione.

Parete ovest. Nella nicchia di destra sorge, sopra una base rettangolare di colore verde, la statua policroma di una tigura temminile alata. Poggia sulla gamba sinistra. Indossa un chitone giallo-viola di cui sorregge un lembo con entrambe le mani davanti al grembo in modo da lasciare scoperte le gambe. In questo lembo si vedono frutti. In testa ha una corona d'uva e foglie di vite. Questi ultimi attributi, piú i frutti e il colore della veste caratterizzano la figura come una Stagione, cioè l'autunno. Solo le ali costituiscono una particolarità, dato che si trovano normalmente in rappresentazioni di Stagioni a partire dal secondo secolo d.C. ${ }^{562}$ Quindi un'interpretazione della figura come Abbondanza, Pace o Flora non è da escludere completamente. ${ }^{563}$

Parete nord. Nell'edicola centrale si trova un pinax raffigurante Omero e i pescatori. Omero è seduto contro una colonna sormontata dalla statua gialla di Poseidon. Solo le gambe, di cui la destra è di appoggio, sono rimaste visibili. Regge un timone nella sinistra presso la spalla e una patera nella sinistra tesa in basso. La figura segue in modo libero il modello lisippeo che si incontra

562 Hanfmann 1951, I, 136. Cf. Wrede 1982, 5, 17-18, Taf. 12.2.

563 Cf. Hanfmann 1951, II, 139. 
anche nella Casa dell'ara massuma

Nel campo destro si vede un pinax raffigurante del pescator che dedicano le loro reti a Pan La divinita eretta sopra una base rotonda, e rappresentata secondo il tipo dell'aposkopon, conosciuto dallo studio di Inez Jucker Anche nella Casa di 11 Lucretius Fronto (cat 195 3) incontriamo una statua dipinta di questo tipo Nella Casa di $M$ Lucretius e stata trovata una statuetta di marmo 564

Parete est Questa parete, la meglio conservata, c importante soprattutio per la zona supeisore Nell'edicola contrale della zond mediana si vede un paesaggio sacro-idlliaco nel centro del quale e la statua gialla di frianna sopra und base rotonda L'amante $d_{1}$ Dioniso siede in posa abbancionata all indietro su di un bloc co Regge nella sinistra un tirso, sotto ll braccio un timpano e la destra e in riposo sopra la testa $t_{1}$ suol piedi si vede una pantera La figura e modellata conforme al tipi ellenistic

velle nicchie a sinistra e a destra sono sopra basi rettangolar in verde imitanti lamine di bronzo statue in posizione sumle a quolla sulla parete ovest A sinistra si distingue una figura $1 \mathrm{cmmin} l e$ alata vestita di un chitone senza manche Joggia uulld gamba smistra Suona una celid nolld sinstra con la mano destra Sembra che avanzl verso clestra La cetra permetlerebbe un'interpretazione della tigura come Musa se non vi tossero le alı

Vell'dltra nicchia si distingue una ligura femminle vestita di un chitone rosso e di una stola bianca In testa ha una corona il peso del corpo poggia sulla gamba destra la mano destra e coperta dal mantello, e la sinistra distesa a meta regge un oggetto rotondo Se questo e un globo, si puo pensare a Lianid Di all non 11 sono tiacce

Vella zond superiore si vedono figure maschilı alate che fungono contemporaneamente da acroterı per l'edicola centrale e da atlantı per l'orlo superiore della decorazione Inclossano una tunica corta La mano esterna sostiene l'architrave, mentre l'altra e in riposo lungo ll tianco La posizione e simule a quella delle tigure nel frigidario della Casa del criptoportsco (cat 148/3) Il colore e giallo Anche sulle altre paretı talı figure devono avere ravvirata la zond superiore

Bibl Jeutsch 195j Schefold 1956, 223-224, Schefold 19ji, 65-66, Beyen $1958,351,4 b b 4$, Beven 1960, 199-233, Abb 85-91, Reutersward 1960,132 n 327, Schetold 1962, 4s-47, Taf 25, Peters 1963, 26-27, 64 Schmidt-Colinet 1977146267 no P4. Piretschner 1977, no 36 . 71-72, Gigante 1979, 7175 , Sulberberg 1981 , no 12, tig 10. Leach $1982,158-159$ fig 8 , Repertor 10 1983,14-1j

V 1, 26 Casa di L Cacclius Jucundus

Cat 186/1 (1) Pareto nord, quadro centrale, III stule (ora $M N$ 111439)

lfigenia in Tauride Davant al tempio sl trova un tavolino con un rassoio rosso a due anse Su di esso s] distingue una statuetta di torma schematica raffigurante lo xoanon di Artemide Il vassoso sembra adibito per $\downarrow$ l trasporto di questa statua sacra Lo s'incontra anche sul pinax con lo stesso tema nella Casa del Vettu (cat 2179)

Bibl Sogliano 538, RP 1695 , Herrmann I, 118, Phuppart 192518 no 25. Elıa 1932, no 73 , Schefold 1957 , 66, Dexter 1975, 119-120, fig 26, Repertor1o 198320

\footnotetext{
564 Dwyer 1982, [1g 43
} 
Cat. 186/2. (1) Parete sud, zona mediana; IV stile +

Sulla lesena rossa attorno alla rappresentazione di un paradeisos si delineano statue di ninfe che reggono un bacino con entrambe le manj davanti al grembo. Un mantello copre la parte inferiore del corpo. Si tratta del tipo normale derivato dalla Venere Landolina.

Sopra il paradejsos si vede un iregjo con una veduta di mare. Fra due navi sorge un'isoletta con la statua bronzea di Posejdon. Le descrizioni non permettono un'analisi del tipo.

Bibl.: A. Mau, lidl 1876, 231-232; llelbig 1580: Sogliano 477, 700 (o 6933); RP 379. 2; Schefold 1957, 68; Grimal 1969, 426 no. 7; Jashemski 1979, 71-72; De Vos 1979, 92; Repertorio 1983, 22-23. 8; De Vos 1979, 90; Repertorio $1983,67$.

V 2, d (Mau 9)

Cat. 187. (m) Parete est, quadro centrale; IV stile +

Afrodite solleva un lembo del suo mantello con la sinistra inentre con la destra poggia su di un pilastro. Alla sua sinistra sorge un'erma di Priapo. Il torso é coperto da un mantello. Il dio ha il cranio calvo, ornato con una corona, e una barba lunga. Lo sguardo è volto verso Afrodite.

L'Afrodite rappresenta una libera copia del tipo Frejus. IJ motivo dell'appoggio al pilastrino sembra derivato dall'Urania o dall"Afrodite nel giardino' di Alcamene. S. Settis accenna che il motivo viene adoperato in tutti i periodi dell'arte antica e lo considera come ripetizione di questo tipo, con le infinite varianti possibili. 565

Bibl.: Schefold 1957, 74; Repertorio 1983, 31.

$\mathrm{V} 2$, g (Mau C)

Cat. 188. (h) Parete est, quadro centrale: III stile +

Paesaggio sacro-idilliaco. Sopra una base rettangolare vi è una colonna sormontata dalla statua di Eracle. L'eroe, reso in giallo, è nudo e poggia sulla gamba destra. Regge una patera o uno scifo nella destra tesa e con la sinistra una clava sopra la spalla. L'Eracle è raffigurato secondo il tipo del bibax, popolare dall'Ellenismo in poi.

Sulla base a destra si trova la statua gialla di una figura vista di profilo. La figura - non si puó distinguere la veste e quindi non è possibile stabilire il sesso - regge con la sinistra, davanti al petto, una patera, mentre l'altra mano non è visibile.

Ancora a destra si vede la statua gialla di una figura maschile vestita di una tunica. La gamba destra è di appoggio. Essa regge una lancia nella destra, mentre la sinistra $\dot{e}$ in riposo lungo il fianco. Le due statue presso la colonna sono offerte votive.

Bibl.: A. Mau, RM 8(1893) 10-11, Abb. 10; RP 387.6: Schefold 1957, 75 (vano a);Schefold 1962, 90. Taf. 54.1; Pfretschner 1977, no. 65; Repertorio 1983, 32.

565 Settis 1966,16-17. Per la statua attribuita a Alcamene Delivorrias 1968; Lohmann 1979,29 n. 208. 
V 2, i (Mau E) Casa delle nozze d'argento

Cat. 189\%1. (q) Pareti est e ovest, zona mediana; IV stile

Le prospettive rosse accanto aj campi laterali contengono atlanti snelli. Carlatidi vestite di un peplo. rese in modo lineare, occupano il centro delle architetture. Reggono patere in tutte e due le mani. Solo sulla parete est si vede un satiro in guisa di sostegno di un candelabro accanto ai suddetti candelabri. Il corpo si leva da un calice d'acanto.

Bibl.: Schefold 1957, 77-78; Schefold 1962, 131, Taf., 121.1; Repertorı 1983, $42-44$

Cat. 189/2. (y) Pareti est e ovest, zona mediana; II stile, fase Ila

La cornice della zona mediana consiste di due serie di mensole fra le quali si distinguono dei centauri miniaturistici di colore giallo. Reggono la serie di mensole superiore con la testa o con una mano. In ogni intercolunnio si trovano cinque figure. L'ombra gettata e stata delineata con tinte scure. Tali costruzioni vengono menzionate da Vitruvio (VI 5,5 ; cf. p. 22) e sono state studiate da Von Hesberg.

Bibl.: Beven 1938, 249-250, 334, Abb. 2, 3, 183; Schefold 1957, 78, Beyen 1 (ง) $43-71$, Abb. 5-6; Schefold 1962, 43-44, Taf 5.2; Schmidt-Colmet $1977,146,266$ no. P2; Pompei 1748-1980, 181 no.47, B1; Von Hesberg 1980, 134; Repertorio 1983, 49.

Cat. 189'3. (5) Parete ovest, zona mediana; IV stile +

Ina vignetta su campo rosso rappresenta un Eracle seduto su di un fusto di colonna. La mano sinistra sorregge il mento, mentre la destra e in riposo sul grembo. L'atteggiamento è quello dell'Eracle di Taranto, l'ultimo lavoro di Lisippo. ${ }^{566}$ Altre copie dipinte di questa opera, tante volte copiate in forma di statuette di bronzo, si trovano nella Casa del Menandro (cat. 163/5) e nella Casa di Epidio Sabino (cat. 277/1).

Bibl.:G. Spano, NSc 1910, 324-325, fig. 4; Schefold 195̄, 79; Dorig 1957, 32; Beyen 160, 48-56, Taf. 3.6; Moreno 1981, 182, fig. 18; Repertorio $1983,53$.

V 2, 1 Casa della regina Margherita

Cat. 190. (o) Parete ovest, quadro centrale; IV stile +

In un'edicola semplice sorge la statua policroma di Leda sopra una base bigia. Leda indossa, sopra il dorso e attorno alla testa, un mantello rosso, un lembo del quale sorregge con la mano destra. ${ }^{567}$ Con la sinsstra abbraccia il cigno che si preme al lato sinistro. L'uccello stende il suo capo fin al collo di Leda. Il peso del corpo poggia sulla gamba destra.

La statua rappresenta una copia summetrica della famosa Leda di Tumoteo, un'opera d'arte conosciuta da ben 28 copie romane. Non solo questa pittura ma anche pinakes nella Casa della caccia antica (cat. 245; 1) e nella Casa di

566 Moreno 1978.

567 Il mantello, sorretto in velificatio, mette l'accento sulla vista frontale della statua. Cf. Borbein 1973, 109. 
Giuseppe II (cat 269/4) sono ispiratı da questo gruppo, solo in questo caso il gruppo dipinto viene caratterizzato come statua attraverso la base E' strano che Herrmann non attribuisca un carattere plastico a questo gruppo opponendolo al gruppi vivi sui pinakes 560

Bibl Gusman 1890. Taf 1-2r (a color 1 ), Herrmann I, 45-46 Abb 13, RP 174 , Schetold 1957, 69, Moreau 1960, Taf 24, Schefold 1962, 125, Taf 89, Rieche 1978, 51, Repertorio 198355

$V$ 2, 4 Casa del triclinuo

Cat $191 / 1$ (r) Parete ovest quadro centrale, IV stule (ora MN 120030)

Scena di banchetto A destra in avantı si vede la statua ierde di un fanciullo sopra una base bassa irıangolare Poggia sulla gamba destra e regge nelle manı tese in avantı un vassoio con piccolı vası d'argento Ora solo le gambe sono vagamente ruconoscibli

La statua riproduce un efebo classico del quinto secolo a $\mathrm{C}$, conosciuto attraverso numerose copıc dell'epoca augustea e dedrianea Nella Casa dell'etebo - stata trovatd una tale statud riadattata come lychnouchos (lampadoloro) Inche da Juttich (Gormania) si conosce una tale statud, ors a Berlmo, mentre und higura trovata a Antequera (l'antica Anticaria in Spagna) puo essere ricostruita allo stesso modo $D_{1}$ recente e stata trovata und copia nella Casa di Julius Polvbius 569

Bibl A Sogliano NSc 1884 49-50, A Vau BdI 1885, 243, pl 11, Herrmann II. $22 \mathrm{Abb} 1$ Taf 212, Amelung 1927, 142-143, Abb 6-7, Schefold 1957, 71, Reuter 5ward 1960, 136, Garcia y Bellido 1969, 73, Repertorio 1983,60

Cat $191 / 2$ (u) Parete sud, zona mediana III stile + Paesaggio sacro-idullaco sul campo destro Presso un albero sacro si vede la statua di Artemide-Ecate che regge nelle mani tese in avantı una flaccola

Bibl Schelold 1957, 71 Schefold 1962 Taf 58, De Vos 1979, 39 Bastet De Vos 197991 , Repertorio 1983, 61

$\mathrm{V} 2,9-12$

Cat 192,1 (n) Parete nord, quadro centrale, III stile +

Pasifae nella bottega di Dedalo Lo scultore e la principessa stanno davantı ad una statua bianca di una Vittoria alata, vista di prof Lo verso sinıstra $E^{\prime}$ vestita di un mantello lungo Nella destra regge un oggetto non leggibule sul disegno di Discanno Mau lo descrive come uno scettro mentre interpreta la figura come Aura La statua non svolge un ruolo nella storia ma fa parte dell'ambiente

568 Sulla statua Schlorb 1965, 51-56, Taf 16, Rseche 1978

569 (Porta del Vesur 10) Jashemski 1979, fig 184 (Berlino) Wolters 1928, (Antequera e Volublis) Garcia $v$ Bellido 1969, (J Polybius) G Torcó Sciarell, CMGr 18 (1978) 267-268 Per la terminologia of M Mannı, Lychnuchus, in Dizionario epigrafico di antichita romane, IV tasc $72(1983)$ 
Bibl. A Mau, RM 5 (1890) 262, Lippold 1951, Taf 20 111, Schefold 1957. 71-72, Van der Meer 1977, 65, Repertorjo 1983, 63

Cat. 1922 (q) Paretı sud e ovest, quadro centrale, IIl stule (ora MN 924J) $+$

Parete sud Paesaggio sacro-ıddliaco. Tre uomini tanno un'offerta alla statua $d_{1}$ una divinita femminile vestita di un mantello lungo. Regge una bacchetta o una lancia nella sinistra alzata e und patera nella destra stesa in giu In testa ha un modjo che potrebbe caratterizzare la figura come Iside 4 destra si vede una $H^{d}$ lftherme con la parte superiore del corpo uguale a quella di Isıde Quest'ultima e und statua votıva Daw'son interpreto la scena come la storia di Ippolito, la statua destra rappresenterebbe Afrodite

Parete ovest 570 Dedalo e Icaro. In primo piano su uno scoglio $v$ e la statua di Poseidon, seduto su una roccia che regge nella mano destra una lancia. E' sumle ai Poseidones nelle altre rappresentazion (p cat. 161/2). Bibl.. A Mau, RM 5(1890) 263-266. RP 387 j, 184 4, Jdwson 1944, 80 89, pl 7-8, Dawson 1950, 300-303; Schetold 1957, 72, Beyen 1960, 443-444, Schctold 1962, 84: Pelers 1963, 82-84, fig 68, 70, Tran Tam Tunh 1964, 126 no 9 , Von Blanckenhagen 1968, 110-111, pl. 361 ; Berard/Horstetter 1979, pl. 78, t1g 9; Repertor10 1983.64.

$\mathrm{V} 2,15$

Cat 193 (p) Parete ovest, zona mediana: IV stile +

Una rappresentazione $d_{1}$ un grardino $e$ delimitata da due fasce rosse $S u$ quella di sinistra si delinea un'erma, la cul testa era gia andata perduta prima della scoperta forse nel giardino stesso si trovava una stmge

Bıbl. A Mau, RM 9(1894) 52, Schefold 1957, 73; Grumal 1969, 446-447.

$\mathrm{V} 3,12$

Cat. 194. (2) Parete est, zona mediana, IV stile +

Una decorazione non compluta nel 79 avrebbe contenuto sccondo la descrizione dı Schefold la statua bianca di un cavallo, visto di profilo la composizione e il contesto sono 1 gnoti. La fotografia citata da questo autore non mostra nessun traccio di una statua del genere. In ogni modo sarebbe l'unica rappresentazıone dl una statua di questo anumale, a parte le statue equestru nel Praedia di Julıa Fels (cat. 179/1) e nella Casd della cacesa antica (cat. $245 / 4)$.

Bıbl.. Schefold 1957, 83; De Vos 1977, 42, pl. 43 fig 58 (fotografia D IIR 56 1276), Repertorio 1983, 81

570 Nonostante la collocazıone menzıonata nella biblografia, mı sembra verosimile che il quadro provenga da (n), come parte di una serie di scene del ciclo dedalico 
V 4, a Casd dı $M$ Lucretsus Fronto

Cat 195/1 (j) Parcte ovest, quadro centrale e zona superiore, III itule Il quadro centrale raffigura Teseo e Arianna nel labirinto Teseo e stato modellato secondo l'ipollo Licio con la mano destra in riposo sulla testa $E^{\prime}$ sumile all'Apollo in 1 12, 3 e torse e stato dipinto dallo stesso pittore (cat 169)

L'edicola centrale della zona mediana sormontata da un'altra edicola al di sopra della quale due cariatidi sostengono ld trabedzione superiore Sono vestite di un chitone verde senza manıche Lo stule e classico e la posizione e summetrica

Anche la parete sud ha avuto una decorazione sumle, sl dinstinguono ancora tracce della caridtide sul lato sinistro

Bibl A Mau, RM 16 (1901) 344, fig 5, Herrmann 1 221-222, Taf 159 Schefold 1957, 84, Bastet De Vos 1979, 65-66, tav AX1Y 51 . Repertorio 1983, 85, Bragantinı Parise Badonı 1984, 124, fig 17-20

Cat 1952 (7) Pareti sud e nord, zone inediana e superiore, III stle Nel campl latelalı al delineano quadrettı con v lle marme che contengono strisce bianche, for se statue solo in quello a sinistra sulla parete sud si distinguono erme bianche che tanno parte di un'Hermenzaun 571

Vella zona huperiore due erme fanno parte del prospettı centrali I fusti, al qualı sono sospesı corone e bende, sono bianchi, le teste gralle Esse sono di stle arcaizzante e rappresentano Dionso

Bibl Rostowzew 1911, 108 109, Taf 6 2 RP 397 1, Herrmann I, 226 Tat 163, Spinazzola 19j3, 858, fig 859, Schefold 19j7, 84, Schefold 1962, 71-72, Taf 102148 , 50, Peters 1963, 115, Lorenz 1965, 39-40. Bastet De Vos 1979, 66-67. Repertorio 1983, 87-89

Cat 195/3 (10) Parctı nord e est, 7ona mediana, It stule

Sulle lesene fra 1 pirradessol si delineano statue bianche sopra bası rerdi Sulla parete nord si vedono tre ninfe sumli che reggono daianti al grembo. con entrambe le manı un bacino le gambe sono coperte da un mantello Solo la figura centrale e rimasta conservata discretamente

La parete est ha una sola lesena lin satiro danzante si regge sulla gamba destra, quella sinistra e alzata Sopra la spalla sinıstra ha una clamide La mano destra e sopra la testa l'altra in riposo lungo ll fianco ton si tratta di una variante del Fauno della Casa del fauno, come sostiene il Sogliano ina di un aposkopon, simule alla statuetta marmorea trovata nella Casd $d_{1} M$ Lucretius 572

Bıbl A Mau, RM 16 (1901) 356, A Sogliano, ASc 1901, 165, fig 15-16, RP $123 \mathrm{~b}$, Schefold 1957, 87, Jucker 1957, 70, Jashemsk1 1979, 71, Repertor 10 1983, 90

$\mathrm{V} 4, \mathrm{~b}$

Cat 196 (K) Paretı est e sud, zona mediana, IV stile +

\footnotetext{
571 Cf Wrede 1972 , qui $p$ 24)

572 Dwyer 1982, [ig 43a-b Per il tipo Jucher 1957
} 
Rappresentazione dı giardino Su entrambe le paretı si trova una fontana bianca sorretta da una stinge teminule alata

Bıbl A Sogliano, $\lambda S c 190 j$ 135, Grumal 1943, 483-484, tig 33, Scheiold 1957 88. Grimal 1969, 447 no 9, tig 38 (pianta), Jashemshı 1979, 59, Repertorio 1983,94

$V$ j 3 Caserma des gladiator

Cat $197 / 1$ (d) Portico est, pluteo interno, IV stue

Sul primo pluteo dd nord sı trova un paesaggio sacro-ıdıllaco quası lleggibile Secondo Mau qui si trovara la statua di una tigura temminule munita di una fiaccola in entrambe le manı Un'unterpretazione come Ecate e la piu plausible

Sul quarto pluteo da nord si trova un paesaggio sacro-idلlidco svanito In un portico con und nicchia si vede una statua dorata In primo piano sarebbe stato visible, secondo Mau, la statua di un atleta nell'atteggiamento del Doriforo di Policleto

Bibl A Sogluano, NSc 1899, 350, fig 10, A Mau RII 16 (1901) 297 299, 300 , schefold 1957 89, Schefold 1062, 148-149, Taf 148 1, Repertorio 1983,99

Cat 197'2 (n, anticamera) Paretı sud e est zona mediana, II stule tase IIb Sopra una fascia che divide la zona mediana dallo zoccolo sorgono $H^{\text {úfthermen }}$ bianche femminll Esse reggono ghirlande con entrambe to mani Sulla parete est una ha I capellı rossı e anellı glalli attorno alle biaccia Le due figurc sulla parete sud hanno un drappo sulla testa, quella di sinistra e andata perduta per la maggior parte

Le tigure entrano nelle serie della Casa del criptoportico (cat 148 1-!), della Casa del Menandro (cat 163/4) e della Casa di Caesjus Blandus (cat 233,2) sono pero piu stlizzate e hanno perso un'ambientazione aschitetionica cosicche sembrano figure qualsıası messe 11 fla davanti ad una parete cleca

Bibl Schefold 1957, 90 Beven 1960, 240-241, Abb 256-266, Schefold 1962, 56-57, Taf 36, Repertorio 1983, 101

Cat 197/3 (n) Parpti nord, est e ovest, quadro contrale e zona superiore, III stule

Il quddro centrale della parete nord rappresenta un paesaggio sacro-idıllaco Su una base vi e la statua di una figura femminule chr indossa un vestito lungo Al piedistallo e appoggiata un'erma di Priapo Tutto e stato dipinto in modo scheinatico

La zona superiore delle paretı est e ovest contiene ilgure femminil sedute e stanti sopra bası tantastıche o su element, vegetalı sono policrome e rese in modo ivace, I affiguranti menadi del tiaso bacchico Le figure su ogm parete sono simmetriche sulla parete est si vedono da sinistra a destra le seguenti figure

1) Donna con le gambe unite, vista di profulo e rivolta verso sinistra Indossa un peplo e un mantello con l lembı divaricat cetra lungo il fianco

2) Donna inginocchiata su di una mensola che sorge sopra una colonna snella, rivolta verso destra e vista $d_{1}$ profilo Indossa un peplo e un mantello Regge delle bende in entrambe le manı distese in avantı 
3) Donna vestita di un peplo sta sorge sopra una base rotonda ed e vista di fronte Ha le braccia distese e regge esil giralı

4) Donna in simmetria rispetto alla figura 2

Von possono essere messe in reldzione con delle statue L'acroterio di una stele luneraria attira del qual to secolo a $C$, provensente dal hrrameitos ad Itene $e$ ora a Berlino, ha un acroterio con tralcs sul qualı sono sedute due donne in posizione simmetrica 573 Anche sul rillevi neoattici sl vede ripetuto il tema della donna seduta su un tralcio 574 In forma di pittura possiamo rumancise alle tıguie nella Vlla umperiale (cat 3053 ) e quelle della Villa di larano (cat 010 1)

Bibl Schetold 1957, 89-90, Schetold 1962, 57, Taf $i 1,36-37$, Bastet De Vos 1979, 31-32, tav IV 7 , Pompel $1748-1980,58-59$, fig 1-3, Repertorio 1983, 102-103

VI 1,7 Casa delle Vestalı

Cat $198 / 1 \quad$ (39)Tutte le paretı, zoccolo e zona mediana, IV stle +

Le colonne della zona mediana sono sorrette da stingl alate temminulı accosciate di colore bianco La plasticita di queste figure e delle colonne fa pensare a costruzionı sunul di II stile per esempio sull'Insula occidentalis (cat 225 2), ma senza dubbıo si tratta dı un'umitazıone dı IV stıle

Suı pannell fra le stingı sı vedono appliques gialle, composte da protomı dı anumalı fantasticı che escono da calicı vegetalı Von imitano esempı esistentı, ma possono essere confiontatı con protomi di bronzo, ratfigurantı tor $e_{5}$ prore di navi, trovati nella Casa del citarista e nella Casa di Obellus Firmus 5 /s

l campı della zona mediana sono ornatı con guerrierı nudi armatı dı una lancia e un elmo che stanno sopra mensole Sulle spalle hanno una clamide Entrano nella serıe di guerrierı modellatı secondo lipı classici, specialmente quellı policletes ( $\mathrm{cf} p$ 43)

Bibl Helbig 1836, Beven 1938, Abb 114, Spinazzola 1953, II, 528-529, fig 586-587. Schefold 1957, 91 (vano d), Beyen 1960, Abb 269, Schefold 1962 , 123-124, Taf 109, Repertorio 1983, 108

Cat $198 / 2$ (e) Par ete ? quadro centrale. III stule +

Medea e le figlie di Pelia Medea regge nella destra la statua lignea di Artemide che non puo essere descritta nel dettagli per mancanza di un'llustrazione della decorazione, ora perduta

Bıbl Soghano 554. Schefold 1957, 91.

Cat 198/3 (h) Parete?, zoccolo, IV stule (ora Louvre P 2)

Davanti ad un campo bianco si delineano due ninfe acquatiche e un Sleno sdraiato in guisa di liume Tutte e tre le figure sono state rappresentate come esser vivi in colori naturalı, ma non sono inseriti in un ambiente per loro reale, cloe un giardino o un ninfeo Una striscia sotto 1 pledi indica modestamente la spazialita. Meravigha il fatto che 1 bacinı delle ninfe e la

573 Blumel 1966, 46-47, 4bb 72.

574 Froning 1981, 63-64, 68-69, Taf. 12, 161,171 (Napolı, New York, Roma rulevi con Paride) Cf anche ll cratere di Dervenı Qui p 22)

575 Dwyer 1982, 87, fig 126, Spinazzola 1953, I, 342, fig 389-390 
brocca del Sileno contengano acqua corrente.

Le ninfe hanno un mantello viola attorno alle reni Reggono con entrambe le manı davantı al grembo un bacino giallo La gamba al lato interno e di appoggio.

Il Suleno indossa un mantello azzurro-verde che lascia scoperto il petto Regge una canna nella destra e poggia con l gomito sinistro su una brocca dalla quale esce dell'acqua. Chiona e barba sono di colore verdastro-azzurro, la pelle e marrone

Lina combinazione di queste tre figure sı trova anche nella Casa di Romolo e Remo Il tipo orıginale delle ninte e la Venere Landolına; per il fiume si conoscono numerose statue e statuette, fra l'altro una dalla Casa di Octavius Quartio 576

Bibl. Schefold 1957, 92, Tran Tam Tinh 1974, 35-37 no 10, fig 11; lluthmann $1975^{\circ}, 92$ nota $4 j$

VI 1,10 Casa del chirurgo

Cat 198a/1 (19) Parete sud, quadro centrale; IV stule +

Un uomo e due lanciulle In tonde vi e und Mantelherme

Bibl Helbig 1459, Schefold 1957, 92, Repertorio 1983, 114.

Cat 198a/2. (21) Parete ?, quadro centrale: IV stile (M\9018)

Una pittrice, nel suo studio, dipinge una $H^{d}$ aftherme su di un pinax. L'erma ha la barba ed ll fusto rosso-rosa. La parte superiore e di colore naturale. Regge nella destra davanti al petto un cantaro, nella sinistra un tirso I due colors potrebbero indicare una scultura composta da due tipl di marmo.

Nel giardino che si vede fuorı dalla tmestra aperta sta una statua bianca sopra una base rettangolare Alla sua destra c'e un vaso su una colonna $S_{1}$ tritta dell'arredamento normale di un giardino.

Babl Helbig 1443, Elıa 1932, no 2j1. Ihlich 19j3, 76, Abb 27, Schefold 1957, 92-93, Reutersward 1960, 181; Ling 1971, 267-268, pl 47b; Pfretschner 197i, no. 87

VI 2, 4 Casa di Sallustio

Cat. 198b/1. (24) Parete est, zona mediana: IV stule +

Rappresentazione di giardino Sulla lesena fra $i$ due pannellı al lato nord e dipınta una colonna sormontata dalla statua gialla di un cervo, visto di fronte. Ha un'armatura larga Uriginariamente davantı alla lesena si trovava una fontana marmorea sorretia da una stinge $5>7$

Lna statua di un cervo dipinta si trova in un pinax nella Casa del marinaio (cat 259): Una scultura topiaria in bronzo provene dalla Casa del citarista. ${ }^{5}$

576 Becattı 1971; Spınazzola 1953, 1, fig 456; Jashemski 1979, fig.77.

57 Kapossy 1969, 33 Forse si tratta della sfinge di VI 16a (MN s.n., Ruesch 1704.

579 Dwyer 1982, fig. 136 
Bibl.: Mau 1908, 297, Abb 154; Beyen 1938, 52 Anm 3, Malurı 1952, fig 1; Schefold 1957, 94 (1v vano f): Grimal 1969, 11, 207, 210, 247-248, 288, fig 39, Peters 1971 9, Michel 1978, 401-402, Abb 4, Jasliemsk, 1079 , 168, fig $250-251$.

Cat $198 \mathrm{~b} / 2$. (31) Parete nord, zona mediana, IV stile +

In des pannell e abbcllito da un satro volante nell'a1tegglamento dell'Hermes ds Olımpla. I colorl sono naturalı e la ligura non e stata considerata conce und statua come nella Casa del naviglio (cat 207,2) e nella Casa di Giasone (cat. 288).

Bıbl. Helbig 373; Van Buren 1932, 38-40, Antonsson 1937, 118-121, f1g 19, Schetold 1957, 93.

Cat 198b/3 (32) Parete nord, zona mediana, IV stule

Il campo centrale con Artemide e Atteone e hancheggiato da ninte acquatiche sinmetriche che stanno su piedistalli rettangolari gialli I $\AA$ gamba sinistra o di appoggio Indossano una tunica rossa senza maniche e hanno una corona sulla testa Il bacino ddvanti al grembo e molto largo Le fattezze delle donne sono naturalı e vivaci La figura sinistra e andata perduta Come sempre si tratta di figure secondo il tipo della Venere Landolina (cf p 000)

Bibl Helbig 1055, Grimal 1943,484-485, fig 10, 34; Jucker 1950, 8; Schefold 1953-1954, 117-118; Wullemsen 1956, 43-45, Schelold 1957, 93, Reutersward 1960, 182, Pfretschner 1977, no 46, De Vos 1979, 92; Pompel 1748-1980, 45, tig 1, 187; Repertorio 1983, 121; Bragantın1/Parıse Badonı 1984, 121, fig 11

VI 3, 7 Accademia di musica

Cat 198c. (14) Parete est, zona superiore, III stule +

Sulla trabeazione dell'edicola centrale della zona mediana $v$ sono donne vestite $d_{1}$ un chitone senza maniche verde e una sopravveste glalla d stue arcaizzante. La figura a sinistra regge una patera nella destra, l'altra e stesa per la meta L'altra ligura tiene uno specchio nella mano destra presso l viso, mentre alza con l'altra un lembo del mantello nell'atteggramento dell'Afrodite di Frejus. Entrambe hanno una corona sulla testa Dietro di esse vi sono delle colonne

Bibl Schefold 1957, 96; Bastet,De Vos 1979, 85, tav. XLV.81, Repertorio 1983,141 .

VI $5,2(?)$

Cat 198d. (?) Parete ?, quadro centrale, IV stile +

Itıgenia ad Aulide Dietro l'Agamennone afflitto si vede un tempio, sul cui tımpano sta un Eros arciero Per quanto si possa stabulıre in base al disegno si tratta dell'Eros di Lisippo. ${ }^{579}$

579 Cf. Dohl 1968 
Bibl.. Helbig 1305; RP 1692 (a rovesc1o); Schelold 1957, 97; Pompei $1748-1980,41, \mathrm{tig} 13,37-38$.

VI 7.8 Bottega del protumiere

Cat 198e (entrata) Plastro, IV stile (MN 8991)

Des falegnami portano in processione un ferculum con i padronı del loro mesiere A sinistra si vede la statud di Atena Ergane, andata perduta a parte uno scudo Apparentemente la dea non e raftigurata secondo l'jconografia usata in I 8,10 (cat. 157), ma come Parthenos.

Accanto stanno lavorando tre falegnam, due segano una trave, un altro avanza con un pezzo ds legno A destra c'e la statua di Dedalo che guarda una figura sdraita davantı a se, forse scolpita da luı. E' una figura rigida con le braccia lungo 1 tanch. Torse si tratta di un kouros che indice la velusta di Dedalo.

I fercula venivano mostrati durante le feste. Si vede un altro esempio nella Casa delle nozze di Ercole (cat 254) Gia su delle urne etrusche si incontra una rappresentazione simule $50 \mathrm{Si}$ tratterebbe di una tradizione comune del'arte glittica e della pittura. Si possono aggiungere alcune lerrecotte 581

Malten accenna all'analogia con Efesto, nelle cus pompae si portavano altrottanto immaginı del genere $\mathrm{La}$ ligura $\mathrm{d}_{1}$ Dedalo infatti mostra le caratteristiche di questa divinita ${ }^{\circ} \theta_{2}$

Bıbl : Helbıg 1480; Malten 1912, 232-264; RP 236.4; Bianchı Bandınell 1950, 175-176, tav. 82, Schetold 1957, 99, Van der Meer 1975, 97-98, pl. XLI.2; Van der Meer 197 7 . 65, fig 30, Felletti Mal 1977, 334-335, fig. 169 a-b, Adan Varene 1980,235 , fig 18

VI 7,18 Casa dell'Adone ferito

Cat 198f (14) Parete nord, zona mediana, IV stile

Sul plastri fintı che dusudono un paradeisos, una rappresentazione dell'Adone terito a un glardino con un Eros dormiente sono stati applicati gruppi scultorel di Chirone e Achlle che stanno su piedistallı rettangolar gialli. A sinistra si vede il centauro al lato sinistro del glovane che lo cinge con il braccio destro, mentre regge nella mano destra la cetra. Solo la parte superiore e rumasta conservata

La coppra a destra e delineata summetricamente. Achlle ha messo la sua destra sulla spalla del centauro che suona con la destra la cetra posta nella sinistra Il corpo del cavallo si nasconde dietro ll giovane

Le due statue sono pendants, raffiguratı in tale modo secondo una pratica consueta ${ }^{53}$ Un sarcofago nel Museo Nazıonale Romano ha due gruppi di Achlle e Chirone fiancheggiantı un tiaso marino. ${ }^{5}$

\footnotetext{
580 Van der Neer 1975; 1977.

381 Dwyer $1982,36,122$ nota 4 , fig. 20

5.2 Cf. Brommer 1978, Taf. 42, 43.

5 日 Jucker 1950, 8, Becattı 1951, 32.
} 
Sono variazionı o copie di un gruppo esposto nel Saepta a Roma descritto da Plinio, del quale anche ll gruppo famoso della cosiddelta Baslica ad Ercolano (cat no 019) e una copia Il gruppo sul puastro sinistro a pul simule a quello ercolanese

L'Lros dormiente sul campo destro non e dissimile a statuette lopiarie $E^{\prime}$ dı colore bianco e indossa una clamide verde Jon e chiaro be il pittore abbia avuto l'intenzione $d_{l}$ rappresentare una statua oppure una figura vira L'archetipo, pero e senz altro chiaro 505

Dietro l'Eros sorge un pinax marmoreo a rilevo sopra un pilastrino $S_{1}$ rede la lotta tra Eros e Pan Ora questa parte della decorazione e quasi illeggibile

Bibl Helbig 436, 1295, Herrmann I, 65-70, Taf 52, Langenskiold 1930. 138-139 Abb 7-8, Jucher 1950, 8, 31-32, Schefold 195i, 101 Grimal 1969, 448-449 no 12, Peters 1971, 10-11, Jashemsh1 1979, 66-6i, LIMC 1 (1981) 226 no 35 s.v. Adonis, Repertorio 1981, 152-153

VI 7, 19 Casa dı Inaco ed Io

Cat $198 \mathrm{~g}$ (a) Parete sud, quadro centrale, IV stule +

Paesaggio sacro-idilliaco con una statud presso una porta sacra Secondo Helbig, che non pote vedere ll paesaggio, esso sarcbbe stato simule al presaggi nel giardino della Casa della fontana piccola (cat 200/2)

Bibl Helbig 1562, Schefold 1957, 101

VI 7, 23 Casa di Apollo

Cat $198 \mathrm{~h} / 1$ (7) Parete sud, zona superiore, IV stule +

L'edicola centrale e corretta da due Hoffthermen La loro parte superiore rappresenta del guerrieri nudı armatı Sull'architrave si trovano centaurı messi in posizione summetrica

Bıbl Curtıus 1929, 155, Abb 100, Schefold 1952, 134-135, Taf 37, Spinazzola 1953. III, tav 104, Schefold 1957, 102 (vano c), Pfretschner 1977, no 8 , Schmit-Colınet 1977, 147, 272 cat P16

Cat $198 \mathrm{~h} / 2$ (10) Parete ovest, zona centrale, IV stile +

Rappresentazione di giardino Dietro o sopra una fontana marmorea vi e la statua di Artemide, vestita di un chitone corto e stıvalı da caccia $\mathrm{Ha}$ una corona sulla testa Regge un arco nella unistra distesa c prende con la destra una freccia dalia fasetra che ha sul dorso il tipo potrebbe essere quello dell'Artemide nella Vula Borghese attribuita a Leochare vel contesto la divinita rappresenterebbe una tigura fluviale Non e dispoinibule un'illustrazione.

504 Sichtermann 1957, 100, Taf 15, CatRoma I 2, II,90-93, 4la II no 6

585 P e kapossy 1969, 40 Cf un esemplare policromo trovato a Cirene Paribenı 1959, 111 no 308 , tav 147 (alı verdı pella di leone arancione) 
Una lotografia del primo Novecento (Thedenat 1910) mostra una figura femminule nuda che ha la mano destra presso le tempie e la sinistra nel fianco La gamba destra e di appoggio. Apparentemente rappresenta un'Atrodite paragonabile a quella in $I 11,6$ (cat. 166/2) o 113,16 (cat 173). L'ummagme e l'unica tuttora conosciuta, mai citata in questo contesto e per tale ragione non e sıcuro se essa sia una totogratia di questo glardino a meno.

Bibl. . Helbig 240; 'Thedenat 1910, 89, fig 51; Schefold 1957, 102 (vano d); Grmal 1969, 449 no. 13; Muthmann 1975, 51-52; Pfretschner 1977. no. 16, Jashemski 1979, 133; Repertorı 1983, 160.

Cat. 198h/3. (f) Parete ovest, zona mediana; IV stile +

Rapprentazione di giardino. Fra le prante sarebbero state riconoscibili fontane sorrette da sfing 1 lemminili. Grimal ammette la loro presenza in base alla descrizione di Breton. Anche qui potrebbe essere stata presente la figura di Afrodite, precedentemente citata (vano d)

Bibl.. Breton 1854, 334-335; Grimal 1969, 119.

Cat. 197/3. (25) Pareti nord e sud, zona superiore; IV stile

La parte superjore della decorazione e sorretta da figure nude snelle. Una mano regge un platto presso il fianco,mentre l'altra e lungo il fianco. Hanno le gambe unite e stanno sopra l'architrave deglı hospitalia della zona mediana. Il loro sesso non a definibile Non sono state menzionate in nessuna descrizione della scaenap trons.

Bıbl.: Schefold 1957, 103 (vano g), Repertorio 1983, 163-164; Moormann $1983 b, 84-91$, fig $7-12$.

VI 8, 3 Casa del poeta tragico

Cat. 1981/1. (3) Parete sud, quadro centrale; IV stile (MN 9559)

Zeus e Hera sul monte Ida. Dietro la coppia vi è una colonna coronata da tre piccoli leoni dorati. Secondo Peters si tratta del sumbolo di Cibele. La scena rappresenta la cosiddetta hierogamia descritta nell'Iliade (IIV 280-281).

Bibl. Peters 1963, 14j, tig. 138, Schert 1967, 54-59, Vermaseren 1978, 14 no. 32, Repertorio 1983, 166.

Cat. 198i/2. (10) Parete nord, quadro centrale; IV stile (MN 9112)

Ifigenia in Aulide. Dietro l'Agamennone afflitto c'e una colonna con la statua gialla $d$ irtemide. La dea è vestita di un peplo e un mantello. Sulla testa ha un modıo. Regge due fraccole con le mani distese in avanti. Dietro di essa, ai due lati, si trovano due cani accosciatı.

Il gruppo rappresenta il tupo della potnia theron. ${ }^{5.6}$ Nello stesso momento potrebbe rappresentare Ecate, vista la presenza delle due fiaccole. Lo stule è arcaistico, ma non si puo trovare un confronto nella scultura finora conosciuta.

Van der Meer osserva certi elementi comuni ai rilievi di alcune urne di Volterra.

Bibl. . Herrmann I, 23, Taf. 15; Elia 1932, no. 30; Schefold 1957, 105; Pfretschner 1977, no 19; Van der Meer 1977-1978, 71 nota 228, fig. 49; LIMC I, 265 no. 41, s.v. Agamemnon; Repertorio 1983, 167.

586 Cf. Christou 1968. 
Cat $198_{1 / 3}$ (15) Tutte le paretı, zona mediana, IV stule

I] pinar sulla parete nord mostra Marsia seduto su una base grigla a gradinı

Il piede clestro poggia su una piccola icala e la gamba sinistra e alzata Suona con entrambe le manı un flauto A sinistra sta in piedi Olunpo Specie Varsia ricorda la figura sul pinax della cosiddetta Baslica di Ercolano (cat 019), ma l'insieme non riproduce il medesimo gruppo

I campi lateralı al lato ovest delle paretı nord e sud sono abbellit guerrieri nudi che stanno sopra piccoli segmenti rotonch visti di scorcio Entramo nel genere del guerrierı d umpronta policletea (ct $p$ k3) Hanno un elmo sulla testa e reggono nella sinistra lungo il fianco lo scudo, la lancia e il mantello La figura sulla pasete nord tiene un gladio nella destrd abbassata, I peso del corpo poggia sulla gamba sinıstra L'altro ha la sua destra presso la spalla, la gamba sinistra e di appoggio

Nes prospettı glallı sono inseritı atlantı e cariatidı sulle paretı nord a sud (mal conservata) si vedono delle donne vostite di un chitono senza muniche in ordine summetrico Reggono con la mano destra und colonnd hulla lesta o con la sinistra un lembo della veste all'altezsa della vita Sulla testa hanno una corona Le gambe sono unite

Sulla parete est gli uomini nudi con una clamide sopra le spalle sono interpretablı come guerrierı La figura a sinistra regge un gladio nella mano destra distesa, mentre l'altra e in riposo lungo il fianco Lod gamba destra e di appoggio La figura a destra ha un gladio nella sinistra lungo ll fianco e ha l'altra distesa La gamba sinistra e di appoggio Ora non si puo piu vedere che esse sorreggono sulla testa la colonna

Bibl Helbig 225 hP 32 2, Schefold 1957, 106 Schefold 1962, 108, 203, Taf 122 , 69, Schmidt-Colınet 1977 147, 271 cat P11, Repertorio 1983, $169-172$

VI 8, 20 Fullonica

Cat 199 (b) Plastro IV stule (MN 9774)

Rappresentazione della fullonica Su un lato si trova un'edicola occupata dalla statua di Afrodite Essa e dipinta a colori naturali $L^{\prime}$ nuda Poggia con Il gomito sinistro su un pilastrino, la mano destra e stesd verso ll basso c regge un drappo attorno alle renı La gamba destra e di appoggio Si tratta di un'imitazione di un tipo ellenistico, forse derivato da und statua prassitelea come si incontra nella scaenae frons in 13,25 (cat 146)

La presenza di Afrodite si spiega dal fatto che essa sia la padrona des fullon che svolgono l'industria maggiore di Pompei, in tale veste si spiega l'associazione con la Venere pompelana

Bibl Helbig 1502, Schefold 1957, 107, Hoeller 1976, 44-46

VI 8, 23 Casa della fontana piccola

Cat 200/1 (7) Paretı nord, est e ovest, zona mediana c superiore; IV' stile Sull'architrave del prospetto attorno al quadro centrale della parete nord si trovano acroter a torma di sfingl temminil alate in posizione simmetrica La figura a sinistra e andata perduta 
L'edicola centrale della zona superiore su tutte le paretı e accupata da figure gialle Sulla parete nord si vede una Fortuna seduta che regge una patera nella mano destra distesa e uno scettro nella sinistra presso ل L lidnco

sulla parete est un uomo nudo, forse tres, regge una lancia con entrambe le manı daiantı al petto La gamba destra e di appoggio L na ligura umile si trova nella scaenae trons del trigidario della Casa del criptoportico (cat $148,4)$

Sulla parete ovest un Apollo nudo suona und cetra nella sinistra Le gambe sono unite Sulla testa ha una corona

Bubl Schefold 1957, 108 (rano d), Schetold 1962, 125, Tal 83, Repertorio 1983,177

Cat $200 \% 2$ (10) Paretı est e ovest, zona superiore. IV stle

Paesaggı sacro-ıdlliacı dı grandi dimensionı, ora quası lleggıbıli vel paesaggio a destra della parete ovest $y$ sono due statue rosse presso un ecliticio lndossino mantelli lunghi La ligura sillstra regge un piatlo nella mano sinistra distesa e una corona nella destra La tigura destra ha la destra all'altezza della lesta e la sinistra lungo l f fanco

Il paesaggio della parete est contiene la statua gialla di Cibele E' seduta su un trono flancheggiato dal consuetı anunalı Regge nella destra stesa una patera L'atteggiamento e quello iconografico, derix dto dalla statua $d_{1}$ Igoracrito, usato per molte rappresentazionı della divinita, fra l'altro a Boscotrecase (cat oof 2) Un bronzetto del tipo e stato trovato nella Casa del citarista 587

Bıbl Helbig 15571561 Herrmann 1, 232-234, Abb 69, Taf 160, 168, Spincizzola 1953, Il. 843-844, tig 829, Schefold 195i, 109 (vano e), Peters 1963, 173-176, tig 17j Pfretschner 1977, no 32, Vermaseren 1978 , 15 no 33179 , Silbex berg 1981 , no 125, fig 101-103, Repertorı 1983, 178-180

VI 91 Casa del duca d'Aumale

Cat 201 (') l'arete sinistra, quadro centrale. IV stule (M 9555)

Io arriva da Iside in Egitto 4 sinistra si trova una base retlangolare con una simge femminle bianca senza alı $\mathrm{Ha}$ una piccola corona sulla lesta ed e del tıpo egizio La sua presenza deve accennare l'ambientazione della scena mitica 5 a

Bibl Helbig 139, Herrmann I, Taf 58, Elıa 1932, no 102, Elıa 1941, 28, fig 28, Schefold 1957, 110, Tran Tam Tinh 1964, 128 no 14, pl XVI 2

VI 9, 2 Casa dı Meleagro

Cat 202/1 (1) Paretı nord e sud, zona medıana, IV stıle

\footnotetext{
5:7 Dwyer 1982, fig 88

5 : Per quanto riguarda la funzione della "casa" vedi Pacher 1978, 24-30
} 
I prospetti gialli su uno sfondo rosso contengono figure maschili simmetriche. La gamba esterna e di appoggio. Sull'avambraccio interno hanno un mantello. Il contrapposto è ben accentuato e il corpo è articolato secondo i moduli lisippei Non è da escludere che le figure non siano state dipinte come statue, ditio che l'intera decorazione è monocroma.

Bibl.; Schefold 1957, 110; Repertorio 1983, 183-184.

Cat. 202/2. (16) Pareti ovest e ?, quadro centrale: IV stile (MN 9320) +

Sileno con il Dioniso fanciullo. Su una colonna a destra sta una statuetta di Prıapo, il cui colore è ignoto. Indossa un mantello che alza con entrambe le mani. Vel grembo ha dej frutti. Il tipo e stato studiato di recente da $\mathrm{H}$. Blanck. ${ }^{589}$

$\mathrm{Di}$ una parete ignota proviene $\mathrm{MN}$ 9320, raffigurante Fetonte o Imeneo (Schefold). Egli è modellato secondo il Pothos di Scopa, posto in posizıone summetrica.

Bibl.: Helbig 377, 855; RP 68.6, 105.4; Herter 1932, 129 no. 7; Kerenyi 1955, 145; Schefold 1957, 112; Stewart 1977, 109-120, 146 no. H4.

Cat. 202/3. (20) Parete est, zona superiore; IV stile

Un candelabro nella parte centrale viene sormontato da una baccante vestita di un mantello giallo volante che lascia scoperto il petto. Con entrambe le mani essa suona un timpano che brandisce verso sinistra. I piedi stanno nell'atteggiamento della danza.

Bibl.: Schefold 1957, 111.

Cat. 202/4. (24) Tutte le pareti, zoccolo e zona superiore; IV stile

Dei satıri in rosso chiaro occupano delle edicole su uno stondo rosso scuro. Vista la monocromia rossa non è sicura che le figure siano senz'altro da considerare statue. Sono tutte del tipo ellenistico del fauno danzante della Casa del fauno. Ogni parete contiene una coppia di satiri le cui gambe di appogg 10 sono in posizone simmetrica.

Parete nord, ovest. Ha una nebride sull'avambraccio sinistro e regge una bacchetta nella sinistra e un cantaro nella destra: il peso del corpo poggia sulla gamba sinistra. Solo questa figura ha la barba.

Parete nord, est. Ha una clamide avvolta attorno al braccio sinistro. Regge una siringa con la destra. La gamba destra è di appoggio.

Parete ovest, nord. Ha una nebride sul dorso, regge un pedum nella sinistra e una siringa nella destra; la gamba destra è di appoggio.

Parete ovest, sud. Ha una nebride sulla spalla e un pedum nella destra presso la spalla; la mano destra è andata perduta; la gamba destra e di appoggio.

Parete est, nord. Ha un mantello sull'avambraccio sinistro e regge un pedum nella sinistra e una siringa nella destra. La gamba sinistra è di appoggio.

Parete est, sud. Ha una nebride sopra l'avambraccio sinistro e regge un pedum nella sinistra e una siringa nella destra. La gamba destra $\dot{e}$ di appoggio.

La zona superiore della parete est contiene due candelabri sormontati da statue gialle. Solo la parte inferiore è rimasta conservata. La gamba esterna regge il peso del corpo.

509 Blanck 1979. 
Bibl.: Schefold 1957, 113; Schefold 1962, 128, Taf. 96.2, 98-99; Schmidt-Colinet 1977, 273 cat. P18; Repertorio 1983, 190-191.

Cat. 202/5. (26) Parcti nord e ovest, zoccolo; IV stile

Su ogni parete si vede una figura maschile marrone accovacciato che sorregge l'orlo superiore dello zoccolo. La figura della parete est poggia sul ginocchio destro e sorregge lo zoccolo con la destra, mentre l'altra $\dot{e}$ in riposo sul ginocchio. Sulla parete nord l'atteggiamento è simmetrico.

In pittura raramente si incontrano figure in questo atteggiamento, mentre nell'architettura se ne conoscono vari esempi. Il pulpito di Fedro nel teatro di Dioniso ad Atene ha due satiri di questo tipo. Nellarchitettura del sarcofago di Velletri essi sono presenti nel registro inferiore e i due barbari nel Museo nazionale di Napoli devono aver avuto una funzione simile. 590

Bibl.: Schefold 1957, 113; Schmidt-Colinet 1977, 273 cat. P18; Repertorio $1983,193$.

Cat. 202/6. (27) Pareti est e ovest, zoccolo; IV stile

Dei satiri brunastri sorreggono con una mano l'orlo superiore dello zoccolo. Sono nudi e tengono una clamide sulle spalle. Le gambe stanno nell'atteggiamento della clanza come quelle del fauno danzante della Casa del fauno.

Parete ovest, sud. Regge l'orlo con la destra e ha nella sinistra distesa una patera. La gamba destra sta davanti alla sinistra ed è di appoggio.

Parete ovest, nord. Ha un pedum nella sinistra e una corona sulla testa, mentre per il resto e simile alla figura precedente.

Parete est, nord. Ha un pedum nella sinistra e sorregge con l'altra. Sulla testa si vede una corona. Le gambe sono nella stessa posizione di quelle dei satiri precedenti.

Bibl.: Schetold 1957, 114; Repertorio 1983, 196-198.

Cat. 202/7. (29) Tutte le pareti, zoccolo; IV stile

Figure gialle fungono da atlanti e cariatidi per l'orlo superiore dello zoccolo. Ugni parete contiene due figure, a meno che vi sia una porta.

Parete nord. A destra sta una figura maschile con una clamide sopra le spalle. Sorregge con la mano sinistra l'orlo mentre l'altra i in riposo nel fianco. La gamba destra e di appoggio.

Parcte est. A destra c'è una figura simile, ora reggente l'orlo dello zoccolo con la destra. A sinistra vi è una figura femminile vestita di un peplo, ora quasi invisibile.

Parete sud. Due figure femminili vestite di un peplo simmetriche. Reggono l'orlo superiore con la mano esterna, mentre l'altra è lungo il fianco. La gamba esterna è di appoggio.

Parete ovest. A sinistra si vede una figura simile a quella della parete opposta.

La posizione degli atlanti sul rilievo di Göreme, ora a Berlino, e la loro composizione sono simili a quelli delle nostre figure. ${ }^{5}{ }_{1}$

Bibl.: Schefold 195̄, 111; Repertorio 1983, 198-200.

\footnotetext{
590 Schmidt-Colinet 1977, cat. M73, M74, M77.

591 Schmidt-Colinet 1977, cat. M69.
} 
VI 9, 3-5 Casa del centauro

Cat. 203/1. (2) Parete nord o sud, quadro centrale; IV stule +

Su una base composta da quattro gradinı vi e la statua arcaica di Apollo sotto torma di un kouros. Le braccia sono in riposo lungo il fianco, il piede sinistro sta un po' in avanti.

Purtroppo, per mancanza di documentazıone sufficiente, non e possibile stabilire il contesto originale, cioe pinax o vignetta Si tratta dellunico kouros volutamente copiato. A. Furtwangler lo paragono ad una statua di Tenea 592

Bibl. Fiorellı 1875, 133; A. Furtwangler, Archaologische Zeitung 40 (1882) 58, Overbeck,Mau 1884, 331, Overbech 1887, 15-16, Fig 2; Maluri 1933, 418, Schetold 1957, 114 .

Cat. 203/2. (26) Parete nord, quadro centrale; III stile (MN 8980)

Meleagro e Atalanta Su una colonna dictro Meleagro si vede la statua verde di Ecate. Indossa un chitone senza maniche e ha una corona sulla testa. In entrambe le mani stese in avanti regge una fiaccola. Sulla spalla destra ha una taretra che la associa immediatamente ad Artemide.

Bıbl.: Helbig 1165, Elıa 1032, no. 46; Herrmann Il, 36, Taf 223; Schefold 1957, 115; Peters 1963, 102, fig. 89, Pfretschner 1977, cat. 17; Bastet/De Vos 1979, 58-59; Repertorio 1983, 206.

VI 9,6 Casa dei Dioscuri

Cat.'204/1. (35) Tutte le paretı, zone mediana e superiore, IV stile

I campl lateralı contengono guerrieri nudı sopra mensole, dipintı in colori naturalı Sono molto danneggiati.

Parete est, sud L'uomo ha una clamide sopra le spalle Ha la destra sulla testa e regge nella mano sinistra lungo il tianco un oggetto non identificabile.

La gamba sinistra e di appoggio.

Parete sud, est e ovest Illeggiblli.

Parete ovest, nord L'uomo ha una clamide sulle spalle. La destra è in riposo nel franco, l'altra regge un gladio che sta sulln terra La gamba sluistra e di appoggio.

Parete oves1, sud Solo le gambe e un lembo della clamude sono rumaste conservate Il peso del corpo poggia sulla gamba sinistra.

Le edicole central della zona superiore sono occupate da statue policrome di divinita. Sulla parete ovest si vede una Fortuna, seduta su un trono. Indossa un mantello verde $\mathrm{Ha}$ un pratto nella destra distesa e uno scettro d'oro nella sinistra davantı al petto.

Sulla parete ovest si trova un Poseidon modellato secondo il tipo Laterano, che si vede anche nes paesaggi nella Villa della Farnesina e nella Vulla di Arranna (cat. 010/5, 319/6)

$392=$ Richter 1960, no. 39, fig. 151-153. Si confronti una figura sumile in un riluevo rappresentante Paride, ora nei Musei Vaticanı, datato nel secondo quarto del primo secolo a.C. (Froning 1981, 64, Taf 12.2).

593 Cf. Walde 1978; Walde-Psenner 1979; Moreno 1978. 
Sulla parete nord $s_{l}$ vede solo la sagoma $d_{l}$ una figura femminle Forse si tratta di Atena

A sinistra della Fortuna sulla parete sud sorge un candelabro vegetale con la statua di un guerriero nudo che regge uno scudo nella simistra e ha la destra alzata Le gambe sono unite

Bıbl Richardson 1955, 18-20, pl V, Schefold 1957, 116-117, Repertorio $1983,208-210$

Cat 204/2 (37) Paretı ovest e sud, zone mediana e superiore, IV stıle

Paretı est e sud, zone mediana e superiore, IV stule (ord $M N$ e $B M$ )

I campi della zona mediana delle paretı ovest e sud contenerano vignette a forma dı divinita che sono ord nel Museo vazionale e nel British Museum Stanno su segmentı ovoldalı

Parete ovest Zeus e seduto su un trono e viene incoronato da una Iike che sta dietro di lui ( $M(\lambda$ 9551) Il dio quindl e rappresentato come persona viva, ma corrisponde ai modell, normalmente usatı per le divinita sedute Vicino al piedı si trovano un'aquila e un globo Le gambe sono coperte da un drappo azzurro Nella sinistra regge uno scettro, la destra giace in riposo sulla coscia

Parete sud, campo sinistro Demetra e seduta su un trono (MN 9454) $E^{\prime}$ vestita di un peplo bianco e ha una corona sulla testa circondata da un numbo ccruleo Regge nella destra una fiaccola e nella sinistra una patera. L'iconogralia e quella consueta per divinita qualı Cibele e Fortuna

Parete sud, campo destro Saturno sta in piedi(MN 8837 ) Indossa una toga bianca $c$ ha la testd coperta Nella destra distesa regge una falce, la sinistra e nascosta dentro la veste La gamba clestra e di appoggio L'iconogratia e quella di un sacerdote romano

Da una parete ignota proviene 1 frammento, ora a Londra, che rappresenta un Apollo Citaredo $\mathrm{Ha}$ un mantello azzurro sulle spalle e regge nella sinistra una cetra, nella destra un plettro A sinistra vi e la statua arcaistica di un uomo ammantato

Sopra l'entrata della parete ovest si trovava MV 27700 raffigurante Ermafrodito e Pan A destra si vede la statua rossa di Priapo Ha un mantello sulle spalle e un berretto sulla testa Nella destra regge una cornucopia, nella sinistra una bacchetta Sotto $\perp$ mantello si vede 1 fallo $E^{\prime}$ rivolto verso sinistra

Il gruppo di Ermafrodito e Pan potrebbe essere stato modellato secondo un modello ellenistico di cui sono conosciutı alcunı esempları 594 Non e chiaro se l'archetipo sia stato una scultura o una pittura 595

Bibl Helbig 96, 102, 176, Herrmann I, 124, Taf 121-122, II, 30, Abb 6, RP 50 2, 99 2, Herter 1932, 138 no 38, Elıa 1932, cat 233, 234, 236; Hinks 1933, no 32 , pl 13, Beyen 1951, 5, pl 13, Richardson 1955, 15, pl 23 1, Schetold 1957, 116, Peters 1963, 138, Pfretschner 1977, no 92. Repertorio 1983, 211

Cat 204/3 (38) Parete ?, quadro centrale, IV stule +

If igenia in Tauride La sacerdotessa regge lo xoanon di Artemide davanti al grembo che ha la torma di un palladio armato con scudo, lancia e elmo Phlippart ritiene la pittura dubbiosa 596

\footnotetext{
594 Marconı 1924 Cf p 000

595 Cf hrahmer 1926

596 Forse proviene dalla parete est (cf Repertorio 1983, 211)
} 
Bibl Helbig 1336b, RP 170 4, Phlippart 1925, 24 no 37, Richardson 1955, 22 , Schefold 1957, 117

Cat 204/4 (42) Tutte le paretı, zona mediana, IV stule +

Le predelle del campl lateral contengono acroterı a forma di centaurı Jei prospett. possibly there were figures or statues in pavillons over the side panels but no traces of these survive (Richardson)

Bibl Helbig 504b, Richardson 1955, 34-36, Schetold 1957, 117-118

Cat 204/5 (43) Tutie le paretı, zone mediana e superiole. IV stule

Vei prospetti della zona mediana si trovano alcune statue In primo piano una donna vestita $d_{1}$ un peplo $d_{1}$ colore giallo funge da acroterio L'unica ligura conservata e quella sulla parete nord al lato ovest che regge un piatto con la destra presso la spalla e tiene con la mano sinistra un lembo del mantello all'altezza della coscra

Ael secondo plano su entrambe le pareti una pergola vene sorretta dalla $H^{*}$ úftherme di un satiro Sorge da un calice vegetale e ha una corona sulla testa Regge nella sinistra un pedum Sulla spalla destra siede ll Dioniso fanciullo

Anche le pareti est e sud devono aver avuto quest prospettı $_{1}$

Il fregio fra le zone mediana e superiore contiene scene del culto dionisiaco Su quella al lato ovest della parete nord si vedono delle donne presso la statud rossa di Dioniso, vestito di un mantello Il fregio al lato est contiene una statua schematica posta su un altare

Un pinax nel British Museum, apparentemente proveniente da questo ambiente, mostra l'erma itıfallica di Priapo su una colonna

Le edicole centralı della zona superiore sono occupate da statue o figure vive a forma di statue

La parete nord ha una Fortuna seduta su un trono uguale alla figura nel vano 35 , che regge una cornucopua davantı al petto ll trono sorge su una bise rettangolare gialla

Sulla parete ovest $S_{1}$ vede Dionsso, seduto su un trono $E^{\prime}$ vestito di un mantello e ha una corona sulla testa Regge nella destra alzata un tirso, nella sinistra stesa una patera Solo la sagorna e rumasta visible

Bibl Hinks 1933, no 49, fig 29. Richardson 1955, 31-32, pl 82 , Schefold 1957, 118-119, Repertor1o 1983, 213-215

Cat 204/6 (45) Pareti nord e ovest, zona mediana, IV stile

Paesaggio monocromı rossı e guall su campı lateralı bianchı, dıpıntı in modo schematico Le statue non sono state rese dettagliatamente

Parete nord, campo ovest Statua gialla di un uomo che regge una lancia nella sinistra sulla spalla La gamba destra e di appoggio ll modello e Il Doriforo policleteo

Parete ovest, campo nord $S u$ un piedistallo rettangolare vi e la statua gialla di un uomo nudo che regge una lancia nella simistra alzata e un cantaro (?) nella destra lungo ll fuanco La gamba destra sorregge ll peso del corpo

Parete ovest, campo sud Su una base rettangolare sta la statua gialla ds una figura ammantata In un lembo della veste alzata davantı al grembo tiene del frutt 1 La figura puo essere Pomona o Priapo (L sesso non e visibile)

Bibl Richardson 1955, 38-40, Schefold 1957, 119, Suberberg 1981, no 79, fig. 75 , Repertorio 1983, 217-218

Cat 204/6 (53) Paretı nord e est, zona mediana, IV stule (ora MN) + 
Sulla parete nord, a sinistra e a destra della porta dell'atrio 37 erano vignette a forma di guerrieri nudi sopra mensole. L'uno ( $M N$ s.n. 33) regge uno scudo sopra la testa con la mano sinistra e un gladio nella destra distesa. L'altro (+) regge uno scudo e una lancia nella sinistra e ha la destra alzata. Il loro atteggiamento ricorda i guerrieri pirrici. Il primo puo essere confrontato con MX 9372 (cat. 076).

Sui pilastri al lato est si trovavano due gruppi gialli rappresentanti la strage dei figli di Niabe davanti a uno sfondo rosso.

IN 9302 conticne i sette figli, MN 9304 le sette figlie. La composizione è simmetrica. Al piede di un tripode apollineo si trovano tre figure, una di fronte, due al fianco. Sui due anelli dentro di esso sono sempre sedute due figure. Tutte sono ferite dalle frecce mortali e si muovono in un'agonia atroce, tentando di togliere le frecce o cercando la fuga. Delle ligure femminili quella nel centro davanti al tripode ripete se pur liberamente la Niobide Chiaramonti nei Musei Vaticani e un altro esemplare a Firenze; le braccia sono state cambiate. ${ }^{597}$

I] figlio inginocchiato a sinistra è una variazione del cosiddetto Narciso negli Lffizi: la posizione delle gambe e stata cambiata e il braccio destro (restaurato!) i alzato, mentre la testa (altrettanto restaurata) è volta in basso. 59 Secondo Snuer la figura maschile centrale copierebbe il vulneratus deficiens di Cresila, come lo descrisse Plinio (NH XXXIV 74.1). Per le altre figure non si possono trovare dei confronti.

In un rilievo, ora nei Musei Vaticani, si vede anche un tripode usato ai fini di un'ambientazione. ${ }^{59}$ Inoltre la combinazione è sconosciuta, purche si interpreti un passo di Pausania (I 21,3) come la descrizione di una rappresentazione simile. Si tratta della tomba di Trasillo in una cava ad Atene:

(Qui dentro/sopra sta un tripode, dentro di essa (scil. la grotta) Apollo e Artemide stanno per uccidere i figli di Niobe.) Sauer credette che si trattasse di un rilievo all interno della cava; $J$. Travlos invece ritiene che $i$ Niobidi fossero dipinti, mentre il tripode sarebbe stato posto sopra la tomba come segno di Trasillo. 600 A. Borbein accetta l'ipotesi di Travlos senza esprimersi sul tipo di decorazione (rilievo o pittura). A. Lesky non si decide per mancanza di indicazioni chiare nel passo di Pausania.

Un gruppo dei Niobidi, seconclo Plinio creato da Prassitele o Scopa attribuzione peraltro dubbiosa -, venne esposto nel tempio di Apollo Sosiano dal fondatore C. Sosio (Plin., N.H. XXXVI 28). Le statue che tuttora sono rimaste conservate (sei figli, quattro figlie, la madre e il pedagogo; il maggiore numero a Firenze) probabilmente copiano una parte di questo gruppo. 601

Forse il pittore ha voluto comporre un gruppo del genere facendo uso di un rilievo o un gruppo non piú conosciuto. $\mathrm{E}^{\prime}$ certo che nessun dei monumenti menzionati è stato l'archetipo.

\footnotetext{
597 Bieber 1961b, fig. 264-265.

598 Mansuelli $1958,120-121$ no. 81.

599 Lippold 1956, 48-j1, Taf. 28.

600 Travlos $1971,562-565$.
}

602 Mansuelli 1958, 101-123, no. 70-83; Bieber 1961b, 74-76, fig. 253-265. 
Sul pilastro meridionale si trovava $\mathrm{MN}$ 8998, rappresentante Perseo e Andromeda. Secondo Phillips il pinax copia un modello scultoreo del quarto secolo, vicino al gruppo nel Kestnermuseum a Hannover.

Bibl.: Helbig 1154, 1834; Sauer, Roscher III 1 (1897-1902) 421-422, s.v. Niobe u. Niobiden; Herrmann I, 180-181, Abb. 51-52. Taf. 131; Richardson 1955, 55, 60; Schefold 1957, 120-121; A. Lesky, RE XVII 683-684 s.v. Niobiden; Borbein 1968, 152 nola 786, Taf. 28.2; Phillips 1968, 5-6, pl. 5 fig. 10; Borbein 1973, 198-199; Repertorio 1983, 221.

VI 10, 2 Casa dei cinque scheletri

Cat. 20J/1. (a) Parete?, quadro centrale; III stile (ora MN 8999)

Cassandra da un oracolo. In fondo sorge sopra una base la statua di Apollo nudo. Poggia con il gomito destro su un pilastrino e ha la mano destra nel lianco. La gamba destra e di appoggio.

Bibl.: Helbig 1391; Herrmann I, 248, Taf. 180; RP 171.3; Davreux 1942, 122 no. 44, fig. 26; Schelold 1957, 123; Bastet/De Vos 1979, 94

Cat. 205/2. (b) Parete ?, quadro centrale; III stile +

Cassandra da un oracolo. Dietro la sacerdotessa si vede la statua policroma di Apollo. $E^{\prime}$ seduto su una seggiola vicino al suo tripode e poggia con il gomito sinistro sulla spalliera. Nella destra tiene un ramo di lauro. Le gambe sono coperte da un mantello.

Nel centro in Iondo, parzialmente nascosta dietro una colonna, c'è la statua di una donna vestita di un peplo. Ha la mano destra presso la spalla.

Bibl.: Helbig 1391b; RP 216.3: Davreux 1942, 123-124 no. 45, fig. 27; Schefold 1957, 123; Pfretschner 1977, no. 48; Bastet/De Vos 1979, 94.

Cat. 205/3. (?) Parete ?, quadro centrale; ? stile +

Schulz scrive: Aias wie er die sch Jne Kassandra bei den Haaren von dem Pallasidol wegreisst, $z u$ welchem diese die $H^{\prime}$ ande erhebt. Secondo Helbig la scena proviene da questa casa e va combinata con una delle scene precedenti.

Bibl.: Schulz, AdI 1838, 181; Helbig 1328.

VI 10, 7 Casa dell'ancora

Cat. 206. (9) Parete ovest, zona superiore; III stile +

Su piedistalli verdi, posti sugli angoli dell'architrave dell'edicola centrale della zona mediana, sono delle donne vestite di un chitone verde senza maniche e di un mantello bianco. Sorreggono una colonna con la testa. Con la mano interna reggono un festone, con l'altra la fíne di esso e un lembo della veste. Sono di Stile Severo e entrano nelle serie consuete (p.00o).

Bibl.: Schefold 1957, 123 (vano b); Bastet/De Vos 1979, 19, 43-44, tav. XIII 24; Repertorio 1983, 233.

VI 10,11 Casa del naviglio 
Cat. 207/1. (2) Tutte le pareti, zona mediana; IV stile (ora MN) +

Le pareti nord o sud hanno una decorazıone a campi ornati con immagini di divinita sedute. Le figure sono state dipinte in modo statico $e$ in atteggiamento statuarjo, ma con una policromia vivace. Non e sicuro che si tratti di statue.

Parete nord, campo est. Hera è andata perduta.

Parete nord, campo ovest. Zeus e andato perduto, ma noto attraverso delle descrizioni. Ha un mantello azzurro sulle gambe e un drappo verde sul trono. Regge uno scettro con la sinistra e ha la destra sotto il mento. Ai di $\dot{e}$ seduta un'aquila.

Parete sud, est. Dioniso (MN 9456) ha una corona sulla testa e un mantello verde-viola sulle gambe. Regge un tirso nella sinistra e un cantaro nella destra. I piedi poggiano su uno sgabello. A destra siede una pantera. a sinistra si vede un timpano. Il trono e coperto da un drappo viola. $H$. Stern considera l'iconografla assez rare.

Parete sud, campo ovest. Demetra (MN 9457) indossa un chitone chiaro e un velo azzurro. Sulle gambe si vede un mantello bianco. Ha la sinistra nel grembo e regge con la destra una fiaccola. I piedi stanno su uno sgabello. A sinistra vi è un canestro pieno di spighe. Il trono e coperto da un panno verde.

I pannelli delle pareti est e ovest sono ornate con statue di guerrieri nudi postı sopra mensole, ora tutte perdute a parte due frammenti che si trovano a Napoli. Entrano nelle serie di statue classicheggianti d'umpronta policletea frequentemente applicate nel IV stile (cf. p. 49').

Ualla parete ovest proviene MN 9123: un guerriero bianco ha 4 braccio sinistro coperto da una clamide e regge una lancia nella destra la cui punta tocca con la sinistra; la gamba destra sorregge il peso del corpo. Lna figura simile si vede nel frigidario della Casa del criploportico (cat. 148/4).

MN 9132 proviene da una parete ignota. $E^{\prime}$ una figura quasi identica alla precedente.

Nel secondo campo da sud sulla parete est si trovava una figura descritta da Helbig (1833) che reggeva una lancia e uno scudo nella mano sinistra e un oggetto non definibile nella destra.

Bibl.: Helbig 101, 162, 175, 392, 1832-1833; Herrmann I, Farbtafel I; RP 7.3, $50.1,107.5,268.1$; Elia 932 , no 237-238; Richardson 1955, pl. 17.1, 22.1; Schefold 1957, 124 (vano a); Stern 1975, 23, fig. 35; Pfretschner 1977, no. 33; Repertorio 1983, 234-235.

Cat. 207/2. (c) Parete ovest, zona superiore; IV stile

Un satiro nudo regge sul braccio sinistro il Dioniso fanciullo e gli presenta con la destra alzata un grappolo d'uva. Il peso del corpo poggia sulla gamba destra. Sulla testa ha una corona e il braccio sinistro $\dot{e}$ coperto da un mantello.

La figura copia in modo libero l'Hermes di Olimpia come avviene nella Casa di Sallustio (cat. 191/2) e nella Casa di Giasone (cat. 288).

Bibl.: Von Rohden 1887, 66-68, Taf. 6; Rizzo 1932, 72, tav. 107; Antonsson 1937, 115-118, fig. 13; Carpenter 1954, 11-12, pl. 2 fig. 5; Schefold 1957, 125 (vano c); Reutersward 1960, 178-179; Schefold 1962, 125, Taf. 94.2; Richter 1970, 199, pl. 713; Pfretschner 1977, no. 69; Repertorio $1983,236$.

VI 11,9 Casa del labirinto

Cat. 208/1. (16) Parete est, zona mediana; IV stile 
La parte superiore di una Venere pompeiana e un Eros e clo che resta di un gruppo rappresentante le divinita $d_{1}$ un larario Le dumensionı sono maggiori di normale Manca ll contesto (edicola, nicchia o architeflura) Lrt dea indossa un mantello i lold o tegfo nella smistra uno bectro

Bibl Schetold 1957, 126, Repertor1o 1983, 240

Cat 208/2 (22) Parete ovest, zoccolo. III stule

Ora quası dleggible e la decorazıone del calidarı Davantı ad uno stondo

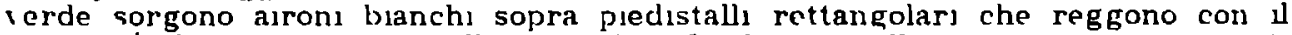
ciuffo J'orlo superiore dello zoccolo 1 due uccellı sono stat sistematı summetricamente con la lesta rivolta verso il centro tnche altrove su uecorazıonı di Ill stye questı uccellı tungono da atlantı, un valido confronto e tornito dalle figure del pistrinum sull'msula orientalis ad Ercolano (cat 038 ) Vere statue di uccelli possono essere servite da inodello per ll pittore (cf p 62-63.

Bıbl Mau 1882, 422, Schefold 1957 127, Peters 1971, 8-9 Fabbricottı 1976, 71-73, tıg 30 Bastet De Vos 1979, 53-54, tav YY-XXI, Repertorio 1983,241

Cat 208/3 (42) Parete ovest, zona mediana, II stule fase I c

L'architrave dell'edicola centrale e sorretto da centaurı o tritoni policrom che stanno su prore keggono con la mano esterna un tumone soprd la spalla c con l'altra la trabeazione Sono sistemate in modo simmetisco Ia pittura ord e molto danneggiata $\downarrow$ l che non permette una descrizione piu dettagliata

Le figure entrano nel genere del centauri epistyla sustinentes descrittı da Vitruvio (of $p$ 225 Nell'architettura reale non si conoscono esempi La nave come base di statue si incontra gia nel quarto secolo a $C$ e rimane in uso anche nell'epocd romana 602

Bibl Beyen 938, 254-267, Schefold 1957, 126, Schefold 1962, 38-39, Engemann 1967, 139, Taf 43-44, Strocka 1980, 16-17, Repertorı 1983, 248

VI 12 Casa del fauno

Cat 209/1 (37) Parete ovest, zona mediana, I stule +

Imitazione di un rilievo eseguita in rosso con linee gralle che imitano le vene del marmo Le sfumature scure contribuiscono alleffetto di plasticita Rappresenta un simposio di cinque figure bacchiche A smistra un centauro e seduto sulle zampe posteriori, nel centro si vedono un Sileno, isto sul dorso, e una donna, mentre a destra sı trovano altrı due centauri Tutte le figure hanno lo sguaido rivolto verso sinistra o stendono una mano in quella direzione Cio potrebbe significare che solo la parte destra del pinax e rimasta conservata La scena viene conclusa al lato destro da un tavolo di marmo o pietra sul quale si trovano piattı e brocche in bronzo

La figura del Sieno con lo scifo nel centro imita le divinita acquatiche, trequentemente presentı nelle rappresentazıonı di giardını ${ }^{63}$

602 P e una base di colonna nel Museo Jazionale Romano (CatRoma I 2, no IV 50)

603 P e cat 1851,251 Cf Bastet 1974, 221, Abb 12-14 
Lo stile delle figure è simile a quello dei rilievi neoattici e delle lussuose coppe quali i due scifi di Berthouville. 604

Bíbl.: Mau 182, 51; Pompei 1748-1980, 190 no. ADS 403, 147 (tav, a colori); De Vos/Martin 1984, 131-135, 139, fig. 3 .

Cat. 209/2. (43) Parcte?, zona mediana; I o Il stile iniziale + Un numero ignoto di páesaggi sacro-idilliaci monocromi su bugne è stato osservato da Mau. Forse si tratta di imitazioni di rilievi.

Bibl.: Mau 1882, 154, 162-163; Dawson 1944, 64; Lehmann 1952, 162 nota 104; Schefold 1957, 128; Von Blanckenhagen, Alexander 1962, 25; Peters 1963, 10; Silberberg 1981, no. 2; Moormann 1984b, 644-645.

VI 13, 2 Casa del gruppo dei vasi di vetro

Cat. 210/1. (8) Parete nord, zoccolo e zona mediana; III stile

Sotto i prospetti della zona mediana si trovano campi occupati da aironi bianchi resi in modo simmetrico. Hanno sulla testa una colonnma che sorregge l'orlo superiore dello zoccolo. Le teste sono rivolte verso l'esterno. Entrano

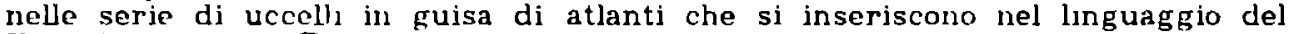
III stile (cf. p.127).

Una vignetta nella zona mediana ora illeggibile avrobbe contenuto secondo Helbig la Statue der Pallas, behelmt, in Chiton, auf der $R$. Eule, in der $L$. Speer. Sembra trattarsi di una Promachos. Si conirontino due statuette bronzee provenienti dalla Casa del citarista. $0^{\circ}$

Bibl.: Helbig 267; Schefold 1957, 129 (vano b); Bastet/De Vos 1979, 121 nota 33, tav. LVIII 107; Repertorio 1983, 263.

Cat. 210/2. (20) Parete ovest, quadro centrale e zona superiore; III stile (ora MN 111477)

Medea e le Peliadi. Medea regge nella destra l'idolo giallo di Atena a forma di una kore arcaica.

Nella zona superiore sarebbe stato visibile, secondo Mau, un paesaggio sacro-idilliaco con una Statue einer Gotheit davanti ad un tempietto.

Bibl.: A. Mau, BdI 1875, 182-188; Soghlano 5j3; Elia 1932, no, 132; Beyen 1938, Taf. 21, 43; Schefold 1957, 129 (vano d): Repertorio 1983, 263.

VI 13, 19 Casa di Sex. Pompeius Axiochus

Cat. 211. (f) Parete est, zona mediana; III stile +

Paesaggio sacro-idilliaco nel quale, secondo la descrizione di Sogliano, un uomo si inchina davanti ad un'erma barbuta, probabilmente di Priapo.

Bibl.: Sogliano 227; Schefold 1957, 131; Repertorio 1983, 271.

604 Cf. Van de Grift 1984.

60s Dwyer 1982, fig. 168-169. 
VI 14, 20 Casa di 11 Vesonius Primus di Orfeo

Cat 212/1 (1) Pareti nord e sud, zoccolo, zone mediana e superiore, III stule $+$

vello zoccolo aironı buanchi sostengono con un calice sulla lesta l'orlo superiore dello zoccolo sotto i campi laterali. Le ali e 1 picdi sono drvarucatı Gl anmals sono visti di tronte e hamno le teste rivolte verso il centro. Entrano nelle scrie di uccell reggenti nel III stue (of $p 27$ ).

- Vella predella sotto L campo centrale della parete nord si vede un giardino in miniatura su sfondo nero. Nella pergola centrale sorge una statua topiarı. indicata solo con una striscia sottile

Le edicole sopra 1 campl laterali - le uniche partı ancora visıbilı - sono occupate da sacerdoti e sacerdotesse egızı sopra piedistall a forma $d_{1} T$ Solo tre di essi possono essere descritti sulla parete nord due figure summetriche inaschli reggono una bacchetta nella destra e un sistro nella sinistra distesa. Sembrano avanzare verso il centro Sulla parete sud la figura femminue a destra e runasta conservata. Ha sulla testa un modıo e regge nelle manı un inodio e una situla

Le figure non corrispondono a statue note, ma entrano nelle serie di ligure che occupano le scaenae frontes nella zona superiore.

Bibl : Sogliano 487-489, Schefold 1957, 132-133; Tran Tam Tinh 1964, 128-129 no. 15: Bastet'De Vos 1979, 62-64, tav. XXV 47, De Vos 1980, 14, tav IX 2, Repertorio 1983, 279.

Cat. 212/2. (o) Parete ovest, zona mediana; IV stile

Rappresentazıoni di giardino al tranco del campo centrale con Orfeo Il telaso che le circonda e abbellito con osclla appesi allorlo superiore che mostrano satırı bianchı su sfondo rosso

Bibl - Herrmann II, Abb. 21, Taf 240, Schefold 1957, 132; Schefold 1962, 146, Grimal, 1969, 450 no. 16, Michel 1978, 396-397, Taf. 74; Zanker 1979a j08-j09, Abb 42; Jashemshı 1979, 72-73, fig 116; Repertorıo 1983, 280 .

VI 14, 22 Fullonica

Cat $213 / 1$. (p) Parete sud, quadro centrale; IV stule +

Ermalrodito e Eros A destra in fondo sorge la statua di Priapo. Indossa un mantello che viene alzato per scoprire il membro. Statuette di questo tupo si incontrano frequentemente a Pompei ${ }^{\circ 05}$ L'Ermafrodito e raffigurato nell'attegiamento dell'Afrodite di Arles

Bibl. A Mau, Bdl 1876, 24-28, Sogliano 74, Schetold 19j7, 133, Schelold 1962, 171, Taf 168.4, Repertorio 1983, 285.

Cat. $213 / 2$ (q) Parete sud, zona mediana; III stlle

Riunione dei lulloni presso un monumento composto da due colonne e una trave sulla quale sta la statua gialla di una figura temminle Regge nelle mani distese delle bacchette.

606 P.e. Dwyer 1982, fig 51, 183, 186. 
Moeller interpreta la scena come J Quinquatrus, festa dedicata a Atena Ergane La statua rappresenterebbe questa divinita Il tipo pero e piuttosto normale per Ecate Le bacchette forse sostituiscono ld lancia e la rocca, attribut 1 present 1 in altre raffigurazionı della disinita Diversa e l'Atena Ergane in 18,10 (cat 157) 607

Bıbl Schetold 19j7, 133, Moeller 1976, 46-49, pl VIII, Jashemskı 1979, 179, Repertorio 1983, 286

VI 1438 Casa di C Poppaeus Firmus

Cat 214 (h) Parete nord, zona superiore, IV stule +

Nelle vecchie descrizionı viene menzionata una figura temminle sopra un piedistallo rosso Indossa un chitone giallo e un mantello verde Regge nella destra un velo e con la sinistra un lembo del mantello forse si tratta di una figurd che occupa un'edicola

Bıbl A Mau, BdI 1878, 115-116, Sogliano 780, Schefold 1957, 136

VI 14,40

Cat 2151 (d) Parete ', zond superiore, I stule +

Due rappresentazioni monocrome descritte da Sogliano devono essere imitazıonı d) rulevi Sulluna di colore verde si vede una donna presso un bacino, accompagnata da un Eros forse si tratta di Atrodite

Sullialtra dı colore giallo una donna alata guida un pavone Anche in questo caso puo essere constatata una relusione con Alrodite

Bıbl Soghano 450, 609, Schefold 1957, 138, Repertorıo 1983, 295, De vos/Martin $1984,135,139$, fig 4

Cat $215 / 2$ (f) Tutte le paretı, zona superiore, IV stule +

Le edicole centralı sono occupate da statue di divinita, conosciute solo attraverso le descrizion di Mau Sulla parete sud si trova Apollo sopra un membro architettonico $\mathrm{Ha}$ una farctra sul dorso, una clamide sulle spalle e una corona sulla testa Regge un plettro nella destra stesa e una cetra nella sinistra

Sulla parete nord sta un Dionıso del tipo del Dioniso di Tivolı

Sopra l'entrata al peristilio, l'edicola contiene una Demetra seduta che regge una fraccola nella mano destra

Bıbl A Mau. BdI 187T, 211, Sogliano 97, 101, 157, RP 24 6, Schefold 19j7, 137

VI 14, 42 Casa del'inperatrice di Russia

Cat 216 (4) Parete ?, quadro centrale, III stule (ora IN 9378)

607 Roscher I, 681-682 Cf D1 Vita 1952-1954 
Elena o Fedra. A destra della figura centrale è la statua marrone di una figura femminile vestita di un peplo e un himation. Regge nella mano sinistra una lancia e nella destra stesa verso il basso una patera. Secondo Pfretscher ha un elmo sulla testa.

Se si tratta veramente di Atena puó essere la personificazione del luogo dell'avvenimento: nel caso di Elena rappresenta la citta di Troia, nel caso di Fedra Atene.

Il formato testimonia del costume di esporre statue di dimensioni ridotte all'interno delle case.

Bibl.: Helbig 1443; Elia 1932, no. 184; Schefold 1957, 138; Reuterswärd 1960, 136 nota 344 ; Pfretschner 1977 , no. 30.

VI 15,1 Casa dei Vettii

Cat. $217 / 1$. (b) Parete nord, zona mediana; IV stile

Il quadretto del campo destro contiene due cervi presso la statua di Artemide. E' dipinta in bianco e ne è visibile solo la sagoma.

Bibl.: Schefold 1957, 139; Pcters 1977, 113-114, pl. 88 fig. 59; Repertorio 1983,304 .

Cat. 217/2. (c) Parete nord, zona mediana: IV stile

Sulla lesena fra le entrate dei vani $g$ e $v$ si trova un quadretto con un'offerta di Eroti a Fortuna. A sinistra è la statua gialla di questa divinita, modellata secondo il tipo consueto. Essa è seduta su un trono c regge nella sinistra uno scettro e nella destra una patera. Sulla testa ha una corona. Accanto al trono si vedono un globo e un timone.

Bibl.: Schefold 1957, 141; Peters 1977, pl. 61.5; Repertorio 1983, 305.

Cat. $217 / 3$. (e) Tutte le pareti, zona mediana; IV stiJe

Le edicole centrali contengono erme in guisa di atlanti. Le loro teste sono, sulle pareti nord e sud, barbue o, sulle pareti ovest e est, inberbi e corronate.

La trabeazione dell'edicola sulla parete sud contiene due centauri in posizione simmetrica. Reggono con una mano la trave e poggiano sulle zampe posteriori.

Bibl.: Schefold 1957, 142; Peters 1977, 104-105, pl. 70-71.22-23; Schmidt-Colinet 1977, 147, 272 no. 13; Repertorjo 1983, 307.

Cat. 217/4. (h) Parete sud, quadretto centrale; IV stile

Due galli stanno presso un erma gialla. Si tratta del genere del ludi gallinari. l'erma simbolizza l'ambiente sportivo dove si presentavano queste gare.

Bibl.: Maiuri 1950, 194 no. 67; Schefold 1957, 143; Croisille 1965, 91 no. 243, pl. 76 ph. 145; Pelers 1977, 97-98, pl. 60.4; Kepertorio 1983, 308.

Cat. 217/5, (i) Parete nord, quadretto centrale; IV stile Natura morta con galli. Dietro la mensa agonistica vi $\dot{e}$ la statua gialla di un uomo nell'atteggiamento dell'atleta westmacott. Ha la mano destra sopra la testa e regge nella sinistra lungo il fianco una lancia. La gamba destra è di appoggio. 
Bibl.: Maiuri 1950, 193 no. 65; Schefold 1957, 143; Croisille 1965, 91 no. 242, pl. 76 ph. 146: Repertorio 1983, 308.

Cat. 217/6. (1) Parcle ovest, zona mediana; IV stile

Il quarto pannello da sud contiene una menade presso la statua verde-bianca di Dioniso. Egli indossa un himation e ha una corona sulla testa. E' barbuto. Regge nella sinistra una patera e nella destra un tirso. E' rivolto verso destra.

Bibl.: Sogliano 1898, 273, lig. 15; RP 128.2.10; Beyen 1950, pl. 14.6: Schefold 1957, 144: Peters 1977, 107-110; Pfretschner 1977, no. 61; Repertorio 1983, 312.

Cat. $217 / 7$. (n) Pareti sud e nord, zona mediana; IV stile

Sfingi lemminili alate di colore verde sono sedute su basi rettangolari sopra la trabeazione attorno all edicola centrale.

Il quadro centrale della parete sud raffigura il supplizio di Dirce. Secondo Sogliano e Mau il gruppo copia il famoso Toro Farnese. Per avvalorare questa ipotesi, pero. mancano argomenti validi. Il pittore non ha voluto copiare una composizione statuaria.

Bibl.: Sogliano 1893-1896: A. Mau, RM 11 (1896) 46: Schefold 195i, 144; Peters 1977, 106-107, pl. 74.27, 80.41; Repertorio 1983, 314.

Cat. 217/8. (p) Parete est. zona mediana; IV stile Pegasi cavalcanti verso il lato esterno delineati simmetricamente si trovano come acroteri nell'architettura gialla delle prospettive fra i campi.

Bibl.: Peters 1977, 105-106, pl. 80.39; Repertorio 1983, 316.

Cat. $217 / 9$. (q) Pareti est c ovest, fregio e zona mediana; IV stile

Il fregio fral lo zoccolo a la zona mediana contiene scene mitologiche e di genere. Nel quadrotto con lfigenia in Tauride sulla parete ovest si vede la statua gialla di Artemide su una colonna. Indossa una tunica corta e avanza verso destra, mentre prende con la cestra una freccia dalla faretra sul dorso e regge un arco nella sinistra. Corrisponde al tipo dell'Artemide di Versailles. Lina statuetta del genere e stata trovata a Pompei. ${ }^{\circ}$ "

La scena degli amorini orefici sulla parete est contiene un armadio nel quale sta la statuetta argentea di Afrodite. Ha la destra presso le tempie e la sinistra davanti al grembo. Poggia con il gomito sinistro su un pilastrino. La gamba destra è di appoggio. Secondo Pfretschner il colore grigiastro imiterebbe l'avorio.

I candelabri fra i pannelli sono sorretti da erme gialle. Ai fusti sono attaccate uraccia che reggono vari oggctti, come pedum, lepre, flauto e patera.

Bibl.: Schefold 1957, 147-148; Peters 1977, 103, pl. 68 fig. 19; Pfretschner 1977, no. 3, 14; Repertorjo 1983, 316-321.

VI 15, 2 Casa di P. Crusius Faustus

Cat. 218. (k) Parete nord, zona mediana; III stile +

608 Bieber 1961b, fig. 285 (=MN 239). 
Rappresentazione di giardino Due pergole sono sistemate l'una dietro l'altra. Quella in primo piano e sorretta da erme semplici In quella in fondo $v 1$ e la statua di una figura temrninile (?) vestita di un lungo mantello e posta sopra una base rettangolare Iutto e noto solo grazie ad un disegno schematico

Bibl A Sogliano, NSc 1897, 19-20, A Mau, RV 13 (1898) 5, Schetold 19iT, 150 (III stule, vano c), Grimal 1969, 451 no 18 , tig 13 (a p 226), Repertorio 1983, 323

VI 15, 7 Casa del principe di Napolı

Cat 219 (m) Pareti sud $p$ ovesl, zona mediana, IV stule

Figure vive, modellate secondo tipi statuarı, riempiono le edicole centralı Sulla parete sud si vede Dioniso nell'atteggiamento del Dioniso di Tivoli che ha un mantello azıurro sopra le spalle Sulla parete ovest ifrodite e modellata secondo il tipo del'Anadyomene

Bibl Lehmann 1953, 69, 170-171, Schefold 1957, 152 (1 ano g), Pompel 1748-1980, 89, fig 1-2, Repertorio 1983, 336, Moormann 1983b, 82, 98, fig $18 \mathrm{a}-\mathrm{b}$, Strocka 1984b,30-31, 4bb 107-108, 119,158-160, 165

VI 16,7 Casa deglı amorin doratı

Cat 220/1 (F) Framments isolatı, III stule (?) +

Sogliano descrive due frammentı isolatı con figure che stanno sopra un membro architettonico In una si vede un satıro che regge sulla spalla destra il Dioniso fanciullo mentre nella mano destra ha un pedum Sulla testa ar robbe appoggiata, ista la presenza di una linea, una colonna snella

Il secondo trammento mostra una mende lestita di un mantello bigıo Regge nella sinistra davantı al petto un cestino e ha l'altra in riposo lungo l fianco Sulla testa si trova un'asta fusa con motivi decorativi

Forse 1 due trammentı provengono da una scaenae frons nella zona superiore di una decorazıone di III stile come si vede fra l altro nella Villa imperiale (cat 305, 3)

Bibl A Soglıano NSc 1908, 32-34

Cat 220/2 (M) Parete nord, zona superiore, IV stile

Imitazioni di rilievi in rosso su uno sfondo giallo si trovano a a sinistra e a destra della porta Su quella a destra sı vede un offerente presso un vaso di proporzioni stravagantı e un'erma Su l'altra $v$ e una statua di Ecate che regge una fiaccola con entrambe le manı stese in avantı Tutto e reso in modo schematico

Bibl Schefold 1957, 155, Repertorio 1983, 351

Cat 220/3 (Q) Parcti nord e sud, zona mediana. IV stile

Le archstetture delle edscole centrali contengono troles come elementı costruttivi Sulla lrabeazione del centauri resi schematicamente lungono da acroters

Bibl Schefold 1957, 155, Repertorso 1983, 353.

Cat 220,4 (R) I'arete sud, quadro centrale, IV stule 
Atteone e Artemide Artermide e stata modellata secondo due tipı statuarı ben

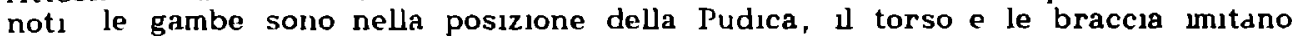
la Venere Medici Questa analısı si deve a Herbig (in Herrmann)

Bibl Herrmann II, 44, Tat 229, Schelold 1957, 156, Repertorio 1983, 354

VI 16,15 Casa dell'ara inassuma

Cat 2211 (B) Parete ovest, zona superiore, IV stle

Scacnae trons composta da elementi eterogenel Nel centro si trova un paesaggio sacro-idilliaco. La schola nel centro e sorretta da colonne e da una cariatide gialla vestita di un chitone senza maniche e un mantello. Ha le braccid stese in avanti ed e rivolta verso destra Sulla testa poggia un elemento vegetale a la trave

A sinistra e d destra degli hospitalia al fianco del paesaggio vi sono, sopra parti aggettantı dell'architettura, due statue verdi A sinistra sta Poseidon che regge nella destra alzata un tiodente e nella sinistra, stesa verso il basso, un delfino II prototipo, attribuito a Lisippo, e noto in numerose copie 609 A destra si trova una Nike posta sopra un globo che regge con la mano destra presso il fianco una palma e nella sinistra una corona Imitd ll tupo della Victoria romana 610

Bibl lppel 1951, Lchmann 1953, 129-130, pl 30, Little 1956, 27-33. Schefold 1957, 156-157 (vano a), Schefold 1962, 126-127, 138 , Peters 1963, 170 , fig 168, henner 1964-1965, 40, Winkes 1969, 202, Allroggen-Bedel 1974a. 149 no 64 , Slber berg 1981, no 130, fig 107, Repertorio 1983,357 , Moormann 1983b, 112-114, fig 26

Cat 221'2 (D) Parete ovest, zona mediana, IV stule

L'drchitettura rossd che circonda il quadro con Narciso contiene due $H^{d}$ úfthermen di satiri. La ligura a sinistra regge nella mano destra stesa un cestino e ha la sinistra davanti al petto L'altro satiro regge un pedum nella destra lungo ll fianco e ha la sinistra sopra la testa nell'atteggiamnto doll'aposkopon

Bıbl Schefold 1957, 157; Schefold 1962, Taf 102, Erıstov 1979, 170-171, Bdrbet 1981, 924, Repertorio 1983, 358

VI 16,28

Cat 222 (F) Pareti sud e est, zona mediana, IV stle +

Paradeisos con tori Sulla parete sud a destra si trova sullo sfondo l'erma itıtallica rossa di Priapo

Sulla parete est sl vede sopra una specie di piranide, una figura umana stante di profilo a sinistra, dipinta in rossa (Priapo?) (Sogliano)

609 E Wbdt, RE XXII 1, j39-j40, s.v. Poseidon, elenca 67 esempları.

610 Holscher 1968, 6-47 Si confrontino le figure di Poseidon e Anfitrite sul mosaico parietale nella Casa di Nettuno e Antitrite ad Ercolano (Sear 1977, $95-96$, pl 40 1) 
11 16. 31

Cat 223 (J) Parete sud, quadro centrale, IV stule +

Pdesaggio sacro-ıdlliaco $A$ sinistra di un albero sacro si trova la statud rossa di Dioniso $E^{\prime}$ rivolto verso destra e regge nella destra stesa in avant un cantaro

Bibl A Soglıano, NSc 1908, 286, Schefold 1957, 159, Kepertorio 1983, 371

VI 16 Castellum aquae

Cat 224 Parete nord sopra l'entrata dellacqua, IV stıle

Sullo sfondo bianco si vedono tre ninfe acquatiche e una divinita fluviale sdraiata, tutte vive. ma modellate secondo tıpı statuarı

A simstra e una ninfa che regge davanti al grembo un bacino giallo Indossa un mantello bianco che lascia scoperta la parte superiore del corpo Segue l tipo della Venere Landolina

Nel centro si trova una donna nell'atteggiamento dell'Anddyomene

A destra si vede una donna con un mantello verde sulle gambe Regge con entrambe le mani una brocca dalla quale esce dell'scqud

Accanto si trova la divinita maschule sdraita che poggia con ll gomito sinistro su una brocca

La combinazione di queste divinita acquatiche si incontra per esempio su del rihevi a Napoli 611

Bibl R Paribeni, NSc 1903, 29, A Mau, RM 19 (1904) 47, Eschebach $1979 b, 35,4 b b 6$

VI Insula accidentalis 10

Cat 225/1 (3) Paretı ', zone mediana e superıore, II stlle, fase Ic o IIa (ora MN 8846, 9301, 9541)

Davantı ad una parete composta dl ortostatı rossı (ora scoloritı e diventatı nerı) vi sono, sopra piedistall rettangoları verdi, statue policrome di Apollo e Artemide Il verde delle basi imita una copertura di bronzo 612

Artemide (MV 9301) indossa una corta tunica gralla con il bordo viola e strisce bianche e un mantello verde La gamba destra e di appoggio Ha una corona sulla testa Regge una lancia nella destra alzata e un arco sotto ll braccio sinistro Spinazzola confronta la ligura con una statuetta proveniente dalla Casa di C Arrius Crescens 613

611 MN 6706, 6707, 6710 Cf Muthmann 1975 Si vede anche Trillmich 1983

012 Cf Drelhaus 1969

613 Casa del moralista Spinazzola 1953, II, 759-761, fig 740-741, Zanker 1979, 488-489, Abb 19-21 
Apollo (MN 9j41) ha un mantello rosso sopra le gambe e sopra l braccio sinistro, ha sulla testa una corona Il gomito sinistro poggia su una cetra che e addossata a un omfalo. La gamba destra e di appogg10 La destra regge un ramo d'olivo lungo l fianco. L'spollo di Cirene tuene menzionato da Allroggen-Bedel rome contronto, doch gibt es unter dem erhaltenen Bestand keinen genau entsprechenden Typus Ind statucta pompoiana potrobbs llustrare un'ditma birlillone 614

Un pmax della zona superiore (MA 8816) mostra Zous. Churone e Ipollo presso un tripode 1 pollo e modellato nell'atteggiamento del Licio.

Bibl. Helbig 184, 236, RP 23 4, 52.3, Spinatzola 1953, II, 761, fig 742; Schefold 1957343 (Vespasianisch), 350 (um 50). Allroggen-Bedel 1975b, 117, Allroggen-Bedel 1976a, 149, 160-161, Pfretschner 197i, no 23

Cat. 225/2 (9) Paret1 ?, zoccolo, Il stle. fase IIa o b (ord IV 8691, 8817)

Due trammentı provenıentı dallo zoccolo, forse dagli scompartı sot to le colonne della zona mediana In uno (M\8G91) sl vede un grito policromo che sorregge con la testa l'orlo superiore dello zoccolo

L'altro trammento ( $M N$ 8817) mostra una sfinge femmmule alata di colore bianco-giallo che sorregge l'orlo superiore $S_{1}$ controntı la decorazsone di IV stile nella Casd delle vestah (cat 198 1)

Bibl Allroggen-Bedel 1976a, 167-169, Abb 4-5.

Cat. 225/3 (11) Paretı ', zona mediana, IV stule (ora II 9476, 9512)

Duc passaggl sacro-1dlllacl provementı dal campl lateral 1 L'uno (MN 9176) raffigura un asino presso la statua itifallica rossa di Priapo Attorno al tallo si vede una corona

L'altro (HN 9J12) mostra una porta sacra e una statua schematica sopra una colonna

Bıbl Rostowzew 1911, 86, Schefold 1957, 347; Allroggen-Bedel 1976a, 178-179

VI Insula occidentalis 19-26

Cat 226. (?, piano sotterraneo) Parete ?, quadro centrale, ? stle +

Una donna suona la lira Mau e Sogliano videro dretro di essa un pilastro quadrato sormontato da una statuetta, che forse porta delle fiaccole. Forse si tratta du Ecate.

Bıbl. A Mau, BdI 1878, 236-237, Soglıano 645; Schetold 1957, 160.

VI Insula occidentalis Masseria Cuomo

Cat 227. (?) Parete? quadro centrale; IV stle (ora IN 9231)

Le tre Grazie stanno in un paesaggio sommariamente delineato Le due figure estorne raggono malzi di plante acquatiche nelle mane stese. Il gruppo e stato modellato secondo un esempio ellenistico, forse creato da un artista neoattico nel primo secolo a $C$. Si conoscono vari esempi in scultura (Siena,

614 Dwyer 1982, 66, fig 16 Per $\downarrow$ tipo of Pinkwart 1965, 154-157. 
Louvre, Vaticano, Cirene, Side), mosaico (MN 10004, cat.115) e pittura (cat. $004,5,011,135,279,3,298$ ).

Il dibattito sul medio originale, pittura o scultura, non ha avuto un risultato conclusivo. Herrmann e Schwarzenberg suppongono che una statua fosse l'archetıpo, secondo Schmidt si tratterebbe di una pittura. Trillmich considera la questione priva di fondamento sulla base di argomenti estremamente validl (cf. p. 76 ). ${ }^{625}$

Bibl.. Helbig 856b; Hermann I, 62-64, Taf. 50; RP 158.2: Elia 1932, no. 135; Von Salis 1947, 164, Taf. XLVlb; Schefold 1957, 161; hellner 1974, Taf. 14.1

VI Insula occidentalis

Cat. 228. (?) Parete ?, zona mediana; IV stile (ora antiquarium 17736)

Tre figure stanno presso una colonna sormontata da una statua ora di difficile interpretazione.

Bibl.: inedito.

VI Strada augustalis

Cat. 229. (?) Parete ?, , zona ?; IV stile +

Paesaggio sacro-1dillaco nel quale sorge al lato sinistro una statua delineata schematicamente posta sopra un piedistallo alzato.

Bibl.: Rostowzew 1911, 84-85, fig. 51; Silberberg 1981, no. 72 , fig. 169.

VI Strada della Fortuna, lato ovest

Cat. 230. (atrio) Parete sinistra, zoccolo: IV stile (ora Antiquarium Pompei dove irreperibile)

Paesaggio sacro-idilliaco di alto livello artistico. Sugli appoggiatoi della rampa che metle nell'edificio centrale sono disposte due figure temminili simmetriche vestite di lunghi mantelli. Reggono un oggetto nella mano distesa al lato interno.

Bibl.; Mau,Ippel 1928, 213-214, Abb. 115; Spinazzola 1953, II, 845, fig. 831-832; Poters 1963, 162, fig. 161; Ślberberg 1981, no. 74, fig. 72 .

VII 1,8 Terme stabiane

Cat. 231/1. (IV) Tutte le pareti, zona mediana; IV stile +

615 Sulla statua esiste una vasta bibliografia: Schmidt 1925, Becatti 1937; Lullies 1948; Paribeni 1959, 108-110; Schwarzenberg 1966, 39-42; Muthmann 1975, 92 nota 45: Trullmich 1983: inoltre la bjblingrafia per la pittura . 
Solo attraverso vecchie descrizioni si conoscono le rappresentazioni del glardino di questo bagno Le figure umane di colore bianco forse sono state dipinte come statue A sumstra e a destra della porta e accanto alla micchid di tronte ad essa si trovano due ninfe vestite $d_{1}$ un peplo e una sopravieste Forse si tratta delle ninie a bacino ( cf $p$ 61).

Fra le nicchie del lati sud e est sono dipinti un ermatrodito e un sleno in guisa di fontana L'ultimo e sdralato, appoggiato su una biocca, si presenta secondo il tipo consueto del fiume sdralato che si incontra altrove (ct p 64 )

Bıbl Helbig 1057, 1868, Schetold 1957, 163, Veuerburg 196j, 127-128, Grmal 1969, 193-194, 451-452 no 19, Peters 1971, 12, IJe los De Vos 1979, $85-86,90-93$, Taf $7 b, 11 a-b, 66 a-c, 67 a$

Cat $231 / 2$ (F. G) Tutte le paretı, IV stle +

Rappresentazionı di giardinı conosciute grazie a vecchie descrizion e fotografie non molto chiare Sulla parcte ovest una nicchid da ninfeo e fancheggiata da ninfo bianche che reggono tra le mani datanti al grembo una conca Hanno le gambe coperte da un mantello e poggiano su bası rettangolarl Le conche sostituiscono le vasche normalı

Statue bianche di alungl sdraiate sia femminul the maschul sono disposte fra i cespuglı saturı in bianco, probabumente di stue ellemstico, vengono menzjonati per le altre pareti

ln di un paesaggio nulotico si vedeva secondo Minerini (citato da De Vos). l'immagine di una divinit \pm a virile avviluppata in ampio panno e col capo coronato

Bibl Schetold 1957, 163, De Vos/De Vos 1979, 85-89, Tat 56b, 65a (con bibl )

Cat 231/3 (palectra) Parete ovest, zona mediana, IV stule

Rlievo a stucco dipinto rappresentante una scaenae frons lella parte sumstra di essa l'edicola centrale a due piani e occupata da due ingure statuarie, l'una sopra l'altra Quella interiore e un Eracle ebbro che tenta di scendere da una scaletta Nella sinistra regge una clava appoggiata alla spalla, nella destra tesa tiene un cantaro un piccolo satiro dccorre per sostenere l'eroe sul lianco sinistro L'Eracle, rappresentato da two corrisponde al tupo ellenistico del bibax di cul sl conoscono varie cople in marmo, p e nella Casa di $\mathrm{I}$ Lucictius e nella Casa del cervi ad Elcolano

Vel registro supei iore un'Arlemide arcasstica vestita di un peplo sta sopra un candelabro con le gambe unite Rappresenta ll tipo della potnia theron ' $\mathrm{E}^{\prime}$ tiancheggiata da un cane e da un cerro Regge nella destra una patera, mentre con la sinistra solleva un lembo del vestito von e simile alle rappresentazion veramente arcaiche, ${ }^{616}$ ma potrobbe raffigurare un nuovo tıpo arcaistico finora sconosciuto

Negli archı che mettono nelle sale $F$ e G. deglı atletı, di nuovo a stucco, sı reggono soprd un canclelabro Sono rivolti verso sinistra c sono completamente nudi

Bibl Schefold 1957 162, Mielsch 1975, 59-62, 142-146 no k54, Nielsch $1979 a, 74-75$, Taf 24c, 56, 58-59, Yoormann 1983b, 111-112, ing 25

VII 1, 25 Casa dı Sirıco

626 Cf Christou 1968 
Cat 232 (10) Tutte le pareti, zone mediana e superiore, IV stle

Statue policrome raffigurantı Muse si delineano su 8 campl lateralı Poggiano su mensole viste di scorcio $\mathrm{Vel}$ prospettı della parete nord una nona Musa e Apollo Vusagete completano la serie

Parete ovest La figura nel campo sud e andata perduta, quella nel campo nord rappresenta Polinnia (Ilelbig 888) in chitone rosso e mantello bianco Il peso del corpo poggia sulla gamba sinistra Tiene la destra nascosta nel mantello e regge con la smistra un lembo del chitone all'altezza della coscia

Parete nord campo ovest Euterpe (Helbig B63) - ormal quasi lleggible indossa un chitone rosso e un mantello verde Entrambe le mani reggono un flauto davantı al petto

Parete nord, prospetto ovest 4pollo Musagete (Helbig 186), nudo eccetto le spalle coperte da un mantello rosso sta in piedi l peso del corpo poggia sulla gamba sinistra La mano destra e in riposo lungo ll Lianco, la sinistra regge una cetra che poggla a terra accanto al suol pied.

Parete nord, prospetto est Callope o Clio (Helbig 860), vestita di un chitone bianco e un mantello dzzurro, regge nella destrd tesa un rotolo e tiene la sinistra in riposo davanti al petto

Parete nord campo est Melpomene (Holbig 872) indossa un chitone e un mantello, cuı color sono svanitı Regge nella sinistra una cetra che suona con la destra

Parete est, campo nord Talia (Helbig 882), vestita di un chitone verde e un mantello bidnco poggia sulla gamba sinistra vella destra tesa regge una maschera tragica, con la sinistra la clave erculea vicino al lianco

Parete est, campo sud Lrania (Helbig 890), ormal svanita, indossa un peplo rosso e un mantello verde Indica con una bacchetta nella destra un punto sul globo che tiene con la sinistra

Parete sud, campo est Tersicore (Helbig 869) avanza la gamba destra a indossa un chitone rosso e un mantello verde Suona la lira posta nella destra con la mano sinistra

Parete sud, campo ovest Erato (Helbig 865) indossa un chitone bianco e un mantello verde con orlo rosso Regge una cetra nella sinistra La testa piu una parte del corpo supcriore sono andate perdute

Nel prospettı sulla parete nord si distinguono due acroteri simmetrici in forma di gruppi bronzel un centauro e seduto sulle zampe posteriori e agita la clava sopra ld testa Il leone ricorda la statua di bronzo della Casa del citarista is

Nella zona superiore delle paretı nord e sud una statua di colore grallo posta sopra un piedistallo rettangolare occupa un'edicola Quella della parete sud a andata perduta, dell'altra e rimasta solo la sagoma Secondo Helbig (273b) quest'ultima figura rappresenta un Ares Indossa una clamide e ha un elmo in testa Regge una lancia e uno scudo nella sinıstra e nella destra ha un oggetto a forma di ramo, che pus probabilmente va identificato come una spada

Bibl Helbig ( $v$ numerı per figura), Schefold 1957, 164-166, Schefold 1962, 120-121, Taf 151,100 , Pfretschner 1977, no 53

VII 1, 40 Casa di Caesius Blandus

Cat $233^{\prime} 1$ (6) Parete est, quadro centrale, II stule, fase IIb +

617 Dwyer 1982, fig 137 
Scena tigurativa con una statua di bronzo di cui non si conoscono descrizioni o disegni Mau contronta ll sistema della parete con quello della Lisa degli epigranum

Bubl Mau 1882, 211212 , Schefold 1957, 167, Beyen 1960, 239, Abb 99.

Cat 233'2 (12) Paretı nord, ovest e sud, zone mediana e superiore, II stule, fase IIb

Su un podio ddvantı ad una serie di ortostatı una coppla di erme femminilı sostiene l'architrave, costruzione simule a quella della Casa del criptoportico (cat 148/2-3) Sulle paretı sud e nord le donne, interpretabil come menadi. reggono un testone, sulla parete ovest esso e sospeso alle spalle delle erme

Paretı nord e sud Menadı nude, sistemate simmetricamente, dipints in color 1 naturali, sorgono da plastrı rosso-rosa a loro volta coperti da loghe verdi In testa hanno una corona e un piccolo calice verde che sostiene l'architrave Le loro alı sono di colore rosso chuaro

Parete ovest Donne vestite di tuniche di colore vermiglione con orli in rosso cupo poggiano su plastrı come le erme delle altre paretı La figura a sinistra suona una tıbıa lunga Manca la parte superıore dell'altra tigura ton posseggono alı

Nel registro superiore della parete sud si trova una brena figurativa, ora illeggibile, sulla quale alcune tigure temminuli eseguono und danza orgiastica davantı ad un'erma itifallica di Priapo Si puo confrontare questa scena con l mosaico in opus sectlle di I $2,6,61$ P

Bıbl - Sogliano 757, Mau 1882, 210, Schefold 1957, 167-168, Beyen 1960, 234-259, Abb 95-101, Schefold 1962, 41, 52, Tef 24, 27, Schmidt Colinet 1977, 147, 267 no P6

VII 2,14

Cat 234/1 (a) Parete sud, quadro centrale, IV stle +

Giudizio di Parıde Sullo stondo si distınguono una porta sacra e una colonna sormontata da una statua resa in modo sommario Le flaccole appese alla colonna potrebbero identificare la figura come Ecate, qui presente soltanto per rinforzare l carattere idlliaco del paesaggio e priva di un ruolo nella scena mitologica

Bıbl Helbig 1283b, Taf 25, Herrmann II, 28-29. Taf 216. Schefold 1957, 169, Beyen 1960, 442-443, Paar 1962, 123, Abb 24, Peters 1963, 129, thg 105

Cat 234/2 (b) Paretı est e ovest, zona mediana, IV stıle + Rappresentazione di giardino, nota solo da un acquarello Sulla parete ovest si distıngue una statua bianca sopra una base quadrangolare accanto ad una fontana $S_{1}$ tratta di und ligura femminile che indossa un mantello solo sulla parte inferiore del corpo La gamba sinistra sostuene il peso del corpo Le manı alzate accanto alla testa ricordano il tipo dell'Anadvomene Sogliano distinse uno scettro nella mano destra Schetold interpreta la figura come ninfa fluviale, Jashemshi invece pensa a Atrodite, ipotesi piu probable in quanto l'atteggiamento del corpo e la collocazione nel giardino sono tipici di questa divinita Statuette di questo modello in marmo sono state trovate $p$

618 Dohrn 1965, Taf 562 
in VII 3, 6 e VII $12,23,{ }^{69}$ Le dimensioni ridotte corrispondono al formato della scultura da giardino in generale. ${ }^{620}$

All'orlo superiore della scena di giardino sulle pareti est e nord sono attaccati un oscillo con testa in rilievo bianco, una maschera policroma e una pelta bjanca. Sono sinili ai tanti esempi trovati a Pompei.621

Bibl.. Sogliano 694; Schelold 1957, 169; Grimal 1969, 452 no. 20; De Vos 1977, 40; Jashemski 1979, 62, fíg. 100.

VII 2, 16 Casa di Gavius Rufus

Cat. 235/1. (u) Parete sud, quadro centrale; IV stile +

Licurgo e una menade (?). A sinsstra sorge l'erma itilallica di Priapo.

Bibl.: Sogliano 614; RP 194.5; Herter 1932, 160 no. 198, Schefold 1957, 170

Cat. 235/2, (o) Parete sud, zona superiore; IV stile +

Sogliano menziona le statue di uomini sotto il paragrafo ritratti realistici. Ci si puo domandare se si abbia a che fare con rappresentazioni di personaggi reali simili ai cosiddetti membri della famiglia giulio-claudia della Villa di $S$. Marco (cat. 009/4).

Bibl.: Sogliano 682, 683; Schefold 1957, 171.

VII 2, 18 Casa di Vibus Italus

Cat. 236. (1) Pareti?, quadro centrale; III stile +

Due paesaggi sacro-idillaci. Sull'uno si vede un'erma di bronzo presso un ruscello vicino ad un gruppo di tre statuette temminili sopra una base comune. Queste tre possono rappresentare sia un hekataion sia un gruppo di divinità fluviali.

Il secondo paesaggio mostra una porta sacra all'interno della quale si trova una statua di bronzo di Ecate. Essa regge in entrambe le mani tese in avanti due fiaccole. In fondo a sinistra sta l'erma itifallica di Priapo, coperta da un cappello.

Bibl.: Sogliano 228, 686; Rostowzew 1911, 40-41. Abb. 17, 18; Herter 1932, 159 no. 193; Lehmann 1953, 108, fig. 60; Schefold 1957, 171; Von Blanckenhagen/Alexander 1962,35-36, pl. 52.1, 53.2; Muthmann 1975, 28-29, Abb. 4; Pfretschner 1977, no. 78; Bastet, De Vos 1979, 84; Silberberg 1981, no. 31. fig. 33-34.

VII 2, 20 Casa di Popidius Priscus

Cat. 237. (o) Parete nord, zona mediana; II stile, fase Ic

\footnotetext{
619 Jashemski 1979, fig. 197-198.

620 Cf. Merker 1973, 7.

621 Cf. Dwyer 1981.
} 
Fra due colonne ve una $H^{\mathcal{L}}$ úftherme bianca sopra un piedistallo che imita un blocco di giallo antico un satiro regge un'oinochoe nella destra (ora perduta) lungo I fianco e nella sinistra, daranti al petto, un piatto di frutti L'arambraccio e coperto da un panno azzurro Diversamente dalle serje di erme di II stle l'crma funziond qui come statua a se stinte, esposta in un intercolunnio, contesto che troviamo raramente, per escmpio nelle Terme suburbane di Ercolano 622

Bıbl Helbig 442b, Mau 1882, 274-276, Schefold 19j7, 172, Beyen 1960, 113-414, Abb 185, De Vos 1976, 51, pl 54-55

VII 2, 25 Casa delle quadrighe

Cat 238 (k) Parete sud, zona mediana, IV stule

Le lesene rosse che dividono una scena di giardino in due partı, sono ornate da erme in bianco A sinistra si vede un erma con la testa do un giovane umberbe, coronato, che secondo Helbig rappresenta il Doriforo di Policleto 623 I trattı del 1150 pero si presentano molto schematics sonza che $v$ sia una carattorizzazione specitica A destra sorge und Mantelherme sumle a quella delle Terme stabiane $5 a_{4}$ Il mantello non copre solo ll corpo e le braccid, ma anche la testa

Bubl Holbig 17j6, Gismal 1913 490-491, pl IA 119 3 Schefold 1957, 173. wimal 1969, 4j3-15.1 no 21 . Mic hel' 1978,398

VIl 2, 45 Casa dell'orso terito

Cat 239 (m) Paretı est e nord, zona medand, IV stule +

Ratigurazione $d_{1}$ un glardino di cul restano visıblı solo tracce vaghissume Sulla parete est una ninfa su di una base rettangolare regge, daiantı al grembo, una vasca nella quale zamplla acqua $L_{n}$ drappo vola copre le gambe Corrisponde alle numerose statue clel tipo clella Venere Landoluna

Sulla parete nord una sfinge alata femminule regge una vasca rettangolare sulla testa Anche qui la lontana e ratfigurata funzıonante L'anmale e rivolto a sinistra ed e inginocchuato Le alı lunghe tocano l'orlo della vasca, ma non la sostengono Il colore monocromo rosso e particolare c ta pensare alla moda del porído nel tardo umpero Si pensi alla fontana con sfinge nel Museo ndzionale a $\backslash$ apolı 625

La fontana a mosaico sulla parete nord ha un tregio con una reduta di mare, nel centro della quale sorge la statua gidlla di Posejdon, modellato secondo il noto tipo lisippeo della statua di honchreas sta con ll prede simistro su di una roccia e poggia con la inano smistra hul ginocchio sinistro,

\footnotetext{
622 Malurı 1958, fig 120-121, Manderscheid 1981, 82 no 126, Taf 23

623 CF l'erma della Vlla del papirı (Pandermalis 1971, Taf 84 1, Zanher 1974, Taf 77,312 )

624 Eschebach 1979a, 17, Taf 52b, Manderscheld 1981, 82 no 27, Taf 23

625 Gnolı 1971, fig 40 Cf un sostegno di tarola a Roma Von Hesberg 1980b, Taf 851
} 
mentre regge un tridente nella destra levata. ${ }^{26}$

Bibl.: Helbig 1060, 1778; Schelold 1957, 175; Reuterswárd 1960, 182; Neuerburg 1965, 128-12! no. 31, fig. 117; Grimal 1969, 454 no. 24; Srar 1977, 77 no. 38, pl. 2.2, fig. 22; Jashemski 1975, fjg. 72.

VII 3, 25

Cat. 240. (i) Parete ?, quadro centrale; III stile +

Paesaggio sacro-idilliaco. In un tempietto si trova la statua gialla di Dioniso. Il doo siede su di un trono, e vestito di un chitone lungo e ha una corona sulla testa. Nella destra alzata regge un tirso, mentre con l'avambraccio sinjstro poggia su un tumpano. Il tipo statuario non è noto per Droniso, ma va paragonato con le statue ellenistiche di divinita come Cibele. Infatti Schefold crede che vi sia rappresentato Cibele/Iside.

A sinistra in tondo $e$ a destra, accanto a un fiume, si trovano erme itifalliche di Priapo, la prima ornata con un cappello.

Bibl.: Soghano 246; Rostowzew 1911, 45 Abb. 25; Schefold 1957, 17; Von Blanckenhagen, Alexander 1962, 35-36, pl. 53.1; Peters 1963, 72, fig. 56; Schefold 1965, 116, Taf. 47.2; Silberberg 1981, no. 30. fig. 32.

\section{3, 30 Casa del panettiere}

Cat. 241. (k) Parete ?, zona mediana; IV stile

happresentazione di giardino molto svanita. Fra le piante sono esposte una vasca marmorea e due sfingi bianche femminili alate, appena visibili. All'orlo superiore sono appesi due oscilli, sul destro dei quali sı distingue una testa di Medusa.

Bibl.: ''. Helbig, BdI 1864, 120-121; Schefold 1957, 179, Grumal 1969, 454 no. 23; Jashernski 1979 , 59, fig. 96 (senza statue).

VII 4,2

Cat. 242. (facciata esterna) zona mediana; IV stile +

Hermes e Afrodite. Vel centro sorge una colonna sormontata dalla statuetta rossa di Priapo. Appoggia su un pilastrino con il gomito sinistro, mentre regge un ramo di palma nella sinistra.

Bibl.: Helbig 20; RP 97.3; Herter 1932, 138 no. 36; Boye 1937, 111 no. 6; Schefold 1957, 179 .

VII 4, 10 Casa di Bacco

Cat. 243. (?) Parete?, quadro centrale; ? stile +

626 Cf. Walde 1978; Walde-Psenner 1979; Moreno 1976. 
Dionıso e Irınna Hinter auf einer Basis die Statue eines bortigen Dionysos in langem Gewand, in der $L$. einen Thyrsos, daneben auf einer anderen Basis, eln die Doppelfl ${ }^{\circ}$ ote spielender Pansknabe (Helbig)

Bibl Helbig 40:, Schetold 1977179

VII 416

Cat 244 (1acciata esterna), nicchia, II stule

La statua gialla di Zeus sta su un segmento rotondo $\mathrm{Ha}$ una clamide sulle spalle e regge nella sinistra alzata uno scettro, nella destra distesa un tulmine Il peso del corpo poggia sula gamba destra ll tipo e quello dei gunirseri nudi d'impronta policletea Solo la sagoma e ancord visibile La statua dipinta or viamente sostitussce und statuetta da larario. facile preda di passantı

Bibl Helbig 8, Boyce 1937, 111 no 15 Schefold 1957, 179

VII 4,48 Casa della caccia antica

Cat $245 / 1$ (4) Palcte sud, quadro centrale, IV stile +

Leda con il cigno Sccondo Herrmann il gruppo copia in modo tedele la statua di Timoteo Certo l gruppo vivo puo essere derivato dallopera tamosa (cf p 000 )

Bibl Herrmann II Taf 229 Schefold 1957, 180

Cat 245'2 (6) Tutte le parets zocolo, zone mediana e superiore, Il stlie Le edicole centralı dello zoccolo sono occupate da figuie gralle, mentre le costruzioni sono sorrette da cariatidı gialle Esse sorreggono la cornice con la mano esterna e porgono una patera con l'altra distesa Solo sulla parete ovest le cariatidi sono ancora visibil

Vell'edicola dolla parete ovest sta un Eros alato con un mantello sopra le spalle La gamba destra e di appoggio Con entrambe le manı regge una fiaccola darantı al petto Sulla parete sud si vedono tracce di una figura maschle nuda che poggia con ll gomito destro su un pulastro Il plede smistro sta davantı al destro Regge sull'dvambraccio sinistro una clamide La figura sulla parete nord e lleggibule

vella scaenae frons nella zona mediana della parete nord si distingue vagamente la statua gialla di una figura femminule che ha la mano destra presso la spalla For'se si tratta di Atrodite

Nella zona superiore della parete ovest la posizione centrale viene occupata dalla statua di Hermes $E^{\prime}$ secluto su di una roccia coperta da un drappo La mano sinistra e un I iposo sul grembo, l'altra regge un caduceo Sulla testa ha un cappello Le spaLle sono coperte con una clamide azzurra Il tipo originale e la statua colossale di Zenodoro fatta su committenza degh Averm e nota atti averso bronzetti 627

Bibl Ilelbig 360,1301 , RP 96 6, Curtius 1929, Abb 110, Jucher 1950, 8, Herrmann II, Taf 229, Schefold 1957, 180-181, Sclimidt-Colmet 1977, 165,273 no P19, Ptretschner 1977, II, no 68, Moormann 1983b, $105-111, \mathrm{t} 1 \mathrm{~g} \quad 21-24$

627 Braemer 1978 
Cat. 245/3. (10) Paretı est e ovest, zona mediana, IV stle

In nicchie sopra lo zoccolo sorgono statue bianche su piedistall rettangolari, purtroppo quasi illeggibuli Sulla parete ovest a smistra si distingue un Dioniso barbuto vestito di un chitone lungo Regge un tirso nella sinistra alzata e una patera nella destra distesa. La figura a destra e illeggibile.

Sulla parete est le due tigure sono simmetriche Indossano lunghi mantelli e reggono nella mano esterna una laneid o un tirso e nell'altra una patera Lo stato di conservazione non permette un'interpretazione accurata.

Nelle prospettive sopra le nicchie sono statj inseritı satir) gialli Reggono con entrambe le manı sopra la testa la cornice nellatteggianento dell'Atlanten-Schema

Bibl . Schefold 19ji, 181, Schefold 1962, Taf. 16.2.

Cat 245/4. (12) Parete ovest, quadro centrale; IV stile +

Paesaggio sacro-1dulliaco. A sinistra in avantı sorge la statua di Ecate sopra una base rettangolare. Inclossa un peplo e ha una corona sulla testa Nella destra regge una bacchetta. A destra di un tempio si trova la statua di Apollo seduto su una seggiola o una roccia. Un mantello copre la parte inferiore del corpo. La mano sinistra e in riposo sul grembo, l'altra regge una cetra posta vicino al fianco.

Peters attribuisce la pittura alla bottega che era attiva nella Casa dei Ceii. Bibl. Helbig 1555, Rostowzew 1911, 95, RP 392.5; Schefold 1957, 182, Peters 1963, 177, Pl retschner 1977, II, no 52.

Cat. 245/5. (15) Pareti est e ovest, zona mediana, IV stule Nelle architetture rosse fra , pannell si distinguono delle statue equestri in guisa di acroterı. Solo la figura nel prospetto sinistro della parete est e rimasta conservata. Essa e rivolta verso destra. Con la sinistra ll cavaliere guida ll cavallo, la destra e alzata nell'attegramento dell'adlocutio.

Il pinax della parete ovest rappresenta Artemide e Itteone. La dea e seduta in modo simle alla Afrodite accosciata attribuita a Doldalsas. "20

A sinistra vi e un cane dipinto in giallo, posto sopra un plinto che assomiglia un pezzo di roccia $E^{\prime}$ rivolto verso destra e ha la testa alzata. Non c possibile trovare precisı confrontı per questa statud

Bibl Helbig 250, Dawson 1944. 111 no 65; Scheiold 1962, 182: Schelold 1962, 112, Tal 76 1, Paar 1962, 101, Vichel 1982, 563-566, lbb. 18-18a .

VII 4, 51 Casa di Arianna/dei capitelli figurati

Cat. 246. (1) Parete ?, quadro centrale, IV stile (ora MN B894)

Eracle libera Prometeo das vincoli. A sinistra si trova un tempio dentro il quale sta una statua rossa, ora Invisibile $E^{\prime}$ delineato in modo schematico e rappresenterebbe secondo Helbig una Mantelherme.

Bibl.: Helbig 1128, RP 212.2; Dawson 1944, 110 no. 63, fig. 23; Schefold 1957, 184-185; Schefold 1962, 155-156, Taf. 155.2; Peters 1963, 133-134.

62. Attribuzione contestata da Linfert 1969. 
VII 4, 59 Casa del bronzı/della parete nera

Cat 247 (f) Tutte le paretı, zona mediana, IV stle

I prospettı giall 1 ra I campı nerı contengono elementı scultorel Sulla parete sud si trova un candelabro nel quale $e$ inserito un tros che regge con entrambe le manı una bacchetta davantı al petto La gambo sinıstra e di appoggio

Sulla trabeazione del primo piano sono sedute figure femminll con l piedı a forma di tralci che roggono un platto con la mano interna Sono flanchegglate da centaurı che reggono un pedum nolla mano interna e sono rivolt verso l esterno

lel secondo pıano vi sono del satirı con una nebride sopra le spalle, un pedum nella mano interna e un ritone o una sirınga nell'altra

Sulle paretı est e ovest sopra lo zoccolo sorgono le statue di Dioniso dietro cancell 1 Entrambe le figure reggono un tirso nella sinistra lungo il tianco e un cantaro nella destra stesa Sulla testa hanno una corona La gambd destra e di appoggio

I pinakes, ora nell'Antiquarium di Pompei (inv no 20879), rappresentano scenc di offerte

1) Psiche e tre Eroti nutrono un pavone vicino ad una colonna attorno alla quale vi e una corona Sopra la colonna si distingue la statua di und figura temminile alata vestita di un peplo Regge un oggetto ignoto nella destra stesa in aranti Helbig interpreta la scena come un'offerta a Afrodite, la statua sarebbe vike Il pavone pluttosto e un'indicazione di Hera come lo e la corona reale La figura alata quindi sarebbe da interpretare come Iride, una divinita che e sempre associata ad Hera 629 inche Psiche v iene raffigurata in questo modo come su un pinad nella Casa di 11 Lucretius (cat 282 7 )

2) Degli Erotı stanno presso la statua gialla di Dioniso che indossa un chitone $E^{t}$ barbuto Regge un tirso nella sinistra e un cantaro nella destra distesa

3) Ofterta a Priapo (ora perduta) La statua del dio incorona una colonnd $\Sigma^{\prime}$ sul punto di scoprire \& membro Queste statuette sono conos ciute da Pomper 630

Le tre scene sono simili per quanto riguarda l tema e lo stile ai pinakes della Casa di M Lucretius (cat 282/7)

Bıbl Helbig 773, 776, RP 94 1, Bıeber 1917, 30, Herter 1932, 120 no 36, 138 no 37, Grimal 1943, 280-281 pl 19, Schefold 1957, 187, Peters 1963,144 , Pfietschner 1977, no 45,66

VII 5 Terme del toro

Cat 248 (II) Due nicchie, IV stule

Rappresentazione di un giardino, ormal quası lleggible, su uno sfondo ceruleo Nelle quattro nicchie si vedono delle fontane poste fra 1 cespugli Le fontane nella nicchia a simstra della porta e in quella seconda a destra della

629 Cf MN 9559 da VI 8, 3 (cat 198'1) L'iconogratia e simule a quella di Nike Cf $M$ Maler, Roscher II 1, 320-357, s.v. Iris

630 Dwyer 1982, fig 51, 163, 186 Cf Blanch 1979 
porta hanno un supporto a forma di una sfinge $1 \mathrm{cmmin} l e$ alata.

Bibl. Uverbeck/Mau 1884, 206, Scheiold 1957, 189; Pelors 1971, 13

VII 6,28

Cat. 249 (104) Parete nord, zona mediana; IV stile +

Pseudoportico diviso in tre intercolunnı con rappresentazion di giardini. Vel centro di ogni intercolunnio, davanti a un parapetto ligneo, si trova una fontana rotonda bianca sorretta da una stinge temminle alata. Esse sostengono il bacino sia con un calice sulla testa che con le alı. I loro visi sono delineati in modo realistico con colori vivaci. Spicca l'interesse del decoratore per $\mathbf{j}$ dettagli.

Bıbl.. A. Sogliano, VSc 1910, 468-469, fig. 10-11: Grimal 1943, 480, pl. IV fig. 3-4, Schefold 1957, 191, Grumal 1969, 45j no. 28: Vichel 1978, Taf. 73.3 (non menzionato nel testo), Jashemshu 1979, 56-57, fig. 92, De Vos,'De Vos 1979, 92, Tal. Gd.

VII 7, 1 Tempio di Apollo

Cat. 250. (portico) Parete ?, zona mediana; IV stile +

Sot to alcuni del quadrı a soggetti mitologici sı vedono prospetti paesistici con statuette schematiche, specie erme di Priapo.

velle scene con Pigmei, in parte conosciute da disegni, si trovano altre statuette ravijuantı il paesaggio. Su uno di essi si distinguono due sfingı femmindı sulla rampa dı un tempıo, su un altro sta una statua rozza sopra una base rettangolare.

Bibl.. Helbig 154, Rostowzew 1911, 57, Abb. 32; RP 377.5-6, Schefold 1957, 192 .

VII 7, 10 Casa di Romolo e Remo

Cat. 251. (p) Paret 1 avest e nord, zoccolo e zona mediana, IV stile

Nel paradeisos sulla parete nord si trova a sinistra la statua bianca di una sfinge femminle accosciata. $E^{\prime}$ rivolta verso destra e siede su di un plinto bianco sopra le roccie Il tipo e quello delle sfingi greco-romane. L'anumale e presente come indicazione del luogo, cioe come sumbolo dell'Lgitto.

Sulla parete ovest si vede la rappresentazıone di un giardino. Lo zoccolo consiste di un parapetto rosso con una abside nella quale sta una tontana a forma di cantaro. Questa fontana e francheggiata da due ninfe acquatiche che reggono bacini davantı al grembo. La parte inferıore del corpo e coperta da un mantello bianco Stanno su piedistallı gialli, la gamba esterna e di appogg10. Guardano verso il centro. Il tipo e quello della Venere Landolina. ${ }^{31}$

Sopra l'abside, in mezzo ai cespugli, e sdraiato un Sileno bianco in guisa di divinita fluviale. Regge un tirso nella destra alzata e poggia con il gomito sinistro su di una brocca dalla quale esce acqua. Appena visibule e una base

631 Becatti 1971. 
bianca, la quale caratterizza la figura senz'altro come statua, identificazione rafforzata dalla monocromia bianca.

Lina simile combinazione si incontra nella Casa delle Vestali (cat. 198/3), anche se l'ambientazione é quella di un ninfeo, vista la presenza del parapetto e dell'abside.

Bibl.: Sogliano 169, 476; Jucker 1950, T; Schefold 1957. 194-195; Reuterswärd 1960, 182; Schefold 1962, 178, Taf. 151.2; Grimal, 1969, 287-288, 155; Peters 1971, 9: Bastet 1974, 224, Abb. 11 (considerato perduto); I'f retschner 1977, no. 47: Michel 1978, 397, Taf. 75.2; Jashemski 1979, $70-71$, fig. 115; Pompei $1748-1980,176$ no. 38 .

VII 9, 4-12 Macello

Cat. 252\%1. (portico) Parete ovest, zoccolo, zone medinna e superiore; IV stile

Cariatidi gialle sorreggono l'orlo superiore dello zoccolo nel Karyatiden-Schema con una mano e reggono nell'altra distesa una patera. Sono rivolte verso il centro e viste di profilo. Sono affini a quelle della Casa della caccia antica (cat. $24 \bar{j}, 2$ ).

Le edicole che fiancheggiano il pannello centrale contengono acroteri a forma di bighe condotte da Nikai di colore giallo. Nell'una mano tengono una frusta, nell altra le redini. Le bighe sono rivolte verso il centro.

Nella zona superiore, molto danneggiata, vi sono candelabri sormontati da figure. $\Lambda$ sinistra sono rimaste conservate le gambe di una figura femminile. Sopra il prospetto a sinistra delledicola centrale sta una donna con un manto rosso che le avvolge la parte inferiore del corpo. Regge un piatto nella destra c tiene la sinistra davanti al petto. Il suo sguardo é volto verso il contro. La gamba destra sta in avanti ed è di appoggio. Sopra l'altro prospetto si vede un uomo nudo con una sola clamide sulle spalle. La mano sinistra e nel fianco, l'altra presso la spalle.

Bibl.: Schefold 1957, 196.

Cat. 252/2. (?) Parete ?, zona mediana; IV stile +

Pinax con duc ninfe e una divinita lluviale maschile. Sullo sfondo si distingue l'erma di un satiro che regge un pedum.

Bibl.: Helbig 1014.

VII 9, 33 Casa del re di Prussia

Cat. 253. (viridario) Parete ovest, zona mediana; IV stile

Paradeisos. Nella parte sinistra sopra una roccia vi è la statua rossa di Priapo. Ha la mano sinistra distesa e la destra nel fianco. I dettagli non sono piú visibili.

Una rappresentazione di un larario taglia il paradeisos nel mezzo. In un'edicola vi sono Afrodite e Ares presso due altari. Sono dipinti a colori vivaci. Ares regge una lancia nella destra alzata e un mantello sul braccio sinistro: sulla testa ha un elmo. La gamba destra e di appoggio. Afrodite è nuda. Il peso del corpo poggia sulla gamba destra. Ha la mano sinistra nel fianco e regge nella destra uno specchio. 
ion sj possono trovare confronti precisi, ma lo stile delle figure avvicina quello di statue lisippee (contrapposto, gambe lunghe, testa piccola). lnoltre lAres si inserisce nella serip di guerrieri nudi e fa pensare alla statua nella Casa della Venere in conchiglia (cat. 178).

Bibl.: Helbig 70; Herter 1932, 114 no. 101; Boyce 1937, no. 303; Schefold 1957, 197; Barbet/Allag 1972, 1020-1021, fig. 42-43; Uwyer 1982, 118.

V'I !, 17 Casa delle nozze di Ercole

Cat. 254. (triclinio) Parete nord, zona superiore; III stile

Ebe e Eracle stanno presso il tempio della Venere pompeiana. A sinistra e a destra si vedono dele processioni. Ora purtroppo il fregio e quasi completamente illoggibile.

Nell'entrata del lempio si vede Afrodite vestita di un chitone senza maniche. Nella sinistra presso la spalla regge una bacchetta, l'altra è davanti al petto. 'E' fiancheggiata dalle statuette di Priapos e Eros. Il primo scopre il suo membro alzando il mantello con entrambe le mani. Eros è vestito di una tunica corta e ha sulla testa un cappello frigio. Regge uno scudo e una lancia nella sinistra e tiene con l'altra la mano sinistra di Afrodite.

A sinistra si vede un ferculum sul quale si trova una serpente attorcigliato attorno ad un albero. Questa statua si puó confrontare con due sculture che decoravano delle fontane nella palestra di Ercolano e nella Casa del citarista. ${ }^{632}$

L'interpretazione della scena non e del tutto chiara. M. Della Corte pensó alla processione del Juventus pompeiano durante le feste della Venere pompeiana. Le figure sarebbero state rappresentate in forma di una pantomima, nel quale l'eroe greco Eracle verrebbe sostituito dalla nuova divinita cittadina. Herbig (in Herrmann) invece interpretó la scena come una trasgressione della colonia greca (Eracle) nei confronti della colonia romana (Venere pompeiana). P. Castren ha potuto stabilire con argomenti validi che non è mai esistito un Juventus a Pompei. ${ }^{63}$ Inoltre non è indiscussa l'origine greca, presunta da Della Corte e Maiuri. "3، Per l'identificazione della scena come una pantomima mancano delle indicazioni.

Per quanto riguarda la datazione si constata, contro l'ipotesi di Maiuri secondo la quale la decorazione era posteriore al 62 , che non vi sono tracce di uno stacco fra la decorazione di III stile nella zona mediana e il fregio nella zona superiore.

Bibl.: Helbig 1479; Herrmann II, 53-54, Taf. 234; RP 190.1; Della Corte 1924; Herter 1932, 120 no. 33; Maiuri 1942, 186; Schefold 1957, 198; Tran Tam Tinh 1964, 132-133 no. 24; pl. XI 1.2; Pfretschner 1977, no. 4; De Vos $1980,20$.

\footnotetext{
632 Jashemski 1979, fig. 247; Dwyer 1982, fig. 139.

633 Castrén 1975, 33,112, 115.

634 Maiuri 1973; cf. De waele 1982, 32.

635 La decorazione non viene discussa né elencata in Bastet.'De Vos 1979.
} 
VII 12, 18 Lupanare

Cat. 255 (corridoio) Parete nord, zona superiore; IV sile

Sopra la porta mediana si trova un paesaggio nel quale vi e la statua polıcroma di Priapo su una base gialla lndossa un chitone blu senza maniche Alza un lembo per mostrare un doppio fallo La gamba destra e di appoggio Il modello e sumile al cosidetto Maripara 636

Bibl Helbig 505, Herter 1932, 120 no 35, Schefold 1957, 201, Pfretschner 1977, no 80

VIl 12, 26-27 Casa di L Cornelius Diadumenus

Cat 257 (h) Parete di fondo, zona superiore, IV stule +

Nel campo centrale sl trova la statua blanca di . Ires sopra un piedistallo Ha un clmo sulla testa e sulle spalle una clamide Nella sinistra regge uno scudo, nella dostra un gladıo Entra nelle serıe di gucrrieres classicheggiantı d'umpionta policletea, ma per mancanza di un'lllustrazıone non puo esserne stablito ll tipo

Bıbl Helbig 272, Schefold 1957, 202

VII $12 \quad 28$

Cat 257 (f) Parete?, zona mediana; IV stule +

Paradeisos Secondo Herter nel centro sorge un'erma du Priapo taeniis ornata

Bibl Helbig 1585, Herter 1932159 no 192, Schefold 195i, 203

VII 13, 4 Casa dı Ganimede

Cat 258/1 (a) Parete?, zona?. IV stule +

Soprd un pledistallo, base o striscia sta un guerriero nudo che regge una lancia nella destra Sulle spalle ha una clamide. La gamba destra e di appoggio Sulla spalla destra e seduto un Eros 637

Bibl Helbig 1395, RP 784 , Schetold 1957293

Cat $258 / 2$ (b) Parete ?. zoccolo, IV stule +

Nello zoccolo si trovano Pinakes mit goldenen Delphinen und Statuen ${ }^{6}{ }^{\circ}$

Bıbl Schefold 195i, 204

\footnotetext{
636 Blanck 1979

637 Per le decorazıonı ancora present, De Vos 1982, 316

638 Per le decorazion ancora presentı De vos 1982,316
} 
VII 15, 2 Casa del marinaıo

Cat 259. (1) Parete nord, quadro centrale; IV stile (ora MN 111479)

La strage del figl di Niobe, situata in un paesaggio selvatico Nel centro sorge un sacello occupato dalla statua gialla a macchic rosse di un cervo, apparentemente sumbolo di Artemide. L'animale e rivolto verso destra, ma ha la testa volta indietro. I colorı giallo e rosso indicano due tipl di marmo, nel corpo giallo sono state inserite delle piccole pietre rosse con una tecnica che secondo Plinıo (N.H. XXXV 1) sarebbe stata in voga all'epoca di Claudio Ĺn cervo cavalcante, ora nel Muses Vaticanı, dumostra 1 medesims colori ${ }^{19}$ Il tipo della statua e quella des due daini della V'lla des papiri.

A una colonna posta sulla destra del sacello e appoggiata una statua bianca dı Pan, ora lleggibile, che indica la collocazione della scena, croe $ل$ l Citero. Regge un pedum e una nebride nella mano sinistra, la destra e alzata.

II pinax non e stato tolto completamente dalla parete: al lato superiore si vede un solo braccio di una delle Niobidi

Dalla descrizione di Mau si conclude che il pinax veniva sorretto du una coppra di figure temmmul. La stessa cosa sarebbe stata rappresentata sulle altre pareti, anche se la sincretica descrizione non permette conclusioni La situazione forse era simule a quella nella Vulla della Farnesina (cat. 319/2).

Bibl.: A. Mau, BdI 1873, 232; Sogliano 218, 452-454, 505, 753-756, Herrmann I. 208-209, Taf. 151, Elia 1932, no 107, Dawson 1944, 92, pl. 10; Schefold 1957, 206. Paar 1962, 111-112, Abb. 20, Peters 1963, 74-76, fig. 58, Grunal 1969, 339.

VII 15, 12 Casa di Octavius Primus

Cat 260. (d) Parete nord, quadro centrale; IV stile

Paesaggio sacro-ıdilliaco con un tempıo Dentro l sacello si trova una statua di Artemide. Essa e seduta su un trono rosso e indossa un chitone grigio. Sulla testa ha una corona.

Un'erma di Dionıso e appoggiata contro una colonna. Regge un cantaro nella sinistra e un tirso nella destra

Bibl.: A Mau, BdI 1873, 235; Sogliano 687; Rostowzew 1911, 41, Herrmann 1, 205, Schefold 1957, 208.

VII Insula occıdentalıs 17 Casa dı Fabıus Rufus

Cat. 261/1. (2) Parete est, quadro centrale, III stule

Paesagg 10 sacro-idilliaco. Presso una porta sacra vi e la statua gialla di Ecate. Nelle manj stese in avanti regge una fiaccola. Sulla testa ha una corona.

Bibl. ' Inedito.

Cat 261/2. (12) Pareti nord e sud, zona mediana; IV stule

Su un pinax sulla parete nord si vede una ninfa spiata da un satiro $E^{\prime}$ seduta nell'atteggramento del'Atrodite attribuita a Doidalsas 640

639 Amelung 1908, 340, Taf. 33. Cf. una pantera (Helbıg, 175. Gnoli 1971, fig 287).

640 Ct. Linfert 1969. 
I prospettı fra 1 pannell sulle paret sorreggono colonne snelle Indossano un chitone senza maniche e hanno in una mano una patera e nell'altra la colonna appoggiata alla testa

Bibl inedito

Cat 261/3 (21) Paretı est, nord e sud, zone mediana e superiore, IV stule Il quadro centrale della parete est rappresenta Lracle e Unfale L'eroe e raffigurato nell'atteggianento dell'Eracle di Tarento di Lisippo

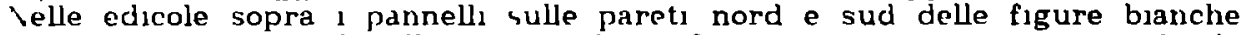
sono poste sopra piedistall rettangolari Sono 3 unaste conservate solo le hagome Le tigure dell una parete ruspecchiano quelle della parete opposta Al lato ovest si vede un uorno nudo con una clamide sulle spalle che regge una paterd nella inano sinistra e un oggetto non identificabile nella destra sopra la testa vell'edicola centrale e una donna ammantata con la mano destra alzata Al lato eat si troia una tigura simmetrica a quella del lato ovest

Bıbl Pompe 1748-1980, 28, fig 11-12

Cat 261,1 (22) Parete nord, quadro centrale, III stule

Pdesaggio sacro-idlliaco Lna porta sacra $P$ occupata dalla statua verde di una figura temminule vestita $d_{1}$ un peplo Daiantı ad essa $W^{2}$ sopra una base rettangolare, la statua gralla di Lcate che regge in entrambe le mani, stese in avantı, una fiaccola $f$ destrd si vede un altare con l'erma rossa itifallica di Prisipo Ha un cappello sulla lesta ed e raffigurata nella chordosis

Bibl medito

Cat $261 / 5$ (24) Tutte le pareti, zona mediana, IV stile

Sul campi lateralı si trovano le Muse come vignette Non stanno su delle mencole, ma si inseriscono orviamente nelle serie delle Yuse nella Casa di Sirico (cat 232) e nei Praedia dı Julıa Felıx (179/2)

Parete sud, campo ovest Perduta

Parete sud, campo est Solo una sagoma

Parete est, campo nord Tersicore indossa un chitone verde chiaro $e$ un mantello a strisce rosse Regge con la destra una cetra che suona con la sinistra Il piede sinistro e appoggiato su uno sgabello

Parete est, campo sud Callope o Polinnia e vestita dl un chitone verde e un mantello giallo IIa la destra presso la spalla e la sinistra avvolta nel mantello

Parete nord, campo ovest Perduta

Parete nord, campo est Talia e vestita di un chitone rosso e un mantello glallo Regge una maschera satiresca nella sinistra.

Il pinax della parete sud rappresenta Dioniso e Arianna a Nasso Vicino al mare si trova la statua di Atena Indossa un peplo e tiene nella una freccia e un arco

I prospettı delle paretı est e ovest contengono del satırı giall che hanno una colonnd sulla testa Le figure simmetriche reggono un pedum nella mano interna e un ritone nella mano esterna Rispetto all'elemento archiettonico aggettante sono in posizione avanzata 641

Bibl De Vos 1977, 44, Pompe1 1748-1980, 24, fig 4-7

Cat 261/6 (28) Parete nord, zoccolo, IV stule

641 Cf gli atlanti trovatı presso il teatro di Pompeo a Roma Schmit-Colınet 1977, cat 1176 
Palestra con lottatori A destra si trova un'erma marrone incoronita I

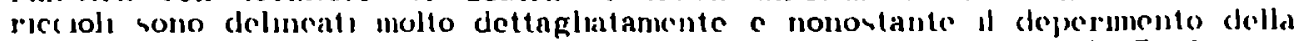
pittura e quasi sicuro che slamo di tronte ad un ritrat to come in 117,4 (cat. $176)$

Uno deglı atlet stitue che si vedono nello zoccolo della scaenae frons nella cosiddeta palestra (cat. 267) ed e simile aglı atletı bronzeı della Vlla dei papırı

Bibl. inedito.

VII Insula occidentalis 20

Cat $262 / 1$ (9) Parete nord, zoccolo, II stule, fase IIa

Una figura femminle vestita di un mantello verde-viola sorregge l'orlo superiore dello zoccolo Le gambe terminano a forma di giralı Regge nella mano destra un'oinochoe gialla, la sinistra e il volto sono andati perdut La ligura puo essere controntata con le teste femminili nella Casa di ubcllus Firmus (cat. 302 1)

la parcte di fronte, completamente perduta, dove aier avuto una decoralione summetrica

Bibl medito

Cat. $262 / 2$ (d) Parete est, zona mediana, II stile, fase IIa

Davantı ad uno sfondo di ortostatı rossi sopra un podio verde vi sono, a

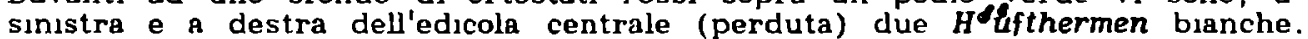
L'ombra e stata indicata in verde. Lu tigura a sinistra há entrambe le manı sopra la testa nell'atteggiamento dell'Afrodite Anadyomene L'altra figura ha un pedum e una clamide nella mno sinistra, mentrenella destra distesa tiene una lucerna appesa a tre corde Sulla testa si vede una corona, attorno al fusto una corona du edera

Le figure sulla trabeazione di sopra sono quasi completamente sparite. Sull'architrave dell'edicola centrale al lato destro vi e una tigura temminule che avanza verso destra $E^{\prime}$ vestita di una tunica corta. Il colore e bianco. Bibl inedito.

Cat. 262/3. (b) Pareti est e ovest, zona mediana; II stile, fase Ila Sul podıo verde, davantı alle micchic, vi sono rappresentati due uominı vivi, modellat secondo tipı statuari. Sulla parete est si vede un poeta vestito di una toga bianca e con una corona sulla testa. Su una panca, dictro di luı, sono una capsa e una cetra Ha le manı davantı al grembo. Il piede simistro e di appoggio. Lo sguardo e rivolto verso destra. Il tipo e quello dell'Eschine della Villa del papirı 642

Sulla parete sud si trova un fllosofo vestito con una toga bianca e regge un bastone nodoso. Ha una barba. La gamba destra e di appoggio. Puo essere confrontato con il fllosofo della Villa del papirs. 643

Il vano potrebbe essere servito da gabinetto di studio o lettura, visto l'accento sulle figure culturali. 644

642 Sgobbo 1972, tav. XII.

643 Sgobbo 1972, tav. IV -V.

64 Cf. Lorenz 1965 Gli stessi temı sono presenti nella Vlla dei papıri e in 
Bıbl Pompel 1748-1980 167, fig 28A, Bragantinı Parıse Badonı 1984, 121 , tIg 13

Cat 2624 ( 7,2 plano) Parete sud, zona mediana II itle fase IIb vel campo sinistro rosso si delinea una Vike sopra un globo sotlo لl quale si tıova un segmento che funzıona da mensola il tipo e quello della Vittorid romana che reggc con entrambe le mani un trofeo appogglato alle spalle Il puede destro sta un po' in diants 643

Il quadro centrale contiene un paesaggio sacro-ıdıllaco Presso una porta sacra sta l'erma itılallica rossa di Priapo $E^{\prime}$ delineata nella chordosis. A smistra della porta $v$ sono tre statuette su una base comune lormano un Ecateo Tutto e reso in modo schematico

Bibl inedito

Cat $26 j 3$ (tablino, 3 piano) Parete?, zona superıore. II stule, tase IIb Paesaggio sacro-ıdlliaco A sinıstra e la statua itifallica dı Priapo nella chordosis Sulla testa ha un cappello

Bibl inedito

Cat 262/6 (terrazza inferiore, nunfeo) Tutte le paretı, zona mediana. III stue

Rappresentazionı di glaidinı, ord in restauro, in due vanı adiacenti che costituiscono un ninteo Jon e ancora possible studiare l'intera decorazione lella sala VII le paretı lateralı mostrano l'articolazıone di un portico nel cul intercolunn lateral delle orme bianche sor reggono del pmakes sulla testa $\mathrm{Al}$ lato superiore del portico sono appese delle maschere Sulla trabeazione si vedono delle imagines clipeatae e del pinakes Al di sopra entro ll telaso che curconda le prospettie sono appese delle pelte

iel innfeo $V$ si vede una slinge sulla parete sud

Bibl Jashemsh1 1979 82, fig 132, S de Caro. CronPomp 5 (1979) 178, fig 3. Id , Pompen lferculaneum Stabıae 1 (1983) 316-318, fig 4

Cat $262 / 7$ (?) Parete ?, zona mediana II stule, fase IIa

In trammento sporadico mostra und figura temminle alata che sorregge con la testa una trave aggettante $\mathrm{Ha}$ un mantello giallo-violaceo attorno alle ren

Forse la parte inferiore che manca e 11 lusto di un'erma Attorno al collo ha un monde composto $d_{1}$ I ubini o altre pietre preziose Con cntrambe le manı regge un festone La figura e sumule alle erme nella Casd del criptoportico (cat 148/2-3) e nella Casa di Caesıus Blandus (cat 233 2)

Bibl inedito

Cat 2628 (15, 2 pıano) Tutte le paretı, zona mediana, IV stule Lelle Muse sono delineate su campl bianch 1 Stanno su del segment rettangoları, che sono delle mensole schematizzate Sono policrome e vive

alcune case pomperane (Pandermalıs 1971, Sgobbo 1971, 1972) Cf moltre $\mathrm{p} 000$

${ }^{645} \mathrm{Cr}$ Holscher 1968 Lo stesso tipo si trova nella Casa dell'ara massuma (cat 221 1) 
Parete nord, cinque pannell con da sinistra a destra le seguenti figure Polinma nell'atteggramento della aufgelehnte Muse, una donna vestita di un mantello rosso che regge una cetra, un poeta laureato vestito di una toga bianca una figurd andata perduta, Melpomene che regge una clava e und maschera

Sulla parete ovest l'unico pannello mostra un satiro che suona due flauti lunghissimı, forse e Marsia

Deı tre pannellı sulla parete est solo quella a sinistra con la figura di Urania che indica con una bacchetta un globo vicino al piedi, e rimasta conservata

Suı quattro campl della parete sud si vedono da sinistra a destra una figura andata perduta una testa temminile, un poeta togato, donna con una cetra presso un'erma

Solo la aufgelehnte Muse e 1 poetı possono essere collegat 1 ad esemp1 statuarı A proposito della prima figura bisogna notare che essa e l'unıca figura dipınta di questo tıpo, usata proprio come Musa, altrove l'incontiamo come Ahte ( $p$ e cal $161 / 2,294$ )

Si tenga presente che le descrizioni di queste e alcune altre decorazioni non sono esaurienti viste le circostanze difficili nelle quall i e siolto 11 lavoro

Bibl inedito

VII Insula occidentalis 39

Cat 263 (?) Paretı?, zona mediana, Il stile. fase Ic (ora MN 8594, 8715)

Scaende frontes delle qualı l'una e rimasta intatta pel la maggior parte (MN 8594), l'altra per un solo pezzo del freg10 (MN 8715) M 8594 quası sempre e stato collocato nel Praedia di Julia Felıx sulla base della data di ritrovamento Dobbiamo a Allroggen-Bedel la collocazione corretta MN 8715 finos d non e stato inserito

La parte centrale e occupata da un prospetto con unu tholos nella quale si vede la statua bianca-grigıa di Afrodite nuda Con entrambe le manı regge J capellı nell'atteggiamento dell'Afrodite Anadyomene Il colore indica l'ar gento La tigura rentrd nell'iconografia della parete gli clement acquatici (pesci, anatre piante acquatiche) potrebbero essere un indizio di Cnido come sede del santuario di Nitrodite Pelagia

La cornice dell'edicola centrale (e rimasta conservata anche un frammento della decorazione della parete opposta IV 8715) e composta di due fughe di mensole fra le qualı si vedono del cignı che escono da calıcı vegetalı verdi Sono composte in modo simmetrico delle otto figure le quattro a sinistra sono rivolte verso sinistra, le altre quattro verso destra Tali uccelli stilizzati sono inseritı nella cormice del vano 5 della Casa di Augusto (cat 316 1) 646

Bibl Helbig 79, Elıa 1932, no 376, Beyen 1938, 268-278, Abb 100 Vallois 1944, 353-354, Lehmann 1953, 120, Schefold 1957, 53, Peters 1963, 19. Crolsille 1965, 28 no 7 , Lyttelton 1974, 23, pi 27, Allroggen-Bedel 1974a, 155 nota 78, Allroggen-Bedel 1976a, 144 nota 2. Allroggen-Bedel $1976 \mathrm{~b}$, 88 nota 21 , Fittschen 1976, 546, Abb 5, Silberberg 1981, no 11, fig 9, Voormann 1984b, 640 nota 14

${ }^{645}$ Cf per questı elementı architettonıcı Von Hesberg 1980a (qui p . 22) 
VIII 2, 1 Casa di Championnet

Cat 264 (n) Parete ?, quadro centrale. III stule +

Una menade viene assalita da un satiro A sinistra si vede l'erma bianca itifallica di Priapo Il suo sguardo e rivolto verso la coppia.

Bıbl.. Helbig 550; RP 125 6, Noack/Lehmann-Hartleben 1936, 118, Schetold 1957, 210; Architects 1981, 216, 224 no. 67

VIII 2, 17-20 Terme dol Sarno

Cat. 265,1 (b) Parete destra, zona mediana, IV stule +

A sinistra e a destra di un'ummagine di Bellerotonte si trovano del prospetti nol cuall sorge sopra un basamento una statua equestre a monocromo bianco (simolante il marmo) la quale progetta la sua wmbra sul fondo.

Bibl Viccolini'viccolın 1851-1896, IV, 56-57, Beyen 1938, Abb 147; Schefold 1957, 211.

Cat 266/2 (7-7a) Parete est. lunetta, IV stule

Due donne vestite di un chitone bianco senza maniche reggono con la mano sinistra un piatto posta sulla testa, sula quale, a sua volta, poggia la trabeazıone. Fuancheggiano il campo centrale Nell'altra mano tengono un lembo della veste Il loro stule e classico e ricorda le horal dell'Eretteo Sono eseguite in stucco colorato

Bibl Schefold 1957, 213-214, Schefold 1962, 118, Taf 87-88, Mielsch 1975, $37-38,12 j$ no K26, Srhmit-Colınet 1977,272 cat P15.

Cat 26/3 (tepidario) Parete est, lunetta. IV stule +

Secondo Schefold qui sarebbe stato visible un apoxyomenos.

Bibl Schefold 1957, 214

VIII 2, 24 Casa del palestrita

Cat 267 ( $\left.f^{\prime}\right)$ Parcti est e sud, zoccolo e zona mediana, IV stule

Davantı allo zoccolo della scaenae frons vi sono delle statue gialle. Sulla parete est si vedono due ninte acquatıche con bacinı davantı al grembo che francheggiano un Hermes Le donne sono derivate dal tipo della Venere Landolina 647 Hermes poggia sulla gamba sinistra Ha una clamide sull'avambraccio sinistro e un cappello sulla testa. Regge con la sinistra una borsetta, con la destra distesa un caduceo Questo atteggiamento e raro; normalmente la mano destra distesa regge 11 marsupium 648

Sulla parete sud all'estrema smistra e all'estrema destra vi sono due statue di atletı poste simmetricamente che si allenano con manubri. Il loro

\footnotetext{
647 Becatti 1971

64 Rarı esempı sono statuette da Trier (Menzel 1966, no 28) e da Augst (Kautmann 1977, no 35) Per ll tipo consueto cf. Boucher 1976, 89-90. $105-106,370-371$
} 
atteggiamento e quello dei corridori della Villa dei papiri. 64

Accanto a loro sono due Eroti, anch'essi in posizione simmetrica, che tengono fra le mani davanti al petto un'oca. Sono sunili alle statuctte da fontana, trovato tra l'altro nella Casa dei Vettii e nella Casa della lontana Erande. 650

Nel centro si trova l'arbitro modellato secondo il tipo del filosofo seduto. Indossa una toga e tiene la destra sotto il mento e la sinstra nel grembo. Ina statuetta del genere e stata trovata nella Casa di Octavius Quartio. ${ }^{61}$

Nella scaenae trons della zona medrana le edicole sono occupate da figure vive provensenti dallambsente sportivo. Alcune di esse sono delineate secondo modelli scultorei.

L'atleta nell'edicola sinistra della parete est ha la mano destra presso la fronte nel atto di pulirla con una strigile, mentre il braccio sinistro $\dot{e}$ in riposo lungo il fianco. La gamba destra e di appoggjo. Richrama alla mente l'atleta Westmacott di Policleto. 652

Il gruppo di lottatori sul pinax centrale ricorda il noto gruppo ellenistico negli Uffizi a Firenze che si incontra anche su sarcofagi, pitture e mosasci. ${ }^{653}$

La figura a sinistra sulla parete sud e un altro apoxyomenos, ora modellato secondo lo schema lisippeo: pulisce il fianco destro del corpo con una strifile che ha nella mano sinistra. Il braccio destro è in riposo (atteggiamento Jell'Apollo Licio) sulla testa. Ovviamente il pittore non era in grado di dipingere una figura con un braccio steso in avanti.654

Bibl.: Von Cube 1906, 28-32, Taf. IV: Maiuri 1950, 170-176, Herrmann II, 32-33, Taf. 220-221; Schefold 1957, 215; Schelold 1962, 111-112; Bieber 1961a, 232, fig. 777-778; Lorenz 1972, 37 nota 175; Lyttelton 1974, 202, pl. 116; Moormann 1983b, 100-105, fig. 19-20.

VIII 2, 26 Casa del cinghiale

Cat. 268. (6) Pareti est e ovest, zona superiore; III stile

Sopra i campi laterali al lato nord si trovano edjcole occupate da statue poste sopra candelabri. La figura sulla parete est $\dot{e}$ andata perduta. L'altra parete contiene una figura femminile vestita di un chitone verde-blu senza maniche. alata. Regge un piatto (?) nella mano destra presso la spalla e la sinistra è appoggiata al fianco. Lo stile e classico.

649 Pandermalis 1971, 203, Beil. 9-10. Cf. pitture nella Casa di Fabius Rufus (cat. 261/6), Castellammare di Stabia (cat. 009/6) e MN s.n.3 (cat. 127).

650 Kapossy 1969, 39, 43-44; Dwyer 1982, fig. 112, 194. Cr. Andreae 1976, 299-304; De Caro 1976, 187-198, fig. 2-3.

651 Jashemski 1979, fig. 84; Dwyer 1982, Cig. 212. Cf. il Posidippo nei Musej Vaticanı (Helbig ", 129 Richter 1965, III, 238, fig. 1647.

632 Zanker 1974, 19-24, Taf. 21-26; Berger 1978.

653 Mansuelli 1958, I, 92 no. 61, fig. 61-62; Palma 1974. 8 (= Helbig " , 3130) Alvino 1976; De Vos; De Vos 1979 , 84-85 nota 11.

654 Lauter 1967 per il modello lisippeo: cf. Manderscheid 1981, 87 cat. 163, Taf. 26; Moreno 1983-1984, 64-66, lig. 4, 12, 13, 15, 22, 25, 27, 33. 
Essendo una decorazione simmetrica, probabumente la parte sud della parete conteneva delle ligure simul La maggior parte della decorazione e andata perduta

Bibl Voach Lehmann Hartleben 1936, 81, Taf 42 5, Schefold 1957, 216, Pompel 1748-1480, 174, 36d

VIII 2, 38-39 Casa di Giuseppe II

Cat $269^{\prime} 1$ (h) Parete nord, zoccolo. IV stule +

Due donne lavorano in un orto Nel centro vi e la $H^{\circ}$ úftherme di Priapo Indossa un mantello nel cul lembo alzato cl sono del fruttl Sulla testa ha una corona, con la mano sinistra regge un pedum, con la destra una falce Si tratta del tipo Formiello analizzato da Blanck

Bibl $\Lambda$ Mau, RY 2 (1887) 119-120, Schelold 1957, 218, Schefold 1960, 100, Taf II, Schetold 1962, 15j, Blanch 1979342 nota 18 , no 25

Cat 269'2 (r) Paretı est e ovest, quadro centrale, III o IV stule + Paesaggi sacro-idlliaci la cul datazione e sospesa fra ll III e il IV stile (Mau IV Schefold III) 655 Visto il tormato del pinakes ld posizione in una decorazione di III stule come nella Villa imperiale o nella Casa del frutteto (cat $305,1-2,161 / 2$ ) e verosimle

Il pinax della parete est contuene la statua di Apollo Citaredo presso un tripode Questı indossa un lungo mantello e regge nella mano sinistra una cetra La gamba destra e di appoggio La parte destra e la testa non sono visibili nemmeno nel disegno 556 Sopra il tripode si vede una statuetta schematica

L'ultro pinax contiene un betilo sormontato dalla statua di Artemide Indossa un peplo e ha ll braccio destro disteso

Bibl A Mau RM 2 (1887) 123, Rostowzew 1911, 43, Schefold 1957, 218, Pfretschner 1977, no 51 , Suberberg 1981 , no 77

Cat 269/3 (?) Parete ?, quadro centrale. III stle (ora Mi inv no 8968)

Sotomsba beve la coppa avvelenata nel palazzo dı Massinissa Dictro tende sospese, davanti ad un colonnato, si trovano due statue A simistra vi e un Dioniso verde barbuto con una corond sulla testa. lndossa un lungo mantello e regge nell sinistra un tirso e nella destra un cantaro A destra vi e un Apollo nudo che regge un ramo di olivo nella destra distesd e un arco nella sinistra lungo il fianco le gambe sono unite in modo arcaico Il colore e grigio e indica l'argento (ct p 105).

Le due statue non svolgono una parte specifica nella scena, ma sono elementı nell'arredamento sontuoso del palazzo Il Dioniso a di stule arcaistico, l'A pollo invece classicheggiante 657

655 Ld decorazione non viene discussa ne elencata in Bastet, De Vos 1979

656 Si confrontı la ligura con una statuetta da VII 2, 15 (Dwyer 1982, fig 207)

659 Sulla storia si trovano varie opinıonı U kahrstedt, $R E$ 31 (1927) 1099-1100, s.v.Sophonisba non si esprime sull'attendiblita, Schur, $R E$ XIV 2 (1930) $2156-2157$, s.v.Massinissa la ritiene un elemento romantizzato nella storiografia antica halbank 1967426 dubita e la ritiene un elemento 
Bibl . Helbig 1385, RP 221 3, Elıa 1932, no 193, Schefold 1957, 219, Borda 1958,240 , Reutersward 1960, 132 nota 325, Ptretschner 1977, no 50. CatKoln $1979,48,50,486-487$

Cat 269/4 (?) Parete ?, quadro centrale, IV stule (ora IIN 9550)

Leda con il cigno e stata modellata, pur in modo libero, secondo lo schema del gruppo di 'Tumoteo (ct cat 190 per ulterior commentı)

Bubl Helbig 147. Clia 1932, no 175 , Schefold 1957, 219

Cat 269/5 (2) Parete ?, quadro centrale, IV stule +

Eracle e Acheloo Eracle e ratigurato come l'Eracle con le mele delle Lsperidi proveniente dal tempio du Hercules Victor a Koma, ora nel Palazzo del Conservator 658 Questa statua sarebbe una variazione ellenistica su un modello lisippeo

Jel prospetti sı vedono capre gialle sumll a quelle su MN 8900 (cat 059 ) Nello zoccolo $v 1$ sono Hodfthermen in torma di sacerdotesse di Iside velle njcchie si trovano piccolı elefantı Poiche tutto e dipinto in giallo, non $p$ sicuro il loro cardtere scultoreo

Bibl Curtius 1929, 168, 175, Abb 108, Schefold 1957, 219-220

VIII 4,4

Cat 270 (28) Parete di fondo, quadro centrale. IV stile +

Narciso e Eros Un'erma di Priapo e appoggiato ad un altare in fondo a destra Dietro l'altare $v$ e la statua bronzea di Dioniso barbuto, vestito di un lungo mantello Regge un cantaro nella mano destra e un tirso nella sinistra

Bıbl Helbig 136j, Herter 1932, 159 no 194, Schefold 19j7, 224

VIII 4,15

Cat 271 (f) Parete?, quadro centrale; IV stule +

Due frigi in colloquio Quello a destra rogge con la sinistra un'erma semplice Schefold l'interpreta come das heilige Mal der Kybele. I due trigı potrebbero simbolizzare ul paese d'origine di Cibele, anche se di per se l'erma non e un simbolo di questa disinita

Bibl Helbig 1398, RP 217 6, Schefold 1957, 225, Schefold 1962, 169

VIII 5,15

Cat 272 (b) Parete ovest, zona superıore, III atle

lantastico inserıto da Ennıo (cf la sua biblıografia)

65. Nash 1961, 472, fig 581, Helbig\&s 4, 1804 
Un'edicola al lato destro e occupata dalla statua, ora perduta di una ligura maschile che indossd un mantello sulle spalle Mau descrive del llauti nelle sue manı, elemento che potrebbe identiticarld come Marsia

Bibl A Mau, BdI 1883, 203, Schetold 1957, 227

VIII 5, 24 Casa del medico

Cat 273 (peristllio) Parete est, quadro centrale, IV stue (ora MN 11319j)

Paesaggio egizio con P'igmei Suglı appoggiatol della rampa di un tempio vi sono due sfingi blanche Hanno le all e sembrano di sesso temminule

Bibl A Soglano, ISc 1882, 322-323, A Mau, BdI 1883, 229-230, Schefold 1957, 227, Peters 1963, 180. fig 178

VIII 7,3

Cat 274 (f) Parete di fondo, quadro centrale, III stile +

Paesaggio sacro-idlliaco Mau menziona un sacello con statua di Pane simile a quello del quadro Sogliano 197 659

Bibl A llau, Bdl 1884, 137, Schefold 1957, 230

VIII 8,28 'I empio di Iside

Cat $275 / 1$ (I) Parete sud, zona mediana, IV stule (ora MN) +

In tiammento MI 8975, e un quadro perduto provengono dal pannelli Sul primo si vede un'offerta ad Arpocrate La divinita sta dentro una nicchia e sembra raffigurata come persona viva resa in colori natural L'atteggiamento e quello normale con l'indice destro presso la bocca 660

Il quadro perduto rappresenta un paesaggio sacro-ıdlllaco, nel quale a destra si vedono due statue di donne vestite ds un mantello lungo sopra una base comune Nella tholos al centro e sopra una base rettangolare la statua di una figura maschle che regge una cornucopia cn la mano destra e una bacchettd con la sinistra Ln mantello copre le gambe Clid l'interpreta come Osiride

I frammenti MN 8517,8518 e 8528 provengono dalle prospettive fra 1 pannell Si vedono architetture glalle su uno sfondo rosso nelle qualı sono presentı del centauri in torma di acroterı, raffigurati in coppia $S_{1}$ reggono sulle zampe posterior e tengono un timone o una conca nelle mani

MN 8519, parte di una prospettiva, mostra una biga gialla, vista di fronte, guidata da una Nike o un Eros con le alı $\mathrm{M} \backslash 8529$ contiene una biga conciotla da una figura alata, vista di profilo rivolta verso destra Infine le figure nel framinento 8527 sono diventate illeggibili

Bibl Elia 1941, 7-8, 12-13, fig 6-7, tat I, Schefold 1957, 232, Tran Tam Tinh 1964, 135, pl V 1, no 29

\footnotetext{
659 Cioe l'aposhopon nella Casa deglı epigramm (cat 185 2)

6s0 Cf statuette, per esempio Tran Tam Tinh 1964, pl. YII-VIII
} 
Cat. 275/2. (VI) Tutte le pareti, zona mediana; IV stile (ora MN)

I prospetti contengono dej candelabri sormontati da statue di sacerdotesse di Iside vestite di un chitone senza maniche e con un modio sulla testa. Reggono degli oggetti di culto nelle mani distese. Lo stile è arcaizzante. Tutto è eseguito in giallo. MN 8913 e 8928 sono pendants: in una mano hanno un ureo, nell'altra una situla. 9768 è quasi simile e proviene da una coppia paragonabile. La sacerdotessa nel frammento 8926 regge un piatto con una cassetta e bende con la mano sinistra e una bacchetta con la destra.

Gli altri frammenti sono i quadri dei pannelli. Contengono paesaggi sacro-idillaci; la loro situazione originale puo essere ricostruita in base alle incisioni dell'epoca.

l'arete nord. A sinistra si vede un paesaggio con una porta sacra (MN $8575)$. Su un muretto semirotondo è seduta una sfinge femminile bianca.

Nel campo centrale si trova il quadro con Io in Egitto (MN 9558). A sinistra è seduta una sfinge maschile barbuta di colore rosso. Ha un polos sulla testa. Elia lo ritiene un ritratto di dinastia ptolemaica. Si tratta piuttosto di una figura decorativa come nella Casa del duca d'Aumale (cat. 201).

Il campo a destra contiene un paesaggio con a sinistra una base con decorazione a rilievo (MN 8574). Sullo slondo verde si delincano due ligure femminili vestite di un chitone senza maniche. Sulla tosta hanno un modio e nelle manj reggono oggetti non identificabili. Elia descrive la base come tripode.

Nel campo sinistro della parete ovest si vede un paesaggio con una tholos (MN 1265). A sinstra sopra una base è seduto un uomo vestito di un mantello lungo e con una corona a forma di ureo sulla testa. Nella destra distesa regge un sistro, nell'altra un lembo della veste. Si tratta probabilmente di Ebè, il figlio di Hathor. Alla sua destra si vedono due sfingi bianche barbute, poste sopra basi rettangolari.

Vel campo a destra si vede la tomba di Osiride (MN 8570). A sinistra si vedono due muretti paralleli sui quali si distinguono statuette di donne vestite di un mantello lungo.

Il pinax sinistro della parete sud, ora perduta, contiene un tempietto vicino al quale è una statua resa in modo schematico. Davanti ad essa si vede un piedistallo basso con una sfinge.

Bibl.: Elia 1941, 23, 27-35, fig. 2i, tav. I-II, 2, 4-7, C; Schefold 1957, 233-234; Peters 1963, 167-169, fig. 163-165; Tran Tam Tinh 1964, 138-143 no. 39-47, pl. VI, VIII, X, XVIII; Silberberg 1981, no. 73, $75-76$, fig. 70-71, 73-74.

Cat. 275/3. (IV) Parete nord, zona mediana; IV stile

Rilievi a stucco a sinistra e a destra dell'entrata. Sopra mensole a forma di calici vegetali vi sono due statue di sacerdotesse isiache di stile classicheggiante. Indossano un chitone senza maniche e un mantello. Le mani sono nei fianchi. Sono paragonabili alle figure sui candelabri nel vano VI.

Bibl.: Schefold 1957, 231; Malaise 1972, 276-277, pl, 43b; Mielsch 1975, 62, 146 no. 55; De Vos 1981, 61, tav. XXXVIII.

IX 1,7

Cat. 276. (e) Pareti nord e sud, zona superiore; IV stlle 
La trabeazione che chiude la zona superiore al disopra isene sorretta da un'erma con una testa femminule Al fusto sono statı appesi degl uccell, prede di caccid La testa e gli anumalı sono statı delineat in modo dettagliato

vella prospettiva centauri che reggono uno scudo nella mano esterna sorreggono la trave di un portico con la testa Attorno alle spalle hanno uns clamide Vella mano interna reggono una spada Hanno una coda di pesce verde, il corpo e di colore marrone

Ld trave sorretta dar centauri e sormontata da una pantera seduta sulle zampe posteriori, dipinta in colori naturali $E^{\prime}$ rivolta verso ll centro e vista di profilo

La prospettiva al lato sinistro della parete nord e andata perduta quası completamente Della parete sud non e rimasto conservato nulla

Bibl Schefold 1957, 235-236, Schefold 1962, 122, Taf 84, Schmidt-Colnet $1977,147,273$ no P17

IX 1, 22 Casa di Epidius Sabinus

Cat 277'1 (t') Paretı ovest e nord, quadro centrale. III stule +

sulla parete ovest si trova un pinax raligurante diteone e Artemide vel centro $v_{1} \rho$ un tempietto con decanto, ul una base rettangolare, la statua di un cervo L'anumale dianza verso destra con la Lampa destra anteriore alzata La testa e volta inchetro Ln cervo sumle di bronzo serviva da elemento topiarıo nella Casa del citarısta 602 Come nella Casa del marınaı (cat 259) ll cervo in questo contesto definisce il sacello come luogo sacro di Artemide, connesso con la scena raffigurata

II quadro della parete nord mostra Omfale e Eracle Eracle e seduto su uno scoglıo con l dorso riolto allo spettatore L'atteggiamento della figura viva e quello dll'Eracle di Taranto

Bıbl Helbig 252a, RP 52 2, Schefold 1957, 238, Schefold 1960, Pdar 1962, $105-106$, $\mathrm{lbb} 18$, Peters 1963, 87, flg 73 ; Bastet De Vos 1979 60, fig 11 , Swinkels 1984,45 , fig 2

Cat $277 / 2$ (y) Parete ovest, zona superiore, I stule +

Una serie di bugne mostra elementı figuratıvi monocrom, del qualı almeno $ا$ seguente rappresenta l'umitazione di un rlievo. Ln tauno danzante avanza verso destra Il piede destro e sollevato La mano destra sopra la testa ricorda l'aposkopon Vella mano sinistra regge un pedum presso la spalla Sulla testa forse si trova una corona $S_{1}$ contronti ll satiro nel cubicolo 4 della Vlla del misteri (cat 306/2)

Secondo Schefold la decorazione di questa parete non e di I stile, ma un restauro o un'mitazione dell'epoca vespasianea, mentre solo la parete nord conterrebbe ancora la decorazione orıginale

B1bl Mau 1882, 101-102, Schefold 1957, 239, Eristor 1979, fig 3, Pompe1 1748-1980, 181, cat 47A1, De Vos Vartin 1984, 131, 139 (vano a), fig 1

IX 2, 7 Casa della fontana d'amore

662 Dwyer 1982, fig 136. 
Cat. 278 (h) Tutte le paretı, zona mediana; IV stile +

Kappresentazione di un giardino sulle pareti che circondano il giardino Le statue tuttora sono lleggibil e sono conosciute solo attraverso vecchie descrizionı.

Parete sud. La nicchia absidale al lato destro e occupata da una ninfa acquatica bianca che regge un bacle in entrambe le mani poste davantı al grembo. Lna veste copre le gambe Nel muro, sopra $\downarrow$ bacino, si trova un tubo dal quale puo uscire dell'acqua, da collegare in una vasca marmorea darant alla tiguri dipinta lonostante cio anche il bacino dipinto mostra l'acqua zampllante Il pittore ha slruttato la situazione attuale nella combinazıone $d_{1}$ una vera lontana con una statua dipinta

Vella nucchia quadrangolare a sinıstra vi e sopra una base rettangolare la statua bianca di un uomo con una clamide sopra le spalle Regge nella destra una clava, nella sınıstra un oggetto invisıbule, dal quale esce dell'acqua; forse e un ritone o un cantaro Sulla base della descrizione si potrcbbe pensare a un Eracle del tipo bibax sumle a quello nelle Terme stabiane (cat 231'3).

A sinistra delle due nicche si trova la statua bianca di un satiro che suona un flauto doppio Ha una barba e sulla testa una corona

Parete est. Nel centro vi e und lontana blanca horretta da una sfinge femminile alata Sul bacino poggiano dei piccionl che fanno pensare al tamoso motıvo di Sosos ${ }^{6}{ }_{2}$ La combinazıone di una vasca con questı uccell 1 con un plede a torma di stinge e unica.

Grumal menziona (la statua $d_{l}$ ) un tritone che gioca con una pantera marina sulla parete sud

Parete nord Forse dirımpetto al satiro della parete sud si trova un satiro posto sopra un piedistallo Avanza verso sinistra e regge nella sinistra un pedum e nella destra una siringa Sulla testa ha una corona

lella zona superiore si trova un tregio con un paradeisos nel quale sarebbe stata presente un'erma

Dell'arredamento dello stesso giardino e rumasta conservata la statuetta di un Eros che cosi si inserisce facilmente nel tiaso delle figure dipinte 663

Bıbl G Minervin, BullArchNap 1 (1852-1853) 27-30, Helbig 1058, 1119 , 1587, 1778, Schelold 1957, 240, Ciprott 1957, 156-158, Schelold 1962, 90-91, Grimal 1969, 456 no 32 ; Muthmann 1975,92 nota 45, Pfretschner 1977, no. 66, De Vos'De Vos 1979, 90-93; Jashemski 1979, $186-187$.

IX 2,16

Cat. 279/1. (b) Parete ?, freglo e zona superiore; III stule +

Nel fregio tra le zone mediana e superiore si trova un paesaggio sacro-iduliaco. Vicino ad un albero vi $e$ un'erma con la testa di Dioniso barbuto. Sulla testa ha una corona. E' rivolto verso destra.

Nella zona superiore si trova una scaenae trons, conosciuta attraverso descrizioni tra le qualı viene riportata quella di Mau:

Die kleineren Architecturen der oberen Wandtheile sind viel reicher und bunter als sonst auf Woanden dritten Stils der Fall zu sein pflegt. Wir sehen da cannelirte solule und Pfeiler. karyatidenartig verwandte Figuren, unter denen Amazonen;

\footnotetext{
662 Parlasca 1963.

663 Jashemski 1979, 186.
} 
ausserdem Vdfgel mit Menschenkdpfen, beide nicht in den nat firlichen Farben, sondern in grtun und gelb gemalt, und einen nackten Apoll mit dem Bogen. auch diese Figuren sind karyatidenartig verwandt. Der Fries ist violett, mit oligochromen Darstellungen

Forse l paesaggio descritto sopra appartiene a questa serıe di raffigurazion monocrome Potrebbe tiattarsi di imitazioni di rilievi

Bibl Helbig 579b, Sogliano 543-546, Mau 1882, 441, Schefold 1957, 242, Schefold 1962 67-68. Taf 444 , Pfuctschner $197 \dagger$ no 38

Cat 279 ' (e) Parete nord, quadro centrale, III stule +

Bellerotonte $e$ un'Amazzone A destrd in tondo $w$ e sopra una base la statua di Artemide Indossa un chitone senzd maniche e un mantello Avanza verso destra e tende il suo arco Il colore non e noto

Bibl Soglano 547, Schefold 1957, 243

Cat 279/3 (h) Parete sud, quadro centrale, IV stule (ora M 9236)

Le tre Grazie sono collocate in un paesaggio con roccie Ld figura centrale, vista sul dorso, abbraccia le altre due che stendono la mano esterna Le loro gambe esterne sono di appoggio, mentre ll peso della ligura centrale poggia sulla gamba sinisira Hanno una corona in testa Le figure esterne reggono un mazzo di tiorı verso il quale rolgono lo sguardo

Imitano il gruppo ellenistico frequentemente copiato nel mondo romano $\mathrm{S}_{1}$ rimand alla discussione a proposito del pmax con lo stesso tema dell'insula occidentalıs. Masserıa Cuomo (cat 227)

Bibl Helbig 856, Herımann I, 61-62, Taf 49, Elid 1932, no 137, Becnttı 1937. Lullies 1948, 45. Scheiold 1957, 242, Schwarzenberg 1966, hellner 1974

IX 2,18

Cat $280 / 1$ (f) Parete nord, quadro centrale, III stule +

Paesaggio sacro-ıdlllaco Su una base presso una porta sacra e la statua bronzea di Dioniso Indossa un chitone e regge nella mano sinistra un tirso e nella destra und patera

Bibl Sogliano 245, Rostowzew 1911, 41, Dawson 1944, 88 no 17a, Schefold 1957,244 , Silberberg 1981, no 41

Cat $280 / 2$ (I) Parete nord, quadro centrale, III stule +

Pailde, Elend e Afrodite sono raffiguratı in un paesaggio, probabulmente a Sparta Presso una porta sacra vi jecola erma di Priapo

Bibl Soglıano 568 RP 1643 , Dawson $1944,87-88$ no 17 , pl 5, Schefold 1956, 226, Schefold 1957, 244, Schefold 1960d, 92, fig 11, Paar 1962, 121-122, Peters 1963, 89-90, Sichtermann 1984, 299, Taf VII

IX $2 \quad 21$

Cat 281 (m) Parete ?, quadro centrale, III stlle + 
Lna donna sta presso la statua gialla di una divinita Sogliano la descrive Il dio rappresentato in maniera arcaica, prendendo con la dr. abbassata un lembo della sua veste, e tenendo fra il pollice e l'indice della sin. un breve tirso Trendelenburg chiama l'ult imo oggetto un caduceo.

Bibl. A. Trendelenburg, BdI 1871, 194; Sogliano 243, Mau 1882, 387; Schefold 195\%, 244 .

IX 3,5 Casa di $\mathrm{M}$ Lucretius

Cat 282/1 (2) Tutte le pareti zona mediana e superiore, IV stile +

Vel prospetti di colore giallo della zona mediana sono state inserite delle lereidi come acroterı \ella zona superıore sı trovano, sopra l'edicola centrale dı ognı parete, due centaurı che reggono una clava o brandiscono una pietra sopra la testa

Bibl . Viccolıni/Niccolını 1854-1896, I 2, 6; Helbig 504c, Schefold 1957, 246.

Cat 282/2 Pareti ovest, sud e est, zona mediana, IV stule I campı lateralı blanch sono stati abbellitı con Erotı che stanno sopra def piedistalli vistı di scorcio Hanno una clamide sopra le spalle e reggono delle armı, imitando e caricaturizzando cosı i guerrıerı nudi ( $c f$ p 43 ).

Parete ovest, campo nord $\mathrm{Ha}$ un elmo nella destra e uno scudo a una lancia nella sinistra La gamba destra e di appoggio.

Parete ovest, campo sud $\mathrm{Ha}$ un elmo nella destra e uno scudo nella sinistra Il peso del corpo poggia sulla gamba sinistra

Parete sud, campo ovest lino scudo vicino al pledi i lene sostenuto con la mano sinistra Un gladio viene tenuto presso ll fuanco destro La testa e coperta da un elmo

Parete sud, campo est. Lin elmo copre la testa. Ha uno scudo nella sinistra e una lancia nella destra La gamba destra e di appoggio.

Parete est, campo sud hegge uno scudo con la mano sinistra e ha la destra distesa. Il peso del corpo poggia sulla gamba sinistra.

Parete est, campo nord. Un elmo copre la testa. Ha uno scudo nella sinistra e una lancia nella destra La gamba destra sorregge il peso del corpo

Il quadro centrale della parete sud mostra Chirone e Achlle in un ambiente paesistico Sono ratigurati nell'atteggiamento del gruppo nella cosiddetta baslica di Ercolano (cat. 019), al quale si rimanda per la discussione dell'archetipo.

Bibl. Helbig 624, 1294; Schefold 1957, 248.

Cat. 282/3. (6) Tutte le pareti, zone mediana e superıore, IV stille l pannell nella zona mediana delle paretı nord e sud contengono Erotı sopra piedistallı. Sono nudi e reggono attributı di divinita

Parete nord, campo est. 'Dioniso' regge un cantaro nella destra stesa verso il basso e un tirso sopra la spalla sinistra La gamba destra e di appoggio.

Parete nord, campo ovest 'Hermes' regge una benda nella destra stesa verso ll basso e un caduceo sulla spalla smistra. Sulla testa ha ll petaso Ia gamba destra $e d_{1}$ appoggio L'atteggiamento e sunde a quello dell'Hermes Ingeminı di Polıcleto

664 Zanker 1974, Taf 15. 
Parete sud, campo ovest. 'Eracle' ha un ramo d'olivo nella destra distesa e una clava sopra la spalla sinistra. La gamba sinistra è di appoggio. L'atteggiamento è quello del Doriforo.

Parete sud, campo ovest. 'Apollo' regge un plettro nella destra davanti al petto e una cetra nella sinistra presso la spalla. Il peso del corpo poggia sulla gamba destra.

I prospetti di tutte le pareti contengono delle figure, ora quasi illeggibili. Sulle pareti sud e ord si vedono alcune donne vestite di un peplo in guisa di acroteri. Sulle parcti est e ovest donne simili alle prime a uomini nudi stanno sull'architrave delle edicole che sono state inserite nelle prospettive. Le ligure sono delineate in Stile Severo e dimostrano un'evidente somiglianza con le figure nelle scaenae frontes di III stile.

Sulla parete est si vede a sinistra un uomo con la mano destra sopra la testa e la sinistra stesa per metà. La gamba destra è di appoggio. A destra vi è una donna che regge nella destra stesa una patera e nella sinistra stesa verso il basso una fiaccola (?). Sulla testa si distingue un modio.

Sulla parete ovest è a sinistra un uomo con una clamide rossa che regge una bacchetta nella sinistra alzata, mentre la destra e in riposo lungo il fianco. La gamba destra è di appoggio. A destra vi è una donna con un modio sulla testa che regge con la destra un lembo del mantello presso la spalla e un piatto nella sinistra.

L'edicola centrale della zona superiore della parete nord contiene un quadretto con un paesaggio sacro-idilliaco sormontato da una biga, guidata da una Nike. Ha una palma nella mano sinistra e una corona nella destra distesa. $E^{\prime}$ rimasta conservata solo la sagoma.

Bibl.: Helbig 603.605-607, 939; Niccolini/Niccolini 1854-1896, I 2, 3; RP 144. 7 ; Schefold 1957, 247.

Cat. 282/4. (7) Pareti est e ovest e posti della porta; zona mediana; IV stile Gli stipiti della porta e i campi laterali contengono Eroti sopra mensole. I due nella porta sorgono sopra candelabri. Come nel vano 4 le t'igure imitano i guerrieri nudi.

Parete est, campo nord. Regge uno scudo nella sinistra presso la spalla e una lancia nella destra. La gamba destra è di appoggio.

Parete est, campo sud. Regge uno scudo e una lancia nella sinistra, mentre la destra è in riposo sulla testa. La gamba sinistra è di appoggio.

Parete ovest, campo nord. Regge una lancia nella destra e un oggetto non identificabile nella sinistra. Il peso del corpo poggia sulla gamba sinistra.

Parete ovest, campo sud. Ha uno scudo vicino alla spalla sinistra e regge una lancia nella destra. Il peso del corpo poggia sulla gamba destra.

Stipite est. Regge una fiaccola nella sinistra sopra la spalla e un oggetto non piu visibile nella destra stesa. La gamba destra è di appoggio.

Stipite ovest. La figura è andata perduta.

Il quadro centrale della parete est contiene Polifemo. Esso è seduto nell'atteggiamento dell'Herakles Epitrapezios di Lisippo.

Bibl.: Schefold 1957, 247.

Cat. 282/5. (8) Parete ovest, zona mediana; IV stile

Sull'edicola centrale sj vedono acroteri a forma di centauri. Sono di colore rosso. La figura sinistra regge un pedum nella sinistra, mentre la mano destra copre il petto. L'altra ha una conca presso la bocca.

Bibl.: Helbig 106; Schefold 1957, 249.

Cat. 282/6. (9) Pareti est, ovest e nord, zone mediana e superiore; IV stile 
I prospettı nella zona mediana delle pareti est e ovest contengono figure reggentı, collocate sopra predistallı rettangoları. Sorreggono una trave con la testa Le figure sulla parete est sono quası completamente svanite

Sulla parete ovest si vede a sinistra una donna vestita di un mantello lungo che regge un piatto nella sinistra e un lembo del mantello nella destra La higura a destra indossa una veste simile e regge due piattı all'altezza delle spalle

L'edicola centrale nella zona superiore della parete nord contiene una donna che sta su un pjedistallo rettangolare Indossa un chitone del quale alra con la destra un lembo presso la spalla Regge nella sinıstra un piatto

Bubl Schefold 195\%, 248

Cat 282/7 (16) Tutte le pareti, quadro centrale, IV stule (ora MN)

MV 8992 Eracle e Omfale Secondo Lippold le figure sono state modellate secondo tipi scultore L'Eracle ripete l'Eracle Farnese di Lisippo 665 Umiale e stata derivata dall'Artemide Waroque Eros imita l'Fros della Pantoffelgruppe di Delo Lippold conclude dass das Bild als Ganzes, als recht getreue Nachbildung wenigstens in den Formen, der Nachfolge Lysipps, der Zeit gegen 300 angeh ort

I quadrı seguentı rappresentano Erotı festeggiantı similı a quells nella Casa della parete nera (cat 247) Le statue sono state inserite come element topiarı senza significato specifico Le figure rientrano nel tiaso bacchico

MN 9207 Sopra una base rotonda verde vi e la statua verde di Dioniso barbuto Indossa un chitone ed ha una corona sulla testa Regge un tirso nella sinistra

MN 9208 Sopra una base rotonda verde vi e la statua verde di Psiche. vestıta di un peplo e di un mantello Ha le manı stese per meta

UA 9193 Sopra una base rotonda verde sta la statua di una figura maschile che regge una palma nella sinistra stesa per meta e ha la destra distesa La gamba destra e di appoggio lon e chiaro se si trattı di Apollo o Hermes

MN 9255 Su una base rotonda sta la statua, ora appena visibule, di un uomo con una clamide sopra le spalle Poggia su una clava che sta alla sua sinistra La testa e inchinata, il peso del corpo poggia sulla gamba destra Forse si tratta di un Eracle Farnese o di una copıa ritlessa dell'Cracle Lansdowne

Bibl Helbig 757, 759, 760, 767, Herrmann 1, 83-86, Taf 64-65, Abb 22-24, RP 93 3-4, 401 2, Bueber 1927, 30, Eha 1932, 25, 227, 229-230, Lippold 1951, 250, Schetold 1957, 249, Pfretschner 1977, no 61b, 65a, 88, Jashemshı 1979, $97-98,177$, fig 262-263.

IX 5, 2 Casa di Achulle

Cat $283 / 1$ (e) Parete nord, zona mediana, IV stile

L'edicola centrale ha acroterı a forma di centaurı doratı Reggono una clava sulla spalla al lato interno, mentre l'altro braccio e alzato Le figure sono state dipinte in modo plastico come in II 1,7 (cat 27(s)

Bibl Schefold 1957, 252

Cat $283 / 2$ (n) Parete ovest, zona superiore; IV stule

665 Cf Moreno 1983 
I prospetti laterali contongono pergole di legno abbellite con tralci di vite, che vengono sorrette da quattro erme. Le due in avanti sono marroni, le altre verdi. Ilanno teste barbute, forse di Dioniso. I fusti sorgono da calici vegetali. Ad essi sono attaccati piccoli pezzi di legno.

Le costruzioni imitano vere pergole come si vedono raffigurate in varie pitture. Von sono state trovate erme in queste costruzioni pompeiane. ${ }^{66}$ Bibl.: Schefold 1957, 252.

IX 5, 6 Casa dell'Ercole ebbro

Cat. 284/1. (6) Pareti accanto all'entrata, zona mediana; IV stile +

Le entrate verso l'atrio e l'esterno sono fiancheggiate da erme con teste feminili, ornate con festoni. Mau le paragono alle erme di II stile nella Casa di Caesius Blandus (cat. 233:2); Schefold invece le inseri nel IV stile. Probabilmente si tratta di figure simili all'erma a ritratto in I 16, 3 (cal. 174) che sostituiscono erme reali nell'atrio o vestibolo. $E^{\prime}$ piu probabile una datazione nel IV stile.

Bibl.: A. Mau, Bdl 1879, 113-116; Sogliano 758; Schefold 1957, 253.

Cat. 284/2. (c) Parete nord, quadro centrale; IV stile +

Chirone e Achille. I due sono nell'atteggiamento del gruppo della cosiddetta basilica ad Ercolano (cat. 019). Sarebbe quindi un'imitazione del gruppo nei Saepta a Roma; per la discussione su questo problema sl rimanda al complesso citato.

Bibl.: Sogliano 571: Schefold 1957, 254.

Cat. 284:3. (t) Pareti est e ovest, zona superiore; IV stile

Scaenae frontes fornite di siparia e tre statue per parete sopra piedistalli rettangolari. I colori sono andati perduti.

Parete ovest. Nell'aula regia vi e una donna vestita di un chitone senza maniche che ha una corona sulla testa. Il peso del corpo poggia sulla gamba destra. Regge con entrambe le mani stese in avanti una corona.

A sinistra si vede un uomo vestito di una toga romana che ha una corona sulla testa. La gamba sinistra e di appoggio. Le mani sono in riposo davanti al petto, avvolte nella toga.

A destra vi é una ligura ammantala di sesso indefinito. La gamba destra è di appoggio. Regge una corona nella mano destra stesa in avanti.

Parete est. Nell'aula regia vi è Atena vestita di un chitone senza maniche che copre la spalla destra con uno scudo. Regge una lancia nella mano destra. E' rivolta verso sinistra e poggia sulla gamba destra.

L'hospitale sinistro e occupato da un uomo togato incoronato. La gamba destra è di appoggio. La mano sinistra si trova nella toga davanti al petto, l'altra $\dot{e}$ in riposo lungo il fianco. L'atteggiamento è quello dei ritratti di oratori e lilosofi quali l'Eschine della Villa dei papiri. ${ }^{66}$

Anche a destra si trova un uomo togato e incoronato. La mano destra è alzata. La figura ricorda l'Arringatore nel Museo $\Lambda$ rcheologico di Firenze.

\footnotetext{
666 Cf.Beyen 1938, 122; Van Buren 1938; Jashemski 1979.

667 Sgobbo 1972, tav. XII.
} 
Bibl. Soliano 623, Herrmann I, Taf 9b; Schefold 1957, 254; Pfretschner 1977 , no 29

IX 5, 9 Casa del Pigmei

Cat. 285 (g) Paretı nord e est, zona mediana, IV stile

L'intero ambiente e decorato con un paesaggio nulotico. Al lato ovest della parete ovest si vede un sacello con un plastro sul quale e la statua gialla di un coccodrillo. L'animale e rivolto verso destra e risalta per l'esecuzione dettagliata. Rappresenta il dio Sobeh che viene venerato sotto lorma di un coccodrillo. Il colore indica la doratura. Ion e necessario attribuire un valore apotropeico alla bestia, come fece G. Spano, in quanto la sua funzione come divinita giustifica sufticientemente la sua presenza

Un altro coccodrllo che rappresenta Sobek si vede su MA 8512 (cat. 049)

Statue che possono servire da contronto sono state trovate in complessi sacrali $e$ in nunfel, fra l'altro nel Canopo della Villa Adriana a Tivoli 660

Il plastro imita ll sarcofago di Osiride, sia in modo stilizzato e allungato, come sj trova su $\mathrm{MN} 8570$ dal tempio di lside (cat. 275/2)

Presso un altare sulla parete est c'e l'erma rossa itıfallıca di Priapo, raffigurata in modo schematico.

Bibl. Spano 1954-1955, 335-369, fig 1, 10, Schefold 1957, 256, Schefold 1962, 150-151, Tat 146-147, Peters 1963, 173-174, fig 171, Whitehouse 1977, 61-63.

IX 5,11 Casa delle Muse

Cat. 286/1. (a) Parete nord, zona superiore; IV stule

vell'edicola della prospettiva destra vi e una figura femminile vestita di un chitone senza maniche rosso. Ha la mano sinistra presso le tempie e regge nella destra alzata una pisside Lo stile e arcaizzante.

Il pendant a sinistra e andato perduto tranne alcune vaghe tracce La parete opposta deve aver contenuto tigure simli.

Bibl. A. Mau, BdI 1879, 197, Mau 1900, 200, Taf. 7; Schetold 1957, 257

Cat. 286/2. (e) Paretı est e ovest, zona mediana; IV stıle +

Sui campi grall, sopra mensole, si delineano guerrierı nudi d'umpronta policletea. La parete est e conosciuta attraverso una vecchia totografia Sul campo sinistro si vede un figura il cul peso poggla sulla gamba destra Regge un gladio nella destra distesa e uno scudo nella sinistra lungo il fianco (cf. Casa di Octavius Quartio, parete ovest campo nord, cat 177/1; Terme suburbane ad Ercolano, cat 024).

La figura sul campo centrale regge uno scudo e una lancia nella sinistra e ha la destra alzata La gamba sinistra e di appoggio (ct. Terme suburbane, (at. 024)

Il guerriero a destra ha uno scudo nella sinistra lungo il fianco e un glndio nella destra Il peso del corpo poggla sulla gamba sinistra Sembra una copla dell'Ares Borghese con la posizione delle braccia cambiatd

668 Roullet 1972, 127-128 no. 254-259, fig. 260-262. 
Bıbl Soghano 768, Schefold 1957, 258, Schefold 1962, 135, 177, Taf 13:-133, Pompel 1748-1980, 56, lig 13-14.

Cat $286 / 3$ (f) Tutte le paretı, zona mediana, IV stule

Quasi llegibul sono le vignette a forma di Muse sui campl grall Le figure stanno su strisce sottuls

Parete sud, campo sinistro una donna in chitone rosso e mantello giallo e rimasta conservata solo per quanto riguarda la parte inferiore

Parete sud, campo destro Donna vestita allo stesso modo ha la destra stesa per meta Secondo Sogliano (422) rappresenta Polınna

Parele ovest Tre sagome di Erato, Callope e Terpsichore (Sogliano 409, 412), non piu leggibili

Parcte nord, campo sinistro Sagoma di Melpomene (Sogliano 405)

Parcte nord, campo destro Incisions di una figura che regge una cetra, forse Euterpe (Sogliano 406)

Parete est Tre sagome dı Clıo, Talıa e Urania dı cuı sono ancora visıblı l globo, la bacchetta e 1 piedı (Sogliano 103419 , 424)

Bibl Sogliano (v numero per tigura), Schetold 1957, 258

IX $5,14-16$

Cat $287 / 1$ (a) Parate est, quadro centrale IV stule

Diomso e Arjanna in tondo, sopra una base rettangoldre, i e la statua rossa di un satiro Kegge nella mano destra dizata un ritone e nella mano smistra un pedum Le grambe sono divaricate

Bibl Sogliano 167, Schetold 1957, 259

Cat $287 / 2$ (c) Tutte le paretı, zone mediana e superiore, IV stule +

I campı della zona mediana contengono paesaggı sacro-ıdılliacı In uno di essi sta presso un albero sacro un'erma coperta da un elmo Regge una lancra nella mano sinistra e un oggetto non identificabile nella destra Schefold l'interpreta come Isis als Kriegsgoftin

Secondo Mau s prospettı nella zona superiore contengono tigure sopra una base Sarebbero distinguibulı un uomo con una cetra (Apollo?) e una donna vestita di un chitonc che regge un platto con la mano sinistra e un lembo della veste con la destra

Bıbl. A Mau, BdI 1879, 261, Schefold 1957, 260

Cat 287/3 (p) Parete ovest, quadro centrale. IV stle

Ifigenıa in Tauride La sacerdotessa regge nella mano sinistra lo xoanon di Artemide che e stato modellato come un Palladio, elmo, lancia e peplo sono gli attributı concuetı di Atena Solo la sagoma c rimasta conservata

Bibl A Mau, BdI 1879, 262, Sogluno 584, Phllppart 1925, 20 no 28, Schefold 1957, 260

IX 5,18 Casa di Glasone 
Cat. 288. (d) Parete ovest, zona superiore; III stile (ora Antiquarium Pompei inv. 17718)

Dal campo centrale nella zona superiore proviene un frammento nell'Antiquarium. Sopra una base bianca, ornata da strisce rosse per indicare il rilievo, vi è un satiro di colore marrone, nudo con una corona di pigne sulla testa. Lina nebride copre le spalle. Ha il Dioniso fanciullo sulla spalla sinistra e gli porge un grappolo d'uva con la destra alzata. E' una variazione ellenistica dell'Hermes di Olimpia, noto da varie copie. ${ }^{669}$ In forma dipinta si incontra anche nel portico della Villa imperiale (cat. 305.3).

Bibl.: Schefold 1957, 263; Zevi 1964, 31; Bustet/De Vos 1979, 49-51.

IX 6 lato sud 4-5 (Mau)

Cat. 289. (c) Parete?. zona mediana; IV stile +

Ln'H ufherme di Eracle sta accanto alla porta che porta al vano e. Ha una corona sulla testa e una pelle di leone gialla attorno alle reni. Regge una clava rovesciata nella destra. Secondo Schefold il viso è von stolzem Ausdruck. Erme di Eracle sono abbastanza frequenti e un esemplare di marmo giallo antico e stato trovato a Pompei. 670

Bibl.: Sogliano 492; Schefold 1957, 266.

IX 6 lato sud 7 (Minu)

Cat. 290. (n) Pareti sinistra e destra, zona mediana; III stile +

Paesaggi sacro-idilliaci sono stati inseriti nelle prospettive. Sulla parete sinistra esso contiene una statua di Priapo, secondo Herter in litore prope varia aedificia. Inoltre si vede la statua di Ecate che regge fiaccole in entrambe le mani stese in avanti.

Sull'altra parete il paesaggio contiene la statua della Fortuna. Il modello non è conosciuto.

Bibl.: A. Mau, Bdl 1880, 268; A. Mau, Bdl 1881, 24-25; Rostowzew 1911, 54 nota 1; Herter 1932,158 no. 183 ; Schefold 1957, 266.

IX $7,12(?)$

Cat. 291. (triclinio) Parete sinistra, quadro centrale; III stile +

Artemide e Atteone. A destra di una fonte stanno tre statuette sopra uno scoglio. Rappresentano divinita fluviali. La figura centrale è appena piú grande delle altre.

Bibl.: Sogliano 1115 (casa IX 7, 16); Dawson 1944, 88 no. 5; Schefold 1957, 268; Michel 1982, 565-566, Ab. 19.

669 Erim 1974.

670 MN inv. no. 6368 (CatLondon 1976, no. 192). Cr. il cosiddet to Alexikakos nel Museo Nazionale Romano (CatRoma I 5, 170-172 no. 72. 
IX 716

Lat 292 (b) Parels est e ovest, quadro centrale, III stue +

Sulla parete est si vedono Bellerolonte e Pegaso Nel centro sorge una schola presso la quale sta un'erma di Priapo

Sulla parete ovesi si trovano Artemide e Atteone A destra sta un gruppo di tre statuette di ninte acquatiche presso una fonte Afrodite e stata modellata secondo l'Afrodite di Doidalsas

Bibl Helbig 98, Sogliano 688, RP 1815 , Dawson 1944, 83, pl 2, Schefold 1957 269, Paar 1962, 100-101, Abb 17, Peters 1963, 80-81, fig

65-66 Muthmann 1975, 28, 32 33, Abb 3, Pfretschner 1977, no 89

IX 7,19

Cat 293 (h) Pareti sinistra e ?, quadro centrale, IV stule +

Paesaggi sacro-idلliaci Sulla parete sinistra esso contiene un'erma di Priapo, secondo Mau evidentemente tagliato di legno

sull'altra parete sorge la statua di Eracle con una pelle di leone sulla spall sinistra e una corona sulla testa Regge una clava nella sinistra

Bibl A Mau, Bdl 1883, 80 Schetold 1907, 270, Plretschner 1977, no 67, 79

IX 7, 25 Casa della fontana a colonne

Cat 294 (viridario) fontana a mosaico, IV stile

La fontana e stata decorata con un mosaico, da datare alla seconda meta del primo secolo d C La lunetta contiene una veduta di mare nella quale si vede un'Akte modellata secondo ll tipo della aufgelehnte Muse (ct cat 262/8)

Bibl Jolv 1965, 67-68 fig 2 27-28, veuerburg 1965, 132-133 no 37 , Sear 1977, 71, pl $181,191 \mathrm{lg}$ is, Jashemskı 1979, tig 73

IX 8, 3-6 Casa del centonarjo

Cal 295/1 (4) parcti nord e est, quadro centrale, III stle +

Paesaggl sacro-idlliaci con statue di titemide Pfretschner crede, basandosı sulle descrizion sommarie, che si trattı del tipo della 1 ike nel Palazzo dei Conservatorı a Roma Ln Artemide indossa un chitone e regge con la mano sinistra un arco, mentie prende con la destra una freccia dalla faretra posta sul dorso

Bibl A Mau, BdI 1882, 52, Schefold 1957, 274, Pfretschner 1977, no 13

Cat $295 / 2$ (8) Parete est, zona superiore, III stule +

La trabedzione del'edicola centrale viene sorretta da due sfingl femminulı alate Sono viste di fronte

Bibl Schefold 1957, 275, Elia 1974, 164, tav VII fig 7 
Cat. 295/3. (11) Parete di fondo, quadro centrale; IV stile +

Paesaggio sacro-idilliaco. Davanti ad un tempietto sorretto da due cariatidi vi è un'erma di Artemide. resa plasticamente. La mano destra é presso le tempie. Sullo sfondo, sopra una base comune, vi sono tre statuette che reggono fiaccole. Si tratta di un hekataion.

Sulla parete sinistra sarebbe stata visibile una statua di Priapo in un paesaggio sacro-idilliaco.

Bibl.: Rostowzew 1911, 43, Abb. 22; Schefold 1957, 280-281; Schefold 1962, 53. Taf. 153.2; Pfretschner 1977, no. 10; Silberberg 1981, no. 33, fig. 37.

Cat. 295/4. (33) Pareti est e ovest, zona mediana; IV stile

Rappresentazione di un giardino. Fra i cespugli si vodono delle fontane sorretle da sfingi leminili alate. Sulla parete ovest si vede una lontana. Sulla parete est se ne vedono due, modellate in modo simmetrico; le sfingi hanno lo sguardo rivolto verso il centro.

Bibl.: Schefold 1953-1954, 117, Taf. 50; Schefold 1957, 277; Jashemski 1979, 111, fig. 181; Pompei $1748-1980,176$ no. 38D.

Cat. 295/5. (40) Parete destra, quadro centrale; III stile +

Paesaggio sacro-idilliaco. Una donna presenta un'offerta all'erma itifallica di Priapo.

Bibl.: Rostowzew 1911, 40, Abb. 16; Schefold 1957, 278; Silberberg 1981, no. 32 , fig. $35-36$.

Cat. 295/6. (61) Tutte le pareti. quadro centrale; IV stile

Parete nord. Teseo riposa dopo aver ucciso il Minotauro. $E^{\prime}$ seduto nell'atteggiamento dell'Eracle Epitrapezios.

Le prospettive a sinistra e a destra, ora perdute, contengono erme.

Parete est. Ermatrodito e Sileno. A sinistra vi é un'erma di Priapo.

larete sud. Ifigenia in Tauride (ora perduta). La sacerdotessa scopre con la mano destra lo xoanon bianco-giallo di Artemide che regge nel braccio sinistro.

Bibl.: Sogliano 530, 585, 596; Herrmann I. Taf. 119; RP 98.3: Philipart 1925, 20 no. 27; Herter 1932, 160 no. 19; Schefold 1957, 278; De Vos 1980, tav. LVII; Silberberg 1981, no. 34, fig. 38.

IX 8,4

Cat. 296. (?) Pareti ovest e sud, quadro centrale; IV stile +

Paesaggi sacro-idilliaci. In quello della parete ovest si vede un tempio in cui due pilastri frontali sono stati sostituiti da cariatidi rappresentate schematicamente. A destra del tempio vi sono tre statuette di figure femminili poste sopra una base rettangolare comune. Reggono delle fiaccole nelle mani stese. Rappresentano un hekatation.

Il paesaggio della parete sud contiene una statua di Priapo.

Bibl.: Rostowzew 1911, 43, Ab. 23; Schelold 1957, 281; Schetold 1962, 153, J'af. 153.2; Pfretsehmer 1977, no. 9 
IX 8,6

Cat. 297. (9) Parete ovest, quarto quadro da nord; IV stile

Eracle e Omfale. Sullo stondo vi sono un tempio e una colonna sormontata da una statua schematica. Su uno scoglio in primo piano sorge un altra statuetta disegnata schematicamente.

Bibl.: A. Mau, BdI 1882, 105; Dawson 1944, 109 no. 61, pl. 24: Schetold 1957, 276; Beyen 1960, 445; Paar 1962, 64-65.

IX 9,8

Cat. 298. (b) Parete di fondo, quadro centrale; IV stile +

Le tre Grazie, modellate secondo lo schema consucto. Mancano sia una descrizione accurata che un'ummagine. Vedı Insula occsdentalis (cat, 227).

Bibl.: A. Mau, RM 4 (1889) 30; Schefold 1957, 282.

IX 9,12

Cat. 299. (i) Pareti sinistra e destra, quadro centrale: IV stile +

Paesaggi sacro-ıdilliaci. Sulla parete smlstra si vede un'erma di Priapo presso un altare. Sulla parete destra sorge una statua di Fortuna che indossa un vestito violaceo e regge una cornucopia nella sinistra e un tumone nella destra. Il modello è sumle a quello delle statuette nei larari.

Bibl.: A. Mau, RM j (1890) 270; Herter 1932, no. 187; Schefold 1957, 283.

IX 9.17

Cat 300. (k) Pareti sud c ovest, quadro centrale; IV stile +

Paesaggi sacro-idilliaci. Sulla parete sud i pescatori stanno presso un tempietto con statue. A destra vi e la statua di Priapo sopra uno scoglio. Tutto e stato delineato in modo schematico.

Sulla parete ovest si vede una statua non definibile posta sopra uno scogho.

Bibl.: Schefold 1957, 284; Schefold 1962, 153-154, Taf. 152.3, 153.1; Silberberg 1981, no. 35, fig. 39-40.

IX 9, 18 Casa del maiale

Cat. $301 ; 1$. (e) Pareti ovest e sud, quadro centrale: III stile

Prospetti con edifici. Sulla parete ovest nel centro del quadro sorge un tempictto con la statua gialla di Ecate, ora invisibile. E stata modellata secondo il modello consueto con due fiaccole nelle mani stese in aranti.

Sulla parete sud due cariatidi sorreggono i tetti del 'proplei' accanto a una porta. Sono state delineate in modo simmetrico con lo sguardo rivolto verso il 
centro. Con una mano sorreggono la trave sopra la testa, nell'altra stesa in avanti hanno una patera. Sono simili alle cariatidi negli zoccoli del Macello (cat. 252/1) e della Casa della caccia antica (cat. 245/2).

La porta centrale mette in un giardino nel quale vi c̀ un pinax bianco, posto sopra un pilastrino. Su di esso e stata rappresentata la lotta fra Lros e Pan.

Bibl.: A. Mau, RM 4 (1889) 101-117; Rostowzew 1911, Anm. 267; Beyen 1938, 290-291, Abb. 131-132; Lehmann 1953, 163, fig. 78; Spinazzola 1953, II, 840, fig. 824: Schelold 1957, 285; Reuterswärd 1960, 183, 190. Ab. 27; Schefold 1962, 68-71, Taf. 10.1, 45.1; Bastet,De Vos 1979, 89-90, tav. XLIX-L.

Cat. 301.2. (h) Parcte smistra, zona supcriore; IV stile + In quadretto rappresenta una statuetta di Priapo fiancheggiato da due maschere.

Bibl.: A. Mau, RN 4 (1889) 115-116; Niccolini/Niccolini 1854-1896, IV 2, tav. 10 ; Schefold 1957, 286.

IX 10, 1-4 Casa di Obellius Firmus

Cat. 302/1. (3) Tutte le pareti, zoccolo, zone mediana $a$ superiore; II stile, tase IIb

Gli zoccoli delle pareti est e ovest (ove illeggibile) hanno erme bianche sotto i campi laterali. Le teste sono svanite. Sotto il pannello centrale funge da atlante un uccello rosso con le ali verdi aperte e le zampe a forma di tralcio. Precede gli esempi di III stile (cf. p. 27 ).

Sulla parete sud due teste temminili che sorgono da calici vegetali verdi sostengono l'orlo superiore dello zoccolo. Le teste sono simili a quelle delle erme nel criptoportico della Casa del cruploportico (cat. 148/2-3). Il motivo rumane senza contronti, ma ricorda il genere del Bildnis im Blotterkelch. 61

Il campo centrale della parete est contiene la figura isolata di una donna vestita di un chitone branco e di un mantello verde che sta sopra una mensola ora invisibile. Ha un diaclema sulla testa e tiene la destra distesa e la sinistra davanti al petto. Spinazzola interpreta la ligura come Giunone.

Le colonne che tiancheggiano questo pannello sono state abbellite con rilievi sottili, resi con strisce gialle e rosse. Donne vestite di una veste lunga reggono, davanti al petto, una patera con una mano, mentre l'altra $\dot{e}$ in riposo lungo il fianco.

Sopra il campo sinistro della parete est si vede un pinax con Afrodite e Eros. Su un altare quadrangolare vi sono due erme semplici.

Bibl.: Spinazzola 1953, I, 352-353, fig. 400; Schefold 1957, 287; Schefold 1962, 53, Taf. 4, 28-33; Bastet/De Vos 1979, 53; Moormann 1984b, 649 .

Cat. 302/2. (15) Parete ovest, zona superiore; II stile, fase IIb

Pinax con una donna offerente modellata secondo il tipo della Fanciulla di Anzio. Forse si tratta di If igenia.

Bibl.: Schefold 1962, Taf. 32 .

671 Jucker 1961 . 
IX 13, 1-3 Casa dı Julius Polybius ${ }^{672}$

Cat 303\%1. (I) Parete est, quadro centrale. III stule

Paesaggio in bianco su uno stondo nero. Ln'edicola vene f sancheggiata da due sting. femminl Sono sedute sopra bası rettangolar e sono rappresentate trontalmente.

Bibl inedito.

(II, antisala) Tutte le parcti, zona superiore, III stule

Le edicole centralı sono occupate da figure femminili che stanno su piedistalli. Sulle paretı nord e sud (delle quali manca la parte superiore) le donne indossano un mantello violaceo che lascia scoperto ll petto Sulla testa hanno una corona. Con entrambe le mani reggono, davantı al petto, un festone Hanno le gambe unite Le vesti sono agitate da un vento immaginario come presso le Aurai dello Stile Ricco.

Le tigure delle pareti est e ovest stanno con le gambe chiuse Indossano del chitonı rossi senza maniche e dei mantellı azzurrı. Con una mano sollevano un lembo del mantello presso la spalla, con l'altra un lembo del chitone presso la vita Lo stıle e plu rigido in contronto con quello delle due tigure antecedentı e ricorda le Danaidı della Villa deı papiri.

Bıbl inedito.

Cat 303/2. (II) Paretı nord, ovest e sud, zona mediana, III stule

Sulla parete nord si vede un paesaggio sacro-ıdlllaco di colore marrone. Sul lato destro vi e la statua di Ecate che regge le consuete fraccole nelle mani distese

Le altre pareti contengono la rappresentazione summetrica di un giardino. Bacinı di fontane sono francheggiate da banconı bianchı sus qualı sono sedute sfing, femminll alate. Su ogni parete ve ne sono due, disposte simmetricamente con lo sguardo rivolto verso la fontana Chioma, occhi, alı e una collana sono statı dipintı in rosso (cf I 9, 13, cat 162'2).

Vista la decorazione dell'antisala la datazione e stata data al III stıle, ma le figure sopra descritte $e$ il paesaggio appartengono pluttosto al IV stile. Quando la decorazione e interamente visibile, possono essere dette parole decisive.

Bibl Ifichel 1978, 382, Taf. 70 1-2.

Cat $303 / 3$ (AA) Paretı est e ovest, zona superiore, IV stule

Nel centro, sopra basi azzurri rettangolari, sono le statue policrome di Afrodite e Ares Sono state dipinte in modo vivace e non si puo piu distinguere la differenza tra statua e figura viva.

Afrodite, sulla parete ovest, poggia sulla gamba sinistra. Regge conl la destra il lembo di un mantello azzurro che copre il dorso, presso la spalla. Sulla sinistra sollevata e seduto un piccolo Eros che le presenta uno specchio. Ha una corona sulla testa e una collana attorno al collo L'atteggarnento e simile a quello dell' Ifrodite di Arles, ma la figura del'Eros tanciullo ricorda piuttosto gruppi qualı l'Hermes di Olimpia e l'lrene di Cefisodoto.

672 Il restauro di questa casa, scavata negli ultımi annı, e ancora in corso cosicche e tuttora impossibile uno studio esauriente delle decorazion. 
L'Ares sulla parete est e stato modellato come gli atletı polıcletel La gamba sinistra e di appoggio Un mantello rosso copre le spalle, un elmo giallo la testa Con la sinistra regge uno scudo rotondo grallo, nella destra alzata una lancia Lo stesso atteggiamento si incontra presso I guerrieri in I 3, 25 (cat 146) nella Casa della Venere in conchiglia (cat 178), nella Casa di Octavius Quartio (cat $177 / 1$ ), nelle Terme suburbane ercolaness (cat 024) e su MN 116086 (cat 119)

Bibl Pomper 1748-1980, 107, fig 8-9

Cat 303/4 (LE) Paretı sud, nord e ovest, zoccolo e zona mediana, III stue Glı zoccolı delle paretı sud e nord contengono menadı in guisa dı carıatıdı Stanno su piccoli piedistallı rettangolar e hanno una colonnina sulla lesta sulla quale pogga l'or lo tra zoccolo e zona mediana Indossano mantellı volantı che lasciano scoperto il seno La figura sulla parete nord ha le gambe unite e regge nella mano destra alzata un tumpano, mentre il braccio sinistro e in riposo lungo il fianco

La ligura sulla parete sud avanza verso destra La mano sinistra e vicino alla spalla, l'altra al'altezza delle renı

Per quanto riguaida ll loro modello e la loro funzione sono simili alle cariatidi delle scaende frontes nelle zone superiorı di III stule, ma per la loro posizione non vi sono del controntı

I pannellı della zona mediana contengono paesaggı sacro-ıdlliacı Quello sul campo settumo della parete est mostra un editicio presso ll quale $v$ e un'erma marrone

Il quadro centrale della parete est raffigura ll supplizio di Dirce Lna porta sacra e occupata dalla statua bianca di Dioniso barbuto Ha sulla testa una corona e indossa un chitone Regge nella destra stesa in avanti un caritaro nella sinistra alzata un tirso

B ibl Pompes 1748-1980, 165, fig 22B

IX 13,5

Cat 304 (facciata esterna) Zona mediana, IV stule

Accanto all'entrata sl trovano due gruppl statuarl di colore marrone A smistra si vede Romolo vestito come un guerriero romano Avanza verso destra, mentre regge sulla spalla sinistra un troteo

A destra e stato dipinto Enea con il padre Anchise sulla spalla sinistra e con il figho Ascanio che avanza verso sinistra, dasanti agl altri Enea indossa un'drmatura romana, Ascanio una corta tumcd, mentre Anchise e vestito con una toga

I due gruppi fungono da pendants e imitano in modo plu o meno fedele le sculture che stavano esposte nelle nicchie della facciata dell'edificio di Eumachia sul foro Esse a loro volta copiano 1 gruppı del foro di Augusto L'iconografia valeva per l'intero Impero Infattı sono state trovate copie in varie dimensionı e materialı $W$ Fuchs, nella sua analisı dell'iconografia, sostiene che questa si basa su una descrizione di Oridio (Fasti V 563-j64), mentre Virglio (Aen 706-707, 721-726) sarebbe la tonte per un tipo diserso, per esempio per il famoso Affenaeneas 673 A Pompel possolio essere menzionati due esempı una terracotta trovata nella Casa del marmı e un rilievo su una clsta di piombo 674

673 MN 9089 (Brendel 1953 1954) 
Bibl.: Spinazzola 1953, I, 147-155, fig. 183-184; Schefold 1957, 289; Zanker 1972, 17, Abb. 40; Fuchs 1973, 628-630; LIMC I, 388 no. 98.

$V$ illa imperiale

Cat. $305: 1$. (A) Tutte la pareti, quadro centrale e zona superiore: III stile Il quadro centrale della parete est mostra Teseo che ha ucciso il Minotauro. Lá scena si svolge in un paesaggio roccioso nel quale e la statua verde di Atena Promachos. Essa de rivolta verso destra. Inclossa un peplo e ha un elmo a pennacchio sulla testa. Regge nella sinistra uno scudo e nella destra una lancia.

Su una piccola base ai piedi di Atena c'è una statuetta grigia raffigurante un'Atena Promachos simile. Probabilmente si tratta di un'offerta votiva d'argento. Lna statuctta simile è stata trovata nella Casa del citarista. ${ }^{65}$ La presenza di Atena accanto a Teseo e frequente nelle decorazioni in ceramica. ${ }^{676}$

L'architettura nella zona superiore contiene delle cariatidi. Sulla parete est si trovano due donne rafligurate simmetricamente vestite di un chitone rosso e un mantello verde. Stanno con le gambe unite su un elemento architettonico aggettante e sorreggono un candelabro vegetale. Con entrambe le mani reggono una bacchetta. Sono di stile classico e possono essere paragonate con le kanephoroi nella Villa Albani e nella Villa Torlonia. ${ }^{\prime \prime} 7$ Brunilde $S$. Ridgeway le confronta con la figura destra sul rilievo Chiaramonti e data l'archetipo, in base alla veste, nel quarto secolo.

Sulle altre parcti sono raffigurate simmetricamente dell donne poste sopra pilastri che sorgono sopra la lesena fra il campo estremo e quello adiacente. Indossano un peplo e un mantello rosso. Reggono con la mano interna un festone e con quella esterna un ramo d'olivo. Sono paragonabili alle Danaidi della Villa dei papiri.

Dei centauri costituiscono gli acroteri delle edicole centrali. Sono rivolti verso l'esterno. Il centauro al lato ovest regge una patera nella destra, quello al lato sud nell'una un ritone e nell'altra una corna.

Caroline E. Dexter attribuisce la decorazione alla bottega che ha lavorato nella Casa di Caecilius Jucundus.

Bibl.: Beven 1956, 54-57; Schefold 1957, 290-291; Reuterswärd 1960, 181; Peters 1963, 108-110; Lauter-Bufe 1967, 40-44; Ridgeway 1970, 119; Dexter 1975, 124-128; Allroggen-Bedel 1975a, 225; Pfretschner 1977. no. 26; Bastet/De Vos 1979, 37-39.

Cat. 305/2. (C) Pareti nord e ovest, quadro centrale; III stile Paesaggi sacro-idilliaci. Sulla parete nord, sopra un piedistallo rotondo, vi è presso un albero sacro la $H$ Gftherme di Dioniso. Il dio ha una barba e sulla testa una corona. Una clamide copre la spalla sinistra. Regge nella sinistra

674 Spinazzola 1953. I, fig. 187; Adamo Muscettola 1982b, 712-714, fig. 17. Cf. per l'iconografia inoltre F. Canciani,LIMC I s.v. Aineias; Petrillo Serafín 1982.

675 Dwyer 1982, fig. 168. Cf. Harrison 1965, G3, pl. 63b (statua di Nicia).

676 Brommer 1982.

677 Schmidt-Coline1 1977, W69. 
un tirso, mentre ha la destra presso il mento. Tutto e di colore bianco.

Sul primo pjano a destra e la statua blanca di Ecate che regge due fiaccole nelle mani stese in avanti. Ora la figura e quasi illeggibile.

Sulla parete ovest si vede la stringe dei Nobidi. Vel centro sorge un sacello nel quale vi sono un tripode e una statua di Artemide della quale e rimasta conservata soltanto la parte inferiore. Indossa un peplo bianco. Questo quadro e stato tolto della parete, ma si trova ancora nello stesso ambiente.

Bibl.: Schefold 1952, 108; Schefold 1957. 291; Peters 1963, 95; Pfretschner 1977, no. 73 .

Cat. $305 / 3$. (c) Parete est, zona superiore: III stile

Scaenae frons su uno sfondo bianco, ora sbiadita. $E^{\prime}$ stata articolata in scomparti che corrispondono ai campi nella zona mediana. Alternamente si vedono architravi triangolari (a tumpano) e rettangoları e costruzioni che non dipendono dalla struttura adiacente. Nel primo caso le figure servono da acroters, nel secondo caso sono figure reggenti o statue che occupano un'edicola. La descrizione comincia con il primo scomparto conservato da sinistra (lato nord).

1) Sul tumpano sono sedute donne che reggono un ventaglio, addossate a un sottile candelabro. Degli acroteri laterali quello a sinistra e andato perduto, qunllo a destra rappresenta un Eros con una gazizella.

2) Carıatidi summetriche stanno sopra un pilastro e sorreggono un epistilio. Sono vestite dj chitoni rossi. La mano interna è presso la spalla, l'altra stesa per meta rogge una patera. Le due edicole che circondano questa costruzione sono incoronate al lato esterno da sfingi femminilj alate e al lato interno da donne sedute su girali.

3) Viel centro sta Apollo Citaredo con una clamide sopra le spalle. La gamba destra è di appoggio. Ha una corona sulla testa e regge nella sinistra presso il fianco la cetra e nella destra distesa il plettro. Sugli angoli laterali si vedono donne inginocchuate. Indossano un chitone verde e un mantello giallo del quale sollevano un lembo al lato interno presso la spalla. La mano esterna porge un piatto. Sono simili alle figure femminili nella Caserma dei gladiatorı (cat. 197/3).

4) Nell'edicola centrale sorge sopra un pilastro un satiro nudo dipinto in colori naturali che regge sulla spalla sinistra un Eros e nella mano destra distesa una siringa. Ha le gambe unite. Il gruppo è una variazione ellenistica dell'Hermes di Olimpia. ${ }^{67}$ Sulle edicole laterali si trovano acroteri a forma di donne sedute sopra girali. Esse sono vestite di chitoni rossi senza maniche. Reggono con entrambe le mani un timpano.

5) Il timpano è incoronato da una Fortuna che indossa un peplo verde. Regge con entrambe le mani una cornucopia davanti al petto. Gli acroteri laterali consistono in centauri marroni che sono rivolti verso l'esterno. Reggono nella mano interna un pedum, nell'altra un oggetto non chiaro. La figura sinistra è andata perduta:

6) Nell'edicola centrale si vede sopra un pilastro la statua di Dioniso. La gamba destra è di appogg1o. Regge un tirso nella destra e ha la sinistra che è coperta da una clamide lungo il fianco in riposo. Sulle edicole laterali sono sedute sfing femminili alate; solo la figura sinistra è ancora visibile.

7) La struttura è simile a quella del quarto scomparto. Su un pilastro sorge una menade che undossa un chitone giallo senza maniche e un mantello azzurro. Regge un timpano rosa nella sinistra sulla quale batte con la destra.

678 Cf. Erim 1974. 
Bibl : Schefold 1957, 291, Bastet 1964, 150, Abb 2, Allroggen-Bedel 1975a, 227.

Ylla del misteri

Cat 3061 (atrio) Parete nord, zona superiore, II stile, fase Ib Paesaggio egizio Al lato est si vede su un'isola nel Nilo una statua bianco-grigia di Ecate Regge in entrambe le mani stese in avantı una fiaccola Sopra una colonna $v_{1}$ sono tre figure femminll recant $f_{1}$ laccole. Or vamente si tratta di un hekataion

Bibl Malurı 1931，4 $7-48,198,200-201,235$, fig 11, 83-84, Beyen 1938, 61-62; Schefold 1957, 296, Beyen 1960, 19, Peters 1963, 7-9, fig. 1, Von Blanchenhagen 1963, 112-113 nota 48, Fittschen 1975, 542 ; Slberberg 1981 , cat. 1 ..

Cat $306 / 2$ (4) Tutte le paretı, zone mediana e superiore; II stile, fase Ib Davani dd una serie di ortostatı rossı vi sono statue bianche di figure del tiaso bacchico. Poggiano sopra bası rettangolar verdı e gialle, che a loro volta stanno su un podio vercle

Parete sud, lato est Un satiro nudo sl regge sulla gamba destra, mentre quella sinistra e alzata La mano destra e in riposo nel fianco, la mano simistra e poggiata sulla testa.

Parete est, lato sud. Lina donna vestita di un chitone viola e un mantello giallo, che lasciano scoperta la spalla sinistra, avanza verso destra Regge nella sinistra, davanti al petto, un volumen, mentre l'altra e in riposo davanti al petto Sulla testa ha una corona L'atteggramento e imule a quello della Fanciulla di Anzio Bastet l'interpreta come Callope che, in certo modo, annuncierebbe le scene del grande tregio nella sala achacente.

Parete est, lato nord Dioniso ebbro viene sorretto da un satiro. Il dio ha la mano destra sulla testa e poggia la sinistra sulla spalla del satiro Esso circonda le reni di Dioniso con entrambe le mani Il gruppo copia in modo libero un gruppo ellenistico del quale si conoscono varie copie 6 is

Parete nord, la1o est Sileno e un satiro. Il Sieno indossa un mantello giallo che lascia croperto ll torso Ha la mano destra alzata e regge nolla sinistra un tirso Da sinistra avanza un satiro che tiene con entrambe le mani il mantello del Sieno Ha un panno violaceo attorno alle reni e una nebride sulle spalle.

Parete nord, centro. A destra della porta, posteriormante inserita, ${ }^{60}$ s! vede la meta di una tigura teminule vestita di un mantello bianco e una sciarpa gialla La mano destra e distesa.

A sinistra della porta su vede una figura sumile In entrambi $i$ casi potrebbero essere menadi danzantı

Parete ovest, lato nord Un satiro nudo avanza verso destra. E' stato modellato nell'at teg gramento dell'aposkopon

vella zona superiore della parete est si trova un pinax. Un uomo e un fanciullo portano un'ofterta allerma gialla di Priapo. Sulla testa e attorno al membro sono appese delle corone.

679 Fra l'altro un gruppo in grovacca a Parma (Sieveking 1941 lo considera una copia della pittura); Mileto (Manderscheid 1981, Kat 210, Taf. 30), Terme di Caracalla (Marvin 1983, 336)

6\&: Maluri 1931, 54-5j dopo il 62 
Bıbl Malurı 1931, 174-181 fig 64-69, tav XVI, Herter 1932, 158 no 182 , Beyen 1938, 59-88, Jucher 1956, 29-30, Schetold 1957, 294, Peters 1963, 9-10, Frova 1965, 14-15, 17, Bastet 1974, 217, Abb 4, Allroggen-Bedel 1976a, 160 nota 52, Ptretschner 1977, no 76

Cat 306/3 (14) Paretı est, ovest e sud, zona superiore, II stule, fase Ib (un frammento ora $M N$ s $n$ )

L'architrave che incorona la decorazıone sulle paretı est e ovest viene sorretto da gruppı statuarı dipintı con color vivacı Le figure sembrano personaggi vivı, presentı nel'ambiente I due gruppi sono summetricı quello della parete ovest si trova nel Yuseo Nazionale Ln grifo bianco assalte un Arumaspo che si copre con una pelta rossa, dlzata con entrambe le manı sopra la testa Eglı indossa una tunica gialla e ha la testa coperta da un drappo giallo L uccello sorregge con le alı apeite l'orlo superiore della decorazione Non e piu visibile come l'Arimaspo fosse inserito nell'architettura

L'insieme ricorda l gruppo marmoreo d) Ganimede assalito dall'aquila che era appeso sopra l'entrata della grotta a Sperlonga e che provocava un effetto simule 601

La parete sud contione un prospetto Nel centro vi e un uomo dipinto in colore giallo posto sopıa un piedistallo verde, andato perduto per meta $\mathrm{Ha}$ una clamide verde sulle spalle Si pensi all'Eros nella Villa di P I ammus Synistor (cat $004 / 3$ )

Bibl Malurı 1931, 188, fig 75-77, Beven 1938, 79-80, lbb 20 b-c, Levi 1947, I, $138-139$ fig 55 b-c, Schefold 1957, 295, Flagge 1975, 58-59 (datazıone sbaglunta)

Cat 306/4 (16) Parete sud, zona mediana, II stle, fase Ib

Il prospetto centrale contiene una tholos occupato dalla statua grigia di una figurd ammantata La parte inferiore e nascosta dietro la parete divisoria Lehmann interpreta la figura ora difficumente discernibile, come Afrodite, specie la Cnidia, sumle a quella nel trammento dell'Insula occidentalis (cat 263) Engemann invece la descrive come un $b^{d}$ artiger Heros

Bibl Malurı 1931 189-190, t1g 73 Lehmann 1953, 119 nota 155, Schefold 1957, 296, Cngemann 1967, 55-56, Taf 232 , Love 1970, 154-155

Fondo Barbatell (extra muros, lato nord)

Cat 307 (?) Parete?, zona?, IV stule (ora MN 12514)

Su un campo rosso b clelinea una fortuna vestita di un peplo verde (he sta sopra una mensold retlangolare liegge una cornucopla nolla mano sinistra e un timone nella destra Ai piedi si trova un globo ll tipo si incontra anche nelle statuette bronzee del lararı

Il trammento ha forme bizzare, agl angolı sı vedono segmentı di cerchi che potrebbero essere un'indicazıone di una cassetta appartenente allo schema decorativo di un soffito ll sommario rapporto di scavo cl lascia perplessi in merito, la fotografia pubblicata mostra gia ll frammento nello stato attuale

Bibl A Sogliano, $\lambda S c$ 1899, 494, tig 1

6 I Jacopl 1963, 114-117, fig 109-111. 
Antiquario

Inv. no. 17718: > IX 5, 18 (cat. 288).

Inv. no. 17736: > VI Insula occidentalis (cat. 228).

Inv. no. 20879: > VII 4, 59 (cat. 247).

Inv. no. ?: > I 8, 10 (cat. 157).

Ins. no. ?: > VI Via della Fortuna (cat, 230). 
Villa sotto l'"Escuderla Real"

Cat 308 (?) Parele". zond mediana, Il stule, fase IIb (ora MN 9276, 8593, 9413)

Il trammento YV 8593 rappresenta un pdesaggio sacro-idlllaco. Sopra una base rettangolare rossa sorge ld statua bronzed a macchie gialle e verdi di Dioniso. Inclossa un chitone del tipo cheirodotos a regge un tirso nella sinistra alzata e un cantaro nella destra stesa verso ل basso Sulla testa si vede una corona, a) pjedı sandalı Il peso del corpo poggla sulla gamba destrd

Secondo Bieber il modello originale va cercato nel quarto secolo a.C Ella credette di riconoscerlo in una descrizıone di Pausania (VIIl 31).

Il I rammento e stato trovato contemporaneamente ad un gruppo di frammenti ricostruiti da Allroggen-Bedel, il 20 luglio 1755 sotto le scuderie reall a Portici, tuttora conoscjute con il nome spagnolo (MN 8.593, 9413) 6a 2 I due paesaggi sono monocromı e costituiscono imitazionı di rulevi marmorel. Gli elementı paesistici sono stati delineati in modo sommario

Bibl - Helbig 568, Bicber 1917, RP 1164 , Elıd 1932, no 178, Herrmann II, 31, Taf. 219. Beyen 1960, 368-369, tbb. 13j. Reutersward 1960, 132, Abb 23, Schefold 1962, 41, 47-48, Taf 21 2, Peters 1963, 33-34, 51-52, 73-74, fig 4, 60, CA4 III, 112, Haubner 1971, 143; Allroggen-Bedel 1975b, 115-116, Abb 95a, Ptretschner 1977, no 55, Bastet'De Vos 1979, 24-25, tai I, Slberberg 1981. cat. 17, fig 16. Moormann 1981b. 64j -616 
Villa

Cat. 308a. (portico) Parete?, zona mediana; IV stile

Lue dei pannelli che adornano il portico di una villa, in corso di restauro dal 1972, contengono delle prospettive architetoniche nelle quali si trovano delle erme, delineate in modo schematico. La mancanza di una pubblicazione con una totografia non permette una descrizione piu dettagliata di questa decorazione, che e importante poiché costituisce uno dei pochi esempl di IV stile nelle province romane.

Bibl. . Fuchs 1984, 64 . 
RICHMOND

Virginia Museum

Inv. no. 66-35: > Boscoreale, Villa di Popidius Florus (cat. 005). 
Regio I; casa privata presso il Colosseo Cat. 309. (?) Parete ?, zona superiore: $320-340+$
Soltanto da una vechia incisione si conosce la parte superiore di una decorazione parictale e la decorazione di un soffito. Nella zona superiore si vede un prospetto con pilastri davanti ai quali sorgono, sempre fra duc mensole, quattro figure. Al lato esterno si vedono due satiri nudi, posti simmetricamente, che reggono un grappolo d'uva con la mano esterna stesa per metá e un pedum nell'altra. La gamba al lato interno e di appoggio.

Le due figure centrali, altrettanto simmetriche, rappresentano menadi vestite di un cluitone e di un himation che viene alzato con la mano esterna. La gamba esterna è di appoggio.

Mielsch commenta che l'incisione è wohl nicht zuverlössig.

Bibl.: Mielsch 1978, 164-165, 'Taf. 86.1.

Regio II; Domus Aurea

Le decorazioni descritte in prosieguo costituiscono una piccola parte del complesso originale, trattandosi soltanto dei resti del padiglione sul Colle Oppio. Nel caso di decorazioni perdute o illeggibili possiamo servirci delle incisioni edite da L. Mirri e G. Carlettj nel 1776. L'attendibilita di queste illustrazioni può essere controllata e, quindi, sostenuta: esse soddisfano bene per le strutture architettoniche e per le combinazioni di vari elementi, ma per quanto riguarda i dettagli e le figure non sono inolto precise. ${ }^{63}$ A parte cio ho potuto studiare il materiale raccolto e gentilmente concessomi da Peters e Meyboom; alcune delle descrizioni percio precedono la loro pubblicazione definitiva. ${ }^{6} 4$ Le mie visite nel 1980 e nel 1981 non hanno aruto ulteriori risultati.

Le figure che popolano i paesaggi sacro-idilliaci, spesso di formato ridotto, non si distinguono facilmente. Perció esse non sono state inserite nel catalogo. 1 pinakes monocromi possibilmente raffigurano imitazioni di rilievi ora illoggibili $e$ in base alle incisioni non si puo stabilire se essi lo fossero stati veramente.

Le decorazioni di alcuni soffiti sono state ugualmente descritte, in quanto esse si mostrano come un'entita indivisibile dalle decorazioni murali e rappresentano un dei rari esempi di volte che sono rimaste conservate.

L'intero complesso e di IV stile, iniziato dopo l'incendio di 64 e terminato per la maggior parte ancora durante il regno di Nerone.

I numeri degli ambienti sono quelli della pianta piú recente. ${ }^{6} 6$

683 Le incisioni di intere pareti possono contenere elementi delle due pareti opposte, in quanto simmetriche, mentre le figure appartengono ad una sola parete. In tali casi si vede l'indicazione 'parete est o ovest'. Il siglo + indica anche pareti illeggibili o soltanto controllabili per gli schemi decorativi.

634 Cf. Peters/Meyboom 1982.

685 Cf. però Peters/Meyboom 1982. Cf. inoltre Weege 1913; Dacos 1969.

686 Fabbrini 1983, tar. IIl. 
Cat. 310/1. (23) Parete sud, lunetta +

Nell'edicola centrale vi e la statua di Hermes sopra un tripode. Il peso del corpo poggia sulla gamba destra. Regge un caduceo nella mano destra alzata e ha la sinistra stesa in avanti. Ha un petaso sulla testa e una clamide sulle spalle e sul dorso.

Ai lati estremi due menadi seminude stanno su calici vegetali sopra un arco sottile. Le figure simmetriche hanno nella mano esterna, vicino alla testa, la fine del festone che reggono con la mano interna. La gamba interna is di appoggio. lin mantello copre la parte inleriore del corpo.

Bibl.: Mirri/Carletti 1776, tav. 38, tav. 41; Peters/Meyboom 1982, 42. pl. 2.4.

Cat. 310/2. (25) Parete sud, lunetta +

L'edicola centrale è incoronata da un satiro danzante che avanza verso destra. Il piede sinistro $\dot{e}$ sollevato. Regge nella destra un grappolo d'uva. Ln mantello svolazza lungo il dorso. Una figura simile si vede nella Casa del colonnato tuscanico ad Ercolano (cat. 035/4). Per tutte e due le figure l'archetipo va cercato nell'ambito ellenistico, mentre in entrambi i casi e vaga la distinzione fra statua e persona viva.

Bibl.: Mirri/Carletti 1776, 34-35, tav. 39; Peters/Meyboom 1982, 42.

Cat. 310/3. (32) Parete est o ovest, zona superiore +

I dettagli sono ancora vagamente riconoscibili.

La trabeazione del portico dietro il porticato in primo piano è sorretta da

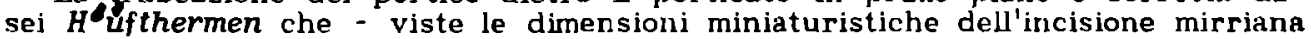
- non possono essere descritte in dettaglio. Le quattro edicole dietro gli hospitalia contengono sempre copple di erme simili.

I due hospitalia a sinistra e a destra dell'aula regia hanno, al lato osterno. una base aggettante sopra la quale sta una statua cquestre. ] cavalieri nudi hanno la destra alzata nell'atteggiamento dell'adlocutio. Statue simili si vedono nei Praedia di Julia Felix $(179 / 1)$ e nella Casa della caccia antica (cat. 215/4). Bibl.: Mirri/Carletti 1776, 29-30, tav. 5; Peters/Meyboom 1982, 41, pl. 2.6.

Cat. 310/4. (33) Pareti sud, est o ovest, zona mediana e lunetta +

Nell'attico di una scaenae frons si trovano, su ogni parete, due figure che reggono un candelabro sulla testa. Il candelabro sorregge sempre l'architrave. La parete est o ovest mostra un uomo nudo a destra e una una donna vestita di un panno che lascia scoperto il seno, a sinistra. Il loro atteggiamento è simmetrico: la gamba interna è di appoggio e la mano interna si trova lungo il fianco, mentre l'altra regge una patera.

Sulla parete sud si trovano due figure femminilj che indossano un chitone. Stanno nel medesimo atteggiamento delle figure sulle altre pareti. Le sei figure possono esscre considerate come statue solo per la loro funzione di sostegno.

La lunetta della parete sud contiene sei cariatidi che stanno sopra le tre edicole. L'edicola centrale è sormontata da due menadi simmetriche di Stile Ricco che indossano una tunica volante. La gamba interna è di appoggio. La mano esterna si trova vicino alla testa, l'altra pende lungo il fianco. Sopra le edicole laterali vediamo due coppie di donne simili di stile arcaistico. Isse sono vestite di una tunica. Le gambe unite forse sono sostituite dal fusto di un'erma. Le mani sono davanti al petto.

Bibl.: Mirri/Carletti 1776, 36-42, tav. $7-9$; Peters/Meyboom 1982, 42, pl. 2.3.

Cat. 310/5. (50) Parete est o ovest, zona mediana e attico + 
La scaenae frons e articolata in due planı e un attico Nel centro del primo piano si vede la statua di una divinita maschule seduta su un trono sopra una base rettangolare I $n$ mantello copre le gambe Regge nella sinistra uno scettro - o se si tratia di Dioniso - un tirso La mano destra e in riposo nel grembo

Contro le quinte delle due edicole estreme vi sono delle statue sopra mensole A sinistra c'e un uomo barbuto, togato Il peso del corpo poggra sulla gamba destra Lglı regge una bacchetta o un rotolo da papro nella sinistra stesa, mentre la destra solleva un lembo della reste Nella seconda edicola da sinistrd e una donna il cui mantello lascia scoperto il seno

All'estrema destra e la statua di una figura maschule interamente nuda che forse rappresenta Apollo Regge con la destra una cetra, mentre la sinistra e distesa in avanti Alla sua sinistra un uomo nudo tende le mani in dvanti II peso del corpo poggia sul piede destro

Sotto 1 prospetti, nel qualı si osservano degli attori, sono presentı appliques a forma di copple simmetriche di tritonı Essı reggono una patera o un ritone in una delle mani

Il secondo piano a privo di figure a parte una menade volante nell'edicola centrale L'attico invece contiene una serıe di sel guerrierı nudi che tungono da atlant simmetrici Le due figure a sinistra e la seconda a destra reggono uno scudo nella mano sinistra e hanno la destra sollevata La gamba destra $f$ di appoggio Gli altrı guerierı hanno la pirte superiore del corpo summetrico e le gambe divaricate

La loro posizione e senza parı nella pittura parietale, perche normalmente talı figure d'impronta polıcletea sı trowano sulle mensole davantı a pannellı semplici (cf $\mathrm{p} 435$.

Bibl Mirrı, Carlettı 1776, 59-60, tav 27, Peters/Meyboom 1982, 39, pl 21

Cat 310/6 (54) Parete sud, lunetta: soltitto +

Le quattro higure femuninl sedute che incoronano la trabeazione della acrenae frons nella lunetta fanno pensare alle statue che si incontrano nelle scaenae frontes di III stile, $P$ e nella Villa imperiale (cat $305 / 1,3$ ) Le figure centralı sono summetriche Pogglano la mano esterna sulla trave, mentre l'altra e distesa in avantı lo altre due tigure, anch'esse simmetriche, tengono le mani bel grembo

Le edicole centrali des lati corti del solfito sono occupate da statue sopra basi rotonde Su di un lato si vede una higura femminule vestita di un chitone senza maniche che regge un ramo d'olivo nella mano destra alzata e una corona nella sinistra distesa verso il basso La gamba destra e di appoggio L'altra e una donna vestita di una tunica Regge un tirso nella sinistra e solleva un lembo della veste con la destra

Bibl Mirri/Carlettı, $5 j-60$, tav 19

Cat $310 / 7$ (55) Parete est o ovest, zona mediana e attico, soffitto +

La scaenae frons conta due piani e un attico Le nove figure nelle edicole del primo pıano sono personaggl WJi, in parte modellatı secondo modellı scultore La figuia centrale, un uoino che versa del vino, e lo due tigure fomminl non coplano tıpi statuarı l sel uominı baibutı e vestitı con und loga entrano nel genere del ritratti dl filosof, retorie poeti Reggono un volumen o stanno nell'atteggiamento dell'adlocutio Tali figure sono gia presentı nel II stile (cat. $262,3)$ e ritornano nel IV stule, $p$ e nella Casa dell' Ercole ebbro $(284 / 2)$. Ia decorazione della Domus Aurea contiene un altro punto di confronto con questa casa, cıoe 1 drappı dietro 1 qualı sorgono delle figure vive I sel poetı, 
retori o flosofi ripetono modelli noti attraverso numerose copie. ${ }^{67}$

Il secondo piano non contiene delle statue. I'nttico is composto da edicole con figure umane dietro cancelli e quinte ornate alternamente con imagines clipeatae e satiri sdraiati. Tanto per $\mathbf{i}$ satiri che per le figurc nelle edicole non possiamo concludere in senso assoluto sul loro carattere scultoreo. Satiri del genere si trovano fra l'altro nella Casa di Meleagro (cat. 202/5).

La prima, terza, sesta, ottava e decima nicchia sono occupate da una figura femminile che indossa un peplo o un chiton, mentre nella quarta e quinta nicchia si vedono uomini togati: la terza, settima e nona edicola contengono un guerriero nudo. Se si tratta di statue, i loro archetipi vanno cercati nell'Ellenismo.

Le edicole centrali di tutti i lati del soffitto sono occupate da statue. Ai lati lunghi si vedono divinità sedute su troni sopra una base rettangolare. La distinzione tra statua e personaggio vivo è vaga. L'una figura è Dioniso, riconoscibile grazie al tirso nella sinistra. Ún mantello copre quasi comletamente il corpo. L'altra figura ì Apollo che regge con la destra una cetra, mentre la sinistra e sulla testa nell'atteggiamento dell'Apollo Licio. Il dio è completamente nudo.

I lati corti sono abbelliti con scene di genere. Offerenti si trovano presso la statua di una divinita, sorgente sopra una base rettangolare. Ad un lato si vede una menade vestita di un chitone che lascia scoperto il seno sinistro. Regge un tirso nella destra, mentre la sinistra $\dot{e}$ in riposo nel fianco. Il quadro al lato opposto contiene la statua di Dioniso nudo che regge un cantaro nella sinistra sollevata e un tirso nella destra. La gamba destra è di appoggio.

Bibl.: Mirri/Carletti 1776, tav. 45, 51; Peters/Meyboom 1982, 45, pl. 2.5.

Cat. 310/8. (77) Porta sinistra nella parete nord

ln un'edicola sorge un candelabro con la statua di una donna alata che fuida una pantera alla sua sinistra. Tutto è di colore giallo.

Bibl.: inedito.

Cat. 310/9. (80) Soffitto

Un fregio con personaggi che assistono a scene rituali contiene la statua di Dioniso. La figura marrone viene sollevata da un'offerente femminile e deve essere fatta di un materiale poco pesante, probabilmente legno. Il dio indossa un mantello lungo nel quale il braccjo sinistro è nascosto. La destra alzata sembra reggere un tirso. Ha il visto barbuto e una corona vegetale sulla testa.

Bibl.: Weege 1913, 179-180, Taf. 9; Meyboom 1984, 38, fig. 3.

Cat. 310/10. (117) Parete ovest, zona superiore

Su un pinax si vedono tre donne che fanno un'offerta alla statua di Atena Promachos. La dea sta sopra una colonna e regge uno scudo nella sinistra e una lancia nella destra alzata. La testa e coperta da un elmo a pennacchio. La figura di stile arcaizzante è simile a quella in I 2,6 a Pompei (cat. 140).

Bibl.: Wirth 1934, 11, Taf. 9; Bordia 1958, 224.

Cat. 310/11. (118) Parete est, zona mediana

607 Cf. P. e. Richter 1965; Lorenz 1965; Sgobbo 1972. 
Due figure femminili che occupano un'edicola forse imitano modelli scultorei, mentre in realtà sono figure vive. A sinistra si vede una donna con una patera nella sinistra; la gamba destra è di appoggio. Puó essere associata con la Fanciulla di Anzio. A destra vi è una menade che poggia sulla gamba destra. Regge un timpano con la destra accanto alla testa e un tirso nella sinistra. Lo stile e elenistico.

Bibl.: inedito.

Cat. 310/12. (119) Pareti nord e est, zona superiore

Lo stretto scomparto a destra dellabside nord contiene la statua di una menade che sta su un piedistallo rettangolare. La gamba destra è di appoggio. Batte su un timpano alla sua sinistra. Lna veste copre il dorso e le gambe. Si può confrontarla con una figura nella Casa di Meleagro (cat. 202/3).

La parete est è divisa in tre scomparti da pilastri stuccati. Soltanto lo scomparto a sinistra è ancora leggibile. Le tre grandi figure davanti alla struttura architeltonica rappresentano due filosofi come sopra (cat. 310/i) e uno seduto, altrettanto paragonabili ai ritratti di filosofi e scrittori. Le figure, peró, sono personaggi vivi.

L'edicola centrale di ogni scomparto è sormontata da un candelabro con sopra la slatua di Eros alato. La figura conservata avanza verso destra e regge un pedum nella mano destra.

L'edicola sinistra contiene un candelabro con un altro Eros che avanza verso sinistra.

Le edicole accanto ai pilastri stuccati sono occupate dalle statue simmetriche di korai che stanno su candelabri vegetali. Le figure indossano un peplo e hanno una mano sollevata, mentre l'altra $\dot{e}$ in riposo lungo il fianco. Lo sguardo è rivolto verso il pilastro.

Bibl.: Bianchi Bandinelli 1969, fig. 143-144.

Cat. 310/13. (131) Parete est, zona mediana

L'aula regia della scaenae frons e occupata dalla statua marrone-rossa di una figura [emminile vestita di un peplo. Regge una bacchetta nella destra alzata e ha la sinistra in riposo nel fianco.

Nei prospetti a sinistra e a destra si intravedono le sagome di statue equestri simili a quelle nel 32 (cat. $310 / 3$ ). Le altre figure si muovono come attori sul podio.

Bibl.: Peters/Meyboom 1982, 39.

Cat. 310/14. (cortile pentagonale) Lato est, zona superiore

Sopra le porte si trovano scaenae frontes, ora molto rovinate. In parte le strutture architettoniche e le figure dentro le edicole sono state eseguite a stucco. Sopra la porta a sinistra l'architettura di un'edicola con una figura illeggibile è sormontata da due guerrieri nudi simmetrici. Essi servono nello stesso momento da atlanti aventi una colonna esile sull'elmo. Sono rivolti verso il centro. Il peso del corpo poggia sulla gamba che sta in avanti. Il torso e rivolto verso lo spettatore. Il tipo è quello dei guerieri pirrici.

Nell'edicola al lato destro the corrisponde a quella suddetta con la figura illeggibile si vede un guerriero nudo eseguito a stucco. La gamba destra è di appoggio. Regge uno scudo con la sinistra lungo il fianco, mentre la destra è stesa verso il basso. Il tipo è quello classico, policleteo.

Bibl.: inedito. 
Regio IV; Via Cavour, casa privata

Cat 311. (M) Parete est, zona superiore; II stile, fase IIb (ora Muse1

(dacanı)

Nel sesto campo da sinistra con 1 paesaggı dell'Odissea si vede il palazzo di Circe. Accanto all'entrata e un'erma bianca che funge da elemento apotropasco La testa e stata delineata detlafliatamente.

Bibl Beyen 1960, 260, Gallina 1961, 25, tav VII 13, Andreae 1962, Belage, Abb 4, Paar 1962, 133-134, Abb. 5, B Andreae, In Helbig 465 von Blanckenhagen 1963 (con bibl)), Taf. 48; Peters 1963, 27-32, fig 20

Regio V; Casa di Fausta sotto ll Palazzo dı Laterano

Ca1 312 (?) Parete? 312-314 d.C (ora Museo lazıonale Romano)

Cossddetta Dea Barberinı, proveniente da una zona ignota della decorazione di questa casa La dea Roma e seduta su un trono in atteggiameno leratico. lndossa una sottoveste bianca, un colubium di broccato " lasce violacee e un mantello purpureo Regge nella simistra una lancia c nella destra stesa (la statuetta di) una tike alata Sulla spalla destra siede una figura femmmile alata La testa e coperta da un elmo dorato.

Si tratta dell'ummagine di culto di Roma esposta nella Basilica di Massenzıo dopo l'incendio del 307, dedicata nel 314 da Costantino La copia dipinta andrebbe datata tra ll 312 e ll 314, cioe dopo l'arrivo di Costantino a Roma e prima del trapasso della casa di Fausta allepiscopato romano Altre immagini della statua si trovano in forma di statuette, su contornatı e nel calendarıo di Filocalo per l'anno 354 .

Bibl - Cagiano de Azevedo 1954, hraus 1967, 212, Abb. 145; Andreae 1973, 356, Abb 152; Spatantike $1983,72,489-490$ rat. no 91 (D. Stutzinger).

Regio V; Casa privata sotto S Giovannı in Laterano

Cat. 313. (ff) Pareli est e ovest, zona mediana, IV stile

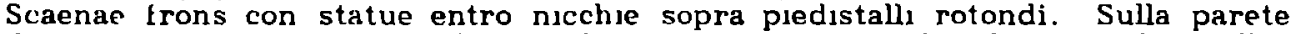
destra si vede un uomo nudo di colore rosso con una clamide sopra le spalle. La gamba sinistra e di appoggio. Gli oggetti che hanno nelle mani distese non sono riconoscibilı. E. Stevenson descrisse inoltre un Mercurio con il petaso in testa e ll caduceo nella mano, da vedere sulla medesuma parete.

Sulla parete $d_{1}$ fronte si distıngue la sagoma $d_{1}$ una figura femminile ammantata. Regge nella destra stesa verso il basso un ramo e nella sinistra alzata forse un tirso

Il complesso datato nell'epoca giulıo-claudia venne ristrutturato nel tempo di Settimio Severo per essere la caserma degll Equites singulares "a,

6. Per l'iconografia di Roma cf. Mellor 1981, 1011-1017, spec 1016-1017 (cosiddetta Roma Eterna, statua esposta da Adriano e sostituita da un esemplare portireo da Massenz10). Spatantike 1983, 72, Abb 49, Kat. no. 84 .

609 Nash 1961, I, 214-218, Abb. 244-250 
Bibl.: E. Stevenson. Annlst 1877, 351-353, tav. RS; Coarelli 1974, 187, 348-349 (con bibl.); Joyce 1981, 27-28, fig. 14 .

Regio VI: casa privata sotto la Villa Negroni

Cat. 314:1. (B) Parete con nicchia, zona mediana; dopo 134 d.C. +

Nell'edicola centrale sorge, sopra una base rettangolare, un uomo nudo. La gamba sinistra e di appoggio. Kegge un lancio nella destra alzata e tiene la sinistra stesa per meta. Il torso e rivolto verso destra e la testa e rivolta in basso. Lo stile è policleteo. L'interpretazione della figura come Adone può essere accettata viste le due scene figurative presenti sulle altre pareti.

Le edicole centrali sulle pareti laterali sono sormontate da sfingi femminili alate di colore bianco. Sono rivolte verso l'esterno.

Il complesso può esssere datato con precisione per la presenza dei bolli laterizi. La maggior parte delle sue decorazioni è stata documentata attraverso incisioni di A.R. Mengs e $A$. von Maron.

Bibl.: Joyce $1083,422,428$, fig. $3-5$.

Cat, 314/2. (C) Pareti ?: zona superiore; dopo 134 d.C. +

Tritoni simmetrici incoronano l'architrave delle edicole centrali delle pareti corte. Reggono un timone sulla spalla esterna con entrambe le mani. Le zampe e la coda sono verdi, la parte superiore del corpo è di colore naturale.

Sulla terza parete si vedono due grifi stilizzati.

Bibl.: Ḱriegel 1919; Borda 1958, 98-99; Joyce 1983, 429, fig. 6-7; Bastet 1984 .

Regio $\mathrm{X}$; Aula isiaca

Cat. 315. (aula) Pareti sud e est; II stile, fase IIb/III stile

Su mensole inserite nella cornice che incorona la zona mediana, vi sono delle statue. 1 colori sono quasi indistinguibili.

Sulla parete sud si vedono due erme composte da un fusto e un busto. Il vecchio disegno di Bartoli le rappresenta come $H$ l fthermen. $_{\text {f }}$

Sulla parete ovest korai vestite di un peplo sorreggono una trave. Hanno le alj avinghiate intorno alla trabeazione dell'epistilio. Sollevano con la mano esterna un lembo del peplo, mentre l'altra mano e stesa. Lo stile è arcaizzante. La posizione assomiglia quella delle cariatidi nella Casa di Livia (cat. $317 / 3$ ).

Ai lati estremi della decorazione si trovano edicole occupate da ligure sopra mensole. Sulla parete sud si trovano statue egizie simmetriche (cf. gli atleti nudi nel disegno di Bartoli!). Avanzano verso il centro e hanno le braccia in posizione di riposo. Attorno alle reni si vede un drappo. Statue del genere erano molto diffuse a Roma e ognitanto erano inserite in strutture architet toniche. ${ }^{690}$

Sulla parete ovest le edicole contengono korai con le mani stese in avanti, vestite di un peplo.

690 Cr. Roullet 1972. no. 139, fig. 158 e no. 101-102, fig. 119-120 (Osiridi in guisa di telamoni dalla Villa Adriana). 
Bibl.: Rizzo 1936, 35, tav. II, VI, fig. 4, 14-15 (nessun riferimento nel testo): B. Androae, in Helbig", 2092 Romanelli 1974, 19-20, fig. 12-13: Bastet/De Vos 1979, 22-23; De Vos 1980, 60, tav. 34.

Regio X: Casa di Augusto

Cat. 316/1. (5) Pareti est e ovest, zona superiore; 36-28 a.C.

Sullo sfondo bianco sono state applicate lastre rettangolari di colore grigio e viola che imitano lastre a rilievo. L'efetto di profondita è suggerita dá linee scure. Tralci sinmetrici sembrano initati da lastre fittili, per esempio lastre Campana. Sulla parete ovest la parte maggiore del rilievo destro è andata perduta.

Bibl.: Carcttonj 1983a, 377-378, tav. 94, tav. a colori 2.1; Carettonj 1983b, 26, 'Taf. C, G.

Cat. $316 / 2$, (7) Parete nord, zona mediana; 36-28 a.C.

Nel campo destro di colore nero si vede un paesaggio sacro-idilliaco in tinte gialle. A sinistra si vedono un altare e una colonna sormontata da una statua schematica.

Bibl.: Silberberg 1981, cat, 13, fig, 11; Carettoni 1983a, 379-381, fig. 1, tav. 95; Carettoni 1983b, 32, Taf. 9, Abb. 2.

Cat. 316/3. (14) Pareti nord, sud e est, zona superiore; 36-28 a.C.

Sugli architravi delle edicole nella zona mediana vi sono ippocampi in posizione simmetrica. Sono dipinti in bianco, giallo e vjola, colori che imitano precisamente il marmo a gli effetti del rilievo. Solo la figura sinistra della parete sud è rumasta conservata. Una nebride copre le spalle. Regge con la destra un pedum presso la spalla e ha la sinistra alzata sopra la testa.

L'ippocampo sinistro della parete est regge un tumone nella sinistra e lo solleva sopra la testa con la sinistra. L'altro animale marino ha le zampe in riposo davanti al petto.

Sopra le travi, fra gli ippocampi, si trova un fregio con animali marini e ippocampi bianchi su uno stondo rosso scuro. Anche in questo caso quasi sicuramente si tratta di un'imitazione di rilievi. I pannelli sono inquadrati da una cornice a rilievo.

Bibl.: Caretton 1983a, 399-402, fig. 18, tav. 101-102, tav. a colori 10.1: Carettoni 1983b, 61, 63, 88, Taf. 15-17, Q-R, T, V.

Cat. 316/4. (15) Parete nord, quadro centrale; 36-28 a.C.

Paesaggio sacro-idiliaco. A destra vi è l'erma marrone di Priapo presso una colonna o pietra a forma conica.

Bibl,: Carettoni 1983a, 402, tav. 104; Carettoni 1983b, 67, Taf. W4.

Cat. 316/5. Frammento sporadico: 36-28 a.C. (ora Antiquario del Palatino)

$\mathrm{Su}$ uno sfondo ceruloo si delinea Apollo Citaredo in colori naturali, seduto su un trono a spalliera alta. Regge con la sinistra sopra il ginocchio la cetra, mentre la destra poggia sulla spalliera. Le gambe sono coperte da un mantello viola, la testa da una corona. Sul dorso tiene una faretra. La chioma scende fin alle spalle. Un omphalos sta presso $\mathrm{j}$ piedi. 
La figura viva ricorda, piú di altre immagini di Apollo, un tipo tardoellenistico di cui si conoscono due copie, ora nel Museo Nazionale Romano. L'originale sarebbe stato concepito nel primo secolo a.C. ${ }^{691}$

Bibl.: B. Andreae, in Helbig ${ }^{4}$. no. 2075 Romanelli 1974, 30, fig. 40; Coarelli 1974, 156 (fig. a colori); Carettoni 1983b, tav. X.1 (non menzionato nel testo).

Regio $X$, Casa di Livia

Cat. $317 / 1$. (II) Pareti B e C, zone mediana e superiore: Il stile, fase Ilb

Nel pinax centrale della parete $B$, rappresentante Polifemo e Galatea, si vede un'erma rossa di Priapo su uno scoglio a destra. Lin cappello copre la testa. Le braccia sono distese.

Il quadro con Io e Argos della parete $C$ contiene una colonna sormontata dalla statua verde di una figura femminile. $E^{\prime}$ vestita di un peplo di stile classico. La mancanza di attributi specifici impedisce un'interpretazione della figura. Lina fiaccola, appesa ala colonna potrebbe essere un'indicazione di Artemide. Secondo Peters la statua è un elemento inserito dal pittore romano, mancante nell'originale greco, attribuibile forse a Nicia di Atene nel quarto secolo a.C. ${ }^{692}$

Il pinax viene fiancheggiato da due figure femminili simmetriche che stanno su una cornice a rilievo e che sorreggono la trave che chiude la decorazione al lato superiore. Indossano un peplo e reggono nella mano interna stesa verso il basso uno scettro e una brocca nell'altra distesa. Lin calice sulla testa serve da capitello. Lo stile i severo.

Sopra i campi laterali della parete B st trovano statue di figure femminili vestite con mantelli fantastici. Stanno sopra colonne doriche. Solo la figura destra è rimasta conservata.

Sull'epistilio nel centro si vedono figure femminili sedute su tralci che escono da una figura grottesca nel centro. Precedono figure simili nel III stile, per escmpio nella Caserma dei gladiatori (cat. 197;3) e nella Villa imperiale (cat. 305/3) a Pompei.

Bibl.: Rizzo 1936, 15, 25, 32-33, fig. $3-4,11-13,16,18-19,21$, tav. 1-III; Peters 1963, 46-47, fig. 35; Romanelli 1974, 28-29, fig. 31, 33-34; leach 1982, 159-164, fig. 9 .

Cat. $317 / 2$. (III) Pareti a e b; fregio fra zone mediana e superiore; II stile, fase IIb.

Un fregio di colore giallo contiene una serie di paesaggi sacro-idilliaci. Ogni parete presenta una suddivisione in quattro intercolunni. Il fregio imita un rilievo in marmo, usato come cornice sopra una parete. La fuga di paesaggi potrebbe essere una copia intera di un Bilderbuch. Altrove, per esempio nella Villa di Poppaea a Torre Annunziata (cat. $341 / 2$ ), tre registri l'un sopra l'altro con paesaggi rappresentano un simile rotolo decorato, qui tagliato a misura.

Parete a, primo intercolunnio. A sinistra una statua di Priapo. rivolto verso destra, sta sopra una base rettangolare elevata. Il dio è itifallico. Nel centro vi è la statua di Apollo, fiancheggiata da due tripodi, posta sopra un

691 Aurigemma 1970, 82 no. 205, 208, tav. XXXIb; w. Fuchs, in Helbig ", no. 2347,2350 .

692 Cf. Neutsch 1939 
piedistallo. Il peso del corpo poggia sulla gamba destra. Apollo regge una cetra nella sinistra e ha la destra stesa verso il basso. Contro il piedistallo e appoggiata un'erma.

A destra di questo gruppo centrale si vede un hekataion a forma di tre statuette sopra una base comune. Stanno davanti ad una colonna. All'estrema destra vi e una statua schematica che non puó essere descritto in modo dettaglio.

Secondo intercolunnio. Sopra uno scoglio sta la statua di Atena vestita di un peplo e con la lesta coperta da un elmo. Regge una lancia nella destra.

A destra di un fiume si distingue, sopra un podio, un edificio fiancheggiato da statue. A sinistra si vede una figura ammantata sopra un'alta colonna, a destra una figura maschile nuda con la mano destra alzata e la sinistra stesa verso il basso. Presso un altro edificio a destra si vede una striscia che, secondo le descrizioni, sarebbe un hekataion. Vicino alla base si vede una statua della Fortuna con la cornucopia nelle mani.

Terzo intercolunnio. Nel centro sorge la statua della Fortuna seduta su un trono. Regge una cornucopia nella sinistra e un sistro nella destra. Sulla testa si trova un modio. La figura quindi rappresenta nello stesso momento Iside. A destra vi sono due colonne e una statua di una figura seduta sopra una base rettangolare.

Quarto intercolumnio, ora quasi completamente perduto. Presso un tempio nel centro si distinguono le statue di una figura in piedi a di una sfinge.

Parete b, primo intercolunnio. Nel centro si trova la statua di una figura maschile nuda che regge una lancia con la destra, mentre la sinistra $\dot{e}$ in riposo lungo il fianco. La gamba destra è di appoggio. Sotto lo scoglio alla sua sinistra è stato posto un hekataion a forma di tre statuette sopra una base comune.

Secondo intercolunnio, ora quasi completamente perduto. Lina figura a destra di una nave va forse interpretata come statua.

Bibl.: Rizzo 1936, 43-51, tav. $V-X$; Peter-s 1963, 35-47. fig. 26-31; Romanelli 1974, 29, fig. 32, 36-37; Silberberg 1981. cat. 15, fig. 14; Moormann $1984 \mathrm{~b}, 645$.

Cat. 317/3. (IV) Parete C, zone mediana e superiore; II stile, fase IIb (ora anche MN 9303)

vell'edicola centrale è stato dipinto un paesaggio sacro-idilliaco con un sacello di Artemide. Presso un betilo sta un piedistallo rotondo con un hekataion a forma di tre statuette. Le figure indossano un peplo e reggono nelle mani stese delle fiaccole. Sulla testa hanno un loto.

La cornice fra la zona mediana e la zona superiore è composta di due plinti fra i quall sono state applicate sirene bianche. Strisce marroni accentuano la loro plasticità. Le figure assomigliano a quelle fra le cornici a mensole doppie come ad esempio quelle nella villa di P. Fannius Synistor a Boscoreale (cat. $004 / 1-3)$.

La zona superiore contiene due cariatidi vestite di un chitone e con un modio sulla testa. La parte inferiore del corpo finisce in un'erma. Stanno sopra mensole rettangolari e sostengono la trabeazione che chiude la decorazione nel lato superiore. Reggono nella mano interna una fiaccola, in quella al lato esterno una brocca. La figura sinistra e stata staccata nel Settecento, trasportata a Napoli come offerta al re, dove tuttora riene custodita nel Museo Nazionale.

La parete di fronte probabilmente conteneva una disposizione simile.

Bibl.: Rizzo 1936, 51-58, fig. 35-39; Schefold 1957, 343; Peters 1963, 42-44, fig. 34; Romanelli 1974, 29, fig. 38; Silberberg 1981, cat. 14, fig. $12-13$; Swinkels 1984,44 , fig. 2 . 
(cat 318) Reg10 XIlI Tempio di Iside, circa 200 d C

Ln pundx nella zona superiore mostra degli orintı presso un'edicola occupata dalla statua di Iside Indossd una tunica verde a strisce rosse e regge nella destra alzata una cornucopia c nella sinistra stesa in avantı una patera, ora dittıculmente identif ıcabule

Misch data l complesso dccoratıvo im fortgeschrittenen 3 Jahrhundert, basandosi sul colori e sulla mancanza di attenzione artistica per le singole tigure

Bibl Darsy 1968 44-48, pl A, Melsch 1981, 212

Regio YII, Vllla delld Farnesina

Tuttı I frammentı superstitı sl trovano nel Museo Nazionale Romano (inv no 1460-1462, 1464 1467-1471, 1477-1479) \ella descrizione si segue lordine tenuto nel catalogo di Bragantinı/De Vos 1982, dove si trovano anche 1 rilerimentı bibliograficı

Cat 3191 (A) Zona mediana e superiore, II stle, fase IIb

Alcunı deı 15 pinahes della zona mediand contengono statue Su AT si vede un sacrificio a una $H$ Q Aftherme di Pridpo che sorge sopra una colonna Essa ha la destra sollevata in alto e regge un tirso nella sinistra

Su AB tre persone s) trovano vicino alla statua verde di Artemide Sta sopra una base rettangolare e ha la gamba sinistra avanzata Indossa un chitone senzd maniche Regge un arco nella sinistra Il prototipo si ritrova nell'Artenide arcaistica della Casa di $M$ Holconius Rufus a Pompel ${ }^{6} 3$

Ogni intercolunnio della cona superiore contiene la statua di una figura lemininle sopra una base rotonda, flancheggiata da costruzioni sorrette da Vittorie alate e sormontate da sfingl un solo frammento e rimasto conservato La figura centrale di colore verde indossa un chitone senzd maniche e regge una llaccola nella sinistra e una patera nella destra Sulla testa ha una corona dentata Delle altie statue l'estrema dostra, secondo l'acquerello di G Mannero, e gialld, le altre verdi Le Vittorie, iniece sono giallo-marroni e sono rivolte verso 1 centro Le sfingl temminili sono policrome Toccano con ld testa l'orlo superiore delld decorazione

Bıbl Suberberg 1981 cat 21 fig 20 , Bragantını'De Vos 1982, 78, 87-88, tav 6-7, 10,20-21, 30

Cat $319 / 2$ (B) Zoccolo, zone mediana e superiore, II stle, fase IIb

Nel'anticamera si delineano $H^{\circ}$ ufthermen bianche che rappresentano del Silenı sulle basi gialle dolle lescie laterali Indossano una tunsca corta e hanno una corona sulla testa, il fusto finisce in un tralcio Reggono un pedum e un tirso Il rulevo non e stato accennato in modo forte, forse si tratta di imitazioni di appliques

Nella zona mediand statue di Iside escono da elementi regetal Sono di stule arcaico Sulla parete sinistra sl vede a sinistra un'Iside vestita di un chitone verde e di un mantello wolaceo con un diadema sulla testa Presenta, con le manı distese una coppia di patere a pantere al due latı, anch'essı

693 Fuchs 1979, 241-242, fig 261-262, Dohl Zanker 1979, 205 
uscentı dal giralı A destra la divinita egizia indossa un chitone viola e un volo chalano. mentre la testa e coperta da un calice con chadema Regge nelle manı stesc in arantı due cornucopie glalle La liancheggano due anumalı tantastici che escono dai girali

Sulla parete destra Iside indossa un chitone giallo e un mantello bianco-viola Sulla testa ha un diadema d'oro e un numbo bianco Regge nella destra distesa un sistro e nella sinustra un ventaglio Sui tralci sono sedutı del falchı che rappresentano ll dio egizio Horus

Sulla parete con la porta, davantı a un campo rosso, e un candelabio sormontato dalla statua policroma di Giove Ammone vestito di un chitone blanco-verde e di un mantello viola Il volto e di colore giallo dorato Regge nelle manı una benda che circonda la testa Sopra la lesta ha una colonna snella

La decorazione dell'alcova e indipendente Davantı aı campi rossı nella zona mediana vi sono le statue di lside e Giove Ammone Sulla parete sinıstri sı vede Isıde vestita $d_{1}$ un chitone violaceo $\mathrm{Ha}$ intorno alla testa un nimbo ceruleo e un diadema Nelle mani distese tiene 1 giralı che escono dalla base Il suo pendant della parete opposta ha lo stesso atteggiamento Giove Ammone indossa un chitone verde e un mantello purpureo e ha sulla testa und corona composta dalle corna abituah e da un calice

I campl della parete di fondo contengono quadri monocrom sorrett 1 da sirene policrome Esse hanno una sola pinna bianca e sono parzidimente coperte da un panno, la parte superiore del corpo e nuda Le all sono verdi Reggono il pinax con entrambe le manı sopra la testa nellatteggiamento dell'Atlanten-Schema 694

Il pinax centrale raffigura Dioniso allattato da Leucotea A sinistrd, sopra un plastro bianco, e la statua viola di Dioniso barbuto, vestito ds un chitone lungo e rivolto verso ll centro Sulla schola dietro la ninta si vede un Sileno di colore verde soraiato, forse immdginato come statua (cf Famars, cat 040)

L'edicola ha dcroteri a forma di Eroti sedutı su pantere I gruppl simmetricı sono dı colore bianco Fanno pensare a gruppl qualı le vereidı di Formia 695

Sopra l'edicola centrale e una figura femminle alata vestita di un chitone sopra una base vegetale

Sopra 1 pinahes nella zona mediana sono appoggiate su mensole gialle delle cariatidı vestite di un chitone verde e maniello bianco viste irontalmente Sorreggono una colonna di altezza modesta con un capilello composto, mentre hanno nella mano interna una brocca lungo ll tıanco e in quella esterna, presso la spalla, una patera

$\mathrm{Al}$ loro lato esterno si trovano edicole occupate dalle statue gialle arcaizzantı $d_{l}$ Ecate, vestite $d_{1}$ un chitone e reggentı flaccole nelle manı distese

Sulla parete a sinistra vi e una Nike alata posta sopra un segmento rotondo che fa parte della struttura architettonica $E^{\prime}$ vestita di un chitone e regge davantı al petto un oggetto lleggibule, lorse due tavolette ceree Ha un diadema alto poggiato sulla testa, torse in origine elemento aschitctionico $E^{\prime}$ vista di proflo

Bıbl Bragantını/De Vos 1982, 128-139, tav 35-70, Leach 1982 162-164, fig $10-11$

Cat $319 / 3$ (D) Alcova zone mediana e superiore, II stule, fase IIb

694 Schmidt-Colinet 1977, 31-35

695 Bielefeld 1969 
Davanti agli ortostati rossi delle pareti sinistra e destra si trovano statue policrome di figure maschili sopra elementi vegetali, simili alle figure nell'ambiente B. Sulla parete sinistra due sacerdoti di Giove Ammone si ergono fra tralci vegetali, mentre sulla parete destra si vede un'lside bianca che regge una patera e un sistro nelle mani distese. Sulla testa tiene un cantaro.

Sui pinakes a fianco dell'edicola centrale sulla parete destra delle donne giocano con lepri davanti alle erme bianche di Pan. Tutte e due le figure sono avolte in mantelli gialli.

Sullaltra parete una donna è seduta davanti alla statua bianca di una figura femminile, parzialmente coperta da un vestito giallo. Lo sgabello di marmo bianco, sul quale é seduta la donna, ha piedi a forma di sirene alate.

Vella zona superiore poggiano sulle edicole alcune cariatidi classicheggianti, due su quella centrale, una su ogni edicola laterale. Le ligure centrali fungono da acroteri a allo stesso tempo sorreggono l'orlo superiore della decorazione. Indossano un chitone e hanno nella mano esterna distesa un piatto. Mentre queste due sono viste di fronte, le figure laterali hanno lo sguardo rivolto verso il centro. Esse indossano vesti simili e sorreggono la cornice delle edicole laterali.

Le figure della parete di fondo sono conosciute attraverso la ricostruzione grafica di Mau e lessing. Il pinax centrale contiene la piccola statua di una figura vestita di un mantello lungo che porge una patera con la destra.

Su un pinax nella fascia fra zone mediana e superiore si distingue appena un'erma itifallica.

Bibl.: Bragantini/De Vos 1982, 188-194, tav. 83-109.

Cat. 319/4. (C) Zone mediana e superiore; II stile, fase IIb I paesaggi sullo sfondo nero della zona mediana qua e la contengono erme e statue di dimensioni miniaturistiche, ora quasi illeggibili. ${ }^{696}$

Nel quarto scomparto del fregio, ove sono dipinte delle scene mitologiche, forse rappresentanti il faraone Bocchoris, si distingue un'erma.

Nella zona superiore si vedono figure femminili e maschili policrome che stanno sui capitelli delle colonne che suddividono la zona mediana. I due sessi si alternano e distinguono stilisticamente. Le statue di uomini barbuti che indossano un chitone giallo e che reggono un tirso e una patera, sono di stile arcaizzante. Le figure femminili, invece, sono di Stile Ricco. Esse sono vestite di chitoni bianchi e mantelli svolazzanti, mentre reggono una fiaccola, una brocca o un altro attributo dionisiaco. Tutte le figure, sia maschili che femminili, sorreggono con la testa una mensola che fa parte della cornice superiore.

Bibl.: Silberberg 1981, cat. 22, fig. 21; Bragantini/De Vos 1982, 234-239, tav. $122-165$.

Cat. 319/5. (E) Alcova, zoccolo, zone mediana e superiore; Il stile, fase IIb Il davanzale dell'edicola centrale viene sorretto da sirene alate monopodi. Hanno un drappo attorno alle reni. Le ali sono bianche. Reggono la cornice con entrambe le mani e con un calice che poggia sulla testa.

I paesaggi sacro-idilliaci nelle edicole delle due pareti conservate (quella di sinistra e andata perduta) contengono statue di bronzo sopra colonne. Sulla parete di fondo si distingue una figura femminile vestita di un lungo mantello e con un elmo sulla testa, probabilmente Atena. Tiene con la sinistra uno scudo. Vel piano inleriore è un'erma di l'riapo.

696 Cio vale specialmente per chi non stia di fronte alla pittura; si rimanda alla descriziono di Bragantini be Vos. 
Il paesaggio sulla parete destra contiene la statua arcaistica di colore azzurro di una divinita femminile, interpretata come Tyche. Davanti alla base vi è un'erma violacea di Priapo.

Al fianco dell'edicola sula parete di fondo si trovano due statue sopra piedistalli verdi rettangolari, visti di scorcio. A sinistra Selene avanza verso sinistra, vestita di un mantello verde che lascia scoperto il petto. Il crescente lunare è ben visibile sul cranio. Solleva con la destra un lembo del mantello sopra la testa.

A destra c'è Helios vestito di una lunica corta. Si dirige verso destra. Un nimbo circonda la testa. Regge una lancia e una frusta. Entrambe le statue sono di stile barocco e non permettono un confronto con tipi conosciuti.

Sopra la stessa edicola sorge, nella zona superiore, un acroterio a forma di un uomo barbuto che sta seduto e regge davanti al petto una cornucopia, insieme a due figure maschili in piedi, vestite in modo orientalizzante che nello stesso momento sorreggono con la testa la cornice superiore.

Su tutte e due le pareti le edicole laterali nclla zona superiore sono sorrette da cariatidi che inclossano un chitone verde e una sopraveste gialla. La coppia sulla parete di fondo regge una brocca nella mano esterna $e$ un piatto nella mano interna, mentre gli oggetti tenuti dal'altra coppia non sono piú leggibili.

La parete sinistra dell'anticamera mostra la struttura completa. Sotto i candelabri che suddividono la zona mediana si vedono, a sinistra, una donna vestita di una tunica rosa $\bullet$, a destra, un satiro accosciato che suona una siringa. Indossa un mantello giallo.

Su di una mensola sopra la colonna destra, sopportata dal satiro, vi è un satiro marrone che indossa un mantello corto sopra le spalle. Il peso del corpo poggia sulla gamba destra. Regge un lungo pedum nella destra.

Della parete destra si è conservata la figura di un Sileno barbuto che indossa un mantello giallo-bianco. Regge un tirso nella sinistra.

Bibl.: Silberberg 1981, cat. 20, fig. 19; Bragantini/De Vos 1982, 284-293, tav. $166-196$.

Cat. 319/6. (F, G) Zone mediana e superiore; II stile, fase IIb

La zona mediana e articolata in campi attraverso colonne sormontate da figure femminili che a loro volta sostengono colonne nella zona superiore. Indossano chitoni e sopravesti di vari colori e hanno le mani in atteggiamento diverso, talora reggenti attributi.

Sopra i pannelli della zona superiore si trovano nature morte e paesaggi sacro-idilliaci nei quali si vedono delle statue.

I1, scomparto 2. A sinistra sta un'erma. Nel centro sorge la statua marrone di una divinita seduta, forse Apollo con arco e cetra.

F2, scomparto 2. Veduta di porto con a sinistra la statua gialla di Poseidon, modellata secondo il tipo Kenchreai. ${ }^{69} 7$

F2, scomparto 4. La statua gialla di un animale accovacciato sta di fronte ad un tempio. Vicino sta un gruppo marrone di Iside, Arpocrate e Anubi sopra un alto piedistallo.

F2, scomparto 6. Cinque erme, fra l'altro un'erma di Pan, stanno dentro il recinto di un giardino. Presso un tempio sorge la statua bronzea di una divinita non identificabile.

F2, scomparto 8. A destra sorge una colonna con la statua marrone di una figura maschile che regge un oggetto nella sinistra. Forse si tratta di Herines. Al'estrema destra si vede l'erma itifallica di Priapo.

G1. scomparto 1. Natura morta con un'crma rossa, forse raffigurante Pan.

697 Cf. Walde 1978; Walde-Psenner 1979. Per il tipo statuario Moreno 1976. 
G2, scomparto 2 Un'erma di Priapo sta in primo piano a sinistra A destra vi a la statua di uni diumita maschule, lor se Zous

G2. scomparto 4 Lna coppia di erme lunge da acroterso sopra una porta

G3, scomparto 2 ln dipulo viene occupato dalla statud marrone di Iside seduta, modellata secondo ll tıpo delle statuette del lararı Regge una cornucopia e ha un loto sulla testa Il diplo ha acroterı a forma di due stingl feinminili alate 1 destra si distingue un'erma di Priapo

G4 scomparto 1 A destra sorge un'erma itıfallıca dı Prıapo dı colore $v$ sola Bibl Silberberg 1981 , cat 23 , fig 22 a-d, Bragantinı/De Vos 1982, $337-341$, tas $20 \bar{i}-241$

Cat $319^{\prime 7}$ (?) Zona mediana, II stule, lase IIb

L'edicold centrale contiene un paesaggio sacro-idullaco nel centro del quale sorge una colonna con la statud di Atena Promachos Avanza verso destra e regge uno scudo nella sinistra, mentre biandisce una lancia sopra la testa con la destra $E^{\prime}$ di colore verde Assomiglia all'Atena della Villa imperiale a Poinper (cat $305 / 1$ )

A tianco si trovano duc statue di figure femminlı sopra bası rettangoları verdi A sinistra si vede una Musa, vestita di un chitone bianco e di un mantello dello stesso colore Ha una corona d'edera sulla testa La gamba destra e di appoggio Regge nella destra, all'altezza delld spalla, un volumen, nell'altra lungo il íanco un calamo verde

La figura destra ha la mano destra dviolta nell'himation bianco e la sinistra rlassata lungo il fanco L'attegglamento e summetrico Le due statue corrispondono a tipi ellenist

Bibl Bragantmi De Vos 1981, 293-295, tar 203-206

Ipogeo presso la Porta Maggiore

Cat 320 (vestibolo) Parete sud, zoccolo. III stule

Tra i pannell, con nature morte e uccell vi sono erme bianche, ora quası lleggibilı, che escono da calici L'unica visibile nelle pubblicazioni ha la mano destra sulla testa e la sinistra rilassata lungo ll fianco

Bıbl Bendinell 1926, 610-611, tav II, Bastet 1958, 48, Bastet/De Vos 1979, 51-52, fig 7

Colombario sotto la Villa Pamfll

(colombario) Paretı A-E, II stule, fase IIb (ora Museo Mazionale Romano s n )

Una serie di rappresentazionı mitologiche e paesistıche, descritte nell'ordine seguilo da Bendincllı

Parete A, VII A destra dell'edificio centrale una donna porge un'offerta alla statua di una divinita femminule sopra und base rettangolare La ligura schematica e di colore gi gio-verde con macchic azzurre

Parete A, XII A destra dı un edificıo sta la statua dı una donna sopra un alto piedistallo contro il quale poggia una fiaccola Il colore e blu-verdastro A sinistra vı e l'erma itifallica dı Pan che regge un pedum nella sinistra 
Parete B, XI. A destra di un tempio tetrastilo sta la statua di una divinità femminile che regge uno scettro. A destra si trova un'altra statua mal visibile.

Parete B. XV. A destra di un'edicola si vede una statua di Priapo.

Parete B. XVIII. Eracle lotta con un centauro. A sinistra sta una colonna sormontata da una statua schematica.

Parete $C$, XVII. A destra sorge sopra una base rettangolare la statua di Fortuna-lside seduta. Sulla testa si vede un modio, nella mano sinistra un timone. nella mano destra stesa in avanti un oggetto lleggibile. Le gambe sono incrociate.

Parete C, XXV. A clestra si trova la statua di una figura seduta non molto chiara posta sopra una base rettangolare. Indossa un mantello.

Parete C, XXX. A destra un uomo porge un'offerta alla statua di una dea vestita di un chitone. La divinità regge uno scettro nella sinistra alzata e una patera nella destra stesa verso il basso.

Parete C, XXXIIl. A destra si vede una statua schematica, forse uno xoanon come dice Bendinelli. Poggia contro una colonna.

Parete D. VII. A destra di un altare si distingue un'erma.

Parete E, X. Un'erma sta presso una porta sacra. A destra di un edificio si vede sopra un'alta base la statua di una divinità seduta. E' rivolta verso sinistra e porge una mano in avanti. Piú a destra si intravede un'altra erma.

Parete F, IX. A sinistra si trova la statua di una clonna seduta che regge in una mano stesa in avantj una patera. E' rivolta verso destra. La figura e conosciuta attraverso un disegno.

Su una 'tavola aggiunta' si vede un paesaggio con la statua di una donna ammantata a sinistra di un tempio tetrastilo. Ha una corona sulla testa e regge nella destra stesa in avanti una patera, mentre la sinistra è davanti al grembo. A destra si trova la statua di un uomo con drappo attorno alle reni. La gamba destra è di appoggio. Regge una clava nella sinistra presso il fianco e tiene la destra stesa. Sulla testa si vede una corona. Sta sopra una base composta da tre blocchi.

Il complesso viene datato in base a dati epigrafici nell'epoca augustea. Stilisticamente i paesaggi assomigliano a quelli nella Villa della Farnesina (cat. 319) e nella Casa di Livia (cat. 317). Peters dice che le statue mostrano the painter's skill in painting the human body. I pinakes sarebbero imitazioni di lastre marmoree, sia o meno a rilievo.

Bibl.: Bendinelli 1941, 7-8, 10-1, 17-19, 21, 23, 24-25, 29, tav. III, V, VI, VII, tav. aggiunta 4, c, fig. 11: Borda 1958, 209-210; Peters 1963. $55-59$; B. Andreae, in: Helbig ", 2490 Silberberg 1981, cat. 145 , fig. 122 .

Catacombe di Cyriaca

Cat. 322. Tomba di Zosimianus; quarto secolo d.C.

Io zoccolo sotto l'arcosolio $\dot{e}$ decorato con un prato con pecore. La siepe contiene pali e erme con teste di figure ignote.

Bibl.: Wrede $1972,132-133$ cal. 3 (con bibl.).

Catacombe di S. Sebastiano

Cat. 323. Domus Petri; quarto secolo d.C. 
L'arcosolio destro ha una rappresentazione di un giardino nello zoccolo. La siepe contiene al posto di pali tre erme con teste barbate. Si tratta dell'imitazione dj un Hermenzaun.

Bibl.: Wrede 1972, 132, Tat. 76, 77.1-3 (con bibl.); Mielsch 1978, 192, Taf. 98.1 .

Cat. 324. Refrigerio; epoca antoniniana

(A) Parete est, quadro centrale

paesaggio con torre e portico lungomare. Sulla torre vi è una colonna con una statua schematica marrone chiaro. Un uomo (?) indossa un lungo mantello e regge nella sinistra alzata una lancia e nella destra stesa verso il basso una patera. A destra della torre e del portico si vede una statua indicata con una sola striscia sopra una bassa base.

All'estrema destra si vede un edificio sormontato da una biga giallo-marrone. Il carro e rivolto verso sinistra e viene condotto da un irionfatore. Egli e incoronato da una figura, probabilmente Nike. La rappresentazione segue l'iconografia normale, discussa da Darling.

Bibl.: Bianchi Bandinelli 1969, fig. 334; Ferrua 1979, 46-47, fig. 13; Darling $1981,24 i, 265-266,269-272$, ill. 28-33.

Necropoli sotto il Vaticano

Cat. 325. Tomba phi ; epoca severiana

Nelle lesene fra gli arcosoli si vedono erme rappresentanti dei Sileni. La parte superiore del corpo è delineata in modo plastico. I fusti sorgono da calici vegctali. Reggono un tirso nella sinistra presso la spalla e un cantaro nella destra distesa. Attorno al viso hanno un numbo, mentre le spalle sono coperte da una clamide svolazzante.

Le figure sembrano ispirate da esempi di II stile conosciute da frammenti nei Musei Vaticani (cat. 012) e soprattutto da Pompei (cf. p. 20). Specie le erme della Casa di Caesius Blandus sono molto affini (cat. 148/2-3, 233/2).

Bibl.: Toynbee/Ward Perkins 1957, 80, pl. 18; Borda 1958, 322; Mielsch 1981, 206 (con bibl.).

Cat. 326. Tomba dei Nasonii; seconda metà del secondo secolo d.C. + Fra gli arcosoli si trovano edicole dipinte, occupate da statue. Ogni parcte contiene due figure maschili nude simmetriche. Quelle sulle pareti lunghe hanno una clamide sulla spalla interna e reggono un cestino con frutti con la mano esterna lungo il fianco. La gamba esterna e di appoggio. Le figure sulla parete di tondo hanno lo stesso atteggiamento, ma sono disposte simmetricamente. Tutte e sei le figure hanno una corona sulla testa.

Andreae, nella sua descrizione accurata della decorazione tombale, non parla di queste figure. Nella Casa delle volte dipinte ad Ostin (cat. 129'1) figure simili possono essere interpretate come Stagioni, ma in questo caso il nunero di sei impedisce una tale identilicazıone. Aon si conoscono contronti possibili nella scultura finora conosciuta, ma lo stile e certamente elenistıco.

Il satiro che si trova su un frammento a Londra viene considerato una variazione dell'Hermes di Olimpia da Antonsson. ${ }^{\circ 9}$

699 Hinks 1933, cat. 72a; Antonsson 1937, 134-136, fig. 22. 
Bibl.: Andreae 1963, 88-130 (descrizione della tomba), Taf. 41-70, Beilage, spec. Taf. 45, 46, Bollage; Andrear 1973, 240, Abb. 483.

Cat. 327. Tomba presso S. Paolo fuori le mura; epoca severiana

vell'arcosolio si trova una scena con Atena e Prometeo. Questi sta per finire un modello fittle del corpo umano che $e$ reso in un attegglamento rigido con le gambe unite e le braccia strette lungo il corpo. Il colore e marrone.

Bibl.: B. Andreae, AA 1957, 225, Abo 45 (con bibl.).

Cat. 328. Tomba dei Pancratii lungo la Via Latina; epoca antoniniana

(2) Paesaggi sacro-ıdillıaco abbelliscono le lunette. Sono descritti secondo I'ordine seguito da Dirling.

A-1. Sulla sinistra, accanto ad un albero, sta la statua schematica di colore giallo di una tigura che indossa una veste corta. Regge una cornucopia nella destra stesa, mentre tiene la sinistra sul fianco. Come suggerisce I)arling, puo trattarsi di un lar vialis.

Sopra la porta sacra nel centro si trovano due acroler gialli a forma di tritoni. La figura a sinistra regge uno scettro nella destra stesa, l'altra una bacchetta nella sinistra. In questo caso gli anmali fungerebbero da elementi funerarı come $\mathrm{j}$ mostri marını sui sarcofagi 'marini', essendo la porta un inonumento funerario Figure sumili sono presenti su MN 9514 (cat. 097).

A clestra contro la porta sta una figura femminile nellatteggiamento dell'aufgelehnte Muse. I'robabilmente la ligura rappresenta una statua viste le dimensioni delle altre figure umane sul pinax.

All'estema destra una donna porge un'ofterta alla statua della Fortuna che regge un tumone con la destra. Il colore e giallo.

B-1. Nel centro si vede la statua di Poseidon di colore giallo sopra una base rettangolare. Poggia con la destra sul ginocchio della gamba sinistra che sta su una prora. leegge nella sinistra alzata un tridente. Si tratta del modello Latcrano, originariamente esposto a henchreai. ${ }^{700}$

B-2. A sinistra, davanti ad una colonna vi $p$ la statua marrone di Priapo nell'atteggiamento della chordosis. La striscia al lato sinistro potrebbe raffigurare uno scettro o un pedum. Il colore, menzionato expressis verbis da Darling indica chiaramente il materiale, cioe il legno. Sotto l'albero a destra si vedono tre statuette sopra una base comune, rappresentanti un hekatation o le tre Grazie.

C-1. A destra si trova una colonna sormontata dalla statua di una figura che regge uno scettro o una lancia. Fra la porta e la tholos nel centro e una statua schematica. Le mani stese che reggono bacchette o fiaccole. con ogni probabilita caratterızzano la figura come Ecate.

C-2. Accanto alla colonna a sinistra si trova una statua quasi illeggibile sopra una base rettangolare.

D-1. A destra vi sono tre statuette sopra un solo piedistallo. Come su B. 2 si tratta di un hekataion o un gruppo delle Grazie.

Bibl.. RP 389; Andreae 1973, 227, 240, Abb. 470-476; Silberberg 1981, cat. 147. fig. 124: Darling 1981, 64-65, 70-71, 72, 74-75, 98-100, 113, $126-127,160-161,177,196$, ill. $4-6,10-23$.

$700 \mathrm{Cr}$. Walde 1978; Walde-Psenner 976. 
Miclsch contronta il frammento per quanto riguarda lo stile con le decorazioni della T'omba des lancratis nella necropol vaticana assegnata allepoca adrianca, ma lo considera posteriore sulla base des colori.

Bıbl : H. Mielsch, in Mlfreschi 1976, 36-37, no. 4'2. tav 4.2.

Musso Vazionale Romano

Cat. 333 In . no" ": IV stile o epoca adrianea

Quadro della zona mediana, facente parte della collezione Rospigliosi Un alleta si incorona con la destra, mentre regge una palma nella simistra La gamba smistra o di anposgio Ld figura copla. come l'atleta nella scaenae trons nella Casa del palestrita a Pompes (cal. 267) l'alleta Westmacott e altro statue del gencre (tria l'altro il famoso Getty Bronze) L'oggetto nella destra potrebbe esserc inportante nella ricostruxione dell'originale, problema linora non risolto $C \cdot C$ Vermeule penso ad Lracle che si incorona. Berger ad una statua descritti da Plmio come I nudus talo incessens. ${ }^{70} 3$

La clatariono del frimmento a controversa Borda a Felletti Maj Moreno anunettono und datis/onc nell'epoca adrianca in base alla classicita della figura, Mielsch inserusce il pinax hell'epoca tlavia Il confronto con la figura nella pittura pompelana $f$ nel pinax $11 \backslash 9051$ potrebbe essere un argomento a favore della clatazione piu alta

Bibl. G Benchinellı. BdA 1925-1926, 147; RP 238.2. Rizzo 1929, 68. Borda 1938. 270, Ielletti Val Moreno 1967. 56 ; Mielsch 1981. 233.

Ins.no. 1460-1462, 1464, 146i-14i1, 147i-1479. > Roma, Villa della Farnesina (cat. 319).

Inv.no. ?', Koma, Casa di Fausta (cat. 312)

Inv.no. ?: , Roma, Columbario sotto la Villa Doria Pamfil (cal. 321).

lns no." Roma Tomba degli Octaril (cat. 329)

703 Vermeule 195\%, Berger 1978; cf. Moormann 1983b, 103. 
Cat 329 Tomba deglı Octavil lungo la Via Triontale, 200-22j d C (ora Museo Vazionale Romano)

Nella lunetta si vede un gruppo di bambini in un giardino con rose vel centro sorge una colonna con la statua di Ecate trilorme In tutte e bel le manı regge una fiaccola Darling contronta la figura con una statua trovata sull' $\rightarrow$ gora di Atene 701

Bıbl G Bendinellı. ISc 1922, 433, Wirth 1934, 148-149, Taf 38, Borda $1958,119,314-315$, Darling 1981, 36-37

Vulla di Numisia Procula presso Tor Marancio

Cat 330 (?) Parete?, zone mediana e superıore, epoca antoniniana +

Solo da un'incisione si conosce una decorazione architettonica Altre parti delle pitture di questa villa si trovano nei Musel Vaticani 702

I campl della zona mediana vengono separati attraverso candelabri sormontati da figure Le quattro figure sono da smistra d destra un satiro due inenadi simmetriche e un altro satiro, immagine simmetrica della prima figura I satirı reggono nella mano esterna un pedum e hanno l'altra sopra la testa nell'atteggiamento dell'aposkopon Le menadi indossano un humation Reggono una patera $\left({ }^{(0)}\right.$ nella mano internd mentre quella esterna e alzata

入ella zond superiore kordi classiche sorreggono con entrambe le mani l'orlo supei iore della decorazione Stanno sopra , capitell corinzi delle colonne della zond mediana Indossano un peplo

Bibl Joyce 1981 , jo, fig 49

Antıquarıum Comunale

Cat 331 Inv no?, IV stile

Paesaggio sacro-idlllaco proveniente da una zona mediana Nel centro davanti ad una porta sacra vi e un'erma bianca A destra di fronte ad un portico si distinguono tre statuette bianche $d_{1}$ tigure temminll Forse rappresentano un hehataion, altrimentı si tratterebbe delle tre Grazıe, meno plausibul vista l'assenza di acqua

Bıbl $H$ Mielsch, in Affreschı 1976, 36, no 4/1, tav $X 1$

Cat 332 Inv no ?, secondo secolo d C

Paesaggio sacro-ıdlliaco nel campo bianco di una zona mediana Presso una porta sacra nel centro e la statua marrone $d_{1}$ un uomo nudo sopra una base rettangolare La gamba destra e di appoggio Regge in entrambe le manı distese in avantı una flaccola

\footnotetext{
701 Cf Harrison 1965, pl 32-39, per ll tipo kraus 1960

702 Nogara 1907, 55-61, B Andreae, in Helbig ", 463
} 


\section{SABRATHA}

V 3 Casa dell'attore tragico

Cat 334 Parelı I e III, zoccolo e zona mediana, epocd? (ora Sabratha, Museo Archeologico)

Parcte I contiene uno roccolo composto da campi separati da edicole Queste sono occupate da figure alate poste sopra piedistall, sirene o sfingı Una sola figura e rimasta conservata Ha un polos sulla testa

La trabeazıone dell edicola centrale della zona mediana e incoronata da due $H^{*}$ Ufthermen che sostengono la colnice superiore della decorazione Il tusto e marrone, il corpo di colore naturale Attorno alle reni si vede una corona Le figure reggono nella mano esterna presso il fianco un mantello, mentre l'altra e stesa per meta Le teste barbute sono rivolte verso ll centro

Sopra l'edicola centrale della terza parete vi sono due figure femminul vestite di un peplo che sorreggono la cornice superıore della decorazione Il colore della veste e bluastro verde attorno al petto e sulla testa hanno una corona Reggono nella mano interna alzata un ramoscello, mentre l'altra tiene una benda lungo il tanco Fra le due donne di stule classico si trova un gruppo composto da una sfinge dlata inginocchiata e un Lros nudo in piedi Ne sono rmaste conservate solo alcune partı

lurigemma sostenne che la datazione del complesso pittorico non tosse possibule in base a dati archeologici o stuhsticl $S_{1}$ puo pensare alle scaenae frontes dell'epoca adrianea nella Vlla adrianea (cat $339^{\prime} 1$ ) e nella Villa Negrom (cat 314/1) conosciute attraverso disegnı

Un frammento sporadico rappresentd una villa marittima Sul molo in avanti vi e la statua schernatica della Fortuna, vestita di un peplo e reggente un tunone con la mano sinistra

Bibl turigemma 1962, 103-104, 105-106, 107, tav 93-95, 99-100, 104-105, 112, Johnston 1982, 196 , pl $141-2$ 
-339-

\section{SELGCH}

Efes Muzesı

Inv no. 1572-1574. , Eteso, $\mathrm{H} \mathrm{2/7a,H} 2 / 14$ (cat. 014/, 017). 
Casa di Loda

Cat 335 (triclinio) Paretı nord e ovest. zona mediana, IV stue

Nel campi si delineano statue sopra piedistallı rettangoları in giallo e nero Nel secondo campo di sinistra delld parete nord si vede Leda che preme con la destra ll cigno contro ll fianco, mentre tiene con la sinistra l mantello scivolato dal dorso l senı sono copertı da uno strofion La gamba destra e dı appoggio Il cigno tiene le zampe vicino al fianco di Ledd e la bacia sulle labbra Come nella Casa della regina Margherita a Pompel (cat 190) l pit tore ha voluto dipingere la statua di Timoteo, anche se a Solunto l gruppo ditferisce notevolmente

I campi lateralı della parete ovest contengono Erotı summetrıcs ad alı cerulee e con una clamide marrone sulle spalle Reggono nella mano esternd una ficcold gialla mentre quella interna (perduta) e alzata De vos li interpreta ce ne Imeneo sulla base dell'iconografia e della combinazione con un paesaggio sacro-idlliaco Lna ligura M\ 9320 proveniente dalla Casa di Meleagro (cat 202 2) serva da confronto

Bibl Le Vos 1975 201-203, fig 17-19 
Cat. 336. Tomba sull'Acropolı; inizio del secondo secolo d.C.

Le pareti sono suddrve in campi neı quali si delineano Apollo Musagete e le nove Muse. Dopo lo scavo furono ancora visibilı solo tre pareti su quattro e due figure.

Apollo sta nell'atteggiamento dell'Apollo Licio di Prassitele: la destra poggia sulla testa, mentre la sinistra e stesa un avanti. leggermente in alto. e sembra sollevarc un lembo del mantello sulle spalle La gambil destra e di appoggio Il corpo ha un contrapposto pesante ed e allungato secondo le torme prassitelee. Lo scavatore A. Adamantiou paragona la tigura con l'Apollino a Firenze. ${ }^{\circ}$

La sola Musa conosciuta indossa un peplo $P$ un himation del quale solleva un lembo con la destra. La mano sinistra $e$ in riposo lungo ll fianco. La gamba sinistra e di appoggio. Moreno contronta la figura con la Cho nella Casa delle Muse ad Ostia (cat. 130/1).

Delle tigure di Melpomene e Euterpe sono rimaste conservate le epigrafi greche.

Bibl.: A Adamantiou, Praktika 1931, 92-96, pin. 1-2; C. Karo, AA 1932, 144; Borda 19j8, 272-273; rellettı Maj/Moreno 1967, 25.

704 Mansuelli $1958,74-76$ no. 46 , fig. 46 a-b. 
Villa di Tiberio

Cat. 337. (Vano absıdale a sin. dell'antro) Parete ?, zona superiore; III stile

Un frammento sporadico mostra una donna vestita di un peplo di colore giallo sopra una trave. Ha la destra presso la spalla e ha la losta coperta da un polos. Lo stile e classico e rucorda le Danaidi della Villa dei papiri ad Ercolano.

Bibl.: Jacopi 1963. 21, tav. III. 
Collezione privata

Cat. 338. Tre frammenti di provenienza ignota; IV stile

Rappresentazione $d_{l}$ un giardino. Nel centro del frammento maggiore, quello centrale, si vede una stinge che sostiene una vasca da fontana. E' rivolta verso destra. I campi laterali sui irammenti minori hanno nell'orlo superiore uno scrigno che contiene due maschere.

Bibl.: Dorig 1975. 370-372, cat. no. 370-372. 
Villa di Adriano

Cat $339 / 1$ (?) Parete ?, zona mediana e superıore, epoca adrianea +

Ln disegno nella collezione di Eton rappresenterebbe una decorazıone parietale della Villa adriana L'edicola absidale di una scaenae frons e occupata dalla statua di tfrodite che sta sopra una base rettangolare La gambd destra e di appoggio Regge uno specchio nella destra alzata e una bacchetta nella sinistra I'n mantello copre le gambe

Due statue sopra del piedistall rettangoları fiancheggiano la nicchia A sinistra si vede Hermes che regge un caduceo nella sinistra, mentre la destra e davanti al petto Ha un petaso sulla testa e una clamide sulle spalle La gamba sinistra e di appoggio

A destra sta rros con una clamide sulla spalle Regge nella destra stesa in avanti una fiaccola e tiene un lembo delld clamide con la sinistra presso le renı La gamba destra e di appoggio

vell edicola centrale della zona superiore si trova la statua di Zeus sopra una base a forma di $\Gamma$ Il dio regge uno scettro nella smistra e un tulmine nella destra Il tipo e quello delle statue di divinita sedute, concepito nell'epoca ellenistica o usato per le statuctte nei lararı

Bibl Swindler 1929, fig 593, Borda 1958, 269

Cat $339 / 2$ (?) Solfito epoca adrianea +

Due soffitti, pubblicati da Ponce, sono interessanti per la presente ricerca SI tratta di decordzioni da lungo perdute senza collocazione esatta.

Sulla tavola 5 si vede un soffitto con quattro coppie identiche di erme che sorreggono il centro della decorazione Ogni coppia e simmetica, in modo che l) braccio esterno e datantı al petto, mentre l'altro e musibile Hanno le ioste barbute c lunghe orechie da satir

Il centro della volta contiene un globo stellare sorrctto da quattro atlantı nudi che sorreggono l'oggetto con entrambi le manı sollevate

Tavola 10 mostra una volta con quattro edicole ad ogni lato con statue sopra bası curılinedri I latı oppostı hanno figure simılı, ciop due uominı e due donne

La prima figura maschle nuda poggia sulla destra Regge un drappo nella destra stesa in avanti e und bacchetta nella destra lungo l lianco II suo pendant ha 1 piede destro sollevato e poggia sulla gamba sinistra L atteggiamento della mano destra e simle a quello dell'Hermes di Olimpid Un mantello copre il fianco sinistro

Le altre due edicole contengono le statue $d_{1}$ donne ammantate Per entrambe la gamba destra e di appoggio Lna tigura regge un platto nella sinistra L'altra e priva di attributi

Bibl Ponce 1786, tar 5, 10 
Vlla di L. Crassius Tertius ("Villa B")

Cat 340. (Vano I primo piano) Parete nord, zona mediana; IV stule

Sopra un piedistallo si delinea Eracle davantı ad un campo bianco Regge nella mano sinistra stesa per meta un cantaro e poggia con la destra sulla clave al suo fianco Il peso del corpo poggia sulla gamba sinistra L'atteggramento della figura e simile a quello dell'Eracle Lansdowne, ora nel Paul Getty Museum a Malibu, ma contiene anche elementi del tipo 'Ludor 1si-Lembach'

Bibl. inedito 706

Villa detta di Poppaea

Cat 341/1 (11) Nicchia nord, parete nord, quadro centrale, II stile, lase Ic In un tempietto vi e la statua gialla di una tigura temminle vestita $d_{1}$ un peplo e un himation. Regge nella sinistra distesa per meta una patera, mentre la destra e alzata. Sulla testa ha una corona. Lo sguardo e rivolto verso destra La mancanza di attributı specilici impedisce un'interpretazione della divinita raffigurata.

Nel campo a destra si trova un paesaggio su sfondo verde. A sinistra del secondo edificio di sopra sta una statua marrone-viola sopra una base circolare. Tutto e stato disegnato in modo schematico.

Bibl De Franciscis 1975, 11, tig. 15.

Cat. 341/2. ( 14 antisala) Pareti est e ovest, zona mediana; II stile, fase Ic Paesaggi giall su campı grall imitano del rllevi. I paesaggi sono sempre divisi in tre registri, forse riproducendo tre parti di un Bilderbuch (ct p. 53) 707 Ogni parete contiene due lastre accanto alla porta. Nel paesagg10 a sinistra della parete ovest si vede una statua di Nike presso una torre rotonda. Il modello e quello della Vittoria romana

Bibl Bastet/De Vos 1979, 24 nota 6; Moormann 1984b, 646, fig. 11-12.

Cat. 341/3. (14) Tutte le pareti, prospetti centrali, II stile, fase Ic I prospetti mostrano dei larari campestri. Le edicole delle pareti est e ovest contengono figure reggent1.

Parete ovest. Dietro alla porta sta una tholos con la statua gralla di Afrodite che indossa un chitone senza maniche e ha una corona sulla testa Solleva 1 lembı della veste con entrambe le mani.

705 Per l'Eracle Lansdowne Howard 1966: Moreno 1977, 416. Per il tipo Ludovisı-Lembach Todisco 1979.

706 Sulla villa G. Magg1, CronPomp 2 (1976) 249: Malandrmo 1977, 81-85, fig 13, 27-28, Jashemshı 1979, 320-322, fig. 497.; A Lagl di Caro, Pompeii Herculaneum Stabiae 1 (1983) 369-375, tig. 37-42.

707 Cf. Bastet/De Vos 1979, 24. 
Sulle paretı disisorie a sinistra e a destra della porta che mette nel santuarıo si vedono grifi glallı, vistı di profilo Hanno lo sguardo rivolto verso la porta l na disposizione simle e nota dal monumento funeraiso di Belev 1 dove fiancheggiano un'urna, si conoscono inoltre modelli scultores di bronzo che possono servire da confronto 70

Al di sotto della cornice sul bordo superiore della decorazione si vedono slingl temminli alate sedute su una trave aggettante Hicordano alcunı elementi plastici inseriti nell'architettura della basulica di Leptis Magna 109

Parete nord Aol centro e una colonna con la statua verde di Ecate Indossa un peplo e regge in entrambe lo manı distese una traccold Dalla testa ormal perduta scendono delle vitte come si vede nelld Villa di P Fannius Synistor a Boscoreale (cat 004/4) Lo stule e arcaizzante

Le colonne dell'edicola attorno al paesaggio sono abbellite con rilievi rappresentantı tritoni e figure temmind alate

Parete est Ripete lo schema della parete ovest La figura nella tholos e andata perduta per la maggior parte Indossa un chitone senza maniche e regge nella sinistra un tirso o una fidccola Un'interpretazione non e possibule visto lo stato frammentario della figura I grifi e le sfingi si presentano in forma poco piu plastica e voluminosa

Bibl De Franciscis 1975 11, $t_{1 g} 17,20$ 23, Picard 1977, 246-247, fig 7 , Tybout 1979, 269-270, fig j-G, Lerch 1982, 147

Cat $341 / 4$ (20) Paretı est e ovest, zona mediana, IV stule

Rappresentazionı di giardini su sfondo rosso Fra 1 cespugli si delıneano su ogni parete due fontane simmetriche sorrette da centaurı accolacciatı Gli anumali hanno lo sguardo rivolto verso il centro e sorreggono un bacino rettangolare nel quale zamplla acqua Il tipo e simule a quello delle quattro statuette trovate presso la piscina grande della vlla Ord le decorazioni sono difficlmente leggibul

Bıbl De Franciscis 1975 11, fig 3, Michel 1978, 393-394 Jashemski 1979, 290-292, fig 139-441

Cat $341 / 5$ (23) Parete ovest, quadro centrale, II stile, fase Ic

Paesaggio di colore verde su una lastra verde $S_{1}$ tratta dell'umitazione di un rilievo simile a quellı nel vano 14 Un pdesaggso dello stesso colore e stato Irovato nella Villa sotto l'Escuderia Real a Portici (cat 308) peplo

$S_{1}$ distingue vagamente sopra una torie una statud di donna che indossd un

Bibl De Tranciscis 1975, 12, fig 13 Tybout 1979, 270, 11g ta-b, Moormanı 1984b, 646

Cat 341/6 (32) Parcte nord quadro centrale, IV stule Una natura morta di colore bianco-giallo su uno sfondo rosso contiene un'erma copra una colonna al lato destro

Bıbl inedito

Cat $341 / 7$ (Corridoio a sud di j) Parete nord, zona mediana, IV stıle

70. Flagge 1975, Abb 139-141
709 Lyttelton 1974, 291, pl 215 
Nel campo a sinistra della porta che mette nell'atrio si trova un paesaggio sacro-sdlliaco schematico. Sulla trabeazıone di un edificio sorgono due tritoni marronı. Macchie gialle suggeriscono un ccrto rilievo.

Bibl . inedito.

Cat. 341/8. (Portico a sinistra di 4) Parete sud, zona mediana; IV stle

Nel seconclo campo da sinistra si trova una natura morta in rosso e verde A destra sorge un'erma sopra una colonna come in 32.

Bibl. . Inedito.

Cat. 341/9. (68) Paretı est e ovest deglı horti conclusi, IV stule

In tuttı e due gli horti conclusi si vedono rappresentazıon di giardinı su uno sfondo giallo Ogni parete contiene un paio di tontane sorrette da un centauro - una sfinge. Lina combinazione uguale si vede in I 17, 4 a Pompei (cat, 176).

Inoltre si delineano fra le plante crateri abbelliti con rlievi, unitazioni dei crater neoattici $E^{\prime}$ stato trovato un esemplare presso la piscina 710

Bibl : Michel 1978, 391-394, Jashemski 1979, 306-309, tig. 470-475

710 Jashemski 1979, fig. 480 . 


\section{TREVIRI}

Cat 342 Basllica

Mielsch menziona una decorazione ancora inedita con ligure plastiche fra 1 pannell della zona mediana La decorazione si potrebbe datare nel tempo di Costantino sulla base della costruzione dell'edificio

Bibl Mielsch 1978, 174 


\section{$-349-$}

\section{TRIPOLI}

Museo Archeologico

lnv. no. ?: > Zliten, Villa di Dar Buc Ammèra (cat. 344). 


\section{TUSCOLO}

Cat. 343. Frammento sporadico; primo quarto del secondo secolo d.C. (ora Louvre inv. no. $P$ 55)

Sopra una base rotonda gialla vi è la statua di una donna vestita di un chitone verde senza maniche e di un mantello verde. Le gambe sono unite. Ha sulla testa la parte estrema di un arco. Regge nella destra stesa verso il basso una patera gialla, mentre la sinistra è sollevata.

Probabilmente il frammento proviene dalla zona superiore nella quale una fuga di archi veniva sorretta da cariatidi. Si confronti un frammento con archi dallo stesso Tuscolo (Louvre inv, no. P. 56).

Bibl.: RP 405.6; Tran Tam Tinh 1974, 99-100 no. 85, fig. 97. 
Villa di Dar Buc Ammèra

Cat. 344. (Criptoportico) Parete ?, zona ?; 69-96 d.C. (ora Tripoli, Museo Archeologico)

Su uno sfondo bianco si delinea la figura verde di Ares. Il dio ha un elmo sulla testa e una clamide sulla spalla sinistra. Regge nella sinistra alzata una lancla, mentre la destra poggia nel fianco reggente un gladio. La gamba sinistra è di appoggio. Con linee rosse sono stati accentuati dettagli quali gli occhi, la chioma e le armi.

Il colore verde imita la patina di una statua verde. Si tratta dell'unica rappresentazione di Ares apparentemente concepita come statua a parte l'Ares nella Casa della Venere in conchiglia (cat. 178). La testa piccola e le dimensioni del torso e delle gambe sembrano imitate da esempi lisippei.

Bibl.: Aurigemma 1962, 49-50, tav. 36-37; Johnston 1982, 196-204, spec. 203. 
Le riviste sono citate con le abbreviazıon della Arch \&ologische Bibliographie, per le riviste non piu esistentı, opere generalı e serie sono stati adoperate le sigle della Arch Cologische Anzeiger 1982, 809-813.

Abad Casal, L , 1982 La pintura romana en España, Alıcante-Sevlla

Adam, J - P / P Varene, 1980 Une peinture romane representante une scene de cantier, $R A, 213-238$

Adamantiou, A , 1931 Andshaphas en Sparte1, Prakt, 91-96

Aclamo Muscettola S . 1979 Bronzett 1 raffigurantı dinastı ellenistici al Museo Archeologico di Napolı, in Bronzes hell enistiques et romaines. Tradition et renouveau, Lausanne, 87-94

Adamo Muscettola, S, 1982a Nuove letture borboniche 1 Vonll Balbı ed I Foro di Ercolano, Prospettiva 28, 2-16

Adamo Muscettola, $S$, $1982 b$ Le ciste di piombo decorate, in Attı 1982, $701-752$

Adrian, A , 1959 Divagazioni intorno ad una coppa paesistica del Museo di Alessandria, Roma

Allroggen-Bedel, A, 1974a Maskendarstellungen in der robmisch-kampanischen Wandmalerei, Munchen

Allroggen-Bedel, A 1974b Das sogenannte Forum von Herculaneum und die borbonischen Grabungen von 1739, CronErcol 4, 97-109

Allroggen-Bedel, A, 1975a Zur Daticrung der Wandmalereien in der Vulla Imperiale in Pompei, BABesch 50, 225-236

Alloggen-Bedel, A, 1975b Herkunft und ursprunglicher Dehorationszusammenhang einiger Malerei-Fragmenten auf der Ausstellung in Essen, in NF 1975, 115-224

Allroggen-Bedel, A, ig7jc Der Hausherr der "Casa del Cervi" in Herculaneum CronErcol 5, 99-103

Allroggen-Bedel, A, 1976a Die Malereien aus dem Haus Insula Occidentalis, 10, CronPomp 2, 144-183

Allroggen-Bedel, Á, 1976b. Eln Malere1-Fragment aus der Villa del Papirı, CronErcol $6,85-88$

Allroggen-Bedel, A, 1977 Die wandmaleresen aus der Villa in Campo Varano, $R M 84,27-89$

Allroggen-Bedel, A, 1983 Dohumente des 18 Jahrhunderts zur Topographie von Herculaneum, CronErcol 13, 139-158

Alvino, $G, 1976$ Tentativo di ricostruzione di un sarcofago con scene di palestra, ArchCl 28, 257-266

Amelung, W, 1908 Die Skulptwren der Vaticanischen Museen, II, Berlin

Ancrent Gardens 1981 E B MacDougall/ $W$ Jashemskı (edd), Ancient Roman Gardens Dumbarton Oaks Colloquium on the History of Landscape Architecture, VII, Dumbarton Oeks 1981

Andersen, F G, 1977 Intorno alle origini del secondo stule, AnalRom 8, $71-78$

Andreae, B, 1962 Der Zyhlus der Odysseefreshen im Vatıkan, RM 69, 106-117

Andreae, B., 1963 Studien zur r\%mischen Grabkunst, Heidelberg

Andreae, B , 1974 Die romischen Repliken der mythologischen Skulpturgruppen von Sperlonga, AntPl 14, 61-110.

Andreae, B, 1975 Rekonstruhtion des grossen Oecus der Villa des P Fannius Synistor in Boscoreale, in NF 1975, 71-83

Andreae, B, 1976 Schmuck eines Wasserbeckens in Sperlongs, $R M$ 83, 287-309

Antonsson, O , 193T The Praxiteles Marble Group in Olympia, Stochholm 
Architects 1981: Pomp!ei. Traveaux et envois des architects français au XIXe siècle, Naples.

Arnold, B., 1969: Die Polykletnachfolge, Berlin.

Art 1981: 'L'art d.coratif ta Rome ta la fin de la République et au début du Principat, Rome.

Atti 1982: La regione sotterrata dal Vesuvio: Studi e prospettive, Atti del convegno internazionale 11-15 nov. 1979, Napoli.

Aurigemma, S., 1962: L'ltalia in Africa. Le scoperte archeologiche. Tripolitana I, 2. Le pitture d'et a romana, Roma.

Aurigemma, S., 1970: Le Terme di Diocleziano e il Museo Nazionale Romano, Roma (6. ed.).

Bacchielli, L. 1980: La Tomba delle "Cariatidi" ed il decorativismo nell'architettura tardo-classica di Cirene, QuadALibia 11, 11-34.

Bacchielli, L., 1984: Una nuova lettura della facciata della Grande Tomba di Leukadia, DArch terza serie 2, 55-58.

Baccini Leotardi, P., 1978: Pitture con decorazioni vegetali dalle terme, MonPitt III, Ostia 5, Roma

Baldassarre, 1., 1975: Contribuzione alla precisazione cronologica de 'Las Incantadas' di Salonicco, Studi Miscellanei 22, 21-36.

Baldassarre, I., 1981: Piramo e Tisbe: dal mito all'immagine, in: Art 1981, $337-351$.

Baldassarre, I., 1984: Pittura parietale e mosaico pavimentale dal IV al II sec. a.C., DArch terza serie 2, 65-76.

Bammer, A., 1978-1980: Elemente flavisch-trajanischer Architekturfassaden aus Ephesos, OeJh 52, 67-90.

Bammer, A./R. Fleischer/D. Knibbe, 1974: Fứhrer durch das Arch åologische Museum in Selçuk-Ephesos', Wien.

Barbet, A., 1971: Peintures murales de deuxième style provenant de la terrasse Sud-Est, zone A, couche 3, in: Fouilles de l'Ecole française de Rome à Bolsena (Poggio Moscini), II. Les architectures, Rome, 321-388.

Barbet, A., 1974: Recueil gónfral des peintures de la Gaule, I. Province de Narbonnaise. 1: Glanum, Paris.

Barbet, A., 1982: La diffusion du III style pompéien en Gaule, I, Gallia 40, $53-82$.

Barbet, A., 1983: La diffusion du III style pompeien en Gaule, II, Gallia 41, 111-165.

Barbet, A./C. Allag, 1972: Techniques de préparation des parois dans la peinture murale romaine, MEFRA 84, 935-1069.

Barbet, A./M.-O. Savarit-Dubbick, 1983: Peinture murale romaine en Gironde, Bordeaux 1983.

Bastet, F.L. 1958: De datum van het grote hypogaeum bij de Porta Maggiore te Rome, Leiden.

Bastet, F.L., 1964: Wann fing der Vierte Stil an?, BABesch 39, 140-143.

Bastet, F.L., 1966: Simulacrum valde ridiculosum, Amsterdam 1966.

Bastet, F.L., 1971: Domus Transitoria, I, BABesch 46, 144-172.

Bastet, F.L., 1972: Domus Transitoria, II, BABesch 47, 61-87.

Bastet, F.L., 1974: Fabularum dispositas explicationes, BABesch 49, 206-240.

Bastet, F.L., 1984: Romeinse wandschilderingen uit de Villa Negroni, in: Herinneringen aan Italies. Kunst en toerisme in de $18 e^{\text {eerw, }}$ 's-Hertogenbosch-Zwolle, 170-173; cat. no. 107 I-II.

Bastet, F.L./M. de Vos, 1979: Il terzo stile pompeiano. Proposta per una classificazione del terzo stile pompeiano, 's Gravenhage.

Bauer, H., 1983: Porticus Absidiata, RM 90, 111-184.

Becatti, G', , 1951: Arte e gusto negli scrittori latini, Firenze.

Becatti, G., 1971: Ninfe e divinità marine, Studi Miscellanei 17.

Belot, E., 1984a: La peinture murale romaine provinciale dans le Nord/Pas-de-Calais, Valenciennes. 
Belot, E, 1984b Les peintures gallo-romanes, ArcheologiaParis 189 (avrl), $39-42$

Belot, E, 1984c Le Vord de la Gaule, ArcheologiaParis Dossier 89, 41-44

Bendinelli, G , 1926 Il monumento sotterraneo di Porta Maggiore in Roma, MonAnt $31,601-847$

Bendinell, G, 1941 Le pitture del Columbario di Villa Panfili, MonPıtt, III, Roma 5, Roma

Berard, Cl /Hofstetter, M , 1979 Dedale et Icare tradition ou renouveau, in Bronzes hell 6nistıques et romaines. Tradition et renouveau, Lausanne, $121-126$

Berger, E, 1978 Zum von Plinıus ( $\mathrm{N} \mathrm{H} \mathrm{34,55)} \mathrm{uberlıeferten} \mathrm{"Nudus} \mathrm{talo}$ incessens" des Polvhlet, AntK 21, 55-62

Bertı, F, 1972-1973 Poseidon ed Amymone Un mosalco romano di Chania, ASAtene $50-51,451-465$

Besch1, L , 1962 I bronzetti romani di Montorio Veronese, Venezia

Beschi, L , 1974 Una nota in margine al "Mercurio di Montorio", Bulletin des Mus Ees royaux d'art et d'histoure $46 \quad 171-183$

Beven, H G , 1938, 1960 Die Wanddekoration vom zweiten bis zum vierten Stil, I-II, Haag

Beyen H G, 1940 Das chronologische Verhaltnis der letzten drei pompelanischen Stile in Berichte uber den VI. Internationalen Kongress feur Arch \&ologie, Berlin 21-26 Aug. 1939, Berlin, 504-505

Beyen, II G 1951 The Workshops of the "Fourth Stvie" at Pompen and in Its Neighbourhood, 1, in Studia Van Hoorn, Leiden, 53-65

Beyen, H G 1958 Das stylustische und chronologische Verhaltnis der letzten drei pompejanschen Stlen, Antiquity and Survival 2, 349-372

Beven, H G , 1965 Pompelani, Still, in EAA VI, 356-366

Bianchı Bandinelli, R, 1950 Storicitd dell'arte classica, Firenze

Branch1 Bandinell, $\mathrm{R}, 1969$ Roma. L'arte romana nel centro del potere, Mulano

Bianchı Bandinellı, R, 1977 La pittura in Storia e civilt \& dei Greci, 10 La cultura ellenistica; le arti figurative. Mulano, 461-513

Bieber, M , 1917 Die Herkunft des tragischen Kostums, JdI 32, 15-104

Bieber M, 1961a The History of the Greek and Roman Theater, Princeton (2 ed )

Bieber, M, 1961b The Sculpture of the Hellentstic Age, vew York (2 ed)

Bielefeld, E, 1969 Drei Ahroter-Statuen reichen Stils, AntPl 9, 47-64

Blanc, ' $\mathrm{N}, 1983$ Les stucateurs romains lemoignages litteraires, epigraphiques et juridiques, MEFRA $95,859-907$

Blanck H , 1979 Il maripara Eıne Priapstatue in Formello, RM 86, 339-350

Blinkenberg, Chr, 1933 Knidıa. Beitr \&ge zwr Kenntnis der praxitelischen Aphrodite. Kopenhagen

Blumel, C, 1948 Der Hermes eines Praxiteles Baden-Baden

Blumel, C', 1966 Die klassischen griechischen Skulpturen der Staatlichen Museen zu Berlın, Berlın

Borbein, A H , 1968 Campanareliefs, Heidelberg

Borbein A H, 1973 Die griechische Statue des 4 Jahrhunderts v Chr, JdI 88,43212

Borda, M , 1958 La pittura romana. Mulano

Boyce G , 1937 Corpus of the Lararia of Pompell, MemAmAc 14

Boucher, S, 1976 Recherches sur les bronzes figur Cs de Gaule pr S-romaine et romaine, Rome-Paris

Bowersock, G W, 1979 Historical Problems in Late Republican and Augustan Classicism in Classicisme 1979, $57-78$

Braemer, F, 1978 Reflexions sur les sculptures antiques de la Basse lalle de la Seine, in Actes du colloque international d'arch ologie, Rouen, II, 191-215 
Bragantinı, I $M$ de Vos, 1982 Museo Nazionale Romano, II Le pitture, 1 Le decorazioni della villa romana della Farnesina, Roma

Bragantinı, I /F Paise Badonı, 1984 Il quadro pompeiano nel suo contesto decorativo, DArch terza serie 2, 119-130

Brendel, O J , 1953-1954 Der Affen-Aeneas, RM 60-61, 153-159

Breton, E, 1854 Pompl wo, Paris

Brinkerhof, D M 1978 Hellenistic Statues of Aphrodite: Studies in the History of their Stylistic Development, New York-London

Brommer, F, 1978 Hephastos, Mainz

Bruneau, $\mathrm{Ph}^{\prime}, 1982$ L'"Ares Borghese" et l'Ares d'Alcamene ou De l'opinion et du rayonnement, in Rayonnement grec. Hommages $C$. Delvoye, Bruxelles, $177-199$

Bruno, V J, 1969 Antecedents of the First Style, AJA 73, 305-317

Bruno, V J ; 1981 The Panted Metopes at Lefkadia and the Problem of Color in Doric Śculptural Metopes, AJA 85, 3-11

Budde, L , 1965 Imago clipeata des Kaisers Traian in Ankara, AntPl 4, $103-117$

Burr Thompson, D /R L Griswold Garden Lore of Ancient Athens, Princeton $\mathrm{N} \mathrm{J}$

Caglano de Azevedo, M , 1954 La Dea Barberını, RIA n s 3, 108-146

Callmer, Chr, 1944 Antike Bibliotheken, OpArch 3, 145-193

Calza, G. 1940 La necropoli del porto di Roma nellílsola sacra, Roma

Calza, R : 1977 Antichite di Villa Doria Pamphilj, Roma

Calza, R 'M Florianı Squarciapino, 1962 Museo Óstiense, Roma

Caputo, G, 1955 Note sugh edifjci teatralı della Cirenaica, in Scritti di archeologia in onore di Carlo Anti, Furenze, 281-291

Carettoni, G 1983a La decorazione pittorica della Casa di Augusto sul Palatino, RM $90,373-419$

Caretton1, G , 1983b Das Haus des Augustus auf dem Palatin. Manz

Carroll, $\mathrm{Ph} \mathrm{M}$, 1983 Greek Classical and Hellenistic Stone Relief Sculpture with Landscape Motifs, Ann Arbor

Carpenter, R, 1954 Two Postscripts to the Hermes Controversy, AJA 58, $1-12$

Casparı, F , 1916 Das Nilschiff Ptolemaıo' IV, Jdl 31, 1-78

Castiglione, 'L 1975 Zur Plastik von Pompejı' in der fruhkolonialen Zeit, in NF 1975, 21i-224

Castren, P, 1975 Ordo populusque pompeianus. Policy and Society in Roman Pompeii, Roma

CatEssen 1973 Pompeji. Leben und Kunst in den Vesuvstádten, Rechlinghausen Die Numider, Reiter und Koónige nibrdlich der Sahara, $K$ oln-Bonn.

CatLondon $1976 \mathrm{~J}$ Ward-Perkins/A Claridge (eds), Pompeii AD 79, London 1976

CatMlano 1981 I cavalli di San Marco, Mulano 1981

CatRichmond Ancient Arts in the Virginia Museum, Richmond Virg 1973

CatRoma I 1- A Giulıano (ed ), Museo Nazionale Romano, I, Le sculture, Roma 1979-

CatZurich 1974 Pompeji. Leben und Kunst in den Vesuvstädten, Recklinghausen

Cerull Irell, M G , 1969 Ercolano, Cava del Tirrenı-Napolı

Cerul Irell, M G ; 1971 Le pitture della Casa dell'atrio a mosaico, MonPitt III, Ercolano, 1, Roma

Cesuli Ircll, M G . 1982 Il ritratto romano ad Ercolano, in Attı 1979, 697700

Chevalier, $\mathrm{R} / \mathrm{Cl}$ Lemaitre, 1969 Note sur une bague d'Izerneres (Ain), in Hommages \& Marcel Renard, Bruxelles 1969, III, 124-145 
Christou, Chr A., 1968- Potnia theron. Eine Untersuchung Púber Ursprung, Erscheinungsform und Wandlungen der Gestalt einer Gottheit, Thessaloniki.

Ciprott1, P., 1957-1959: Pitture pompeiane medite, RendPontAcc 30-31, $153-160$

Civilta 1983. Civilta dell'antico Egitto in Campania. Per un riordinamento della collezione egiziana del Museo Nazionale di Napoli, Napol

Classicisme 1979. Le Classicisme - Rome aux Iers sidcles avant et apr.?.s J.-C., Entretiens Hardt, XXVI, Geneve

Coarell,' F., 1972. Il complesso pomperano del Campo Marzio e la sua decorazione scultorea, RendPontAc 44, 99-122

Conrell, F, 1974 Guida archeologica di Roma, Verona

Collart, P., 1937 Philippes, ville de Mac!edoine depuis ses origines jusqu'la la fin de l'é poque romaine, Paris.

Comparett1, $D / G$ de Petra, 1883. La villa ercolanese dei Pisoni, Torıno

Conticello, B., 1974. I gruppı scultoreı dı soggetto mitologico a Sperlonga, AntPl i4, 7-59

Cook, R M 1977 The Aberdeen Head and the Hermes of Olympia, in. Festschrift $f^{d}$ er Frank Brommer, Mainz, 77.

Corchia, $R$, 1980. Rulevi con venationes dall'anfiteatro di Lecce. problem e proposte di lettura, in. Studi di antichita. Lecce, 117-204

Corswand, I., 1982 Oscilla. Untersuchungen zu einer rómischen Reliefgattung, Berlin

Cosenza, G., $190 \bar{i}$ Stabia. Studi archeologici, topografici e storici, Trani

Croisllle, J.M, 1965 Les natures mortes campaniennes, Bruxelles

Croislle, J M', 1966 Les toulles archeologiques de Castellamare Stabia: decouvertes recentes, Latomus $25,245-257$.

Croisille, J M, 1969 Stabiana, in. Hommages \& Marcel Renard, Bruxelles, III, $145-170$

Croislle, J Y., 1983 L'art décoratif au temps de NÉron aux Flaviens, Bruxelles

Cumont, F, 1926 Fouilles de Doura-Europos (1922-1923), Parıs.

Cunlilte, B., 1971 Fishbourne. A Roman Palace and its Garden, London

Cunlilfe, B. Roman Gardens in Britain A Review of the Evidence, in: Ancient Gardens 1981, 95-108

Curtius, L. , 1929 Die Wandmalerei Pompejis, Leıpzig.

Curtıus, L., 1947. Interpretationen von sechs griechischen Bildwerken. Bern

Lacos, N, 1969 La df couverte de la Domus Aurea et la formation des grotesques z la Renalssance, London-Leiden

D'Amore, L e a , 1983 Premier rapport sur l'etude de la Villa San Marco a Stabies, MEFRÁ 95, 909-936.

Darling, J K., 1981. Sacro-idyllic Landscape of the Antonine Dynasty in Rome, Ann Arbor

D'Arms, J.H, 1970: Romans on the Bay of Naples, Cambridge Mass.

D'Arms, J.H., 1977 Proprietarı e vلle nel golfo dı Napols, in I Campi flegrei nell'archeologia e nella storia, Roma. 347-363.

D'Arms, J.H., 1979 Ville rustiche e ville dy "otıum", in. Pornpei 79, 65-86.

Darsy, F.M.D., 1968 Recherches arch ologiques a Sainte Sabine sur l'Aventin, Citta del Vaticano.

Davreux, J., 1942 La le gende de la proph!etesse Cassandre, Paris

Dawson, Chŕ M, 1944 Romano-Campanian Mythological Landscape Painting, New Haven

Dawson, Chr M., 1950 Postscript to Yale Classical Studies Vol. IX, YaleCtSt 11 (1950) $297-303$

De Caro, S., 1976 Sculture della Villa di Poppea in Oplontis, CronPomp 2, 184-225.

Dechers, J.G., 1979. Die Wandmalereien im Kaıserkultraum von Luxor, JdI 94, 600-652.

De Franciscıs, A., 1951: Il ritratto romano a Pompei, Napoli. 
De Franciscis, A., 1963: Il Museo Nazionale di Napoli, Napoli.

De Franciscis, A., 1975: La villa romana di Oplontis, in: NF 1975, 9-18.

De Franciscis, A., 1979: Beryllos e la villa "di Poppea" ad Oplontis, in: Studies in Classical Art and Archaeology. A Tribute to P.H. von Blanckenhagen, Locust Valley N.Y., 231-233.

De Franciscis, A.. 1980; La dama di Oplontis, in: Eikones, Studien zum griechischen und rofmischen Bildnis. H. Jucker zum 60. Geburtstag gewidmet, Bern, 11j-117.

Delbrueck, R., 1932: Das spätantike Kaiserornat, Die Antike, 1-21.

Delivorrias, A., 1968: Die Kultstatue der Aphrodite von Daphni, AntPl 8, 19-32.

Delivorrias, A., 1974: Attische Giebelskulpturen und Akrotere des foúnften Jahrhunderts, Tübingen.

Della Corte, M., 1924: Juventus.

Delorme, J., 1960: Gymnasion. Etude sur les monuments consacr $6 s$ a l'Education en Grice, Paris.

Delplace, Chr., 1980: 'Le griffon de l'archa'tsme ta l' Bruxelles-Rome.

Demisch, H., 1977: Die Sphinx, Stuttgart.

Demisch-Quatember, M. 1980: Das Mädchen von Antium, RM 8i, 57-80.

Leubner, O., 1977-1978: Das Heiligtum der alexandrinischen Gottheiten in Pergamon genannt "Kizil Avli" ("Rote Halle"), IstMitt 27-28, 227-250.

De Visscher, F., 1962: Heracles Epitrapezios, Paris.

De Vos, M., 1975: Pitture e mosaico a Solunto, BABesch 50, 195-224.

De Vos, M., 1976: Scavi nuovi sconosciuti (I 9, 13): pitture e pavimenti della Casa di Cerere a Pompei, MededRam 38, 37-75.

De Vos, M., 1977: Primo stile figurato e maturo quarto stile negli scarichi provenienti dalle macerie del terremoto del 62 d.C. a Pompei, MededRom $39,29-47$.

De Vos, M. 1980: L'egittomania in pitture e mosaici romano-campani della prima et 2 imperiale, Leiden.

De Vos, M., 1982: Die Casa di Ganimede in Pompeji VII 13, 4. Pavimenti e pitture, $R M \quad 89,315-352$.

De Vos, M./A. de Vos, 1975: Scavi nuovi sconosciuti (I 11, 14; I 1, 12): pitture memorande di Pompei. Con una tipologia provvisoria dello stile a candelabri, MededRom 37, 47-85.

De Vos, M./A. de Vos, 1979: Die Wanddekorationen der Stabianer Thermen, in: Eschebach 1979, 81-95.

De Vos, M. e.a., 1982: A Painted Oecus from Settefinestre (Tuscany): Excavation, Conservation and Analyses, in: J. Liversidge (ed.), Roman Provincial Wall Painting of the Western Empire, B.A.R. Int. Series 140, London, 1-32.

De Vos, M./A. Martin, 1984: La pittura ellenistica a Pompei in decorazioni scomparse documentate da uno studio dell'architetto A.A. Parland, DArch terza serie 2, 131-140.

De Waele, J.A., 1982: De "Dorische" tempel op het Forum Triangulare te Pompeii, Hermeneus 54, 27-35.

Dexter, C.E., 1975: The Casa di L. Cecilio Giocondo in Pompeii, Ann Arbor 1975 .

Dimitriou, D., 1982: The Polychromy of Greek Sculpture to the Beginning of the Hellenistic Period, Ann Arbor.

Di Vita, A., 1952-1954: Atena Ergane in una terracotta dalla Sicilia e il culto della dea in Atene, ASAtene 30-32, 141-154.

Döhl, H., 1968: Der Eros des Lysipp. Frolhhellenistische Eroten, Göttingen.

Döhl, H., 1976: Plastik aus Pompejt, Göttingen.

Döhl, H., 1982: Pompejanische Plastik zwischen Kunst und Konsum, in: Pompeji 79-1979, Stendal, 66-76.

Döhl, H.,P. Zanker, 1979: La scultura, in: Pompej 79, 177-210. 
Dorig, J , 1957 Lysipps letztes Werk, JdI 72, 19-43

Dorig, J , 1975 Art antıque. Collections privées de Susse Romande, Geneve

Dohrn, T', 1960 Die Tyche von Antiochia, Berlin

Dohrn, T , 1965 Crustae, RM 72, 127-141

Dorigo, W, 1966 Pittura tardoromana, Mulano

Dreihaus, J 1969 Bronzeverkleidungen romischer Statuenbasen aus weissenthurn, Landkreıs Koblenz, BJb 169, 424-436

Drerup. H, 1957 Zum Ausstattungsluxus in der rämischen Architektur, Munster

Drerup, H 1959 Bildraum und Realraum in der romischen Architektur, RM $66,147-174$

Dunbabin, K M D , 1982 The Victorian Charioteer on Mosaics and Related Monuments AJA 86, 65-89

Dwyer, E J . 1981 Pomperan Osclla Collectıons, KM 89 247-306

Dwver, E J , 1982 Pompeian Domestic Sculpture. A Study of five Pompeian Houses and their Contents, Rome

Ellich, W, 1953 Bild und Rahmen im Altertwn Leipzig

Ehrhardt, $W, 1977$ Die Entwicklung der $r$ dgmischen Wandmalerei von der spden Republik bis zur Zeit Neros, Bonn (diss dattloscritta)

Ehrhardt, W , 1982 recensione Bastet 'De Vos 1979, Gnomon 54, 577-588

Elchler, F, 1953 Forschungen in Ephesos, VI, Die Bibliothek, wien

El Fahharan, F, 1975 Das Theater von imman in Jordanien, AA, 377-403

Elıa, $O, 1931$ L'ipogeo di Calvano, MonAnt 34, 421-492

Elıa, O , 1932 Pitture romane nel Museo Nazionale di Napoli, Vapolı

Elia, O; 1938 I conogratid aulica in pitture stabiane, BdA 23, 101-114

Elıa, O , 1941 Le pitture del Tempio di Isıde, MonPitt III, Pompei 3-4, Roma

Elıa, O , 1947 Le pitture della Casa del citarista, MonPıtt III, Pompel 5, Roma

Elıa, O , 1957 Pitture di Stabia. Napols

Elia, O 1974 Vota sul IIl Stle pompelano, RendAccNapoli 49, 155-166

Elia, O , 1975 La scultura pompeiana in tufo. CronPomp 1, 118-143

Lnea 1981 Enea nel Lazio. Archeologia e mito, Roma

Engernann, J, 1967 Architekturdarstellungen des frolhen zweiten Stils. Heidclberg

Erım, K T , 1974 The Satyr and Young Dionysus Group from Aphrodisıas, in Mansel' e Armangan, Ankara, II, $767-775$

Eristov, H , 1979 Corpus des faux-marbres peints a Pompe1, MEFRA 91, $693-771$

Eschebach, H , 1979، Die Stabianer Thermen in Pompeji. Berlm

Eschebach, H , 1979b Probleme der Wasserversorgung Pompejs, CronPomp 5 , $24-60$

Fabbricottı, E, 1976 I bagnı nelle prime ville romane, CronPomp 2, 29-111.

Fabbrinı, L, 1983 Domus Aurea una nuova lettura planmetrica del palazzo sul colle Oppio, in Citt $\mathrm{e}$ architettura nella Roma imperiale, X Suppl AnalRom, Roma, 169-186

Fears, J R , 1981 The Theology of Victory at Rome. Approaches and Problems, in ANRW II $172,736-826$

Fellettı Maj, B M, 1961 Le pitture della Casa di Giove e Ganimede, MonPitt III, Ostia 1, Roma

Fellett, Maı, B M, 1977 La tradizione italica nell'arte romana, Roma

Fellettı Maj, B M /P Moreno, 1967. Le pitture della Casa delle Muse, MonPitt III, Ostia 3, Roma

Ferrua, A , 1979 La basilica e le catacombe di San Sebastiano, Roma

Fiorellı, G', 1875 Descrizione di Pompei, Napolı

Fittschen K, 1976 Zur Herkunft und Entstehung des 2 Stuls - Probleme und Argumente, in $\mathrm{P}$ Zanker (ed), Hellenismus in Mittelitalien, Gottingen, $539-563$ 
Fittschen, K., 1977 Katalog der antiken Skulpturen in Schloss Erbach, Berlin

Flagge, I., 1975: Untersuchungen zur Bedeutung des Greifen, Sankt Augustin.

Fleischer, $R$, 1973. Artemis von Ephesos und vemwandte Kultstatuen aus Anatolien und Syrien, Lesden

Fleıscher, R., 1983 Neues zu klemasiatıschen Kultstatuen, AA, 81-93.

Floren, J., $1981 \mathrm{Zu}$ Lysipps Statuen des sitzenden Herakles, Boreas 4, $47-60$

Froning, H 1980 Die lkonographische Tradition der kaiserzeitlichen mythologischen Sarkophagreliets, JdI 95, 322-341.

Froning, H., 1981 Marmor-Schmuckreliefs mit griechischen Mythen im 1. Jh. v. Chr. - Umtauschungen zur Chronologie und Funktion, Mann.

Frova, A., 1965 La Villa del Misteri a Pompei, Mulano-Ginevra.

Fuchs, G, 1954. Zur Wanddekoration der Casa del Bell'Impluvio in Pompej1, $A A, 10-29$.

Fuchs, Michaela, 1982. Eine Musengruppe aus dem Pompeius-Theater, $R M$ 89, 69-80

Fuchs, Michel, 1984 La Sulsse, Archeologia Paris Dossier 89, 61-68

Fuchs, W., 1958 Der Dornauszieher, Bremen

Fuchs, W., 1959 Die Vorbilder der Neuattischen Reliefs, Berlun.

Fuchs, W., 1963 Der Schiffsfund von Mahdia, Tubingen

Fuchs, W., 1979 Die Skulptur der Griechen, Munchen (2 ed ).

Fuhrmann, H, 1931 Philoxenos von Eretria, Gottingen

Gabelmann, H', 1977 Zur Tektonik oberitalischen Sarkophage, Altare und Stelen, BJb 177, 199-244.

Gabriel, M.M. . 1951 Masters of Campanian Painting, New York

Gabriel, M M. 1955 Livias Garden Room at Prima Porta, New York

Gallina, A., 1961 Le pitture con paesagg1 dell'Odissea, Studi Miscellanei 6

Garcia y Bellido, A , 1969 Der bronzene Mellephebe von Antequera, AntPl 9, $73-78$

Gard, J -M , 1974 L'Ilermes juvenule du Palais 11 d'Eretrie AntK 17, 50-59.

Ghall-Kahl, L B $195 j$ Les enl vements et le retour d'H. Giane dans les textes et les documents figur Es, Paris.

Gloure, E, 1978 Ho krateras tou Derbeniou, Athenal.

Gigante, M' 1979 Civilta delle forme letterarie nell'antica Pompei, Napoli

Giordano, C /G V Pelagall, 1957 Canı e canll nella antica Pompei, Atti dell'Accademia Pontiana, in s VII

Gnolı, R., 1971 Marmora romana, Roma.

Gottlicher, J , 1981 Nautische Attribute \& $^{\prime}$ mischer Gotheiten, Bremen

Grumal, P., 1943 Les jardins romains ta la fin de la $r$ e publique et aux deux premiers si đ̀ cles de l'Empire. Essay sur le naturalisme romain, Parıs.

Grimal, P., 1969 Les jordins romains, Paris (2 ed revue).

Guadagno,' G , 1983. Herculanensium Augustalium Aedes, CronErcol 13, 159-173.

Gualandı, G, 1969. Artemı-Hekate Un problema di tıpologia nella scultura ellenistıca, RA, 233-272

Gualand,, G., 1976 Sculture di Rodi, ASAtene 54, n.s 38, 7-259.

Gusman, $P$ ' 1899 . Pomp ối, Paj's

Hadaczek, K', 1907 Marsyas, JJh 10, 312-326.

Hantmann, G', 1951 The Season Sarcophagus in Dumbarton Oaks, Cambridge Mass

Harl-Schaller, F., 1976-1977: Figurlıche Gerat- und Mobelfbdse, OJh 51, 45-56

Harrison, E B., 1965 The Athenian Agora, XI. Archaic and Archaistic Sculpture, Princeton N.J 
Haubner, D M, 1971 Die Tracht des Gottes Dionysos in der grlechischen Kunst, Wien (diss dattiloscritta).

Heidenreich, R, 1966 Zur Tyche des Eutychides, in $M$ langes offerts $\pm a K$.

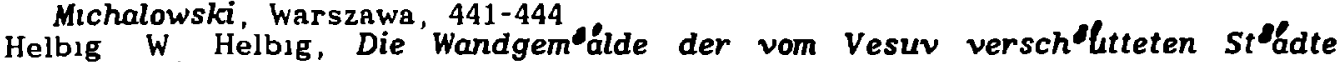
Campaniens, Leipzig 1868

Helbig " W Helbig, Fuhrer durch die offentlichen Sammlungen klassischer Altert umer Roms, 4 Aufl hrsg von $\mathrm{H}$ Speier, Tubingen 1963-1972

Herbig, R, 1949 Pan, Frankfurt

Herbig, R, 1962 Nugae Pompeianorum. Unbekannte Wandmalereien des dritten pompejanischen Stils, Tubıngen

Herrmann. P , I-II Denkm Aler der Malerei des Altertums, Munchen 1906-1950

IIıggins. R A 1954 Catalogue of the Terracottas in the Department of Greek and Roman Antiquities, Britısh Museum I, London

Hull, D K , 1981 Some Sculpture trom Roman Domestıc Gardens, in Ancient Gardens $1981,81-94$

Hinks, R P, 1933 Catalogue of the Greek, Etruscan and Roman Paintings and Mosaics in the Britısh Musewn, London

Holscher, T, 1968 Victoria Romana, Mainz

Holscher, T ; 1970 Die Victoria von Brescia, AntPl 10, 67-80

Horı, M, 1979 Dea Syria. Studien zur religiosen Tradition der Fruchtbakeitsg ottin in Vorderasien, Kevelaer

Hornbostel-Huttner, G，1979 Studien zur romischen Nischenarchitektwr, Leiden

Horster, G , 1970 Statuen auf Gemmen, Bonn

Howard, S, 1966 The Lansdowne Heracles, Malibu

Iacopı, G , 1963 L'antro di Tiberio a Sperlonga, Roma

Impegno 1983 Un impegno per Pompei, Mulano

Inan, J , 1973 Roman Copies of some Famous Greek Statues from Side, AntPl $12,69-79$

Ippel, A, 1939 Statuetten aus Pompej1, AA, 350-376

Ippel, A , 1951 Figurata sumlitudo, in G E Mylonas (ed ), Studies Presented to David Moore Robinson, Saint Lou1s Missouri, I, 808-812

Jacob-Felsch, M , 1969 Die Entwicklung griechischer Statuenbasen und die Aufstellung der Statuen, Waldsassen

Jashemsk1, W F 1979 The Gardens of Pompeii, Herculaneum and the Villas Destroyed by Vesuvius. New Rochelle

Jashemskı, W F , 1981 The Campanian Peristyle Garden, in Ancient Gardens $1981,29-48$

Jeppesen, K, 1979 Where was the So-Called Erechtheion, AJA 83, 381-394

Jeppesen, $K, 1983$ Further Inquiries on the Location of the Erechtheion and its Relationship to the Temple of the Polias I Prostomaion and Prostomion, AJA 87, 325-333

Johnson, F P, 1927 Lysippos, Durham

Johnston, D E , 1982 Some Mosaics and Murals in Roman Tripolitana, in $J$ Liversidge, (ed ), Roman Provincial Wall Painting of the Western Empire, B A R Int Serjes 140, 193-208

Joly. D , 1965 Quelques aspects de la mosarque parietale au Ier siecle de notre ere d'apres trois monuments pompeiens, in $L a$ mosa ique gr!eco-romaine, Parıs, 57-71

Joyce, H, 1981 The Decoration of Walls, Ceilings and Floors in Italy in the Second and Third Centuries A.D. . Roma

Joyce H, 1983 The Ancient Frescoes from the Villa Negronl and their Influence in the Eighteenth and Nineteenth Centuries, ArtB 65, 423-440

Jucker, $H, 1950$ Vom Verh ditnis der $R$ omer zur Bildenden Kunst bei den Griechen, Frankfurt a M 


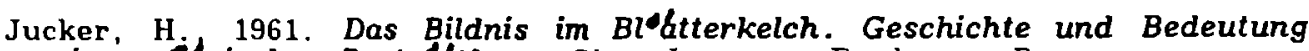
einer romischen Portrdtform, Olten-Lausanne-Fresburg $1 \mathrm{Br}$

Jucker, I., 1956. Der Gestus des Aposkopein. Ein Beitrag zur Geb'brdesprache in der antiken Kunst, Zurich

Kampen, N.B , 1979: The Ancient Use of the Spada Reliefs, AntCl 48, $583-600$.

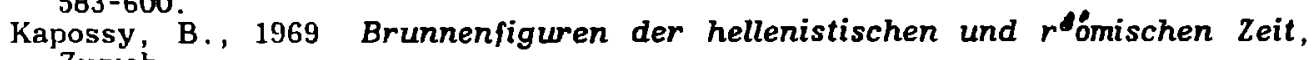
Zurich.

Karusu, S , 1974. Neues zur Aphrodite "Frejus", AM 89, 151-172.

Karwiese, S., 1967 Attis, jn der antiken Kunst, Wien.

Kaster, $G$, 1974' Die G\&rten des Lucullus. Entwicklung und Bedeutung der Bebauung des Pincio-Hels $H^{*}$ in Rom, Munchen.

Kater-Sibbes, G J F,M Vermaseren, 1975: Apis, II. Monuments from Outside Egypt, Leiden.

kautmann-Heinmann, A., 1977: Die rómischen Bronzen der Schweiz, I. Augst, Mainz.

Kellner, H-J, 1974. Drej Grazıen aus Bayern, in: Festschrift fofr Joachim Werner zum 65. Geburtstag. Munchen, I, 191-196

kelsey, F.W., 1908 Codrus's Chiron and a Painting from Herculaneum, AJA $12,30-38$.

Kemp-Lindemann, D, 1975 Darstellungen des Achilleus in griechischer und $r$ ómischer Kunst, 'Bern-Frankturt

Kenner, H. 1966. in R. Egger, Die Ausgrabungen aut dem Magdalensberg 1962 bis 1964, Carinthia 1, 156, 435-447.

Kenner, H 1967 Wandmalerejen augusteischer Zeit vom Magdalensberg in Karnten, Gymnasium 74, 437-444

Kenner, H., 1970 Romische Fresken vom Magdalensberg, AW 1, Heft 3, $42-46$

Kenner. H., 1976: Romische Fresken vom Magdalensberg, II, AW 7, Heft 4, $21-27$

Kolendo, J 1982: Le port d'Alexandrie sur une peınture de Gragnano?, Latomus 41, 305-311.

Kleın, W., 1910. Zum Grundproblem der pompejanischen Malereı, ${ }^{8} \mathrm{Jh} 13$, 123-149.

Klein, W., 1919. Pompejanısche Bulderstudien, II. OfJh 22, 268-295.

Koch, G./H. Sichtermann, 1982 - $R \cdot$ ofmische Sorkophage, Munchen.

Krahmer, G , 1927. Die einansichtige Gruppen und die spathellepistische Kunst, Nachrichten von der Gesellschaft der Wissenschaften zu Gdottingen. Phil. Hist. Klasse, 53-92.

Kraus, Th., 1960 Hekate, Heidelberg.

Kreeb, M., 1978: Zur statuarischen Ausstattung delischer Privathauser (Zusammenfassung), in: Wohnungbau im Altertum, DiskAB 3, Berlin, $145-146$.

Kreeb, M., 1980. Fig Urliche Ausstattung sp äthellenistischer H\&user, Munchen (diss. dattuloscritta)

Kreeb, M., 1984 Studien zur figurlichen Ausstattung delischer Privathauser, $B C H$ 107, 317-343.

Krieger, H, 1919 Dehorative Wandgemalde aus dem 2 Jahrhundert n.Chr., RM 34, 24-52

Kron, U., 1977 Eine Pandion-Statue in Rom. Mit einem Exkurs zu Inschriften auf Plinthen, JdI 92, 139-168.

Kunzl, E., 1968 Fro thhellenistische Gruppen, Koln.

Kunzl, E., 1970 Venus vor dem Bade - ein Neufund aus der Colonia Ulpra Tralana und Bemerkungen zum Typus der "sandalenlosenden Aphrodite", $B J b 70,102-162$.

Lacrolx, L , 1949 Les reproductions de statues sur les monnaies grecques. La statuaire archaique et classique, Liege. 
Lafon, X., 1981: A propos des "villae" républicaines. Quelques notes sur les programmes decoratils et les commanditaires, in: Art 1981, 151-172.

Lahusen, G., 1978: Goldene und vergoldete romische Ehrenstatuen, $R M$ 85, $385-396$.

Laidlaw, A., 1975: Reconstruction of the First Style Decorations in the Alexander Exedra in the House of the Faun, in: NE 1975, 39-52.

Laidlaw, A., 1976: Reconstructions of the First Style Decorations in the House of Sallust in Pompeii, in: In memoriam Otto Brendel, Mainz, 105-114.

Langenskiold. E., 1930. Torso di Belvedere, ActaArch 1, 121-146.

Langlotz, E., 1968: Beobachtungen uber die antike Ganosis, AA, 470-474.

Langlotz, E., 1969: Beobachtungen über die antike Ganosis, AA, 231.

La Rocca, E., 1984: Fabio o Fannio. L'affiresco medio-repubblicana del'Esquilino come riflesso dell'arte "rappresentativa" e come espressione di mobilità sociale, DArch terza serie 2, 31-53.

La Rocca, E./M. de Vos/A. de Vos: Guida archeologica di Pompei, Verona.

Lattimore, S., 1976: The Marine Thiasos in Greek Sculptwre, Los Angeles.

Laubscher, H.P., 1982: Fischer und Landleute. Studien zur hellenistischen Genreplastik, Mainz.

Lauter, H., 1967: Eine seitenverkehrte Kopie des Apoxyomenos, BJb 167, 119-128.

Lauter, H., 1971: Ptolemais in Lybien. Ein Beitrag zur Baukunst Alexandrias, JdI $86,149-178$.

Lauter, H., 1973: Das hellenistische Sbdtor von Perge, BJb 173, 1-11.

Lauter, H., 1979: Bemerkungen zur spathellenistischen Baukunst in Mittclitalien, JdI $94,390-459$.

Lauter, H., 1983: Kunstliche Unfertigkeit: Hellenistische Bossensaulen, JdI $98,287-310$.

Lauter-Bufe, H., 1969: Zur Stilgeschichte der figُülichen pompejanischen Fresken, Berlin.

Lauter-Bufe, H., 1975: Zur architektonischen Gartengestaltung in Pompeji und Herkulaneum, in: NF 1975, 169-178.

Leach, E.W., 1980: Sacral-Idyllic Landscape Painting and the Poems of Tibullus' First Book, Latomus 39, 47-69.

Leach, E.W., 1981: Metamorphoses of the Actaeon Myth in Campanian Painting, $R M$ B8, 307-327.

Leach, E.W., 1982: Patron, Painters and Patterns. The Anonymity of Romano-Campanian Painting and the Transition of the Second to the Third Style, in: Literary and Artistic Patronage in Ancient Rome, Austin, $135-173$.

Le Corsu, F., 1967: Un oratoire pompéien consacré à Dionysos, RA, 239-254.

Lehmann, Ph.W., 1953: Roman Wall Paintings from Boscoreale in the Metropolitan Musewn of Art, Cambridge Mass.

Lehmann, Ph.W., 1979: Lefkadia and the Second Style, in: Studies in Classical Art and Archaeology. A Tribute to P.H. von Blanckenhagen, Locust Valley N.Y., 225-29.

Leibundgut, A., 1984: Der "Trajan" von Ottenhusen. Eine neronische Privatapotheose und ihre Beziehungen zum Merkur des Zenodorus, JdI 99, 257-289.

Levi, D., 1947: Antioch Mosaic Pavements, Princeton N.J.

Libertini, G., 1927: Centuripe, Catania.

Linfert, A., 1969: Der Meister der kauernden Aphrodite. AM 84, 158-164.

Linfert, A. 1978: Kunstzentren hellenistischer Zeit. Studien an weiblichen Gewandfiguren, wiesbaden.

Linfert, A., 1982: Athenen des Phidias, AM 97, 57-77.

Ling, R., 1971: 'Cylinder' in Campanian Art, Antiquaries Journal 51, 267-280.

Ling, R., 1977: Studius and the Beginning of Koman Landscape Painting, JRS $67,1-16$.

Lippold, G., 1923: Kopien und Umbildungen griechischer Statuen, München. 
Lıpold, G, 1936-1956: Die Skulpturen des Vaticanischen Musewns, III 1-2, Berlin.

Lippold, G , 1950 Die griechische Plastik, ItdA III 1, Munchen

Lippold, G., 1951 Antıke Gemaldekopien, Abhandlungen der Bayerischen Akedemie von Wissenschaften phil.-hist. Klasse, N F 33.

Little, A.M.G., 1956 A Roman Source Book for the Stage, AJA 60, 27-33.

Little, A.M G., 1964: A Series of Notes in Four Parts on Campanian Megalography, AJA 68, 390-395.

Lohmann, H . 1979 Grabm dler auf unteritalischen Vasen, Berlin.

Lorenz, Th 1965 Galerien von griechischen Philosophen- und Dichterbildnissen bei den $R$ ofmern, Manz

Love, I , 1970 Excavations at Knidos 1969, AJA 74, 149-155.

Lulles, R., 1931 Die Typen der griechischen Hermen, Konigsberg Pr

Lullies, R., 1954. Die kauernde Aphrodite, Munchen

Lullies, R./M. Hirmer, 1979 Griechische Plastik, Munchen (4 Aufl.).

Lunsingh Scheurleer. R A, 1979. Elephants in Faience. BABesch 54, 99-110.

Lyttelton, M . 1974 Baroque Architecture in Classical Antiquity, London.

Maaskant-Kleibrink, M, 1982. Five Greek Shallow Reliefs, BABesch 57, $12-24$

MacDermott, W C., 1938. The Ape in Antiquity, Baltumore.

Maluri, A., 1931: La Villa dei Misteri, Roma.

Maluri, A., 1933 La Casa del Menandro e il suo tesoro di argenteria, Roma

Maluri, A., 1938. Le pitture delle Casa di M. Fabius Amandio, del Sacerdos Amandus e di P. Cornelius Teges, MonPitt III, Pompe1 2, Roma

Malurı, A., 1940 Picturae lignels formis inclusae, Reñdiconti della Reale Accademia d'ltalia serie VII, $1,138-160$

Maıurı, A., 1942. L'ultima fase edilizia di Pompei, Roma

Masuri, A, 1953 La peinture romaine, Geneve.

Malur,, A., 1955. Nascita e ratto delle "nove Muse" a Pompei, Napoli 80, T-8.

Malur1, A., 1958 Ercolano. I nuovi scavi (1927-1958), I, Roma

Maiur, A / $\mathrm{R}$ Pane, 1947. La Casa di Loreio Tiburtino e la Villa di Diomede in Pompei, Roma

Malurı, B., 1950. Ludı ginnico-atletici a Pompel, in Pompeiana, Napoli, $167-205$.

Malaise, M , 1972: Inventaire préliminaire des documents legyptiens, d Écouverts en Italie. Leiden.

Malandrıno, C. , 1978 Oplontis, Napolı (2 ed.)

Manderscheid, H., 1981: Die Skulpturenausstattung der kaiserzeitlichen Thermenanlagen, Berlin.

Mannı, M, 1974 Le pitture della Casa del Colonnato Tuscanico, MonPitt III, Ercolano 2, Roma.

Mansuell, G A , 1958 Galleria degli Ufizi. Le sculture, I, Roma.

Marabinı Moeus,' M.T. 1981 Le Muse de Ambracia, BArte 66, 1-58.

Marcade, J., 1969. Au Mus 6 e de DElos, Paris

Marconı, P., 1924. Gruppi erotici dell'ellenismo neı Museı di Roma, BullCom $51,225-298$.

Mariemont 1952: Les antiguit és cgyptiens, grecques, 8 trusques, romaines et gallo-romaines du Mus e de Mariemont, Bruxelles.

Mariemont 1966: Tr!esors inconnus du Mus be de Mariemont, I. Rome, ses origines et son empire, Marsemont.

Martıni. W., 1969: Lehrer und Schuler (zur Achill und Chron Gruppe), in: Opus nobile. Festschrift f Ur U. Jantzen, Wiesbaden, 105-108.

Marvin. M., 1983: Freestanding Sculpture from the Baths of Caracalla, AJA $87,347-384$.

Mau, A., 1882, Geschichte der decorativen Wandmalerei, Lelpzig.

Mau, A.. 1908: Pompeji in Leben und Kunst, Lespzig

Mau, A./A. Ippel, 1928. F uhrer dwch Pompeji. Leipzig (6 ed.).

Mazols, F., 1812-1838 Les ruines de Pomp Éi, Parıs. 
Mellor, $\mathrm{R}, 1981$ The Goddess Roma, ANRW II 17.2, 950-1030

Menzel, V., 1966 Die roómischen Bronzen aus Deutschland, 2. Trier, Manz

Merker, G S , 1973 The Hellenistic Sculpture of Rhodes, Goteborg

Meyboom, P G P , 1984 Fabullus demasque, in Tuin 1984, 31-39

Michel, D , 1980 'Pompejanische Gartenmaleresen, in 'Tainia. Festschrift $R$. Hampe, Manz, 373-404

Miclsch, $H, 1971 \quad R$ Omische Architekturterrakotten und Wandmalereien im Akademischen Kunstmuseum Bonn. Berlın

Mielsch, H., $1975 R$ Ormische Stuckeliefs, Heidelberg

Mielsch, H., 1978 Zur stadtromischen Malerei des 4. Jahrhunderts n Chr., RM 85, 151-207

Mielsch, H, 1979a Die Stukkaturen der Stabianer Thermen, in Eschebach $1979,74-80$

Mielsch, H., 1979b Zur Deutung und Datierung der Knochelspielerinnen des Alexandros, $R M$ 86, 233-248

Mielsch, $H, 1981$ Funde und Forschungen zur wandmalerel der Prinzipatszeit von 1945 bis 1975, mit einem Nachtrag 1980, ANRW II 12 2, 157-264

Mirrı, L /G Carlett1, 1776 Le antiche camere delle terme di Tito delineate da Lod. Mirri, Roma

Moeller, W O, 1976 The Wool Trade of Ancient Pompeii, Leiden

Moormann, EM , 1983a Sulle pitture della Herculanensium Augustalium Aedes, CronErcol 13, 175-177

Moormann, E M, 1983b Rappresentazionı teatralı su scaenae frontes di quarto stıle a Pompel, Pompeii, Herculaneum, Stabiae 1, 73-117.

Moormann, E M , 1984a Een beeld $v$ an een tuin en andere fragmenten, in Tuin 1984, 57-64

Moormann, C M , 1984b Le pitture della Villa deı papirı ad Crcolano, in Atti del XVII Congresso internazionale di papirologia, Napolı, 637-674

Moormann, E M , 1985 Un fior di giardino ed altri framment,, CronErcol 15 (in stampa).

Moreau, J 1960 Das Trierer Kornmarktmosaik, Koln

Morel, J-M , 1975 L'Ilioupersis dans la ct ramique italiote. Les mythes et leur expressions figur Zes au IVe sitecle, Rome.

Moreno, $P, 1976$ Una cretula di Cirene ed l Posidone del Laterano, QuadAlibia 8, 81-98

Moreno P. 1977. Da Lisıppo alla Scuola di Rodı, in Storia e civilt $\pm a$ dei Greci, 10. La cultura ellenistica; le artı figurative, M1lano, 412-460.

Moreno, P 1978 An Alexandrine Bronze Statuette and Lysippos' Heracles in Tarentum, Meddelelser fra Ny Carlsberg Glyptotek 35, 92-96

Moreno, P , 1978-1980 Il bronzo Getty ed una statuetta di Eracle al Muse1 Vaticani, RendPontAcc 51-52, 69-89

Moreno, P, 1981 Modellı lisippel nell'arte decorativa di eta repubblicana ed augustea, in Art 1981, 173-227

Moreno, P, 1982 Il Farnese ritrovato ed altri tipı di Eracle in riposo, MEFRA 94, 379-526

Moreno, P , 1983-1984 Opere di Lisippo, RINASA s III, vol 6-7, 13-70

Moreno, $P$ ', 1984 lconografia lisıppea delle imprese di Eracle, MEFRA 96, 117-174

Muller, H W, 1971 Il culto di Iside nell'antica Benevento. Benevento.

Muthmann, F', 1975 Mutter und Quelle. Studien zur Quellenverehrung im Altertion und im Mittelalter, Basel-Mainz

Nash, E, 1961 Bildlexikon zur Topographie des antiken Rom. I, Tubingen

Naumann, F . 1983 Die Ikonographe der Kybele in der phrygischen und der griechischen Kunst, Tubingen

Neuerburg, $N, 1965$ L'architettura delle fontane e dei ninfei nell'Italia antica, Napoli 
Neutsch, B , 1939 Der Maler Nikias von Athen. Ein Beitrag zur griechischen Kunstgeschichte und zwr pompejanischen Wandmalerei, Borna-Leipzıg

Neutsch, B., 1955 Das Epigrammenzummer in der 'Casa degli epigrammi' zu Poinpejı und sem handbild 'Eros in Ringkampf mit Pan'. JdI 70, 15j-184

veutsch, B.'E. Walde, 1978: Das Achlleusrelief von Maria Saal und seine ıhonographische Verbindung zur Magna Graecia, in: Classica et provincialia. Festschrift Erna Diez, Graz, 141-149.

NF 1975: B Andreae/H. Kyrieless (edd.), Neue Forschungen in Pompeji, Recklinghausen.

Nıccolını, F./F. Niccolıni, 1854-1896: Le case $e$ i monumenti di Pompei designati e descritti, Napolı.

Nicholls, R., 1982 The Drunken Herakles. A New Angle on an Unstable Subject, Hesperia 51, 321-328.

Niemeyer, H.G., 1960: Promachos. Untersuchungen zur Darstellung der bewaffneten Athena in archaischer Zeit, Waldsassen.

Nusson, M.P.. 1967 Geschichte der griechischen Religion, I, 3. ed., Munchen.

Noack, F./K Lehmann-Hartleben: Baugeschichtliche Untersuchungen am Stadtrand von Pompeji. Berlin-Leipzig

Nogara, B , 1907 Le Nozze Aldobrandini, i paesaggi con scene dell'Odissea $e$ le altre pitture murali antiche conservate nella Biblioteca Vaticana $e$ nei Musei Pontefici, Mllano.

Ostrow, S.E., 1979 The Topography of Puteoli and Baiae on the Eight Glass Flasks, Puteoli $3,7 \mathrm{i}-140$

Overbeck, J., 1887-1889: Griechische Kunstmythologie, III 5, Apollon, Leipzig.

Overbeck, J./A. Mau, 1884: Pompeji in seinen Gebóduden und Alterth úmern,

Paar, I., 1962 Ovid und die mythologischen Landschaftsbilder der $r$ ómischen Wandmalerei, hien (diss. dattloscritta).

Pacher, J., 1978. Inns at Pompen. a Short Survey, CronPomp 4, 5-53.

Paduano Faedo, L. . 1981. I sarcofagi romani con Muse, ANRW II 12 2, 65-155.

Paller, J.-M., 1982. Les oscilla retrouves. Du recueil des documents a une theorie d'ensemble, MEFRA 94, 743-822.

Palma, B., 1974 Il sarcofago attico di tiaso di fanciulli dall'Isola sacra. Ostıa.

Pandermalis. D., 1971 Zum Programm der Statuenausstattung in der Villa dei Papirı, AM 86, 173-209.

Panella, C.. 1966-1967 lconografia delle Muse suı sarcofagı romani, Studi Miscellanei $12,11-42$

Panella, C., 1968: Osservazıoni al Corpus dı sarcofagi con Muse di Max Wegner, ArchCl 20,327-351

Pape, M., 1975: Griechische Kunstwerke aus Kriegsbeute und ihre Ooffentliche Aufstellung in Rom von der Eroberung von Syrakus bis in augusteische Zeit, Hamburg.

Parlbenı, E., 19j9- Catalogo delle sculture di Cirene, Roma.

Parlasca, K., 1963: Das pergamenische Taubenmosaik un der sogenannte Nestor-Becher, JdI 78, 256-293.

Parlasca, K., 1977. Iphigenle in Tauris, in Festschrift fón F. Brommer. Mainz, 231-236.

Parlasca, K., 1984: Die Stadtgottin Palmyras, BJb 184, 16i-176.

Pavlovskis, Z., 1973 Man in Artificial Landscape. Leiden.

Pehary, T., 1968 Goldene Statuen der Kalserzeit, $R M 75,144-148$.

Pellegrino, A., 1984. Itinerari ostiensi, $V$, Le necropoli di Ostia sacra e Porto, Ostia.

Perdrizet, P. 1930 : L"Incantada" de Salonique, MonPiot 31, 51-90.

Perhins, A., 1973. The Art of Dura-Europos, Oxford. 
Pernuce, E, 1932 Hellenistische Tische, Zisternenm lundungen, Beckenunterst útze. Alt obre und Truhen, Berlun-Leipzig

Peschlow-Bindokat, 4, 1975 Zur Saulenstrasze ion Pompesopolis in Kuykien, IstMitt 25, 373-391

Peters, W J T , 1963 Landscape in Romano-Campanian Mural Painting, Assen

Peters, W J T , 1965-1966 Mural Painting Fragments Found in the Koman Castra at Nijmegen, BerRijksdOudhBod 15-16, 113-144

Peters, W J Th, 1971 Van Prima Porta naar Pompeii, Alimegen

Peters, W J Th, 1977 La composizıone delle paretı dipinte nella Casd deı Vett 1 a Pompe1, MededRom 39, 95-128

Peters, W J Th. 1979 Mural Painting Fragments Found in the Legionary Fortress and the Canabae Legionis at Nymegen, BerRijksdOudhBod 29, 373-402

Peters. W J Th, 1982a La composizione delle pitture parıetalı dı IV Stıle a Roma e in Campania, in Att 1 1982, 635-659

Peters, W J Th, 1982b Die Landschaftsbulder in den Wand- und Deckenmalereien der Domus Aurea, BABesch 57, 52-69

Peters, W J Th ./P G P Meyboom, 1982 The Roots of Provincial Roman Painting Results of Current Research in Nero's Domus Aurea. in J Liversıdge (ed) Roman Provincıal Wall Painting of the Western Empire, B A R Int Series 140 London, 33-74

Petrillo Serafin, Y, 1982 La pletas di Enea Due monete a confronto, BdA 67 no $13,35-38$

Petsas, Ph M, 1966 Ho taphgs ton Lefkadion, Athenal

Ptretschner, A, 1977 Gebtterstatuen auf pompejanischen Wandgem blden, Innsbruck (diss dattuloscritta)

Philippart, H , 1925 Iconographie de l'"Iphigenie en Tauride" d'Euripide, Revue Belge de philologie et d'histoire 4, 5-33

Phillips. K M , 1968 Perseus and Andromeda, AJA 72, 1-23

Picald, $\mathrm{Ch}$, 1959 Pouzzoles et le paysage portuaire, Latomus 18, 23-51

Picard, G-Ch , 1977 Origine et signitication des fresques architectoniques romano-campaniennnes dites de Second Style, RA, 231-252

Pinkwart D, 1965 Das Relief des Archelaos von Priene und die "Musen des Philiskos", Kallmunz

Pinkwart, D, 1967 Die Musenbasis von Halıkarnass London, Br M 1106, AntPl 6, 89-94

Pinkwart, D, 1972 Drel spathellenıstısche Bronzen vom Burgberg in Pergamon, in Pergamon. Gesammelte Aufs átze (=PF I). Berlin, 115-139

Plommer. $W H, 1979$ Vitruvius and the Origin of Caryatids, JHS 99, 97-102

Pompes 79 F Zevı (ed ). Pompei 79, Napolı 1979

Pomper 1748-1980 Pompei 1748-1980. I templ della documentazione, Roma 1981

Ponce, M , 1789 Arabesques antiques des Bains de Livie, et de la Ville Adrienne, avec les plafonds de la Ville-Madome peints d'apr 2 s les dessins de Raphael, Paris

Preisshofen, F, 1979 Kunsttheorie und Kunstbetrachtung, in: Classicisme $1979,263-282$

Preısshofen, F.'P Zanker, 1970-1971. Reflex einer eklehtischen hunstanschauung beim Auctor ad Herennium, DArch 4-5, 100-119

Presuhn, E, 1878 Pompeji. Die neuesten Ausgrabungen von 1874 bis 1878 , Leipzig

Puccı, G . 1981 La ceramica aretina "Imagerı" e correntı artistıche, in Art $1981,101-119$

Raeder, J, 1978 Eine klassizıstische Frauenfigur in Rom Zur Arbeitsweise hlassizistischer kunstler des 1 Jahrhunderts $v \mathrm{Chr}$ und der mittleren Kasserzelt, JdI 93, 252-276

Raeder, J , 1983 Die statuarische Ausstattung der Villa Hadriana bei Tivoli, Frankfurt-Bern

Ragghıantı, C, 1963 Pittori di Pompei, Muano 
Rakob, F, 1969 Das romsche Quellhelligtum bei Zaghouan in Tunesien, AA, 284-300

Repertor1o 1981, 1983 Repertorio delle fotografie del Gabinetto Fotografico Nazionale. Pitture e pavmenti di Pompei, I-1I, Roma

Reutersward, P 1960 Studien zur Polychromie der Plastik. Griechenland und Rom, Stockholm

Riad, H, 1964 Tomb Paintings trom the Necropolis of Alexandria, Archaeology 17, 169-172

Riad, H, 1967 Quatre tombes de la necropole ouest d'Alexandrie, BArchAlex $42,89-96$

Richardson, L J , 1955 Pompen. the Casa del Dioscurı and its Painters, MemAmAc 22

Richardson, L J , 1970 The Archaistic Diana of Pompell AJA 74, 202

Richardson, L J ; 1977 Hercules Musarum and the Polticus Phllppi in Rome, AJA $81,35,-361$

Richter, G M A . 1960 Kouroi. Archaic Greek Youths. I,ondon

Richter, G M A; 1965 The Portraits of the Greeks, London

Richter, G M A, 1970 The Sculptwre and the Sculptors of the Greeks, London

Ridgway, B S , 1971 The Setting of Greek Sculpture, Hesperia 40, 336-356

Ridgway, B S, 1981 Greek Antecedents of Garden Sculpture, in Ancient Gardens $1981,7-28$

Rieche, A , 1978 Die Kopien der "Leda des Timotheos", AntPl 17, $21-55$

Rizzo, G E , 1929 La pittura ellenistico-romana, Roma

Rizzo, G E, 1932 Prassitele, Roma

Rizzo, G E, 1936 Le pitture della "Casa di Livia", IonPitt III, Roma 3, Roma

Rochette, $\mathrm{R}$, 1844 Choix de peintures de Pomp $\mathcal{E}_{i}$, Parıs

Rodenwaldt, G, 1909 Die Komposition der pompejanischen Wandgem ålde, Berlin

Romanell, P , 1974 Il Palatino, Roma (4 ed )

Roques de Maumont, $\mathrm{H}$ von, 1958 Antike Reiterstandbilder. Berlın

Roscher W'H Roscher, Ausführliches Lexicon der griechischen und robmischen Mythologie, Lelpzıg. 1884-1937

Rossignanı, M P , 1969 La decorazione architettonica in bronzo nel mondo romano, Contributi dell'Istıtuto Archeologico dell'Universiti Cattolica di Milano, 2, 44-98

Rostowzew, M , 1911 Die hellenistisch-romische Architehturlandschaft, RM $26,1-186$

Roullet, A, 1972 The Egyptian and Egyptianizing Monwments of Imperial Rome, Leiden

RP $S$ Reinach, $R$ épertoire des peintures grecques et romaines, Paris.

Ruesch, A. Guida illustrata del Museo Nazionale di Napoli, Vapoli

Ruesch, A, 1936 Griechische, etruskische und robmische Altert'bmer. Auktion Luzern, Luzern

Ruggiero. M, 1881 Degli scavi di Stabia dal 1749 al 1782 notizie raccolte e pubblicate, Napol,

Ruggiero, M 1885 Storia degli scavi di Ercoleno, Napolı

Salza Prina Ricottı, E, 1982 Villa adriana neı suol limitı e nella sua funzionalita, MemPontAcc III 14, 25-55

Sampson, J , 1974 Notes on Theodor Schreiber's Hellenistische Reliefbulder, PBSR 42, $27-45$

Sauron, G, 1982 Templa serena A propos de la "villa del papiri" d'Herculanum Contribution a l'etude des comportements aristocratiques romaines a la fin de la republique, MEFRA 92, 277-301

Scarpı, P, 1979 Le pyrriche o le armı della persuasione, DArch n s 1 , 78-97. 
Schafer, J., 1972 Eine reif-helenıstısche Standerlampe aus Pergamon, in Pergamon. Gesammelte Aufs atze (= PF I), 193-201

Schalter, F, 1973 St utzfiguren in der griechischen Kunst, wien

Schefold, K', 1937 Statuen auf Vasenbudern, JdI 52, 30-75

Schefold, K, 1952 Pompejanische Malerei. Sinn und Ideengeschichte, Basel

Schefold, K , 1953-1954 Pompeji unter Vespasıan, RM 60-61, 107-125

Schefold, K : 1956 Vorbilder romischer Landschaftsmalere1, AM 71, 211-231.

Schetold, K : 1957 Die Wdinde Pompejis, Berlin

Schefold, K , 1960a Lachendes Pompe11, Gymnasium 67, 90-102

Schetold, K' 1960b Herakles, Orpheus und die Vusen, in Theoria. Festschrift W.H. Schuchhardt, Baden-Baden, 209-215

Schetold, K, 1962 Vergessenes Pompeji Berlin

Schefold, K , 1964 Aphrodite von knidos, Isis und Serapis, AntK 7, 56-59

Schetold, $\mathrm{K}$, 1965 Probleme der pompelanischen Malere1, RM 72, 116-126

Schefold, K, 1972 La peinture pomplienne. Essay sur l' volution de sa signification, Bruxelles

Schetold, K, 1975 Der Lweite StL als Zeugnis alexandrunscher Architehtur. in $N \Gamma$ 197j. 53-59

Schefold, K, 1977 Caesars Epoche als goldene Zeit romischer kunst, ANRW I $4,949-953$

Schetold, K . 1980 Zur subernen Periode der Malere Pompejis, Archaiognoseia 1,91-100

Schefold, K, 1982 Romische Visionen und griechische Motive am Fuss des Vesuv, in Attı 1982, 1-40

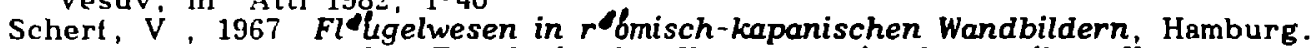

Schiffler, B, 1976 Die Typologie des Kentawren in der antiken Kunst vom 10. bis zum Ende des 4. Jhs. v.Chr.. Frankfurt a M -Bern

Schlling. $\mathrm{R}, 1982$ La r ligion romaine de $V$ Enus depuis ses origines jusqu'au temps d'Auguste Paris

Schlorb, B. 1965 Timotheos, Berlin

Schmidt, Ld 1925 Lber einige Falle der Ubertragun in Rundplastik, in Festschrift Paul Arndt, Munchen, 96-114

Schmidt, E, 1982 Geschichte der Karyatide. Funktion und Bedeutung der menschlichen Träger- und St útzfigur in der Baukunst, Wurzburg

Schmidt-Colinet, A, 1977 Antike St itzfiguren, Frankfurt aM

Schnesder-Herrmann, G, 1972 hultstatue in Tempel auf italischen Vasenbuldern BABesch 47, 31-42

Schrelber, Th, 1888 Die Wiener Brunnenreliefs aus Palazzo Grimanj, eine Studie Oaber das hellenistısche Reliefbild mit Untersuchungen úber die bildende Kunst in Alexandrien, Leipzig

Schreiber, Th, 1894 Die hellenistischen Reliefbilder, Leipzig

Schuchhardt, W - H , 1977 Alkamenes, BerlWinckPr 126

Schwarzenberg, E, 1966 Die Grazien, Bonn.

Schwingenstein, Ćhr, 1977 Die Figurenausstattung des griechischen Theatergeb gude, Munchen

Schwinzer, E, 1979 Schwebende Gruppen in der pompejanischen Wandmalerei, Wurzburg

Scullard, $\mathrm{H} \mathrm{H}, 1974$ The Elephant in the Greek and Roman World, New York-London

Sear, F B , 1977 Roman Wall and Vault Mosaics, Heidelberg

Settis, S , 1966 Chelone. Saggio sull'Afrodite Urania di Fidia, P1sa

Sgobbo, I', 1971 Le "Danzatricı" de Ercolano, RendAccNapoli 46, 51-74

Sgobbo, I , 1972 Statue di oratorı attıcı ad Ercolano dinanzı alla biblıoteca della "Villa del Papiri", RendAccNapolı 47, 241-305

Sichtermann, H , 1957 Zur Achll- und Chirongruppe RM 64, 98-110

Sichtermann, H , 1974 Gemalte Garten in pompelanischen Zummern, AW 5, no $3,41-51$

Sichtermann, H , 1984 Vythologie und Landschaft Gymnasium 91, 289-305 
Sulberberg, S R, 1982 A Corpus of the Sacro-Idyllic Landscape Paintings in Roman Art, Ann Arbor

Sogliano A Sogliano, Le pitture muralı campane scoperte negh ann annı $1867-1879$ descritte, in Pompei e la regione sotterrata dal Vesuvio Vapoli. II, $87-243$

Sogliano, A , 1893-1896 Il supphizio di Dirce in un dipinto pomperano o l Toro Farnese, Atti della Reale Accademia di Archeologia, Lettere e Belle Artı Napoli 17 no 5

Soghano, 1, 1898 La Casa des Vettu a Pompel, MonAnt 8, 233-388

Sommella, P . 1980 Forma e urbanistica di Pozzuolı romana, Puteoli 2

Spatantike 1983 D Stutzinger (ed), Spötantike und frolhes Christentun, Frankfurt aM

Spano, G . 1927 Le rappresentazionl di Artemis e Ahtaion e l'Aphrodite dl Loidalsas, Atti della Reale Accadema di Archeologia, Lettere e Belle Artí Napoli, in s 10

Spano, $G$. 1953 Alcune osservazioni nascenti da una descrizione dell'anfitéatro di Pomper, Vapolı

Spano, G, 1954-195j Paesaggio nulotico con pigmel ditendentisı magicamente del coccodrill, MemLinc $6,335-36^{\circ}$ )

Spinazzola, V, $19 j 3$ Pompei alla luce degli scavi nuovi di Via dell'Abbondanza (anni 1910 1923) Opera postuma a cura di S Aurigemma,

Roma
Stern $H, 1975$ Les mosa iques des Maisons d'Achille et de Cassiopán à Palmyre', Paris

Stewart, A' F 1977 Skopas of Paros, Park Ridge N J

Stllwell, R, 1952 Corinth, II, The Theatre Princeton $\searrow \mathrm{J}$

Strocha, V M , 1965 Die Brunnenreliefs Grimani, AntPl 4, 87-102

Strocka, V M , 1975 Pompelanische Nebenzimmer in NF 1975, 101-114

Strocha, V M; 1977 Die Wandmalereien der HERgh \&user in Ephesos, Wien

Strocka, V M , 1979 Variante, Wiedcrholungen und Serıe in der griechischen Budhauere1, JdI 94, 143-173

Strocka, V M, 1980 Case di Pompei. Un programma di documentazione archeologica, \apolı

Strocka, V M , 1984a Ein missverstandener Terminus des Vierten Stils Die Casa del Sacello lhaco in Pompej! ( 6,4$), R M$ 91, 125-140

Strocka, V M , 1984b Casa del Principe de Napoli (VI 15, 7.8), Tubingen

Stuveras, $\mathrm{R}, 1969$ Le putto dans l'art romain Bruxelles

Swindler, M H 1929 Ancient Painting from the Earliest Times to the Period of Christian Art London

Swinkels, L J F. 1984 Hoe sacraal is deze idvlle?, in Tuin 1984, 40-46

Telisidor, J , 1979 The Pantheon of Palmyra, Leiden

Thedenat, H', 1910 Pompéi. Histoire - Vie priv $e_{\text {, Paris }}$

Theophilidou, E, 1984 Die Musenmosalken der romischen haserzeit, $\operatorname{Tr} Z 47$, $239-348$

Thomas, R, 1982 Die 'schwebende Athena' in Basel Bemerkungen zur Darstellung der Athenagestalt im 5 Jahrhundert $v$ Chr , Jdl 97, 47-63

Thompson, D L . 1979 Painted Portralture at Pompen, in 'Pompeii and the Vesuvian Landscape, Washington D C , 78-86

Todisco, L , 1979 Un frammento di statua al Museo di Lecce e 1 tipı di Eracle e Melpomene con teste taurica sotto la clava, ArchCl 31, 141-157

Tortorella, S, 1981 Le lastre Campana Problem di produzıone e di sconogratia, in Art 1981, 61-100

Touchefeu-Meynier, $O, 1968$ Th Kmes odysckens dans l'art antique, Pdris

Toynbee, J M C , '1973' Animals in Roman Life and Art, Ithaca

Toynbee, J M C 'J B hard Perkins, 1956 The Shrine of Sant Peter and the Vatican Excavations, London

Tran Tam Tinh, V , 1964 Le culte d'Isis a Pompéi, Parıs 
Tran Tam Tinh, V, 1971 Le culte des divinit $\mathbf{e}_{s}$ orientales à Herculanum, Leiden

Tran Tam Tinh, V, 1972 Le culte des divinit Ls ortentales Campanie en dehors de Pomp. $i$, de Stabies et d'Herculanum, Leiden

Tran Tam Tinh, V, 1973 Isis lactans, Leiden

Tran Tam Tunh, $\dot{v} 1974$ Catalogue des peintures romaines (Latiun et Campanie) du Musci du Louvre, Parıs

Tran Tam Tinh, V, 1975 Les problemes du culte de Cybele et d'Attis a Pompe1, in NF 1975, 279-290

Traversarı G, 1982 Il gruppo di Dionıso appoggıato ad un satıro nel Museo Archeologico di Venezia $\operatorname{RdA} 6,43-46$

Travlos, J, 1971 Blldlexikon zur Topographie des antiken Athen, Tubingen

Trillmich, $W$, 1973 Bemerkungen zur Ertorschung der romschen ledealplastık JdI $88 \quad 247-282$

Trillmich, W, 1983 Die Charitengruppe als Grabielıef und kneipenschlld, JdI $98,311-349$

Tuin 1984 J A de Wdele'L J F Swinhels/E M Moormann (edd), Om de tuin geleud. Feestbundel aangeboden aan prof. dr. W.J.Th. Peters ter gelegenheld van zim 65. verjaardag, Nimegen

Tybout R A 1979 Uplontis, Hermeneus $71,263-283$

Lcell, G , 1950 Le navi di Nem, Roma

Vallois R', 1944 L'architecture hell hique et hell Cnistıque a DGlos jusqu'd l'a viction des D Cluens (166 av.J.-C.), I, Paris

Van Buren, A $W, 1932$ Further Pompelan Studies, MemAmAc 10, 9-54

Van Buren, A W' 1938 Pinacothecae, MemAmAc 15, 70-81

Van der Meer, L B, 1975 Archetype-Transmitting Model-Prototype Studies of Etruscan Urns from Volterra, I, BABesch 50, 179-193

Van der Meer, L B , 1977-1978 Etruscan Urns from Volterra Studies on Mvthological Representations, BABesch 52-53, 57-131

Van Grift, J, 1984 Tears and Revel The Allegory of the Berthouvllle Centaurs Scyphı AJA 88, 377-388

Van Wonterghem, F, 1984 Forme Italia. Regio IV I, Superaequun, Corfintum, Sulmo, Firenze

Vermaseren, $\mathrm{M} \mathrm{J}, 1966$ The Legend of Attis in Greek and Roman Art, Leiden

Vermaseren, M J , 1978 Corpus cultus Cybelae Attidisque. IV. Italia - aliae provinciae, Leiden

Vermeule, C C , 1957 Hercules Crowning Himself, JHS, 283-299

Vermeule, C C, 1977 Greek Sculptwre and Roman Taste, Ann Arbor

Vogel M , 1978 Chiron der Kentaur mit der Kithara, Bonn-Bad Godesberg

Von Blankenhagen, $\mathrm{PH}, 1963$ The Odyssey-Frieze, RM 70, 100-146

Von Blanckenhagen, $\mathrm{PH}, 1968$ Daedalus and Icarus on Pomperan Walls, $R M$ $75,106-143$

Von Blanckenhagen, $\mathrm{PH} / \mathrm{Chr}$. Alexander, 1962 The Paintings from Boscotrecase, Heidelberg

Von Cube, G, 1906 Die rómische "scaenae Frons" in den pompejanischen Wandbildern 4. Stils, Berlin

Von Grdeve, V, 1984 Marmorbulder aus Herculaneum und Pompejı, DArch terza serie 2, $89-113$

Von Hers-Landwehr, Chr , 1982 Griechische Metsterwerke in rómischen Abgliss en. Der Fund von Baia. Zur Technik antiker Kopisten. Frankturt a M

Von Hesberg, H, 1980a Konsolengeisa des Hellenismus und der frolanen Kaiserzeit, Vainz

Von Hesberg, H. 1980b Eıne Marmorbasıs im Vatikan, RM 87, 255-282

Von Hesberg, H , 1981 Archaologische Denhmaler zu den romischen Gottergestalten, in ANRW II $172,1032-1199$ 
Von Hesberg, H , 1983 Zur Datierung der Gefangenenfassade in Korinth Eine wederverwendete Architektur romischer Zeit, AM 98, 215-238

Von Petrikovits, H, 1975 Die Innenbauten robmischer Legionslager wấhrend der Prinzipatszeit, Opladen

Von Rohden, H. 1880 Die antiken Terracotten, I. Die Terracotten von Pompeji, Stuttgart-Berlin

Von Rohden, H , 1887 Zum Hermes von Praxiteles, JdI 2 66-68

Von Salıs, A: 1917 Antike und Renaissance. OJber Nachleben und Weiterwirken der Alten in der neuen Kunst. Erlenbach-Zurıch

Von Steuben, H , 1971 Der Kanon des Polyklet, Tubingen

walbanh, F W 1967 A Historical Commentary on Polybius, II, Oxtord

walde, E, 1978 Die Aulstellung des aufgestutzten Poseidon, AM 93, 99-108

walde-Psenner E , 1979 Der bronzene Poseidon aul der Hafenmolc von

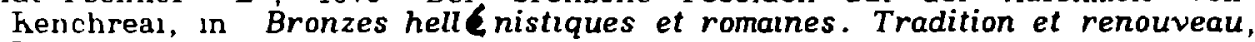
Lausanne, 61-64

Walter, H , 1970 La colonne cisel $e_{e}$ dans la Gaule romaine, Parıs

weege, $F, 1913$ Das Goldene Haus des Nero, JdI 28, 127-244

Wegner, M , 1966 Die Musensarkophagen, Berlın

Wesenberg, B , 1984 lugustusforum und Akropolis, JdI 99, 161-185

Whitehouse, H', 1977 In praedus Juliae Felicis the Provenance of Some Iragments of Wall-Painting in the Museo Nazionale, Vaples, PBSR 40, $52-68$

Willemsen, F, 1956 Ahtaionbulder, JdI 71, 29-58

Winkes, R , 1969 Clipeata imago. Studien $z u$ einer $r$ dómischen Bildnisform, Bonn

Wirth, F $1934 R^{\circ}$ mische Wandmalerei vom Untergang Pompejis bis aufs Ende des dritten Jahrhunderts, Berlin

Wolke, H, 1978 Untersuchungen zur Batrachomyomachie, Meisenhem am Glan

Wolters, P . 1928 Der Knabe von Xanten, Forschungen und Fortschritte 4, $281-282$

Wrede, H, 1972 Die sp dtantike Hermengalerie von Welschbillig. Untersuchungen zur Kunsttradition im 4. Jahrhundert n.Chr. und zur allgemeinen Bedeutung des antiken Hermenmals. Berlin

wunderlich E, 1975 Die Bedeutung der roten Farbe im Kultus der Griechen und $R^{\circ}$ ómer. Giessen

hurster, W W, 1977 Stadtdarstellungen auf lykıschen Reliefs, Architectura, $117-151$

Yadin, Y, 1966 Massada. Herods Fortress and the Zealots Last Stand, London $1824-1852$ Die sch \&nsten Ornamente und merkw ofurdigsten Gem falde aus Pompeji. Herculaneum und Stabiae, Berlin

Zanker, P, 1965 Lwel Ahroterfiguren aus Tvndarıs, RM 72, 93-99

Zanker, 'P', 1972 Forum Romanum. Die Neugestaltung durch Augustus, Tubingen

Lanker P, 1974 Klassizistjsche Statuen. Studien zur Veroanderung des Kunstgeschmacks in der $r$ omischen Kalserzeit, Maınz

Zanker, P, 1979a Die Villa als Vorbild spaten pompejanischen Wohngeschmacks, JdI 94, 460-523

Zanker, P., 1979b Lur Funktion und Bedeutung griechischer Shulpturen in der Romerzeit, in Classicisme 1979, 283-314.

Zanker, P. 1983 Der 4pollotempel auf dem Palatın Ausstattung und politische Sinnbezuze nach der Schlacht von Actium, in Citt $\pm a$ architettura nella Roma imperiale, $X$ Suppl AnalRom, 21-40

Zanher, P. s d Forum Augustum. Das Bildprogramm, Tubingen

Zevi, F, 1964 La Casa Reg IX 5, 18-21 a Pompel e le sue pitture, Studi Miscellanei 5

Zummer, G . 1982 Rdómische Berufsdarstellungen, Berlın 

Eric M Moormann e nato nel 1955 Dopo ll ginnasio nel Boschveldcollege a Venray ha studiato lettere classiche nel'Lniversita cattolica di Nimega, dove sl e laureato nel 1980 con ll prof dr h J Th Peters Materia primaria era l'archeologia classica, materie secondarie erano lettere greche e staliane Ha Insegnato letterc classiche 1980-1981 e ha lavorato come assistente 1980-1981. 1983-1985 nell'L nuersita di Numega 1981-1983 soggiornava a Napoli e Roma graze a borse di studio della Rotarv Foundation e della Organizzazione Olandese delle Rscerche (ZWO) 

Stellingen behorende bij het proefschrift van Eric M. Moormann

I

De term "egittomania" die door Mariette de Vos gebruikt wordt ter aanduiding van het gebruik van Egyptische motieven in de beeldende kunst van de vroege keizertijd, is tendentieus en derhalve onjuist.

Contra: M. de Vos, L'egittomania in pitture e mosaici romano-campani (Leiden 1980).

\section{I}

De gele, rode en zwarte vlakken waartegen zich tuinvoorstellingen op Romeins muurschilderingen uit de keizertijd aftekenen, geven de illusie van verder terug liggende tuinmuren, waarvoor borders zijn aangelegd.

III

De landschapsvoorstellingen in het vestibulum van de Casa Sannitica te Herculaneum moeten worden gedateerd in de periode van de eerste stijl.

Contra: W.J.Th. Peters, Landscape in Romano-Campanian Mural Painting (Assen 1963) 60, 166; A. Barbet, La peinture romaine (Paris 1985) 80; cf. A. Laidlaw, The First Style in Pompeii (Roma 1985) 304.

IV

In een seismisch kritische zone als Campanië moet rekening worden gehouden met aardschokken die niet in de schriftelijke bronnen vermeld zijn. Dientengevolge dient men het voor de Pompejanisten zo belangrijke jaartal $62 \mathrm{n}$.Chr. mer de nodige voorzichtigheid te gebruiken.

De combinatie van twee episodes uit het leven van Achilleus in een voorstelling, namelijk het verblijf bij de Kentaur Cheiron en de ontdekking in het paleis van Lykomedes op Skyros, is niet onlogisch.

Cf. K. Dunbabin, The Mosaics of Roman North Africa (Oxford 1978) 41.

VI

De gevleugelde Amor op het stuk barnsteen uit het grafveld van Ulpia Noviomagus (Nijmegen) heeft als enige attribuut een guirlande.

Contra: J.E. Bogaers/J.K. Haalebos, Ontdekkingen in het grafveld van Ulpia Noviomagus, Numaga 31 (1984) 4; J.E. Bogaers/J.K. Haalebos, Spiegel Historiael 20 (1985) 131.

VII

Bij de bestudering van Romeinse kopieën van Griekse kunstwerken dient steeds het adagium "imitatio et aemulatio" in gedachte te worden gehouden. 


\section{VIII}

Leveau houdt bij zijn interpretatie van de vorm van het amphitheater uit augusteïsche tijd van Iol-Caesarea (Cherchell, Algerije) ten onrechte geen rekening met het amphitheater van Pompeii.

Contra: Ph. Leveau, Caesarea de Mauretanie: une ville romaine et ses campagnes (Rome 1984) 38 .

IX

Catullus, Carmen 4, beschrijft een schilderij waarop een schip te zien is, niet de tocht van een schip.

Contra: L.J. Fordyce, Catullus. A commentary (Oxford 1973) 96-106.

$X$

De commentaren van Jebb en Kamerbeek op Sophokles, Antigone 456, zijn voorbeelden van het onnodig leegschudden van een kaartenbak dat regelmatig in wetenschappelijke werken voorkomt.

Contra: R.C. Jebb, Sophocles, Part III (Cambridge 1900) 90; J.C. Kamerbeek, The Plays of Sophocles, III (Leiden 1978) 97.

$X I$

De huidige indeling van het studiejaar maakt veldonderzoek voor archeologen in de mediterrane wereld vrijwel onmogelijk. Een indeling van het academisch jaar in twee semesters en twee periodes zonder colleges en tentamens is gunstiger.

\section{XII}

In handboeken en populair-wetenschappelijke werken wordt van een kunstwerk veelal alleen een detail afgebeeld. Dit is volstrekt af te keuren.

\section{XIII}

De persoonlijkheid van Pier Paolo Pasolini kan niet alleen op grond van zijn films worden beoordeeid. Het ontbreken van vertalingen van zijn poetisch en essayistisch werk in Nederland heeft tot een verkeerd beeld van deze gecompliceerde kunstenaar geleid.

\section{XIV}

De verplichting de stellingen aan de hoogleraren van de betreffende vakgebieden voor te leggen alvorens ze aan het proefschrift worden toegevoegd, doet afbreuk aan de spanning bij aanval en verdediging. 

